

\section{DISCLAIMER}

This report was prepared as an account of work sponsored by an agency of the United States Government. Neither the United States Government nor any agency Thereof, nor any of their employees, makes any warranty, express or implied, or assumes any legal liability or responsibility for the accuracy, completeness, or usefulness of any information, apparatus, product, or process disclosed, or represents that its use would not infringe privately owned rights. Reference herein to any specific commercial product, process, or service by trade name, trademark, manufacturer, or otherwise does not necessarily constitute or imply its endorsement, recommendation, or favoring by the United States Government or any agency thereof. The views and opinions of authors expressed herein do not necessarily state or reflect those of the United States Government or any agency thereof. 


\section{DISCLAIMER}

Portions of this document may be illegible in electronic image products. Images are produced from the best available original document. 


\section{DO NOT MICROFILM
COVER}

This work was supported by the US Department of Energy, Office of Safeguards and Security, and by the Program for Technical Assistance to IAEA Safeguards. Government. Neither the United States nor the United States Department of Energy, nor any of their employees, nor any of their contractors, subcontractors, or their employees, make any warranty, express or implied, or assume any legal liability or responsibility for the accuracy, completeness or usefulness of any information, apparatus, product, or process disclosed, or represent that its use would not infringe privately owned rights. Further, neither the subject matter nor the content of this report reflects any policy, expressed or implied, by the United States Government. 


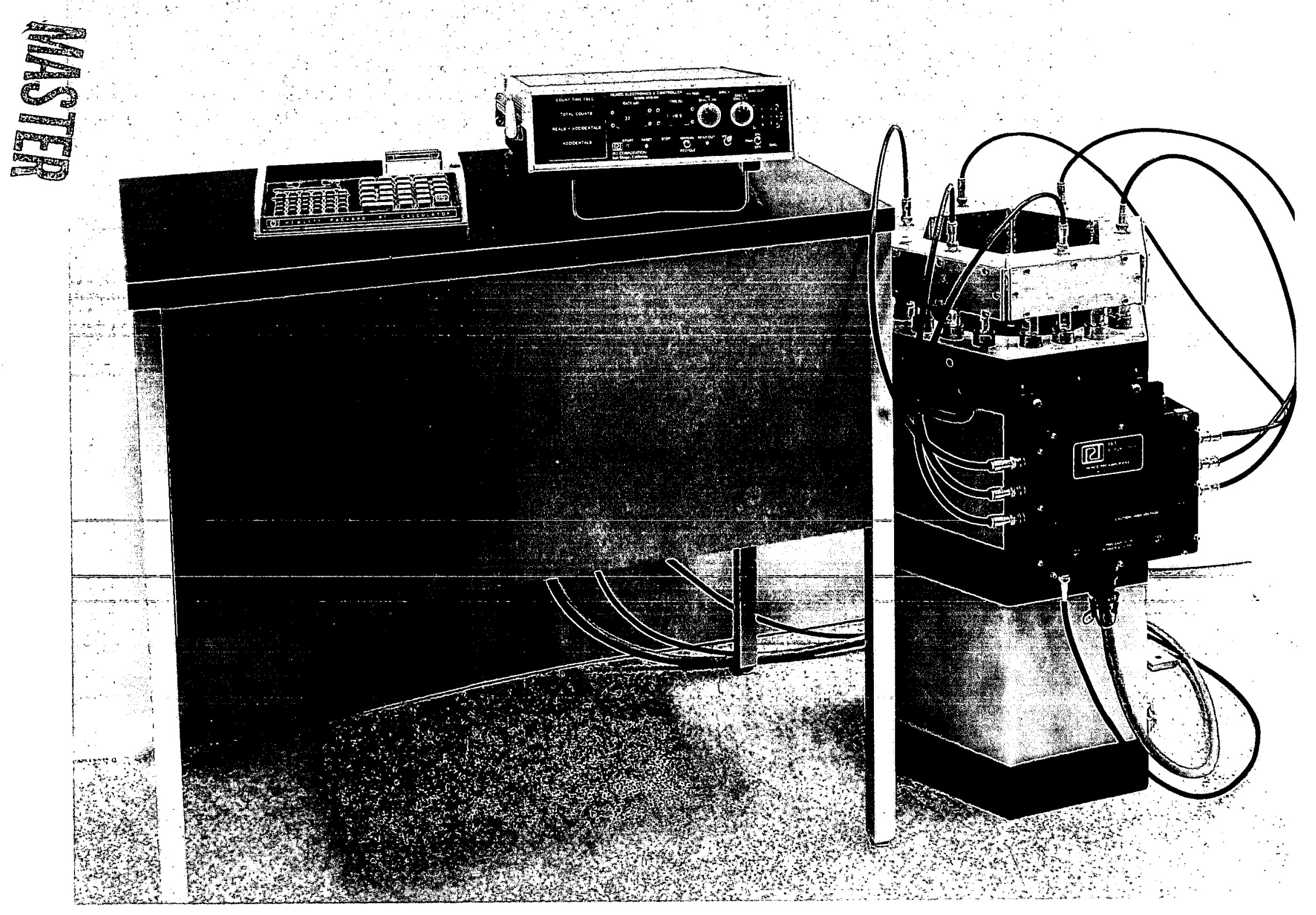

HLNCC assembled for measurement. 



\title{
High-Level Neutron Coincidence Counter Maintenance Manual
}

\author{
J. Swansen \\ P. Collinsworth
}

\section{DISCLAIMER}

This report was prepared as an account of work sponsored by an agency of the United States Government. Neither the United States Government nor any agency thereof, nor any of their employees, makes any warranty, express or implied, or assumes any legal liability or responsibility for the accuracy, completeness, or usefulness of any information, apparatus, product, or process disclosed, or represents that its use would not infringe privately owned rights. Reference herein to any specific commercial product, process, or service by trade name, trademark, manufacturer, or otherwise does not necessarily constitute or imply its endorsement, recommendation, or favoring by the United States Government or any agency thereof. The views and opinions of authors expressed herein do not necessarily state or reflect those of the United States Government or any agency thereof.

This document is

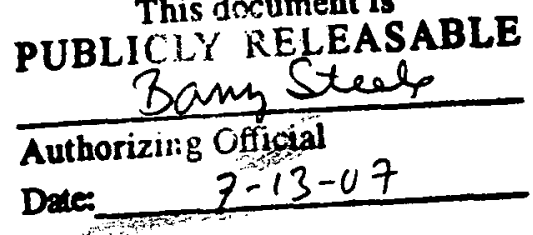

NOTICE

PORTIONS OF. THIS REPORT ARE ILLEGIBLE. It has been reprouluced from the best available copy to permit the broadest possible availability. 


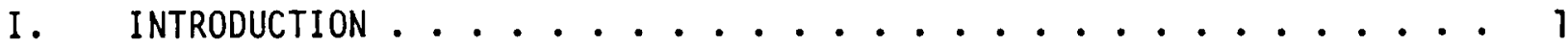

II. VISUAL AND MECHANICAL INSPECTION ............. 2

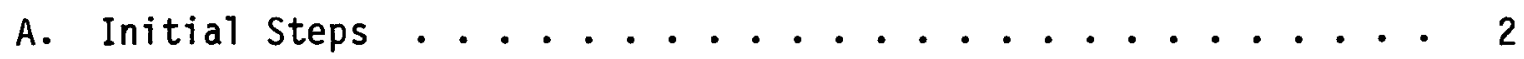

B. $\mathrm{HEC}-100 \ldots \ldots \ldots \ldots \ldots \ldots \ldots$

C. HP-100 Preamplifier Box .............. 6

D. Accessory Cables .................... 7

III. DIGITAL SECTION CHECKS ............. 7

A. Initial Setup and Power-On Check .......... 8

B. Input Synchronizer Check ............. 11

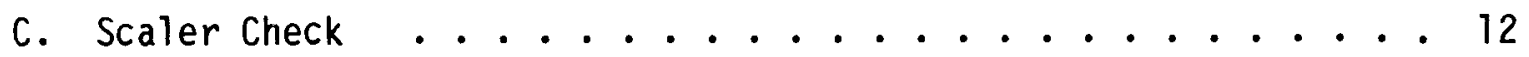

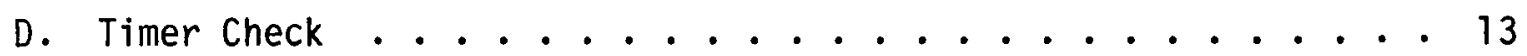

E. Gate and Adder Check ............... 13

F. Predelay Check ...................... 15

G. Accidental Delay Check .............. 19

IV. ANALOG SECTION CHECK-OUT AND CALIBRATION .......... 21

A. Setup and Mechanical Adjustment .......... 22

B. Noise Check ................. 22

C. Discriminator Calibration ............. 22

D. High-Voltage Power Supply Calibration ........... 24

Procedure 1: High-Voltage and Amplifier Calibration with Neutron Source ........... 24

Procedure 2: High-Voltage Calibration without lieutron Source ............ 27

Procedure 3: Amplifier Calibration wi thout Source . . . . 27

E. One-Shot and OR Gate Check ............. 28

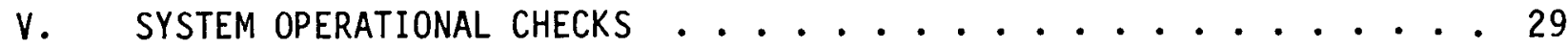

A. Efficiency Check ................. 29

B. Bias Check ..................... 30 
C. Electromagnetic Interference (EMI) Check . . . . . . . . 30

REFERENCES . . . . . . . . . . . . . . . . . 30

APPENDIX A: RP501 GATE AND ADDER TEST PROGRAM . . . . . . . . . . . . 33

APPENDIX B: NEW 1-K PROM FOR COINCIDENCE COUNTER ELECTRONICS PACKAGE • • 39

APPENDIX C: HLNCC(PROM) VERSION 1.2 ; ORIGINAL PROM LISTING. . . • . • 85

APPENDIX D: NONSTANDARD LSI AND HYBRID COMPONENTS DATA. . . . . • • • 98

APPENDIX E: SYSTEM-TIMING ILLUSTRATIONS . . . . . . . . . . . . 163

APPENDIX F: HLNCC SCHEMATIC SET . . . . . . . . . . . . . . 169 


\title{
HIGH-LEVEL NEUTRON COINCIDENCE COUNTER \\ MAINTENANCE MANUAL
}

by

J. Swansen and P. Collinsworth

\begin{abstract}
High-level neutron coincidence counter operational (field) calibration and usage is well known. This manual makes explicit basic (shop) check-out, calibration, and testing of new units and is a guide for repair of failed in-service units. Operational criteria for the major electronic functions are detailed, as are adjustments and calibration procedures, and recurrent mechanical/electromechanical problems are addressed. Some system tests are included for quality assurance. Data on nonstandard large-scale integrated (circuit) components and a schematic set are also included.
\end{abstract}

\section{INTRODUCTION}

During the past few years several reports and manuals ${ }^{1-6}$ have been written for the High-Level Neutron Coincidence Counter (HLNCC) electronics package. Much of this information is duplicative. These reports give adequate information on what is in the HLNCC shift-register electronics and how to use it for normal applications, but they do not address the problem of checking out a new unit or repairing a failed unit.

The present manual gives maintenance and check-out procedures for a new or failed HEC-100 electronics unit. We assume that the basic information in Refs. 1, 3, and 5 is available to the user. This report is addressed to the electronics maintenance personnel rather than the normal HLNCC user, and it is 
assumed that the reader is familiar with the instrument; for example, attendees of a Los Alamos HLNCC maintenance school would usually have the requisite familiarity with the instrument.

This manual primarily deals with checking out a HLNCC electronics package (HEC-100 counter/controller, HP-100 high-voltage junction and preamplifier box, and accessory items) for proper operation. Secondarily, the information in the manual is arranged to assist maintenance personnel in isolating problems to major subcircuits. Illustrations of component locations are included as are illustrations of selected waveforms; a schematic package is included to aid in detailed troubleshooting.

\section{VISUAL AND MECHANICAL INSPECTION}

A visual and electromechanical inspection of incoming HLNCC systems is of critical importance. Our experience has shown that operational problems can be reduced, or avoided altogether, by simple inspection and remedial action. Among the problems, in approximate order of importance, are

- improper shielding and discontinuous or high-impedance grounding, particularly in the preamplifier box, but also at other locations in the analog section of the complete HLNCC system;

- noise because of failure to maintain cleanliness in the high-voltage and high-impedance signal section of the preamplifier box;

- loose, missing, or improperly installed components, circuit boards, connectors, and wiring harnesses;

- poor solder connections (that is, cold solder joints);

- improper component values; and

- faulty cable assemblies.

All six problem areas have been seen both in newly manufactured units and in field (in-service) instruments.

\section{A. Initial Steps}

1. Unpack and check contents of shipping containers (Fig. 1).

2. Rotate and shake HEC-100 to check for loose components.

3. Rotate and shake HP-100 (preamplifier box). 


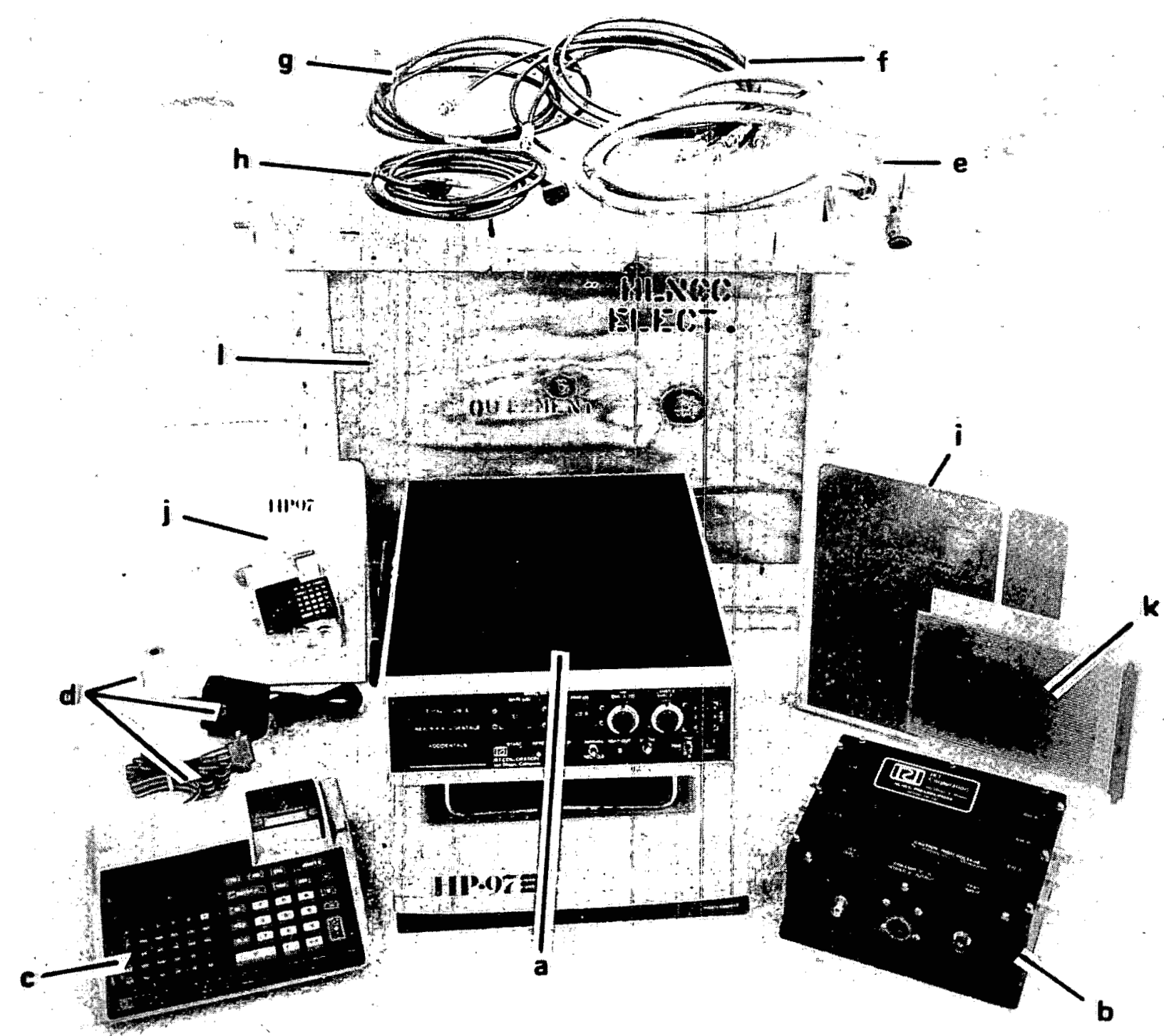

Fig. 1 .

Major HLNCC components and accessories as received, from manufacturer.

a. HEC-100 counter/controller

b. HP-100 high-voltage junction and preamplifier box

c. HP-97 calculator (modified)

d. HP-97 accessories (attached interface cable, recharger, paper tape)

e. HEC-100/HP-100 interconnecting cable

f. HP-100/HEXAPUS (detector) high-voltage cables

g. HEC100/HP-100 high-voltage supply cable

h. ac power cord

i. Manufacturer's HLNCC manual

j. HP-97 User's Manual

$k$. Extender board

1. Shipping crate 
B. HEC-100

1. Remove top cover from HEC-100. Release clamp at rear of board assembly.

2. Rotate and lock board assembly in vertical position (Fig. 2).

3. Remove boards. Boards (from front to back) are amplifier board, shift-register board, and microprocessor board (Fig. 2).

4. Inspect boards for cleanliness, good solder connections, and missing components.

5. Check the following shift-register board components (Fig. 3):

Component

C5

$\mathrm{C} 22$ $\frac{\text { Should Be }}{68-120 \mathrm{pF}}$

$220 \mathrm{pF}$

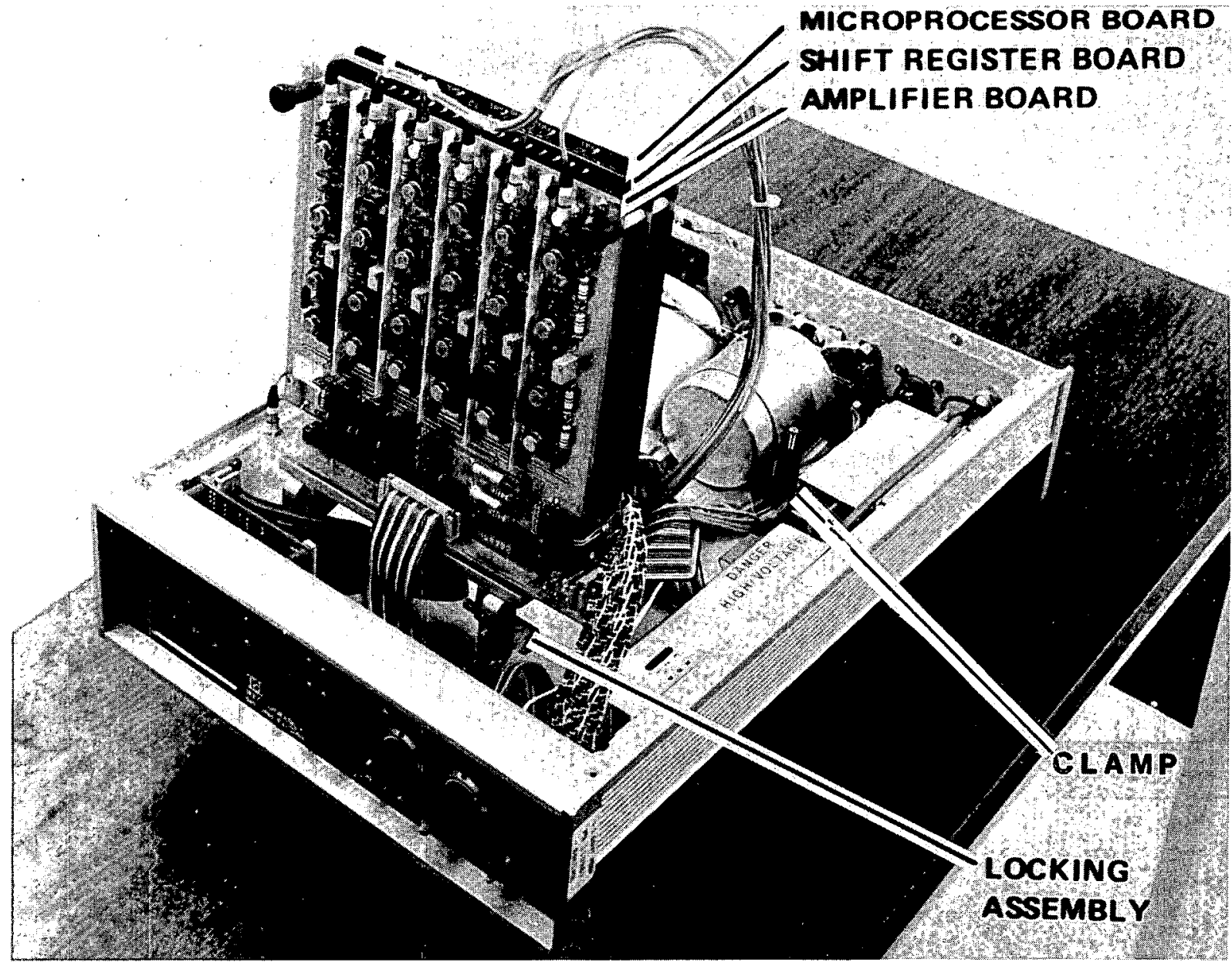

Fig. 2.

HLNCC with boards in upright locked position. 


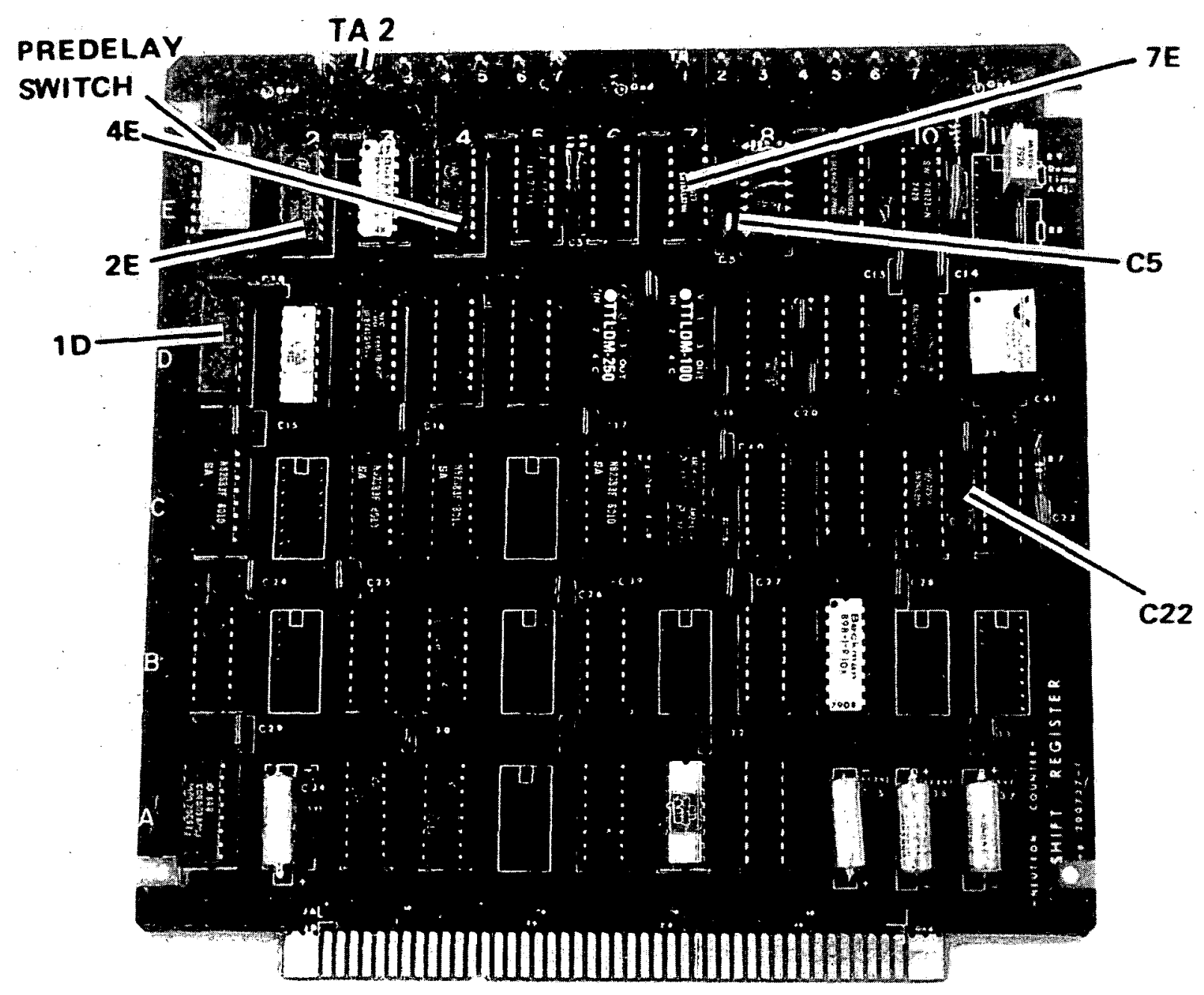

Fi.j. 3.

Shift-register board.

$\begin{array}{ll}\text { 7E (chip location) } & \text { Texas Instrument } 74 L S 74 \\ 4 E \text { (chip location) } & \text { Motorola MC14557 AL or BAL (not CP) } \\ 2 \mathrm{E}, 10 \text { (chip location) } & \text { Motorola MC14517 AL or BAL (not CP) }\end{array}$

6. Check microprocessor clock (microprocessor board location 5E) frequency label; clock frequency must be $921.6 \mathrm{kHz}$.

7. Check the chassis interior for loose or inissing screws and components.

8. Check the front and rear panels for loose or missing controls and conponents.

9. Reinstall the amplifier, shift-register, and microprocessor boards. NOTE: ise caution in inserting boards because socket pins are easily bent. 
10. Check continuity from front pane1 ground test point to rear panel "AMP INPUT" (J15) pins $G$ and $F$.

C. HP-100 Preamplifier Box

CAUTION: High voltage may be present inside the box when disconnected from high-voltage supply; there is no high-voltage bleeder resistor in the box. If it is necessary to connect and operate the box with the cover removed, caution must be taken.

1. Remove top cover from HP-100 (preamplifier box) (Fig. 4).

2. Short the high-voltage input connector (SHV) on the HP-100 preamplifier box to ground while working in the box interior (see preceding cautionary note).

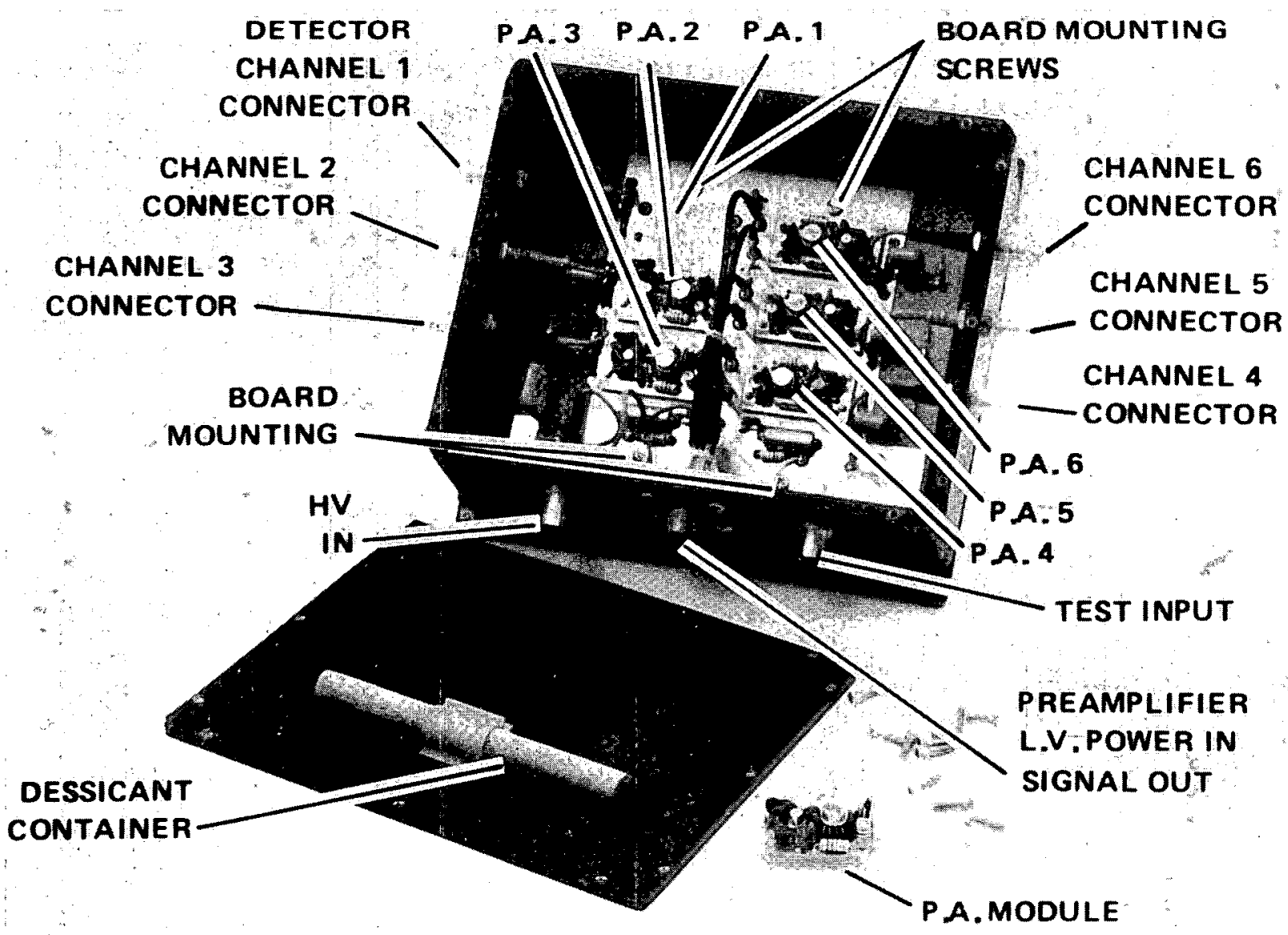

Fig. 4.

High voltage junction and preamplifier box, HP-100. 
3. Check for (solder flux) residue on preamplifiers and main board; boards must be clean.

4. Check main board mounting screws and box connectors (Fig. 4) for looseness; check to see that preamplifiers are seated firmly.

5. Check for continuity from main board ground plane to connector shells.

6. Check desiccant for appropriate color (deep blue) and replace/reactivate, if necessary.

7. Reinstall plastic foam keeper(s) and box cover.

\section{Accessory Cables}

1. Check the power cord for electrical and mechanical integrity.

2. Check grey (multiconductor) cable assembly strain reliefs and ground lugs.

3. Check high-voltage cables for proper connector assembly (SHV) and cable type.

$\begin{array}{ll}\frac{\text { Cable }}{\text { High-voltage supply cable }} & \frac{\text { Type }}{\text { RG59 or RG71 }} \\ \text { High-voltage detector cable } & \text { RG71 }\end{array}$

III. DIGITAL SECTION CHECKS

There are seven procedures to test the digital section of the HEC-100. The following are the major features tested:

- power supplies, HP-97, data transmission, and display;

- input synchronizer and TOTALS (T) scaler;

- reals + accidentals $(R+A)$ and accidentals (A) gate functions and scaling;

- scaler display;

- timer;

- predelay function;

- accidental gate delay shift register.

The coincidence circuitry test relies heavily on the use of a random pulse generator and correctly interpreting the resulting data. Periodic sources, while useful, are inadequate for testing the $R+A$ and $A$-coincidence gates. Data 
generated during the test should be recorded and logged for future reference; a brief HP-97 program is provided in Sec. III. A for recording HEC-100 display data. A second, more comprehensive test program referred to in Sec. III.E is found in Appendix $A$, page 33 .

The following test equipment is used in the digital section checks (Fig. 5).

Tektronix TM506 (mainframe)

Tektronix SC504 oscilloscope (module)

Tektronix PG508 pulse generator (module)

Tektronix DC503A counter (module)

Tektronix DM502A DVMi (module)

Los A1amos/Q-1 RP501.

A. Initial Setup and Power-On Check

This procedure provides initial tests of the power supplies, HEC-100 display, and microprocessor board. Two versions of resident (microprocessor) software are available for the HEC,-100 (PROM chip 10cation E2, microprocessor board). At power-on or microprocessor RESET, the software will transmit a test

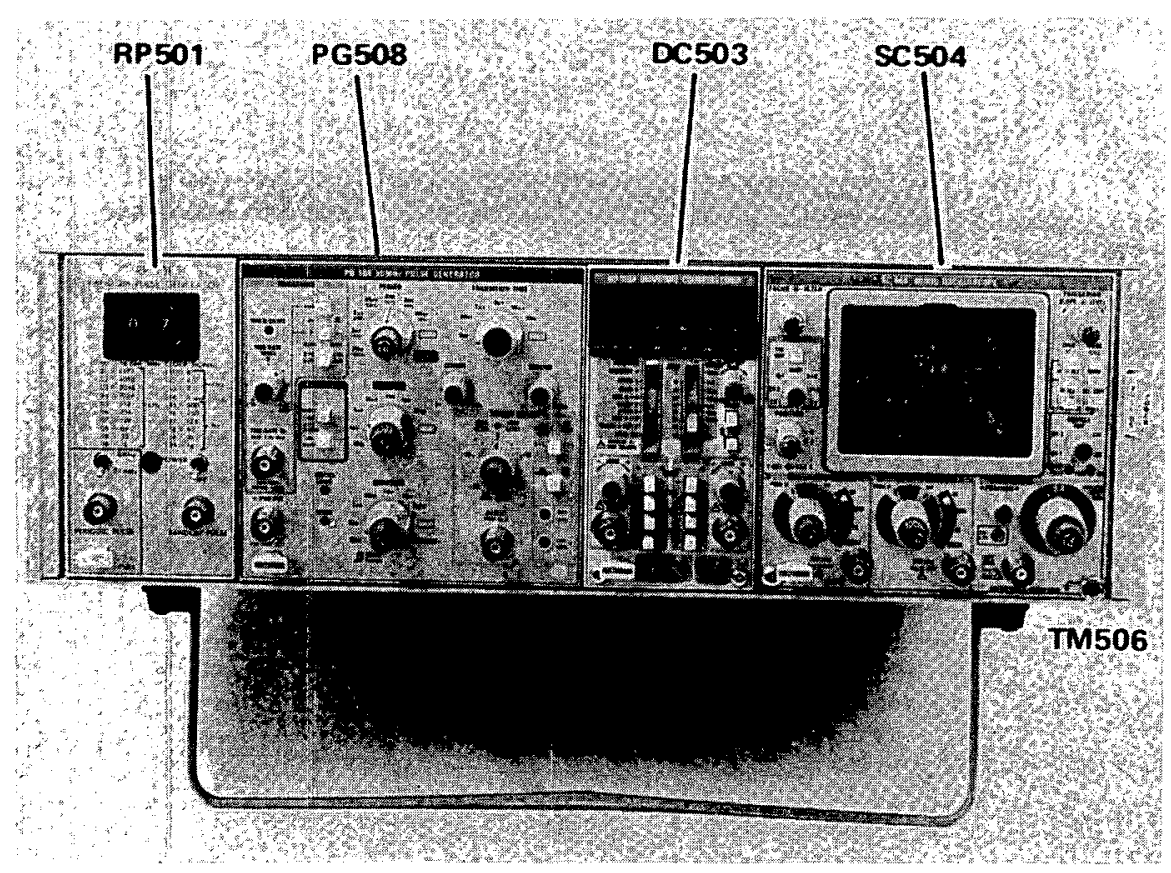

Fig. 5 .

Digital section test set. 
message to the HP-97; the message consists of simulated scaler data (TIME, TOTALS, $R+A$, and $A$ ), which are loaded in HP-97 registers 1 through 4 , followed by an "A" command that will start an HP-97 program at "LBLA." A test message message occurs on the RS232 port at power-on/microprocessor RESET, if the original software is resident. Below is a table of test messages.

$\begin{array}{lcl} & \text { HP-97 MESSAGE } & \text { RS-232 MESSAGE } \\ \text { VERSION } 1.2 & 444444 & \text { HLNCC } \\ \text { (original version) } & 3333333333 & \text { V } 1.2 \\ & 2222222222 & 12 \mathrm{JUL} 78 \\ & 111111111 & \\ & 1111111111 & \end{array}$

VERSION 2.1

543210

(NONE)

(remote control

9876543210

version )

9876543210

9876543210

0

The following are the RS-232 port requirements for a remote receiving device:

$\begin{array}{ll}\text { DATA RATE } & 300 \text { BAUD } \\ \text { BITS/CHARACTER } & 7 \\ \text { STOP BIT } & 1 \\ \text { PARITY } & \text { EVEN } \\ \text { MODE } & \text { FULL DUPLEX for V 2.1; HALF DUPLEX (receive oniy) for } \\ & V 1.2\end{array}$

1. HEC-100 initial conditions (Fig. 6). LINE VOLTAGE SWITCH (REAR PANEL) 110 or 220 (Vac) POWER (FRONT PANEL) OFF

HV (FRONT PANEL) OFF

POWER CORD UNPLUGGED

2. Measure continuity from power-cord ground to the HEC-100 chassis before connecting to ac power. 


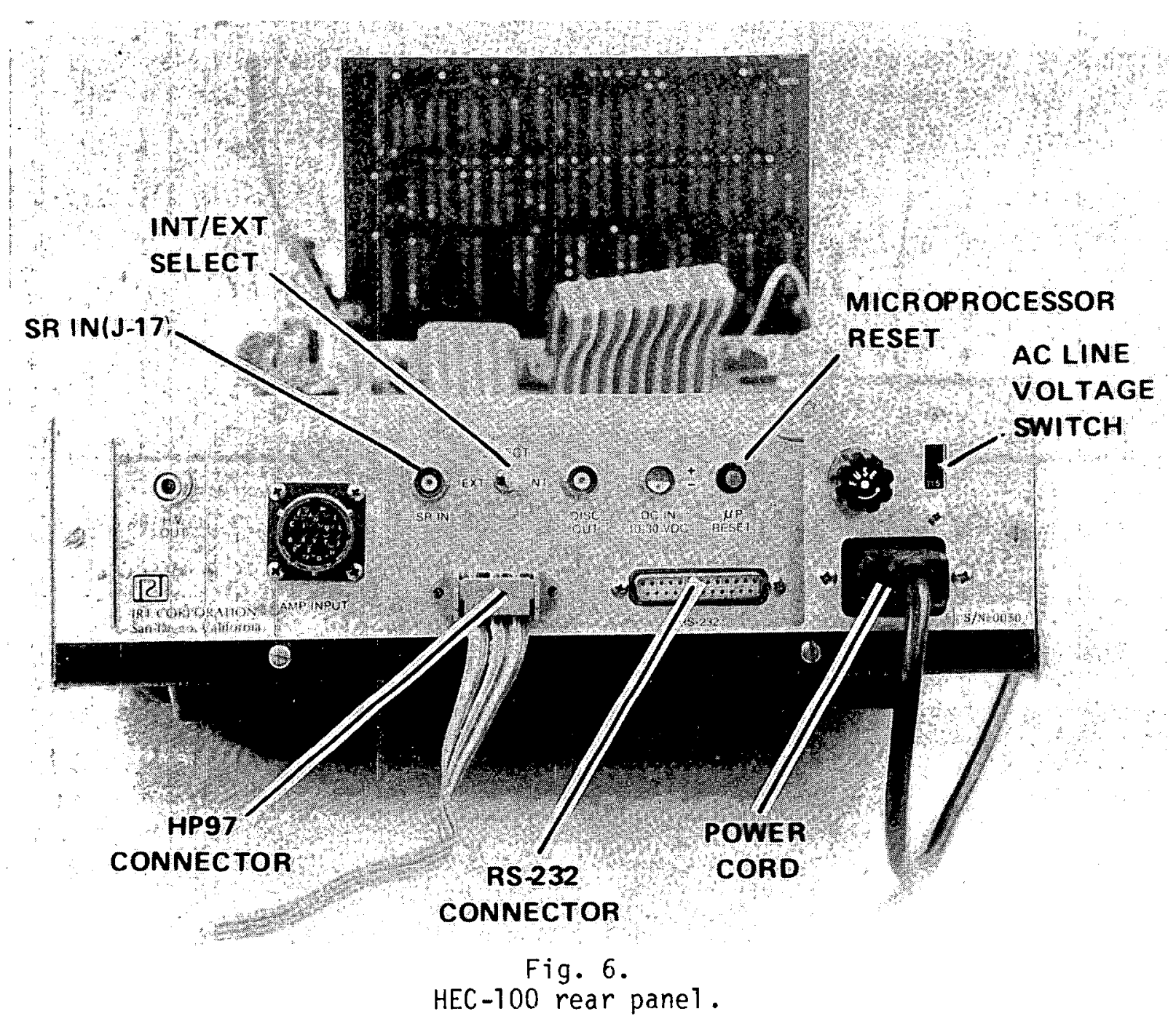

3. Connect HP-97 to HEC-100 (HP-97, J-14, rear panel).

4. Connect HP-97 to charger.

NOTE: Do not run HP-97 without charger when it is connected to HEC-100.

5. HP-97 initial conditions.

POWER

$O N$

RUN/PROGRAM

PRGII

MAN/TRACE

MAN

6. Enter the following program into the HP-97:

LBL A

FIX

DSP 0 
RCL 1

PRINT $X$

$\mathrm{RCL} 2$

PRINT $X$

RCL 3

PRINT $X$

$\mathrm{RCL} 4$

PRINT $X$

RCL 3

RCL 4

(Subtraction)

PRINT $X$

f SPACE

7. Switch HP-97 from PRGM to RUN.

8. Turn on HEC-100 power. Observe that during test transmission the HEC -100 readout 1 ight is on, that the HP-97 prints a valid test message, and that the HEC-100 display shows zeros in the two least significant digit positions.

9. Measure between front panel controls and screws and ground-test point for extraneous power supply voltages $( \pm 15 \mathrm{~V}, \pm 12 \mathrm{~V}, 5 \mathrm{~V})$; presence of voltage indicates wiring error.

10. If a low-voltage dc power supply failure appears to have occurred, the voltages may be measured on the "mother board" (as indicated on Fig. 7).

\section{B. Input Synchronizer Check}

This check tests synchronizer circuit performance. This circuit synchronizes input pulses to the HEC-100 clock. C5 is selected to minimize synchronizer deadtime and prevent loading of an input pulse into adjacent clock cycles. The check verifies that $\mathrm{C} 5$ is greater than the minimum value necessary to prevent double pulsing and also tests TOTALS scaling and timer operation.

1. HEC - 100 setup.

$\begin{array}{lll}\text { TIME (s) } & 1 \times 10^{1} & \text { (front pane1) } \\ \text { MANUUAL/RECYCLE } & \text { MANUAL } & \text { (front pane1) } \\ \text { SELECT } & \text { EXT. } & \text { (rear pane1, SW 10) }\end{array}$




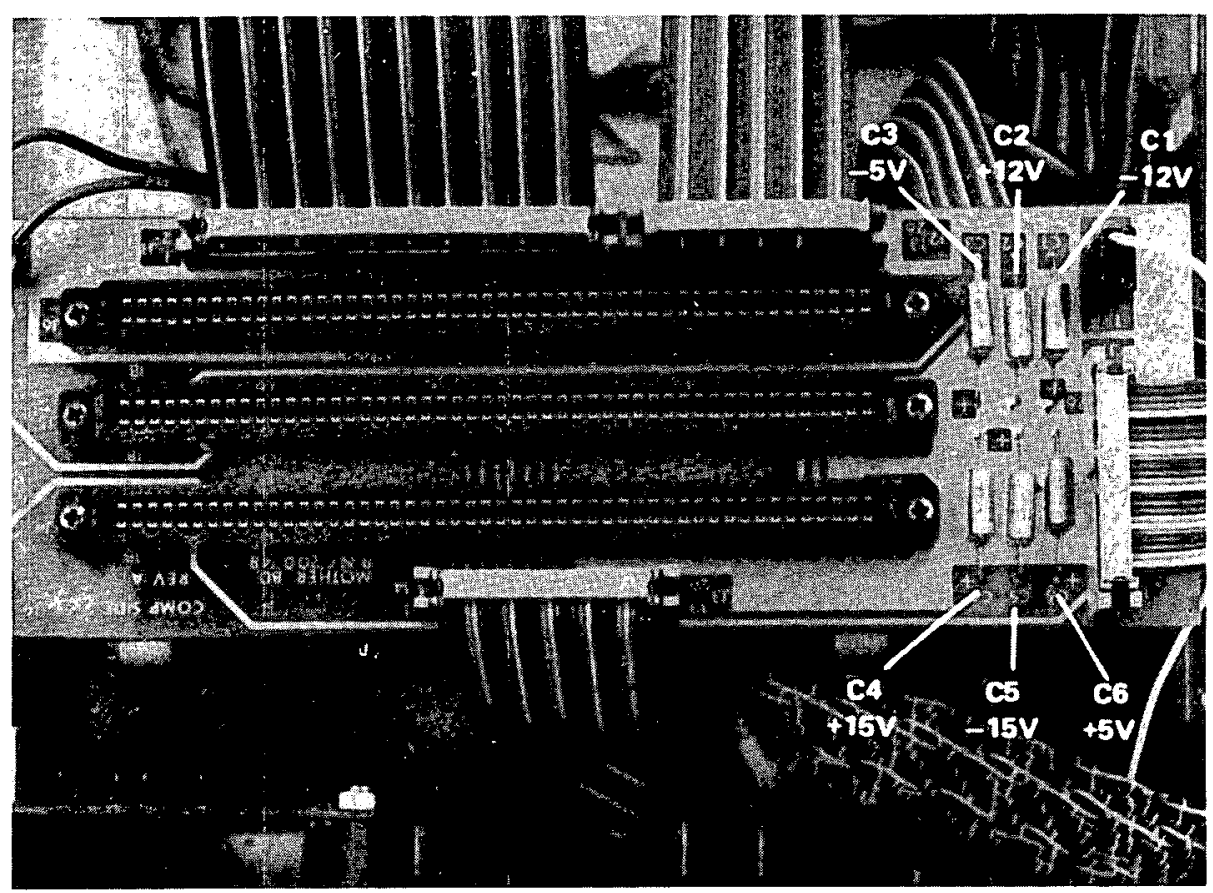

Fig. 7 .

HEC-100 mother board: 10w-voltage dc power supply test points.

2. Los A1 amos/Q-1 RP501 module setup. PERIODIC PULSE SW. $\quad 0.1 \mathrm{MHZ}$

3. Connect RP501 "PERIODIC PULSE" BNC to HEC-100 "SR IN" (J-17)(Fig. 6.

4. Start the HEC-100 (depress RESET push button, then START push button).

5. Observations at completion of run.

$\begin{array}{lcl}\text { SCALER } & \text { DISPLAYED VALUE } & \text { COMMENTS } \\ \text { TOTALS } & 1000000 \text { (counts) } & \pm 100 \text { counts } \\ \text { COUNT TIME } & 10 \text { (seconds) } & --\end{array}$

C. Scaler Check

This check tests that the nine digits of the TOTALS, $R+A$, and A scalers count and display. Reconnect "SR IN" (J-17) to the RP501 "RANDOM PULSE" output. Switch the Los Alamos pulser to 00 and start the HEC-100. Count for a sufficient period of time to observe count accumulation in all digits of the three scalers. 
D. Timer Check

This check tests that the HEC-100 TIME switches properly control tiner operation.

1. Set the HEC 100 TIME to $0 \times 10^{\circ}$ and press the START pusin button. The HEC-100 should immediately switch to STOP and the displayed time should remain at 0 .

2. Set the HEC-100 TIME to $1 \times 10^{\circ}$.

3. Start the HEC-100 and observe that it stops at and displays the set time.

4. Repeat for $2 \times 10^{\circ}$ through $9 \times 10^{\circ}$.

5. Repeat for $1 \times 10^{1}, 1 \times 10^{2}$, and $1 \times 10^{3}$. (Check $10^{4}$ and $10^{5}$ setting if time allows).

\section{E. Gate and Adder Check}

This check tests the HEC-100 coincidence circuit consisting of the shiftregister gate, gate length selector, gate up/down counter, $R+A$ and $A$ gate adders and latches, and associated logic. It also provides testing of $R+A$ and $A$ scaling and additional testing of the TOTALS scaler, timer, synchronizer, and HP-97 operation.

HEC-100 coincidence data are ultimately reduced to the difference between the $R+A$ and $A$ scalers. When the instrument is checked with a random (nonfissioning) source, the difference should be zero $\pm \sqrt{2 A}(1 \sigma)$ and any consistent offset is bias. For a random source, bias is defined as $\{[(R+A)-A] / A\} \times 100$, a percentage quantity that may be positive or negative. A properly functioning instrument should have a measured bias of $0.01 \%$ or less.

A Los Alamos developed random pulser ${ }^{7}$ (module RP501) is used in this check. Tests have shown that its effect on the HEC-100 digital section closely matches the predicted effect of a true random source. About $3 \%$ rate difference between the pulser setting and the TOTALS scaler will be observed at $128 \mathrm{kHz}$ because of the input synchronizer 0.5 - $\mu$ s deadtime.

Enter the HP-97 program referenced in this check and 7 isted in Appendix A, page 33. Its purpose is to expedite a go/no-go decision by reducing the data variance to a level less than or equal to \pm 10 . Ten lines of numerical data are printed. 
Lines 1 through 5: Raw scaler data [IIME, TOTALS, $R+A, A$, and $(R+A)-A$ ].

Line 6 : Gate width calculated from TIME, TOTALS and $A$.

Lines 7 through 9: Percentage deviations of the measured data rates from a random-puiser-based reference rate expressed in tenth per cent units.

Line 10 : A Reals vs Reals-error figure expressed in units of sigma $(\sigma)$.

This program requires a minimum count TIME setting of $1 \times 10^{2} \mathrm{~s}$.

1. Change random pulser setting to $07(128 \mathrm{kHz})$.

2. HEC-100 setup.

$\begin{array}{lll}\text { TIME } & 1 \times 10^{2} & \text { (front pane1) } \\ \text { GATE } & 8 \mu \mathrm{s} & \text { (front pane1) } \\ \text { MANUUAL/RECYCLE } & \text { MANUAL } & \text { (front pane1) }\end{array}$

3. Start the HEC-100. When HEC-100 stops, press READOUT to print the result (NOTE: The printout will not correspond to the sample printout below if the Appendix A program is not entered). Acquire and print several counting intervals if time allows.

4. The following is a typical example of the results printed on the HP-97 (8-us gate).

HP -97

Printout

100

12441158

12388376

12380938

7438

(space)

8

(space)

\begin{tabular}{ll} 
Quantity \\
\hline COUNT TIME & $\left(t_{C}\right)$ \\
TOTALS & $(T)$ \\
$\frac{R+A}{A}$ & \\
$\frac{A}{(R+A)-A}$ & (R)
\end{tabular}

GATE WIDTH $\left(t_{g}\right)$

\section{Tolerance/Comment}

Should agree with TIME setting. Should agree with TOTALS display. Should agree with $R+A$ display. Should agree with A display. $\sim \pm \sqrt{2 \bar{A}}$

Should agree with GATE setting.

$\begin{array}{rll}0 & D_{T^{\star}} & 0 \pm 5 \text { (tenths per cent) } \\ 0 & D_{R+A^{\star}} & 0 \pm 10 \text { (tenths per cent) } \\ -1 & D_{A}^{\star} & 0 \pm 10 \text { (tenths per cent) } \\ 1 & D_{R}^{\star} & 0 \pm 3 \text { (units of sigma) }\end{array}$

* Refer to Appendix A for detailed description. 
5. Repeat 3 and 4 for the other GATE settings. $R+A$ and $A$ should increase in proportion to the GATE setting, and the calculated gate width $\left(t_{g}\right)$ should agree with the GATE setting.

F. Predelay Check

This check tests the predelay circuitry that provides a delay between the $R+A$ strobe and the $R+A$ gate. The delay is necessary to prevent amplifier deadtime from effectively shortening the $R+A$ gate. The predelay is produced by a variable length (64-stage) shift register and is set by a binary-coded switch mounted on the shift-register board. Each predelay shift-register stage equals

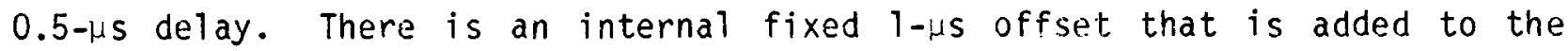
switch setting. Total predelay $($ in $\mu s)=$ (sum of open switch settings) +1 .

The predelay is measured by putting a pulse pair into the instrument input, observing the appearance of counts in the $R+A$ scaler as the second pulse is slowly separated from the first, and measuring the time delay between the two pulses in clock-cycle $(0.5 \mu \mathrm{s})$ increments at the synchronizer output (TA2, shift-register board). This measurement will be facilitated by aligning the pulse leading edge transitions with the oscilloscope graticule lines. The accidental scaler may be ignored during this check.

1. PG508 pulser setup.

MODE

DOIJBLE PULSE. (delay and undelay push-button switches IN)

DURATION

0.1 us (CALIBRATED)

DELAY

$<1$ HS

PERIOD (rate)

$0.1 \mathrm{~ms}$ ( $50 \%$ UNCALIBRATED)

OUTPUT (volts)

+3 to $+5 \mathrm{~V}$

Check pulse pair on scope (Fig. 8).

Connect the pulser output to HEC-100 "SR IN" (J 17).

2. SC504 oscilloscope setup.

VERTICAL

$2 \mathrm{~V} / \mathrm{div}$.

HORIZONTAL

0.5 to $2 \mu \mathrm{s} / \mathrm{div}$.

Connect oscilloscope probe to TA2 (shift-register board)(Figs. 9 and 10). 


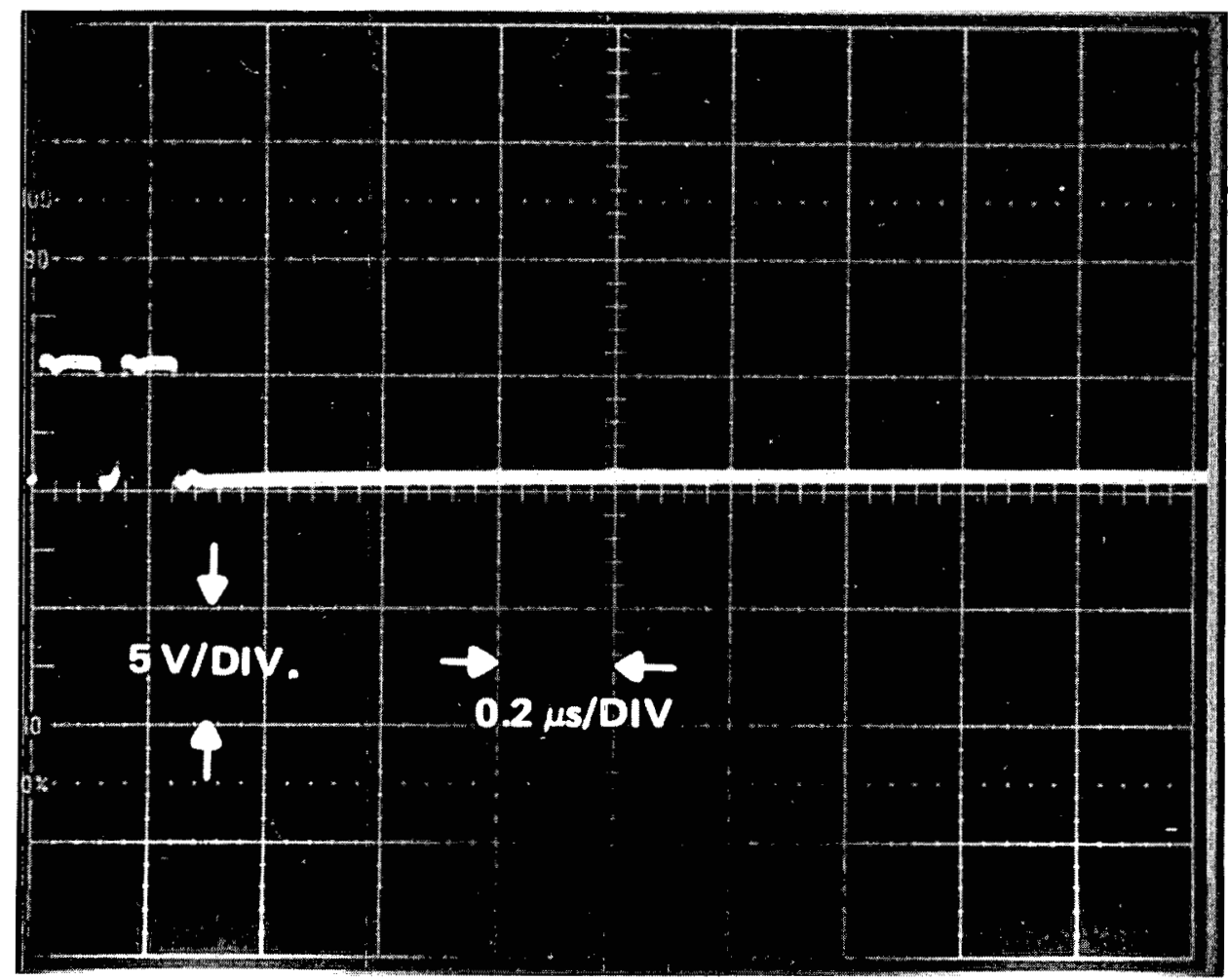

Fig. 8.

Predelay check step 1; PG508 output.

3. HEC-100 setup.

TIME

MANUAL/RECYCLE

PREDELAY
Arbitrarily long initially

MANUAL

All closed (initially) (shift-register board)

4. (a) START HEC-100.

(b) Slowly increase PG508 "DELAY" until $R+A$ scaler just begins to count.

(c) The delayed pulses should now begin to fall in the third $0.5-\mu \mathrm{s}$ cycle (Fig. 11).

5. (a) Increase the delay until all delayed pulses fall in the third or fourth cycle (Fig. 12).

(b) Set HEC-100 time to $1 \times 10^{\circ} \mathrm{s}$.

(c) Restart HEC-100 and count for $1 \mathrm{~s}$.

(d) Observe that Totals $=2(R+A)$ precisely.

(e) This tests the 1 -us internal minimum predelay. 


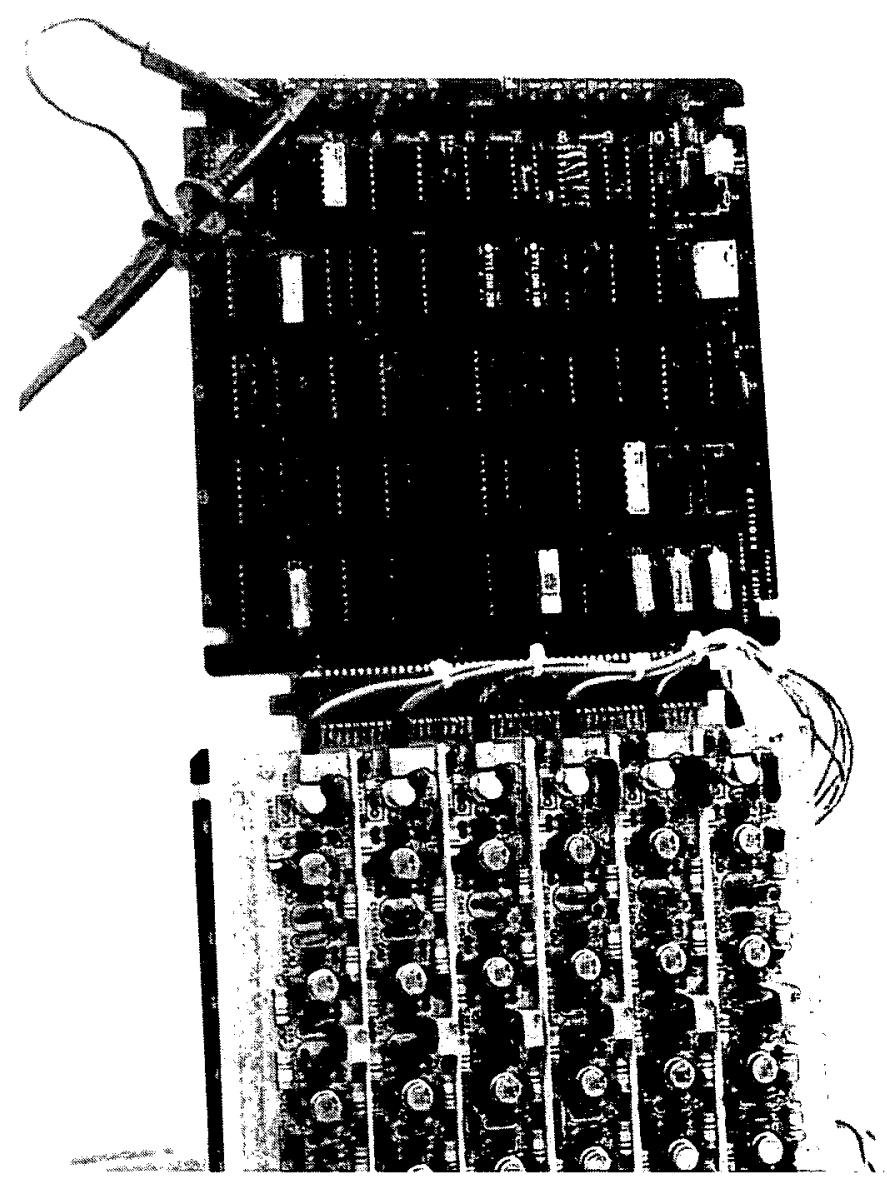

Fig. 9.

Predelay check step 2:

SC504 connected to TA2.
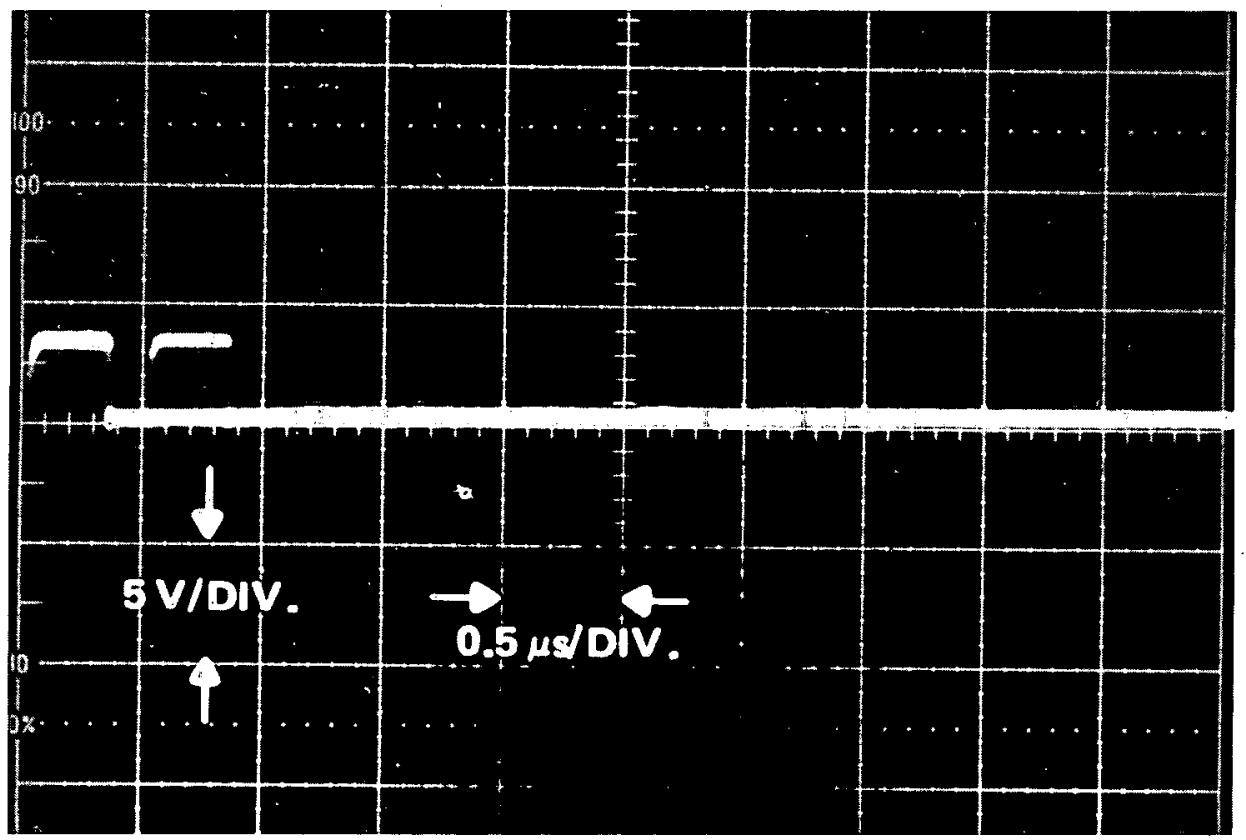

Fig. 10.

Predelay check step 2: synchronizer output at minimum predelay; no $R+A$ counts. 


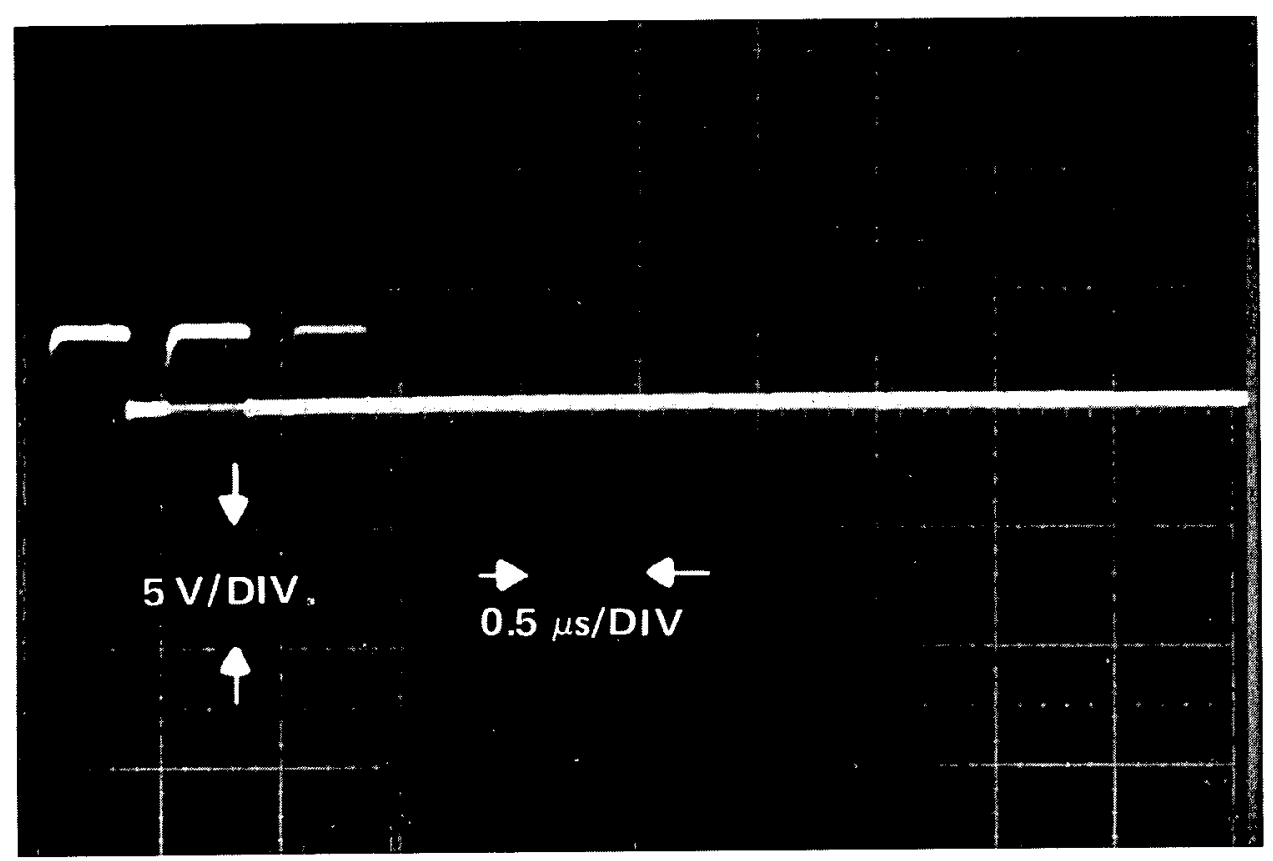

Fig. 11 .

Predelay check step $4(c)$ : synchronizer output at slightly increased delay; $\mathrm{R}+\mathrm{A}$ scaler starting to count.

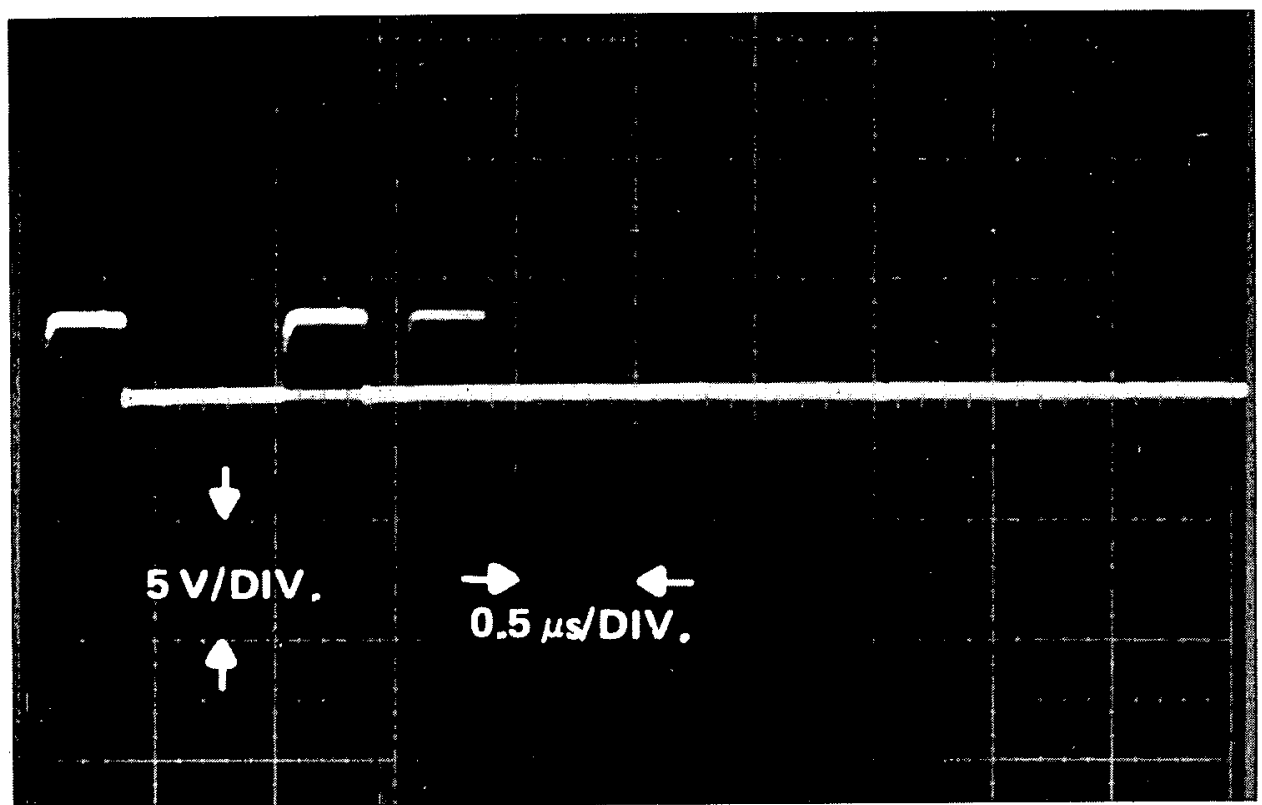

Fig. 12 .

Predelay check step 5(a): synchronizer output with all delay pulses in third/fourth clock cycle; $R+A$ count equals one-half totals count. 
6. Repeat steps 4 and 5, opening the predelay switches $(0.5$ to $16 \mu \mathrm{s})$ in turn and increasing the PG508 delay to satisfy $4(\mathrm{~b})$. Further increase the delay as was done in $5(a)$ and observe that the condition in $5(d)$ is met. The increase in delay will be precisely two clock cycles per microsecond of switch setting.

7. Return the predelay switch setting to $3.5 \mu \mathrm{s}$ for a total of $4.5 \mathrm{\mu s}$ of predelay (Figs. 13 and 14). This completes the predelay check.

\section{G. Accidental Delay Check}

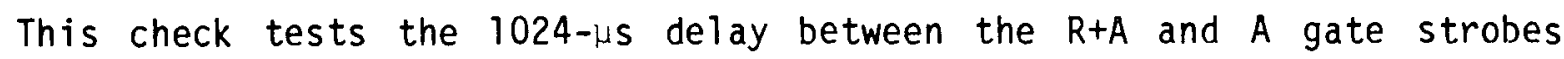
by direct measurement. The $D C 503$ counter/timer module is used for the measurement and the instrument input is supplied by the PG508 pulser. The HEC-100 must be counting during this test.

1. PG508 pulser setup.

$\begin{array}{ll}\text { MODE } & \text { Undelayed (single pulse) } \\ \text { PERIOD (rate) } & 20 \mathrm{~ms}(50 \mathrm{~Hz})\end{array}$

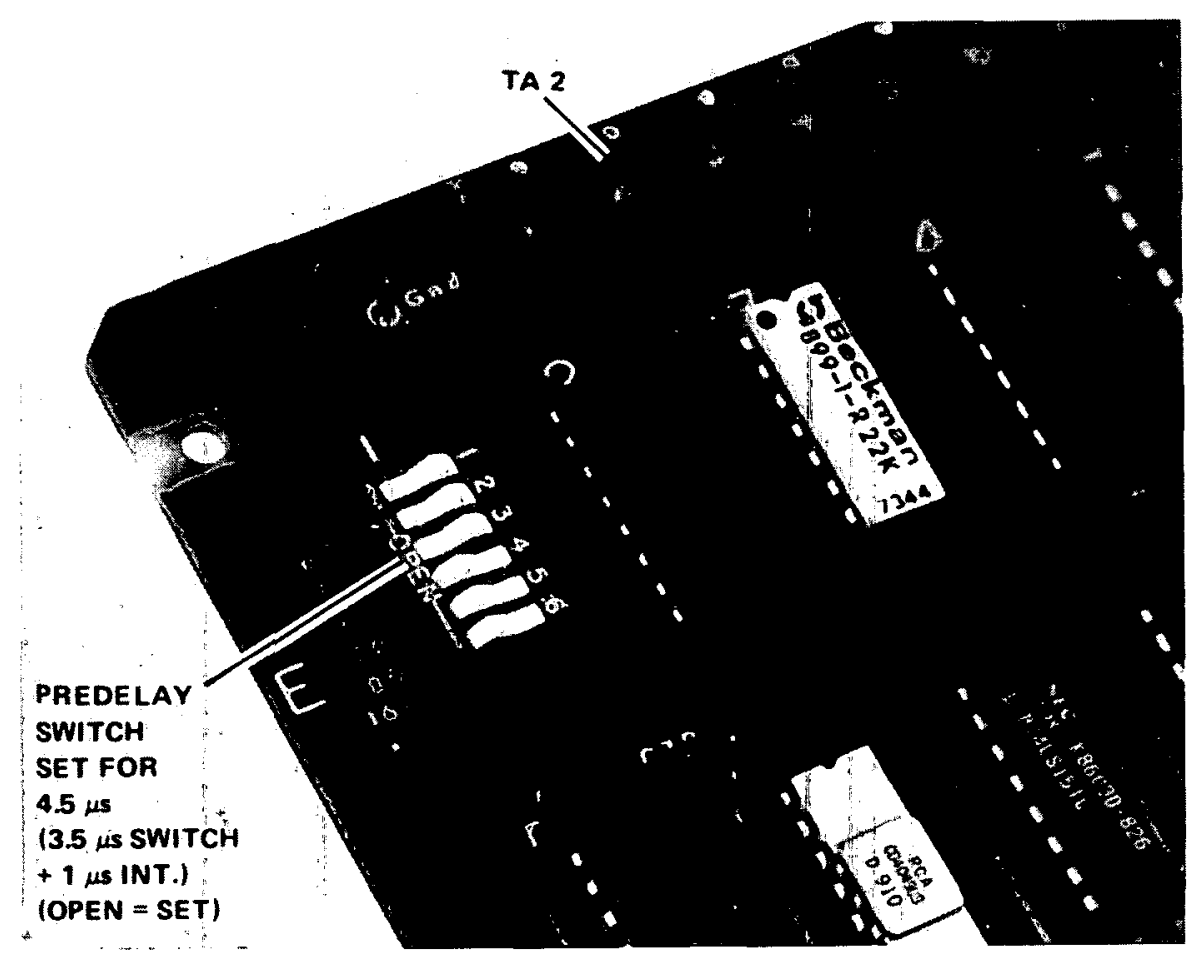

Fig. 13.

Predelay check step 7 ; predelay switch setting at end of predelay check. 


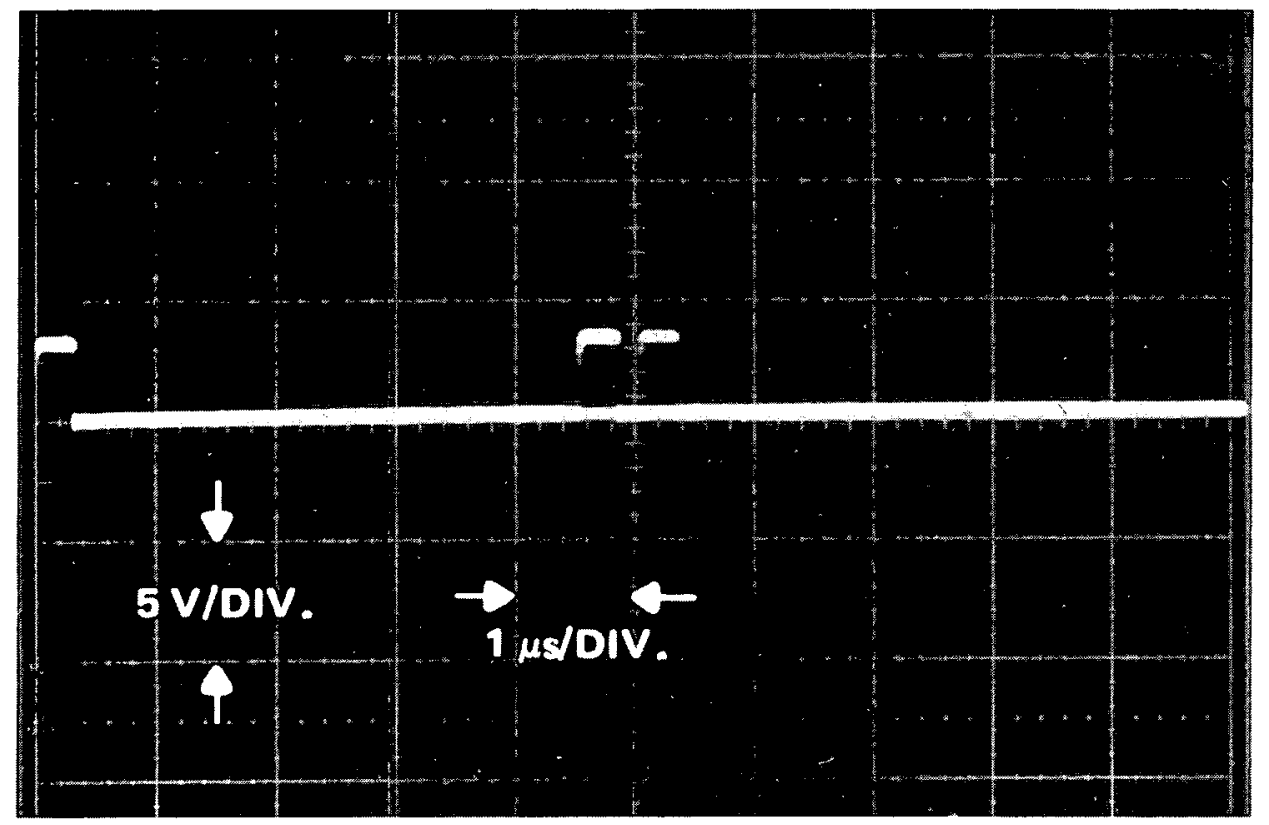

Fig. 14.

Predelay check step 7; SC504 presentation of delay pulse at $4.5 \mathrm{\mu s}$ and predelay set for $4.5 \mathrm{\mu s}$..
DURATION
10 is
OUTPUT (volts)
+3 to $+5 \mathrm{~V}$
Connect pulser output to HEC-100 "SR IN" (J17).

2. DC503 counter/timer setup.

FUNCTION

\section{TIMING}

Ch. $A$ and $C h$. B SLOPE

Ch. $A$ and $C h$. B ATTEN

Ch. $A$ and $C h$. B COUPLING

$\mathrm{Ch}$. $\mathrm{A}$ and $\mathrm{Ch}$. B SOURCE

START/STOP

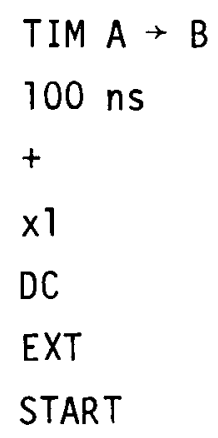

DC

EXT

START

Using oscilloscope probes ( $x$ l probe, if available), connect $\mathrm{Ch}$. A to TB5 (shift-register board) and Ch. B to TB7 (shift-register board).

3. START the HEC-100 (with a "long" TIME setting).

4. Temporarily switch to "freq. A" on the DC503; find the minimum (ccw) and maximum (CW) A LEVEL settings at which the DC503 properly counts $(250 \mathrm{~Hz})$; set A LEVEL midway between these points. Temporarily 
switch to "Period B" on the DC503 and set B LEVEL using the same procedure (measuring $220 \mathrm{~ms}$ ). Return the DC503 function switch to TIM $A \rightarrow B$.

5. Observe a time interval of $1024.0 \pm 0.1$ us on the DC503. This completes the digital section checks.

IV. ANALOG SECTION CHECK-OUT AND CALIBRATION

Several procedures are detailed for testing, calibrating, and verifying the operation of the HEC-100 analog section and the HP-100 preamplifier box. The major subsections and parameters of interest are

- high-voltage power supply voltage calibration, noise, breakdown

- detectors (5 each) noise, breakdown

- preamplifiers (6 each) noise, gain, pulse shaping

- amplifiers (6 each) noise, gain, pulse shaping

- discriminator/Or gate pulse timing and voltage levels

The usual practice is to calibrate and check the system as a whole. Changing any of the above elements may necessitate recalibration.

Noise control is of prime importance for proper operation of the instrument; noise problems have been a major source of instrument downtime. The following types of noise have been encountered:

- electromagnetic radiation (RFI, 1 ine transients, etc.);

- high-voltage breakdown (faulty components);

- printed circuit board surface leakage (dirty boards, humidity, etc.);

- ground loops and power supply noise.

The inajor sources of these problems have been improper grounding and shielding, and failures in maintaining board cleanliness. Several figures illustrating noise have been included on page 23 .

Because detector sensitivity is about $1 \% / \mathrm{V}$, high-voltage calibration requires care.

The following test equipment (Fig. 15) is used in the analog section procedure:

Tektronix

Tektronix

Tektronix
TM506 mainframe

SC504 oscilloscope module

DM502A DVM module

Los Alamos high-voltage supply module 


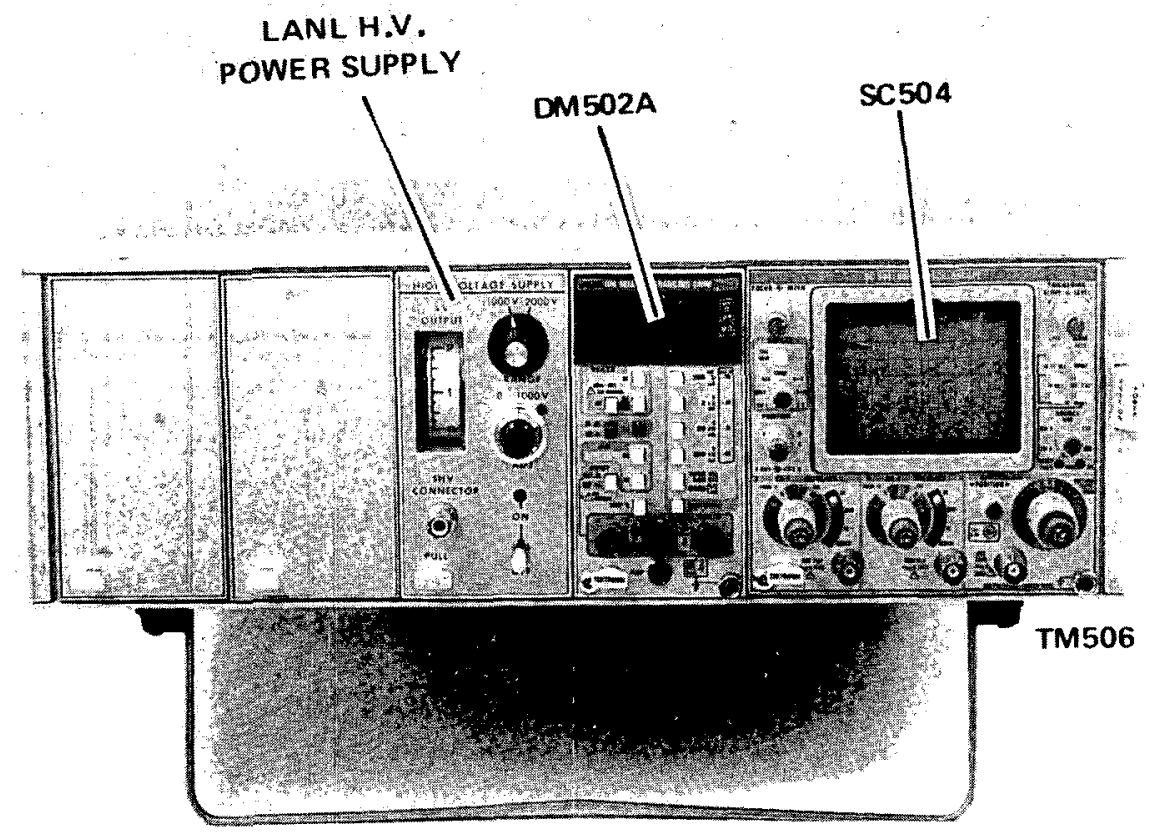

Fig. 15.

Analog section test set.

\section{A. Setup and Mechanical Adjustment}

1. With ac power (PWR SW., front panel) off, interconnect ${ }^{3}$ He detector, HP-100 (preamplifier box), and HEC-100 with supplied cables.

2. Rotate HV and DISC $V$ knobs (front panel) to minimum and observe dial reading of 0.00 . If dial is not at zero, loosen the $k$ nob(s) set screw $(\mathrm{s})$, set the indicator to 0.00 , and retighten the set screw(s).

3. Set the HV and DISC V knobs to $7.50(1500 \mathrm{~V})$ and $3.00(1.5 \mathrm{~V})$, respectively. Switch POWER to ON. Switch SELECT (sw. 10, rear panel) to INT.

\section{B. Noise Check}

1. Using the SC504 oscilloscope, check each amplifier front panel test point. Observe that base-line noise is less than $1 \mathrm{~V}$ peak-to-peak (Figs. 16 and 17). Noise greater than 0.5 to $1 \mathrm{~V}$ peak-to-peak is usually a result of loose mounting screws in the HP-100 preamplifier box or other grounding problems.

\section{Discriminator Calibration}

1. Connect the DM502 (DVM) between the (front panel) DISC $V$ test point and the ground-test point. Observe a reading of $1.500 \mathrm{~V}$ on the DVM. 


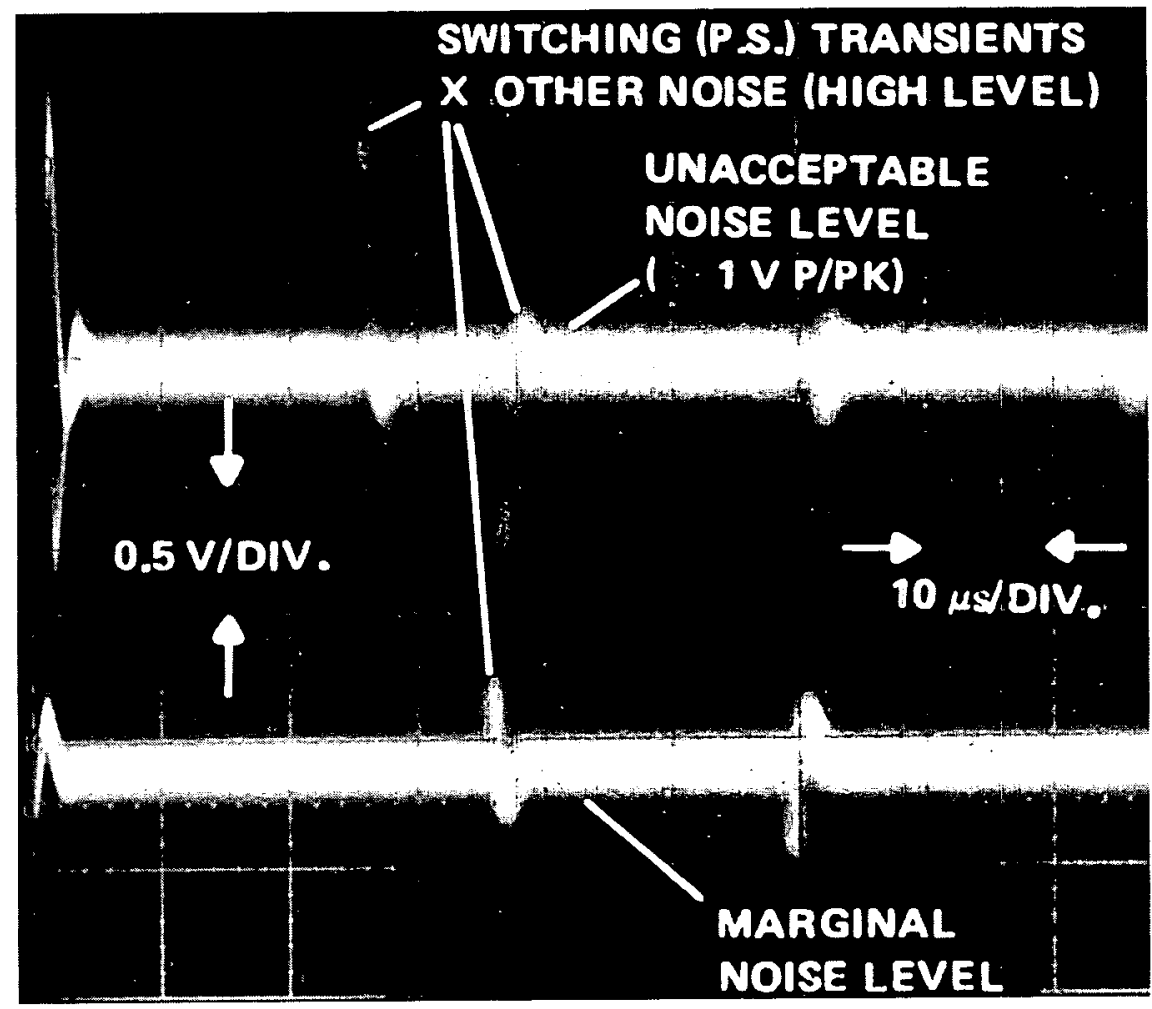

Fig. 16.

Analog check B: unacceptable and marginally acceptable noise levels.

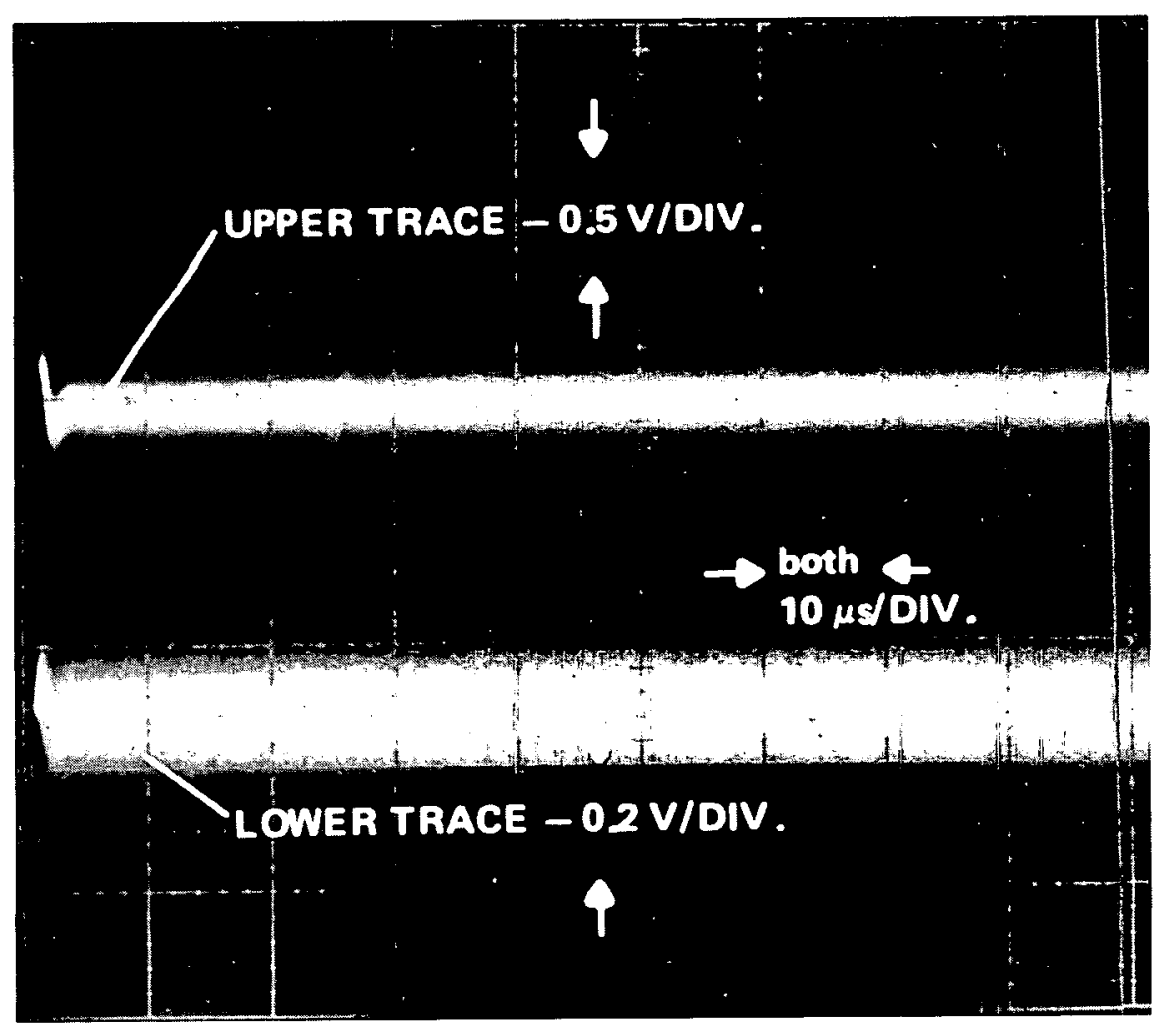

Fig. 17.

Analog check B: acceptable noise level. 
If DVM reading is not $1.500 \mathrm{~V}$, do step 2; otherwise, go to $D$.

2. Adjust RI, the forward potentiometer on the HEC-100 high-voltage supply board, for $1.500 \mathrm{~V} \pm 0.001 \mathrm{~V}$ at the DISC $\vee$ test point (Fig. 18).

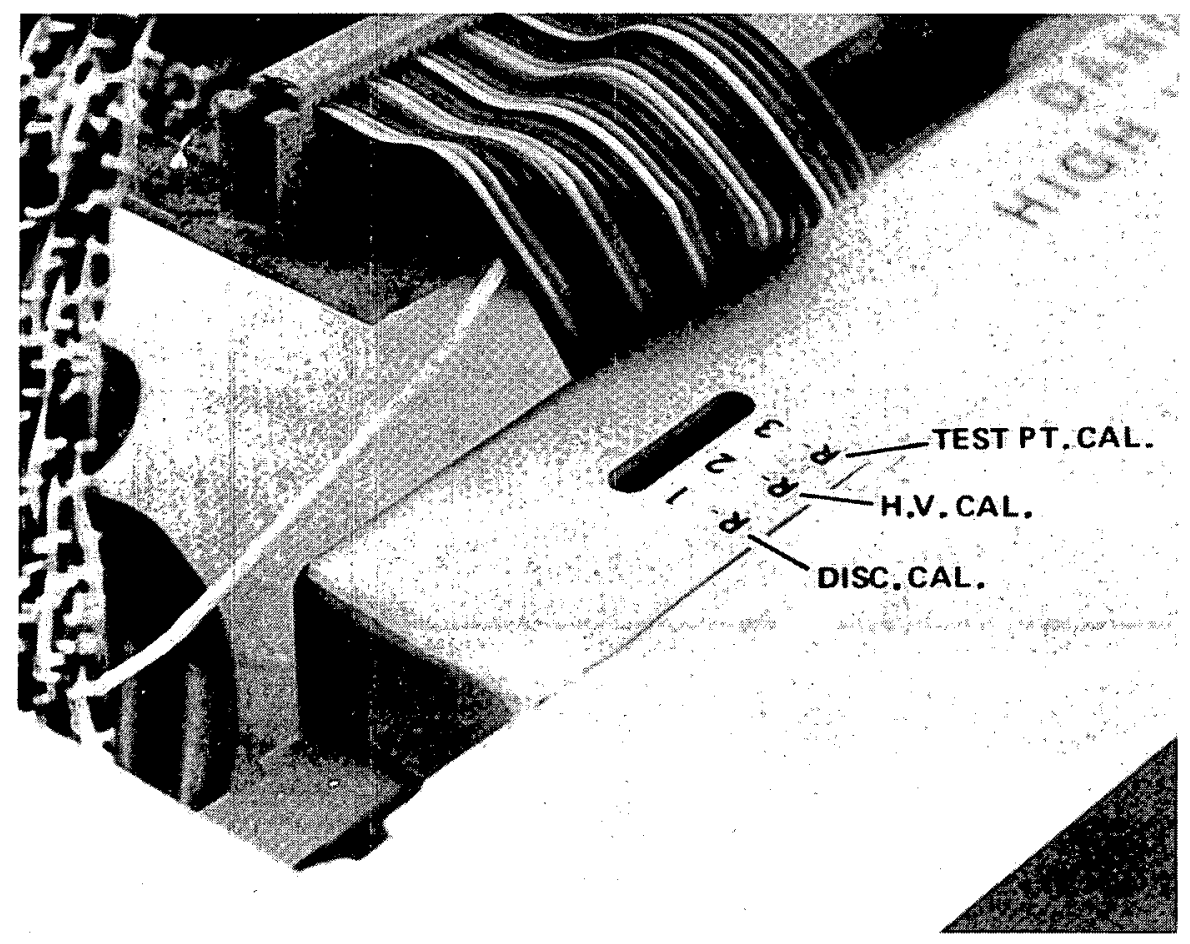

Fig. 18.

Discriminator, high voltage, and high-voltage test point calibration potentiometers.

D. High-Voltage Power Supply Calibration

NOTE: Two procedures are provided for high-voltage calibration: Procedure 1 uses a neutron source and Procedure 2 does not use a neutron source. In addition, Procedure 1 incorporates amplifier gain calibration as an integral part of the procedure, whereas Procedure 2 requires a separate gain calibration. Procedure 1 is recommended strongly as the preferred method (assuming that a source is available) because of accuracy.

Procedure 1: High-Voltage and Amplifier Calibration with Neutron Source

1. Connect Los Alamos high-voltage power supply module to HV IN on HP-100 (preamplifier box) and set to $1500 \mathrm{~V}$. Switch high-voltage module on. 
2. Place (neutron) source in detector.

3. Adjust pole zero potentiometers (Fig. 19) to maximum in direction that gives bipolar pulse at amplifier front panel test points (Fig. 20).

NOTE: Maximum may be either $\mathrm{cw}$ or $\mathrm{ccw}$, depending on the instrument. Later models have no pole zero adjustment.

4. Adjust each amplifier gain potentiometer lat midsection of analog board (Fig. 19) to center the amplifier peak pulses at 6 V (Fig. 20). NOTE: Observe the amplitude level carefully as it is the reference level for high-voltage calibration.

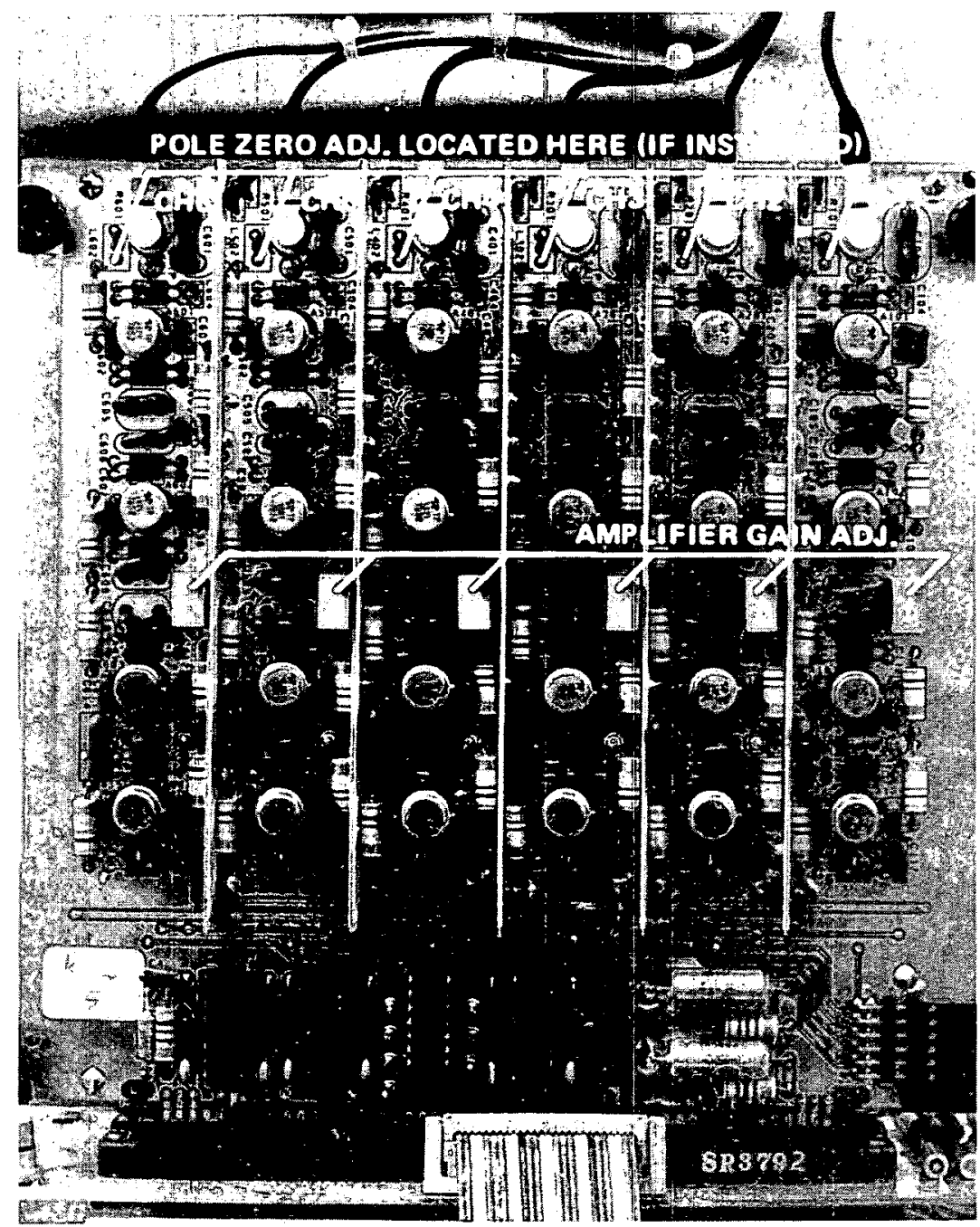

Fig. 19.

Location of gain and pole zero adjustment potentiometers (amplifier board). 


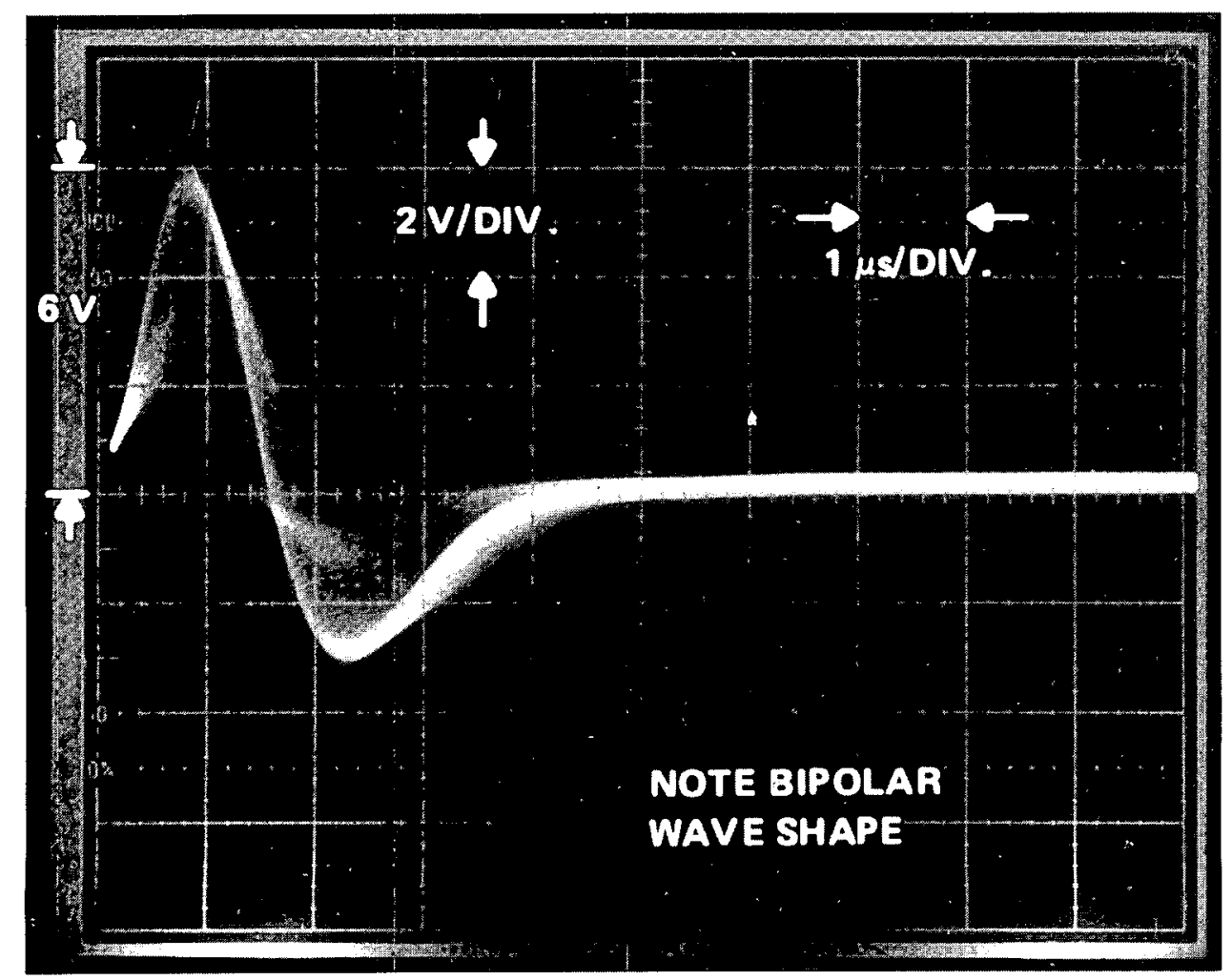

Fig. 20.

Properly adjusted amplifier output front panel test point presentation on SC504. (vertical scale $=2 \mathrm{v} /$ div.)

5. Switch HEC-100 high-voltage switch ON, disconnect high-voltage cable from high-voltage module, and immediately connect it to HEC-100 HV OUT (SHV, rear panel).

6. Observe arnplifier pulse 1evel. If it has changed, adjust R2 on highvoltage board (center potentiometer, Fig. 18) to bring the level back to $6 \mathrm{~V}$. Switch the high-voltage cable back and forth from the HEC-100 high-voltage output to the Los Alamos high-voltage module output several times, observing an anplifier test point and adjusting $R 2$ until no gain change is seen. Final high-voltage connection should be from HEC-100 internal supply.

7. Connect the DM502A (DVM) to HV/1000 and ground-test points.

8. Adjust R3 on high-voltage board (rear potentiometer, Fig. 18) to give a reading of $1.500 \mathrm{~V} \pm 0.001 \mathrm{~V}$ on the DVM.

9. Recheck gain on all amplifier channels and readjust to $6 \mathrm{~V}$, if necessary. Base-line noise should not exceed $0.5 \mathrm{~V}$ peak-to-peak at this time (Fig. 17). 


\section{Procedure 2: High-Voltage Calibration Without Neutron Source}

1. Turn HEC-100 PWR switch ON.

2. Turn high-voltage switch OFF.

3. Connect high-voltage cable from Los Alamos high-voltage module to HEC-100 HV OUT.

4. Set Los Alamos high-voltage module to $1500 \mathrm{~V}$ and turn on.

5. Connect DM502A (DVM) to HV/1000 (front panel) test point and groundtest point.

6. Adjust R3, on high-voltage board (rear potentiometer, Fig. 18) for a DVM reading of $1.500 \mathrm{~V} \pm 0.001 \mathrm{~V}$.

7. Disconnect the Los Alamos power supply from HEC-100; turn on HEC-100 HV.

8. Adjust R2 [middle potentiometer, HEC-100 HV board, (Fig. 18)] until DVM again reads $1.500 \mathrm{~V} \pm 0.001 \mathrm{~V}$.

Procedure 3: Amplifier Calibration Without Source

USAGE NOTE: The following procedure is not recommended for field usage at this time. Due to detector gain variations, an absolute system gain calibration is not possible using a pulser. It is possible, however, to match the gains of the 6-channel amplifier board; the gains can then be raised or lowered simultaneously by adjusting the high voltage. This method is under investigation for system calibration.

PROCEDURAL NOTE: This amplifier gain calibration is done by injecting a calibrated charge into each preamplifier input with a battery-operated pulser. It has been calibrated to provide the appropriate input for a $6-V$ amplifier pulse. Battery condition is determined by output pulse rate; when the rate drops to $22500 \mathrm{~Hz}$, the battery should be replaced.

1. Turn high-voltage sivitch off.

2. Disconnect six SHV cables from detectors.

3. Connect six SHV cables to pulser box.

4. Connect oscilloscope to Ch. I "ArMp" test point. 
5. Adjust jain potentiometer (center of board on right-hand side) for a 6-V output.

6. Repeat 4 and 5 for $\mathrm{Ch} .2$ through 6 , using the appropriate "AMp" test point and gain potentiometer.

Dne-Shot and OP Gate Check

Using either a source input or the allplifier gain calibration pulser and the SC504 oscilloscope, verify that a 150-ns transistor-transistor logic (TTL) pulse is present at TEST POINTS 1 through 7 (Figs. 21 and 2.2), bottoril of amplifier board. TEST POINTS 1 through 6 are the individual channel one-shots and are inverted (TTL) logic; TEST POINT 7 is the OR gate output and is positive (TTL) logic.

This completes the analog section calibration and check procedures.

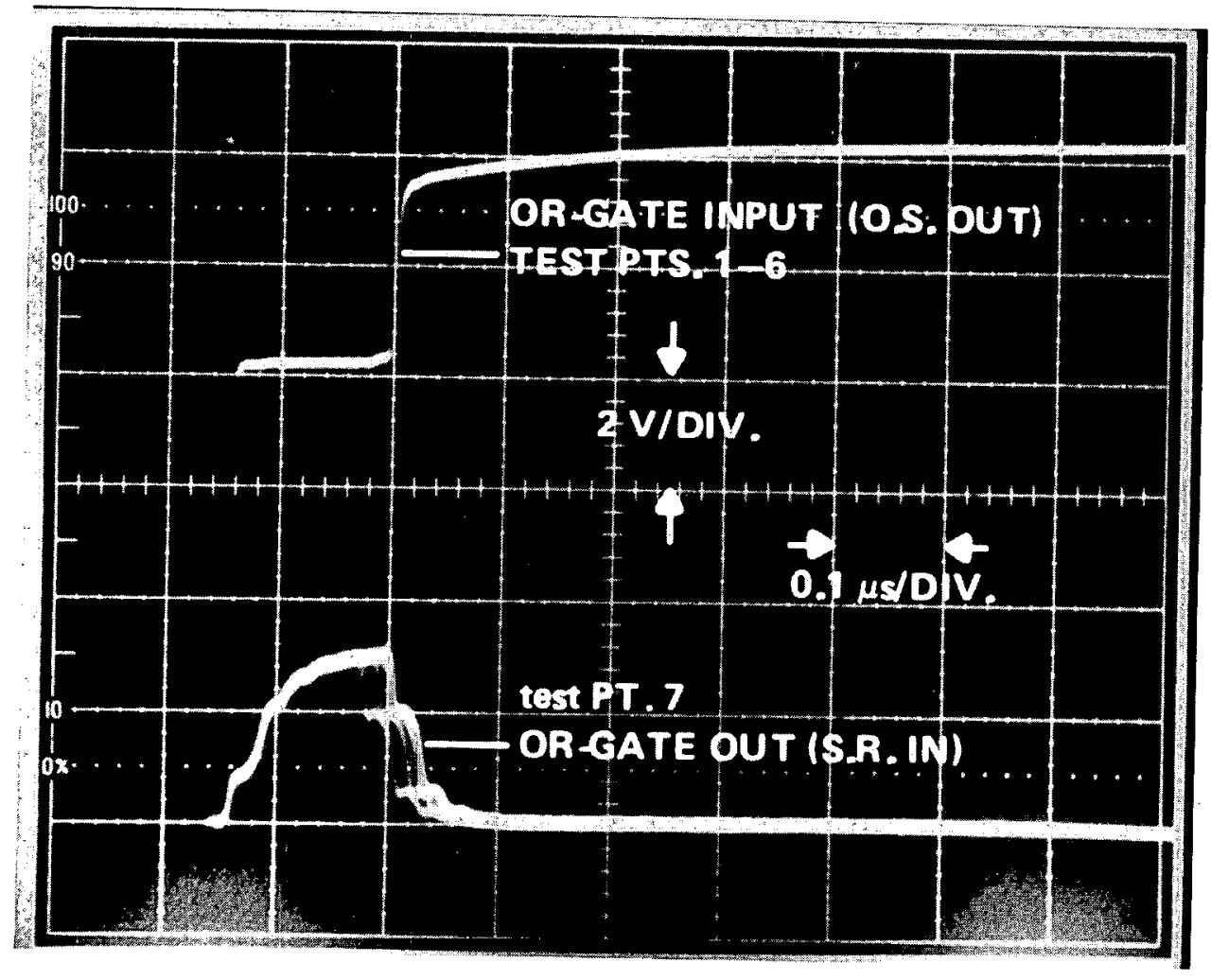

Fig. 21 .

One shot and/or gate pulse presentation on SC504; amp board test points 1 through 7 . 


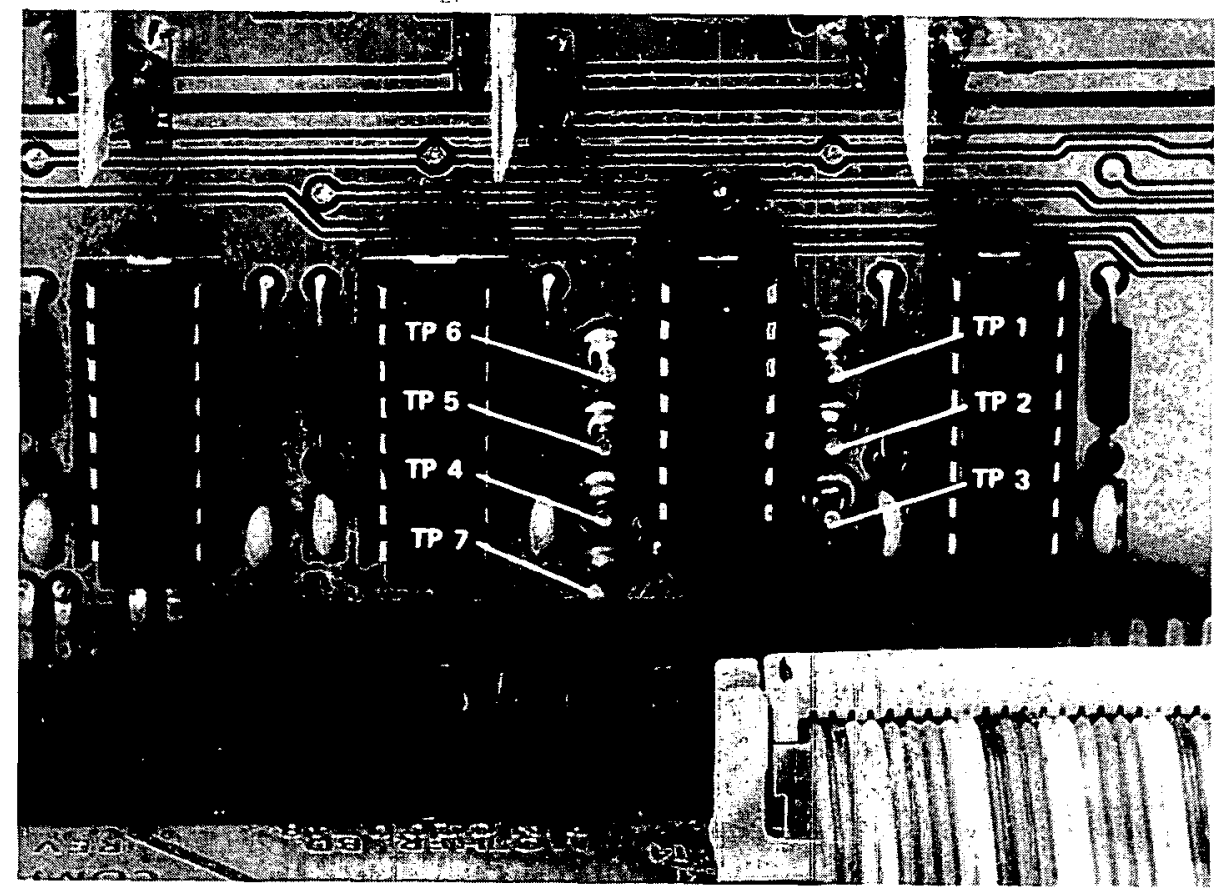

Fig. 22.

Amplifier board one shot and/or gate test points.

\section{SYSTEM OPERATIONAL CHECKS}

These checks should be done when possible; the results of the tests should be recorded for each system for reference (and to compare present with past performance).

\section{A. Efficiency Check}

1. For each system, using a detector complete with end caps/plugs, and with HEC-100 GATE at $32 \mu \mathrm{s}$ and predelay at $4.5 \mu \mathrm{s}$, measure:

a. background totals rate $(\dot{B})$ (no source present),

b. totals rate $(\dot{T})$ (using ${ }^{252}$ Cf source), and

c. reals coincidence rate $(\dot{R}=(\vec{R}+A)-\dot{A})$ (using ${ }^{252} C f$ source).

2. Compute $\dot{R} /(\dot{T}-\dot{B})$. This is a system efficiency measure and it should be recorded for future reference and compared with previous results (if any). 
B. Bias Check

For each system, measure a (known random) AmL $i$ source for a period long enough to determine system bias. Bias $=R / A \times 100$. (For a $0.01 \%$ level of bias, the statistical error must be smaller than $0.01 \%$ to make the determination valid. This requires at least $1 \times 10^{9}$ accidental counts.) once again, record (and compare) the test results.

\section{Electromagnetic Interference (EMI) Check}

Perform an EMI test using the LoS Alamos supplied noise source or other source (Tesla coil, etc.). The test checks cable and preamplifier shielding integrity. It has been useful in finding faulty cables, anodizing on connector holes, etc. Basically, the test consists of attempting to induce spurious counts in the instrument by generating high levels of transient (EM) field around the instrument, particularly around the detector/preamplifier box assembly and the associated cables. At present, only comparative tests will produce meaningful results, and relatively great tolerance must be used in interpreting the results. To perform the test, start the HEC-100; move the "noise" source along each detector cable at a close distance ( $\sim \mathrm{cm}$ ) while observing a totals scaler. Ignore both $R+A$ and $A$ scalers during this test. Differences in induced rate, channel to channel, of less than 10 to 1 should be ignored, particularly if not repeatable. If large (repeatable) differences are observed, substitute another cable, component, or HP-100 preamplifier box in an attempt to isolate the problem. The preceding test procedure is an example; the technician may devise alternative procedures that produce useful results. It should be emphasized that the results of these procedures can be and often are ambiguous. Practice will improve the technician's ability to determine whether the results indicate a real problem and to determine the appropriate corrective action.

\section{REFERENCES}

1. M. S. Krick and H. O. Menlove, "The High-Level Neutron Coincidence Counter (HLNCC): Users' Manual," Los Alamos Scient if ic Laboratory report LA-7779-M (ISP0-53) (June 1979).

2. D. Altman, "Maintenance Manual, High Level Neutron Coincidence Counter (HLNCC)," EG\&G report EGG-1183-5091 (July 1978). 
3. HLNCC-100 Operation and Maintenance Manual, IRT Corporation, San Diego, California, IRT 9441-001 (October 1980).

4. John E. Foley, "Field Manual for the High Level Neutron Coincidence Counter (HLNCC)," Los Alamos Scientific Laboratory draft report (February 1979). This document is available from Los Alamos Group Q-1.

5. W. F. Lindsay, "Instructions for Use of High Level Neutron Coincidence Counter" International Atomic Energy Agency report IAEA-IMI \#29 (Vol. 1, September 1980; Vo1. 2, April 1981).

6. J. Swansen, P. Collinsworth, and M. Krick, "Shift-Register Coincidence Electronics System for Thermal Neutron Counters," Nucl. Instrum. Methods 176, 555-565 (1980).

7. J. Swansen, "A Digital Random Pulser for Testing Nuclear Instrumentation," Nucl. Instrum. Methods 188, 83-91 (1981). 

APPENDIX A

RP501 GATE AND ADDER TEST PROGRAM

This program, in conjunction with the RP501 random pulser, provides a method for rapid testing of the TOTALS, $R+A$, and $A$ counting, and the readout circuitry. To eliminate the need to interpret counting statistics, the minimum recommended counting time is $1 \times 10^{2} \mathrm{~s}$.

At a thumbswitch setting of $07(128 \mathrm{kHz})$, the RP501 should count $124461 \pm 1 \sigma$ TOTALS counts/s in a properly operating HEC-100. This reference constant $(M)$ is already stored in the program. Measured data are compared with data that are calculated using this reference.

Shown below is an example of a printout from this program.

HP-97 Printout

Quantity

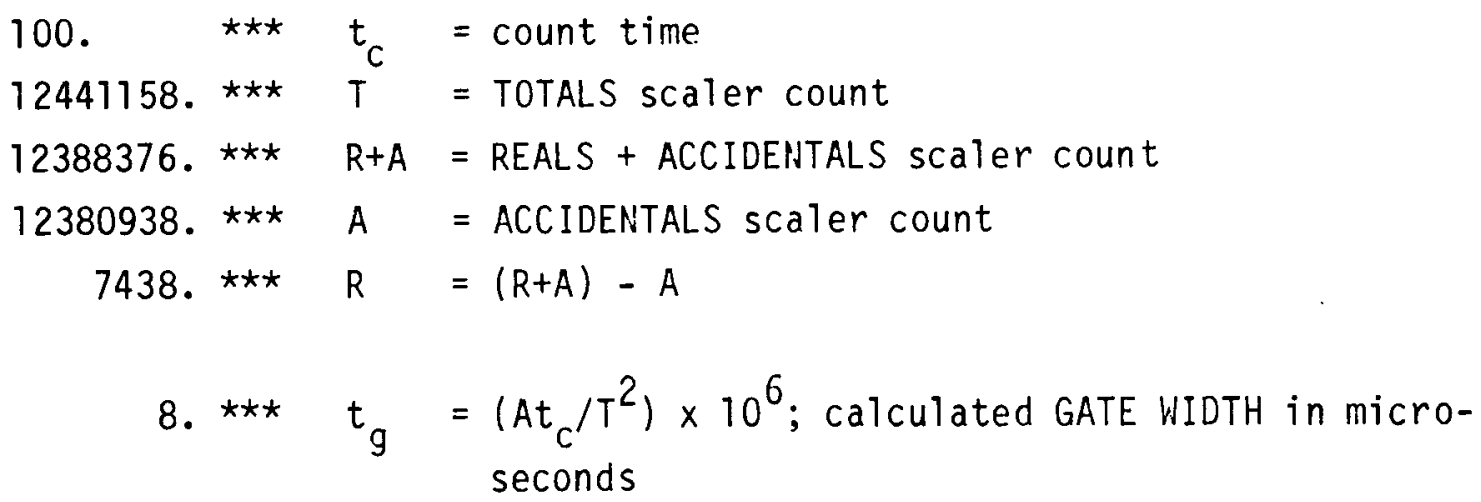

$0 . \star \star \star \quad D_{T}=1000[(T / M)-1]$; fractional $T$ deviation expressed in $0.1 \%$ units

$0 . \star \star * \quad D_{R+A}=1000\left\{\left[(R+A) / M^{2} t_{g}(s)\right]-1\right\}$; fractional $R+A$ deviation expressed in $0.1 \%$ units

$-1 . * \star \star \quad D_{A}=1000\left\{\left[A / M^{2} t_{g}(s)\right]-1\right\}$; fractional A deviation expressed in $0.1 \%$ units

1. $* * \star \quad D_{R}=R / \sqrt{(R+A)+A}$; fractional error in $R$ expressed in $1-\sigma$ units

Normally, the last four lines of the printout are sufficient to determine if the counter is functioning properly. The last four numbers of the printout should be 0 or within the following tolerances: 


$$
\begin{aligned}
& D_{T}= \pm 5 \\
& D_{R+A}= \pm 10 \\
& D_{A}= \pm 10 \\
& D_{R}= \pm 3
\end{aligned}
$$

These tolerances were determined empirically from experience and are necessary to allow for the influence of capacitor $C 5$ on the digital deadtime. If $D_{T}$, $D_{R+A}$, and $D_{A}$ are consistently outside of tolerance, check capacitor $C 5$ on the shift-register board first; it should have a value of 68 to $120 \mathrm{pF}$. An integrated circuit (7E) on the same board should be a Texas Instrument 74LS74.

\begin{tabular}{|c|c|c|c|}
\hline 001 & *LBLA & 2111 & Reference constant (M) \\
\hline 002 & 1 & 01 & \\
\hline 003 & 2 & 02 & \\
\hline 004 & 4 & 04 & \\
\hline 005 & 4 & 04 & \\
\hline 006 & 6 & 06 & \\
\hline 007 & 1 & 01 & \\
\hline$\underline{008}$ & ST07 & 3507 & Stored in $R-7$ \\
\hline 009 & FIX & -11 & Fix display to show integers only \\
\hline$\underline{010}$ & DSPO & -6300 & \\
\hline 011 & RCL1 & 3601 & Printout of raw data \\
\hline 012 & PRTX & -14 & $t_{c}$ \\
\hline 013 & RCL2 & 3602 & \\
\hline 014 & PRTX & -14 & $T$ \\
\hline 015 & RCL3 & 3603 & \\
\hline 016 & PRTX & -14 & $R+A$ \\
\hline 017 & RCL4 & 3604 & \\
\hline 018 & PRTX & -14 & A \\
\hline
\end{tabular}
If $D_{R}$ is consistently outside of tolerance, check $D_{A}$ and $t_{g}$, or $D_{R+A}$, to determine whether the $R+A$ or the $A$ scaler is counting incorrectly.

Program Listing -- GATE and ADDER Test 


\begin{tabular}{|c|c|c|c|}
\hline 019 & $\mathrm{RCL} 3$ & 3603 & \\
\hline 020 & RCL4 & 3604 & \\
\hline 021 & - & -45 & \\
\hline$\underline{0} 22$ & PRTX & -14 & $(R+A)=A$ \\
\hline 023 & $\mathrm{SPC}$ & $16-11$ & Space \\
\hline 024 & RCL4 & 3604 & \\
\hline 025 & $\mathrm{RCL} 1$ & 3601 & Calculate GATE width \\
\hline 026 & $x$ & -35 & \\
\hline 027 & $\mathrm{RCL2}$ & 3602 & \\
\hline 028 & $x^{2}$ & 53 & \\
\hline 029 & $\div$ & $-2 \cdot 4$ & $t_{g}(\mu s)=A t_{C} / T^{2} \times 10^{6}$ \\
\hline 030 & EEX & -2.3 & \\
\hline 031 & 5 & 06 & \\
\hline 032 & $x$ & -35 & \\
\hline 033 & PRTX & -14 & Print $\mathrm{t}_{\mathrm{g}}$ in microseconds \\
\hline 034 & RND & 1624 & \\
\hline 035 & EEX & -23 & \\
\hline 036 & CHS & -22 & Round off \\
\hline 037 & 6 & 06 & \\
\hline U38 & $x$ & -35 & Convert $t_{g}$ to seconds, tilat is, $t_{g}$ (s) \\
\hline 039 & STO8 & 3503 & Store it in $R-\varepsilon$ \\
\hline$\underline{040}$ & $S P C$ & $16-11$ & Space \\
\hline 041 & RCL1 & 3601 & Convert measured $T, R+A$, and $A$ to $B A T E S$ and \\
\hline 042 & $\mathrm{STO} \div 2$ & $35-2402$ & store in $R-2, R-3$, and $R-4$, respectively \\
\hline 043 & STO $\div 3$ & $35-24 \quad 03$ & \\
\hline$\underline{0} 4 \underline{4}$ & $S T 0 \div 4$ & $35-24 \quad 04$ & \\
\hline 045 & PCL7 & 3607 & Calculate refereice "R+A" and "A" rates lifil \\
\hline 046 & RCL7 & 3607 & random source--they are the saine) \\
\hline 047 & RCL8 & 3608 & \\
\hline 048 & $x$ & -35 & \\
\hline 049 & $x$ & -35 & $(R+A)$ or $A=M^{\prime} t_{G}$ \\
\hline 050 & ST09 & 3509 & store result in $R-0$ \\
\hline 051 & RCL2 & $36 \mathrm{ij} ?$ & Calculate $D_{T}=1000[(T / M)-1]$ \\
\hline 052 & $\mathrm{RCL} 7$ & 3607 & \\
\hline 053 & GSBB & 2312 & Print $D_{T}$ expressed in $0.1 \%$ units \\
\hline
\end{tabular}




\begin{tabular}{|c|c|c|c|}
\hline 054 & RCL 3 & 3603 & Calculate $D_{(R+A)}=\left\{\left[(R+A) / M^{2} t_{g}(s)\right]-1\right\}$ \\
\hline \multirow[t]{2}{*}{$\begin{array}{l}055 \\
056\end{array}$} & $\begin{array}{l}\text { RCL } 9 \\
\text { GSBB }\end{array}$ & $\begin{array}{ll}36 & 09 \\
23 & 12\end{array}$ & 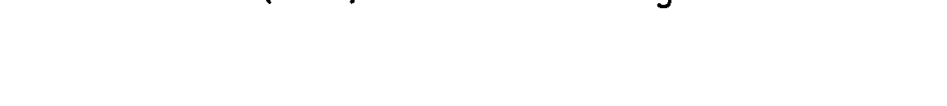 \\
\hline & & & Print $D(R+A)$ expressed in $0.1 \%$ units \\
\hline 057 & $\mathrm{RCL} 4$ & 3604 & Calculate $D_{A}=\left\{\left[A / M^{2} t_{g}(s)\right]-1\right\}$ \\
\hline 058 & RCL 9 & 3609 & \\
\hline \multirow[t]{2}{*}{059} & GSBB & 2312 & \\
\hline & & & Print $D_{A}$ expressed in $0.1 \%$ units \\
\hline 060 & RCL3 & 3603 & \\
\hline 061 & RCL4 & 3604 & Calculate \\
\hline 062 & - & -45 & \\
\hline 063 & $\mathrm{RCL} 3$ & 3603 & \\
\hline 064 & RCL4 & 3604 & $D_{R}=R / \sqrt{(R+A)+A}$ \\
\hline 065 & + & -55 & \\
\hline 066 & $\sqrt{x}$ & 54 & \\
\hline 067 & $\div$ & -24 & \\
\hline 068 & $\mathrm{RCLI}$ & 3601 & \\
\hline 069 & $\sqrt{x}$ & 54 & \\
\hline 070 & $x$ & -35 & \\
\hline 071 & RND & 1624 & Print $D_{R}$ expressed in $1-\sigma$ units \\
\hline 072 & PRTX & -14 & \\
\hline 073 & SPC & $16-11$ & \\
\hline 074 & SPC & $16-11$ & \\
\hline 075 & $R / S$ & 51 & \\
\hline 076 & *LBLB & 2112 & \\
\hline 077 & $\div$ & -24 & \\
\hline 078 & 1 & 01 & Convert ratios of $x$ and $y$ to $0.1 \%$ increments \\
\hline 079 & 0 & 00 & and print \\
\hline 080 & 0 & 00 & \\
\hline 081 & 0 & 00 & \\
\hline 082 & $x$ & -35 & \\
\hline 083 & 1 & 01 & \\
\hline 084 & 0 & 00 & \\
\hline 085 & 0 & 00 & \\
\hline
\end{tabular}




\begin{tabular}{lrr}
086 & 0 & 00 \\
087 & - & -45 \\
088 & RND & 1624 \\
089 & PRTX & -14 \\
090 & RTN & 24 \\
091 & R/8 & 51 \\
\hline
\end{tabular}

Switch calculator back to Run after entering Program. Then record program on card for future use. 

APPENDIX B

NEW 1-K PROM FOR COINCIDENCE COUNTER ELECTRONICS PACKAGE

This document was produced by $J$. E. Swansen in conjunction with a new 1-K PROM that offers the operator of the HLNCC the capability of controlling the instrument by computer in addition to the previously used control methods, which were simple manual and recycling control modes. 
Issued: February 1982

\section{A New 1-k PROM for the Coincidence \\ Counter Electronics Package \\ J. E. Swansen}

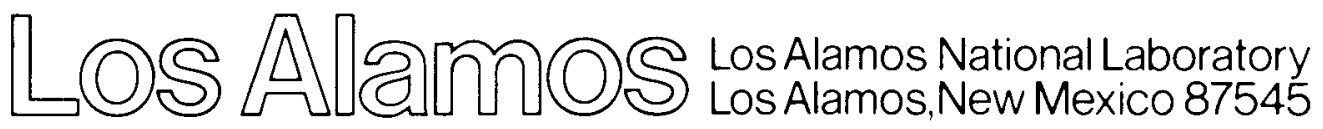


CONTENTS

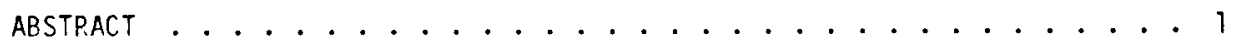

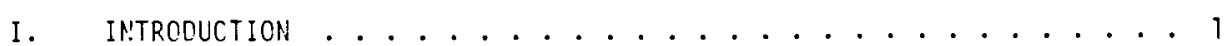

II. BRIEF CESCPIPTICN CF THE NEW OPERATING MOCES . . . . . . . . . . 2

A. Front-Panel Control Mode ................ . 2

B. Computer Control Mode .................. 2

c. Terminal Control Mode .................. 3

III. OPEPATINC INSTPUCTIONS FOP COMPUTER CONTROL MCDE $\ldots \ldots \ldots$

IV. OPERATING INSTPUCTIONS FOR TERMINAL CONTRCL MORE $\ldots \ldots \ldots$

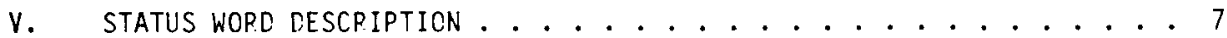

VI. ACKNOWLEDGMENTS ...................... 8

APPENDIX A: INSTALLATION AND ADDITICNAL DETAILS ON USE OF THE NEW PROM • . 9 APPENDIX B: MICROPROCESSOR PROGRAM FLCW CHART . . . . . . . . . . . 13

APPENCIX C: ASSEMBLY LANGUAGE LISTING . . . . . . . . . . . . . . 23

APPENDIX $[:$ FORTRAN-CALLABLE IRIVEP AND FOPTPAN TEST PROGRAM . . . . 37 
A NEW $1-k$ PROM FOR THE COINCICENCE COUNTER ELECTRONICS PACKAGE

by

J. E. Swansen

ABSTRACT

A new programmable read-only memory (PROM) for the Los Alamos-designed neutron coincidence electronics package is described. The new $1-k$ PROM allows remote control of the electronics by a computer or a remote terminal through an R.S-232 serial data port. No modifications of the existing unit are required.

\section{INTRODUCTION}

The portable high-level neutron coincidence counter (HLNCC), the active well coincidence counter (AWCC), and a variety of in-plant neutron counters have been accepted as useful tools for the nondestructive assay of fissionable materials. The electronics package used to operate these detectors consists of a high-voltage power supply, six amplifiers, a shift-register coincidence circuit, and four scalers. This package was designed at Los Alamos National Laboratory and is commercially available through IRT Corporation, San Diego, California.

Although the electronics package was originally designed as a stand-alone instrument with only a front-panel display, modifications were added after several users showed interest in controlling the instrument remotely. The instrument was first modified to send data to a Hewlett Packard HP-97 programmable calculator for local data processing. This modification was implemented by interfacing a small Motorola MC6800 microprocessor system to the existing hardware. In addition to providing data readout capability, this interface allows processor control of the basic hardware functions: start, stop, and reset. A recycle mode was added, and an $\mathrm{RS}-232$ serial data port was also 
provided for auxiliary data output. Pcth of these features are under the control of the MC6800 software. When originally implemented, the FS-232 port was set up only to output data, hut hardware was provided so that this port could later he used to input data. The new l-k programmable read-only memory (PROM) described in this report takes advantage of this provision. Remote control is accomplished by inputting ASC11 command characters and outputting data through the PS-1.32 port. Remote control is possible from any RS-232-compatible device, such as a stand-alone data terminal or a computer that has been configured to accept a second terminal.

Section II of this report provides a brief description of the front-panel, computer, and terminal control modes. The computer and terminal control modes are described more fully in Secs. III and IV. Installation of the new PR.OM and additional details on its use are given in Appendix A. Appendix B shows a flow chart of the microprocessor program, and Appendix C provides an assembly language listing. Appendix $r$ lists a FOPTPAP-Callahle driver and a FORTPAN test program to illustrate operation of the new PROM under computer control.

II. BFIEF TESCRIFTIO! OF THE NFH! CPEPATINC MCRES

A. Front-Panel Control Mode

This mode is the default mode when the pover is turned on. The frontpanel control mode eperates in a similar manner as it did with the previous PRCM, with minor exceptions as noted in Appendix $A$. The front panel controls the operation of the counter. Readout is directed to both the HP-97 calculator and the R.S-232 serial port. The instrument must be in the front-panel control mode to enter either the computer control mode or the terminal control mode. While hecoming familiar with the new PROM, the user can always return to frontpanel control by pressing the restart push button on the rear panel or by turning the power off momentarily.

\section{B. Computer Control Mode}

This mode allows remote control of the electronics package through the PS-i3? serial port. The serial port setup is described in Appendix $A$. The following functions may he controlled remotely. 


$$
\begin{aligned}
& \text { S - Start } \\
& \text { H - Stop (halt) } \\
& Z \text { - Clear scalers and timer } \\
& \text { R - Readout dato to computer } \\
& \text { P - Readout to HP- } 97 \text { caiculator } \\
& X \text { - Request status } \\
& \text { I - Set status interrupt flag (I flag) } \\
& \text { F - Return to front-panel control. }
\end{aligned}
$$

The control functions are achieved by transmitting the appropriate command from the computer to the electronics package. Computer control is designed for ease of control from the remote computer. The electronics package may be operated in a passive mode where information is returned to the computer only when requested or in a pseudo-interrupt mode where a flag (I flag) signals the computer when there is a change in status. Commands from the computer are not echoed back to the computer. All data sent to the computer are terminated by a carriage return/1ine feed (CRLF).

\section{c. Terminal Control Mode}

This mode allows a terminal to function as a remote front panel for the electronics package. The terminal control mode operates similarly to the computer control mode but provides more information to the operator. Commands to the electronics package are the same as listed above for the computer coritrol mode, but are all ASCII control characters (the CTRL key and letter key are depressed simultaneously). Commands echoed back to the terminal are preceded by $T$ and followed by CRLF. Invalid control characters are echoed in the same manner, but are followed hy a question mark and bell. A1l ASCII printing characters are echoed as is, allowing the operator to type identification or other text on the terminal without inadvertently interfering with operation of the electronics package.

\section{OPERATING INSTPUCTIONS FCR COMPUTER CONTPOL MODE}

To enter the computer control mode, transmit $\wedge$ from the computer while in the front-panel mode. The electronics package will respond by returning to the computer: CRLF version number CRLF. This is followed by an 8-character status 
word CRLF (see Sec. V). The electronics package then waits for one of the following commands from the computer.

$$
\begin{aligned}
& S \text { - Start } \\
& \text { H - Stop (halt) } \\
& Z \text { - Clear scalers and timer } \\
& \text { R - Readout data to computer } \\
& \text { P - Readout data to HP- } 97 \text { calculator } \\
& X \text { - Pequest status } \\
& \text { I - Set status interrupt flag (I flag) } \\
& F \text { - Return to front-panel control }
\end{aligned}
$$

All other inputs are ignored. Also, the electronics package will not echo command characters hack to the computer while in the computer control mode. Upon receiving one of the above commands, the electronics package will execute it with the following conditions.

(1) The I command allows the computer to be advised of changes in status. When the I flag is set, the next status change causes the electronics package to send a single character with CPLF to the computer. This character has a numerical value between 0 and 8 , representing the status bit that has changed. A numerical value of 0 indicates no status change; it is transmitted only after an H.P-97 calculator readout. The other status values are defined in Sec. V. Normally, the I command would be used only before a timeout or a data readout to the HP-97 calculator. The I flag is cleared after a status change. The I command must he sent again hefore the next desired status change.

(2) If the electronics package had previously timed out, it must be reset (7) before it. can be restarted (S).

(3) Readout commancis $P$ and $P$ are operable only when the scalers are stopper; otherwise, they are ignored.

(4) The $F$ command allows the computer to return control to the front panel.

Four types of data are sent to the computer:

(1) Version number: CRLF V 2.1 1? Dec 80 CPLF 110 characters). This occurs only once upon entering the computer control mode.

(2) I flag response: a single ASCII digit 0 through 8 CPLF ( 3 characters). This occurs only if the I flag has been set and there is a status change. 
(3) Status word: $x x x x x x x x x$ CRLF $(10$ characters). (See Sec. V for detailed description.) This status word is sent to the computer under the following conditions.

(a) Immediately after version number upon entering computer control mode.

(b) When requested by the $X$ command.

(4) Data String: time, totals, R+A, A CRLF (57 characters). Data are a11 numeric or space characters. This data string is sent to the computer if an $R$ readout command has been received by the electronics package when it is stopped. Note that $(P+A)-A$ is not sent in the computer control mode.

The format for these four types of data is given in Table I.

TABLE I

DATA FORMAT FOR COMPUTER CONTROL MODEa

Data Type

Total Character Count

Version Number:

CRLF V-2.1-12-DEC-80 CRLF

I Flag:

$x$ CRLF

$x=$ any value 0 through 8

3

Status Word:

$x x x x x x x x$ CRLF

$x=1$ or 0

10

Data Readout:

$x x x-x x x---x-x x x x-x x x-x x x-\cdots-x-x x x-x x x-x x x---x-x x x-x x x-x x x \quad$ CRLF

Time

Totals

$\mathrm{R}+\mathrm{A}$

A

ax denotes a numeric character; - denotes a space character.

bLeading zeroes are replaced by spaces. 
IV. OPERATING INSTRUCTIONS FOR TERMINAL CCNTROL MODE

The terminal control mode is used for remotely controlling the electronics package from a teminal only. It differs from the computer control mode in that the control characters are used as commands from the terminal to the electronics.

To enter the terminal control mode, type CTRL $T$ on the keyboard while in the front-panel mode. The electronics package responds by returning CRLF $V$ 2.112 Dec 80 CRLF and an 8-character status word CFLF. The electronics package then waits for one of the following input commands from the terminal.

CTRL S - Start

CTPL H - Stop (halt)

CTPL Z - Clear scalers, timer, and fault condition

CTPL P - Peadout data to terminal

CTRL P - Readout data to HP-97 calculator

CTRL $X$ - Request status

CTRL F - Return to front-panel control

CTRL I - Set status interrupt flag (I flag)

Valid commands are echoed preceded by $T$ and followed by CRLF. Invalid control characters are echoed in the same manner, but are followed by a question mark and bell. Printing characters do not affect operation of the electronics package, allowing typing of headings or other text on the terminal.

Upon receiving one of the above commands, the electronics package will execute it with the following conditions.

(1) The operator may be advised of changes in status, if desired, by setting the status interrupt flag (CTRL I) before any anticipated status change, such as completion of count time or calculator readout. (These two operations require time to complete; if the terminal is located some distance away, the operator will know when to continue.) When the I flag is set, the next status change causes the status character to be printed on the terminal and the $I$ flag to be cleared. See Sec. III, item (1), for additional details on the I command.

(2) If the scalers had previously timed out, the electronics package must be reset (CTRL Z) before it can be started (CTRL S). If stopped for any other reason it may be restarted with a CTRL S. 
(3) Readout commands CTRL $R$ and CTRL $P$ are operable only when the electronics package is stopped.

(4) The command CTRL F causes the terminal to return control to the front panel of the electronics package.

(5) The command CTRL $R$ causes data to be printed on the terminal in the following format.

Time, totals, $R+A, A,(R+A)-A$

Leading zeros are suppressed and digits are separated into groups of three by spaces for ease in reading.

V. STATUS WORD DESCRIPTION

The status word may be requested by the $x$ command at any time except during readout. It is an 8-character word, each character being either an ASCII 1 or 0 . Each character's position (1-8, reading from left to right) within the word determines its function, as shown in Table II. The status word is terminated by CRLF.

TABLE II

STATUS WORD DEFINITIONS

Character Positiona and Function

Status

1 - Run/stop

1 = stopped, $0=$ counting

2 - Time out

1 = count terminated by time out

3 - Fault

1 = fault condition set

4 - Stop push button

1 = button is depressed

5 - Reset push button

1 = hutton is depressed

6 - Start push hutton

1 = button is depressed

7 - Manual/recycle switch

1 = manual, $0=$ recycle

8 - Readout push button

1 = hutton is depressed

${ }^{\mathrm{a}}$ Character position within word $(12345678$ CRLF $)$. 
VI. ACKNOWLEDGMENTS

Parts of the microprocessor program described in this report were developed by M. S. Krick of LOS Alamos for the stand-alone version of the electronics package. S. Johnson provided the FORTRAN-callable driver and the FORTRAN test program. Discussions with N. Ensslin of Los Alamos were also helpful. 
APPENDIX A

INSTALLATION AND ADDITIONAL DETAILS ON USE OF THE NEW PROM

I. INSTALLATION

This operation requires no modification of the existing circuit board.

(1) Remove ac power cord from instrument.

(2) Remove top cover.

(3) Remove screw from clamp at rear of printed circuit board assembly on right side, then swing clamp back out of the way.

(4) Remove plastic pin from hinge assembly.

(5) Pivot board assembly to upright position and reinstall plastic pin to hold boards in this position. Save packing material, if any.

(6) Remove knurled screw and spacer on each side of board assembly.

(7) Unplug microprocessor board (rear board of three) from its connector.

(8) Carefully remove old PROM (located directly under TP1 at top of board) and place it in protective foam.

(9) Locate pin 1 of new PROM and plug it in with pin 1 nearest $C l$ on the board. Verify that all pins enter the socket properly.

(10) For reassembly, follow steps 1-7 in reverse order. Use care when plugging in the microprocessor board to prevent damage to the connector. If foam packing material is provided, reinstall it at the end of the printed circuit board assembly.

After installing the PPOM, verify its operation with the following procedure.

(1) connect HP-97 and charger.

(2) When the power is turned on, the readout light will turn on and the following test message will be loaded into the HP-97 storage registers R1-R4.

$$
\begin{aligned}
& \mathrm{R} 1=543210=\text { time } \\
& \mathrm{R} 2=9876543210=\text { totals } \\
& \mathrm{R} 3=9876543210=\mathrm{R}+\mathrm{A} \\
& \mathrm{R} 4=9876543210=\mathrm{A}
\end{aligned}
$$

The readout light will then turn off. Ignore the ERROR message displayed on the HP-97 calculator. 
II. DIFFERENCE BETMEEN CLC AM:D HEW PFOM: FR.CNT-PANEL CONTROL MODE

(1) The test message to the RS-232 serial port has been eliminated.

(2) The test message to the HP-97 calculator has been changed to include all digits $(0-9)$.

(3) Column headings for the data readout have been removed.

(4) The commas between groups of three digits in data readout have been replaced by spaces.

(5) A CL X is sent to the HP-97 calculator before data are sent.

(6) The manual/recycle switch on the front panel is tested at the end of the data readout, allowing the operator to terminate the recycle mode during readout.

(7) If the electronics package is connected to a terminal either directiy or indirectly through a computer, it will echo back any characters received from the terminal or computer while it is operating under the front-panel control mode. Exceptions are:

(a) $\wedge$ is not echeed but will transfer control to computer mode.

(b) CTRL $T$ is not echoed but will transfer control to terminal mode.

(c) CF or LF is echoed as both; that is, CRLF.

(d) Characters received during data readout are not echoed.

III. RS-232 SEPIAL INTERFACE REQUIREMENTS

Ful 1 duplex.

Baud rate: 300

Data format: 7 bits, even parity, 1 stop bit

Electronics package rear-panel connector: 25-pin "D" type DBM - $25 \mathrm{~S}$

Pin connections:

2. - Receive data from computer or terminal

3 - Transmit data to computer or terminal

$4-8$ ( 4 and 8 are tied together)

7 - Data common

All other pins: no connection 
IV. EXAMPLE OF COMPUTER INTERFACE

Shown below is an example of a Digital Equipment Corp. (DEC) DLVIl-J serial interface board configuration for use with LS1-11 computers. This is a 4-channel serial port board. Port 3 is configured for the console terminal; port 1 is configured to interface with the electronics package. (Refer to DEC publication "Memorys and Peripherals" for more details; see page 2-149 for port 1 jumper locations).

Baud rate selection: port 1 to $T=300$ baud

Data parameters:

$$
\begin{aligned}
& D \times \text { to } 0=7 \text { bits } \\
& S \times \text { to } 0=1 \text { stop bit } \\
& \mathrm{P} \times \text { to } 0 \quad \text { = parity enabled } \\
& \mathrm{E} \times \text { to } 1=\text { even parity }
\end{aligned}
$$

Figure A-1 shows the cable connections to the electronics package and the cable numbers of the Berg connector as viewed from the end of the board.

(a)

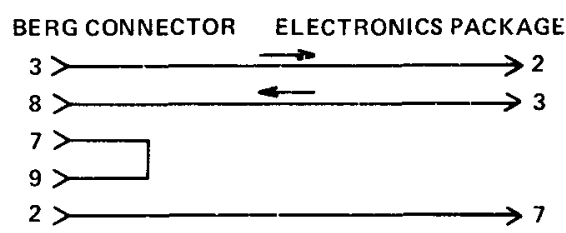

(b)

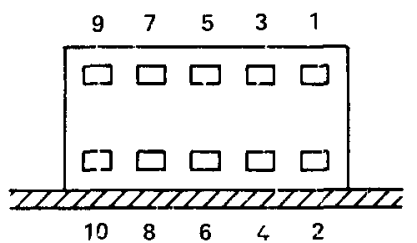

Fig. $A-1$.

(a) Cable between DLV1 -J interface hoard and electronics.

(b) View of Berg connector from end of board.

*Digital Equipment Corp., Maynard, MA 0T574 
d

d

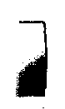

d

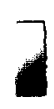

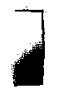

$\square$

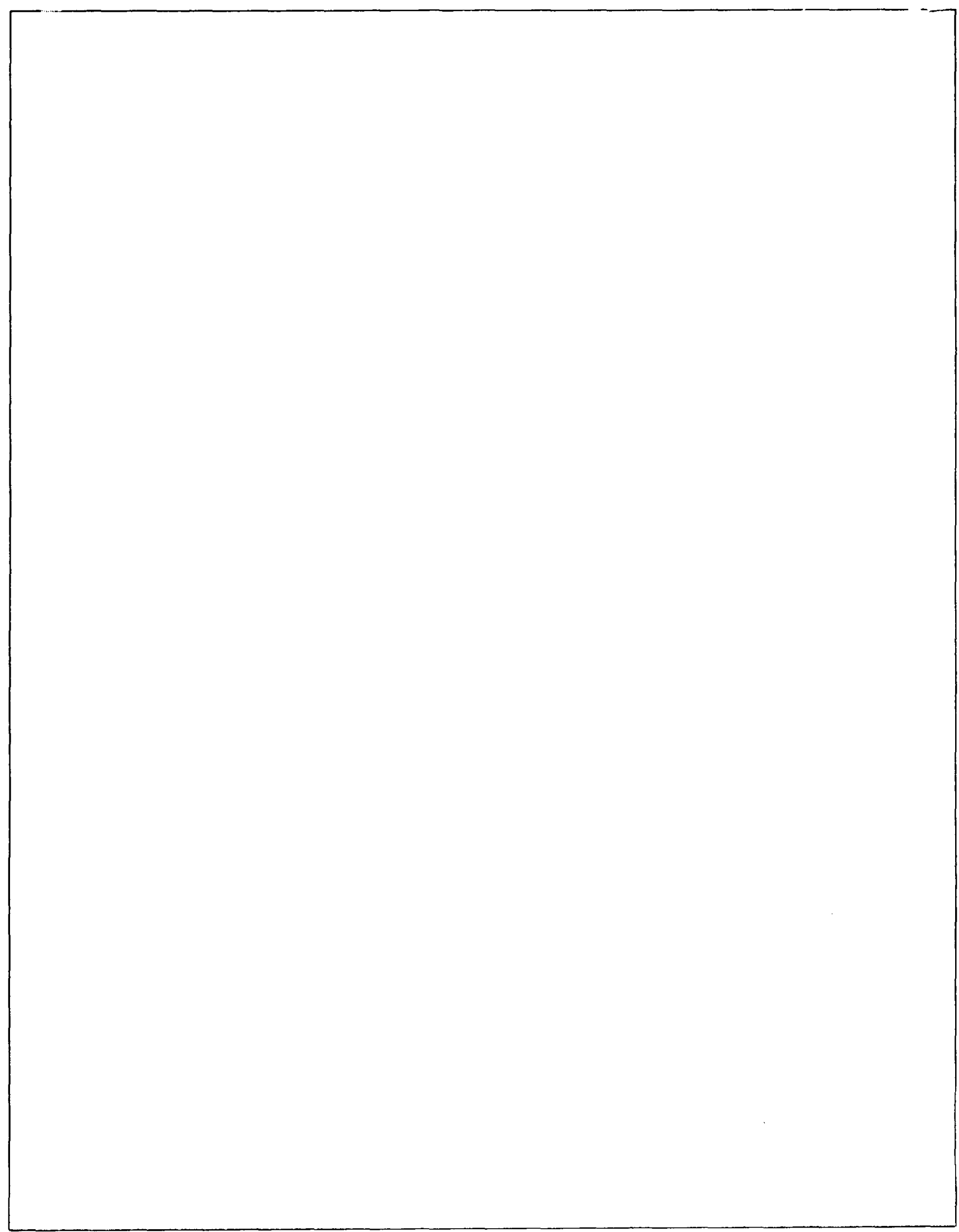




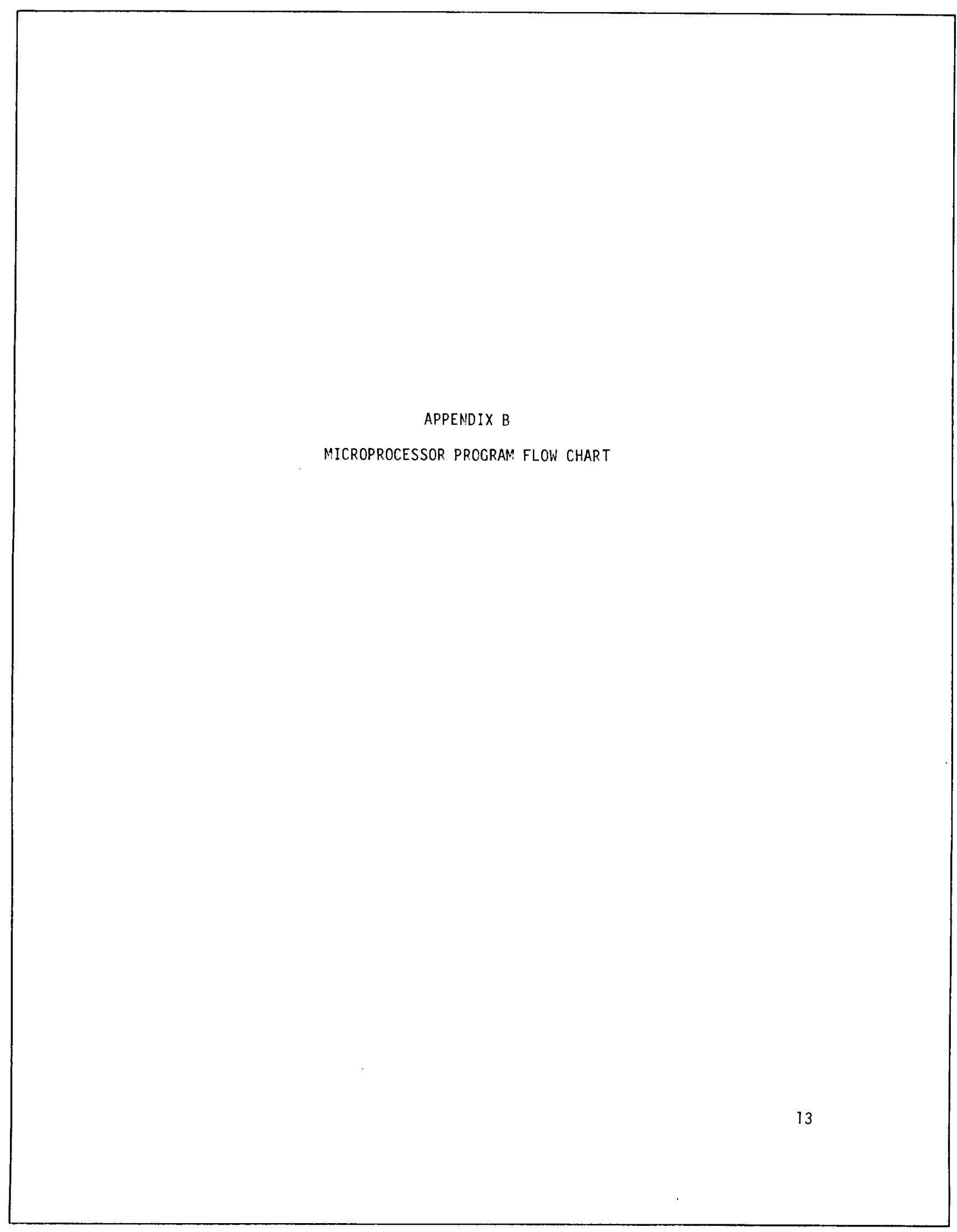




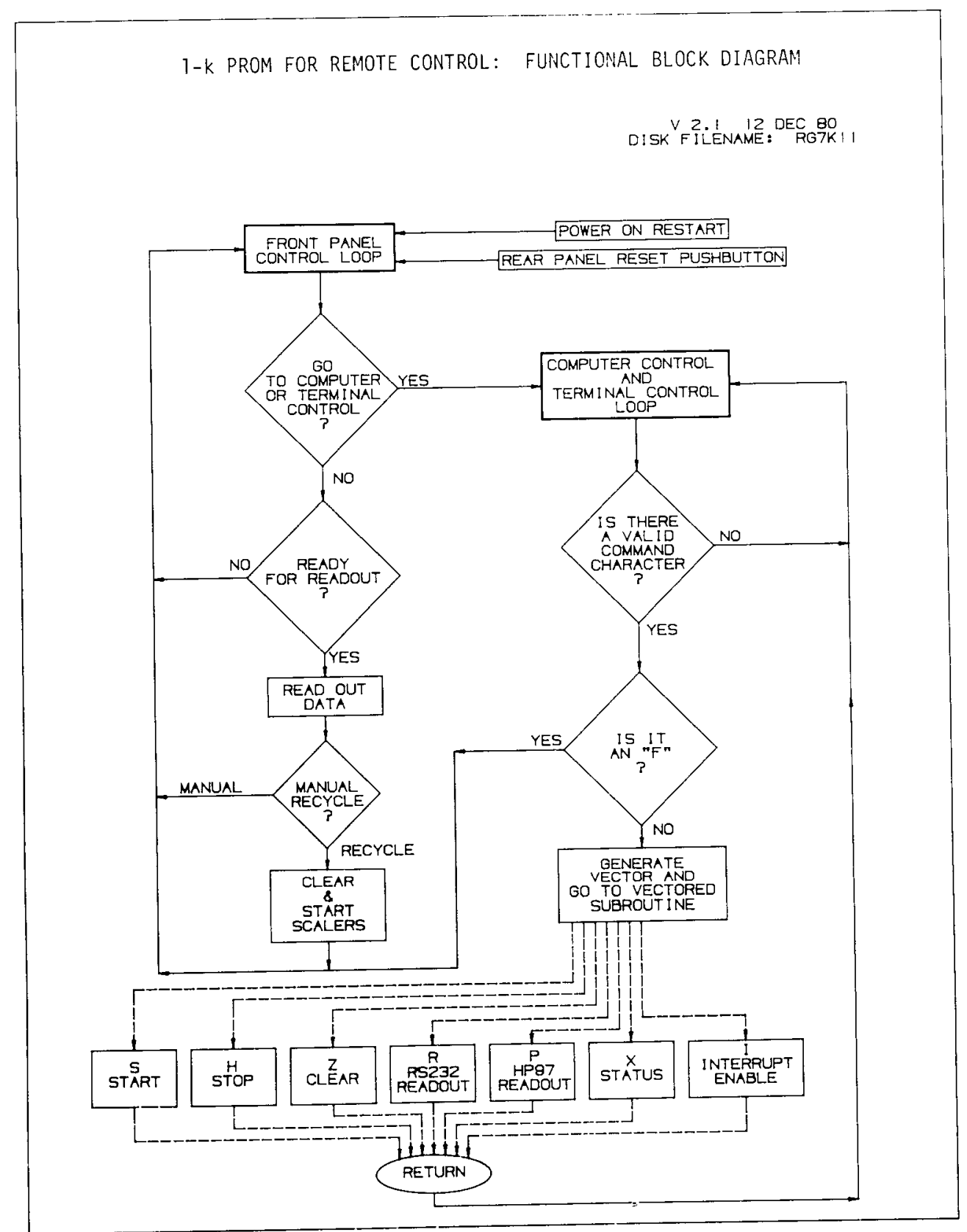

14 


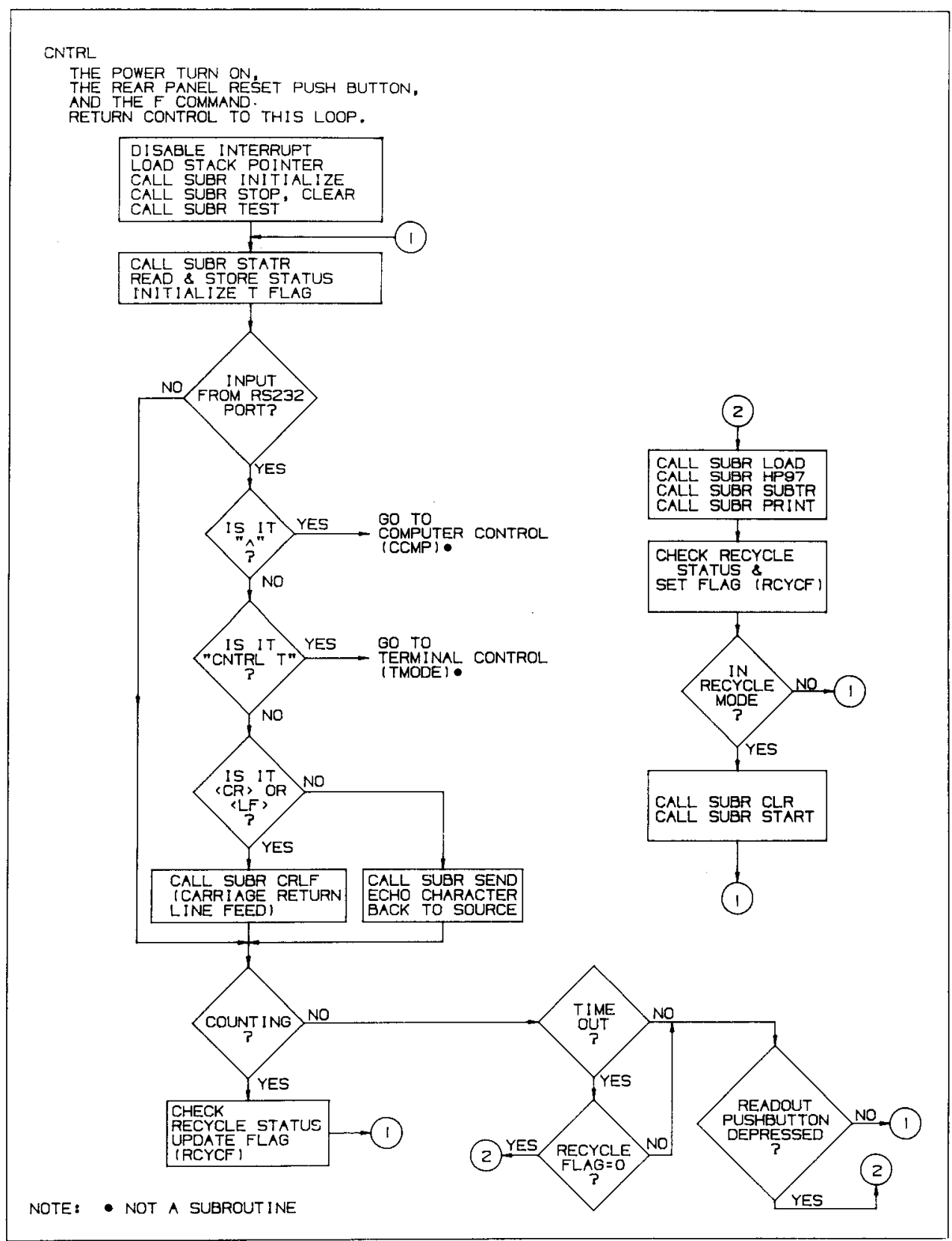




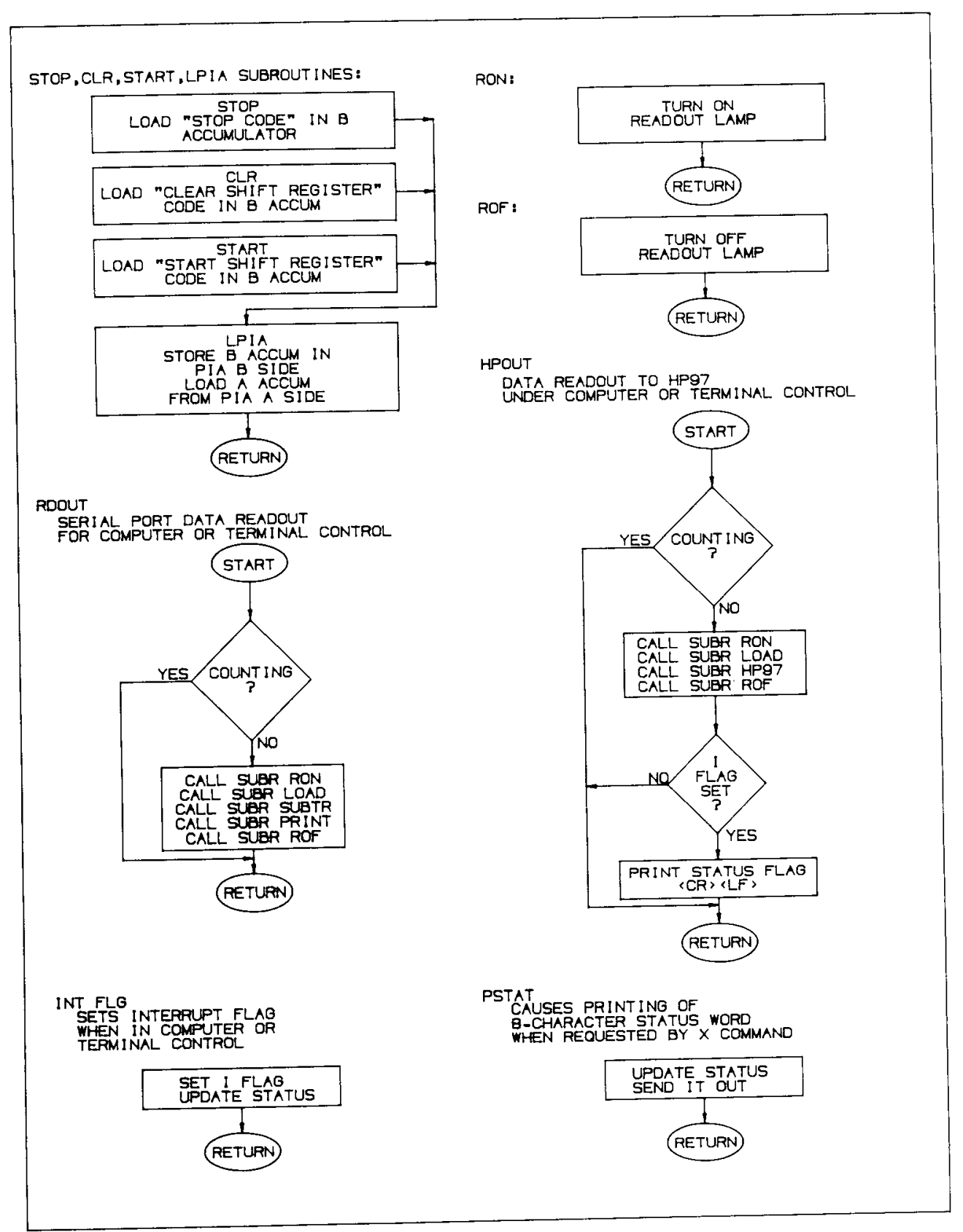

16 


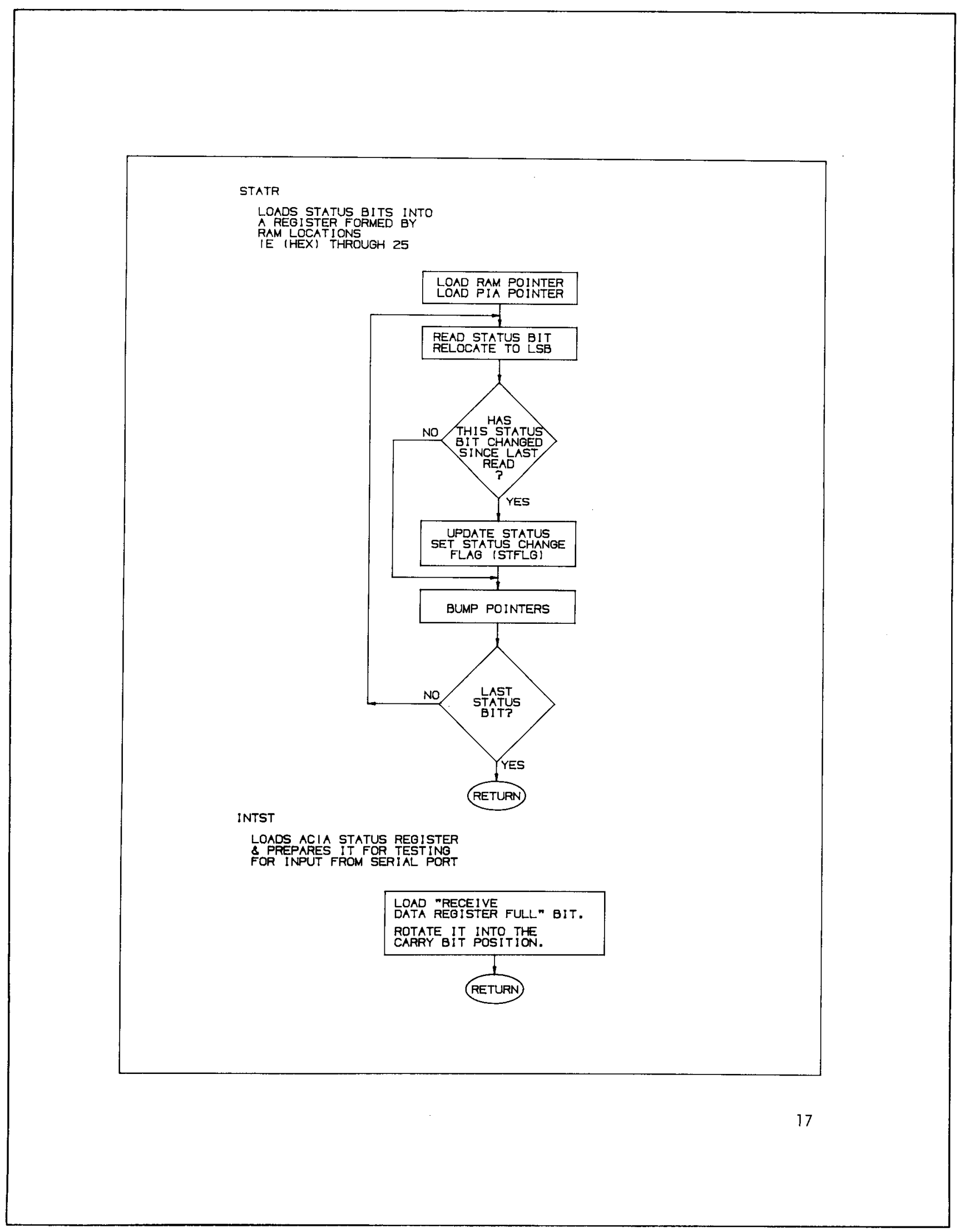




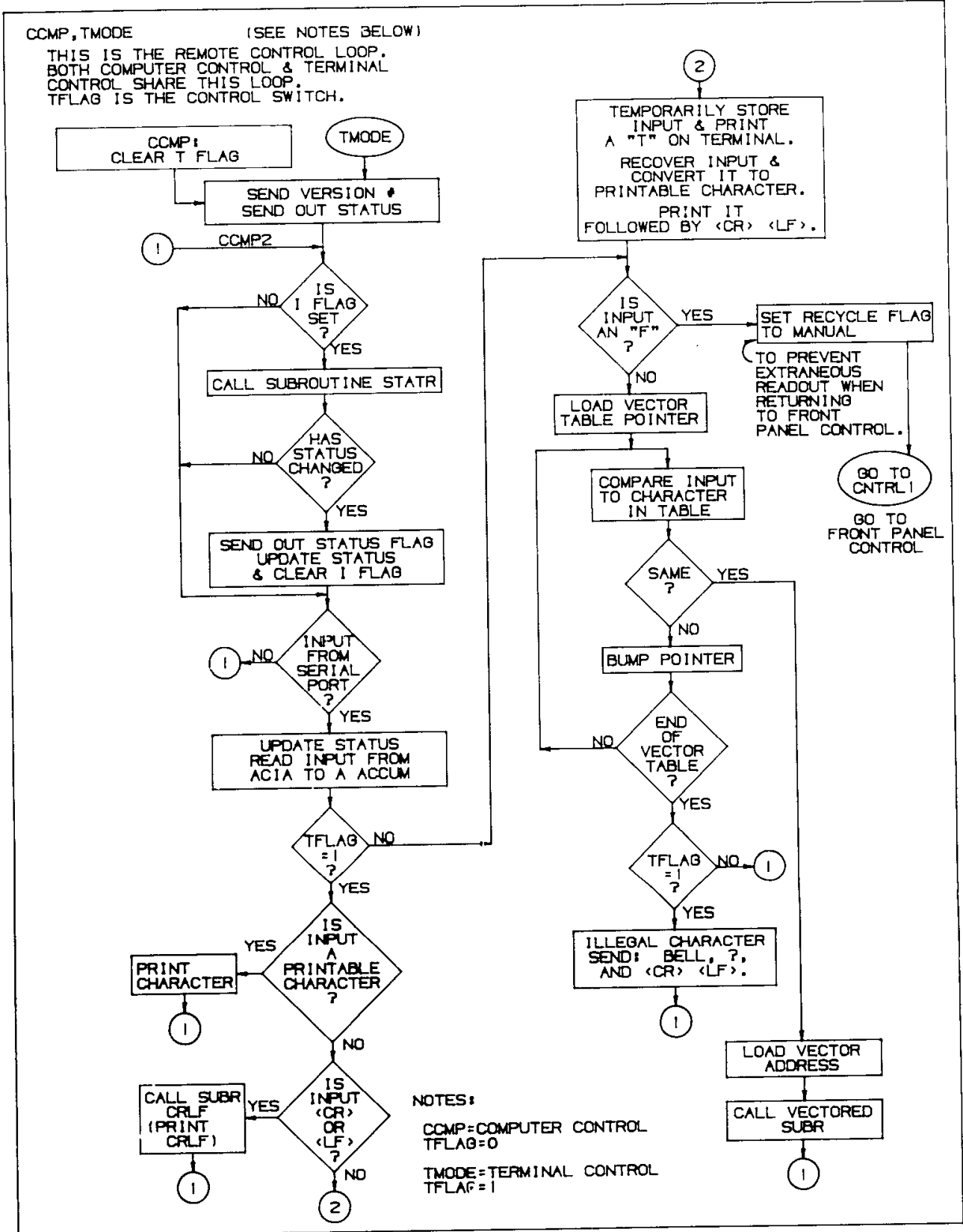

18 


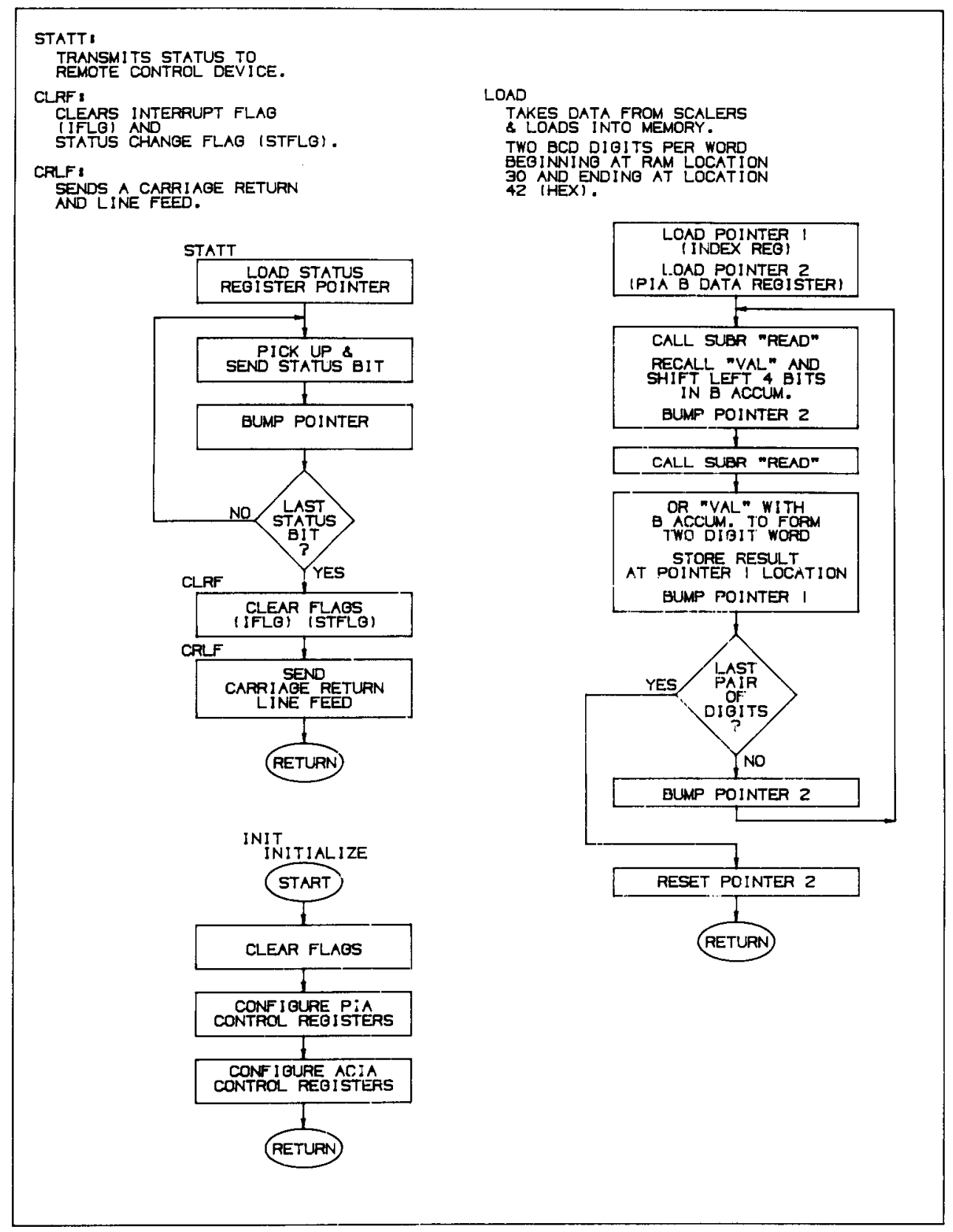




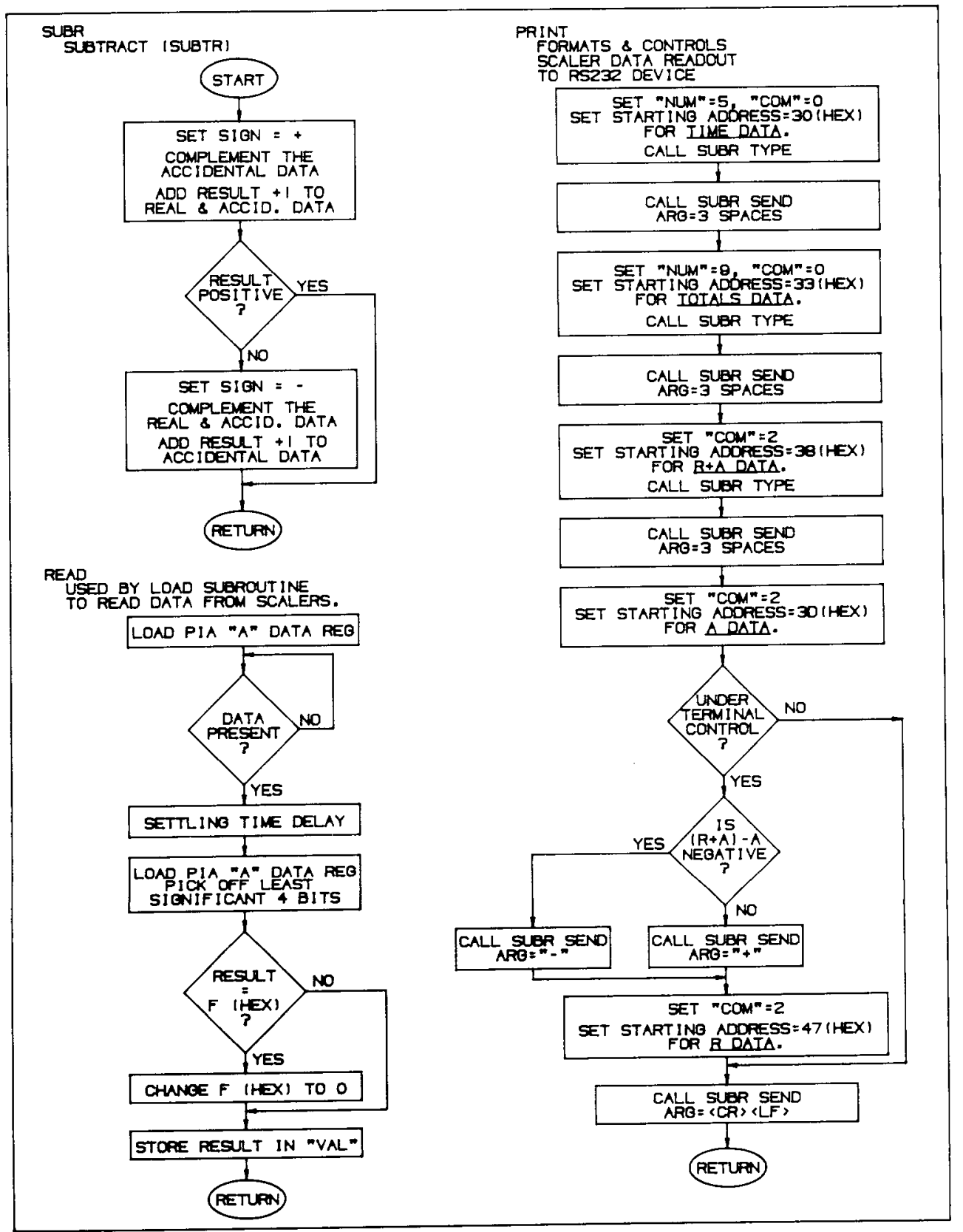




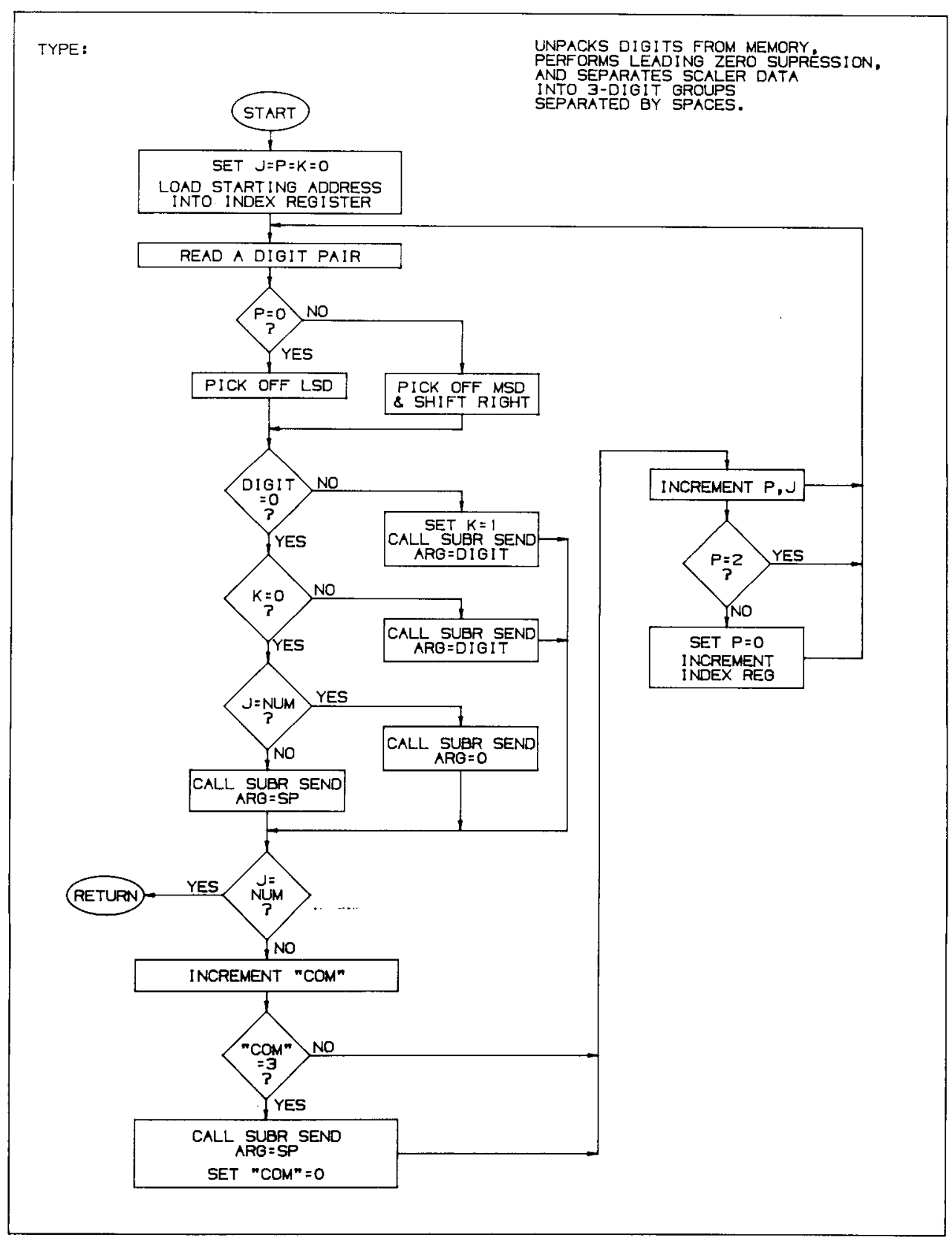

21 


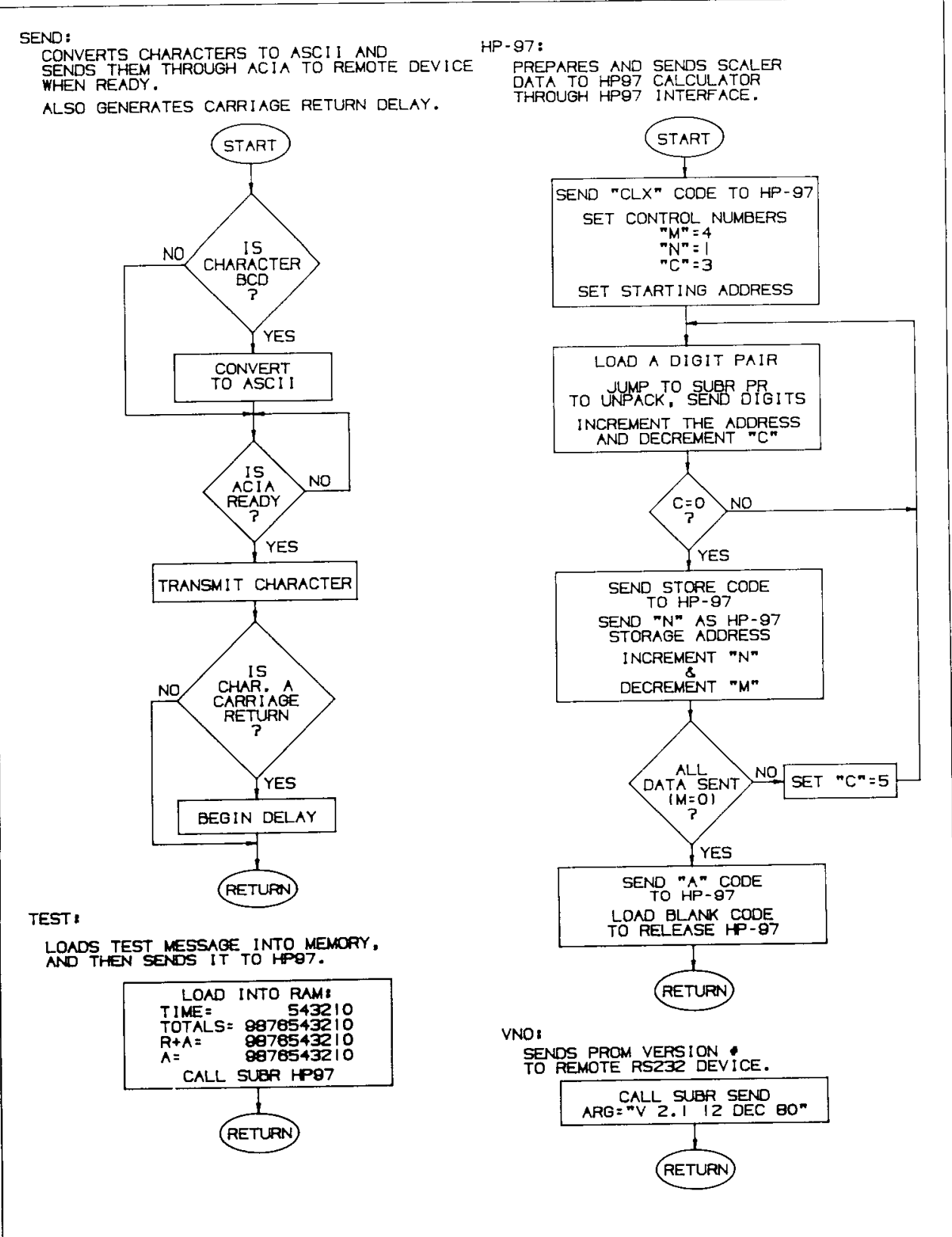

22 


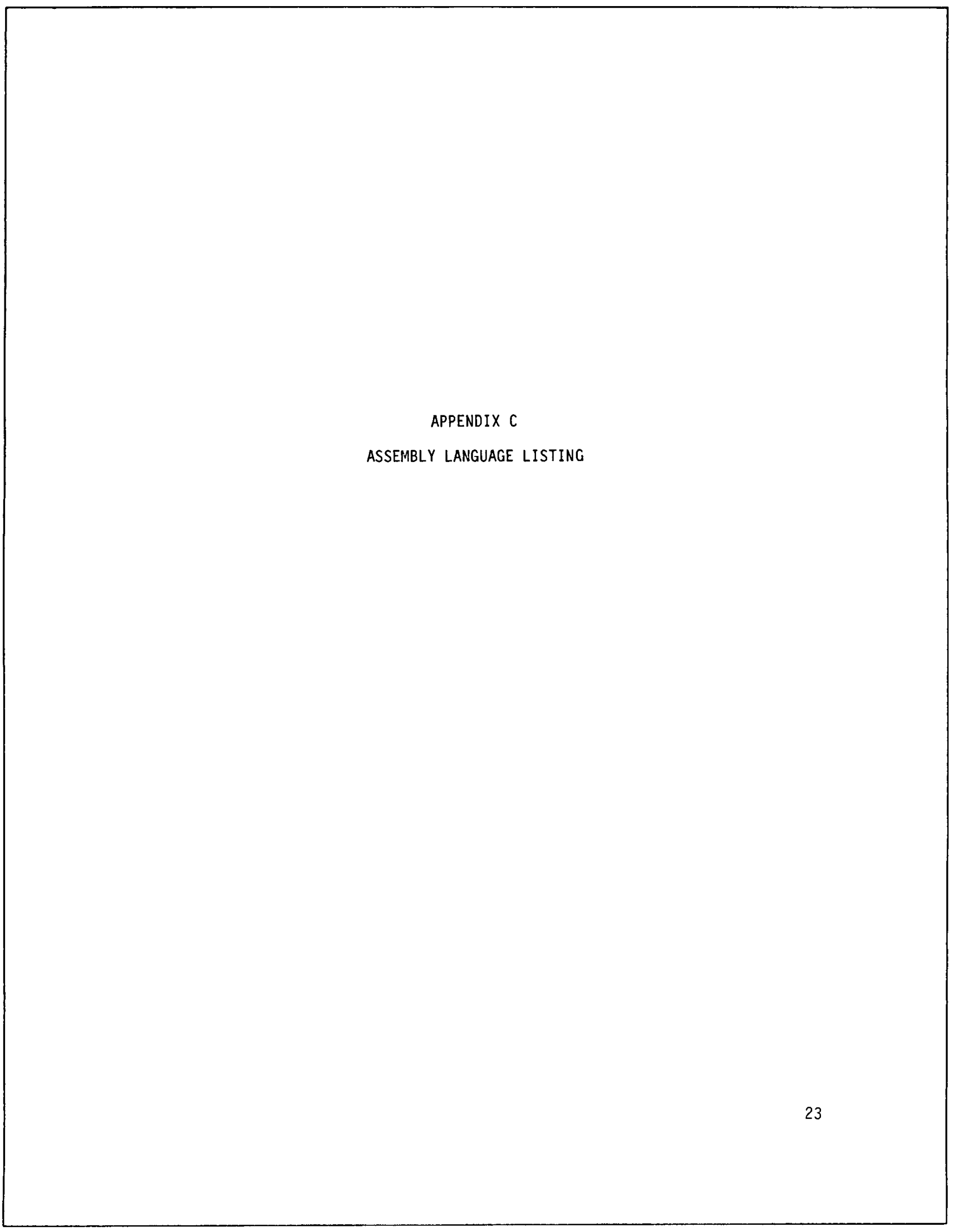




\begin{tabular}{|c|c|c|c|c|c|c|c|}
\hline FHIiE & 6121 & For 11 & $5 \ddot{H}$ & 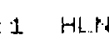 & & Los & lamos Identification No. LP-1358 \\
\hline 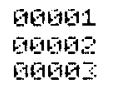 & & & & & 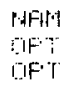 & $\begin{array}{l}\text { HLWHCI: } \\
6 \\
=\end{array}$ & 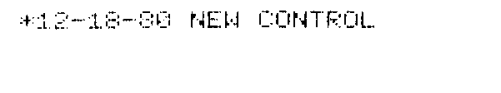 \\
\hline E151964 & & 50015 & Fi & FIAI & EDII & +5650 & :FIH A DHTA FEDISTEF FLDPE \\
\hline 619605 & & 50162 & $\overrightarrow{F l}$ & $F(F-1)$ & E1:1 & It1912 & AFIA E: LFTH FELISTEF ACCFE \\
\hline 매통 & & 50101 & H & FIFIË & E:D!l & 韦5101 & AFIA FA DOPTFOL FEG FODF \\
\hline 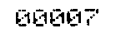 & & 5601 & $\overrightarrow{\mathrm{H}}$ & 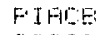 & Ely! & $\$ 560$ & 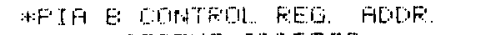 \\
\hline 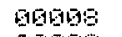 & & 461515 & $\vec{A}$ & HIIHS & Ë1 & 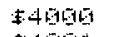 & :AFI IA STATUS FWDFESS \\
\hline 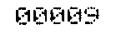 & & 4101 & $\mathrm{FI}$ & FII:TAT & Ei:li & \$4 401 & :AFIIIA TFEMUIIT ADFESE \\
\hline E16日1日 & & 40151 & $\bar{H}$ & AI: IAFE & ENI & $=45191$ & :FHLIA RECESWE AQEESE \\
\hline 101511 & & G19 F & $\mathrm{H}$ & $\Rightarrow$ Hith & EnI & $\$ i F$ & \\
\hline 먼ㄷㄴ & & GDEE & $\ddot{H}$ & FEAF & E1! & $\mathrm{E}$ & 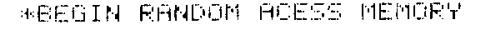 \\
\hline G611: & & & & ; & & & \\
\hline 1014 & & & & :t:it:it:if:at:it & $+\div+5+\cdots+1+4$ & 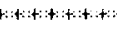 & 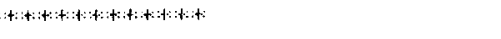 \\
\hline 101915 & & & & :t: & & & \\
\hline EDS1EA & 1950E: & & & & DFig & FAHE & 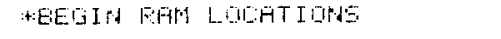 \\
\hline DEN17A & GEAGE: & $51+1$ & $\mathrm{H}$ & [St.' $T$ & FIIE: & 1 & \\
\hline BDE-1:AF & 155151 & 01512 & A & $\because F L$ & RME & 1 & \\
\hline ज1E1 191 & 1 Eâ0 & 범데 1 & $\bar{H}$ & $\because$ I Ijd & FWE & 1. & 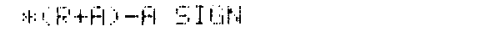 \\
\hline EBLEЕ & GEQE & 9101 1 & $H$ & M.⿲丿丨子⿴囗十 & FWE & $t$ & \\
\hline 트를 $\mathrm{A}$ & SEEF & G651. & $A$ & $10 n$ & FWE & 1. & \\
\hline BE1EZZF & 0016 & 4040 & $\vec{H}$ & $F$ & FME & 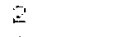 & \\
\hline Q1613 $2 A$ & 01512 & DE61 & $H$ & .7 & FilE: & 1 & \\
\hline $00124 \mathrm{~A}$ & E1E1S & Q615:1 & F & $\Leftrightarrow$ & FME: & 1 & \\
\hline EQ1E2SH & 1014 & 6101 & 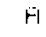 & $F$ & Fin: & t. & \\
\hline EQ1日2B & 6015 & 5051 & $\vec{H}$ & DELI & FHE & j. & 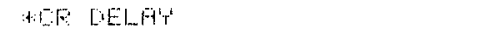 \\
\hline EEAECPA & E1E1E & 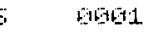 & $\mathrm{A}$ & TELE & FUHE & 1 & \\
\hline 010129H & 1917 & 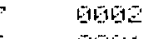 & Ft & $F:$ & Erre & 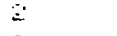 & \\
\hline GE1 & A1013 & 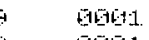 & $H$ & $T$ & FUlE: & 1. & \\
\hline 501526R & Div1t & 51561. & A & $M$ & Folli: & 1 & \\
\hline $06131 \mathrm{H}$ & E1E1E & DET1. & 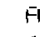 & $t:$ & FEIME: & 1. & \\
\hline G日G & 1910 & 06151 & $\vec{H}$ & $n$ & Fris & 1 & \\
\hline 501521 & 8010 & E1411. & $\ddot{H}$ & TEP & FWE: & 1. & \\
\hline 9615 4 F & QD1E & BE1 & $\mathrm{A}$ & Fum & FINE & 1 & 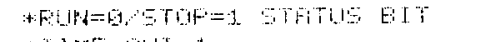 \\
\hline 머먼둔 & EG1F & EAㅓㅓㄴ 1 & $\mathrm{~F}$ & TC111T & FINE: & 1 & H. I WE INIT =1. \\
\hline BQELEEA & 51520 & 51501 & $\bar{H}$ & FAIILT & 5145 & 1. & $: A F F \mid H_{-} T=1$ \\
\hline GE19ZR & E162 21 & E1E151 & $\vec{F}$ & $\because$ GIFE: & FWE & 1 & 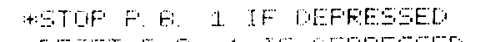 \\
\hline 5016 $3 \mathrm{H}$ & 5192 & E161:1 & $\overrightarrow{\mathrm{H}}$ & $F E S F E$ & FME: & 1 & AFEST F E 1 T PFFESEO \\
\hline 610199A & 0623 & E1101 & $\overline{\mathrm{H}}$ & $\therefore T H F E$ & FHE: & 1 & AOAFT FE I IF CEFEEEE \\
\hline 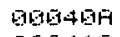 & 0024 & Q151 1 & A & F'L'T': & $\mathrm{FH}$ & 1. & 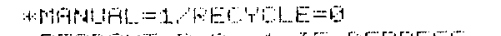 \\
\hline 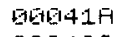 & E162:5 & Eget 1 & $\mathrm{Fi}$ & FOFE & FIIE: & 1. & +FEFWLIT F $E$ I IF LEFEE \\
\hline DQE142A & 1026 & E15151 & Fi & $\Xi T F L D$ & FNE & 1 & WETATUS CHAHAE FLFIL $=1$ \\
\hline E1E1녚 & EE2? & 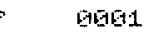 & $\bar{H}$ & F:C:T'CF & ETIE: & 1. & AFEELTLLE FLFIE \\
\hline $515644 \mathrm{H}$ & 밀 & 0101 & F & TFLATI & FF' & 1 & 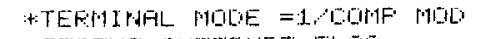 \\
\hline BGEASIF & 1009 & E1501. & $\ddot{H}$ & IFLG & FHE: & 1 & :T:STHTIS IPTEEULFT FLALI \\
\hline 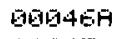 & ब12A & 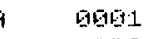 & H & IHAF & FWE & 1 & 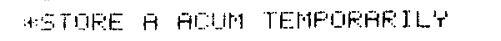 \\
\hline G1647 & & 10120 & $\bar{H}$ & F:HA & ELII & +50 & \\
\hline 60158 & & 6130 & $\vec{F}$ & $\Xi \Xi T$ & EO!! & +5 & \\
\hline 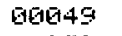 & & & & $: 4$ & & & \\
\hline Q19E5 & & & & :t:it:it:t:t:t & 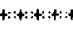 & $4:+\div:+\div$ & $:: t: i t: i t: t: i t: i t+$ \\
\hline E1E16.51 & & & & $:$ & & & \\
\hline 10652 & $7 \mathrm{FE}$ & & & & IIFI & TPFE & AFESTHET RLEESE \\
\hline $\begin{array}{l}\text { Q615:A } \\
\text { 610054 }\end{array}$ & FFE & $\overrightarrow{0} 2 \theta$ & $\vec{H}$ & $\begin{array}{l}F: D T: T \\
:\end{array}$ & FDE: & EATTEL & 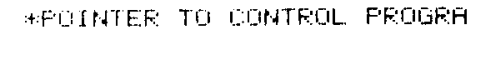 \\
\hline Q90655 & & & & 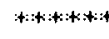 & 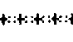 & $t:: t: t:: t:: t:: t$ & 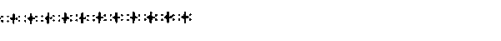 \\
\hline 6165 & & & & *: & & & \\
\hline 0095\% & 7 G日a & & & & GFI & 79001 & \\
\hline 터도 & PEDEG & 5 & $\mathrm{H}$ & UTELL & $F[: L$ & $\therefore=$ & \\
\hline
\end{tabular}




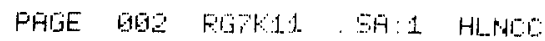

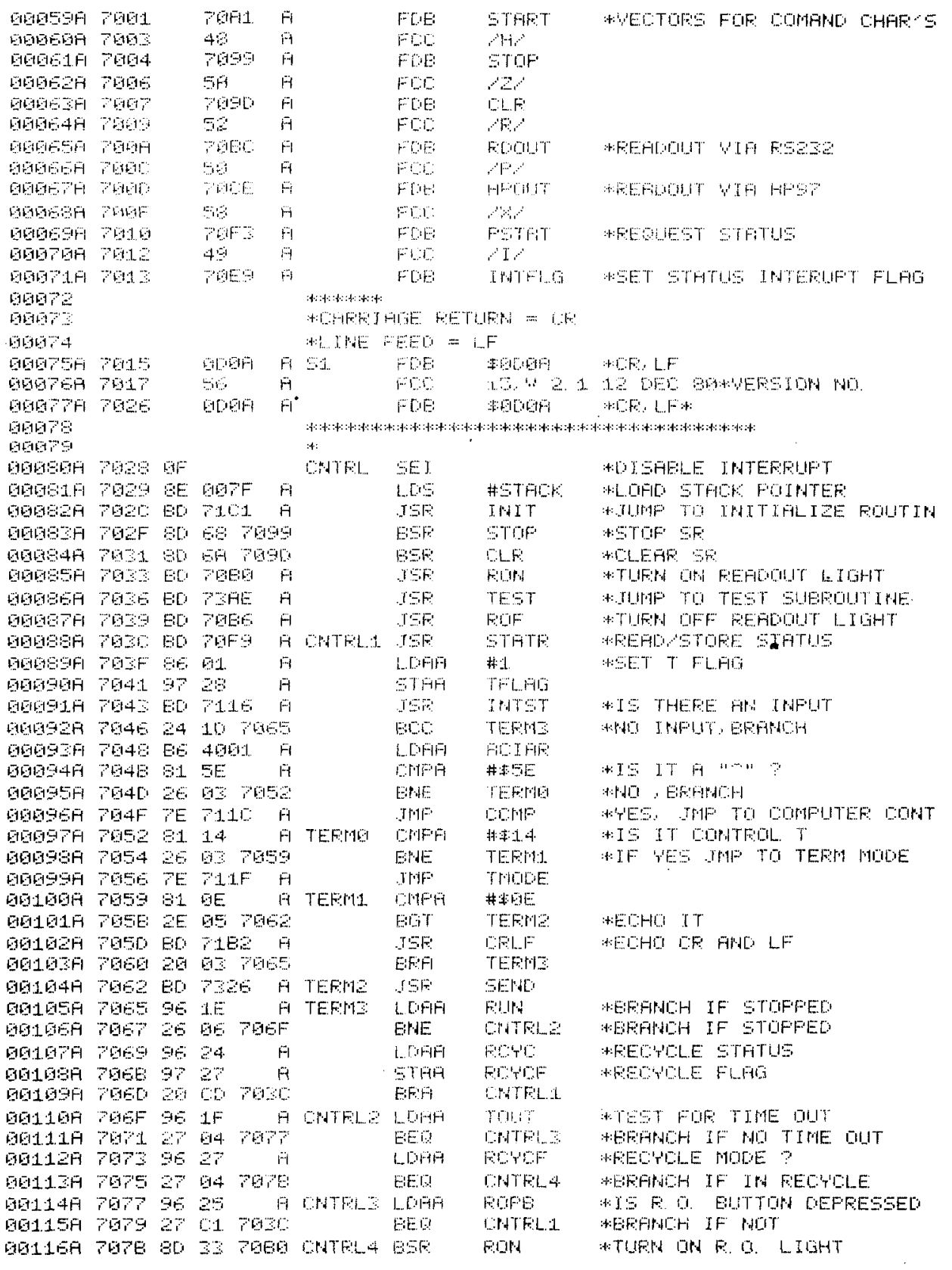




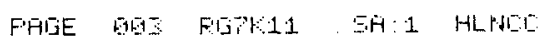

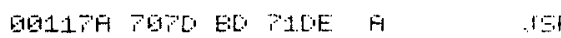

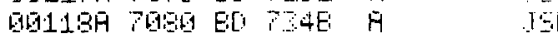

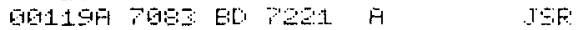

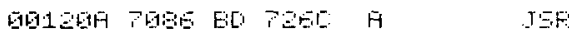

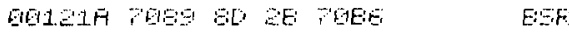

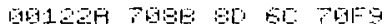

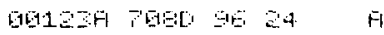

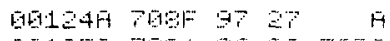

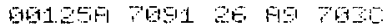

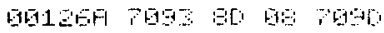

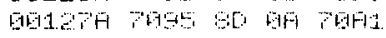

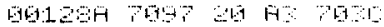

Q1912:-

WG13H

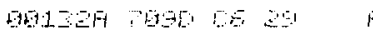

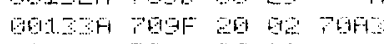

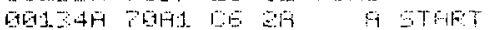

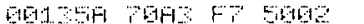

Q192.

HEA

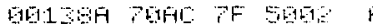

DU1 $39 \mathrm{~F}$ TEFF

610.48

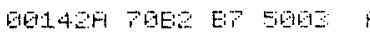

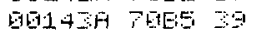

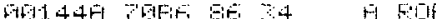

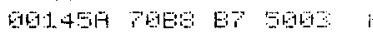

OIIASF NEE

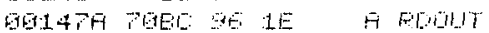

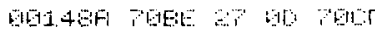

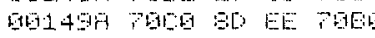

DELEF

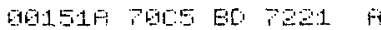

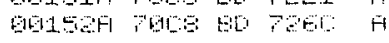

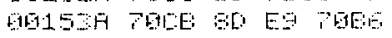

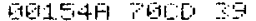

91155

EE

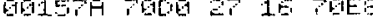

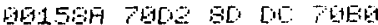

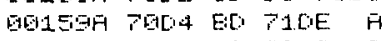

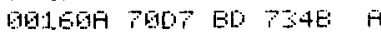

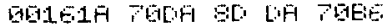

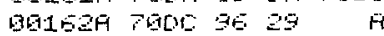

GOLESA TOOE 27 GO TOEO

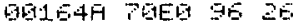

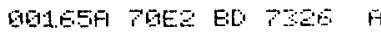

DQ1DEA 7 GES BD $71 \mathrm{AD}$ A

GIELFA PGEE

므넝

$F F$

L.DPFIF

$\because$ TAF

EWE

E:F

E:EF:

EFFH

LIITL

$\mathrm{HF}+9$

EIIETF:

FPIYT

FUIF

STHTF:

Fil:- $r$ 'L.

OCIOF

EATFLLI

i. $\%$

$\because$ HFT

EnTHEA

DFEHC THE EILEFE

*ENO FESLLS TO HF-S

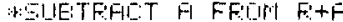

AFIFITT RESILTS

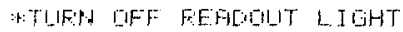

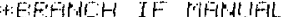

ALEEAR AF

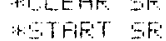

LPE HIE

EFFA $\quad \mathrm{WIF}$

LDEE \#FE

EEFG LF IF

\#5E

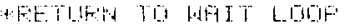

HF IA GTH OME

AF IF ARE GHE

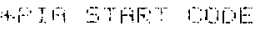

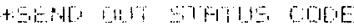

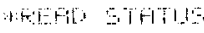

A

:FFEE TIIFAP

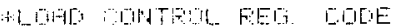

FETd IT OTIT

IFT:TH

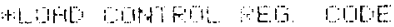

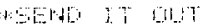

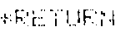

Fi:

L.C.PHA

EE:

Sar

I $\because$ rit:

lon

$y=6$

:t::t::t

HFEIT LEHA

SEE

BEF

ISF

ESF

LDAFH

EEO

LDPHA

ISE

Fits

Fin

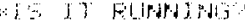

I a t

Fint

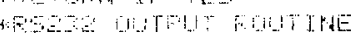

1.10

FF: ind

Filf

Fils.

HFOili:

Find

LIATE

HFO?

HOH

IFLE;

HFLIIT 1

STFLI

SEASC

HFOIITI FITS

E:LF:F

TI STFTIS INTEELAFT FLFIJ

IT IF MUT EFPHAH

AFFINT STATIS FLAL

it:at::t:

GNIEOA PEES EE DL A INTFLO

G0176A 7 BEE 9729

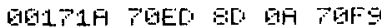

DQ172A TGEF $T \mathrm{~F}$ BQ2E $A$

G017ZA PEFE

THA

EEF:

\#1.

IFL

STHTF:

50174

FT: 


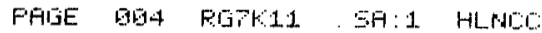

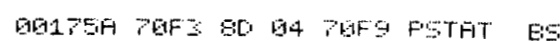
GS17EA TEFE EQ $71: F$ H

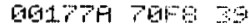

61. 18

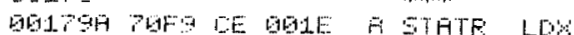

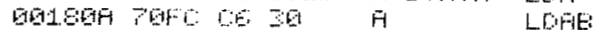

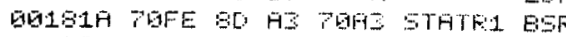

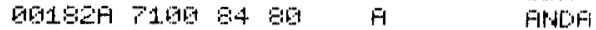

DEISER PIE2 49

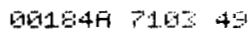

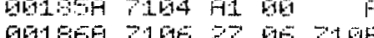

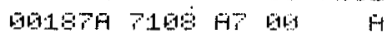

GQ1EEA 7.LAF SC:

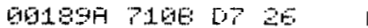

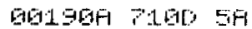

BO191A 71 1EE 50

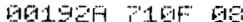

015193A 7120 aD 01625

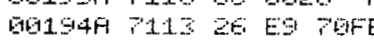

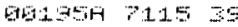

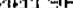

9

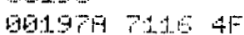

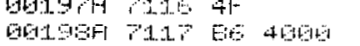

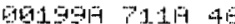

जUसमी $711 \mathrm{E}$

ज1: 11.

160

6020

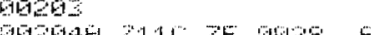

GE

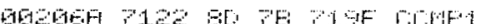

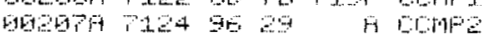

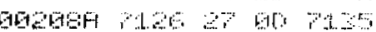

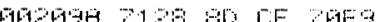

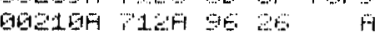

ब0211न 720 \%

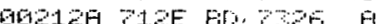

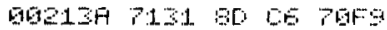

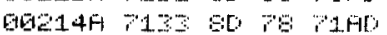

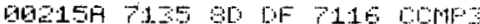

EQ1EA 713724 EE 724

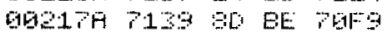

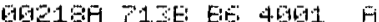

Q6219H $712 \mathrm{E}$ BE 4061 F

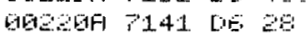

$90201 \mathrm{H} 714 \% 2 \%$

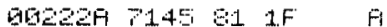

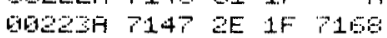

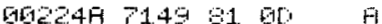

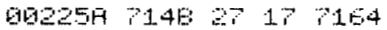

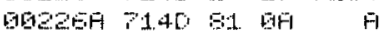

ब19227A $714 \mathrm{~F}$ 27 137154

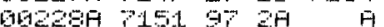

90229A 7152 35 54

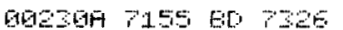

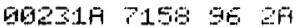

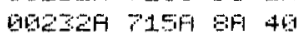

जTFTF:

STHTT

粆10.

\#1:

LFIF

\#*6

E. $\%$

जTATRE

B.

ETFLI

जTHA INAE

dilis:

I.

EHE

F.?

IAT

CLIPA

L. DFFi

Fin

FT:

:

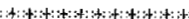

E:F

Loffit

EE:

9

L. I.FAT

EED

$T S F$

BO

ESF:

Baf?

Ei:

ESF

L..DFIF;

L.DAH

L. [NFE:

EIE

CPFFI

EIST

IIFA

$\mathrm{BED}$

CIFA

EE

$\therefore$ TFF

LDAFA

TSF

$\mathrm{LOFHH}$

Carifir
PFL.

प

S. F FT

T. 10

ante

जHA

GTFl.

101

$\because$ FPI

जTA7

C.L.FF

INTST

r. CONF=

Ут̈̈ту:

FiC IFIF:

FII: IFIF:

TFLFIO

FOAPE:

\#1.F

INPF

\#娄

CTPP 4

\#:DFन

LNP4

GHAF?

\#楼5 4

SEPD

OHA

粉4
T:FEQUEST STATLS

WLDFO FEM 57 TTUS FOINTEF :LOFIO PIA STHYIIS FOINTEF:

HNFIS FLL EITS EUTT 7

THOWE TO EIT

WIS EIT SAME FE BEFDEE

A:EFARALH IF SATHE

:F GTORE IF PIOT

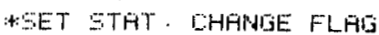

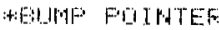

W HET STHTUS EIT?

FEIAT WEFSIOH W

TFFEINT STATLIS

+IS STFTIS INTERUFT SET?

AEEFAlOH IF PHIT

WFEFO STOEE OTATLS

WHFE STATUS CHAMUED?

*IF HI EFHAEH

\#IF 'TES GEND OUT STFTLS FL

WFELUTHART STATUS FEFO

WFE DIMALANT LIOAO

:LOFFC IPAFUT

*IS IT FEINTINL DHFEO

:AEFFNOH IF 'TES

*IS IT FI I:F?

WEPANDH IF TES

iI I $I T$ F LF?

$: T=$ TEFWINAL MDOE FRIMPT

WFTFEE FFIATTPHO CHAF 


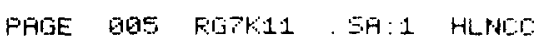

\begin{tabular}{|c|c|c|c|c|c|c|c|c|}
\hline $\begin{array}{l}01233 \mathrm{~A} \\
019234 \mathrm{~A} \\
61225 \mathrm{~F}\end{array}$ & $\begin{array}{l}7150 \\
715 F \\
7152\end{array}$ & $\begin{array}{l}E D \\
E 0 \\
20\end{array}$ & $\begin{array}{l}720 \\
7192 \\
99716\end{array}$ & $\begin{array}{r}\mathrm{H} \\
\mathrm{A} \\
\mathrm{E} \mathrm{C}\end{array}$ & & $\begin{array}{l}\text { TSF } \\
\text { J'SF } \\
\text { ER'A }\end{array}$ & $\begin{array}{l}\text { SEHL } \\
\text { EFLLF } \\
\text { CEMPFE }\end{array}$ & $\begin{array}{l}\text { *FEINT IT } \\
\text { *ELHQ IE FND LF }\end{array}$ \\
\hline EQ123EA & $7: 164$ & $B D$ & $41: 71 E$ & $E$ & $\mathrm{ENMF}_{4}$ & ESF & IFLF & \\
\hline घ182こ7A & $P L E$ & 20 & EI: $\quad 712$ & & & EIFAH & E:CPHE & \\
\hline $0 B 2 Z B A$ & 7160 & $\mathrm{ED}$ & 7326 & $\vec{H}$ & LINPS & T5E & SENO & \\
\hline 브로 & PLE: & 20 & $E F \quad 12$ & & & E:R:H & LOWF & \\
\hline B日E-4BA & PIEO & 81 & $4 E$ & $\mathrm{H}$ & COMFE & EMFA & \#\#官 & : IS IT A "F"? \\
\hline 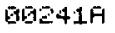 & PLEF & 28 & EF 7 & & & EREE & Y'EET & :IF HO EFAHE:H TE VELT \\
\hline $01042 H$ & 7171 & 36 & 01 & $\mathrm{~F}$ & & LDFFA & $\# 1$ & \\
\hline 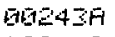 & 7175 & 97 & 27 & $\ddot{H}$ & & ЕTAH & FE:'I'CF & \\
\hline $96244 F$ & 7175 & $P E$ & 7615 & $\mathrm{H}$ & & IPIF & CWTFLL1 & 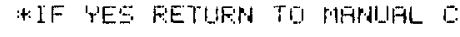 \\
\hline 161245 & & & & & $:+: *: 4:$ & & & \\
\hline GEZAEF & 7178 & EE & 7650 & A & WET & $1 \ldots \%$ & \#VTEL & :LDAL UELTOF FOINTEF: \\
\hline E1B24 $4 A$ & $P 17 \theta$ & F1. & det & Fi & YET:T 1 & E:FF'F & Q. ' 'c' & \\
\hline DE24BA & 7170 & $2 \vec{r}$ & $1 \mathrm{Fi} \cdot \mathrm{F}$ & & & BEO & WEETS & : IF 'T'ES LUFE 'VELTOR \\
\hline $00249 \mathrm{~F}$ & P17F & 89 & & & & IPd: & & *IF NO EUNF FOIMTEF: \\
\hline $00250 \mathrm{R}$ & 7150 & 98 & & & & $\operatorname{IN} x$ & & \\
\hline $101251 \mathrm{~A}$ & $718: 1$ & 98 & & & & IPA & & \\
\hline $\begin{array}{l}00252 \mathrm{~A} \\
00252 \mathrm{~A}\end{array}$ & $\begin{array}{l}7182 \\
7185\end{array}$ & 80 & $\begin{array}{l}7015 \\
\mathrm{~F} 4717\end{array}$ & $\begin{aligned} \mathrm{H} \\
\mathrm{H}\end{aligned}$ & & EF'X & $\begin{array}{l}\text { \#VTEL + } 21 \\
\text { VETTL }\end{array}$ & WLESET DNE ? \\
\hline $90254 A$ & 7187 & 96 & 28 & $A$ & & L.DÄA & TFLAT.j & \\
\hline $\begin{array}{l}90255 F \\
\text { 60255 }\end{array}$ & 7189 & $2 \vec{r}$ & E1: 719 & $\vec{i}$ & :ACKRIIL & $\begin{array}{l}\text { EED } \\
\text { ALEBE }\end{array}$ & $\begin{array}{l}\text { 'YEC:TZ } \\
\text { ILLEEGHL L:HH }\end{array}$ & 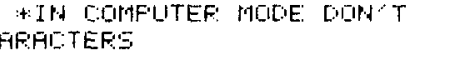 \\
\hline $912 \div 59$ & 7188 & $\theta \vec{\theta}$ & $\theta 7$ & $\bar{H}$ & & L.D:FF & $\# \$ 07$ & \#FIEI:I I "EELL." \\
\hline D1925日 & 7180 & $\mathrm{E}[$ & 7320 & $\mathrm{H}$ & & $\mathrm{r} S \mathrm{~F}$ & E.JP1 & * SENdO EELL GUOE \\
\hline $60259 \mathrm{~A}$ & 7190 & E6 & $\Xi F$ & $\mathrm{Fi}$ & & LDFF & $\#=\mathrm{F}$ & 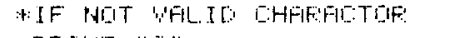 \\
\hline DEEEGA & 7192 & $\mathrm{EC}$ & 7326 & $\vec{H}$ & & TSE & ENE & AFFIINT "?" \\
\hline DEAEAH & 7195 & 80 & $1 \mathrm{E} P 1 \mathrm{E}$ & & & ESF: & EF:LF & AFFINT CFELLF \\
\hline 10EЕ $2 \mathrm{H}$ & 7197 & 26 & BE 71 & $\because 4$ & WLTS & $E F: A$ & EICHEZ & 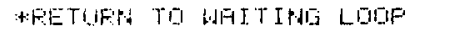 \\
\hline DAEEZEA & 7199 & $E E$ & 1. & $\vec{F}$ & $\because \mathrm{ELT}$ & L.E & 1. $x$ & \\
\hline 브녀 & $719 \mathrm{~B}$ & $\mathrm{AC}$ & E16 & $\bar{A}$ & & $\Gamma$ & 巨. $x$ & 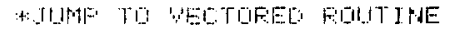 \\
\hline GE265A & 7190 & 20 & FE 19 & & & $\mathrm{EF}: \mathrm{H}$ & WEET: & \\
\hline E16206 & & & & & t: $:<:+$ & & & \\
\hline 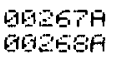 & $\begin{array}{l}719 F \\
\text { P1FE }\end{array}$ & $\begin{array}{l}\therefore E \\
B E\end{array}$ & $\frac{911 E}{616}$ & $\begin{array}{l}\vec{H} \\
\ddot{H}\end{array}$ & $\begin{array}{l}\text { STATT } \\
\text { STHTTI }\end{array}$ & 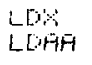 & $\begin{array}{l}\text { \#FUIP } \\
\text { E. }\end{array}$ & :TFEAMS1T STATLS \\
\hline $00259 B$ & TIA4 & $\mathrm{BD}$ & 7326 & $\mathrm{Fi}$ & & TSE & SEND & \\
\hline 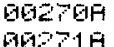 & $\begin{array}{l}71 \mathrm{FP} \\
1 \mathrm{~B}\end{array}$ & $\begin{array}{l}\text { BE: } \\
\text { BI: }\end{array}$ & 6025 & & & $\begin{array}{l}I H \cdot X \\
I F \%\end{array}$ & \#Fldp +3 & \\
\hline $\begin{array}{l}00271 \mathrm{H} \\
01272 \mathrm{~A}\end{array}$ & $\begin{array}{l}71 \mathrm{~B} \\
7 \perp A E:\end{array}$ & $\begin{array}{l}31: \\
26\end{array}$ & $F=71 F$ & $F 2$ & & ENAS & STATTL. & \\
\hline $\begin{array}{l}00272 A \\
00273 \mathrm{~A}\end{array}$ & $\begin{array}{l}P \perp F E: \\
P \perp A D\end{array}$ & $\begin{array}{l}26 \\
4 F\end{array}$ & & & DLFF & ELRA & & \\
\hline $\begin{array}{l}00273 A \\
69274 \mathrm{~A}\end{array}$ & $\begin{array}{l}P \perp A Q \\
\text { PIAE }\end{array}$ & $\begin{array}{l}4 F \\
97\end{array}$ & 29 & $\overrightarrow{\mathrm{H}}$ & & STAH & IFL.Tj & \\
\hline $\begin{array}{l}00274 \mathrm{~A} \\
00275 \mathrm{~A}\end{array}$ & $\begin{array}{l}\text { P IHE } \\
\text { P1EQ }\end{array}$ & 97 & 2 & $\vec{H}$ & & БTमA & STFLS & \\
\hline $\begin{array}{l}00275 A \\
01276 \mathrm{~B}\end{array}$ & $\begin{array}{l}\text { PEV } \\
71 \mathrm{EZ}\end{array}$ & 97 & $2 \mathrm{~A}$ & $\mathrm{H}$ & CFLLF & STFA & DHFF: & \\
\hline $\begin{array}{l}\text { 9E27EA } \\
\text { BQ277A }\end{array}$ & & & QD & $\mathrm{A}$ & C.Th-1 & L_DAFA & \#邦 & :ETLEE A ACLMA TEMFDF!HF:IL'T' \\
\hline 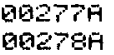 & $\begin{array}{l}71 E 4 \\
7 \perp E E\end{array}$ & EO & 7226 & $\vec{H}$ & & ISE: & $\equiv \mathrm{EN}$ & \\
\hline $\begin{array}{l}90279 A \\
90279 A\end{array}$ & $\begin{array}{l}\text { PAEE } \\
\text { P1B9 }\end{array}$ & $\begin{array}{l}\mathrm{ED} \\
85\end{array}$ & & $A$ & & & \#\#OA & \\
\hline $\begin{array}{l}09279 A \\
00296 A\end{array}$ & $\begin{array}{l}\text { P1B9 } \\
\text { PIEE }\end{array}$ & $\begin{array}{l}85 \\
E D\end{array}$ & $7 \leq 26$ & $\begin{array}{l}\mathrm{H} \\
\mathrm{A}\end{array}$ & & ISE & $\begin{array}{l}\text { \#ELAH } \\
\text { EEND }\end{array}$ & \\
\hline 09281月 & T1EE & 96 & Іே̈ & $\bar{H}$ & & LDAF & DHAF & :PFESTIIFE A AISULMI \\
\hline $\begin{array}{l}062 B 27 \\
00283\end{array}$ & 7100 & 39 & & & : & FITE & & \\
\hline 미용 & & & & & :t:it:it:t:t:t:t::t & ::it:it:it & 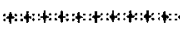 & 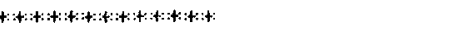 \\
\hline GE2085 & & & & & *: & & & \\
\hline DG286F & 7101 & $4 F$ & & & INIT & ELFÉ & & :RELEF ARUUMUATOF: $\bar{H}$ \\
\hline जब287A & 7102 & $9 \vec{i}$ & 28 & $\mathrm{H}$ & & $\Xi T A F$ & STFLG & 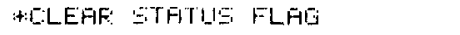 \\
\hline $\begin{array}{l}60288 A \\
616209 A\end{array}$ & $\begin{array}{l}7104 \\
7107\end{array}$ & $\begin{array}{l}E: 7 \\
4 \leq\end{array}$ & 50615 & $\overrightarrow{\mathrm{H}}$ & & $\begin{array}{l}\text { STFA } \\
\text { CIIMA }\end{array}$ & PIAI & :DEF IRE FI EIDE HE IPFLIT \\
\hline 00290A & $71 \mathrm{CB}$ & $B 7$ & 5002 & $\vec{H}$ & & STAR & FIFII & TDEFIPE E SIDE FE IUITFIIT \\
\hline
\end{tabular}

28 


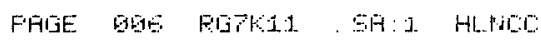

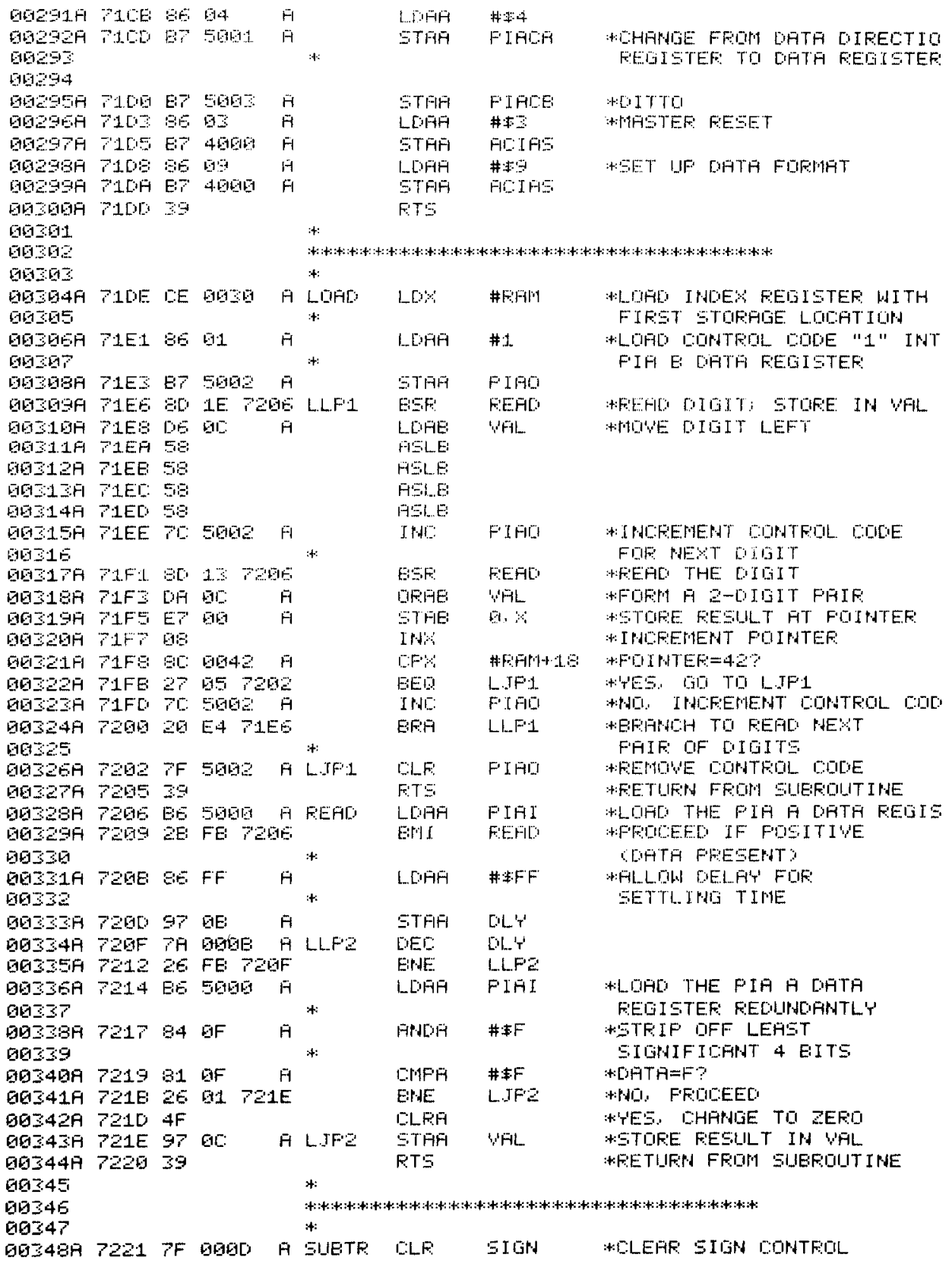




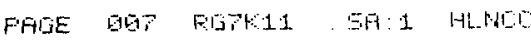

태대은

90150A 7234 IE GOE

G5 14 T27 CE

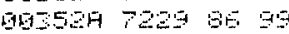

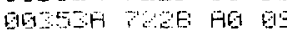

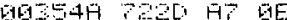

11 5 EF

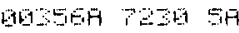

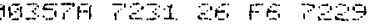

9655

काषण

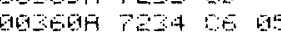

GESEA

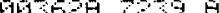

Q19.6.5

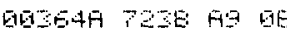

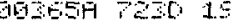

JQIESH $P 2 Z E$ HP 13

ansora 7240 우

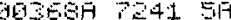

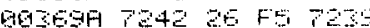

51536

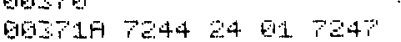

01072

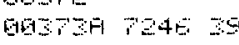

519374

$0055 \% 7245$

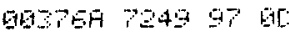

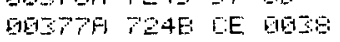

E10 3

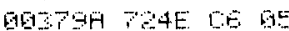

0630H 7250 BE

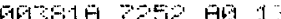

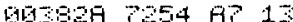

$\begin{array}{ll} & \text { : } \\ A & \\ H & \\ H & =1 \\ H & \end{array}$

$+$

SE

I DHE:

$L$ Lot

$S$ LFE LCOFA

tis

Hi

(1)

3

क

$+$

H $: ., \%$

$\overrightarrow{\mathrm{H}}$

it:

H $\begin{array}{ll}H \\ H\end{array}$

$\theta$

H

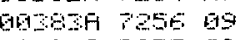

603940 7257 50

QH

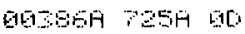

EQSETA P2SE LE

G93:SEA 7250 LE DHSE

GO

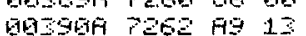

GQSOIA 726413

D09-92月 7255 A7 13

G19

00394A 7263 푸

19295A 7269 26 F5 720

6056म $725 \mathrm{~B} \quad 39$

19597

06398

102399

Q04BOA $726 \mathrm{C}$ BE 05

EG441A $726 \mathrm{E} 97 \mathrm{P}$

00402A $7270 \quad 36 \quad 30$

61049

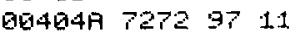

619465i $72 r^{4} 4 \mathrm{~F}$

G0406A 7275 $97 \quad 10$

30

\section{$+$}

A PRINT LOAH

*

STAH
$1, E Y$

LDPE

LDMF

CUEA

STHF

OE:

DEI:E

EPAE

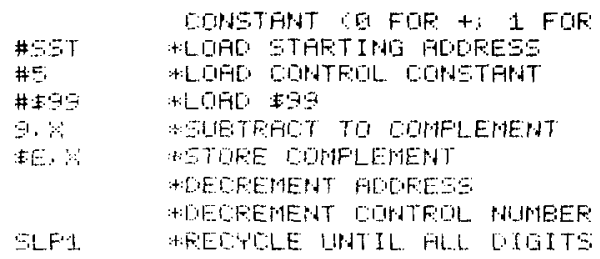

HEF COMPLEMENTEL

A:SET THE IEFFE'

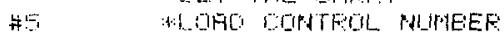

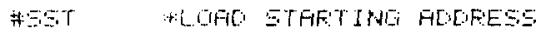

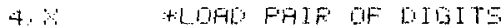

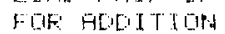

SE. $\because$ : HDD WJTH CHFE'T'

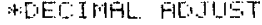

स: GTFE GINA

ADEREETIEHT AOCRESE

WLECFENEMT GOWTFOL NDWEEF:

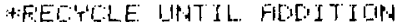

IS GDIFLETE

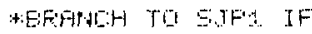

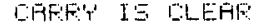

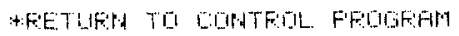

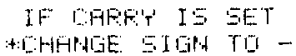

$\# 1$

silid

\#政

LOHE \#
LOAFA \#ASG

EIIE:A DIS

ETHA $\$$ T.

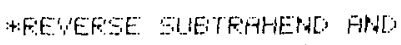

MINUIENO HWU FEFEFT

\begin{tabular}{|c|c|c|}
\hline & $\begin{array}{l}{[E,} \\
{[E, E}\end{array}$ & \\
\hline & ENAE & $S F^{\prime}$ \\
\hline & $\begin{array}{l}\text { SEL } \\
\text { LERE }\end{array}$ & $\# .5$ \\
\hline & L. & $\# S 5 T$ \\
\hline SLF & LEFAA & $\# 0$ \\
\hline & AOCH & $+15, x$ \\
\hline & $\begin{array}{l}\text { DHA } \\
\text { STRA }\end{array}$ & $\$ 13 . \gamma$ \\
\hline & UEK & \\
\hline & $\begin{array}{l}\text { DEEB } \\
\text { ENE } \\
\text { RTS }\end{array}$ & SLFE \\
\hline
\end{tabular}

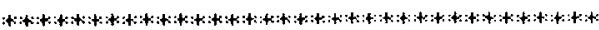

STHF PNLIFI

A LOFA \#FEAI

:SET MUM $=5$

$F+1$

:LLAFD STARTINI ADCRESS

(AEISG) FT F. F+1

A CLFH 


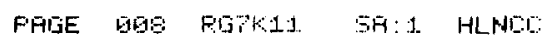

\begin{tabular}{|c|c|c|c|c|c|c|c|c|}
\hline B日1448\% $\mathrm{F}$ & $P 27$ & 97 & SF & $\overline{\mathrm{H}}$ & & STHA & CEIM & :SET [ODH=D \\
\hline 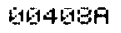 & 7279 & $8 \mathrm{a}$ & $4 F$ & 7201 & & ESE & T'Y'F'E & *T'T'PE DUT' TIME \\
\hline $514459 \mathrm{~F}$ & FTE & $8 \mathrm{a}$ & 46 & 7200 & & ESt: & $\Xi F$ & AFFIPAT $\triangle S F F E$ ES \\
\hline 515419H & 7270 & 35 & 89 & $\mathrm{H}$ & & LDFIA & $\#+9$ & HEET MH_M $=9$ \\
\hline $90411 \mathrm{FI}$ & $727 F$ & 97 & WE & A & & STFIF & NLP & \\
\hline $15412 A$ & 7201 & 86 & 33 & $\overline{\mathrm{H}}$ & & LDFIA & $\# R F+1+Z$ & :SET STAFT ALOF = \$DOBZ \\
\hline 6E41ZH & 7283 & 97 & 11 & $\mathrm{H}$ & & STHF & $F+1$ & \\
\hline $61414 \mathrm{Fi}$ & 7285 & 80 & 92 & $\mathrm{~F}$ & & LБНR & \#丰已 & *5ET $\quad \mathrm{DIN}=2$ \\
\hline Q16415F & 728 & 97 & OF & $\mathrm{H}$ & & STAFI & COOH & \\
\hline 304 $1 E A$ & 7289 & 50 & F & ZA & & Est & $T Y P E$ & +T'T'FE OUIT TOTFLS \\
\hline 961417F & $720 \mathrm{E}$ & $8 \mathrm{D}$ & 201 & $72 \mathrm{EO}$ & & EF: & $S F$ & NFFINT 2 SFALES \\
\hline EE1419A & 7290 & 80 & 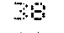 & H & & $\mathrm{I}_{n}$ EIAF & 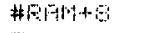 & WEI STHFT FIDLR = =EDSB \\
\hline 튼ㄷㅍ & $7 \mathrm{EF}$ & 9 & 1.1. & $\mathrm{Fi}$ & & 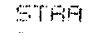 & $F+1$ & \\
\hline 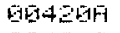 & 7291 & $\theta 0$ & 37 & $P 20$ & & 15 & $T^{\prime} \mathrm{I}^{4} \mathrm{~F} E$ & :\# T'TEE DYT F+F \\
\hline $9942 \mathrm{~L} H$ & 209 & 6 & 26 & $2 \mathrm{EO}$ & & Estis: & $5 \%$ & WFrrTA $\mathrm{S}$ SFACES \\
\hline 694224 & 7205 & 85 & 1 & $\mathrm{H}$ & & DFFA & \#AHC1+ & *SET STFFT ADOF = WEIS \\
\hline ALA4EA & 729 & $9 \%$ & 31 & H & & जTFA & $F+1$ & \\
\hline Q16424F & 7293 & 80 & $2 F$ & $\mathrm{PLOH}$ & & $E \subseteq F$ & T'PFE & : T'R'FE DUT FICIDENTALS \\
\hline 914425,4 & $729 \mathrm{e}$ & 96 & 28 & $F$ & & L.IFH & TFL FIGi & 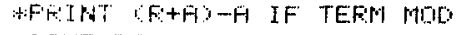 \\
\hline $01942 E A$ & 700 & 27 & $1 \mathrm{H}$ & $72 \mathrm{EG}$ & & $B E$ & FF, IF & 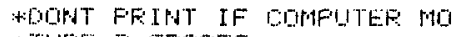 \\
\hline E1E1427H & 79F & 80 & $16:$ & $72 E 0$ & & $E:: F:$ & $S F$ & NTT'TEE $\triangle$ SFACES \\
\hline 61925A & $32 F 1$ & 56 & 81 & $\ddot{H}$ & & H... & $\# 1$ & :IS CFAHD-A FOSITIVE? \\
\hline 619429月 & $7 \mathrm{MS}$ & 91 & 40 & $\vec{H}$ & & DNFF & Eloipt & \\
\hline EE- $40 \mathrm{BA}$ & PaAs & $\therefore 7$ & 07 & $P Q A E$ & & BED & FPIFI & *No. GL TQ FFIFI \\
\hline $60431 H$ & PिF & EE & $2 \mathrm{E}$ & $\mathrm{H}$ & & LDFF & Alat & : ${ }^{\prime} E S$ S. FFIPT + \\
\hline 59423 & 7219 & $\mathrm{ED}$ & 72 & H & & ISE: & $S E N D$ & \\
\hline $914-3 \mathrm{~A}$ & $P \mathrm{HC}$ & 2 & $\mathrm{GE}$ & $P E \mathrm{E}$ & & EFH & $F F:] F=$ & +GO TO FEUTFE \\
\hline$E 15434 \bar{H}$ & PQAE & 36 & 20 & $\mathrm{H}$ & FFIF-1 & L.EFFI & $\# \div 2 \mathrm{D}$ & :FFINT - \\
\hline G9435f & $7 \geq \mathrm{E}$ & $\mathrm{E}: \mathrm{C}$ & 72 & $E$ & & $r E F$ & EENSO & \\
\hline G043В & 7283 & 80 & 47 & $B$ & FFIIFE & LDAFI & $\# P A+1+2 Z$ & :SET START FDOR = $=$ FE1G 4 \\
\hline $09457 \mathrm{~F}$ & $72 \mathrm{E}$ & 97 & 11 & $\mathrm{H}$ & & STAF & $F+1$ & \\
\hline D1542EA & 7287 & $B \mathrm{D}$ & 11 & 7204 & & ESE & $T T^{\prime} F \cdot E$ & :H:T'T'FE OIIT $\because F+F Y-A$ \\
\hline घC4 $29 \mathrm{~A}$ & $7 \mathrm{ES}$ & $\mathrm{EO}$ & $7 \pm$ & 2 & $P F \because F$ & $I=F$ & CFLF & SFFIPAT CFFFIHGE ETN LTHE F \\
\hline $9644 \mathrm{EA}$ & $72 \mathrm{EI}$ & 89 & & & & FTS & & AFEETIAW TO EONTPOL PROIJFRM \\
\hline $619441 \mathrm{H}$ & $72 \mathrm{O}$ & 60. & 26 & A & $6 F$ & LIFA & \#玤 & AFFINA 3 SFFIEES \\
\hline 85442 & & & & & it: & & & GUEFHUT I WE \\
\hline E1E44SA & $72 \mathrm{EF}$ & $E \mathrm{D}$ & 69 & 720 & & ESE: & SENO & \\
\hline D16444A & 201 & $3 E$ & 20 & $\mathrm{H}$ & & LDHA & $\# \div=6$ & \\
\hline E1445F & 70 & 80 & $6 . \mathrm{L}$ & 20 & & ESE: & $S E \mathrm{WO}$ & \\
\hline 19440A & 7205 & 8 & 29 & $\mathrm{~F}$ & & L $\triangle A F A$ & $\#=20$ & \\
\hline $80447 \mathrm{P}$ & $P 20$ & $8 \mathrm{E}$ & $5 \mathrm{C}$ & 726 & & ESE: & SEND & \\
\hline $1544 \mathrm{BH}$ & 720 & 39 & & & & マTS & & WFETURH \\
\hline 91445 & & & & & 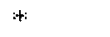 & & & \\
\hline $\begin{array}{l}06450 \\
00451\end{array}$ & & & & & 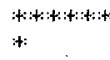 & 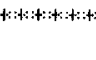 & 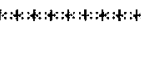 & 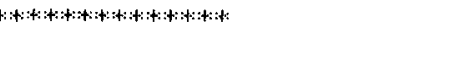 \\
\hline 9154529 & PECA & $4 \mathrm{~F}$ & & & T'Y'PE & $\because$ FFA & & *ILEEFF CONTEOL \#S TA F \\
\hline $9645 \div \mathrm{F}$ & $P \angle E$ & 97 & 12 & $\mathrm{FI}$ & & $\leq|\mathrm{F}| \mathrm{F} \mid$ &. $\mathrm{T}$ & \\
\hline $05454 \mathrm{~A}$ & $P Q D O$ & 97 & 14 & $\mathrm{H}$ & & $\triangle T A M$ & $P$ & \\
\hline DE.45.5F & $7 \mathrm{C}: \mathrm{F}$ & 97 & 13 & Fi & & STAF & r & \\
\hline $1645 \mathrm{HA}$ & 7201 & DE & 18 & $\vec{H}$ & & $\operatorname{Lox}$ & $F$ & WLDEL STAFTIND ADORESS IN \\
\hline $16457 \mathrm{FH}$ & $\because \mathrm{CO}$ & $\mathrm{HE}$ & E10 & $\mathrm{H}$ & $T^{\prime} T^{\prime} L F+1$ & L.DIFA & D. $x$ & :LGAC F FFIF OF OIGITS \\
\hline $69458 \mathrm{~B}$ & 7205 & DE & 14 & $\mathrm{Fi}$ & & L.LHB & $P$ & :ANED LEFST SID. DIGIT? \\
\hline $16459 \mathrm{FI}$ & 7207 & 27 & 94 & 7200 & & $\mathrm{EEO}$ & E1. & HNOE GO TO EL \\
\hline D640.6H & 7209 & 84 & EF & $F$ & & FNDE & $\# \# F$ & W'YES GET LEHST SIG 4 EIT \\
\hline $014401 \mathrm{H}$ & TCDE: & 20 & 96 & TES & & EFA & $\mathrm{Ee}$ & $+10 \mathrm{TO}$ EZ \\
\hline $\begin{array}{l}514 E 2 A \\
5104 E S A\end{array}$ & $\begin{array}{l}720 \mathrm{O} \\
720 \mathrm{~F}\end{array}$ & 54 & $\mathrm{FB}$ & 月 & E1 & $\begin{array}{l}\text { HMLA } \\
\text { FIOHA }\end{array}$ & \#玤F & $\begin{array}{l}\text { :FICK OFF MOST SIT } 4 \text { ETTS } \\
\text { :FFWE RIGHT } 4 \text { EITS }\end{array}$ \\
\hline 016454 & PEE & $4 E$ & & & & FDFH & & \\
\hline
\end{tabular}




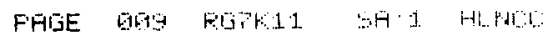

\begin{tabular}{|c|c|c|c|c|c|c|c|}
\hline $601465 \mathrm{H}$ & $\because E 1$ & $4 \vec{r}$ & & & FIFH & & \\
\hline G64E. & $P E E$ & $4 E$ & & & FIF:A & & \\
\hline $66+878$ & $72 E$ & 4[ & & $\mathrm{EE}$ & TETA & & 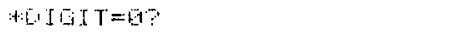 \\
\hline DELSEA & $7 \& E 4$ & $2 \%$ & DE PZEE & & EEE & $E:-3$ & 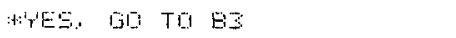 \\
\hline D164:Bज & $\angle E$ & $B$ & [11 & & L. DAFE: & $\#: 1$. & HII. SET $\quad \because=1$ \\
\hline 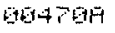 & 725 & OP & 12 & & ETAE & $\vdash$ & \\
\hline 6154 $1 \mathrm{H}$ & $\overrightarrow{P E \mathrm{EF}}$ & $\Xi[$ & $2 \mathrm{H} \quad 72 \mathrm{E}$ & $E$ & ESF: & EEROH & 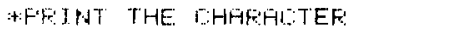 \\
\hline 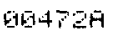 & PEE: & 20 & 正F $P 2 F$ & & E:F:H & $\mathrm{E}: 4$ & +OOH TO E4 \\
\hline$E 1475 A$ & $P E E$ & 70 & HII $\quad H$ & $E:-$ & $T \Xi T$ & $k$ & $+: t=01$ \\
\hline 604747 & $72 F 1$ & $2 E$ & $F P \quad P E E A$ & & ENA & $E: 5$ & :TPSO FRITT THE OIBIT \\
\hline $\mathrm{OW}+7 \mathrm{C} F$ & $\because F=$ & {$[s$} & DEE $\quad A$ & & 1. EPAE & N.W & WEST IS THIS THE LFET [IG \\
\hline 60475 & & & & : & & & IP THE PIHEEFT? \\
\hline 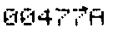 & $7 E F$ & {$[1$} & $1=$ & & CHFE & $y$ & \\
\hline ब154 & $P F F$ & 27 & $F+1 F H$ & & EE:E & EE & :TES, PEINT THE ZEFID \\
\hline 5154799 & $\because \mathrm{F}=$ & $\Xi \theta$ & 20 & & LIFAF & \#五至 & 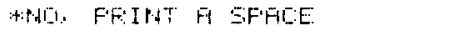 \\
\hline Q볌ㅍ & $\because F B$ & 20 & $\mathrm{E} D \mathrm{P} Z \mathrm{EH}$ & & EFiri & $E: 5$ & \\
\hline D194. $1 \mathrm{FF}$ & $\mathrm{FF}$ & 96 & SE $\quad \bar{A}$ & E4 & L.DFIF & Nuri & AIS THIS THE \&FET DIGIT \\
\hline 64049 & & & & : & & & IA THE PUIEEF? \\
\hline $0164 B: H$ & GEF & 91. & at: & & :NFH & 1 & \\
\hline E1949AF & 7351 & 25 & 011754 & & $B R$ & Eit: & सम\#. GH Ta ER \\
\hline 16455月 & 728 & 3 & & & $\vec{F}+:=$ & & 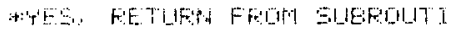 \\
\hline B15486 & 7394 & $P \mathrm{E}$ & G日B!: & EE & $1+1=$ & 10 & 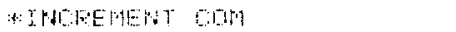 \\
\hline GEAQBF & 79 & $\theta$ & Q & & D...PFH & 4 & : $+0: 010=3$ \\
\hline Q1548:A & 7369 & 81 & EF & & CMFA & Sin & \\
\hline 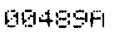 & 736 & $2 \theta$ & E-74. & & ENAE & $E$ & 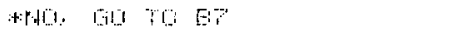 \\
\hline 턴대 & 7365 & 85 & $2 B \quad A$ & & 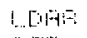 & $4+26$ & A $\%$ CHT $A$ B SFFE \\
\hline G144.9 & $\checkmark \mathrm{BF}$ & 80 & $157 \%$ & & E:s: & EWE & \\
\hline EQ949 & 721 & $\vec{r} F$ & DEtal= & 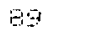 & GL_F & Grm & 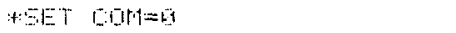 \\
\hline $91493 \mathrm{~F}$ & 214 & $t$ & E112 & $E$ & 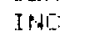 & $y$ & 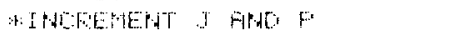 \\
\hline $86494 \mathrm{H}$ & 7217 & $P E$ & 9514 & & $\mathrm{~J}+\mathrm{dL}$ & $F$ & \\
\hline $81445 \mathrm{H}$ & Z1.A & 50 & $1 \%$ & & L.DEFAF & $H$ & $\therefore F=2$ \\
\hline $09455 \mathrm{H}$ & 7315 & 51. & 3.4 & & WFA & $F$ & \\
\hline E164978 & TIE & $2 \theta$ & EI 200 & & EPVE & $T^{\prime} \cdot r^{\prime} L F^{\prime} \cdot 1$ & 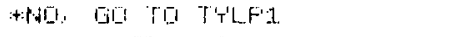 \\
\hline 19498A & 7320 & $P F$ & E15:14 & & GLF & $F$ & W' ${ }^{\prime} E S, \quad S E Y \quad F=5$ \\
\hline GE149G & 75 & $\overline{60}$ & & & Into & & HETEF TO WEWT FHIF OF DILI \\
\hline 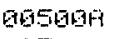 & 7284 & 20 & AD $7 \mathrm{OSO}$ & & EFR & $T^{\prime} \mathbf{r}^{\prime}-F^{\prime} \cdot 1$ & \\
\hline GESEL1 & & & & $:+:$ & & & \\
\hline 01502 & & & & $:$ t:itit: & $t: t:: t:: t:: t:: t$ & $t: i+i t: i t: i t: i t: i t$ & 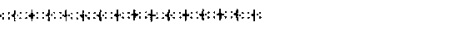 \\
\hline 010562 & & & & : : & & & \\
\hline Ë5БムA & 7326 & 31 & E19 & SEPdi: & WFH & $\#: 9$ & 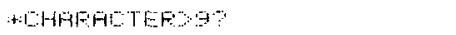 \\
\hline 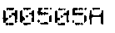 & 728 & $2 E$ & 92732 & & EOT & $5 \mathrm{E} / \mathrm{F} \cdot 1$ & F'TES: F'FOILEES \\
\hline 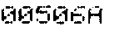 & $7 \Xi 2 A$ & $B \ddot{H}$ & 54 & & DFAA & $\# \div 5$ & WUI. FDFU FEOLI GOCE \\
\hline 보듀 & 7220 & $F E$ & $4 E+15$ & $\Xi E, I F \cdot 1$ & LDFE: & FIE I I & :LOHE STATUS OF FUIF \\
\hline 모듭 & $\overrightarrow{1} 2 \mathrm{FF}$ & $5 i 5$ & & & $F: O F E$ & & \\
\hline 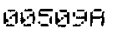 & 739 & 5 & & & FQCEE: & & \\
\hline 615.51日A & 731 & 24 & $F=7-2$ & & $E 1: S$ & $\Xi E \cdot I F \cdot 1$ & :FFOUEES IF ALIH IS FEFW' \\
\hline 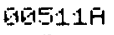 & $7 \leq 3$ & $\mathrm{E}: 7$ & 46121 & & STHA & $\overline{A L}: I B T$ & TEEHE DUT CHAFFIETEF: \\
\hline 015512 & & & & : & & & TO TEFWIPALL \\
\hline ETEDSH & 730 & 81 & 10 & & CIFA & $\#=00$ & :t: IS IT FI CFE \\
\hline $961514 \mathrm{H}$ & 7338 & 26 & $19734 \mathrm{~A}$ & & BPE & $\mathrm{BL}=2$ & \\
\hline $965 \pm 59$ & $P S E A$ & $\theta$ & SiF & & LEFE: & $\# \div: \mathrm{C}_{\mathrm{L}}$ & :A IF 'TEE: GTHFT AI QELEF'T' \\
\hline $6015 \mathrm{~A}$ & 735 & {$[7$} & 15 & {$[1,6$} & STRE & CELL-1 & \\
\hline E16517月 & $P 2 \mathrm{E}$ & {$[\because$} & IE & & STHE & DELLE & \\
\hline Q651BA & 7340 & $P A$ & 61915 & DL1 & DEE: & [EL1 & :EEGJI OA OELF'r' \\
\hline 51515.19A & 724 & 2 & $\mathrm{FE} \quad \mathrm{Y} 3 \mathrm{4E}$ & & ENA & DL1 & \\
\hline Q1952DA & 7245 & $P A$ & E916 $\quad A$ & & DEE & DELZ & \\
\hline $06521 \mathrm{~A}$ & 7348 & 26 & $\mathrm{FE}: 2 \mathrm{E}$ & & ENAE & [L. 1. & :LEELAT' FINISHED? \\
\hline E1052:R & $724+1$ & 39 & & DLE & $F: T S$ & & :X'YES. RETIFHA \\
\hline
\end{tabular}

32 


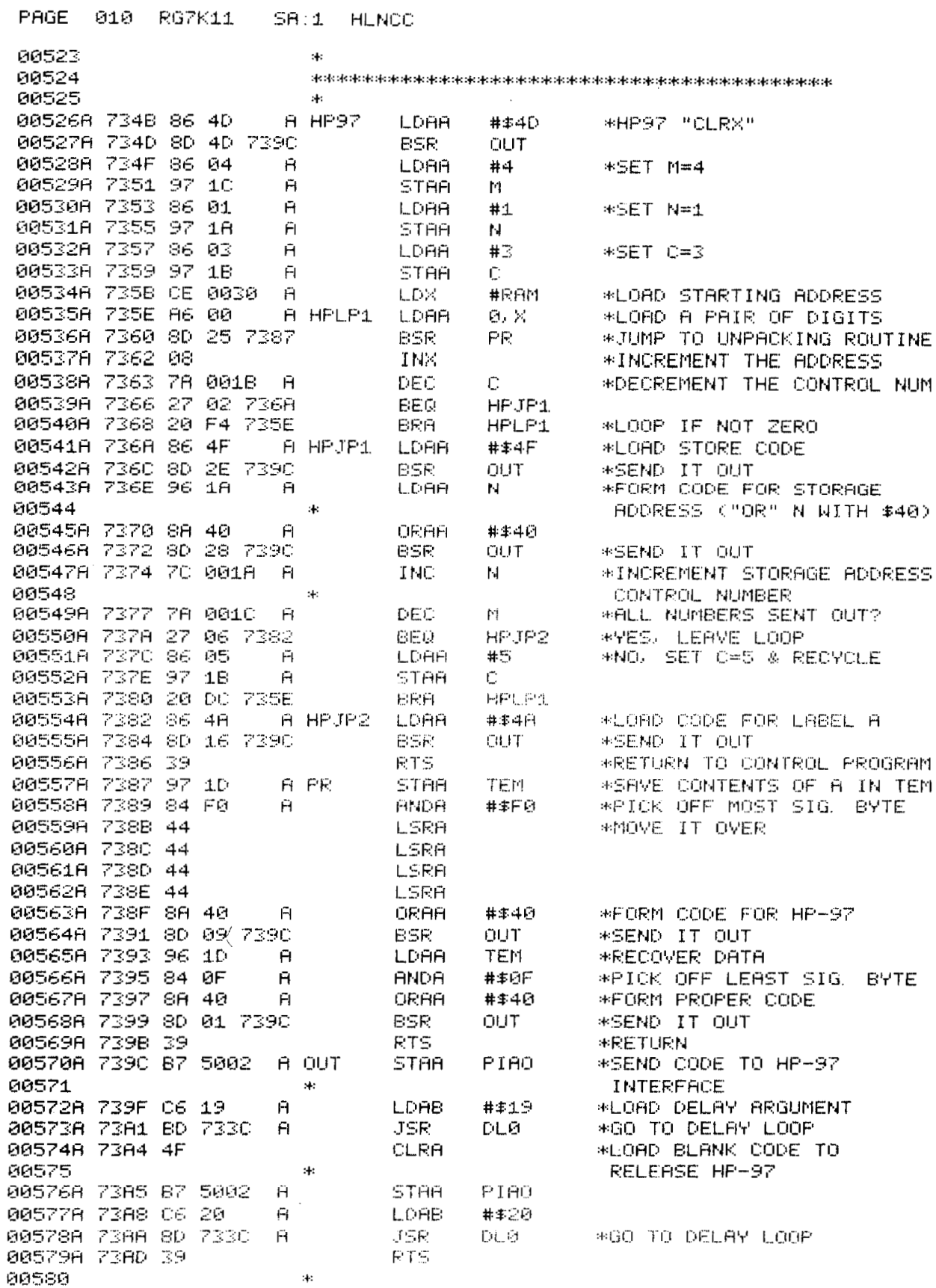

FEENO IT FUIT

TIMIFEMENT STOFEOE ADOFESE DOKTFOL MUIEEF:

WFILL PHITEEFS SERT OUT?

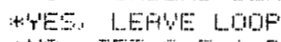

THO GET OES OAECTCLE

C

HFif:

\#\#4A WOHO DOEE FOF LAEEL, $A$

DUT $A$ SEP IT OUT

NFETLEN TO GOMTFIL FFOGFHM WEATE COHTENTS OF FI IN TEM *FICK DFF MOST SIG. BYTE HOWE IT GWEF

AFCIFW COLE FOF HF-9P

:SEND IT OIIT

* FECOTWER DATA

WPICK OFF LEFST SIS. BUTTE

AFUEA FFOFEF: DODE

*SEPHO IT DIIT

:TFETIIFIS

AEENO COOE TO HF-G?

IPTEFEFFCE

\#\#19 WLGFI OELA'T REIJUMENT

* TOO TO DELF'T' LOUF

*LOAD ELFNK CODE TO

RELEFISE HF- 9 ?

WUO TO DELAW LOMF 


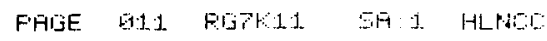

\begin{tabular}{|c|c|c|c|c|c|c|c|c|}
\hline \multirow{2}{*}{$\begin{array}{l}09591 \\
08592 \\
005920\end{array}$} & \multirow[b]{2}{*}{ PЧЁE } & \multirow[b]{2}{*}{ TEE } & \multirow[b]{2}{*}{ 晋 $4+1$} & & \multicolumn{4}{|c|}{ 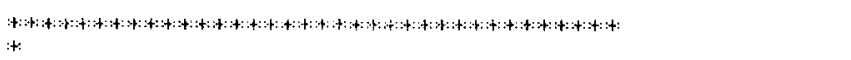 } \\
\hline & & & & $\hat{H}$ & TEET & $\operatorname{Lot}$ & $\#$ \#AIr+17 & :LLDF STFIETIPAS ADOFESS \\
\hline $01564 \mathrm{H}$ & $P \mathrm{BE}$ & 91 & & & TEST:1 & CLE: & & 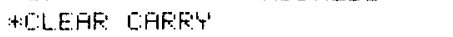 \\
\hline Q605:SA & 7982 & 55 & 18 & $\overline{\mathrm{H}}$ & & LEAH & $\# 10$ & :A:CEEINHAL "1S" \\
\hline 매드여 & $7 \mathrm{E}: 4$ & $\mathrm{H} \vec{i}$ & E15 & $\mathrm{H}$ & TESTE & GTFH & 5. & \\
\hline DOSE7A & TZEE & $\mathrm{SE}$ & 22 & 苚 & & HDOH & $\#=2$ & *CELIHAL "Z" \\
\hline 695:GE & $7 \mathrm{EQ}$ & 15 & & & & DFIF & & WLAWEET SUH TO DECIMAL \\
\hline 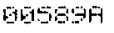 & $78 B 9$ & 9 & & & & [SE & & A:DELFIMENT FIINTEE: \\
\hline 口ब15日A & PEF & $G[$ & 11日2F & $H$ & & $C: F+1$ & $\# F F+1$ & 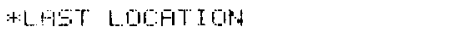 \\
\hline 615591H & $3 \mathrm{BO}$ & $\therefore$ & $014 \quad 720$ & & & BED & TESTS & \\
\hline El15 & $\mathrm{EF}$ & 2 & $F=$ & & & Bis & $T E=T 1$ & 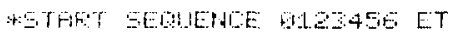 \\
\hline $01653 \mathrm{~A}$ & $7 \leq 1$ & 20 & $F \cdot P E$ & & & $E F$ & TEOTE & \\
\hline 619594FI & 70 & $\mathrm{ECO}$ & $P 4 \mathrm{E}$ & $\ddot{H}$ & TEOS & $I E F$ & 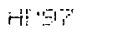 & 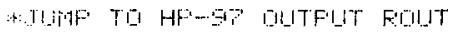 \\
\hline D15951 & 730 & 20 & & & & $F T:$ & & \\
\hline Q1950 & & & & & t:it:-t:-t: & & & \\
\hline DES57A & 7207 & $E E$ & 7515 & $\ddot{H}$ & $\because 1$ & $L r$ & \#1 & WLLHE STAFTIHIS ADLFESS \\
\hline E1EG:GH & PEOH & Hit. & bis & FI & TELF'1. & LDPFA & E. $\%$ & 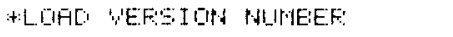 \\
\hline 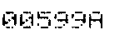 & $7 \mathrm{OLE}$ & $\mathrm{ECO}$ & 7320 & $\mathrm{~A}$ & & $I=F$ & SEPSL & A:FFU IMT $\mathrm{IT}$ \\
\hline 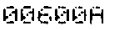 & $P \triangle \mathrm{CF}$ & 6: & & & & ind & & 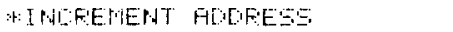 \\
\hline ब1日 & 7.505 & :1: & 760 & Fi & & $F F$ & $\# 1+19$ & 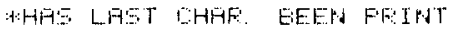 \\
\hline DEEECF & 78 & $2 E$ & $F$ & & & Eirat & TEL.F'1. & 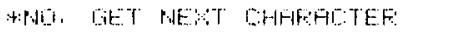 \\
\hline E16 & $7 \mathrm{Br}$ & 3 & & & & F.T: & & ATES. HETUEH \\
\hline Ex66 & & & & & & EPdC & & \\
\hline
\end{tabular}

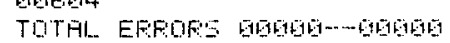

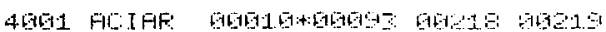

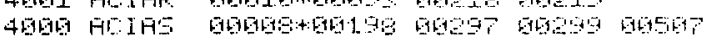

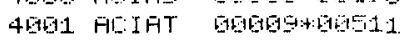

7200 E1 019459 51402.

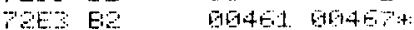

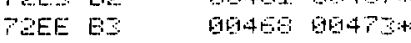

$7 \mathrm{ECO}$ EA

PZEH ES GE

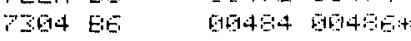

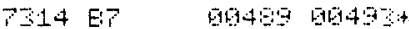

721 ES

WIE E :

7.1.1: L:CHF

$7120 \mathrm{CHF}$

7124 LENA

7135 ENPF

710.4 ECHF'4

PLE GertFs

$7 \in O$ COFE

DIPA L.HAF

TUI LLLR

T1AL ELEF

PEQS CNTFL

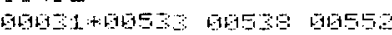

Etages 019244

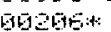

ब1920 +

00208 03211 andit

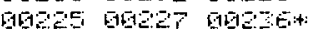

1922- 90250

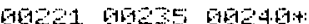

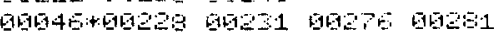

G160.

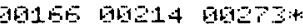

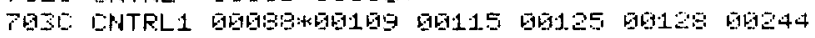

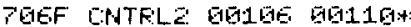

PQT7 CNTRL 3 GD111 50114:

TETE LNTFL 4 G0112 GE11EN

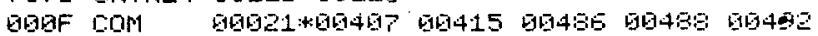

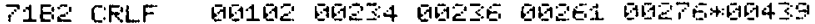

Q015 DEL1 00025:409516 01651:

34 


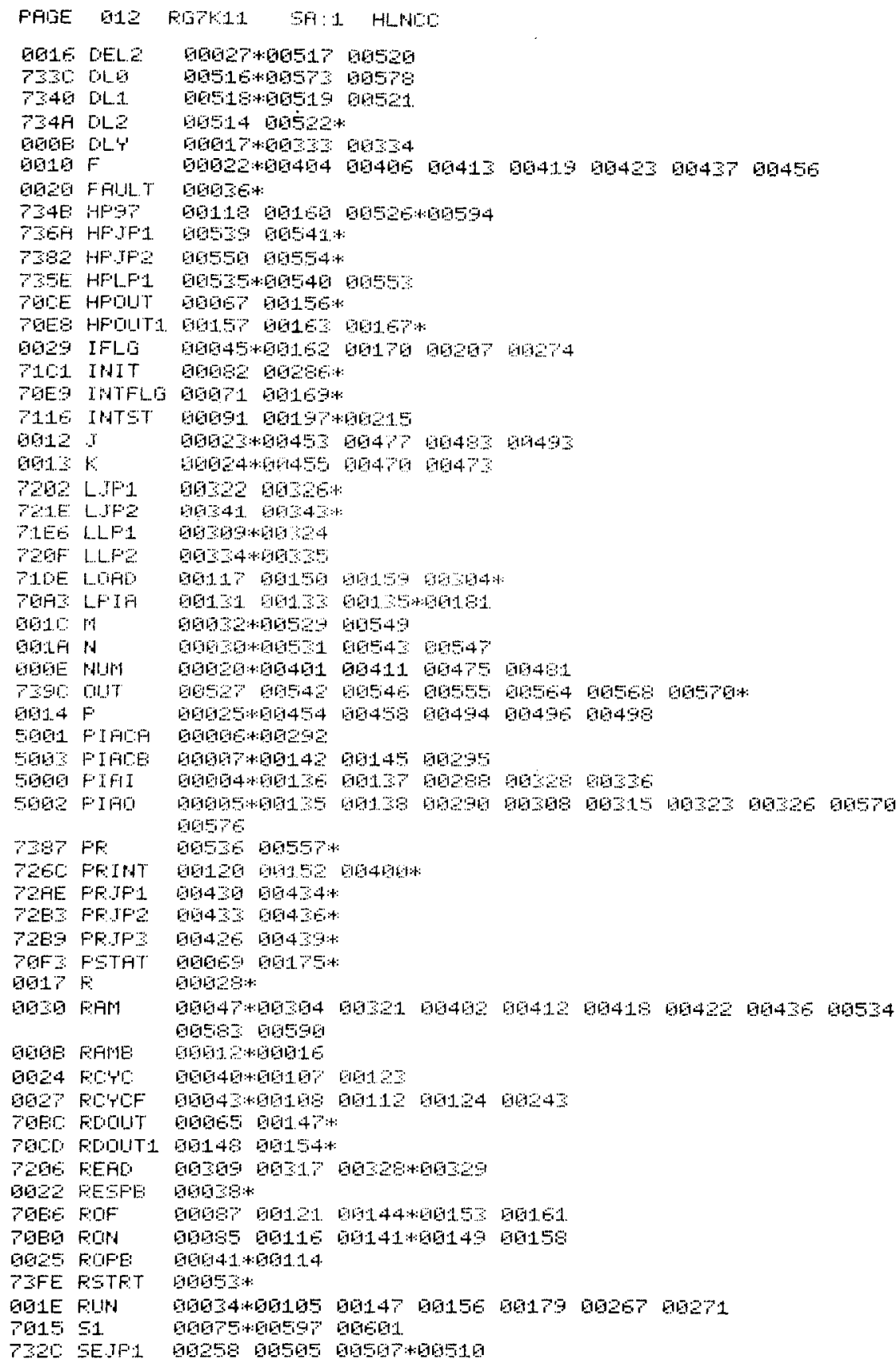




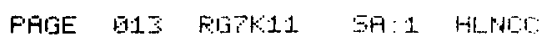

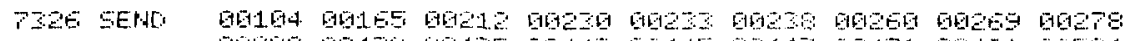

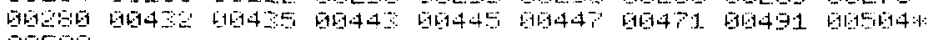
0659

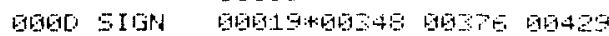

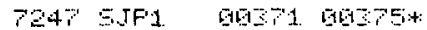

7229 SLFI $01952+1095$

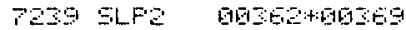

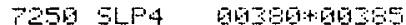

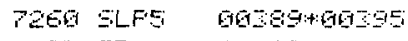

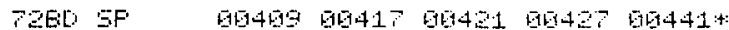

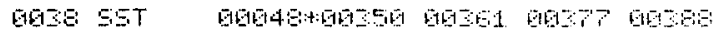

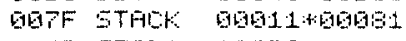

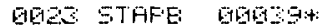

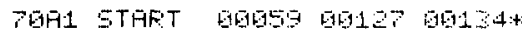

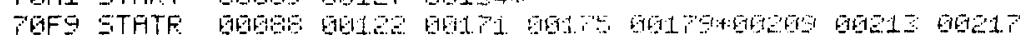

TEFE STHTF1 $019191+6194$

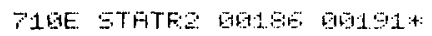

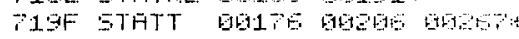

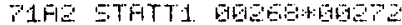

5020 STFL G.j

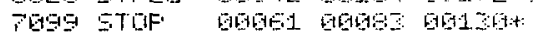

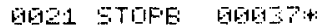

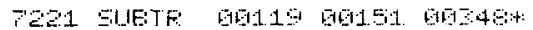

$0819 \mathrm{~T}$.

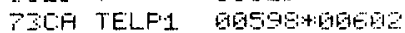

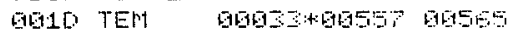

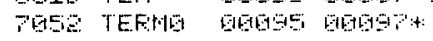

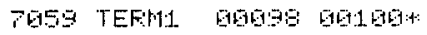

760 TEFil

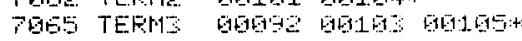

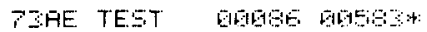

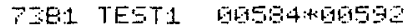

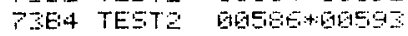

7303 TEST 6159101054

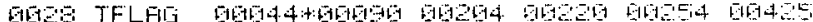

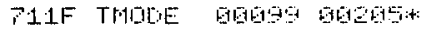

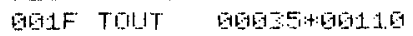

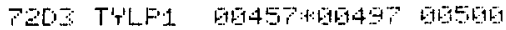

$720 \mathrm{~T}$ TFE

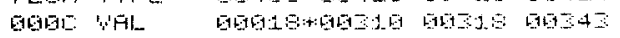

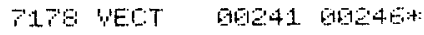

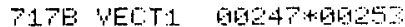

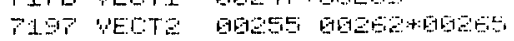

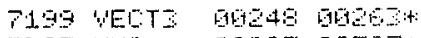

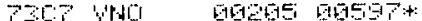

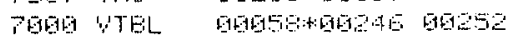


APPENDIX D

FORTRAN-CALLABLE DRIVER AND FORTRAN TEST PROGRAM 


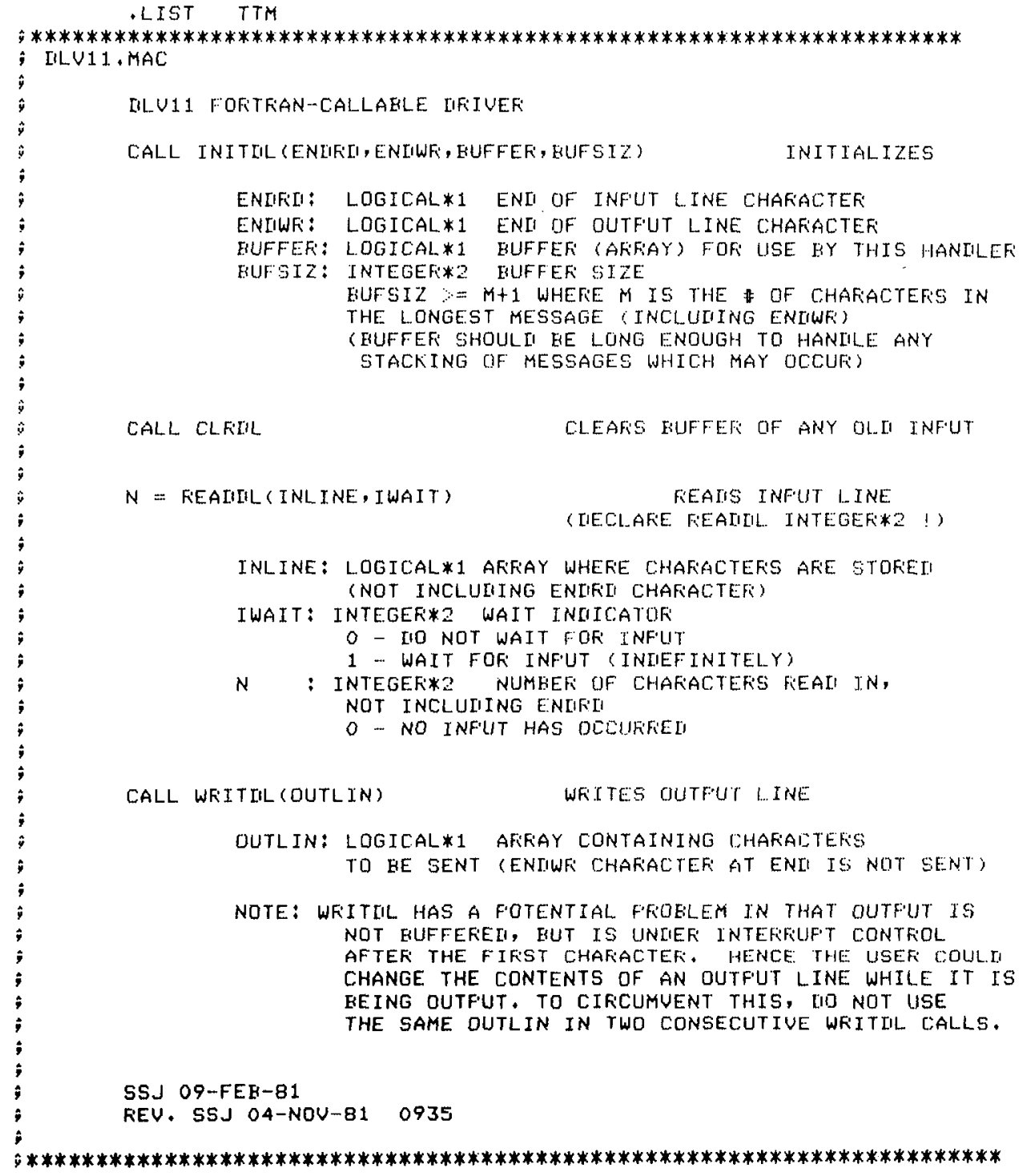

38 
+TITLE IILU11

, GLOEL INITIL, CLFEIL, FEEAIITL, WFITIIL

$\dot{t}$

* FEGISTER REFINITTONS ANI SFECIAL ALIIFESSES

FUEC $=310$

$X \cup E C=314$

FCSK $=176510$

; FECEIUE UECTOR

; TRANSMIT VECTOF:

- FEECIVE CONTROL /STATUS; REGTSTEF

FUEC2 $=$ FIUEC+2

$X \cup E C 2=X \cup E C+2$

FIEUF $=\mathrm{FICSFI}+2$

$X C S F i=F i C S F i+4$

$X \mathrm{E} \cup \mathrm{F}=\mathrm{FC} \mathrm{CSF}+6$

; FECEIUE F.SW

; TFANSMIT PSW

;FECEIUE BUFFER

- TFANSMIT coNTFOL/STATUS

TTFANSMIT BUIFEEK

FAF'AM $=F I S$

SAUF TF: $=$ FiA

COUNT $=F: O$

; FAKAMETEF FEGISTER

- SAVE FOINTEF kEGISTEF

;COUNT OF INFUT CHAFACTEFIS

WAI $T=F^{\prime} 1$

; WAIT FLAG

INTTIALIZATION FOUTINE -.. SET UF INTERFUFTS,

GET FAFIAMETEFSS

INITIL: CLF: U\#FICSF

CLF $\quad \#$ MCSF:

MOV

MOV

MOV

*FINTEF, $Q$ \&FUEC

$\$ 340$, OFFEC2

$X I N T E F$, , $\$ X U E C$

MOV

MOUE

$\$ 340$, [ $\$ X \cup E C 2$

Q2 2 (FAFAM), FIENI

Q4 (F'AFIAM), XENI

MOU O(FARAM), EUFFEF ; GET ALINESS DF EUFFEF

¿CLEAF RECEJUE STATUS

;CLEAR TFANSMIT STATUS

\$SET UF FECEIUE JNTEFFIUFT UECTOF

;IISABLE ALL OTHEF INTERFIUF'TS

; SET UF TFANSMIT INTEEFIUFT UECTOF

- IIISAELE ALLL OTHEF INTERKUFTS

MOV E1O(FAFIAM), EUFENII FGE BUFFEF STZE

ALII

EUFFEFI, EUFENL

JSF $\quad$ F'C, CLFIIL

MOV

$\$ 100$, , DFICSF

- SET UF POINTEFS

; ENAELE THE RECEIUE TINTEFFUFT NOW FIETUFIN

CLEAF' RUFFEF' FIOUTINE

CLFIIIL: MOV

MOV

MOU

EUFFER, FICF'TF EUFFEF, RILIFTF EUFFEF', L.NF'TF

SSET FECEIVE FOINTEF TO HEGIN OF EUFFEF

ISAME WITH FEAI FOINTEF:

; SAME WITH LINE FOINTEF

FTS

$F^{\prime} \mathrm{C}$

; FEETUKN 
;FESET COUNT OF INFUT CHAFIACTEFIS MOV Q4 (FAKAM), WAIT

95: LNFTFI, FIEIFITK ;GET WAIT FLAG

;HAUE WE FEEAII THE LATEST LINE ALFEALIY? BNE $3 \$$

TST WAIT

EEQ $7 \$$

$7 \$$

\NO - A HEW LINE HAS COME IN

TYES -- SHOULII WE WAIT?

;NO -. USEF WANTS AN IMMEIIATE FETUFIN

$3 \$: \quad$ INE $\quad$ INC

GYES - WAIT FOF A NEW LINE

;EUMF FOINTEF TO NEXT CHAFACTEF

FIIFTF, BUFENII ;ARE WE AT THE ENII OF THE BUFFER?

$\begin{array}{ll}\text { CMF } & \text { FIIIF } \\ \text { BNE } & 1 \$\end{array}$

; NO

MOV BUFFEF', FILIF'TF

¿YES - START AT BEGINNING OF BUFFEF:

EFILTF, FIENI ;IS THIS THE ENII OF MESSAGE?

CMF'B
$B E Q$

MOUE ERIF'TF, EINFTF

INC COUNT

INC INFTR:

F: FITS F.C

;YES - IIONE, IION'T INELUIIE THIS CHAR

INO - MOVE THE CHAFACTEF TO USEF'S LINE

- ALIII 1 TO CHAFACTEF COUNT

; EUMF FOINTER TO UEEF'S LINE

; FIEF'EAT

;FETUFN

FIECEIUE INTEFRIUFT ROUTINE

DCCUFS WHEN A CHAFACTEF HAS COME IN ANII $1 S$ FEALIY TO BE FEAI

;

FINTEF: INC FCFTF EUMF FECEIUE FOINTEF:

CMF FICFTR, BUFENI ;HAUE WE WEACHEI THE ENII CIF BUIFEF?

ENE $1 \$$

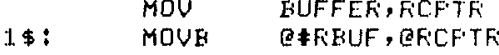

;NO

- YES - STAFT AT EEGINNING AGAIN

;FUT CHAFIACTER II EUFFE

CMFE OECFTF, RENII ;IS THIS THE LAST WHAF IN LINE?

ENE $3 \$$

3\$: $\quad$ FOU

NO

;YES - MAFK THE ENI! OF THE LINE ; FEETUFIN

OUTFUT FIOUTINE

USES TFANSMIT FIEALY INTEFFUF'T FEATUFE

NOTE: EXFEFIENCE WITH IILU11J SHOWS THAT THE FIEALY INTEFFIUFT OCCUFS IMMEIIATELY AFTEF THE ENAELE

(

WFITIL: BIT

; (IE, IS INTERFUFT IIISABLEIT?)

ENE WRITIL \$NO :- JUST WAIT HEFE TILL IT IS

MOU 2(FAFAM), SAUFTF ;GET AIIIFESS OF FIFIST CHAFACTER

MOU SAUFTK,TFFTF "SAVE FIFST AIIEFESS FOF USE LATEF

MOV $\$ 100, Q \$ X C S F$ DENAELE TFANSMIT REAIYY INTEFFUFTS

;WAIT A BIT

NOF.

$\begin{array}{lll}\text { CMF } & \text { TFFTF, SAUFTF } & \text { HAS FEAIY INTEFFUFT OCCUFFEI JUST NOW } \\ \text { BNE } & \text { IYES - OUTFUT HAS STAFTEI ALFEAIY }\end{array}$

JSF FC,SEND ONO - SENI OFF FIRST CHAFACTEF:

S\$: RTS FC

; FETUFIN 


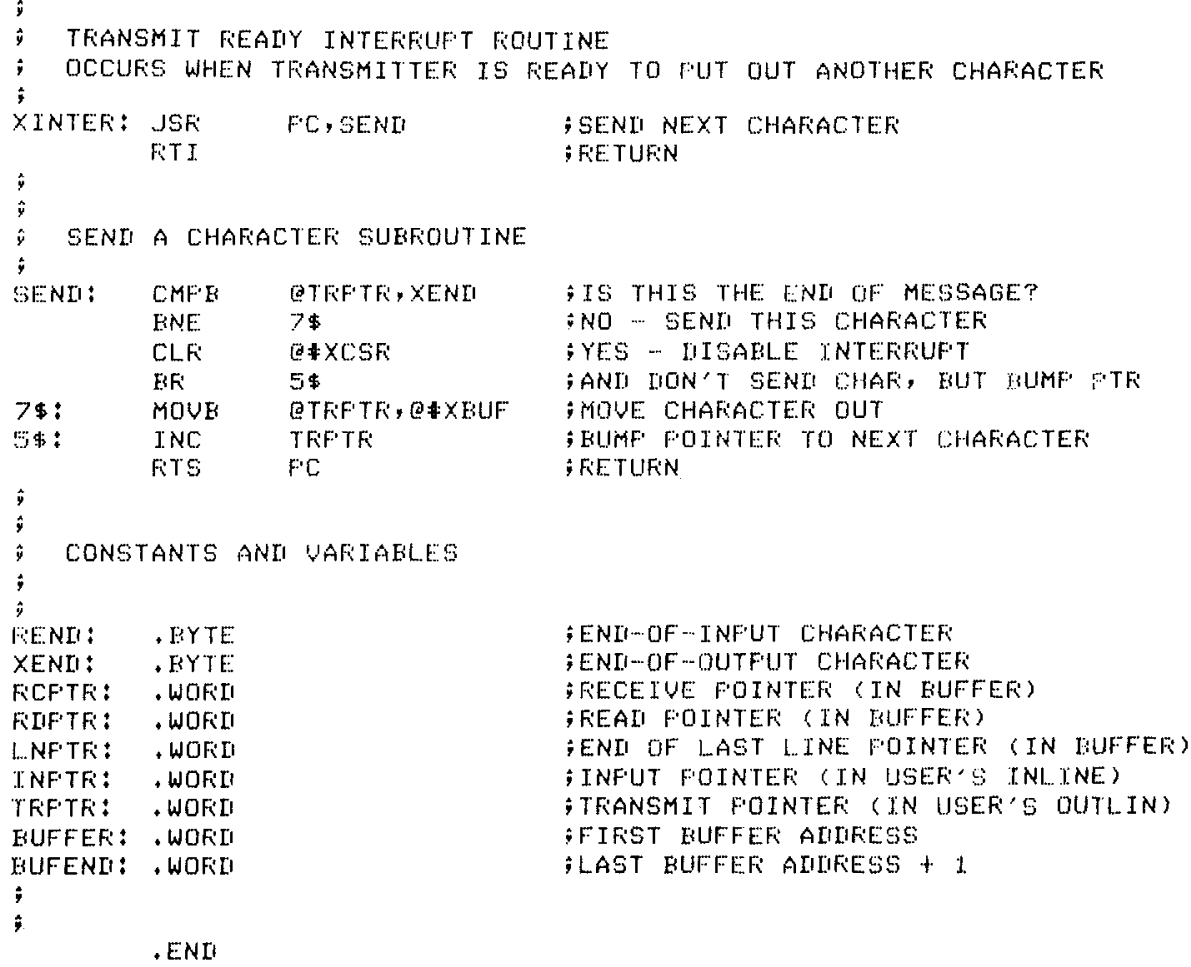

¿ENI-...OF -.. TNFUT CHAFACTEF:

; ENI-ODF-OUTFUT CHAFIACTER

FECEIUE FOINTER (IN BUFFEF)

; FEEAII FOINTEK (TN BUFFEF)

IENI OF LAST LINE FOINTEF (IN RUFFER)

; INFUT POINTEF (IN USEF'S TNITNE)

- TFANSMIT FOINTEF (IN USEF'S OUTLIN)

SFIFST RUFFEF AIILFESS

- LAST BUFFEF AIIIIFESS+ 1 
WOFTFAN JU VOz.1-10 Wed 11-Fed-91 FAGE OO1

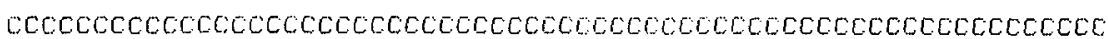
C HLNCC

C TEST INFUT ANII DUTFUT TO HLNCC

C FOF SWANSEN/ENSSLIN

JOHNSON 94-FEE-81

FEEU, $11 \cdots F E F-81$

c:

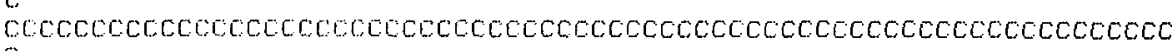

0001

0002

0003

0004

0005

C:

JMFLICIT INTECEE: (A-ZZ)

C.

LOGICAL * CHAFi $(80)$, INL TNE (80)

LOGICAL $* 1$ BUFFEF $(500)$

LOGICAL 1 L.INEFI, BLANK

is

[IATA LINEFE1/"12/, ELANK $; ; /$

USE THE NEW FUFTFAN-CALLAELE DLUI1 HANILEF

0006

0007

5

CALL INITELL (IIINEFII, ELANK, EUFFEF, 5OO)

c

GO TO 20

C LIST THE OFTTINS

$0008 \quad 10 \quad$ TYFE 1.000

00091000 FGFMAT, - WEGIN COMMUNICATIONS,

: 5 - START:

(H - HALT,

$X$

$x$ ' $Z$ - $X$ CLEAF SCALEFS ANI TIMEF'

$x$ 'Fi - FEAL LATA TO COMFUTEF',

$X$ "F. FIEAII OLIT IIATA TO HFQ7\%

$x \quad x$ REQUEST STATUS'

$x$ ' $x$ - SET INTERFUFT FLAG'

$X$, F... FETUFIN TO FFONT FANEL CONTFIOL' $/$

$X \quad T$ - TEST FOF INFUT',

$X$ 'W WAIT FOF INFUT'

$x \quad$ Q $\cdots$ FEINITIALIZE JNFUT',

C

C GET OFEFIATOF INFUT

0010

$\mathrm{C}$

20 TYFE 1001

1001 FOFMAT ('DOFTIONS $\cdots$,

ACCEFT 1.002, C.HAF

001.2

0013

1002 FOKMAT (BOA1)

C TEST FOFI SFECIAL CHAFACTEFS

0014

0016

IF (CHAF (1) , EQ, ' $\left.0^{\prime}\right)$ GO TO 10

IF (CHAF'(1) . EQ. 'T') GO TO 50

IF (CHAF'(1) , EQ. 'W') GO TO 40 


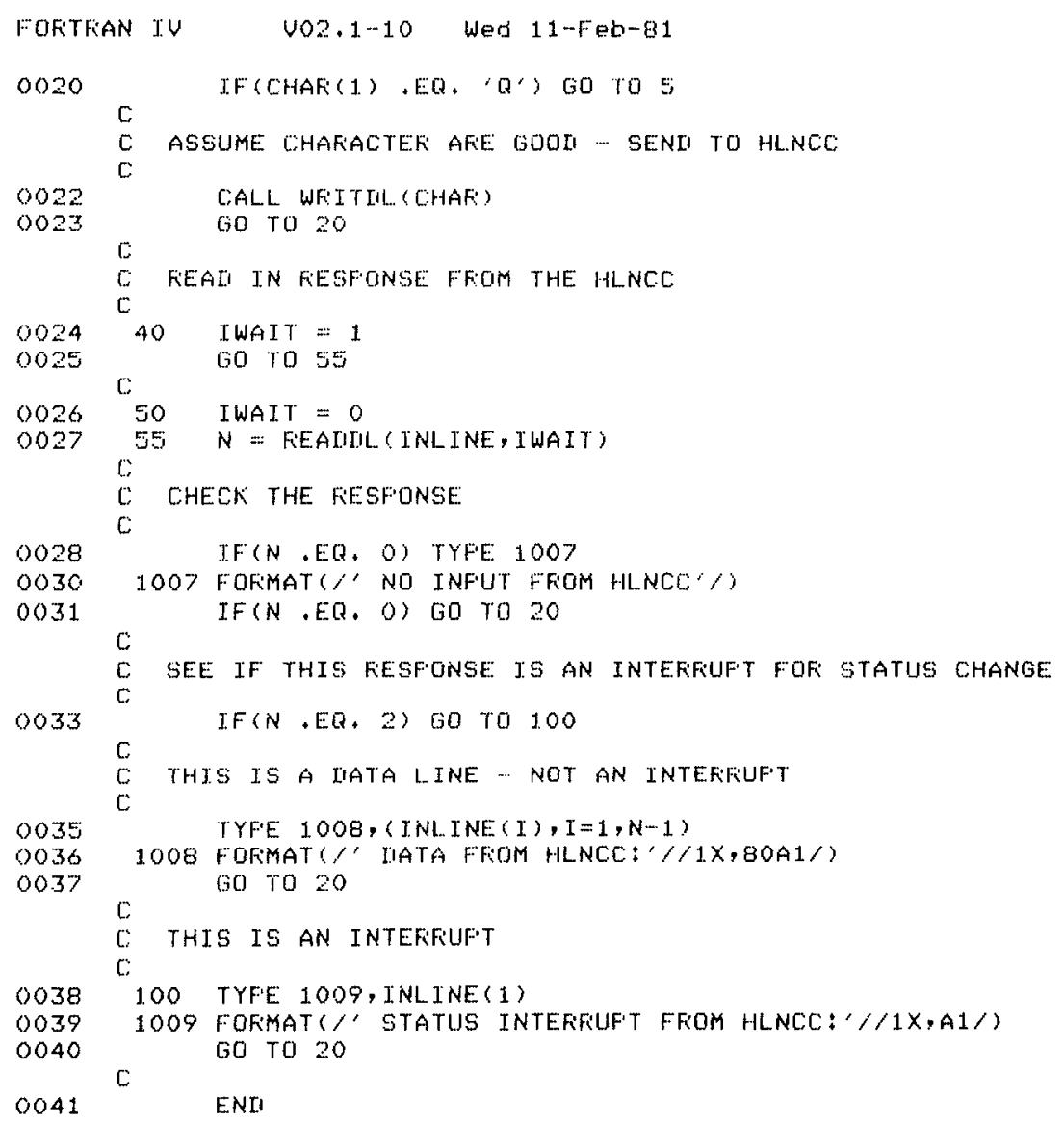




\section{APPENDIX C \\ HLNCC (PROM) VERSION 1.2; ORIGINAL PROM LISTING}

The software in this PROM was developed to provide the following capabilities for the HLNCC:

(a) Transmit data and control statements to a HP-97 calculator.

(b) Transmit data by RS-232 serial port (to a terminal, for "hard copy").

(c) Implement a software "recycle mode" to allow continuous data collection.

This version ( $V$ 1.2) does not provide for remote control of the HLNCC. 


\begin{tabular}{|c|c|c|c|c|c|c|c|c|}
\hline$\because A G E$ & 001 & & TFEOL & & & & & \\
\hline 00001 & & & & & NAH & & OONTFOL & \\
\hline 00002 & & & & & OF'T & & O & \\
\hline 00003 & 7000 & & & & OFG & & 47000 & \\
\hline 00004 & 7000 & $O F$ & & & $S E I$ & & & *IISAELE TNTEFFUET \\
\hline 00005 & 7001 & $8 E$ & $007 \mathrm{~F}$ & & LISS & & \#TACK & KLDAII STACK FUTNTEE \\
\hline 00006 & 7004 & En & $70 \mathrm{E} 5$ & & $J 9 F$ & & INIT & *.JUMF TO INITIALTZE FOUTTAE \\
\hline 00007 & 7007 & 86 & 29 & & LIIA & A & 相生29 & ALOAII FESET COIEE IMTO FIA \\
\hline 00008 & 7009 & $\mathrm{~B} 7$ & 5002 & & STA & $\hat{\mathrm{A}}$ & FTAO & \\
\hline 00009 & $700 \mathrm{C}$ & $\mathrm{HII}$ & $70 A 9$ & & $J S F$ & & FON & KTUFN ON FEATOUT LIGHT \\
\hline 00010 & $700 \mathrm{~F}$ & ESII & 7394 & & $15 \mathrm{Fi}$ & & TEST & * JUMF TO TEST SUEFOUTINE \\
\hline 0001.1 & 7012 & $\mathrm{HII}$ & $7 O A F$ & & $J S F$ & & FIOF & *TUFW OFF FEAIIOUT LISHT \\
\hline 00012 & 7015 & 86 & 28 & & L.IA & $\mathrm{A}$ & 棑 $\$ 28$ & *LOAD STOF COIE TNTO FTA \\
\hline 00013 & 7017 & 57 & 5002 & & STA & A & FIAO & \\
\hline 00014 & $701 \mathrm{~A}$ & $7 F$ & 0017 & L.F $F$ & CLFi & & 5 & $* S E T S=0$ \\
\hline 00015 & 70111 & $7 F$ & 0018 & & CLLF & & $\mathrm{T}$ & *SET T:=0 \\
\hline 00016 & 7020 & 20 & $2 E$ & & EFIA & & $\sqrt[3]{4}$ & *OO TO WAITINO LOOF \\
\hline 00017 & 7022 & 86 & 30 & L.F.1 & L... Ino & $A$ & 标 30 & WWAT FOF SF TO STOF \\
\hline 00018 & 7024 & EII & $709 \mathrm{~F}$ & & $J S F$ & & CHECH & \\
\hline 00019 & $702 \%$ & $2 A$ & $F 9$ & & EF'L & & $L F \cdot 1$ & \\
\hline 00020 & 7029 & 86 & 31 & & $1 \ldots[\mathrm{IA}$ & $\hat{A}$ & : $\$ 31$ & *III THE SF TIME OUT? \\
\hline 00021 & $702 \mathrm{~B}$ & $\mathrm{BI}$ & $709 \mathrm{~F}$ & & $J S F$ & & CHECK & \\
\hline 00022 & $702 E$ & $2 B$ & 05 & & EMI & & $M F 1$ & *YES, 60 TO JF1 \\
\hline $0002 \pi$ & 7030 & $7 F$ & 0018 & & CLFF & & $\dddot{\gamma}$ & *NO, SET $T=0$ \\
\hline 00024 & 7033 & 20 & 05 & & BFIA & & $W F=2$ & \\
\hline 00025 & 7035 & 06 & 01 & $J F 1$ & LIA & $\mathrm{E}$ & : 1 & $* 5 \mathrm{ET} T=1$ \\
\hline 00026 & 7037 & $\mathrm{~F} 7$ & 0018 & & STA & $\mathrm{H}$ & $T$ & \\
\hline 00027 & $703 \mathrm{~A}$ & 86 & 36 & $J F 2$ & $\operatorname{LIA}$ & $\mathrm{A}$ & $* 36$ & *IS SF IN FEEYYLE MOIE? \\
\hline 00028 & 7030 & $\mathrm{BI}$ & $709 \mathrm{~F}$ & & ISF & & CHECK & \\
\hline 00029 & $703 \mathrm{~F}$ & $2 B$ & of: & & BMT & & $I F \cdot 3$ & *No, Gll TO JF \\
\hline 00030 & 7041 & $C 6$ & 01 & & L...A & $\mathrm{B}$ & $\# 1$ & *YES, SET FI=1 \\
\hline 00031 & 7043 & $F 7$ & 0016 & & STA & $B$ & $\mathrm{Fi}$ & \\
\hline 00032 & 7046 & $F 1$ & 0018 & & CPFF & $\mathrm{H}$ & $\mathrm{r}$ & $* T=1 ?$ \\
\hline 00033 & 7049 & 27 & $1 \mathrm{~A}$ & & BEO & & $J F$ & *YES \\
\hline 00034 & $704 \mathrm{E}$ & 20 & 03 & & ERAA & & $J F^{4}$ & 籼O \\
\hline 00035 & $704 \mathrm{II}$ & $7 F$ & 0016 & $4 F 3$ & CLF: & & $\mathrm{F}$ & *SET Fi:O \\
\hline 00036 & 7050 & 86 & 30 & $J F^{\prime} 4$ & $\operatorname{LI} A$ & $A$ & $\$ \$ 30$ & *IS SF STOFFEII? \\
\hline 00037 & 7052 & En & $709 F$ & & $J S F i$ & & CHECK & \\
\hline 00038 & 7055 & $2 \hat{A}$ & $\mathrm{CE}$ & & EF'L. & & L..F' 1 & *NO, GO TO LFI. \\
\hline 00039 & 7057 & 86 & 34 & & L.IAA & $\hat{A}$ & $\# \$ 34$ & WYES, IS SF FIESET IIEFFIESSEII? \\
\hline 00040 & 7059 & BII & $709 ト$ & & JSF: & & C.HECK & \\
\hline 00041 & 7050 & $2 \mathrm{H}$ & $\mathrm{EC}$ & & BMl. & & L.F.3 & *YES, 00 TO LFB \\
\hline 00042 & $705 E$ & 86 & 37 & & LIMA & $\mathrm{A}$ & $\# \$ 37$ & *NO IS SF FEALOUT TEFFESSEII \\
\hline 00043 & 7060 & EII & $709 F$ & & ISF & & CHECK & \\
\hline 00044 & 7063 & $2 A$ & $\mathrm{EE}$ & & EFL & & $J F 4$ & NNO, 60 TO WAIT LOOF \\
\hline 00045 & 7065 & EII & $70 A 9$ & $J F$ & $J S F$ & & FiON & *TUFN ON FEAIIDUT LIGHT \\
\hline 00046 & 7068 & BII & 7136 & & $J S K$ & & LOAII & *FEAI THE IIISFLAY \\
\hline 00047 & $706 \mathrm{E}$ & BII & $730 \mathrm{E}$ & & $J S F i$ & & HFG & *SENI FIESULTS TO HF ...97 \\
\hline 00048 & $706 E$ & EI & 7189 & & $J S F$ & & SURTE & *SUETFACT A FFOM FItA \\
\hline 00049 & 7071 & 4F & & & CLE & $\mathrm{A}$ & & $* 5=0 r$ \\
\hline 00050 & 7072 & E1. & 0017 & & CMF & $\mathrm{A}$ & $S$ & \\
\hline 00051 & 7075 & 26 & 06 & & ENE & & If 6 & *No, Go TO JF 6 \\
\hline $\begin{array}{l}00052 \\
00053\end{array}$ & $\begin{array}{l}7077 \\
707 A\end{array}$ & $\begin{array}{l}\text { EII } \\
7 \mathrm{C}\end{array}$ & $\begin{array}{l}70110 \\
0017\end{array}$ & & $\begin{array}{l}\text { JSF } \\
\text { TNC }\end{array}$ & & $\begin{array}{l}\text { TITLE } \\
S\end{array}$ & $\begin{array}{l}\text { *YES, FFINT THE TITLE } \\
\text { *SET } S=1\end{array}$ \\
\hline 00054 & $707 \mathrm{II}$ & EII & $71 . \Pi \mathrm{IF}$ & JF 6 & $J S F i$ & & TYFE & *FFINT FESULTS \\
\hline 00055 & 7080 & EII & TOAF & & $J S F$ & & FiOF & *TUFIN OFF FIEALOUUT LIGHT \\
\hline 00056 & 7083 & $4 F$ & & & CLEF & $A$ & & $k T=0 r$ \\
\hline 00057 & 7084 & E1. & 0018 & & $\mathrm{CMF}$ & $A$ & $T$ & \\
\hline 00058 & 7087 & 27 & $c 7$ & & $\mathrm{BEQ}$ & & $3 F^{4} 4$ & *YES, 60 TO WAITING LOOF \\
\hline
\end{tabular}




\begin{tabular}{|c|c|c|c|c|c|c|c|}
\hline 00059 & 7089 & E1. & 0016 & & CMF & $\hat{A}$ & $\mathrm{Fi}$ \\
\hline 00060 & 7080 & 27 & C? & & $\mathrm{EEQ}$ & & $1 H^{\prime} 4$ \\
\hline 00061 & $708 E$ & 86 & 29 & & LIIA & $\hat{A}$ & $\because \div 29$ \\
\hline 00062 & 7090 & $\mathrm{~B} 7$ & 5002 & & STA & $\hat{\mathrm{A}}$ & F'IAO \\
\hline 00063 & 7093 & 86 & $2 A$ & & L. [IA & $A$ & $\forall \$ 2 A$ \\
\hline 00064 & 7095 & $B 7$ & 5002 & & STA & $A$ & F'IAO \\
\hline 00065 & 7098 & 86 & $2 \mathrm{E}$ & & LIIA & $A$ & $\# \$ 2 \mathrm{~B}$ \\
\hline 00066 & & & & * & & & \\
\hline 00067 & & & & * & & & \\
\hline $\begin{array}{l}00068 \\
00069\end{array}$ & $\begin{array}{l}709 A \\
70911\end{array}$ & $\begin{array}{l}17 \\
20\end{array}$ & $\begin{array}{l}5002 \\
83\end{array}$ & & $\begin{array}{l}\text { STA } \\
\text { ERA }\end{array}$ & $A$ & $\begin{array}{l}F I A D \\
\text { LFI }\end{array}$ \\
\hline 00070 & $709 F$ & 137 & 5002 & CHECK & $\Xi T A$ & $A$ & $F] A O$ \\
\hline $0007 \mathrm{~J}$ & $70 A 2$ & $B 6$ & 5000 & & LWA & $A$ & F'IAI \\
\hline 00072 & $70 A 5$ & E6 & 5000 & & L..IIA & $\hat{A}$ & FIAI \\
\hline 00073 & $70 A B$ & 39 & & & FITS & & \\
\hline $000 \% 4$ & $70 A 9$ & 86 & $3 C$ & FION & LIIA & $A$ & $\# \$ 3 C$ \\
\hline 00075 & $70 \mathrm{AB}$ & $\mathrm{B} 7$ & 5003 & & $\operatorname{STA}$ & $A$ & FIACE \\
\hline $000 \% 6$ & TOAE & 39 & & & FiTS & & \\
\hline 00077 & $70 \mathrm{AF}$ & 86 & 34 & FiOF & LIIA & $\hat{A}$ & $\$ \$ 34$ \\
\hline 00078 & $70 B 1$ & $\mathrm{~B} 7$ & 5003 & & STA & $\hat{A}$ & F IACE \\
\hline 00079 & $70 B^{4}$ & 39 & & & FITS & & \\
\hline 00080 & & 001 & & $\mathrm{~F}$ & EQU & & $\$ 16$ \\
\hline 00081 & & 00 & & $S$ & EQU! & & $\$ 17$ \\
\hline 00082 & & 001 & & $T$ & EQU & & $\$ 18$ \\
\hline 00083 & & 00 & & STACK & EQU & & $\$ 7 F$ \\
\hline 00084 & & 735 & & TEST & EQU & & 事7394 \\
\hline 00085 & & $70 \mathrm{E}$ & & INIT & EQU & & $\$ 70 \mathrm{ES}$ \\
\hline 00086 & & 71 & & L.OAII & EQU & & $\$ 7136$ \\
\hline 00087 & & 701 & & TITLE & EQU & & $\$ 7010$ \\
\hline 00080 & & 711 & & TYFE & EQU & & $\$ 71 \mathrm{IIF}$ \\
\hline 00089 & & 71.6 & & SUETF & EQU & & 47189 \\
\hline 00090 & & 500 & & FIAI & EQU & & $\$ 5000$ \\
\hline 00091 & & 500 & & FIAO & EQU & & $\$ 5002$ \\
\hline 00092 & & 500 & & $F \perp A C B$ & EQU & & $\$ 5003$ \\
\hline 00093 & & 730 & & HF97 & EQU & & $\$ 730 E$ \\
\hline 00094 & & & & & ENTI & & \\
\hline
\end{tabular}

*Na; $F=0$ ?

*YES, GO TO JF 4

*NO, FESET THE SFI

*STAFIT THI: SFI

* LOAII UNUSEI CONTFOL NUMEEF

TO FEMOUE MICFOFROCESSOF

CONTFOLL

* WAIT FOF SF TO STOF"

* sentr out status colie

* FEAII STATUS

* FEIHUNIIANT FEEAI STATUS

* FETUKR

*loar contFiol rieg. colie

*SENII IT OUT

*FETUFIV

* LOAII CONTFOL FEg, COLE:

SSENII IT OUT

XFETUFI

TOTAL EFFORS 00000

\begin{tabular}{|c|c|c|}
\hline PAEE & 001 & INITI \\
\hline $\begin{array}{l}00001 \\
000012 \\
00003 \\
00004\end{array}$ & & $\begin{array}{l}5000 \\
5002\end{array}$ \\
\hline $\begin{array}{l}00005 \\
00006 \\
00007 \\
00008 \\
00000\end{array}$ & & $\begin{array}{l}5001 \\
5003 \\
4000 \\
7000 \\
73 F E\end{array}$ \\
\hline $\begin{array}{l}\text { ano10 } \\
\text { 00011 } \\
\text { 0001E }\end{array}$ & $\begin{array}{l}\text { 73FE } \\
\text { T3FE } \\
\text { 70E5 }\end{array}$ & 8000 \\
\hline $\begin{array}{l}00013 \\
000194 \\
00015 \\
00016\end{array}$ & $\begin{array}{l}\text { 70E: } \\
\text { TaEs } \\
\text { TOE: } \\
\text { TaEA }\end{array}$ & $\begin{array}{l}4 F \\
E 75000 \\
43 \\
E 7=1002\end{array}$ \\
\hline
\end{tabular}




\section{(1):17 7 CIEI E:E 04 UOOIS TOEF ET 5001 colols

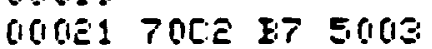

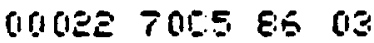

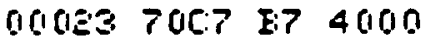 UUUE 7 UICA EE OS CUCE:5 7 OC: ET 4000 UCUE⿱E TUCF 39 COOEZT}

TDTAL EFFDF: OOOGO
LIIR $A$ :\$:4

STA A FIREA

STA F PIACE

LIIF $A$ :\$: 3

STA $A$ RIIAS

LIA $A$ \#\$?

STA $B$ RCIAS

FITS

ENII
-CHANEE FROM IATA IIIFECTIDN REGISTER TD IIFTA FEEISTTER. - IITTO

- MASTER FEESET

-SET UF IATA FDFMAT

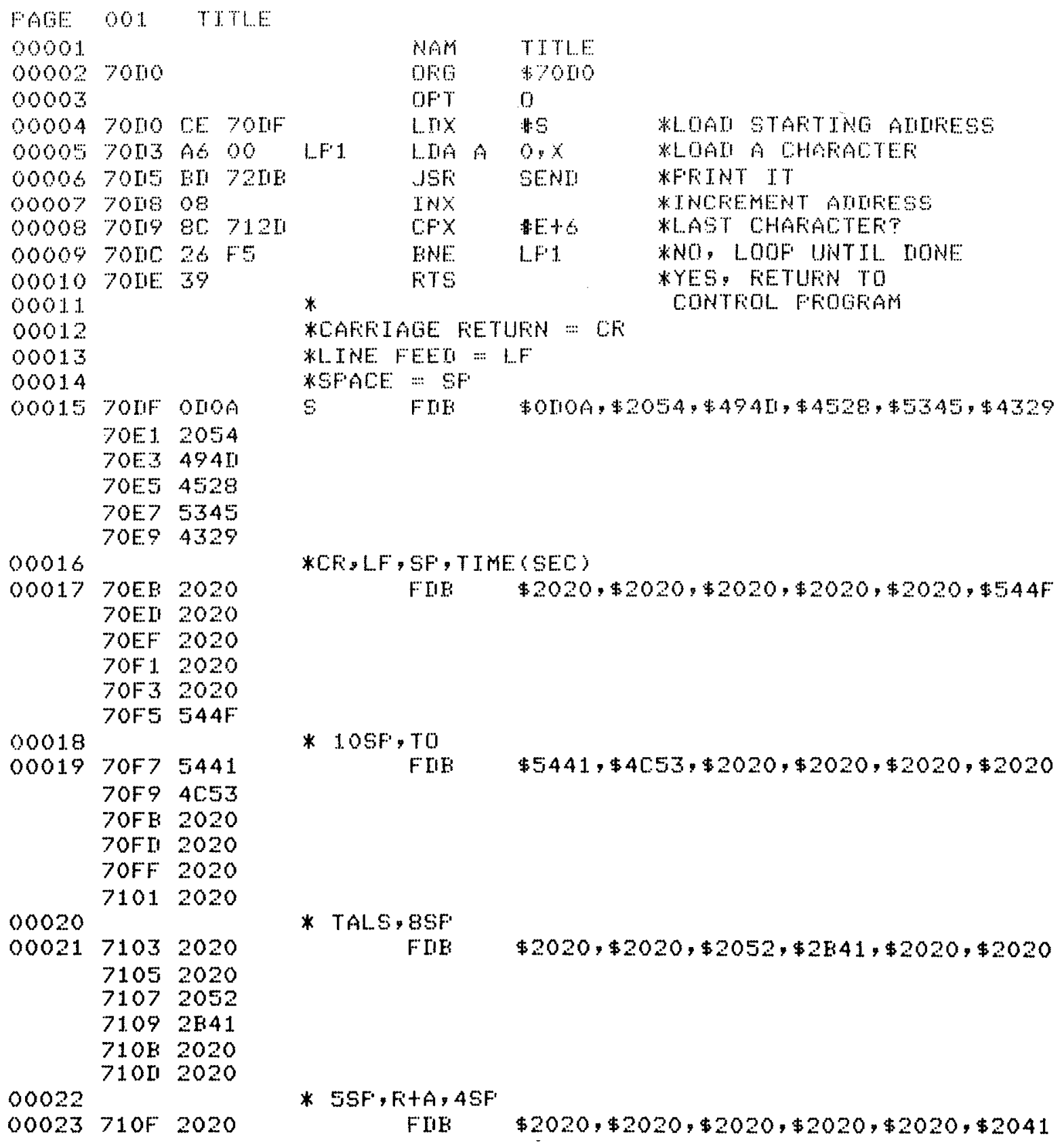

\begin{tabular}{|c|c|}
\hline NAM & TITLE: \\
\hline$O F G$ & *7oro \\
\hline OFT & 0 \\
\hline $\operatorname{LIX}$ & $\# 5$ \\
\hline LWA Aि & $0 . x$ \\
\hline$J S F$ & SENH \\
\hline$I N x$ & \\
\hline$C F X$ & $A E+6$ \\
\hline ENE & L.F' 1 \\
\hline
\end{tabular}

*

*CAFFIAGE FETUFN :-: CF 
71112020

$7113 \quad 2020$

$7115 \quad 2020$

711.72020

$7119 \quad 2041$

00024

$00025711 \mathrm{E} \quad 2020$

711112020

$711 \mathrm{~F} 2020$

71212020

$7123 \quad 2020$

71252020

00026

0002771272020

$7129 \quad 2020$

7125620

00028

00029

00030
* $11 \mathrm{SF} ; \mathrm{A}$

FIF $\$ 2020, \$ 2020, \$ 2020, \$ 2020, \$ 2020, \$ 2020$

* 1.25F

E FIE $\$ 2020, \$ 2020, \$ 5220$

* $4 S \mathrm{~F}, \mathrm{~F}, \mathrm{SF}$

SENO EQU

ENI!

$\$ 72[\mathrm{E}$

TOTAL.. EFFEFS 00000

\begin{tabular}{|c|c|c|c|c|c|c|c|c|}
\hline \multirow{3}{*}{$\begin{array}{l}\text { FGE } \\
00001 \\
00002\end{array}$} & 001 & \multicolumn{3}{|c|}{ L.OAII } & \multirow{2}{*}{\multicolumn{2}{|c|}{ NAM }} & \multirow[b]{2}{*}{ LOAII } & \\
\hline & & & & & & & & \\
\hline & & & & & OFT $T$ & & 0 & \\
\hline 00003 & & 713 & & LOAII & EQU & & $\$ 7136$ & \\
\hline 00004 & & 003 & & FIATI & EQU & & $\$ 30$ & * SET UIF IOOCATTON FOF IMTA \\
\hline 00005 & & & & * & & & & STOFAGE \\
\hline 00006 & 7136 & & & & OFEG & & L. LAFI & \\
\hline 00007 & 7136 & $\mathrm{CE}$ & 0030 & & LIIX & & $\# F A M$ & *LOAII INTEX FEGISTEF WTTH \\
\hline $\begin{array}{l}00008 \\
00009\end{array}$ & & & & * & & & & $\begin{array}{l}\text { FIFET STOFAGE LOCATION } \\
\text { *LOAII CONTFOL CORE " } " \text { INTO }\end{array}$ \\
\hline $\begin{array}{l}00009 \\
00010\end{array}$ & 7139 & 86 & 01 & & LIIA & A & $\$ 1$ & $\begin{array}{l}\text { *LOAII CONTFOL CORE "I" INTO } \\
\text { FIA E MATA FEGISTEF }\end{array}$ \\
\hline $\begin{array}{l}00010 \\
00011\end{array}$ & $713 \mathrm{~B}$ & $\mathrm{~B} 7$ & 5002 & * & STA & $\hat{A}$ & $\$ 5002$ & FIA E IIATA FEGISTEE \\
\hline 00012 & $71.3 \mathrm{E}$ & BI! & 7162 & L.F.1 & $J S F$ & & REAI & *FEEAII IIIGITA STOFE IN VAL. \\
\hline 00013 & 71.41 & $F 6$ & $000 \mathrm{c}$ & & L.IIA & E: & VAL & *MOUE IIIGIT LFFT \\
\hline 00014 & 7144 & 58 & & & ASL. & E & & \\
\hline 00015 & 7145 & 58 & & & ASL & $\mathrm{B}$ & & \\
\hline 00016 & 7146 & 58 & & & ASL & $\mathrm{B}$ & & \\
\hline 00017 & 7147 & 58 & & & ASL & E & & \\
\hline 00018 & 7148 & $7 \mathrm{C}$ & 5002 & & INC & & $\$ 5002$ & *INCFEMENT CONTFOL COLE \\
\hline 00019 & & & & * & & & & FOF NEXT IITGIT \\
\hline 00020 & $714 \mathrm{~B}$ & BII & 7162 & & JSFi & & FEAII & *FEAII THE IIIGIT \\
\hline 00021. & $71.4 \mathrm{E}$ & $F A$ & $000 \mathrm{C}$ & & DFIA & E & VAL & *FOFIM A 2-[IIGIT F'AIF \\
\hline 00022 & 7151 & E7 & 00 & & STA & $B$ & $0, x$ & *STOFE FEESULT AT FOINTEFI \\
\hline 00023 & 7153 & 08 & & & INX & & & * INCFEMENT FOINTEF \\
\hline 00024 & 7154 & $8 \mathrm{C}$ & 0042 & & CFX & & $\$ \$ 42$ & *FOINTEK $=42 ?$ \\
\hline 00025 & 7157 & 27 & 05 & & BEQ & & $J F^{\prime} 1$ & *YES, GO TO JF 1 \\
\hline 00026 & 7159 & $7 \mathrm{C}$ & 5002 & & INC & & $\$ 5002$ & *NO, INCFEMENT CONTFOL COIIE \\
\hline 00027 & $715 \mathrm{C}$ & 20 & EO & & EFIA & & LF 1 & *EFANCH TO FIFAII NFXXT \\
\hline 00028 & & & & * & & & & F'AIF OF IIGITS \\
\hline 00029 & $71 \mathrm{SE}$ & PF & 5002 & JF' 1 & CLFi & & $\$ 5002$ & *FEMOVE CONTFIOL COLIE \\
\hline 00030 & 7161 & 39 & & & FITS & & & *FETUR'N FFOM SUEROUTINE \\
\hline 00031 & 71.62 & Bo & 5000 & FIEAII & LIIA & A & $\$ 5000$ & *I.OAII THE FIA A IIATA FEEGIST \\
\hline 00032 & 7165 & $2 \mathrm{~B}$ & $\mathrm{FB}$ & & BMI I & & FEFAII & *FFOCEEII IF FOSITIUE \\
\hline 00033 & & & & * & & & & (IIATA FRESENT) \\
\hline 00034 & 7167 & 86 & $\mathrm{FF}$ & & L. IIA & A & $\# F F$ & *ALLOW IIEI.AY FOF \\
\hline
\end{tabular}




\begin{tabular}{|c|c|c|c|c|c|c|c|}
\hline 00035 & & & & * & & & \\
\hline 00036 & 71.69 & 57 & DOOE & & STA & A & DIL_Y \\
\hline 00037 & $71.6 \mathrm{C}$ & $7 A$ & $000 \mathrm{E}$ & $1 F 2$ & RIEC & & IILY \\
\hline 00038 & $71.6 \mathrm{~F}$ & 26 & $\mathrm{FE}$ & & ENE & & $L F 2$ \\
\hline 00039 & 7171 & B6 & 5000 & & LIIA & A & $\$ 500$ \\
\hline $\begin{array}{l}00040 \\
00041\end{array}$ & 7174 & & & $*$ & & & \\
\hline 00042 & 1.74 & 84 & or & $*$ & N1. & 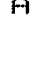 & $*$ \\
\hline 00043 & 7176 & 81 & OF & & CMF' & $A$ & $\# F$ \\
\hline 00044 & 71.78 & 26 & 01 & & ENE: & & $J F^{2} 2$ \\
\hline 00045 & $717 \mathrm{~A}$ & $4 F$ & & & CLFi & A & \\
\hline 00046 & $717 \mathrm{~B}$ & E7 & $000 \mathrm{C}$ & $\mathrm{JF}_{2}$ & STA & $A$ & VAL \\
\hline $\begin{array}{l}00047 \\
00048\end{array}$ & $717 \mathrm{E}$ & 39 & & & FITS & & \\
\hline 00049 & & $\begin{array}{l}000 \\
000\end{array}$ & & $\begin{array}{l}\text { ILY } \\
\text { UAL. }\end{array}$ & $\begin{array}{l}\text { EQU } \\
\text { EQU }\end{array}$ & & $\$ C$ \\
\hline 00050 & & & & & ENII & & \\
\hline
\end{tabular}

SETTLING TIMF:

TOTAL ERFORS 00000

\begin{tabular}{|c|c|c|c|c|c|c|c|c|}
\hline$F A G E$ & 001 & & JETFAO & & & & & \\
\hline 00001 & & & & & NAM & & SUBTFACT & \\
\hline 00002 & & & & & OFT & & 0 & \\
\hline 00003 & 7189 & & & & OFG & & $\$ 7189$ & \\
\hline $\begin{array}{l}00004 \\
00005\end{array}$ & 7189 & PF & 0001 & * & CLF: & & SIGN & $\begin{array}{l}\text { *CLEAF } S T G N \text { CONTFOL. } \\
\text { CONSTANT (O FOF }+ \text { \& FOF } \cdots \text { ) }\end{array}$ \\
\hline 00006 & 7180 & $C E$ & 0038 & & $\operatorname{LIXX}$ & & \#S & *LOAI STAFTING AIIIFESS \\
\hline 00007 & $718 \mathrm{~F}$ & $\cos$ & 05 & & L..LA & $\mathrm{E}$ & H & *LOAAI cONTFOL constant \\
\hline 00008 & 7191 & 86 & 99 & L.F. 1 & LLAA & $A$ & $\# \$ 99$ & *LOARI $\$ 99$ \\
\hline 00009 & 7193 & AO & 09 & & SUE & $A$ & $9, x$ & *SUETFACT TO COMFLEMFNT \\
\hline 00010 & 7195 & A7 & $O E$ & & STA & $A$ & QE, X & *STOFE COMHEMENT \\
\hline 00011 & 7197 & 09 & & & IUEX & & & *IIECFIEMENT AIIIIEESS \\
\hline 00012 & 7198 & $5 A$ & & & IIEC & $\mathrm{E}$ & & *IEECFEMENT CONTFOL NUMEEF \\
\hline 00013 & 71.99 & 26 & FG & & ENE & & $\operatorname{LF} 1$ & *FECYCLE UNTIL ALL TITGITS \\
\hline 00014 & & & & * & & & & AFE COMFLEMENTEI \\
\hline 00015 & $719 \mathrm{~B}$ & on & & & $\mathrm{SEC}$ & & & *SET THE CAFFY \\
\hline 00016 & $719 \mathrm{C}$ & $\cos$ & 05 & & LUA & $\mathrm{B}$ & $\$ 5$ & *LOAI CONTFOL NUMEEF \\
\hline 00017 & $71.9 \mathrm{E}$ & CE & 0038 & & LIIX & & *5 & *LOALI STARTING AIIRIEESS \\
\hline 00018 & $71 A 1$ & $A G$ & 04 & LF2 & LLA & A & $4, x$ & *LOAII FAIF OF IIIGITS \\
\hline 00019 & & & & & & & & FOR ALIIITTION \\
\hline 00020 & $71 A 3$ & A9 & $O E$ & $J F 5$ & AllC & $A$ & DE, $X$ & *AIII WTTH CAFEYY \\
\hline 00021 & $71 \mathrm{AS}$ & 1.9 & & & IIAA & & & *IIECIMAL ATIJUST \\
\hline 00022 & $71 A G$ & A 7 & 13 & & STA & $A$ & $\$ 1.3, x$ & *STORE SUM \\
\hline 00023 & $71 \mathrm{AB}$ & 09 & & & IIEX & & & *IIECFEMENT ATILFESS \\
\hline 00024 & $71 \mathrm{A9}$ & $5 A$ & & & IIEC & $\mathrm{B}$ & & *DECKEMENT CONTFOL NUMBEF \\
\hline 00025 & 71AA & 26 & $F 5$ & & ENE. & & $1 . F_{2}^{2}$ & *FECYCLE UNTIL AHIITTON \\
\hline 00026 & & & & * & & & & IS COMFLETE \\
\hline 00027 & $71 A C$ & 24 & 01 & & $\mathrm{BCC}$ & & $J F \cdot 1$ & *BFIANCH TO JFI IF \\
\hline 00028 & & & & * & & & & CAFFEY IS CLEAF \\
\hline 00029 & $71 \mathrm{AE}$ & 39 & & & RTS & & & *FETUFIN TO CONTFOL FFOGFAM \\
\hline 00030 & & & & * & & & & IF CAFFFY IS SET \\
\hline $\begin{array}{l}00031 \\
00032\end{array}$ & $\begin{array}{l}71 \mathrm{AF} \\
71 \mathrm{BJ}\end{array}$ & $\begin{array}{l}86 \\
17\end{array}$ & $\begin{array}{l}01 \\
000 \mathrm{II}\end{array}$ & $J F^{\prime} 1$ & $\begin{array}{l}\text { LIIA } \\
\text { STA }\end{array}$ & $\begin{array}{l}A \\
A\end{array}$ & $\begin{array}{l}\# 1 \\
S I G N\end{array}$ & *CHANGE STGN TO - \\
\hline 00033 & 7184 & $\mathrm{CE}$ & 0038 & & LIX & & $\# 5$ & *REUEFSE SUETFAHENII ANI \\
\hline 00034 & & & & * & & & & MINUENII ANII FEFEAT \\
\hline 00035 & $71 \mathrm{~B} 7$ & C6 & 05 & & LIIA & $\mathrm{E}$ & $\$ 5$ & \\
\hline 00036 & 7189 & 86 & 99 & $L F \cdot 4$ & LIA & $A$ & $\$ \$ 99$ & \\
\hline
\end{tabular}

*LOAII THE FIIA A IIATA FEEISTEF RERUUNIIANTLY *STFIF OFF LEAST SIGNIFICANT 4 EITS *IATA $=F$ ?

*NO, FROCEEI

*YES, CHANGE TO ZEFO * STOFE FiESULT IN VAL *RETUFIN FFOM SUEROUTINE
*CLEAF: STGN CONTFOL * LoAII STAFTING ATIIFESS *loAI contFol constant * SUETFACT TO COMFLEMFNT TTERE COMFLENFNT *IIECFEMENT CONTFOL NUMBEF *FECYCLE UNTIL ALL TIIGITS AFE COMFLEMENTEI

*SET THE CAFFY

*LOALI CONTFOL NUMAEF

LoAli STARTING alILIRESS LOAII FAIF OF IIIGITS

FOF AIIIITTION

WALII WTTH CAFFY

*IIECIMAL. AIIJUST

*IECFEMENT CONTFOL NUMBEF

ISECLE UNTIL AIIUITTION

CAFFYY IS CLEAF' *FETUFN TO CONTFOL FFOGGAM IF CAFFYY IS SET REVEFSE SUETFAHENII ANI MINUENII ANII FEFEAT 


\begin{tabular}{|c|c|c|c|c|c|c|c|}
\hline 00037 & $71 \mathrm{BE}$ & AO & 13 & & SUE & $A$ & $\$ 13, x$ \\
\hline 00038 & $71 \mathrm{Br}$ & $A 7$ & 13 & & STA & $A$ & $\$ 13, x$ \\
\hline 00039 & $71 \mathrm{BF}$ & 09 & & & IIEX & & \\
\hline 00040 & 7100 & $5 A$ & & & IIEC & $\mathrm{B}$ & \\
\hline 00041 & 7101 & 26 & $F G$ & & BNE & & $L F^{\prime} 4$ \\
\hline 00042 & 7103 & OII & & & $S E C$ & & \\
\hline 00043 & 7104 & C6 & 05 & & $\operatorname{LI} A$ & $E$ & $\$ 5$ \\
\hline 00044 & $71 \mathrm{C} 6$ & $C E$ & 0038 & & L.IIX & & $\$ 5$ \\
\hline 00045 & 7169 & 86 & 00 & LFF & LIIAA & $A$ & $\neq 0$ \\
\hline 00046 & $71 \mathrm{CE}$ & A9 & 1.3 & & $A I I C$ & $A$ & $\$ 13, x$ \\
\hline 00047 & $71 \mathrm{CI}$ & 1.9 & & & $I I A \hat{A}$ & & \\
\hline 00048 & 7.CE & $A 7$ & 13 & & STA & $A$ & $\$ 13, x$ \\
\hline 00049 & 71110 & 09 & & & IIEX & & \\
\hline 00050 & $71[1$. & $5 A$ & & & TIEC & $\mathrm{B}$ & \\
\hline 00051 & 7112 & 26 & $\mathrm{FS}$ & & ENE: & & LF: \\
\hline $\begin{array}{l}00052 \\
00053\end{array}$ & $71[14$ & $\begin{array}{l}39 \\
00\end{array}$ & & $S$ & $\begin{array}{l}\text { FiTS } \\
\text { EQU }\end{array}$ & & 丰38 \\
\hline $\begin{array}{l}00054 \\
00055\end{array}$ & & 000 & & $S I G N$ & $\begin{array}{l}\text { EQLI } \\
\text { ENII }\end{array}$ & & $\$[I$ \\
\hline
\end{tabular}

TOTAL EFFOFS 00000

\begin{tabular}{|c|c|c|c|c|c|c|c|c|c|}
\hline FAGE & 001 & \multicolumn{3}{|c|}{ FFint } & & & & & \\
\hline 00001 & & & & & NAP & & FFitit & & \\
\hline 00002 & & & & & OFT & & 0 & & \\
\hline 00003 & & 000 & & $516 N$ & EQU & & 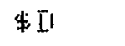 & & \\
\hline 00004 & & 000 & & NUM & EQU & & $\$ E$ & & \\
\hline $0000 \%$ & & 000 & & Conjo & EQU & & $\$ F$ & & \\
\hline 00006 & & 001 & & $F$ & E. & & $\$ 10$ & & \\
\hline $0000 \%$ & & 721 & & SENW & EQU & & $\$ 72 \mathrm{IH}$ & & \\
\hline 00008 & & 725 & & TYFE & EQOU & & $\$ 25 \mathrm{~A}$ & & \\
\hline 00009 & $71 \mathrm{IIF}$ & & & & OFIO & & 牛71. IIF & & \\
\hline 00010 & 71 lif & 86 & $0 n$ & & L..IA & $A$ & $\#$ & WFFINT GAFETAGE FETURN & $(C F)$ \\
\hline 00011 & $71 \mathrm{E} 1$ & In & $72 \mathrm{HH}$ & & JSF: & & SENII & & \\
\hline 00012 & $71 . \mathrm{E} 4$ & 86 & OA & & LIIA & $A$ & $\# A$ & WFEINT LINE FEEII (LF) & \\
\hline 00013 & $71 E 6$ & $\mathrm{EI}$ & $72 \mathrm{IIB}$ & & $J S F:$ & & SENII & & \\
\hline 00014 & $71 E 9$ & EII & 7240 & & $J S F_{i}$ & & $S F$ & *FFINT 3 SFACES & \\
\hline 00015 & 71E: & 86 & 05 & & L. IIA & $A$ & $\$ \$ 5$ & *SET NUM $=5$ & \\
\hline 00016 & $71 E E$ & $9 \%$ & $O E$ & & STA & A & NUM & & \\
\hline 00017 & $71 \% 0$ & 86 & 30 & & L.IIA & $A$ & $\$ 30$ & *LOATI STAFTTHG AIITKEES & \\
\hline 00018 & & & & * & & & & $(\$ 0030)$ AT F, F+1 & \\
\hline 0001.9 & $71 F 2$ & 97 & 1. 1 & & $\operatorname{STA}$ & $A$ & $F+1$ & & \\
\hline 00020 & $71 F A$ & $7 F$ & 0010 & & CLER & & $\mathrm{F}$ & & \\
\hline 00021 & $71 \mathrm{~F} 7$ & $\not F$ & OOOF & & CLF' & & COM & *SET COM $=0$ & \\
\hline 00022 & 71.FA & $\mathrm{HII}$ & $725 A$ & & $J S F i$ & & TYF'E & *TYFE DUT TIME & \\
\hline 00023 & $71 \mathrm{FII}$ & BSI & 7240 & & ISF & & $S F$ & \#FFINT 3 SFACES & \\
\hline 00024 & 7200 & 86 & 09 & & L. IIA & $A$ & $+\$ 7$ & *SET NUM $=9$ & \\
\hline 00025 & 7202 & 97 & $O E$ & & STA & $A$ & NuM & & \\
\hline 00026 & 7204 & 86 & 33 & & LIIA & $\hat{A}$ & $\$ \$ 33$ & *SET STAFTING ATIIFESS & $\because: 4003$ \\
\hline 00027 & 7206 & 97 & 11 & & STA & $A$ & $F+1$ & & \\
\hline $\begin{array}{l}00028 \\
00029\end{array}$ & $\begin{array}{l}7208 \\
720 A\end{array}$ & $\begin{array}{l}86 \\
97\end{array}$ & $\begin{array}{l}02 \\
O F\end{array}$ & & $\begin{array}{l}\text { LIIA } \\
\text { STA }\end{array}$ & $\begin{array}{l}A \\
A\end{array}$ & $\begin{array}{l}\text { E. } 52 \\
\text { COM }\end{array}$ & *SET $\quad \mathrm{COM}=2$ & \\
\hline 00030 & 7200 & BII & $725 \mathrm{~A}$ & & $J S F i$ & & TYF'E & *TYFE DUT TOTALS & \\
\hline 00031 & $720 F$ & EII & 7240 & & JSF: & & $\mathrm{SF}$ & *FFIINT 3 SFACES & \\
\hline 00032 & 7212 & 86 & 38 & & LIIA & $A$ & $\$ 38$ & *SET STAFT ING ATIIIFESS & $=\$ 003$ \\
\hline 00033 & 721.4 & 97 & 11. & & STA & $A$ & $F+1$ & & \\
\hline 00034 & 7216 & EI.! & $725 A$ & & JSFi & & TYFE & *TYFE QUT FItA & \\
\hline
\end{tabular}




\begin{tabular}{|c|c|c|c|c|c|c|c|c|}
\hline 00035 & 7219 & EII & 7240 & & $J S F$ & & $S F$ & *FFINT 3 SFACES \\
\hline 00036 & $721 \mathrm{C}$ & 86 & 3 II & & LIIA & A & $\# \$ 3 \mathrm{I}$ & *SET STAFTING AIILFESS $=\$ 003$ \\
\hline 00037 & $721 \mathrm{E}$ & 97 & 11 & & STA & A & $F+1$ & \\
\hline 00038 & 7220 & $\mathrm{EII}$ & $725 \mathrm{~A}$ & & JSF & & TYFEE & *TYFE OUT ACCIDENTALS \\
\hline 00039 & 7223 & BII & 7240 & & $J S F$ & & SF & *TYFE 3 SFACES \\
\hline 00040 & 7226 & 86 & 01. & & L.IIA & A & $\$ 1$ & *IS (FI+A) - A FDSITIVE? \\
\hline 00041 & 7228 & 91 & OII & & CMF & A & SIGN & \\
\hline 00042 & $722 \mathrm{~A}$ & 27 & 07 & & BER & & $J F 1$ & *NO, GO TO JFI \\
\hline 00043 & 7220 & 86 & $2 \mathrm{E}$ & & LIAA & A & $+2 \mathrm{E}$ & *YES, FRINT + \\
\hline 00044 & $722 \mathrm{E}$ & BII & $72 \mathrm{NE}$ & & JSF & & SENII & \\
\hline 00045 & 7231 & 20 & 05 & & EFIA & & $J F \cdot 2$ & *GO TO JF2 \\
\hline 00046 & 7233 & 86 & $2 \mathrm{II}$ & $J F 1$ & L..IIA & A & $\$ 2 I$ & *FFINT - \\
\hline 00047 & 7235 & $\mathrm{BII}$ & $72 \mathrm{IIF}$ & & JSF & & SENI! & \\
\hline 00048 & 7238 & 86 & 47 & $J F_{2}$ & LLI'A & A & $\# \$ 47$ & *SET STAFITING AIIIIRESS $=\$ 004$ \\
\hline 00049 & $723 \mathrm{~A}$ & 97 & 1.1 & & STA & A & $F+1$ & \\
\hline 00050 & $723 \mathrm{C}$ & EII & $725 \mathrm{~A}$ & & $J S F$ & & TYFE & *TYFE OUT $(F+A)-A$ \\
\hline 00051 & $723 \mathrm{~F}$ & 39 & & & FITS & & & *FEETUFN TO CONTFOL FFOGFAM \\
\hline $\begin{array}{l}00052 \\
00053\end{array}$ & 7240 & 86 & 20 & $\begin{array}{l}S F \cdot \\
*\end{array}$ & LIIA & $A$ & $\$ \$ 20$ & $\begin{array}{l}\text { *FFINT } 3 \text { SFACES } \\
\text { SUEFOUT INE }\end{array}$ \\
\hline 00054 & 7242 & BII & $20 \mathrm{HE}$ & & JSF & & SENII & \\
\hline 00055 & 7245 & 86 & 20 & & LIA & $A$ & \#20 & \\
\hline 00056 & $724 \%$ & EII & 72LE: & & JSFi & & SENII & \\
\hline 00057 & $724 \mathrm{~A}$ & 86 & 20 & & LI & A & $\$ 20$ & \\
\hline 00058 & $724 \mathrm{C}$ & EIII & $72 \mathrm{NH}$ & & JSF & & SENI & \\
\hline 00059 & $724 \mathrm{~F}$ & 39 & & & FITS & & & *FETUFN \\
\hline & & & & & & & & \\
\hline OTAL.. & EFFEOF & 150 & 0000 & & & & & \\
\hline$F A G E$ & 001 & & F'E & & & & & \\
\hline 00001 & & & & & NAM & & TYFE & \\
\hline 00002 & & & & & OFT & & & \\
\hline 00003 & $725 A$ & & & & OFG & & $4725 \mathrm{~A}$ & \\
\hline 00004 & $725 \mathrm{~A}$ & $\mathrm{TF}$ & 0012 & TYFE & ILF & & i & WLLEAR OONTFOL W'S J,F'\& K \\
\hline 00005 & $725 \mathrm{H}$ & $\mathrm{TF}$ & 0014 & & CLF: & & $F$ & \\
\hline 00006 & 7260 & $7 F$ & 0013 & & CLF & & K & \\
\hline 00007 & 7263 & $F E$ & 0010 & & $i \ldots x$ & & $\mathrm{~F}$ & *LOAII STAFTING ADIRESS JN $X$ \\
\hline 00008 & 7266 & As & 00 & L.F'1 & LIAA & A & $0, x$ & *LOAII A FAIFE OF IIIGITS \\
\hline 00009 & 7268 & 711 & 0014 & & $\mathrm{TST}$ & & $F$ & *NEEI LEAST SIG, IIISITT \\
\hline 00010 & $726 \mathrm{E}$ & 27 & 04 & & $\mathrm{BEQ}$ & & Bi & *NO, GO TO B1 \\
\hline 00011 & 7261 & 84 & OF & & ANLI & A & $\# \mathbf{\# F}$ & *YES, GET LEAST SIG, 4 EITS \\
\hline 00012 & $726 \mathrm{~F}$ & 20 & 06 & & EFA & & E2 & \$GO TO $\mathrm{Ba}$ \\
\hline 00013 & 7271 & 84 & Fo & E1 & ANOI & $A$ & $\#$ \#O & *FICK OFF MOST SIG, 4 EITS \\
\hline 00014 & 7273 & 46 & & & FOF: & $\mathrm{A}$ & & WOUE FIOHT A EITS \\
\hline 0001.5 & 7274 & 46 & & & FOF: & $A$ & & \\
\hline 00016 & 7275 & 46 & & & EOF: & $A$ & & \\
\hline 00017 & 7276 & 46 & & & FOF: & $A$ & & \\
\hline 00018 & 7277 & 411 & & $\mathrm{E2}$ & TST & $\mathrm{A}$ & & $* \mathrm{IIGIT}=0 ?$ \\
\hline 00019 & 7278 & 27 & $O A$ & & $\mathrm{BEQ}$ & & $\mathrm{B3}$ & *YES, GO TO BS \\
\hline 00020 & $727 \mathrm{~A}$ & $\mathrm{Cs}$ & 01 & & LIHA & $\mathrm{B}$ & $\neq 1$ & *NO, SET KN=1 \\
\hline 00021 & $727 \mathrm{C}$ & $F 7$ & 0013 & & STA & $\mathbf{B}$ & $k$ & \\
\hline 00022 & $727 F$ & EII & $72 \mathrm{IIE}$ & $\mathrm{B} 5$ & JSF & & SENI! & *FFIINT THE CHARACTEF \\
\hline 00023 & 7282 & 20 & 11 & & BFA & & B4 & *GO TO $\quad$ BA \\
\hline 00024 & 7284 & $7 \mathrm{II}$ & 0013 & E3 & TST & & Ki & $* K=0 ?$ \\
\hline 00025 & 7287 & 26 & FG & & ENE & & $B 5$ & *NO, FFINT THE IIIGIT \\
\hline 00026 & 7289 & F6 & OOOE & & LIAA & $\mathrm{E}$ & NUM & *YES IS THIS THF LAST IIIGIT \\
\hline 00027 & & & & * & & & & IN $\mathrm{T}$ \\
\hline
\end{tabular}




\begin{tabular}{|c|c|c|c|c|c|c|c|}
\hline 00028 & $728 C$ & $F 1$ & 0012 & & CMF & E & $J$ \\
\hline 00029 & $728 \mathrm{~F}$ & 27 & $E E$ & & $\mathrm{BEQ}$ & & ES \\
\hline 00030 & 7291 & 86 & 20 & & LIIA & A & $\$ \$ 20$ \\
\hline 00031 & 7293 & 20 & $E A$ & & EFIA & & BE \\
\hline 00032 & 7295 & E6 & OOOE & BA & $L I I A$ & A & NUMY \\
\hline 00033 & & & & * & & & \\
\hline 00034 & 7298 & B 1 & 0012 & & CMF & $A$ & $\mathrm{~J}$ \\
\hline 00035 & $729 \mathrm{~B}$ & 26 & 01 & & ENE & & B6 \\
\hline 00036 & 72911 & 39 & & & FITS & & \\
\hline 00037 & $729 E$ & $7 C$ & $000 \mathrm{~F}$ & B6 & INC & & COM \\
\hline 00038 & $72 A 1$ & 86 & 03 & & LIIA & A & $\$ 3$ \\
\hline 00039 & $72 A 3$ & B1 & $000 \mathrm{~F}$ & & CMF & A & COM \\
\hline 00040 & $72 A 6$ & 26 & 16 & & ENE & & F. 7 \\
\hline 00041 & $72 A 8$ & 86 & 01 & & LIIA & A & 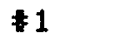 \\
\hline 00042 & $72 A A$ & $\mathrm{~B} 1$ & 0013 & & CMF & A & 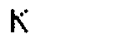 \\
\hline 00043 & $72 \mathrm{AII}$ & 26 & 07 & & BNE & & B8 \\
\hline 00044 & T2AF & 86 & $2 \mathrm{C}$ & & LIIA & $A$ & $\neq \$ 2 C$ \\
\hline 00045 & $72 \mathrm{B1}$ & BII & $72 \mathrm{IIB}$ & & JSF: & & SENII \\
\hline 00046 & $72 \mathrm{BA}$ & 20 & 05 & & BF'A & & B9 \\
\hline 00047 & $72 \mathrm{~B} 6$ & 86 & 20 & B8 & LIIA & A & $\$ 20$ \\
\hline 00048 & $72 B 8$ & BII & $72 \mathrm{IIE}$ & & JSF & & SFNII \\
\hline 00049 & $72 \mathrm{BB}$ & $7 F$ & OOOF & $\mathrm{B} 9$ & CLF' & & COM \\
\hline 00050 & 72BE & $7 C$ & 0012 & B7 & INC & & $J$ \\
\hline 00051 & $72 \mathrm{C} 1$ & $7 C$ & 0014 & & INC & & $F^{\circ}$ \\
\hline 00052 & $72 C 4$ & 86 & 02 & & LIIA & A & $\neq 2$ \\
\hline 00053 & 7206 & B1 & 0014 & & CMF' & A & $F^{\prime}$ \\
\hline 00054 & $72 C 9$ & 26 & $9 \mathrm{~B}$ & & BNE & & LF'1 \\
\hline 00055 & $72 \mathrm{CH}$ & TF & 0014 & & CLF' & & $F^{\prime}$ \\
\hline 00056 & $72 \mathrm{CE}$ & 08 & & & INX & & \\
\hline 00057 & $72 \mathrm{CF}$ & 20 & 95 & & BFiA & & LF' 1 \\
\hline 00058 & & $72 \pi$ & & SEND & EQU & & $\$ 72 \mathrm{II}$ \\
\hline 00059 & & 000 & & NUMY & EQU & & $\$ E$ \\
\hline 00060 & & 000 & & COM & EQU & & $\$ F$ \\
\hline 00061 & & 001 & & $F$ & EQU & & $\$ 10$ \\
\hline 00062 & & 001 & & $J$ & EQU & & $\$ 12$ \\
\hline 00063 & & 001 & & $\mathfrak{k}$ & EQU & & $\$ 13$ \\
\hline 00064 & & 001 & & F. & EQU & & $\$ 14$ \\
\hline 00065 & & & & & ENI & & \\
\hline
\end{tabular}

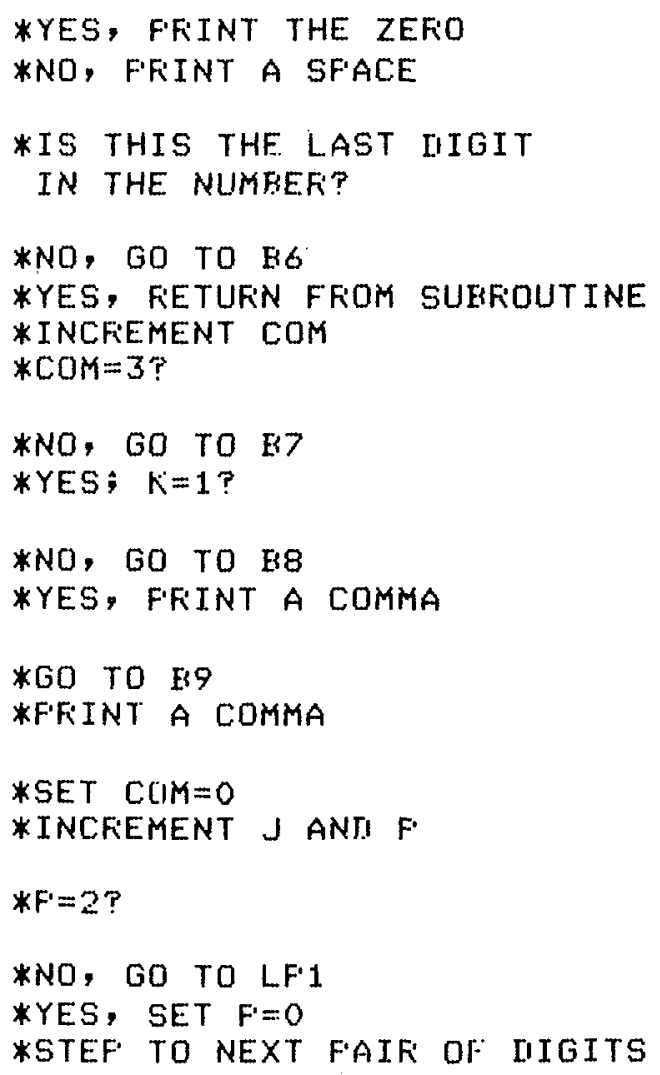

TOTAL EFFOFS 00000

FAGE 001 SENTI

\begin{tabular}{|c|c|c|c|c|c|c|c|c|}
\hline 00001 & & & & & NAM & & SENII & \\
\hline 00002 & & & & & OFT & & 0 & \\
\hline 00003 & $72 \mathrm{InB}$ & & & & DFE & & क72 $\mathrm{LE}$ & \\
\hline 00004 & $72 \mathrm{LH}$ & $8 \mathrm{~J}$. & 09 & & CHF & $A$ & $\$ 9$ & *CHAFACTEFY? \\
\hline 00005 & $72 \mathrm{IHI}$ & $2 E$ & 02 & & EGT & & $F \cdot T$ & *YES, FFOCEEI \\
\hline 00006 & $72 \mathrm{IIF}$ & $8 A$ & 30 & & OFIA & A & $\# \$ 30$ & *NO, FOFIM ASCII COLE \\
\hline $\begin{array}{l}00007 \\
00008\end{array}$ & $\begin{array}{l}72 E 1 \\
72 E 3\end{array}$ & $\begin{array}{l}\text { C6 } \\
F 7\end{array}$ & 01 & F'T & $\begin{array}{l}\text { LIIA } \\
\text { STA }\end{array}$ & $\begin{array}{l}\mathrm{B} \\
\mathrm{F}\end{array}$ & $\begin{array}{l}\# 1 \\
I I E L\end{array}$ & *SET IIELAY CONSTANT $=1$ \\
\hline 00009 & $72 E 6$ & 81 & on & & CMF & $A$ & \#\$OII & *IS CHAFACTEF $\$ O E$ ? \\
\hline 00010 & $72 E 8$ & $2 \mathrm{E}$ & 05 & & EGT & & $J F 1$ & *NO, FFOCEEI \\
\hline 00011 & 72EA & $c 6$ & 08 & & LIIA & E & $\$ 8$ & *YES, SET IIELAY CONSTANT $=8$ \\
\hline 00012 & $72 \mathrm{EC}$ & $\mathrm{F} 7$ & 0015 & & STA & $\mathrm{B}$ & IIEL & \\
\hline 00013 & 72EF & F6 & 4000 & JF 1. & I. TIA & $\mathrm{B}$ & ACIAS & *LOAII STATUS OF ACIA \\
\hline 00014 & $72 F 2$ & 56 & & & FOF & $\mathrm{E}$ & & \\
\hline
\end{tabular}




\begin{tabular}{|c|c|c|c|c|c|c|}
\hline 00015 & $72 F 3$ & 56 & & FIOF: & $\mathrm{B}$ & \\
\hline 00016 & $72 F 4$ & $24 \mathrm{Fq}$ & & $\mathrm{BCC}$ & & $J F^{\prime} 1$ \\
\hline 00017 & $72 F 6$ & E7 4001 & & STA & $A$ & $A C I A T$ \\
\hline 00018 & & & * & & & \\
\hline 00019 & $72 F 9$ & $867 F$ & & $\operatorname{LI} A$ & $\hat{A}$ & $\$ \$ 7 \%$ \\
\hline 00020 & $72 \mathrm{FE}$ & $7 A \quad 0015$ & & IIEC: & & IIEL \\
\hline 00021 & 72FE & $26 \mathrm{EF}$ & & BNE: & & $J F 1$ \\
\hline 00022 & 7300 & 39 & & Fits & & \\
\hline 00023 & & 4000 & $A C] A S$ & EQU & & $\$ 4000$ \\
\hline 00024 & & 4001 & $A C] A T$ & ERU & & $\$ 4001$ \\
\hline $\begin{array}{l}00025 \\
00026\end{array}$ & & 0015 & IIELL. & $\begin{array}{l}\text { EQU } \\
\text { ENI }\end{array}$ & & $\neq 15$ \\
\hline
\end{tabular}

TOTAL. EFIFUFIS 00000

NI.

\begin{tabular}{|c|c|c|c|c|c|c|c|c|}
\hline FAGE & 001 & & 97 & & & & & \\
\hline 00001 & & & & & NAM & & $H F: 9 ?$ & \\
\hline 00002 & & & & & $\mathrm{OF} T$ & & 0 & \\
\hline 00003 & $730 \mathrm{~B}$ & & & & DF: 5 & & $\$ 730 \mathrm{E}$ & \\
\hline 00004 & $730 \mathrm{~B}$ & 86 & 04 & & L. IIA & $A$ & $\sharp 4$ & *SET $M=A$ \\
\hline 00005 & 7301 & $\mathrm{H} 7$ & $001 \mathrm{~B}$ & & STA & $A$ & $M$ & \\
\hline 00006 & 7310 & $8 t$ & 01 & & $\operatorname{LI} A$ & $\mathrm{~A}$ & * 1 & *SET $N=1$ \\
\hline 00007 & 7312 & $\mathrm{~B} 7$ & 001.9 & & $S T A$ & $A$ & $N$ & \\
\hline 00008 & 7315 & 86 & 03 & & $L I \cap A$ & $\mathrm{~A}$ & $\$ 3$ & *SET $\mathrm{C}=3$ \\
\hline 00009 & 7317 & $\mathrm{H} 7$ & $001 \mathrm{~A}$ & & STA & $A$ & E & \\
\hline 00010 & $731 \mathrm{~A}$ & $\mathrm{CE}$ & 0030 & & $\operatorname{LIIX}$ & & $\#=30$ & *LOAII STAFITING ATIIFESS \\
\hline 00011 & $731 \mathrm{II}$ & $A B$ & 00 & L.F'1 & L.IIA & A & $0, x$ & *LOAII A FAIF OF IIIGITS \\
\hline 00012 & $731 \mathrm{~F}$ & EIII & 7350 & & $J S F i$ & & $F F$ & * JUMF TO UNFACKIING FOUTINE \\
\hline 00013 & 7322 & 08 & & & $\operatorname{INX}$ & & & *INCFEYEETT THE ACIIFFSS \\
\hline 00014 & 7323 & $7 \mathrm{~A}$ & $001 \mathrm{~A}$ & & IIEC & & $\mathrm{C}$ & *LECFEMENT THF CONTFOI. NUMEE \\
\hline 0001.5 & 7326 & 27 & 02 & & EEQ & & $J F \cdot 1$ & \\
\hline 00016 & 7328 & 20 & $F 3$ & & BFEA & & $L F \cdot 1$ & *LOOF JF NOT ZEFO \\
\hline 00017 & $732 A$ & 86 & $4 \mathrm{~F}$ & $J F \cdot$ & LIIA & $\mathrm{A}$ & $\# 4 F$ & *LOAII STOFE COIE \\
\hline 00018 & 7320 & BII & 7369 & & $J S F$ & & OUT & *SENDI IT OUT \\
\hline 00019 & $732 \mathrm{~F}$ & B6 & 0019 & & L.IA & $A$ & $\lambda$ & *FOFH COLE FOF STOFIAGE \\
\hline 00020 & & & & * & & & & AIIFESS ("OF" N WITH \$4O) \\
\hline 00021 & 7332 & $8 A$ & 40 & & OFA & $A$ & $\#+40$ & \\
\hline 00022 & 7334 & $\mathrm{BII}$ & 7369 & & $J S F$ & & OUT & *SENI IT OUT \\
\hline 00023 & 7337 & $7 \mathrm{C}$ & 0019 & & INC: & & th & * INCFEMENT STOFAGE AIIIFESS \\
\hline 00024 & & & & * & & & & CDNTFOL NUMEEF \\
\hline $0002 \%$ & $733 A$ & $7 A$ & $001 \mathrm{E}$ & & IIEC & & $M$ & *ALL NUMEEFS SENT OUIT? \\
\hline 00026 & $733 I$ & 27 & 07 & & HEQ & & $J F 2$ & *YES, LEAUE LOOF \\
\hline 00027 & $733 \mathrm{~F}$ & 86 & 05 & & LIIIA & $A$ & $\# 5$ & *NÖ. SET C=5 \& FECYCLE \\
\hline 00028 & 7341 & $\mathrm{E} 7$ & $001 \mathrm{~A}$ & & $S T A$ & $A$ & $\mathrm{C}$ & \\
\hline 00029 & 7344 & 20 & 117 & & BFAA & & LF' 1 & \\
\hline 00030 & 7346 & 86 & $4 A$ & $J F=2$ & $\operatorname{LI} I A$ & $\hat{A}$ & $\# 4 A$ & *LOAII COIIE FOF LAEEL A \\
\hline 00031 & 7348 & $\mathrm{EII}$ & 7369 & & $J 5 F$ & & out & *SENII IT OUT \\
\hline 00032 & $734 \mathrm{E}$ & $4 F$ & & & CLEF & $A$ & & *LOAII BLANK COIIE TO \\
\hline 00033 & & & & * & & & & FIELEASE HF-.97 \\
\hline 00034 & $734 \mathrm{C}$ & BII & 7369 & & $J S F$ & & OUT & *SENII JT OUT \\
\hline 00035 & $734 F$ & 39 & & & FITS & & & *FIETUFIN TO CONTFOL FFOGFAM \\
\hline 00036 & 7350 & B7 & $001 \mathrm{C}$ & $\mathrm{FR}$ & STA & $A$ & TEH & * SAVE CONTENTS OF A IN TEM \\
\hline 00037 & 7353 & 84 & FO & & ANII & $A$ & $\#$ \#FO & *FICK OFF MOST SIG. BYTE \\
\hline 00038 & 7355 & 44 & & & LSFi & $A$ & & *MOUE IT OUEF \\
\hline 00039 & 7356 & 44 & & & L.SFi & $A$ & & \\
\hline
\end{tabular}

* FFUCEEI IF ACIA to fiEariy *SENII OUT CHAFACTEF TO TEFMINAL *..OAII UNUSEII COIIE *IS IIELAY FINISHEII? *NO, LOOF TO JFI *YES, FETUFIN * (acia status alirifiess) * (acta liata alimifiEss)

\author{
TOTAL ERSTOFS 0000
}

$\sqrt{2}$ 


\begin{tabular}{|c|c|c|c|c|c|c|c|}
\hline 00040 & 7357 & 44 & & & L.SFi & $A$ & \\
\hline 00041 & 7358 & 44 & & & LSF: & $A$ & \\
\hline 00042 & 7359 & $8 A$ & 40 & & QFiA & $A$ & $\$ 40$ \\
\hline 00043 & $735 \mathrm{E}$ & $\mathrm{BH}$ & 7369 & & $J 5 R$ & & DUT \\
\hline 00044 & $735 E$ & $B 6$ & $001 \mathrm{C}$ & $J F^{\prime} 3$ & LIIA & $\hat{A}$ & TEM \\
\hline 00045 & 7361 & 84 & OF & & ANII & $A$ & $\#$ \#韦 \\
\hline 00046 & 7363 & $8 A$ & 40 & & OF:A & $A$ & $\$ \$ 40$ \\
\hline 00047 & 7365 & $\mathrm{BI}$ & 7369 & & $J S F i$ & & OUT \\
\hline 00048 & 7368 & 39 & & $J F^{\prime} A$ & FTS & & \\
\hline $\begin{array}{l}00049 \\
00050\end{array}$ & 7369 & $\mathrm{~B} 7$ & 5002 & $\begin{array}{l}\text { OUT } \\
*\end{array}$ & STA & $A$ & $\$ 5002$ \\
\hline 00051. & $736 C$ & EII & 7377 & & JSFi & & II \\
\hline 00052 & $736 F$ & $4 F$ & & & CLF & $A$ & \\
\hline 00003 & & & & * & & & \\
\hline 00054 & 7370 & $B 7$ & 5002 & & STA & $A$ & $\$ 5002$ \\
\hline 00055 & 7373 & BII & 7377 & & $J S \mathrm{~F}^{\mathrm{i}}$ & & II \\
\hline 00056 & 7376 & 39 & & & FITS & & \\
\hline $0005 ?$ & 7377 & 86 & $\because F$ & I & $L I I A$ & $\hat{A}$ & $\notin F F$ \\
\hline 00058 & 7379 & $B ! !$ & 7380 & LF? & JSFi & & IIL. Y \\
\hline 00059 & $737 \mathrm{C}$ & $4 A$ & & & IIEC & $A$ & \\
\hline 00060 & 7371 & 26 & $\because A$ & & ENE & & $L F=$ \\
\hline 00061 & $737 F$ & 39 & & & FITS & & \\
\hline 00062 & 7380 & $c 6$ & 26 & IIL. $Y^{\prime}$ & LIIIA & $\mathrm{E}$ & $\# 26$ \\
\hline 00063 & 7382 & $5 A$ & & L.F 3 & IIEE & $\mathrm{E}$ & \\
\hline 00064 & 7383 & 26 & FII & & BNE & & $L F 3$ \\
\hline $0006 \mathrm{~s}$ & 7385 & 39 & & & FTS & & \\
\hline 00066 & & 001 & & is & EQU & & 束1.9 \\
\hline 00067 & & 001. & & C & EQU & & \$ $1 \mathrm{~A}$ \\
\hline 00068 & & 001 & & $M$ & EQU & & \$ $1 \mathrm{E}$ \\
\hline 00069 & & 001 & & TEY & EQU & & $\$ 1 \mathrm{C}$ \\
\hline 00070 & & & & & ENTI & & \\
\hline
\end{tabular}

*FOFIM COIE FOF: HF-97

* SENII IT OUT

*FECOUEF IMTA

*FJCK OFF LEAST SIG. BYTE

*FOFM FFOFFER COLE

* SENII IT OUT

*FETUR'N

*SENII COTIE TO HF-97

INTERFACE

*GO TO IIELAY LOOF

* loar kllakk colie to

FEL.EASE HF- -97

*GO TO LIELAY LOOF

*LOAII FFF AS TIMING CONTFOL * JUMF TO SECOdIT tIELAY LOOF

* IECFEMENT CONTEOL NUMEEF

*EECYCLE IF NONZEFO

*EETUFN TO OUTFUT SUEFOUTINE * LoAT 226 as TIMING CONTFOL *IECFEMENT $\mathrm{B}$

*FECYCLE IF NONZEEFO

* FETUFiN TO FFIMAFY TIELAY L.OO

TOTAL EFFERS 00000

\begin{tabular}{|c|c|c|c|c|c|c|c|c|}
\hline PAE & $00 \mathrm{l}$ & & $8 \mathrm{~T}$ & & & & & \\
\hline 00001 & & & & & WAPH & & TEST & \\
\hline 00002 & & & & & OFT & & 0 & \\
\hline 00003 & 7394 & & & & OFO & & $\$ 7394$ & \\
\hline 00004 & 7394 & CE & 0030 & & L..IX & & $\# \$ 30$ & *LDAR STAFTING AMUIRES \\
\hline $0000 \mathrm{~s}$ & 7397 & 06 & 03 & & LLAA & E & $\$ 3$ & *LOAI COUTEOL NUAEEF \\
\hline 00006 & 7399 & 86 & 44 & & L. INA & A & $\# \$ 44$ & WOTOFE AAA4A FOF TIME \\
\hline 00007 & $739 \mathrm{~B}$ & BII & 73 IIE: & & $.15 F_{i}$ & & $\mathrm{sT}$ & \\
\hline 00008 & $739 \mathrm{E}$ & 86 & 33 & & LLAA & $\vec{m}$ & 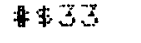 & *5TOFE 3333333333 Fan TOTHL \\
\hline 00009 & 7300 & EI & $73 \Pi \mathrm{E}$ & & $J S F$ & & ST & \\
\hline 00010 & $73 A 3$ & 86 & 22 & & LIAA & A & $\$ 22$ & * STOFE 2222222222 FOF FIA \\
\hline 00011 & 7395 & BII & 73 UIE: & & USF & & $5 \mathrm{~T}$ & \\
\hline 00012 & $73 \mathrm{AB}$ & 86 & 11 & & LIIA & $\dot{A}$ & $\$ 11$ & WSTOFE 111111111 FOF A \\
\hline $\begin{array}{l}00013 \\
00014\end{array}$ & $73 A A$ & EI & $73 \mathrm{IIE}$ & & JSE: & & $\mathrm{ST}$ & \\
\hline 00015 & $\begin{array}{l}73 \mathrm{AII} \\
73 \mathrm{EO}\end{array}$ & $\begin{array}{l}\text { BII } \\
\text { CEE }\end{array}$ & $\begin{array}{l}730 \mathrm{~B} \\
73 \mathrm{BF}\end{array}$ & & $\begin{array}{l}\text { JSR } \\
\text { L.IIX }\end{array}$ & & $\begin{array}{l}H F \\
\# 51\end{array}$ & $\begin{array}{l}\text { * JUMF TO HF-O7 OUTFUT FOUT IN } \\
\text { *LOAII STAFTING AOLFESS }\end{array}$ \\
\hline 00016 & 7383 & $A 6$ & 00 & LFI & L.TIA & $A$ & $0, x$ & WLOAII A CHAEACTEF \\
\hline 00017 & $73 \mathrm{Bg}$ & $\mathrm{BI}$ & $72 \mathrm{LE}$ & & 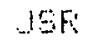 & & SENII & BFFETAT IT \\
\hline 00018 & $73 \mathrm{B8}$ & 08 & & & $\operatorname{INX}$ & & & WIMCFEMENT ATILESS \\
\hline 00019 & $730 \%$ & 80 & $7 \mathrm{THE}$ & & $\Delta F$ & & $8 \mathrm{sT}$ & WHAS LAST CHAE EEE FEUTEM \\
\hline 00020 & $73 \mathrm{EC}$ & 26 & $F 5$ & & ENE & & LF & WNO, GET NEXT CHAFACTEE \\
\hline 00021 & $73 \mathrm{HE}$ & 39 & & & FTS & & & WTES, FETULEN \\
\hline
\end{tabular}




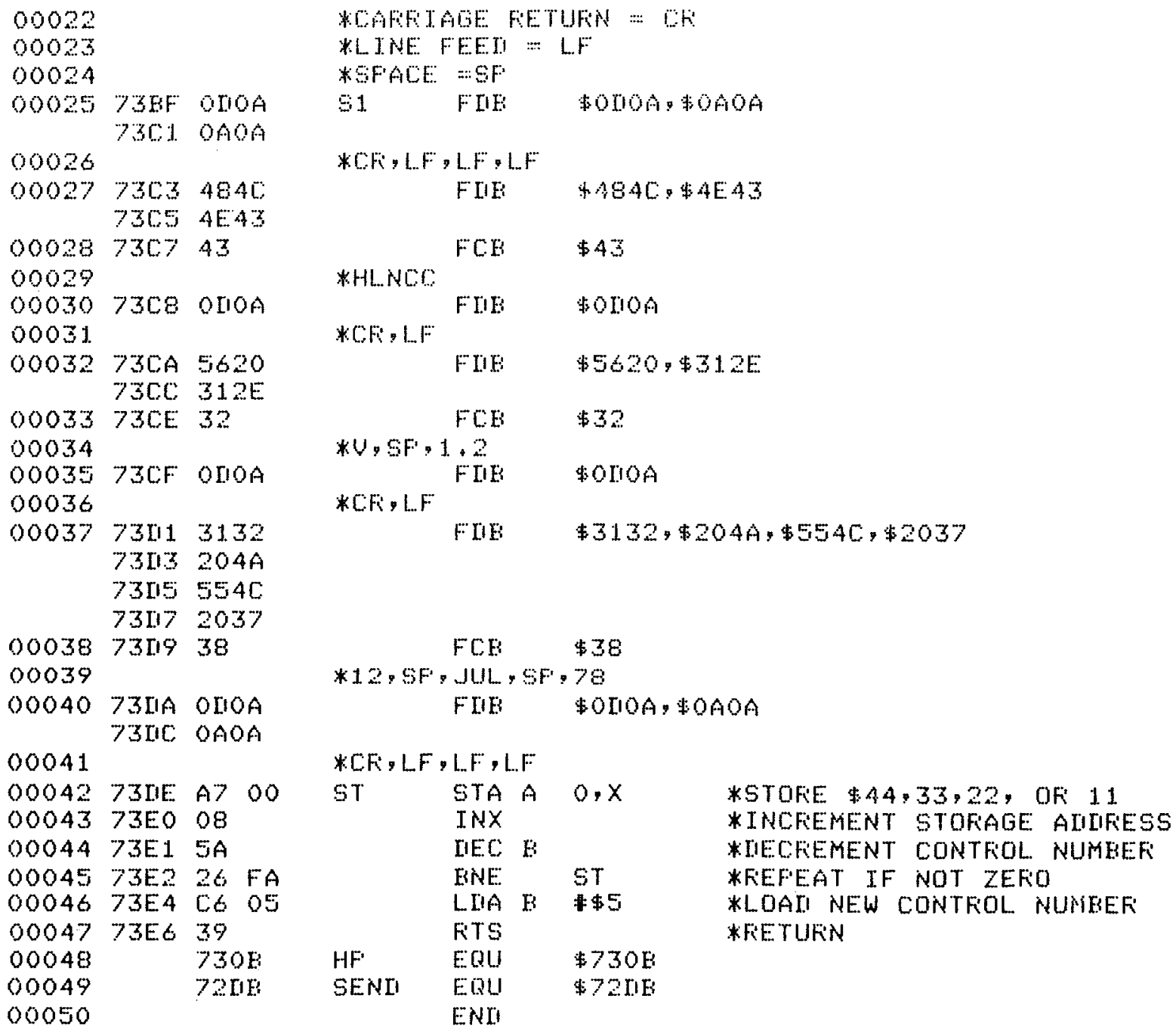

TOTAL EFFOFS 00000 
PROM LISTING

\author{
HLNCC $\vee 1.2 \quad 12$ July 1978
}

7000 OF $8 E$ OO $7 F$ EII 70 E5 $86 \quad 29$ B 750 O2 EI 70 A9 EII $\begin{array}{llllllllllllllllll}7010 & 73 & 94 & \mathrm{BI} & 70 & \mathrm{AF} & 86 & 28 & \mathrm{E} 7 & 50 & 02 & 7 \mathrm{~F} & 00 & 17 & 7 \mathrm{~F} & 00 & 18\end{array}$ $\begin{array}{lllllllllllllllllll}7020 & 20 & 2 E & 86 & 30 & \mathrm{ELI} & 70 & 9 \mathrm{~F} & 2 \mathrm{~A} & \mathrm{~F} 9 & 86 & 31 & \mathrm{EII} & 70 & 9 \mathrm{~F} & 2 \mathrm{E} & 05\end{array}$ $\begin{array}{lllllllllllllllllll}7030 & 7 \mathrm{~F} & 00 & 18 & 20 & 05 & \mathrm{C} 6 & 01 & \mathrm{~F} 7 & 00 & 18 & 86 & 36 & \mathrm{EI} & 70 & 9 \mathrm{~F} & 2 \mathrm{E}\end{array}$ $\begin{array}{lllllllllllllllll}7040 & \text { OC } & C 6 & 01 & F 7 & 00 & 16 & F 1 & 00 & 18 & 27 & 1 A & 20 & 03 & 7 F & 00 & 16\end{array}$ $\begin{array}{lllllllllllllllllllll}7050 & 86 & 30 & \mathrm{ELI} & 70 & 9 \mathrm{~F} & 2 \mathrm{~A} & \mathrm{CE} & 86 & 34 & \mathrm{ELI} & 70 & 9 \mathrm{~F} & 2 \mathrm{E} & \mathrm{EC} & 86 & 37\end{array}$ 7060 EII 70 9F $2 A$ EE EII 70 A9 EII 7136 EII 73 OE BII 71

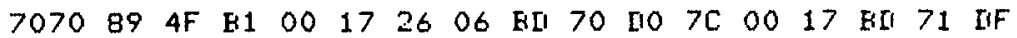
$\begin{array}{lllllllllllllllll}7080 & \text { EII } & 70 & \text { AF } & 4 F & \text { E1 } & 00 & 18 & 27 & \text { C7 } & \text { E1 } & 00 & 16 & 27 & \text { C2 } & 86 & 29\end{array}$ $\begin{array}{lllllllllllllllll}7090 & \mathrm{~B} 7 & 50 & 02 & 86 & 2 \mathrm{~A} & \mathrm{~B} 7 & 50 & 02 & 86 & 2 \mathrm{~B} & \mathrm{E} 7 & 50 & 02 & 20 & 83 & \mathrm{~B} 7\end{array}$ $\begin{array}{lllllllllllllllll}70 A O & 50 & 02 & \text { E6 } & 50 & 00 & \text { B6 } & 50 & 00 & 39 & 86 & 3 C & \text { E7 } & 50 & 03 & 39 & 86\end{array}$ $\begin{array}{lllllllllllllllll}70 \mathrm{BO} & 34 & \mathrm{E} 7 & 50 & 03 & 39 & 4 \mathrm{~F} & \mathrm{E} 7 & 50 & 00 & 43 & \text { E7 } & 50 & 02 & 86 & 04 & \mathrm{~B} 7\end{array}$ $\begin{array}{lllllllllllllllll}70 C 0 & 50 & 01 & \text { B } 7 & 50 & 03 & 86 & 03 & \text { E7 } & 40 & 00 & 86 & 09 & \text { E7 } & 40 & 00 & 39\end{array}$ 7OIJO CE 70 IIF A6 $00 \mathrm{BII} 72$ LIE 08 8C $71 \quad 2016$ $\begin{array}{lllllllllllllllll}7 O E O & O A & 20 & 54 & 49 & 4 I & 45 & 28 & 53 & 45 & 43 & 29 & 20 & 20 & 20 & 20 & 20\end{array}$ $\begin{array}{lllllllllllllllll}70 F 0 & 20 & 20 & 20 & 20 & 20 & 54 & 4 F & 54 & 41 & 4 C & 53 & 20 & 20 & 20 & 20 & 20\end{array}$

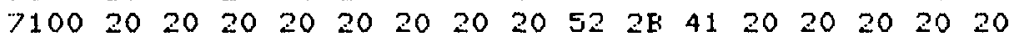
$\begin{array}{lllllllllllllllll}7110 & 20 & 20 & 20 & 20 & 20 & 20 & 20 & 20 & 20 & 20 & 41 & 20 & 20 & 20 & 20 & 20\end{array}$ $\begin{array}{llllllllllllllll}7120 & 20 & 20 & 20 & 20 & 20 & 20 & 20 & 20 & 20 & 20 & 20 & 52 & 20\end{array}$

$\begin{array}{lllllllllll}7130 & \text { CE } & 00 & 30 & 86 & 01 & \text { B7 } & 50 & 02 & \text { EII } & 71\end{array}$ 7140 62 FG 00 OC $585858 \quad 58.7 C 50$ O2 EI 71 62 FA 00

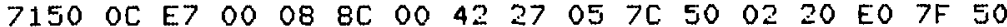
$7160 \quad 0239 \mathrm{~B} 650 \quad 00 \quad 2 \mathrm{E} F \mathrm{FE}$ 86 FF E7 00 OB $7 \mathrm{~A} \quad 00$ OE 26 $\begin{array}{llllllllllllllll}7170 & \mathrm{FE} & \mathrm{H} 6 & 50 & 00 & 84 & \text { OF } & 81 & \text { OF } & 26 & 01 & 4 \mathrm{~F} & \mathrm{~B} 7 & 00 & \text { OC } & 39\end{array}$ $7180 \quad 7 F$ OO OII CE 0038 C6 7190 O5 8699 AO 09 A7 OE $095 A 26$ FG OII C6 OS CE 00 $\begin{array}{lllllllllllllllll}71 A O & 38 & A 6 & 04 & A 9 & O E & 19 & A 7 & 13 & 09 & 5 A & 26 & F 5 & 24 & 01 & 39 & 86\end{array}$ $\begin{array}{lllllllllllllllll}71 \mathrm{BO} & 01 & \mathrm{~B} 7 & 00 & \text { OII } & \mathrm{CE} & 00 & 38 & \mathrm{C} 6 & 05 & 86 & 99 & A 0 & 13 & A 7 & 13 & 09\end{array}$ $\begin{array}{llllllllllllllllll}71 C 0 & 5 A & 26 & F 6 & 0 D & C 6 & 05 & C E & 00 & 38 & 86 & 00 & A 9 & 13 & 19 & A 7 & 13\end{array}$ $7111009 \quad 5 A \quad 26 \quad F 5 \quad 39$

$\begin{array}{lllllllllllllllll}71 E O & \text { OII EII } 72 & \text { LIE } & 86 & \text { OA } & \text { EII } & 72 & \text { IIE EII } & 72 & 40 & 86 & 05 & 97 & \text { OE }\end{array}$

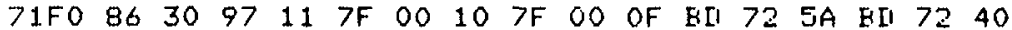
\begin{tabular}{llllllllllllllll}
7200 & 86 & 09 & 97 & OE & 86 & 33 & 97 & 11 & 86 & 02 & 97 & OF & BII & 72 & $5 A$ \\
\hline
\end{tabular} $\begin{array}{lllllllllllllllll}7210 & 72 & 40 & 86 & 38 & 97 & 11 & \mathrm{EI} & 72 & 5 \mathrm{~A} & \mathrm{BII} & 72 & 40 & 86 & 32 & 97 & 11\end{array}$ $\begin{array}{lllllllllllllllll}7220 & \mathrm{BL} & 72 & 5 \mathrm{~A} & \mathrm{BII} & 72 & 40 & 86 & 01 & 91 & \text { OII } & 27 & 07 & 86 & 2 \mathrm{E} & \mathrm{EII} & 72\end{array}$ $\begin{array}{lllllllllllllllll}7230 & \text { IIE } & 20 & 05 & 86 & 2 \mathrm{II} & \mathrm{BLI} & 72 & \text { IIE } & 86 & 47 & 97 & 11 & \text { EII } & 72 & 5 \mathrm{~A} & 39\end{array}$

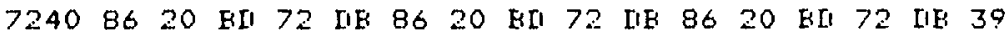
$7250 \quad 7 F \quad 0012 \quad 7 F \cdot 0014$ $\begin{array}{lllllllllllllllll}7260 & 7 \mathrm{~F} & 00 & 13 & \mathrm{FE} & 00 & 10 & \mathrm{AG} & 00 & 7 \mathrm{II} & 00 & 14 & 27 & 04 & 84 & \text { OF } & 20\end{array}$ $\begin{array}{lllllllllllllllll}7270 & 06 & 84 & F O & 46 & 46 & 46 & 46 & 4 I & 27 & O A & C 6 & 01 & F 7 & 00 & 13 & \mathrm{EII}\end{array}$

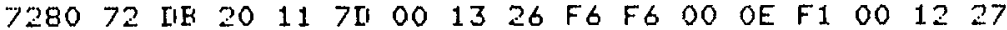

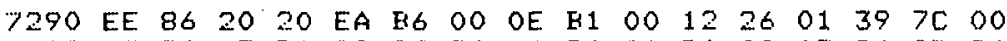
$\begin{array}{lllllllllllllllll}72 A O & \text { OF } & 86 & 03 & \mathrm{~B} 1 & 00 & \text { OF } & 26 & 16 & 86 & 01 & \mathrm{~B} 1 & 00 & 13 & 26 & 07 & 86\end{array}$ $72 \mathrm{EO} \quad 2 \mathrm{C}$ EII 72 IIE 20 OS $86 \quad 20 \mathrm{BII} \quad 72$ IIE $7 \mathrm{~F} \quad 00$ OF $7 \mathrm{C} \quad 00$ $\begin{array}{lllllllllllllllll}72 \mathrm{CO} & 12 & 7 \mathrm{C} & 00 & 14 & \mathrm{~B} 6 & 02 & \mathrm{E} 1 & 00 & 14 & 26 & 9 \mathrm{~F} & 7 \mathrm{~F} & 00 & 14 & 08 & 20\end{array}$ 7211095 81 O9 $2 E$ O2 $8 \mathrm{~A}$ $\begin{array}{lllllllllllllllll}72 E 0 & 30 & \mathrm{C} 6 & 01 & \mathrm{~F} 7 & 00 & 15 & 81 & \text { OII } & 2 \mathrm{E} & 05 & \mathrm{C} 6 & 08 & \mathrm{~F} 7 & 00 & 15 & \mathrm{~F} 6\end{array}$ $\begin{array}{lllllllllllllllll}72 F O & 40 & 00 & 56 & 56 & 24 & F 9 & B 7 & 40 & 01 & 86 & 7 F & 7 A & 00 & 15 & 26 & E F\end{array}$ 730039 $\begin{array}{llllll}86 & 04 & 87 & 00 & 1 \mathrm{E}\end{array}$ $\begin{array}{lllllllllllllllll}7310 & 86 & 01 & \text { B7 } & 00 & 19 & 86 & 03 & \mathrm{~B} 7 & 00 & 1 \mathrm{~A} & \mathrm{CE} & 00 & 30 & \mathrm{~A} 6 & 00 & \mathrm{EII}\end{array}$ $\begin{array}{llllllllllllllllllllll}7320 & 73 & 50 & 08 & 7 A & 00 & 1 A & 27 & 02 & 20 & F 3 & 86 & 4 F & E I & 73 & 69 & \text { L } 6\end{array}$ $\begin{array}{lllllllllllllllll}7330 & 00 & 19 & 8 A & 40 & E D & 73 & 69 & 7 C & 00 & 19 & 7 A & 00 & 1 E & 27 & 07 & 86\end{array}$

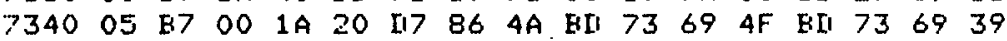

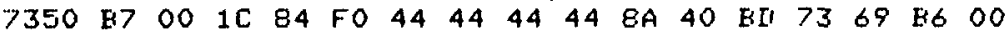
$\begin{array}{llllllllllllllllllll}7360 & 1 \mathrm{C} & 84 & \text { OF } & 8 \mathrm{~A} & 40 & \mathrm{EII} & 73 & 69 & 39 & \mathrm{E} 7 & 50 & 02 & \mathrm{EII} & 73 & 77 & 4 \mathrm{~F}\end{array}$ $\begin{array}{llllllllllllllllll}7370 & \text { E7 } & 50 & 02 & \text { EII } & 73 & 77 & 39 & 86 & \mathrm{FF} & \mathrm{BII} & 73 & 80 & 4 \mathrm{~A} & 26 & \mathrm{FA} & 39\end{array}$ 7380 C6 26 SA 26 FII 39

$\begin{array}{lllllllllllll}7390 & \text { CE } & 00 & 30 & \text { C6 } & 03 & 86 & 44 & \text { ELI } & 73 & \text { IIE } & 86 & 33\end{array}$ $73 \mathrm{AO}$ BII 73 IIE $86 \quad 22$ EII 73 IIE $86 \quad 11$ EII 73 IIE ELI 73 OE $73 \mathrm{BO}$ CE 73 EF A6 $00 \mathrm{BII} 72$ IIE 08 8C 73 LIE 26 F5 39 OII $\begin{array}{llllllllllllllll}73 C O & O A & O A & O A & 48 & 4 C & 4 E & 43 & 43 & \text { OII } O A & 56 & 20 & 31 & 2 E & 32 & \text { OI }\end{array}$ $\begin{array}{lllllllllllllllll}73110 & O A & 31 & 32 & 20 & 4 A & 55 & 4 C & 20 & 37 & 38 & \text { OII OA OA } & O A & A 7 & O O\end{array}$ 73EO O8 $5 A$ A 26 FA $C 6 \quad 0539$

$73 \mathrm{FO}$

7000

Note: Blank areas are unused memory locations that may contain random hexadecimal codes. 


\section{APPENDIX D}

\section{NONSTANDARD LSI AND HYBRID COMPONENTS DATA}

The data sheets in this section were selected primarily on the basis of whether or not the described component was a standard series component. For instance, most of the $74 \times x$ TTL and $4 x \times x / 14 \times x x$ metal oxide semiconductor (MOS) components in the HLNCC are listed in manufacturers' and second source data books, so the data should be readily available. Within the three sections of this appendix, the components are 1isted, first, alphabetically, and then by the initial digits in the device number.

A. Analog Section

1. TP 1321/1322 (Preamp/Amplifier Operational Amp) . . . . 99

B. Digital Section

1. AM 0026 (Level Shifter/Driver. . . . . . . . . 101

2. Am 2804 (Accidental Delay Shift Register). . . . . . 107

3. C0 238 (Shift-Register Clock). . . . . . . . . 113

4. DS 8973 (Display Digit Strobe Driver). . . . . . 114

5. HCTR 4010 (4 BCD Digit Scalers). . . . . . . . 116

6. MC 14411 (Baud Rate Selector). . . . . . . . . 125

7. MC 14517 (Gate Shift Register) . . . . . . . . 128

8. MC 14557 (Predelay Shift Register) . . . . . . . . 132

9. MC 6871A (Microprocessor Clock). ......... 136

10. MK 5009 (Shift-Register Clock Dividers). . . . . . 138

11. 5082-7430 (7 Segment, 3 Digit Displays). . . . . . 142

12. 82583 (Gate Adders). . . . . . . . . . . 146

C. Power Supplies

1. OP-07 (High-Voltage Power Supply Feedback Buffer Amplifier). . . . . . . . . . 150

2. PMS B 2-025-1A (High-Voltage Power Supply

DC/DC Converter) . . . . . . . . . . 159

3. WC (7-40) T 15/165 (Low-Voltage DC Power Supply DC/DC Conv). . . . . . . . . . . 161 


\section{NTELEDYNE PHILBRICK}

\section{$1321 / 1322$ WOEBAND, HOH HLLW RATE OPERATIONAL AMPLIFIERS}

The 1321 and 1322 are amplifiers packaged in T0-99 cases which are second to none in low cost, high frequency, high speed, and precision performance. Each has been optimized for a particular set of applications.

These are true differential input amplifiers with stable 6 $\mathrm{dB}$ per octave gain vs frequency plots from maximum open loop gain to closed loop gains between 3 and 10 . The 1321 should be used for precision settling (to $0.01 \%$ ), high frequency precision closed loop gain, or low bias current. Applications include a low cost, fast 12.bit voltage DAC from a fast current DAC, fast integrators, and high gain precision differential input video amplifiers.

The 1322 should be used when the $120 \mathrm{~V} / \mu$ sec slew rate, or $1.6 \mathrm{MHz}$ full power frequency is required (both@ $\pm 10 \mathrm{~V}$ into $500 \Omega$ ).

\section{APPLICATION}

As with all high frequency devices. optimum performance from the 1321 and 1322 demands care in lead length, bypassing, and stray capacity. When operating at closed loop gains of less than 10 , compensation tech. niques may be required at the bandwidth control pin (pin 8) for stable operation. They are mandatory with closed loop gains below 3 .

Most applications operating at a gain of less than 10 are stabilized by connecting a $20 \mathrm{pF}$ capacitor between pin 8 and ground. The effect on the amplifier's Bode plot by the stabilizing capacitor is shown in Figure 2. When gains of greater than 10 are used, care should be taken to minimize stray capacity to pin 8 . When maximum speed/fre. quency response is required, pin 8 should be cut off close to the TO-99 case.

When optimum response and settling time are required at gains of less than 10 , the amplifier must be "fooled" and operated at a gain greater than 10 at high frequency as shown in Figure 1.

These amplifiers will usually operate better with a $50 \mathrm{pF}$ load capacitor and 5 to $20 \mathrm{pF}$ in parallel with the feed. back resistor. Short, individual lines should be run from the power supply to $\pm V_{c c}$ and to circuit power common. In addition, + and $-V_{c c}$ pins should be bypassed to com. mon at the device with $1 \mu \mathrm{F}$ tantalum capacitors in par. allel with $0.01 \mu \mathrm{F}$ ceramic discs.

Maximum initial $E_{O S}$ is $10 \mathrm{mV}$ for the 1322 and $5 \mathrm{mV}$ for the 1321, so most low gain high frequency circuits do not require zero trim components. However, an op. tional potentiometer may be used as shown. Note, the wiper of potentiometer is connected to $+V_{c c}$.

A feature of both amplifiers is their ability to drive a $\pm 10 \mathrm{~V}$ high frequency signal into a $500 \Omega$ to $1 \mathrm{k} \Omega$ load. This high output current is available with a quiescent current of less than $4 \mathrm{~mA}$ in the 1321 and $6 \mathrm{~mA}$ in the 1322.

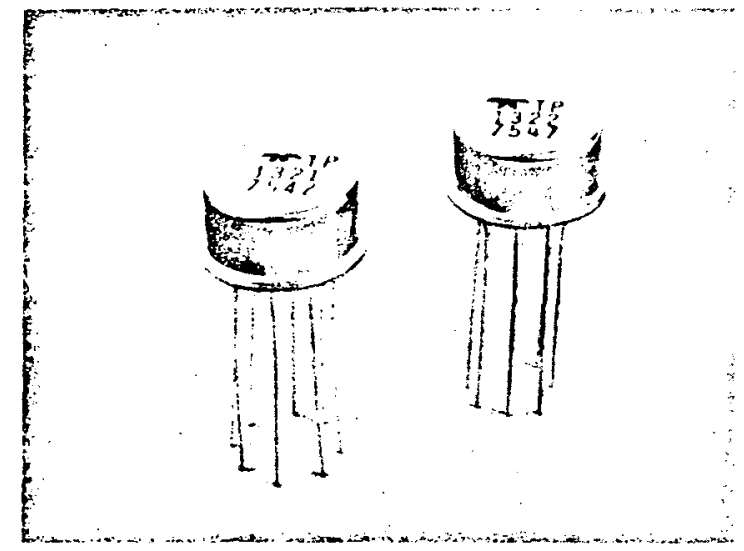

Reproduced with permission from Teledyne Philbrick, Dedham, MA 02026. 
$1321 / 1322$

SPECIFICATIONS (at $25^{\circ} \mathrm{C}, V_{c c}= \pm 15 \mathrm{~V}, R_{\mathrm{L}}=2 \mathrm{~K}$, unless otherwise indicated )

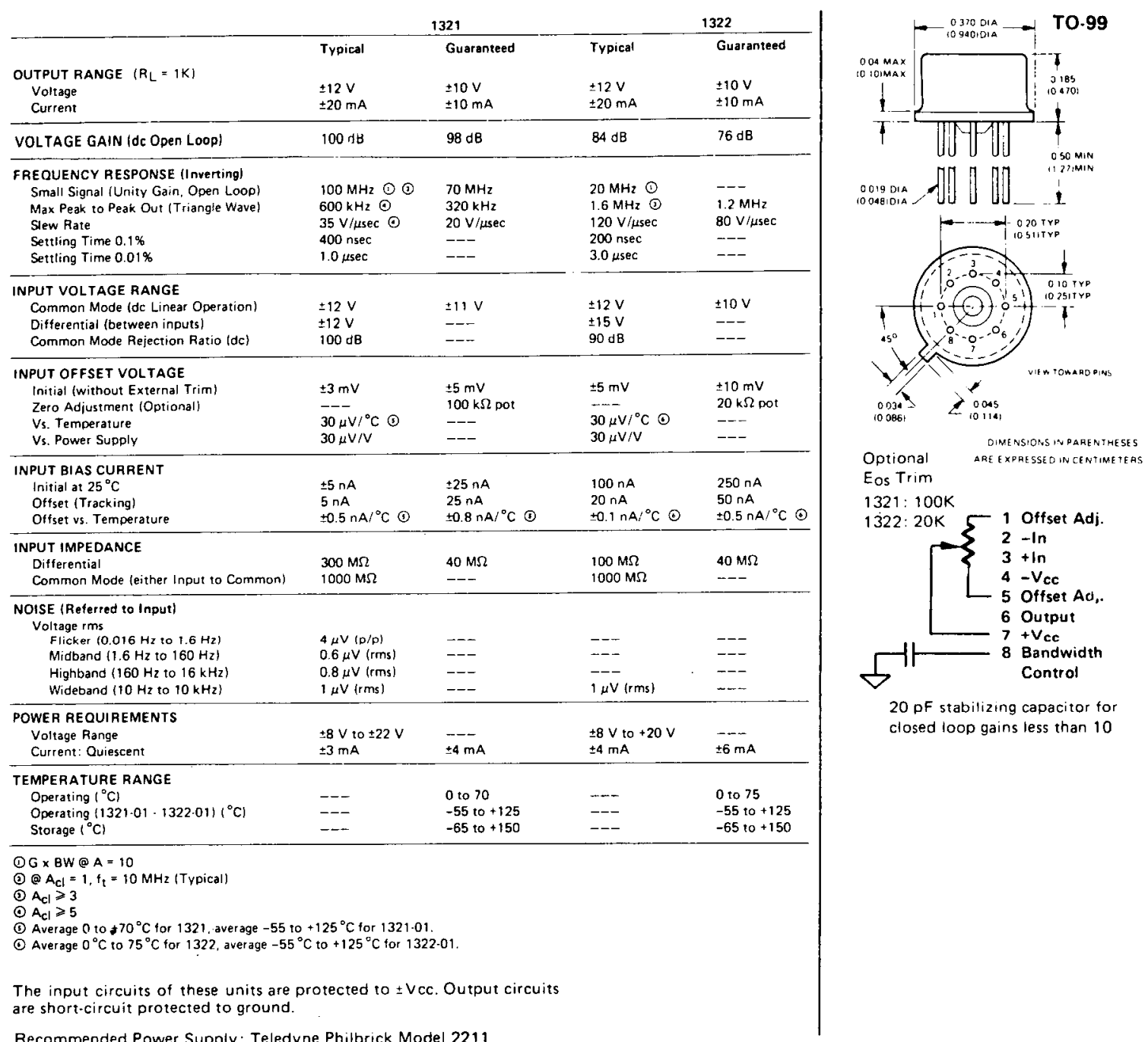
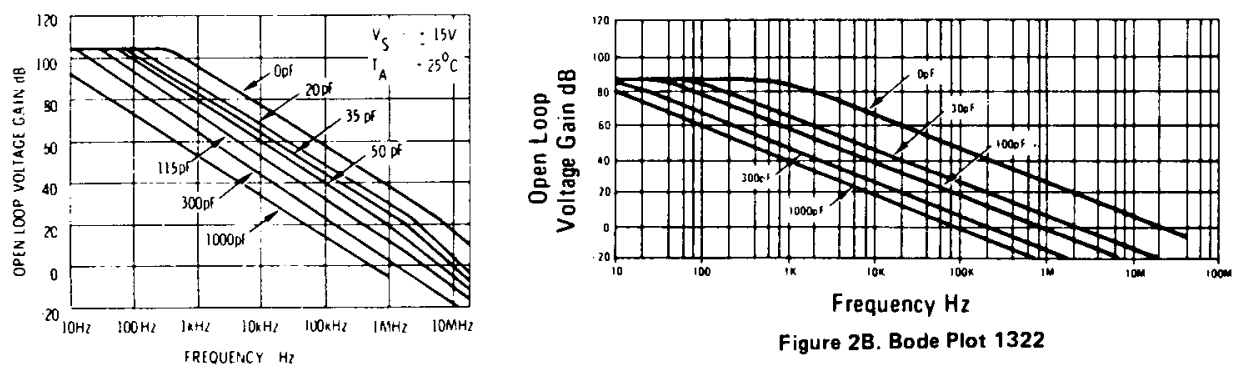

Frequency $\mathrm{Hz}$

Figure 2B. Bode Plot 1322

Figure 2A. Bode Plot 1321

Teledyne Philbrick makes no representalion that use of is modules in the circuits described nerein or use of other tectinical information contained herein will not intringe on existing or tuture pa:ent righis nor do the describlions contained herein imply ine granting of licenses to make. use. or sell equipment consliucted in accordance inerewith. 


\section{Am0026/Am0026C $5 \mathrm{MHz}$ Two-Phase MOS Clock Driver}

ctinctive Characteristics

$20 \mathrm{~ns}$ rise and fall times with $1000 \mathrm{pF}$ load

$20 \mathrm{~V}$ output voltage swing

$\doteqdot 1.5$ amps output current drive

High speed 5 to $10 \mathrm{MHz}$ depending on load
- $100 \%$ reliability assurance testing in compliance with MIL.STD-883

- Mixing privileges for obtaining price discounts. Refer to price list.

\section{FUNCTIONAL DESCRIPTION}

The Am0026 is a dual high speed MOS clock driver and interface circuit. The device is particularly suitable for driving two phase MOS circuits and can provide high speed operation even when driving into hich capacitive loads. The device accepts standard TTL/DTL outputs and converts them to SHOS logic levels. The output puise width of the device is determined by the input pulse width.

The Am0026 can operate with a variety of MOS circuits. A popular application is a two-phase clock timer for driving long silicon gate shift registers such as the $A m 1402 / 3 / 4$ series. A single clock driver is able to drive $10 \mathrm{k}$ bits at $5 \mathrm{MHz}$. The device can also be used with standard dynamic MOS RAMS such as the 1103 to provide address and precharge drive for memories up to $8 \mathrm{k}$ by $16-$ bits.

The device is available in an 8.1 ead TO-5, one watt copper lead frame 8-pin mini.DIP, a one and one-half watt TO-8 package, and a 14-pin ceramic package.

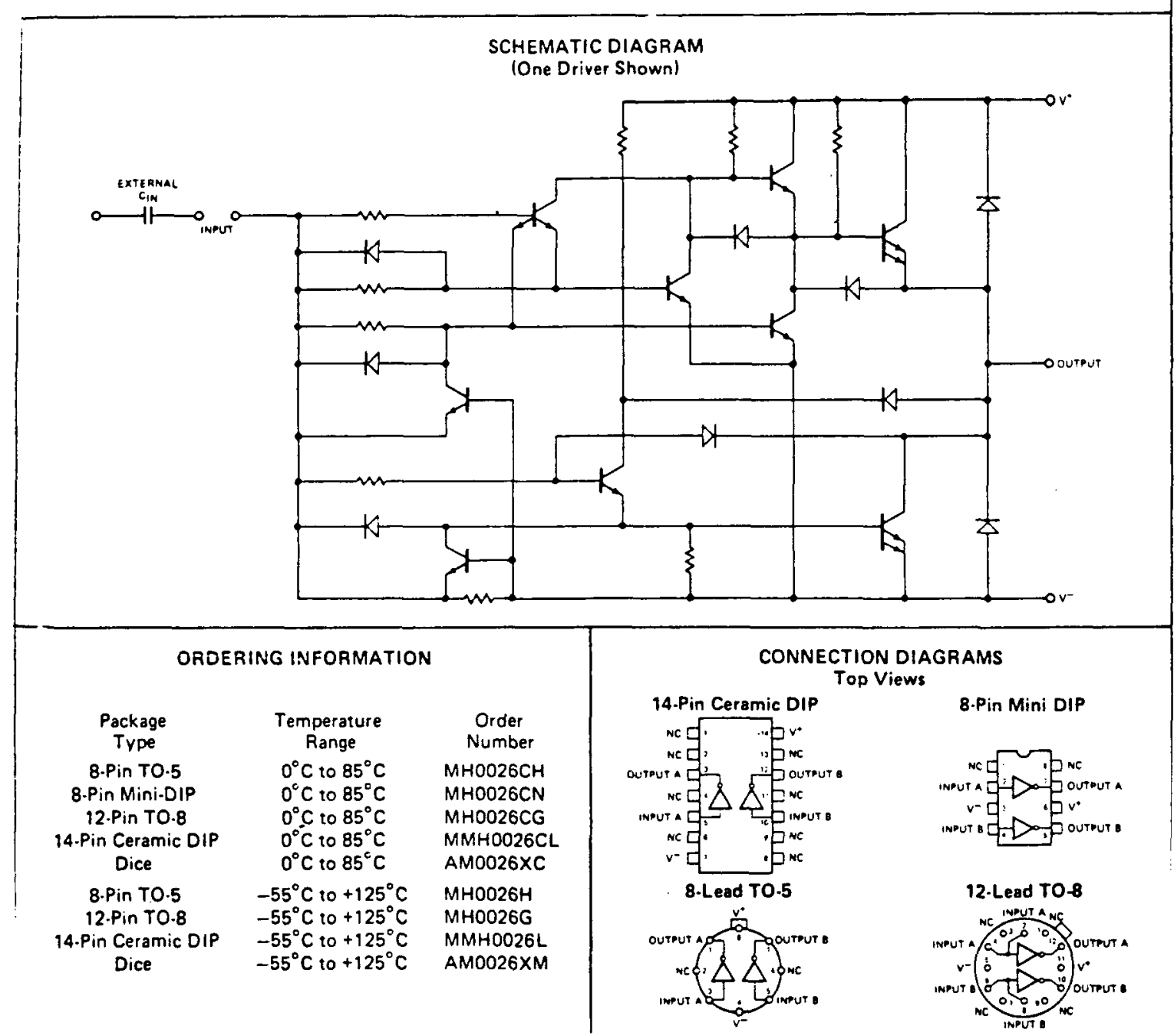

Copyright ${ }^{\circ}$ (1974) Advanced Micro Devices, Inc., Sunnyvale, CA 94086. Reproduced with permission of copyright owner. All rights reserved. 
MAXIMUM RATINGS (Above which the useful life ray be impaired)

\begin{tabular}{lr}
\hline Storage Temperature & $-65^{\circ} \mathrm{C} t 0+150^{\circ} \mathrm{C}$ \\
\hline Temperature (Ambient) Under Bias & $-55^{\circ} \mathrm{C}$ to $+125^{\circ} \mathrm{C}$ \\
\hline $\mathrm{V}^{+}-\mathrm{V}^{-}$Differential Voltage & $22 \mathrm{~V}$ \\
\hline Input Current & $100 \mathrm{~mA}$ \\
Input Voltage $\left(\mathrm{V}_{\mathrm{N}}-\mathrm{V}^{-}\right)$ & $5.5 \mathrm{~V}$ \\
\hline Peak Output Current & $1.5 \mathrm{~A}$ \\
\hline Power Dissipation & See curves
\end{tabular}

ELECTRICAL CHARACTERISTICS OVER OPERATING TEMPERATURE RANGE (Unless Otherwise Noted)

\begin{tabular}{|c|c|c|c|c|c|c|}
\hline $\begin{array}{l}\text { Am0026c } \\
\text { Am0026 }\end{array}$ & $\begin{array}{l}T_{A}=0^{\circ} \mathrm{C} 1085^{\circ} \mathrm{C}(\mathrm{COM} \mathrm{R} \\
T_{A}=-55^{\circ} \mathrm{C} 10+125^{\circ} \mathrm{C} / \mathrm{M}\end{array}$ & $\begin{array}{l}v^{+}-v^{-}=10 \mathrm{~V} \text { to } 20 \mathrm{~V} \\
\text { Unless Otherwise Spocified }\end{array}$ & & & & \\
\hline Parameter & Description & Test Conditions (Note 1 ) & Min. & Typ. (Note 2) & Max. & Units \\
\hline$v_{\mathrm{OH}}$ & Output HIGH Voltage & $\begin{array}{l}v^{+}=+5.0 v, V^{-}=-12.0 v \\
v_{I N}=-9.5 V\end{array}$ & & -11.5 & $-11: 0$ & Volts \\
\hline & & $V_{1 N}-V^{-}=2.5 V$ & & $v-0.5$ & $v^{-}+1.0$ & \\
\hline$-v_{O L}$ & Output LOW Voltege & $\begin{array}{l}V^{+}=+5.0 \mathrm{~V}, V^{-}=-12.0 \mathrm{~V} \\
v_{I N}=-11.6 \mathrm{~V}\end{array}$ & 4.0 & 4.3 & & Volts \\
\hline & & $v_{I N}-V^{-}=0.4 V$ & $v^{+}-1.0$ & $v^{+}-0.7$ & & \\
\hline$V_{I H}$ & Inpul HIGH Level & $V_{\text {OUT }}=V^{-}+1.0 \mathrm{~V}$ & 2.5 & 1.5 & & Voles \\
\hline$v_{I L}$ & Input LOW Level & $V_{\text {OUT }}=V^{+}-1.0 V$ & & 0.6 & 0.4 & Volts \\
\hline$I_{1 L}$ & Input LOW Current & $V_{I N}-V^{-}=0 V, V_{\text {OUT }}=V^{+}-1.0 V$ & & -0.005 & -10 & $\Delta A$ \\
\hline$I_{1 H}$ & Input HIGH Current & $V_{I N}-V^{-}=2.5 V, V_{\text {OUT }}=V^{-}+1.0 V$ & & 10 & 15 & $\mathrm{~mA}$ \\
\hline ICCON & "ON" Supply Current & $v^{+}-v^{-}=20 \mathrm{~V}, v_{I N}-v^{-}=2.5 v$ & & 30 & 40 & $\mathrm{~mA}$ \\
\hline ICC OFF & "OFF" Supply Current & $v^{+}-v^{-}=20 v, v_{I N}-v^{-}=0.0 v$ & & 1.0 & 100 & HA \\
\hline
\end{tabular}

Notes: 1. These specificatione apply for $\mathrm{V}^{+}-\mathrm{V}^{-}=10 \mathrm{~V}$ to $20 \mathrm{~V}, \mathrm{C}_{\mathrm{L}}=1000 \mathrm{pF}$, over the temperature range $-55^{\circ} \mathrm{C} 10+125^{\circ} \mathrm{C}$ tor the $\mathrm{Am0026}$ end $0^{\circ} \mathrm{C}$ to $+85^{\circ} \mathrm{C}$ for the Am0026C

2. All typical values for $T_{A}=25^{\circ} \mathrm{C}$.

Switching Characteristics (Notes 1 and 2 Above)

\begin{tabular}{|c|c|c|c|c|c|c|}
\hline arameter & Description & Test Conditions & Min. & Typ. & Max. & Units \\
\hline PPHL & Turn On Delay & & 5.0 & 7.5 & 12 & ns \\
\hline TPLH & Turn Off Delay & & 5.0 & 12 & 15 & ns \\
\hline \multirow{3}{*}{ 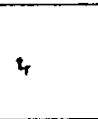 } & \multirow{3}{*}{ Rise Time (Note 3) } & $v^{+}-v^{-}=17 v, c_{L}=250 \mathrm{pF}$ & & 12 & & \multirow{3}{*}{ ns } \\
\hline & & $V^{+}-V^{-}=17 V, C_{L}=500 \mathrm{pF}$ & & $\sqrt{5}$ & 18 & \\
\hline & & $v^{+}-v^{-}=17 v, C_{L}=1000 \mathrm{DF}$ & & $20^{-}$ & 35 & \\
\hline \multirow{3}{*}{ is } & \multirow{3}{*}{ Fall Time (Note 3) } & $v^{+}-v^{-}=17 v, c_{L}=250 \mathrm{pF}$ & & 10 & & \multirow{3}{*}{ ns } \\
\hline & & $v^{+}-v-17 v, c_{L}=500 \mathrm{pF}$ & & 12 & 16 & \\
\hline & & $v^{+}-v^{-}=17 v_{,} c_{L}=1000 \mathrm{pF}$ & & 17 & 25 & \\
\hline
\end{tabular}

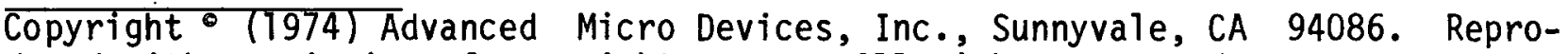
duced with permission of copyright owner. All rights reserved. 
TYPICAL PERFORMANCE CHARACTERISTICS
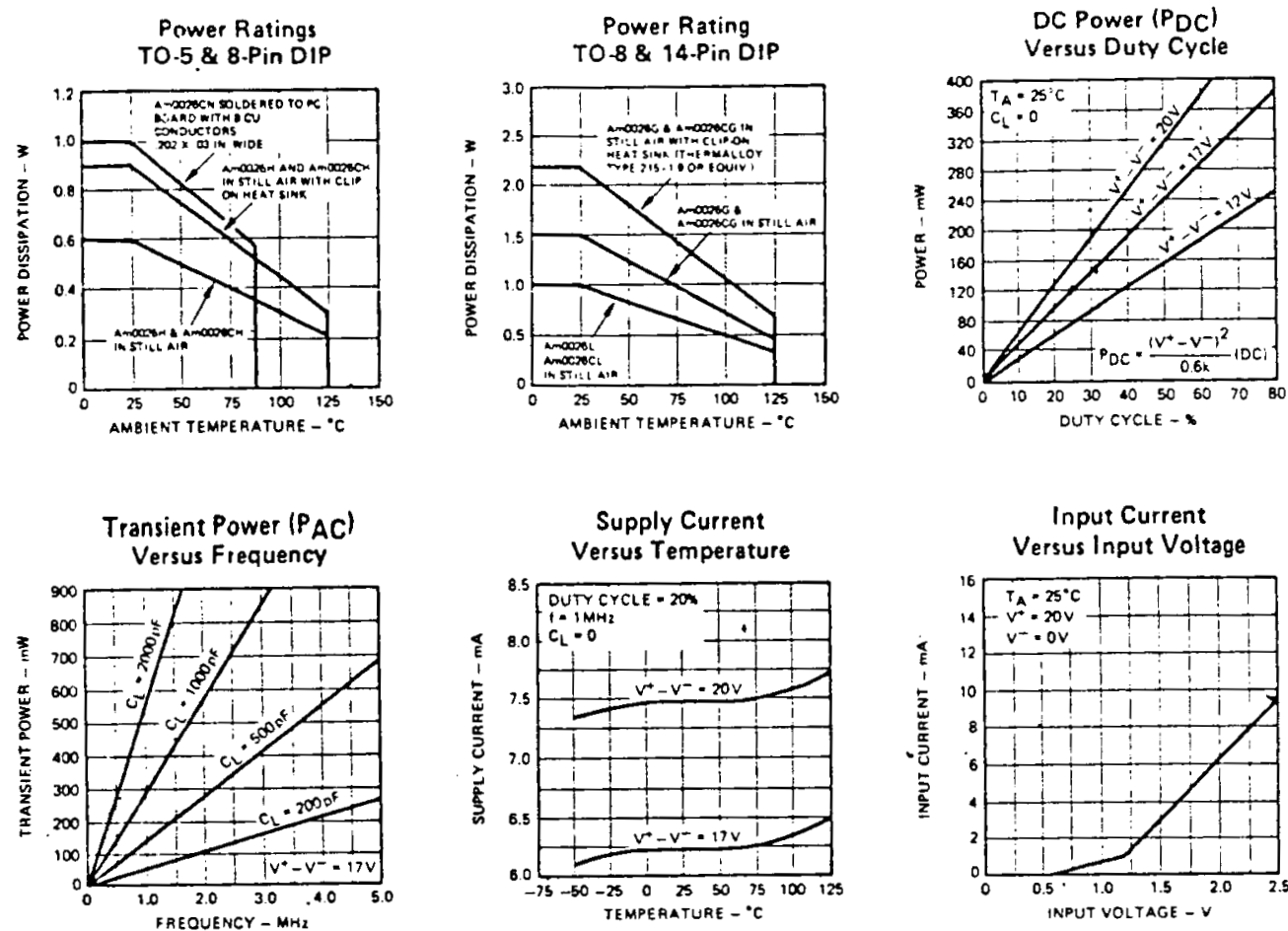

Rise Time

Fall Time Versus Load Capacitance

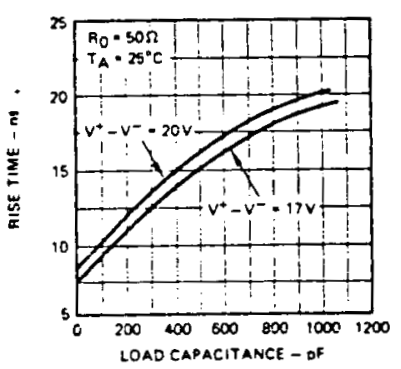

Versus Load Capacitanco
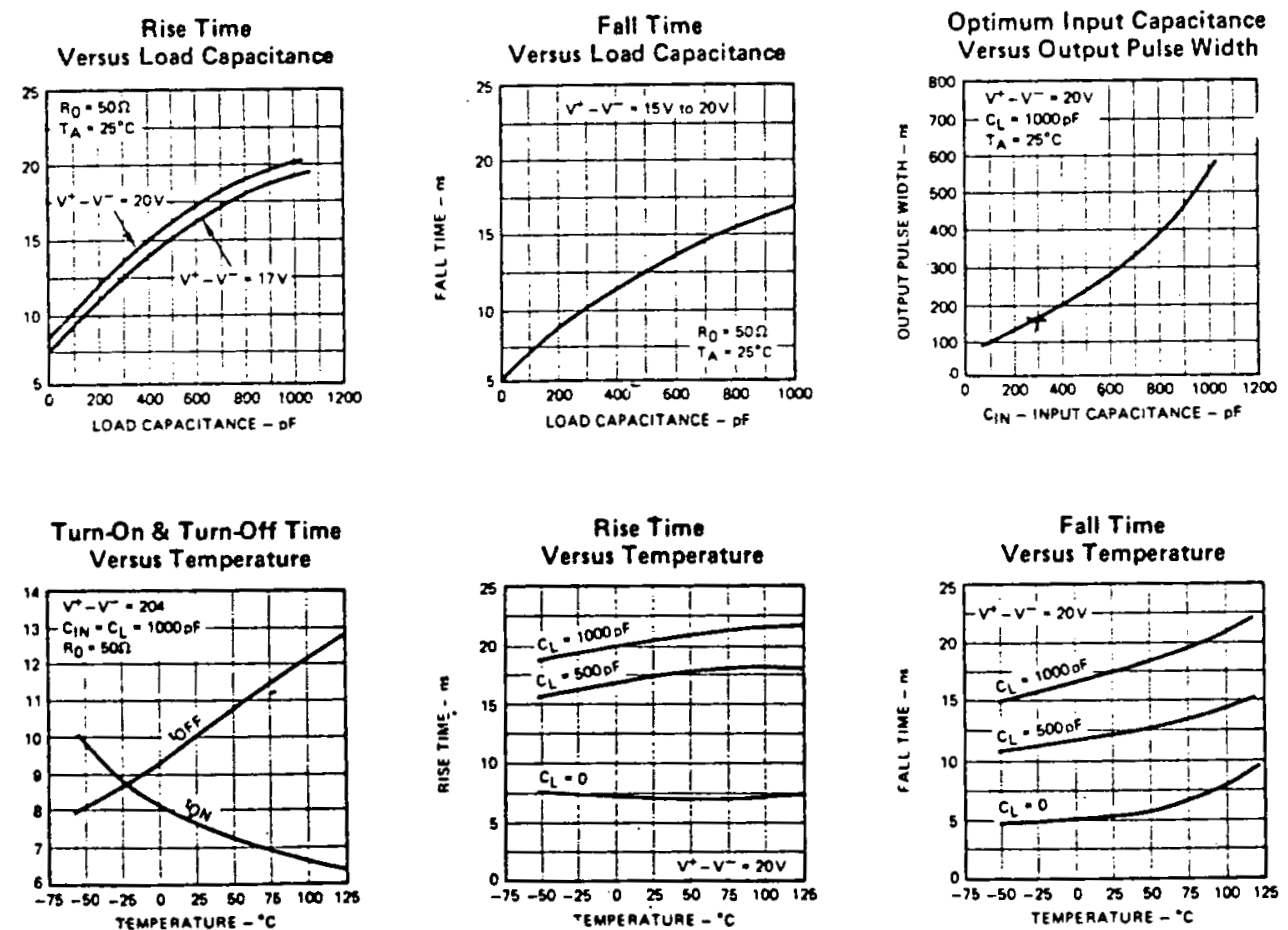

Fall Time

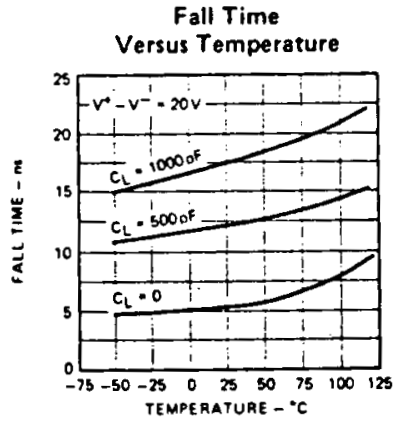

Copyright (1974) Advanced Micro Devices, Inc., Sunnyvale, CA 94086. Reproduced with permission of copyright owner. All rights reserved. 
SWITCHING TIME WAVEFORMS

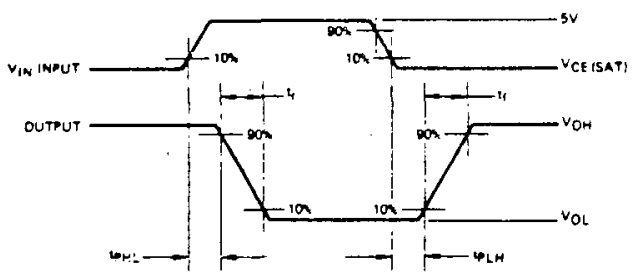

AC TEST CIRCUIT

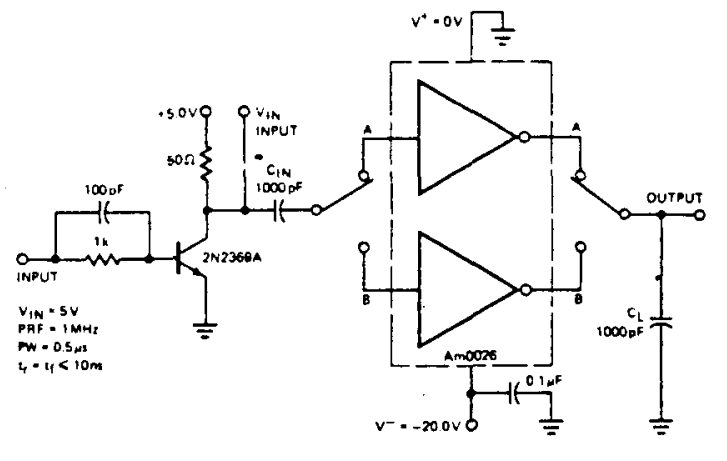

\section{APPLICATION INFORMATION}

\section{POWER DISSIPATION}

The total average power dissipation of the Am0026 is the sum of the DC power and $A C$ transient power. This total must be, less than the given package power rating.

$$
P_{\text {DISS }}=P_{A C}+P_{D C} \leqslant P_{M A X} \text {. }
$$

With the device dissipating only $2 \mathrm{~mW}$ when the output is at a HIGH voltage (MOS logic " 0 "), the dominant factor in average $D C$ power is the duty cycle or fraction of the time the output is at a LOW voltage level (MOS logic "1"). For the shift register driving where the duty cycle is less than $25 \%$, PDC is usually negligible. For RAM address line driver appli: cations $P_{D C}$ dominates since duty cycie can exceed $50 \%$. DC Power per driver:

DC power is given by.

$$
P_{D C}=\left(V^{+}-V^{-}\right) \times \text {Is }_{\text {(LOW }} \times \text { Duty Cycle. }
$$

where IS (LOW) is ISUPPLY ION) at $\left(\mathrm{V}^{+}-\mathrm{V}^{-}\right)$

$$
\begin{aligned}
\text { ISUPPLY(ON) is } 40 \mathrm{~mA} \times \frac{\left(\mathrm{V}^{+}-\mathrm{V}^{-}\right)}{20 \mathrm{~V}} \text { worsi case } \\
\text { or } 30 \mathrm{~mA} \times \frac{\left(\mathrm{V}^{+}-\mathrm{V}^{-}\right)}{20 \mathrm{~V}} \text { typically }
\end{aligned}
$$

AC transient power per driver:

$A C$ transient power is given by,

$$
P_{A C}=\left(V^{+}-V^{-}\right)^{2} \times C_{L} \cdot \times f \times 10^{-3} \text { in mW }
$$

where $f=$ frequency of operation in $M H_{z}$ and $C_{L}=$ load capacitance including all strays and wiring in pF.

\section{PACKAGE SELECTION}

Power ratings are based on a maximum junction rating of $175^{\circ} \mathrm{C}$. The following guidelines are suggested for package selection. Graphs shown in the Performance Curves illustrate derating for various operating temperatures.

T0.5 ("H") Package: Rated at $600 \mathrm{~mW}$ in still air (derate at $4.0 \mathrm{~mW} / \mathrm{C}$ above $25^{\circ} \mathrm{C}$ ) and rated at $900 \mathrm{~mW}$ with clip-on heat sink (derate at $6.0 \mathrm{~mW} / \mathrm{C}$ above $25^{\circ} \mathrm{C}$ ). This popular hermetic package is recommended for small systems. Low cost (about 10d) clip-on-heat sink increases driving power dissipation capability by $50 \%$.

B.pin ("A") Molded Mini.DIP: Rated at $600 \mathrm{~mW}$ still air (derate at $4.0 \mathrm{~mW} / \mathrm{C}$ above $25^{\circ} \mathrm{C}$ ) and rated at 1.0 watt soldered to $P C$ board (derate at $6.6 \mathrm{~mW} /{ }^{\circ} \mathrm{C}$ ). Constructed with a special copper lead frame, this package is recommended for

\$.4 medium size commercial systems particularly where automatic insertion is used. (Please note for prototype work, that this package is only rated at $600 \mathrm{~mW}$ when mounted in a socket and not one watt until it is soldered down.)

$$
C_{L}(\max .)=\frac{10^{3}}{n} \frac{\left(P_{\max } \operatorname{Req}-10^{3}\left(V^{+}-V^{-}\right)^{2} \text { Duty Cycle }\right)}{\operatorname{Req}\left(V^{+}-V^{-}\right)^{2} \times f}
$$

where $n$ is the number of drivers used in the package.

$P_{\text {max }}$ is the package power rating in milliwatts for given package, heat sink, and maximum ambient temperature.

Req is the equivalent resistance $\left(V^{+}-V^{-}\right) / I_{\text {S }}($ LOW $)=500 \Omega$ (worst case over temperature or $600 \Omega$ (typically).

Duty cycle is the fraction of the time that the output signal is in the LOW state.

$f$ is the input signal frequency in $\mathrm{MHz}$.

$C_{L}$ (max.) is the maximum load capacitance per driver in picofarads which can be driven without exceeding device power limits.

When used as a non-overlapping two phase driver with each side operating at the same frequency and duty cycle, and with $\left(V^{+}-V^{-}\right)-17 V$, the above equation simplifies to

$$
C_{L}=\frac{10^{3}}{f}\left[\frac{P_{\text {max }}}{578}-\text { Duty Cycle }\right]
$$

Table I gives maximum drive capability for various system conditions using the above equation.

\section{PULSE WIDTH CONTROL}

The Am0026 is intended for applications in which the input pulse width sets the output pulse width; i.e., the output pulse width is logically controlled by the input pulse. The output pulse width is given by:

$$
(P)_{\text {OUT }}=(P W)_{1 N}+t_{f}+t_{f}=P W_{I N}+25 n s
$$

Two external input coupling capacitors are required to perform the level translation between TTL/DTL and MOS logic levels. Selection of the capacitor size is determined by the desired output pulse width. Minimum delay and optimum performance is attained when the voltage at the input of the Am0026 discharges to just above the devices threshold (about $1.5 \mathrm{~V}$ ). If the input is allowed to discharge below the threshold, tr and $t_{1}$ will be degraded. The graph in the Performance Curves shows optimum values for $C_{\text {IN }}$ versus desired output pulse width. The value for. $C_{I N}$ may be roughly predicted by:

$$
C_{\text {IN }}=\left(2 \times 10^{-3}\right) \text { (PW) OUT }
$$

For an output pulse width of $500 \mathrm{~ns}$, the optimum value for $\mathrm{C}_{\text {IN }}$ is:

$$
C_{I N}=\left(2 \times 10^{-3}\right)\left(500 \times 10^{-9}\right)=1000 \mathrm{pF}
$$




\section{RISE AND FALL TIME CONSIDERATIONS (Note 3)}

The Am0026's peak output current is limited to 1.5A. The peak current limitation restricts the maximum load capacitance which the device is capable of driving and is given by:

$$
l=C_{L} \frac{d v}{d t}<1.5 \mathrm{~A}
$$

The rise time, $t_{r}$, for various loads may be predicted by:

$$
t_{r}=(\Delta V) \quad\left(250 \times 10^{-12}+C_{L}\right)
$$

Where: $\Delta V=$ the change in voltage across $C_{L}$

$$
\cong v^{+}-v^{-}
$$

$C_{L}=$ The load capacitance

for $V^{+}-V^{-}=20 \mathrm{~V}, C_{L}=1000 \mathrm{pF}, \mathrm{L}_{\mathrm{r}}$ is:

$t_{\mathrm{r}} \cong(20 \mathrm{~V})\left(250 \times 10^{-12}+1000 \times 10^{-12}\right)$

$$
=25 \mathrm{~ns}
$$

For small values of $C_{L}$, the equation above predicts optimistic values for $t_{r}$. The graph in the performance curves shows typical rise times for various load capacitances.

The output fall time (see Graph) may be predicted by:

$$
t_{f} \cong 2.2 R\left(C_{S}+\frac{C_{L}}{h_{F E}+1}\right)
$$

\section{CLOCK OVERSHOOT}

The output waveform of the Am0026 can overshoot. The overshoot is due to finite inductance of the clock lines. It occurs on the negative going edge when $Q_{7}$ saturates, and on the positive edge when $a_{3}$ turns OFF as the output goes through $v^{+}-v_{b e}$. The problem can be eliminated by placing a small series resistor if the output of the Ain0026. The critical valve for $R_{S}=2 L C_{L}$ where $L$ is the self-inductance of the clock line. In practice, determination of a value for $L$ is rather difficult. However, $R_{S}$ is readily determined emperically, and values typically range between 10 and $51 \Omega$. R $R_{S}$ does reduce rise and fall times as given by:

$$
t_{r}=t_{r} \cong 2.2 R_{S} C_{L}
$$

\section{CLOCK LINE CROSS TALK}

At the system level, voltage spikes from $\phi_{1}$ may be transmitted to $\phi_{2}$ (and vice-versa) during the transition of $\phi_{1}$ to MOS logic "1". The spike is due to mutual capacitance between clock lines and is, in general, aggravated by long clock lines when numerous registers are being driven. Transistors $O_{3}$ and $Q_{4}$ on the $\phi_{2}$ side of the Am0026 are essentially "OFF" when $\phi_{2}$ is in the MOS logic " 0 " state since only micro-amperes are drawn from the device. When the spike is coupled to $\phi_{2}$, the output has to drop at least $2 V_{B E}$ before $O_{3}$ and $Q_{4}$ come on and pull the output back to $\mathrm{V}^{+}$. A simple method for elimin. ating or minimizing this effect is to add bleed resistors between the Am0026 outputs and ground causing a current of a few milliamps to flow in $\mathrm{O}_{4}$. When a spike is coupled to the clock line $O_{4}$ is already "ON" with a finite $h_{\mathrm{fe}}$. The spike is quickly ciamped by $O_{4}$. Values for $R$ depend on layout and the number of registers being driven and vary typically between $2 k$ and $10 k \Omega$.

\section{POWER SUPPLY DECOUPLING}

Adequate power. supply decoupling is necessary for satisfactory operation. Decoupling of $\mathrm{V}^{+}$to $\mathrm{V}^{-}$supply lines with at least $0.1 \mu \mathrm{F}$ noninductive capacitors as close as possible to each Am0026 is strongly recommended. This decoupling is necessary because otherwise 1.5 ampere currents flow during logic transition in order to rapidly charge clock lines.

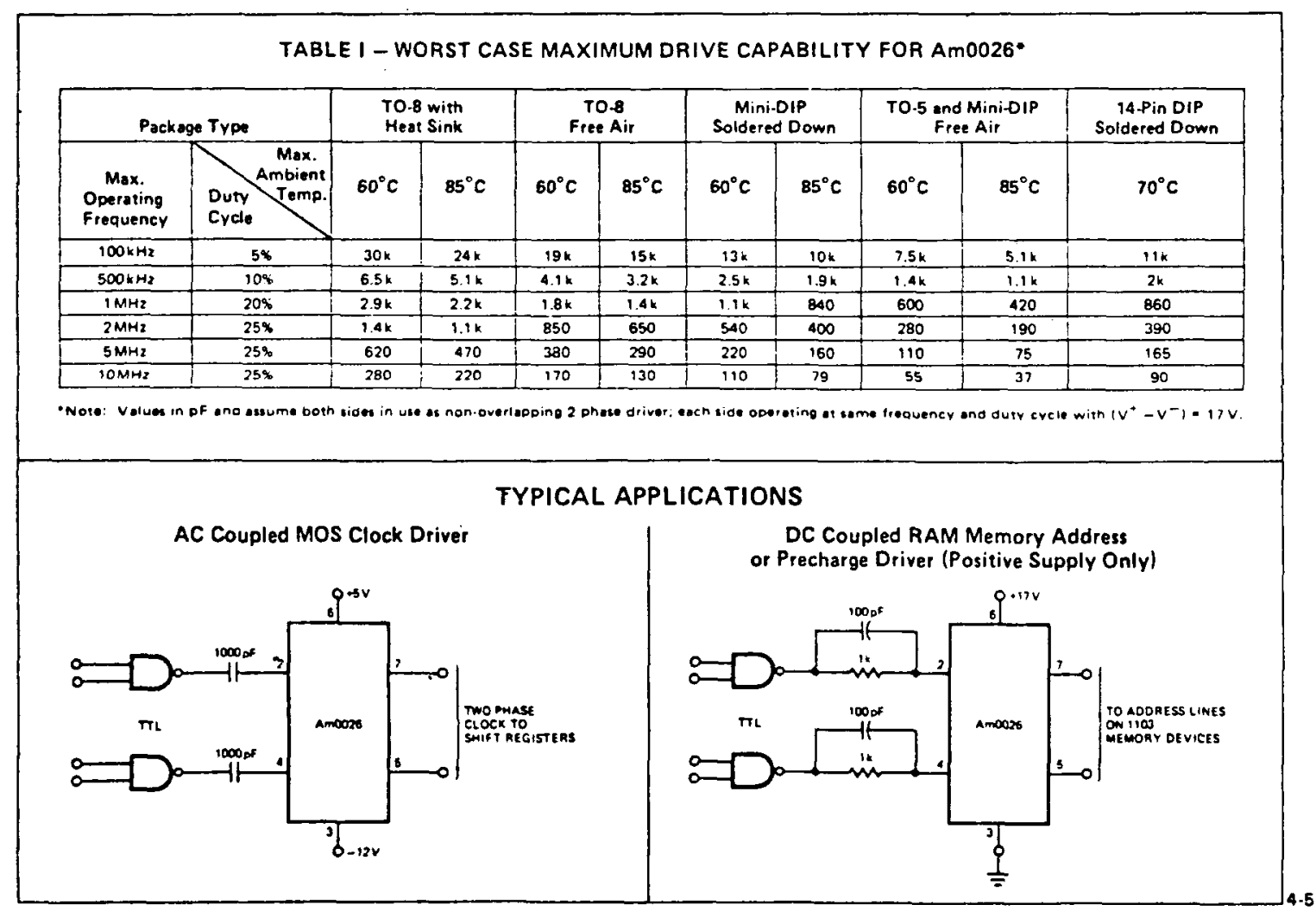

Copyright (1974) Advanced Micro Devices, Inc., Sunnyvale, CA 94086. Reproduced with permission of copyright owner. All rights reserved. 


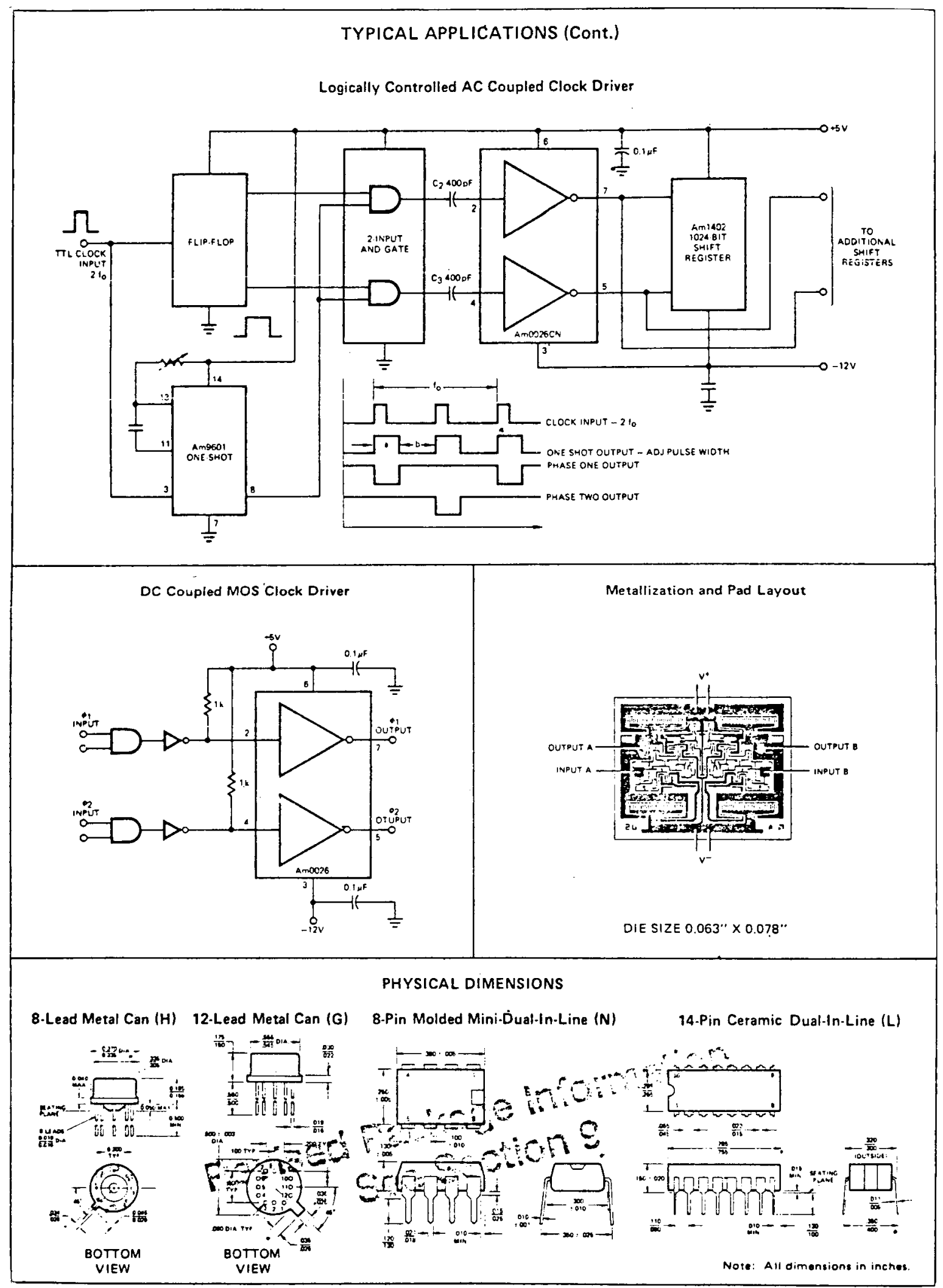

Copyright - (1974) Advanced Micro Devices, Inc., Sunnyvale, CA 94086. Reproduced with permission of copyright owner. All rights reserved. 


\section{Am1402A/1403A/14.04A Am2802/2803/2804 1024-Dit Dynamic Shift Registers}

Distinctive Characteristics

- Quad 256-bit, dual 512-bit, single 1024-bit

- $10 \mathrm{MHz}$ frequency operation guaranteed for Am2802. Am2803 and Am2804.

- Low power dissipation of $0.1 \mathrm{~mW} / \mathrm{bit}$ at $1 \mathrm{MHz}$

- DTL and TTL compatible
- Both military and commercial grade devices available

- $100 \%$ reliability assurance testing in compliance with MIL.STD.883.

- Electrically tested and optically inspected die for the assemblers of hybrid products

\section{FUNCTIONAL DESCRIPTION}

The Am1402A, 3A, and 4A are 1024-bit silicon gate dynamic shift registers. The low threshold characteristics of this technology allow high-speed operation and DTL and TTL compatibility. The Am1402A is a quad 256-bit device; the Am 1403A is a dual 512.bit register; and the Am1404A is a single 1024-bit register. All three devices require two-phase non-overlapping clocks, and provide a one-bit shift on each clock pulse. The Am2802, 3, and 4 registers are functionally identical to the Am1402A, 3A, and 4A, but are guaranteed to operate over frequencies from $400 \mathrm{~Hz}$ to $10 \mathrm{MHz}$.

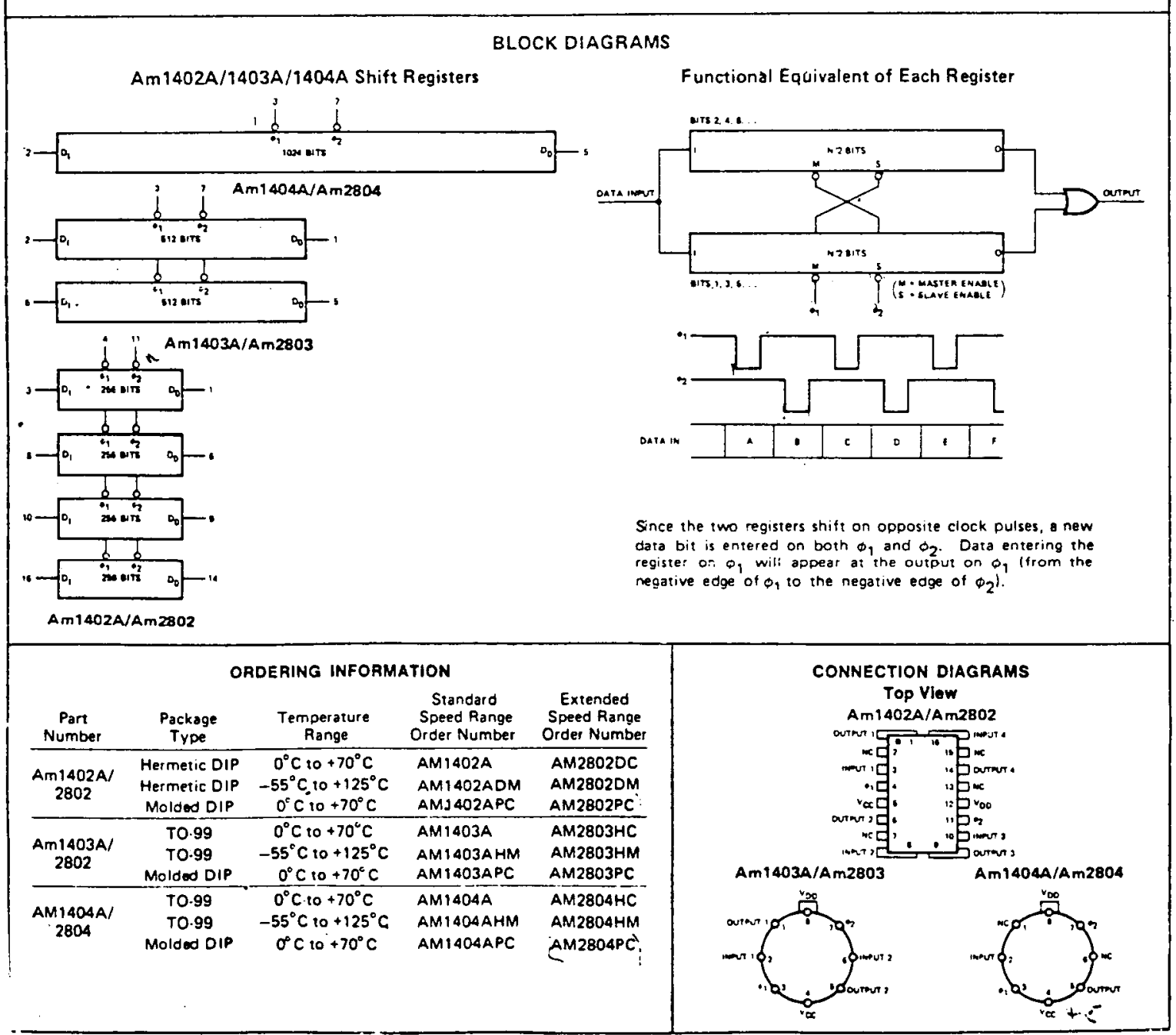

Copyright (1974) Advanced Micro Devices, Inc., Sunnyvale, CA 94086. Reproduced with permission of copyright owner. All rights reserved. 
MAXIMUM RATINGS (Above which the useful life may be Impaired)

\begin{tabular}{|c|c|}
\hline Storage Temperature & $-65^{\circ} \mathrm{C} 10+160^{\circ} \mathrm{C}$ \\
\hline Temperature Under Bias & $-55^{\circ} \mathrm{C}$ to $+125^{\circ} \mathrm{C}$ \\
\hline Power Dissipation (Nole i) & $600 \mathrm{~mW}$ \\
\hline Data and Clock Input Voltages with respect to most Positive Supply Voltage, $V_{c c}$ & $0.3 \vee$ to $-20 \mathrm{~V}$ \\
\hline Power Supply Voltage, $V_{D E}$ with respect to $V_{C C}$ & $0.3 v$ to $-20 V$ \\
\hline
\end{tabular}

\section{OPERATING RANGE}

\begin{tabular}{|c|c|c|c|}
\hline Part Number & $v_{c c}$ & $V_{O D}$ & Temperature Range \\
\hline Am1402A, Am1403A, Am1404A & $5 V: 5 \%$ & $-4.75 V$ to $-9.45 V$ & $0^{\circ} \mathrm{C}$ to $+70^{\circ} \mathrm{C}$ \\
\hline AMI $402 A D M$, AMI 4Q3ATHM, AM1404AHM & $5 V \pm 5 \%$ & $-4.75 V$ to $-9.45 V$ & $-55^{\circ} \mathrm{C} 10+125^{\circ} \mathrm{C}$ \\
\hline Am28020C, Am2803MC, Am2804HC & $5 V \pm 5 \%$ & $-5 V=5 \%$ & $0^{\circ} \mathrm{C}+0+70^{\circ} \mathrm{C}$ \\
\hline Am28020M, Am2803HM, Am2804HM & $5 V \pm 5 \%$ & $-5 \dot{v} \pm 5 \%$ & $-55^{\circ} \mathrm{C}$ to $+125^{\circ} \mathrm{C}$ \\
\hline
\end{tabular}

ELECTRICAL CHARACTERISTICS OVER OPERATING RANGE (Unless Otherwise Noted)

\begin{tabular}{|c|c|c|c|c|c|c|c|c|c|c|}
\hline \multirow[b]{2}{*}{ Parameters } & \multirow[b]{2}{*}{ Description } & \multirow{2}{*}{\multicolumn{2}{|c|}{ Test Conditions }} & \multicolumn{3}{|c|}{ Am1402A, 3A, 4A } & \multicolumn{3}{|c|}{ Am2802, 3, 4} & \multirow[b]{2}{*}{ Units } \\
\hline & & & & Min. & Typ. & Max. & Min. & Typ. & Max. & \\
\hline$V_{I H}$ & Inout HIGH Voltage & & & $v_{c c-2.0}$ & & & $v_{C C-2.0}$ & & 1 & $\mathrm{v}$ \\
\hline$v_{I L}$ & Input LOW Voltepe & & & $v_{C C-10}$ & & $v_{C C}-4.2$ & $v_{C C-10}$ & & $v_{C C-4.2}$ & $\mathbf{v}$ \\
\hline$I_{1}$ & Input Current & $T_{A}=25^{\circ} \mathrm{C}$ & & & $<10$ & 500 & & $<10$ & 500 & nA \\
\hline 10 & Output Leskoge Currẹnt & $T_{A}=25^{\circ} \mathrm{C}, V_{\text {OUT }}$ & - OV & & $<10$ & 1000 & & $<10$ & 1000 & $n A$ \\
\hline$I_{\phi i}$ & Clock Leakage Current & $T_{A}=25^{\circ} \mathrm{C}, V_{Q}=-1$ & $12 v$ & & 10 & 1000 & & 10 & 1000 & $n$ \\
\hline \multirow{4}{*}{$v_{\text {OH }}$} & Output HIGH Voltage Driving TTL & $\begin{array}{l}R_{L}=3 \mathrm{k} \text { to } V_{D D} \\
V_{D D}=-5 \vee=5 \%\end{array}$ & & 2.4 & 3.5 & & $v_{c c}-1.9$ & $v_{\mathrm{Cc}-1}$ & & \\
\hline & Output HIGH Voltage Driving MOS & $\begin{array}{l}R_{L}=4.7 k \text { to } V_{D D} \\
V_{D D}=-5 V_{=5 \%}\end{array}$ & & $v_{C C-1.9}$ & $v_{c c-1}$ & & $\begin{array}{l}V_{C C}-1.9 \\
\text { Note } 2 \mid\end{array}$ & $\begin{array}{l}\left.v_{\mathrm{Cc}-1}\right) \\
\text { (Note 2) }\end{array}$ & & \multirow{2}{*}{$\mathbf{v}$} \\
\hline & Output HIGH Vohage Driving TTL & \multicolumn{2}{|l|}{$\begin{array}{l}R_{L}=4.7 k: 0 V_{D D} \\
V_{D D}=-9 \vee * 5 \%\end{array}$} & 2.4 & 3.6 & & & & & \\
\hline & Output HIGH Voluge Driving MOS & \multicolumn{2}{|c|}{$\begin{array}{l}R_{L}=6.2 \mathrm{k} \text { to } V_{D D} .3 .8 \mathrm{k} 10 \mathrm{~V} C \mathrm{C} \\
V_{D D}=-9 \mathrm{~V}: 5 \mathrm{X}\end{array}$} & $v_{c c-1.9}$ & $v_{c c-1}$ & & & & & \\
\hline \multirow{2}{*}{$\mathbf{v}_{\text {OL }}$} & \multirow{2}{*}{ Output LOW Voltwose } & \multicolumn{2}{|c|}{$\begin{array}{l}V_{O D}=-5 V \pm 5 X \\
R_{L}=3 k 10 V_{D D} .1 O L=-1.6 \mathrm{~mA}\end{array}$} & & -0.3 & 0.5 & & -0.3 & 0.5 & \multirow{2}{*}{$\mathbf{v}$} \\
\hline & & \multicolumn{2}{|c|}{$\begin{array}{l}R_{L}=4.7 \mathrm{~K} \text { to } V_{D D} \\
V_{O D}=-9 \mathrm{~V}=5 \mathrm{X}, 1 \mathrm{OL}=-1.6 \mathrm{~mA}\end{array}$} & & -0.3 & 0.5 & & & & \\
\hline$v_{0} H$ & Clock Input HIGH Level & & & $v_{c c-1}$ & & $v_{C C}+0.3$ & $v_{c c^{-1}}$ & & $V_{C C}+0.3$ & $\vec{v}$ \\
\hline \multirow{2}{*}{$\mathbf{v}_{\mathbf{\phi l}}$} & \multirow{2}{*}{ Clock Inpui LeW Level } & \multirow{2}{*}{\multicolumn{2}{|c|}{$\begin{array}{l}V_{D D}=-5 V \pm 5 x \\
V_{D D}=-9 V \pm 5 x\end{array}$}} & $v_{C C}-15$ & & $v_{C C}-17$ & $v_{C c-15}$ & & $v_{C C-17}$ & \multirow{2}{*}{$\mathbf{v}$} \\
\hline & & & & $v_{c c-12.6}$ & & $v_{C C}-14.7$ & & & & \\
\hline \multirow{6}{*}{$\begin{array}{l}\text { IDD }(-5) \\
\text { (Note 1) }\end{array}$} & \multirow{6}{*}{$\begin{array}{l}V_{D D} \text { Curront, } V_{D D}=-5 V \pm 5 x \\
V_{D D} \text { Curront, } V_{D D}=-5 V \pm 5 x\end{array}$} & \multirow{3}{*}{$\begin{array}{l}5 \text { MHz Date Rate } \\
33 \% \text { Duty Cycle } \\
V_{\phi L}=V_{C C-17 V}\end{array}$} & $T_{A}=25^{\circ} \mathrm{C}$ & & 40 & 50 & & 40 & 50 & \multirow{3}{*}{ ma } \\
\hline & & & $T_{A}=0^{\circ} \mathrm{C}$ & & & 56 & & & 56 & \\
\hline & & & $T_{A}=-55^{\circ} \mathrm{C}$ & & & & & & 70 & \\
\hline & & \multirow{3}{*}{$\begin{array}{l}10 \mathrm{MHz} \text { Data Rate } \\
40 \% \text { Duty Crele } \\
V_{\triangle L}=V_{C C-17}\end{array}$} & $T_{A}=25^{i} \mathrm{C}$ & & & & & 50 & 60 & \multirow{3}{*}{ ma } \\
\hline & & & $T_{A}=0^{\circ} \mathrm{C}$ & & & & & & 68 & \\
\hline & & & $T_{A}=-55^{\circ} \mathrm{C}$ & & & & & & 80 & \\
\hline \multirow{3}{*}{$\begin{array}{l}\text { IOD }(-\theta) \\
\text { (Note i) }\end{array}$} & \multirow{3}{*}{$V_{D O}$ Current، $V_{D D}=-9 V \pm 5 \%$} & \multirow{3}{*}{ 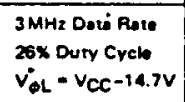 } & $T_{A}=25^{\circ} \mathrm{C}$ & & 30 & 40 & & 30 & 40 & \\
\hline & & & $T_{A}=0^{\circ} \mathrm{C}$ & & & 45 & & & 45 & $m a$ \\
\hline & & & $T_{A}=-55^{\circ} \mathrm{C}$ & & & & & & 60 & \\
\hline
\end{tabular}

Copyright (1974) Advanced Micro Devices, Inc., Sunnyvale, CA 94086. Reproduced with permission of copyright owner. All rights reserved. 
SWITCHING CHARACTERISTICS AND OPERATING CONDITIONS (Over Operating Range) Am1402A/Ami1403A/Am1404A

$V_{\infty}=-5 V=5 \%$

(Test Load 1)

$V_{D O}=-9 \vee \pm 5 *$

(Test Load 2)

\begin{tabular}{|c|c|c|c|c|c|c|c|c|c|}
\hline Parameter & Description & Test Conditions & Min. & Typ. & Max. & Min. & Typ. & Max. & Units \\
\hline$i_{c}$ & Clock Frequency fiange & & INote 11) & & 2.5 & (Note 1$)$ & & 1.5 & $\mathrm{MHz}$ \\
\hline Id & Data Repetition Rate & & (Note 1) & $=$ & 5.0 & (Note 1) & & 3.0 & $\mathrm{MHz}$ \\
\hline SPPW & C!cck Pulse Width & & 0.13 & 1.0 & & 0.17 & & 10 & Hs \\
\hline$b_{d}$ & Clock Pulise Delay (Note 2) & $10 \mathrm{PW}=130 \mathrm{~ns}$ & 10 & & (Note 2) & 10 & & (Note 2) & ns \\
\hline$\pi, v_{r}$ & Clock Pulse Rise/F all Time & & & & 1000 & & & 1000 & ns \\
\hline$t_{1}$ & Date Set Up Time & $t_{r}=\overline{t f}<50 \mathrm{~ns}$ & 30 & & 30 & 60 & & 60 & ns \\
\hline$t_{h}$ & Data Hold Time & $t_{r}=t_{f}<50 \mathrm{~ns}$ & 20 & & 20 & 20 & & 20 & ns \\
\hline$t_{p d}+t_{p d-}$ & Clock to Data Out Deloy & & & & 90 & & & 110 & $\overline{n s}$ \\
\hline $\mathrm{C}_{\mathrm{IN}^{\circ}}$ & Input Capacitence & ब $1 \mathrm{MHz}, 250 \mathrm{mVPP}$ & & 5 & 10 & & 5 & 10 & $\mathrm{pF}$ \\
\hline CoUT: & Output Capacitance & $@ 1 \mathrm{MHz}, 250 \mathrm{mVPP}$ & & $5-$ & 10 & & 5 & 10 & $\mathrm{DF}$ \\
\hline $\cos ^{--}$ & Clock Capacitance & (1) $1 \mathrm{MHz}, 250 \mathrm{mVPP}$ & & 110 & 140 & & 110 & 140 & $\overline{p F}$ \\
\hline
\end{tabular}

SWITCHING CHARACTERISTICS AND OPERATING CONDITIONS (Over Operating Range)

\begin{tabular}{|c|c|}
\hline Am2802/Am2803/Am2804 & $\begin{array}{l}\text { Clock Pulse Width }=70 \text { nsec } \\
\text { Clock LOW Lewel }=\left(V_{C C}-15\right)\end{array}$ \\
\hline
\end{tabular}

\begin{tabular}{|c|c|c|c|c|c|}
\hline Parameter & Description & Test Conditions & Min. & Max. & \\
\hline$t_{c}$ & Clock Frequency Range & $i_{t}=t_{f}=10 \mathrm{~ns}$ & (Note 1$)$ & 5.0 (Note 4$)$ & $\mathrm{MHz}$ \\
\hline$f_{0}$ & Data Repetition Rate (Note 1) & & (Note 31 & $10.0($ Note 4$)$ & $\mathrm{MHz}$ \\
\hline topw & Clock Pulse Width & & 0.07 & 10 & $\mu \mathrm{s}$ \\
\hline कd & Clock Pulse Delay & $10 \mathrm{pW}=70 \mathrm{~ns}$ & 10 & (Note 2) & $n s$ \\
\hline$t_{f}, t_{r}$ & Clock Pulse Rise/Fall Time & & & 1000 & ns \\
\hline 4 & Data Set UD Time & & 30 & & ns \\
\hline$t_{n}$ & Data Hold Time & & 20 & & ns \\
\hline $2 p d+1, p$ & Clock to Data Out Delay & & & 90 & ns \\
\hline
\end{tabular}

Notes:

1. See minimum opersting frequency graph for low limits on date rep. rate.

2. Upper limit on tod is determined by minimum frequency.

3. Soe max clock pulse delay graph for guarantee.

4. For adotitional intormation on $10 \mathrm{MHz}$ operation (5MHz clock rate) ses AMD application note doted Juiy 1973 on "Applications of Dynamic Snifi Registers."

\section{DESCRIPTION OF TERMS}

\section{OPERATIONAL TERMS}

$V_{O H}$ Minimum logic HIGH output voltage with output HIGH current $\mathrm{I}_{\mathrm{OH}}$ flowing out of output.

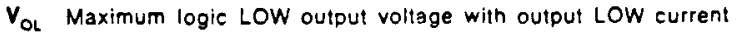
$I_{O L}$ into junction of output and load resistor.

$V_{\text {IH }}$ Logic HIGH input voltage.

$V_{\text {IL }}$ Logic LOW input voltage.

$v_{\mathrm{OL}}$ Clock LOW input voltage.

$V_{\text {OH }}$ Clock HIGH input voltage.

I Input leákage current.

Io Output leakage current.

Power supply current.

$C_{\text {IN }}$ Input capacitance.

$C_{\phi}$ Input clock capacitance.

$\mathrm{C}_{\text {ous Output capacitance. }}$

FUNCTIONAL, TERMS

$\phi_{1}, \phi_{2}$ The two clock phases required by the dynamic shlit register.

I. The clock frequency of the shith register.

f. The input data repetitlon rate.

\section{SWITCHING TERMS}

19. The delay between the LOW to HIGH transition of a clock phase to the HIGH to LOW transition of the other clock phase.

topw The clock pulse widths necessary for correct operation.

$t_{f}, t$. The clock pulse rise and fall times necessary fot correct op. eration.

t. The time required for the input data to be present prior to the LOW to HIGH iransition of the clock phase to ensure correct operation.

4 The time required for the input data to remain present after the LOW to HIGH transition of the clock phase to ensure correct operation.

the propagation delay from the HIGH to LOW clock phase o transition to the output LOW to HIGH transition.

tod- The propagation delay from the HIGH to LOW clock phase $\phi_{2}$ transition to the output HIGH to LOW transition.

Copyright ${ }^{-}(1974)$ Advanced Micro Devices, Inc., Sunnyvale, CA 94086. Reproduced with permission of copyright owner. All rights reserved. 


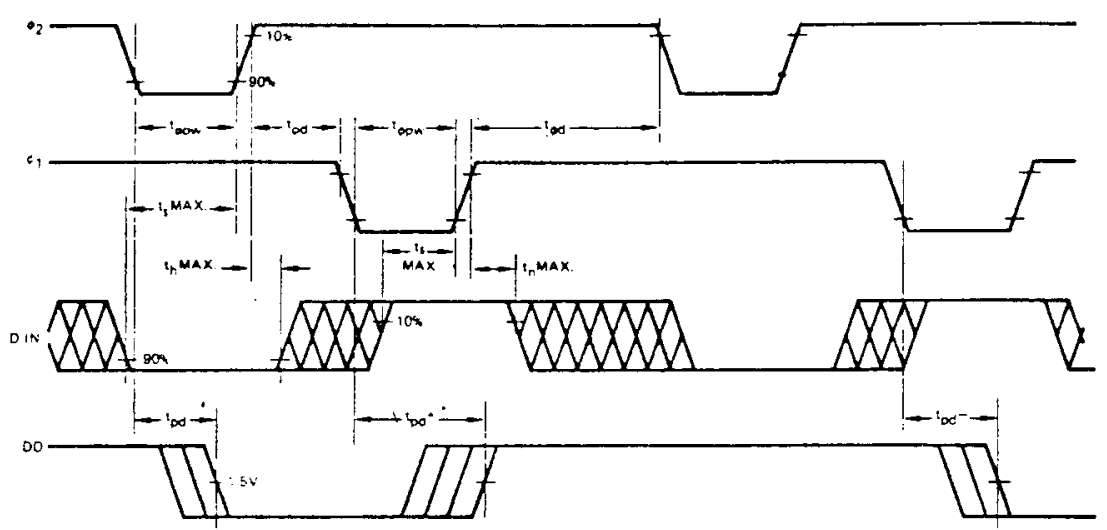

Clock Rise Time 10 ne

Clock Fall Time 10 ns

Output Loso i TLL LOAS

Test Load 1

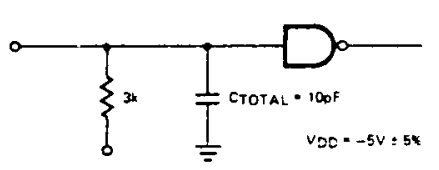

Test Load 2

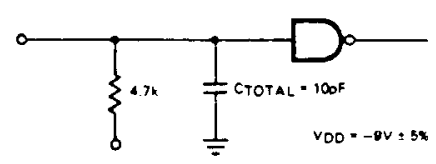

CIRCUIT DIAGRAM

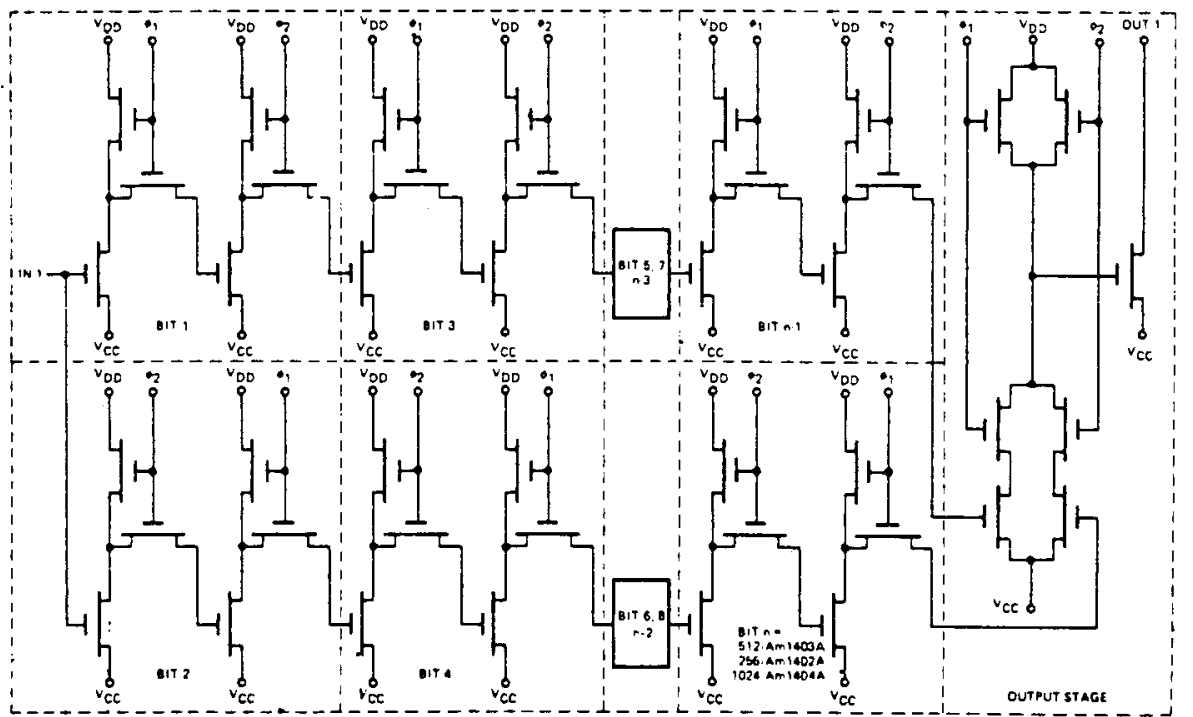

Copyright ${ }^{-11974)}$ Advanced Micro Devices, Inc., Sunnyvale, CA 94086. Reproduced with permission of copyright owner. All rights reserved. 


\section{POWER CHARACTERISTIĆS}
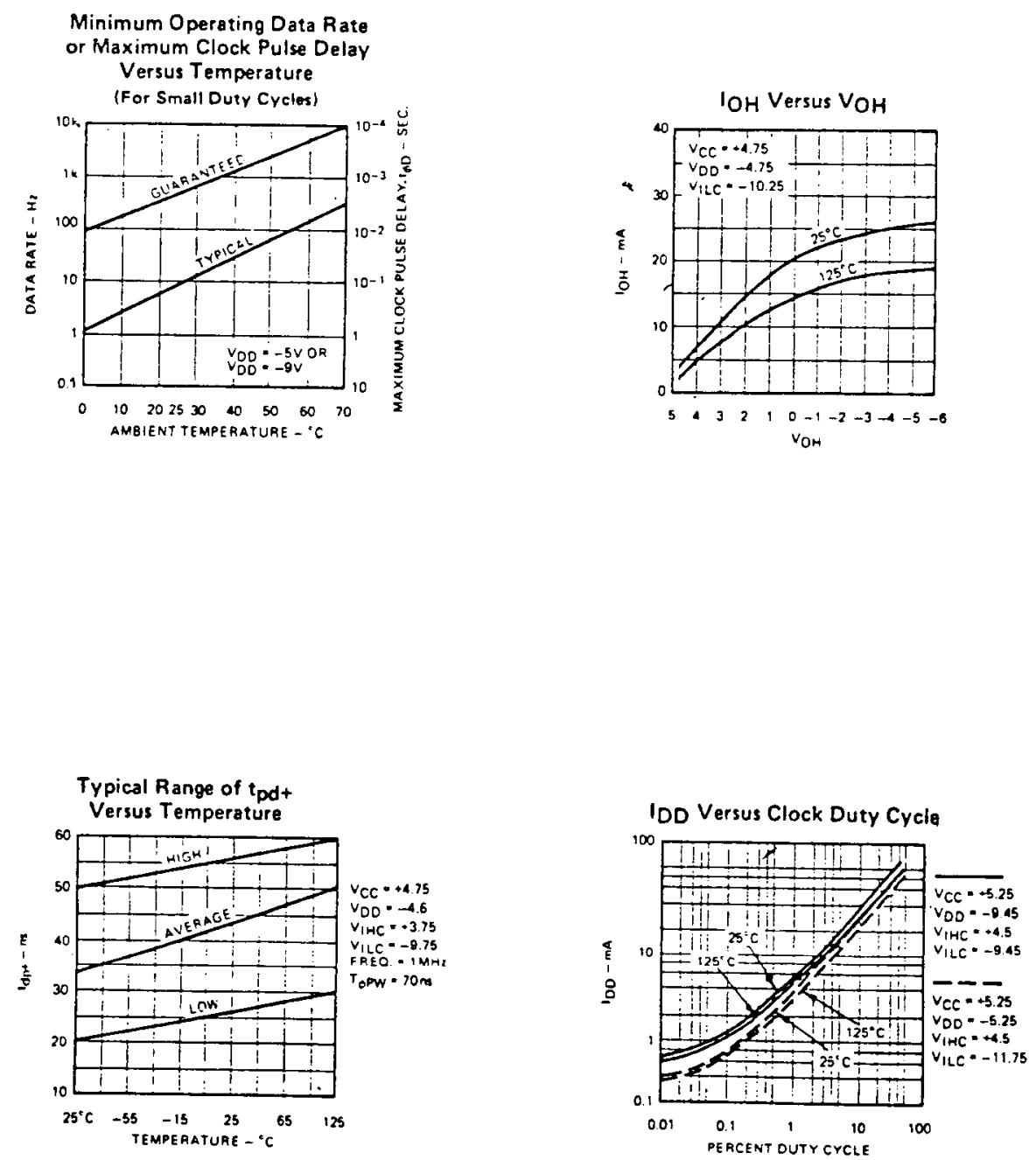

Copyright ${ }^{-}$(1974) Advanced Micro Devices, Inc., Sunnyvale, CA 94086. Reproduced with permission of copyright owner. All rights reserved. 

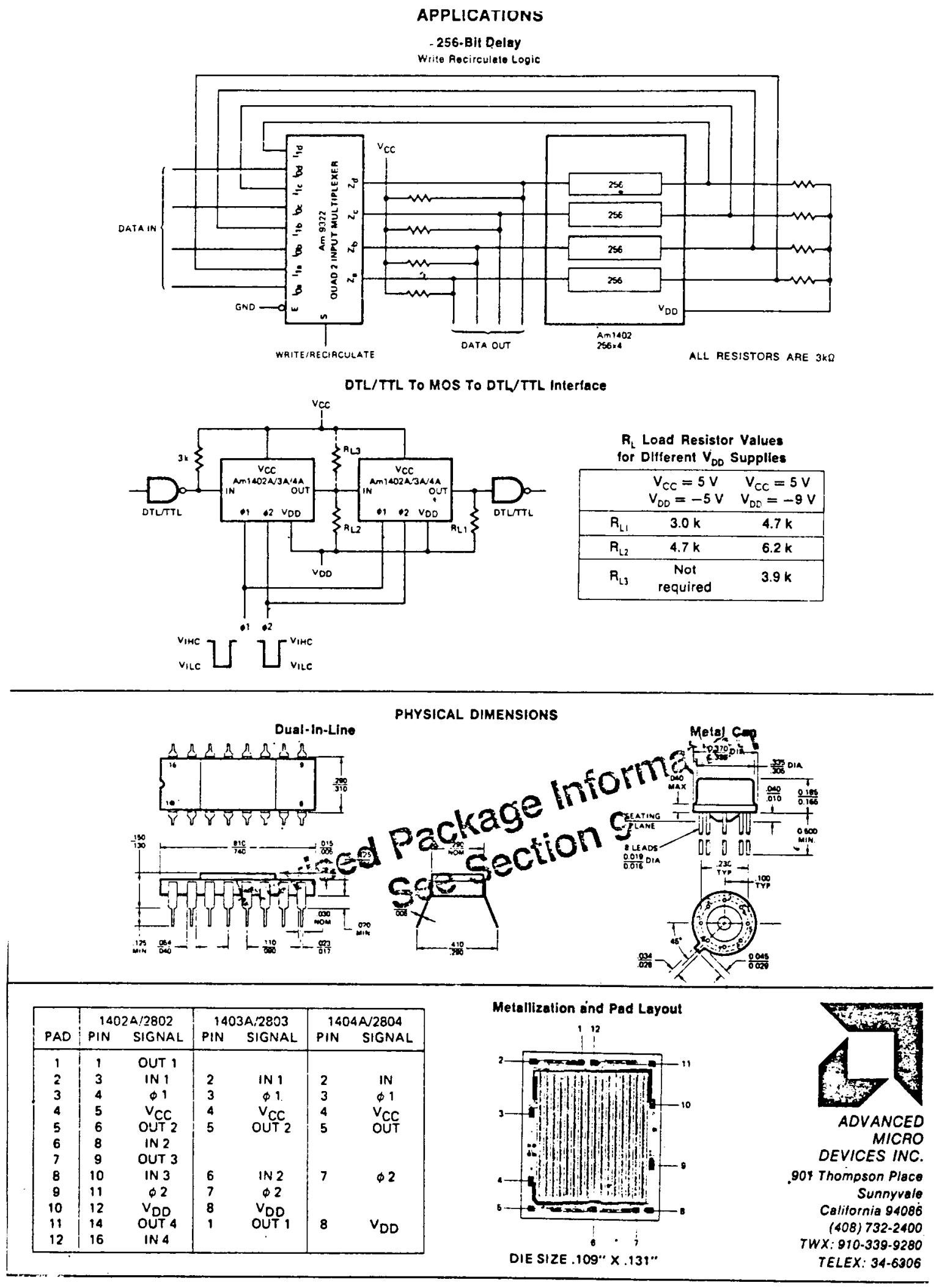

Copyright (1974) Advanced Micro Devices, Inc., Sunnyvale, CA 94086. Reproduced with permission of copyright owner. All rights reserved. 


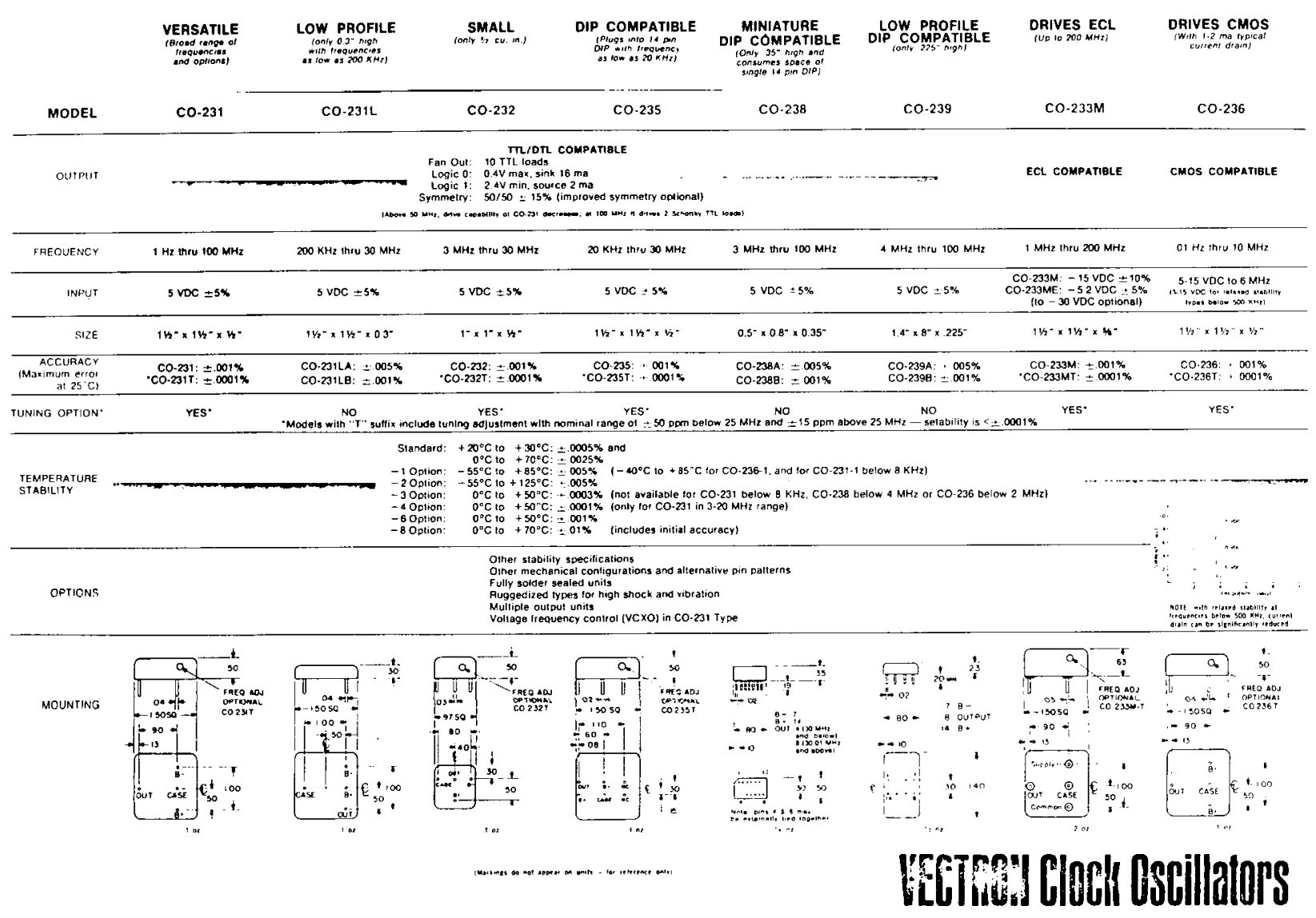

Rebroduced with Dermission from Vectron Labs, Inc., Norwalk, CT 06850. 


\section{Display Drivers}

MATIOKAL

\section{DS8973, DS8974, DS8976 LED 9-digit drivers}

\section{general description}

The DS8973, DS8974, and DS8976 are 9-digit drivers designed to operate from 4 cell (DS8973) or 3 call (DS8974) or 6 cell (DS8976) battery supplies. Each driver will sink $100 \mathrm{~mA}$ to less than $0.5 \mathrm{~V}$ when driven by only $0.1 \mathrm{~mA}$. Each input is blocked by diodes so that the input can be driven below ground with virtually no current drain. This is especially important in calculator systems employing a dc-to-dc converter on the negative side of the battery. If the converter were on the positive sice of the battery. the converter would have to handle all of the display current, as well as the MOS calculator

chip current. But if it is on the negative side, it only has to handie the MOS current. The DS8973 and DS8974 ore designad for the more efficient operating mode. features

- Niné completé digit drivers

- Built-in low battery indicator

- High current outputs-100 mA

- Chioice of 3 or 4 -cell operetion

- Straight through pin out for easy board layout

\section{equivalent circuit and connection diagrams}

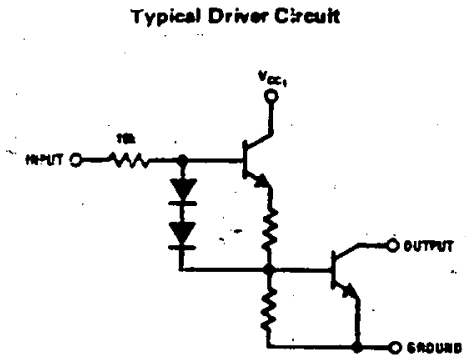

Trpiesl D.P. Out Cireuh

Dual-In-Line Packeas:

oumurs

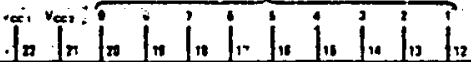
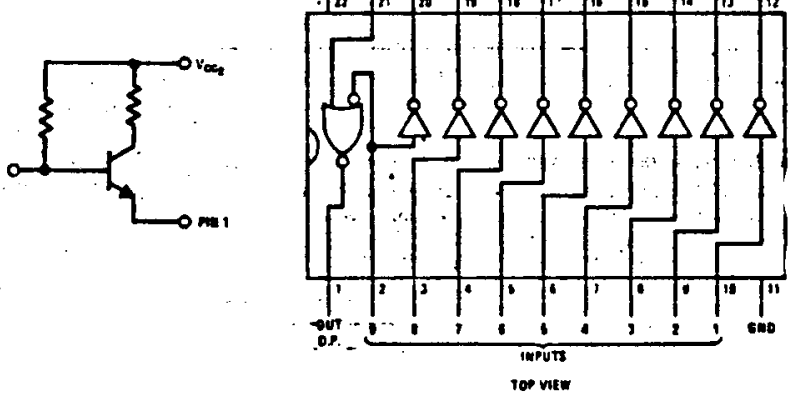

Order Number DS8973N, DS8974N or DS8976N

typical applications

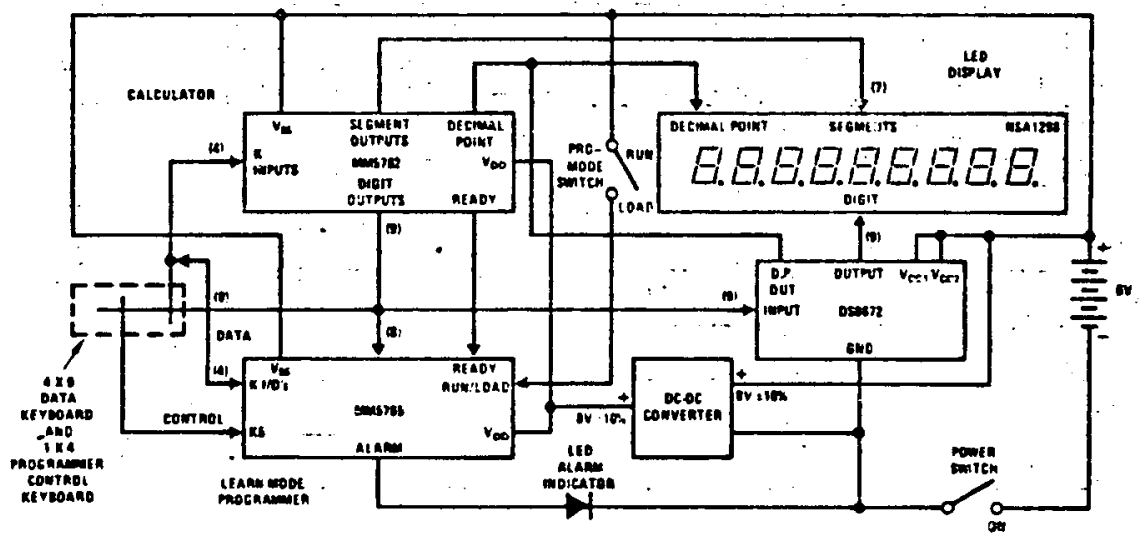

FIGUAE 1. GV Programmabie Statistical Colauletor 
Supply voltuge (VCC) Dcog73

DS8974, DS8976

Tenporuture $\left[T_{A}\right.$ i

$\begin{array}{ccc}\text { MIN } & \text { MAX } & \text { UNITS } \\ 4.4 & 10.0 & \text { V } \\ 3.3 & 4.5 & V \\ 0 & 70 & \text { C }\end{array}$

electrical characteristics

\begin{tabular}{|c|c|c|c|c|c|c|c|}
\hline$v_{\text {an }}$ & Logical " I" Inpur Voteoge & \multicolumn{2}{|l|}{$V_{c c}=\operatorname{Max}$} & 3.8 & & & $\mathbf{v}$ \\
\hline$V_{1 L}$ & Lagical "0" Input Volisge & $V_{c c}=\operatorname{Max} \quad-$ & $\dot{.}$ & . & $\therefore$ & 0.5 & $v$ \\
\hline IIL & Logical " 0 " Input Current & \multicolumn{2}{|l|}{$V_{c c}=M_{a x} V_{1 b}=0.5 V$} & & $\cdot$ & 40 & $\mu \mathrm{A}$ \\
\hline \multirow{2}{*}{$V_{\text {TH }}$} & \multirow{2}{*}{ High Low Battery Threshold } & \multirow{2}{*}{$\begin{array}{c}V_{\text {OT }}(P \text { in } 1)=1 \mathrm{~V}, I_{\text {OT }} \leq-50 \text { MA, } T_{A}=25^{\circ} \mathrm{C} \\
V_{\text {IM }}(\operatorname{Pin} 2)=3.9 \mathrm{~V} \\
\end{array}$} & DS8974 & 4.6 & & & $v$ \\
\hline & & & Dsea76 & .0 & & & $v$ \\
\hline \multirow[t]{3}{*}{$v_{T L}$} & \multirow[t]{3}{*}{ Low Low Bartery Threshold. } & \multirow{3}{*}{$\begin{array}{l}V_{O T}(P \text { in } 1)=2.3 \mathrm{~V}, I_{O T} \geq-6 \mathrm{~mA}, T_{A}=25^{\circ} \mathrm{C} \\
V_{\text {IN }}\left(P_{\text {in } 2}\right)=3.9 \mathrm{~V}\end{array}$} & DS8973 & & & 3.1 & $v$ \\
\hline & & & DS8974 & & & 4.2 & $\mathbf{v}$ \\
\hline & & & DS8976 & - & & 6.2 & v \\
\hline Icc, & Supply Curren: & \multicolumn{2}{|l|}{$V_{c c}=$ Max, One Input "ON" } & & & 6 & $m A$ \\
\hline $\mathrm{Ce}_{2}$ & Pin 21 (Low Barzery Supply) & \multicolumn{2}{|l|}{$V_{c c}=M_{a x}, V_{c c_{2}}=V_{c c \text { max }}$} & & & 1.2 & $\mathrm{ma}$ \\
\hline
\end{tabular}

Note 1: "Absolute Moximum Rating" are those va.ues beyond which the sofety of the dovice cennot be guaranteed. Except for "Operating Tompersture Range" they ore not meant to impiv that the devices should be aperated at these limiz. The table of "Electrical Characteristics" provides conditions for actual device operation

Note 2: Uniess otherwise specified $\mathrm{min} / \mathrm{max}$ limits apply seross the $0^{\circ} \mathrm{C}$ to $+70^{\circ} \mathrm{C}$ range. All typicals are given for $\mathrm{T}_{A}=25^{\circ} \mathrm{C}$.

Now 3: All currents into device pins shown as positive, out of device pins os negative, all vol tages referenced to vround unless otherwise noted. All vilues shown $\mathrm{a}$ max or min on absolute value basis.

typical applications (con't)

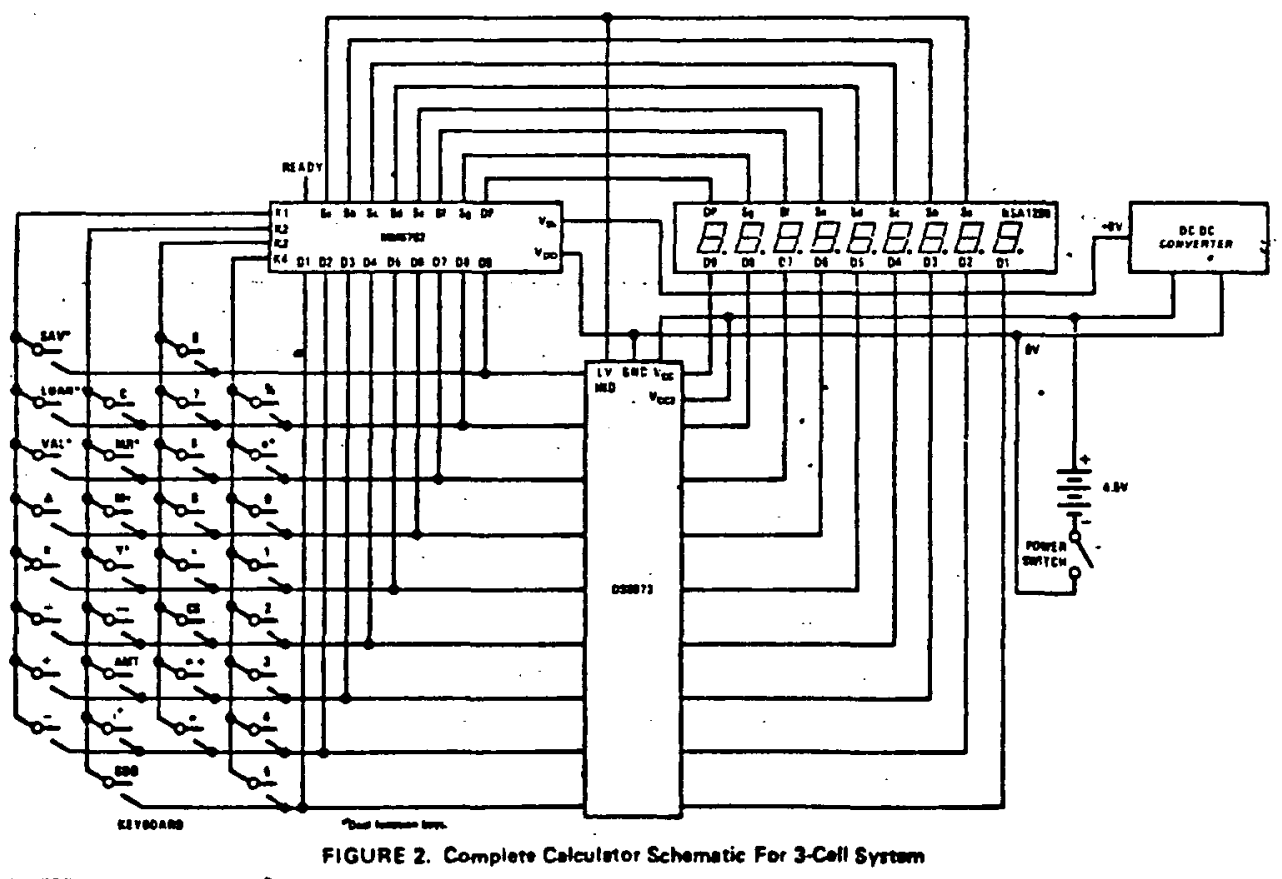

.6 .78

Reproduced with permission from National Semiconductor Corp., Santa Clara, CA 95051 . 


\section{DESCRIPTION}

The Hughes HCTR4010 is a TTL compatible CMOS LSI circuit containing four sychronous decade up/down counters and their associated storage latches. Information from the latches is multiplexed to the ECD outputs, to drive readily available TTL display decoder-drivers.

FEATURES

CMOS techriology for low power Up/down-counting copability Iriternal scan and clock oscillators

All inputs and outputs

TTL compatible ( $V_{D D}=4.5$ to 5.5 volts) Optional 3 or 4 Decade Operation Independent latches on each decode \& overflow $>\mid \mathrm{MHz}$ operation@ $@$ DD $>4.5$ volts Multiplexed $B C D$ outputs with automatic or manual leading zero unblanking Copobility of floating BCD outputs 3 to 10 volts operation

PINS

1. BCD 4 Out

2. BCD 8 Out

3. Int. Clock enable or external clock in

4. Int. Clock frequency control or external clock disoble

5. Decode Strobe True/Comp

6. Decode 2 Strobe

7. Decade 4 Strobe

8. Decade. 1 Strobe

9. Decade 3 Strobe

10. Hold/Load Latches
11. Up/Down Control

12. + VDD

13. Osc. Disable

14. Master Reset

15. Ground $(-V)$

16. 3/4 Decade Select

17. Sean Frequency Control

18. Clock Out

19. Overflow

20. Stored Overflow

21. Chip Enoble

22. Unblank

23. BCD I Out

24. BCD 2 Out

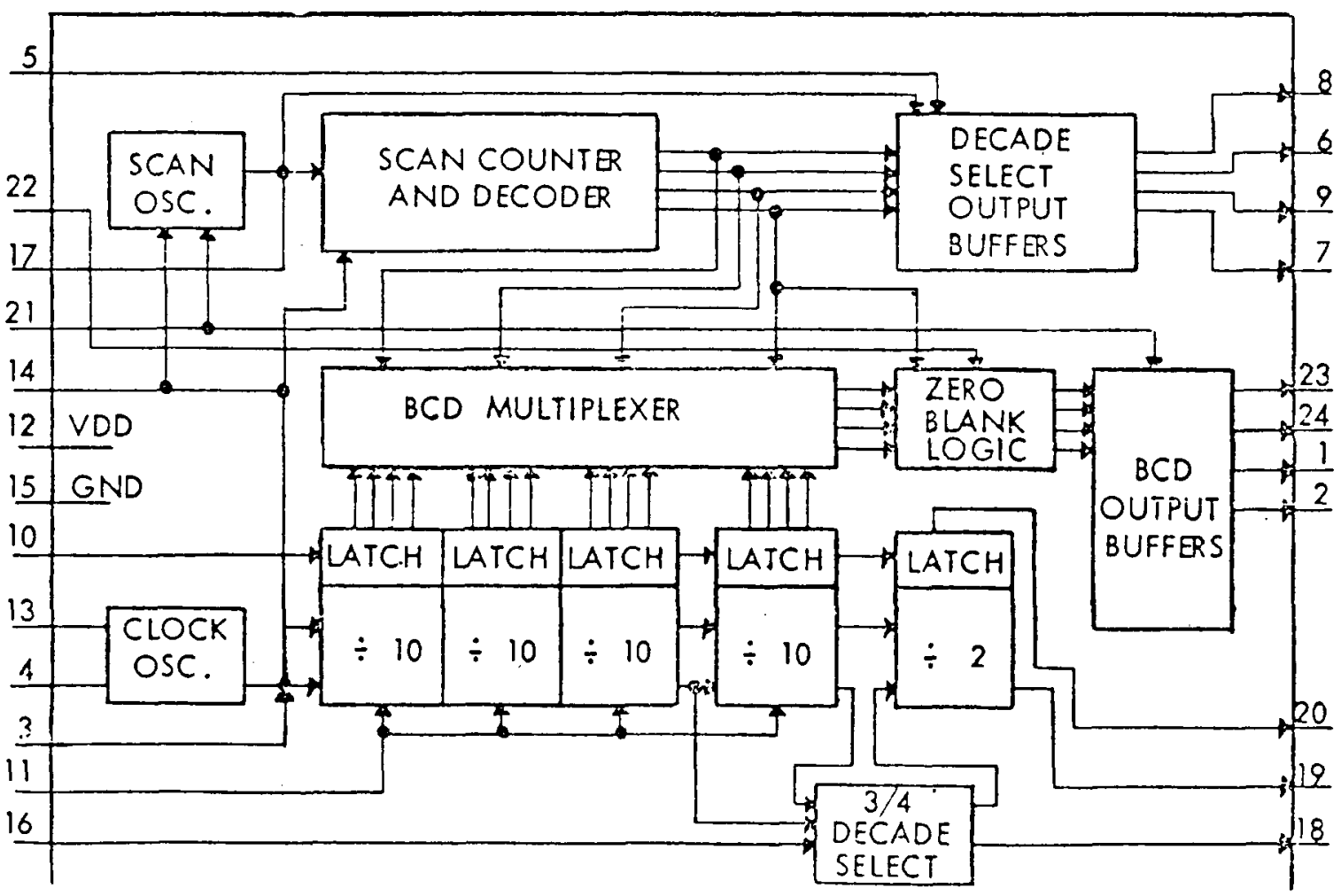

Reproduced with permission from Hughes Aircraft Company, New Port Beach, CA 92663. 
QUAD DECADE CLOCK CONTROL

External Clock Mode ("l" on Oscillator Disable Input):

\begin{tabular}{|c|c|c|}
\hline $\begin{array}{l}\text { Frequency Control/ } \\
\text { External Clock Disable }\end{array}$ & 1 & $\begin{array}{l}\text { Disables External Clock (Internal Clock } \\
\text { Enable/External Clock In must be "O" } \\
\text { when disable is energized). } \\
\text { Enahles External Clock. }\end{array}$ \\
\hline $\begin{array}{l}\text { Internal Clock Enable/ } \\
\text { External Clock In. }\end{array}$ & $1 \rightarrow 0$ & $\begin{array}{l}\text { Advarces counter to next state on "I" to } \\
\text { "0" transition of External Clock applied } \\
\text { at this. input. }\end{array}$ \\
\hline
\end{tabular}

Internal Clock Mode ("0" on Oscillator Ixsable Input):

Frequency Control/
External Clock Disable

Internal Clock Enable/ External Clock In

Unblank

Strobe True/Complement

Chip Enable

$3 / 4$ Decade Select

Hold/Load Latches

$\mathrm{U}_{\mathrm{P}} /$ Down
- Capacitor connected from this input to VDD or GR OUND controls Internal Clock Frequency. If no capacitor is connected, the oscillator will have a free-running frequency of typically $300 \mathrm{KHz}$.

1 Enables internal oscillator to counter. 0 Disables internal oscillator to counter.

\section{QUAD DECADE INPUTS}

$\begin{array}{ll}1 & \text { Pnsitive true. } \\ 0 & \text { Negative true. }\end{array}$

1 Enables BCD information to BCD lines.

0 Floats $B C D$ lines and turns off scan oscillator and turns off all strobe outs. Rest of counter still works.

Displays all decades.

$\begin{array}{ll}\text { Strobe } 1 & \begin{array}{l}\text { Displays all decades. } \\ \text { Displays from first non-zero decade or } \\ \text { just decade } 1 \text {. }\end{array} \\ \text { Strobe } 2 & \begin{array}{c}\text { Displays from first non-zero decade or } \\ \text { just decades } 1 \text { \& } 2 .\end{array} \\ \text { Strobe } 3 & \begin{array}{c}\text { Displays from first non-zero decade or } \\ \text { just decades } 1 \text { \& } 2 \text { \& } 3 .\end{array} \\ 0 & \text { Displays from first non-zero decade. }\end{array}$

1 Stores data in counters (data at $0 \rightarrow 1$ transition is stored).

0 Accepts data from counters.

I Counter counts up.

0 Counter counts down.

NOTE: Up/Down Input should only be changed with. External Clock $=0$ or with External Clock Disable $=1$, otherwise can get false count.

1 Clock Out and overflow driven by output of decade 3.

$0 \quad$ Clock Out and overflow driven by output of decade 4 . 


\section{QUAD DECADE INPUTS (continued..)}

Master Reset

Overflow

Stored Overflow

Clock Out

Jecade $(1,2,3,4)$ Strobe

$B C D 1,2,4$ and 8
1

Resets counter and overflow to zero (latches also reset if HOLD/LOAD $=0$ ). Drives BCD to 1111 , drives strobes off, and resets strobe counter to decade 4.

o Allows counter to operate.

\section{QUAD DECADE OUTPUTS}

$B C D$ information from decade selected by strobe outputs. Blanked information represented by state 1111 . Open state is high impedance to both $+V$ and $-\bar{y}$.

$Q$ from a divide by 2 stage driven by Clock Out.

Stored overflow information output (controlled by HOLD/LOAD input).

A positive pulse with leading edge coincident with first $0 \rightarrow 1$ transition of external (or internal) clock following count 999 or 9999 . The trailing edge is coincident with the $1 \rightarrow 0$ transition of the external (or internal) clock which drives the counter into state 1000 or 10000 (determined by $3 / 4$ Select input).

Tells which decade is being displayed on $B C D$ lines Sequence is 4, 3, 2, 1 with Decade 4 as MSB and decade 1 as LSB. Can be either positive true or negative true depending on state of Strobe $\mathrm{T} / \mathrm{C}$ input. Scan rate determined by scan frequency control input. When scan frequency control $=0$ strobes are driven off, this characteristic can be used to control duty cycle of displays.

\section{QUAD DECADE SCAN OSCILLATOR CONTROL}

External Scan Oscillator Mode

Scan Frequency Control

1 Enables strobe out.

$1 \rightarrow 0 \quad$ Advances strobe crunter.

0 Disables strobe out and drives BCD out to 1111 .

Internal Scan Oscillator Mode

Scan Frequency Control

Capacitor connected from this input to $V_{D D}$ or GROUND controls Internal Scan Oscillator Frequency. If no capacitor is connected, the oscillator will have a free-running frequency of typically $300 \mathrm{KH}$ :

Reproduced with permission from Hughes Aircraft Company, New Port Beach, CA 92663. 


\section{DESCAIPTION}

The 6010 series of monolithic CMOS LSI four decade counters is designed for use in digital panel meters, digital voltmeters and other counting applications.

Four synchronous decade counters and a divide by two overflow stage are included in the 6010 devices to provide a counting range from 0 through 19999 . The counting range can be further extended by cascading devices. Quad latches for each decade and a latch for overflow permit storage of any count in a BCD Format.

An "on chip" scan oscillator and scan counter/decoder generate the timing signals for time division multiplexing of the latched BCD count of each decade to the BCD outputs. The multiplex frequency is controlled by an external capacitor connected to the Scan Oscillator input. The decade strobe duty cycle of the HCTR6010A can be changed from the nominal 12 percent by connecting a resistor to the appropriate supply voltage.

The HCTR6010B is identicai to the HCTR6010A except the scan oscillator has been designed for applications requiring a decade strobe duty cycle of nearly $25 \%$.

In the HCTR6010, an internal delay circuit is placed in series with the Hold/Load Latches input. The delay is sufficiently long to ensure that a count "rippling" through the counter will be stabilized and correctly loaded into the latches even if Hold Latches and clock advance signals are applied simultaneously. This delay circuit is not included on the HCTR6010A and HCTR6010B devices. The HCTR6010 decade strobe duty cycle is similar to that of the HCTR6010B; however, a different range of capacitor values is used for control of the scan frequency.

ABSOLUTE MAXIMUM RATINGS

\begin{tabular}{|l|c|c|c|}
\hline \multicolumn{1}{|c|}{ RATING } & SYMBOL & VALUE & UNIT \\
\hline DC Supply Voltage & $V_{D D}$ & $+10.510-0.5$ & Vdc \\
\hline Input Voltage. All Inputs & $V_{\text {in }}$ & $V_{D D}+0.510-0.5$ & Vdc \\
\hline DC Current Drain Per Pin, All Inputs & 1 & 10 & mAdc \\
\hline DC Current Drain Per Pin, All Outputs & 1 & 20 & mAdc \\
\hline Operating Temperature Range & $T_{A}$ & 01075 & ${ }^{\circ} \mathrm{C}$ \\
\hline Storage Temperature Range & $T_{\text {stg }}$ & $-6510+150$ & ${ }^{\circ} \mathrm{C}$ \\
\hline Power Dissipation & PD & $\begin{array}{l}600 \text { (plastic pkg) } \\
700 \text { (ceramic pkg) }\end{array}$ & mW \\
\hline
\end{tabular}

- CONSULT FACTORY FOR DEVICES WITH HIGHER VOD RATINGS
16 PIN DIP

PIN CONNECTIONS

(TOP VIEW)

BCO B OUT

CLOCK IN

DECADE 2 STROBE

DECADE 4 STROBE

DECADE 1 STROBE

DECADE 3 STROBE

HOLDROAD LATCHES

$v_{D O}{ }^{1+1}$

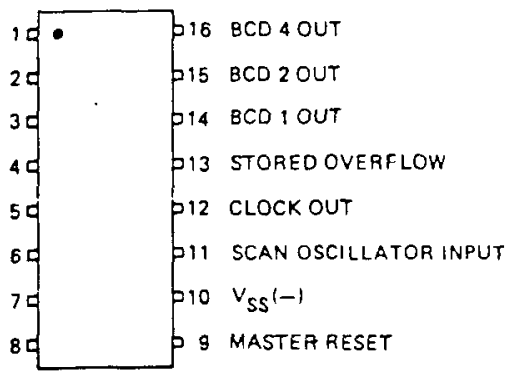

ORDERING INFORMATION:

USE D SUFFIX FOR CERAMIC PACKAGE:

HCTR6O100, HCTREO1OAD, HCTR60108D

USE P SUFFIX FOR PLASTIC PACKAGE:

HCTRGO1OP, HCTRGO1OAP, HCTR6O1OBP

- On Chip Scan Oscillator with adjustable duty cycle and frequency

- Cascadable

- Output latches for four decades plus overflow

- $1 \mathrm{MHZ}$ operation at $5 \mathrm{~V}\left(0\right.$ to $\left.75^{\circ} \mathrm{C}\right)$

GENERAL OPERATING NOTES

1. All high impedance inputs of this device have an input protection circuit to prevent damage due to high static voltage or elestric fields. It is advisable, however, to use normal MOS handling precautions to prevent damage to the inputs resulting from application of voltages in excess of the maximum rated voltages for the device.

2. Erroneous operation may result if unused inputs are allowed to "float". All unused inputs should be terminated to a known logic level such as $V_{D u}$ or $V_{s s}$.

\begin{tabular}{|c|c|c|c|c|}
\hline \multicolumn{3}{|c|}{ INPUTS } & \multirow{2}{*}{$\begin{array}{l}\text { INTERNAL } \\
\text { COUNTER } \\
\text { RESPONSE }\end{array}$} & \multirow{2}{*}{$\begin{array}{l}\text { BCD\& STORED } \\
\text { OVERFLOW } \\
\text { OUTPUT } \\
\text { RESPONSE }\end{array}$} \\
\hline $\begin{array}{l}\text { MASTER } \\
\text { RESET }\end{array}$ & $\underset{\text { IN }}{C \operatorname{LOCK}}$ & $\begin{array}{l}\text { HOLOILOAD } \\
\text { LATCHES }\end{array}$ & & \\
\hline 0 & & 0 & Aovances I Count & $\begin{array}{l}\text { Indicates New } \\
\text { Count }\end{array}$ \\
\hline 0 & & 0 & No Change & No Change \\
\hline 0 & & 1 & Advances 1 Count & $\begin{array}{l}\text { Retains Prior } \\
\text { Count }\end{array}$ \\
\hline 1 & $x$ & 0 & $\begin{array}{l}\text { Resets entire } \\
\text { Counter ISee } \\
\text { note "I }\end{array}$ & $\begin{array}{l}\text { Outputs go to } \\
\text { Zero }\end{array}$ \\
\hline 1 & $x$ & 1 & $\begin{array}{l}\text { Resers entire } \\
\text { Counser (See } \\
\text { note 1) }\end{array}$ & $\begin{array}{l}\text { Retains Prior } \\
\text { Count }\end{array}$ \\
\hline
\end{tabular}

NOTE 1: A master reset has no effect on the scan counter.
TRUTH TABLE NO. 2

\begin{tabular}{|c|c|c|c|}
\hline $\begin{array}{l}\text { SCAN } \\
\text { FREQUENCY } \\
\text { CONTROL } \\
\text { INPUT }\end{array}$ & $\begin{array}{l}\text { INTERNAL SCAN } \\
\text { COUNTER }\end{array}$ & $\begin{array}{l}\text { DECADE STROBE } \\
\text { OUTPUTS }\end{array}$ & $\begin{array}{l}\text { BCD } \\
\text { OUTPUTS }\end{array}$ \\
\hline & $\begin{array}{l}\text { Decrements one } \\
\text { Count (see nore } 2 \text { ) }\end{array}$ & $\begin{array}{l}\text { All strobe outputs } \\
\text { set to Zero }\end{array}$ & $\begin{array}{l}\text { Output changes to } \\
\text { data from next lower } \\
\text { decade isee note } 2 \%\end{array}$ \\
\hline 0 & No change & $\begin{array}{l}\text { All strobe outputs } \\
\text { at Zero }\end{array}$ & $\begin{array}{l}\text { Output data from } \\
\text { decade corresponding } \\
\text { to Scan Counter } \\
\text { Value }\end{array}$ \\
\hline & No change & $\begin{array}{l}\text { Reflects count of } \\
\text { Internal Counter }\end{array}$ & No change \\
\hline 1 & No change & $\begin{array}{l}\text { Decade Strobe corres. } \\
\text { ponding to Scan Counter } \\
\text { Value will be "One" } \\
\text { level }\end{array}$ & $\begin{array}{l}\text { Output data from } \\
\text { decade corresponding } \\
10 \text { Scan Counter } \\
\text { Value }\end{array}$ \\
\hline
\end{tabular}

NOTE 2: Decade strobe scanning secuence is from the most signiticant decade to the least significant decade: i.e., $4 \cdot 3 \cdot 2 \cdot 1.43 \cdot 2 \cdot 1$, etc.

\section{Reproduced with permission from Hughes Aircraft Company, New Port Beach, CA 92663.}




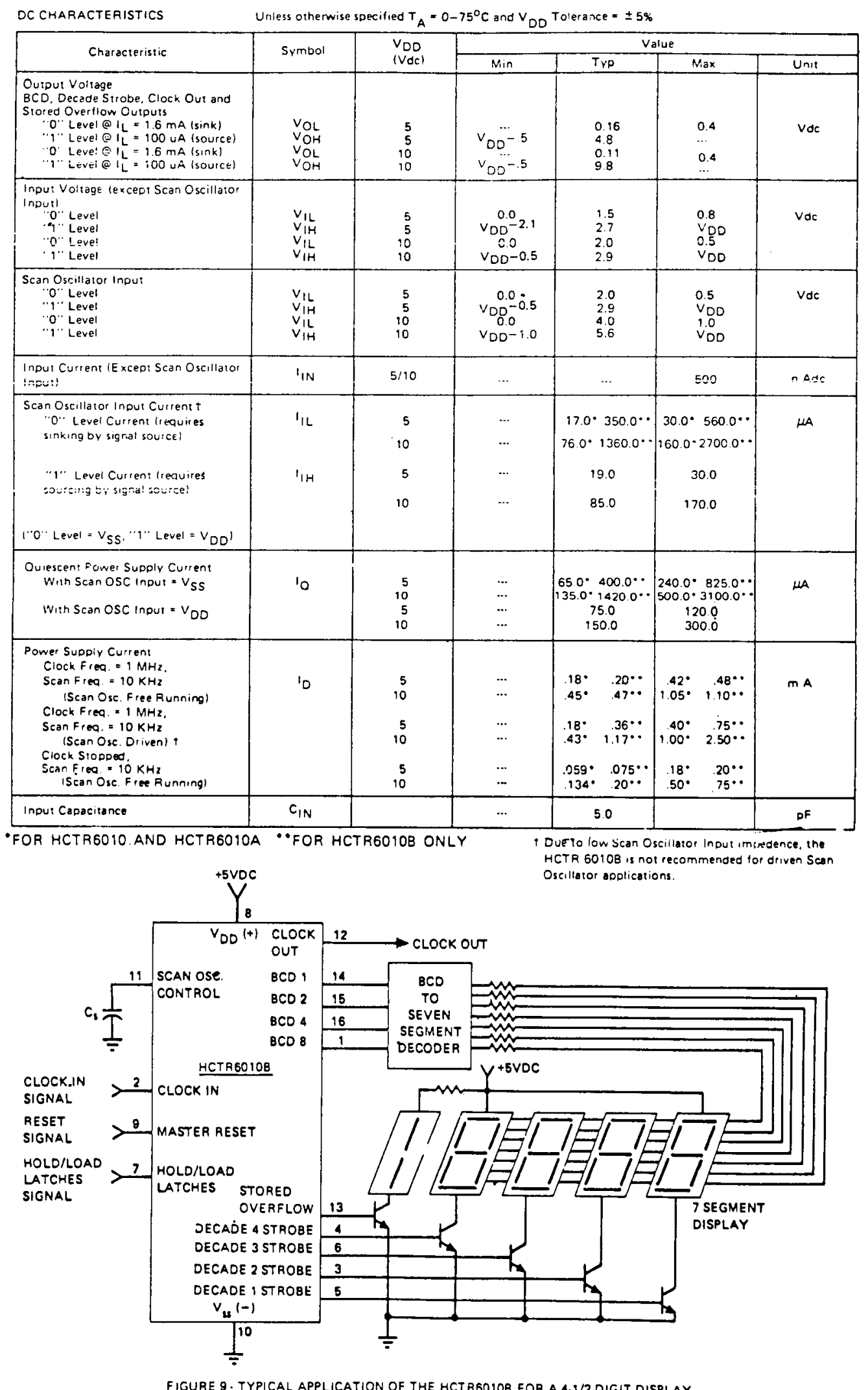

FIGURE 9. TYPICAL APPLICATION OF THE HCTR6O10B FOR A 4.9/2 DIGIT DISPLAY 
AC CHARACTERISTICS Unless Othervise sDecified $T_{A}=0-75^{\circ} \mathrm{C}$ and $V_{\text {DD }}$ Tolerance $= \pm 5 \%$

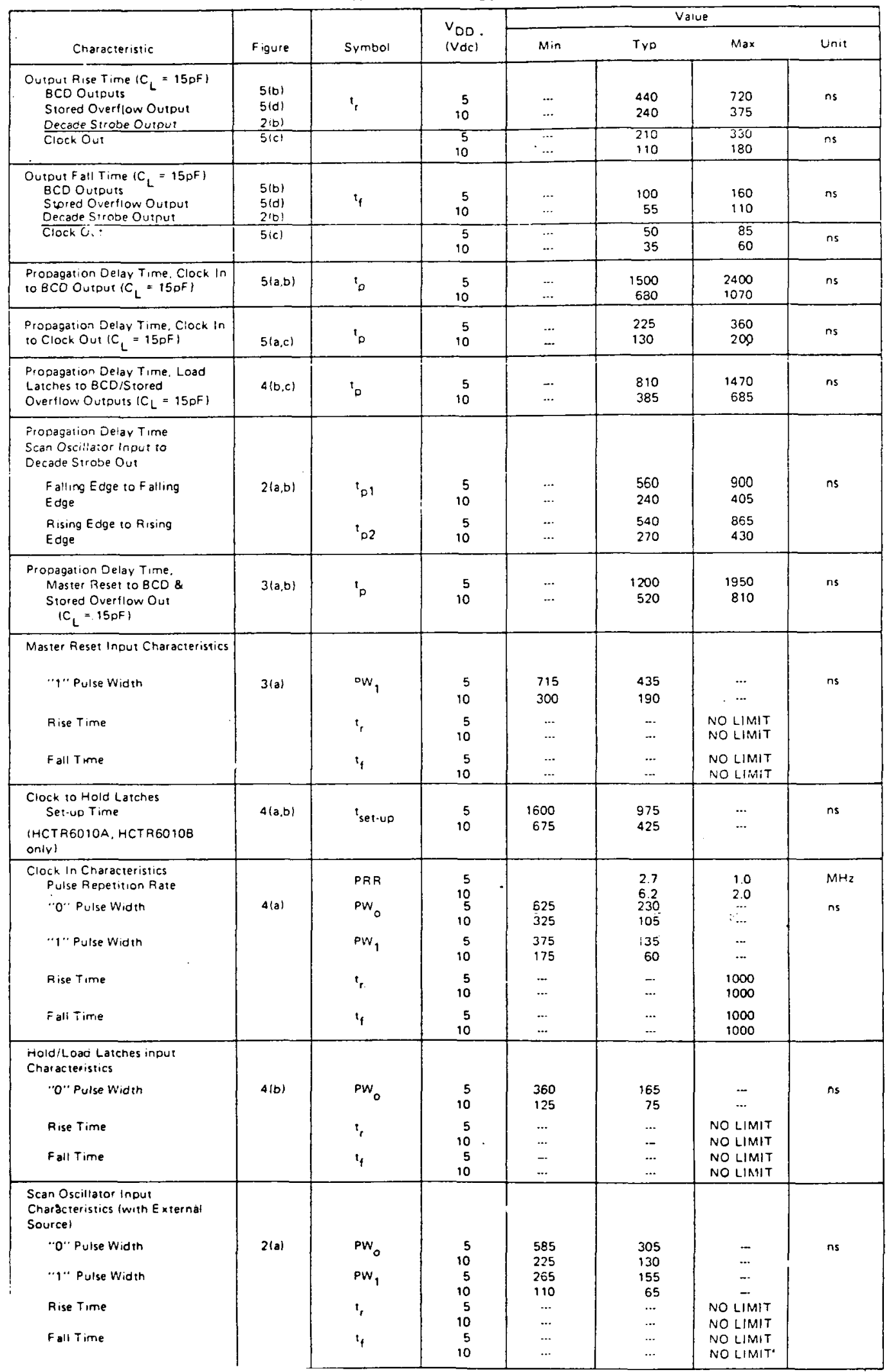

Reproduced with permission from Hughes Aircraft Company, New Port Beach, CA 92663. 

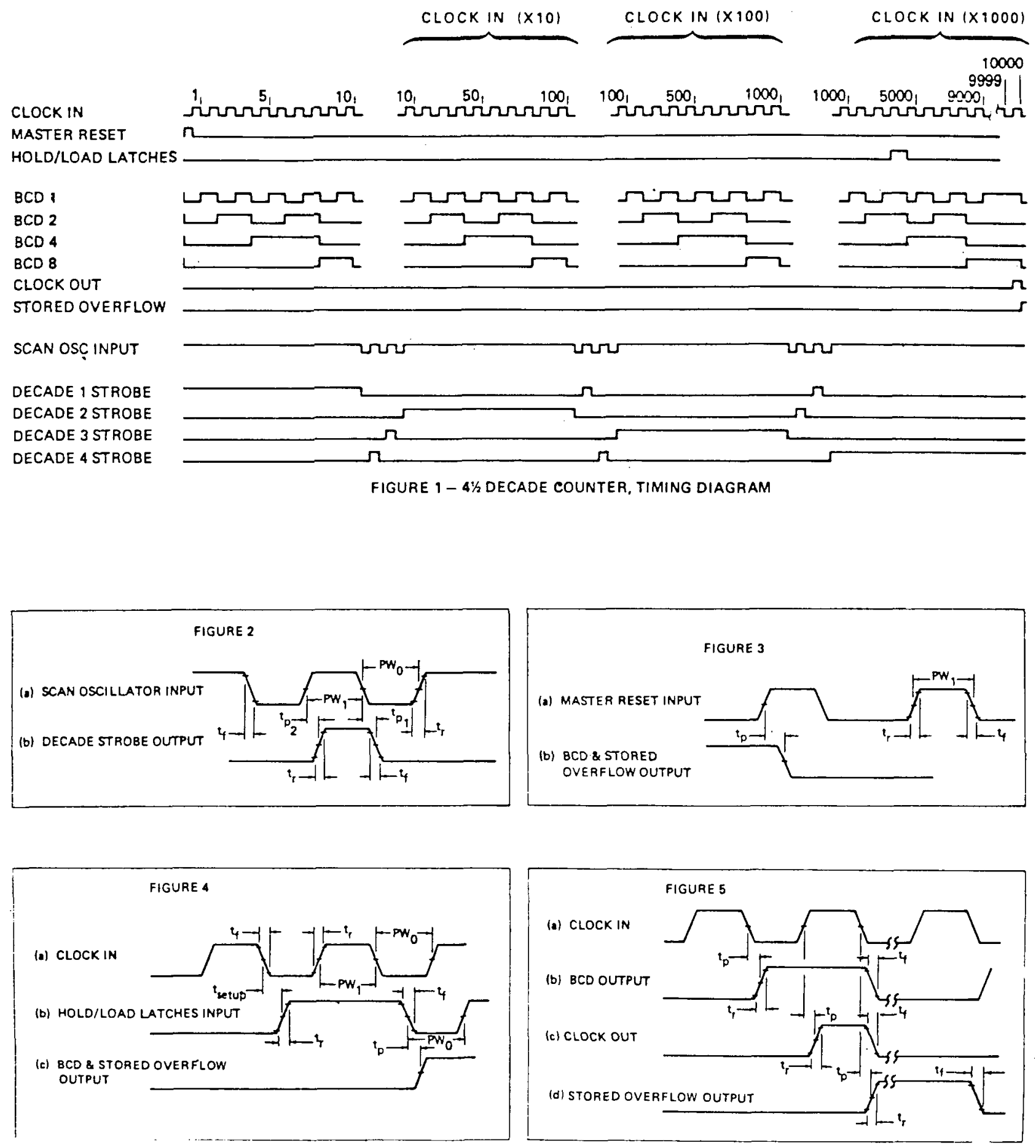

Reproduced with permission from Hughes Aircraft Company, New Port Beach, CA 92663. 


\section{SCAN OSCILLATOR CHARACTERISTICS}

All three devices in the 6010 series have on-chip scan oscillators; however. they are diflerent in some characteristics. All devices are designed to permit adjustment of scan oscillator frequency by adjusting the value of a capacitor connected between the scan oscillator input and $A C$ ground $\left(V_{00}\right.$ or $V_{S S}$ ) as shown in figure 6(a). The nominal duty cycle of each decade strobe output for this configuration is approximately $23 \%$ for the HCTR6010 and HCTR6010B and approximately $12 \%$ for the HCTR6010A. Typical frequency versus capacitance characteristics are given in figures

7 (b) and $7(\mathrm{c})$. The HCTR6010A is designed to permit adjustment of frequency and duty cycle using an external RC circuit connected to the scan oscillator input as shown in figures $6(b)$ and 6 (c). If only the capacitor is connected the duty cycle is approximately $12 \%$. The duty cycle can be increased or decreased by connecting a resistor to $V_{D O}$ or $V_{S S}$ respectively as shown in Figure $6(b)$ and $6(c)$. Typical scan oscillator operating characteristics and waveform definitions are given in Figures $7(a)-7(d)$.

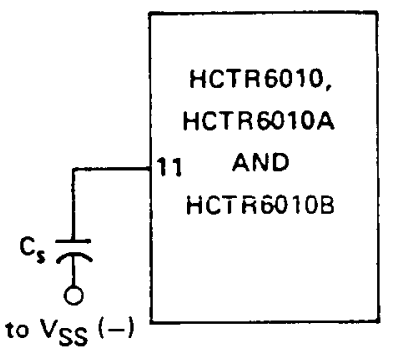

or $V_{D D} 1+1$

(a)

SCAN OSCILLATOR FREQUENCYADJUSTMENT CONFIGURATION

$v_{D D}{ }^{1+1}$

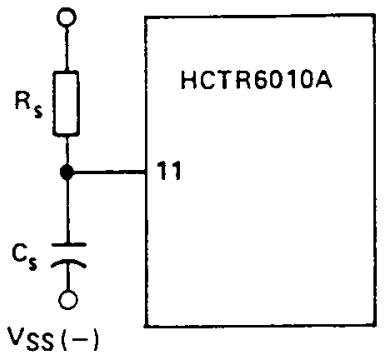

(b)

INCREASED DUTY CYCLE CONFIGURATION

FIGURE 6

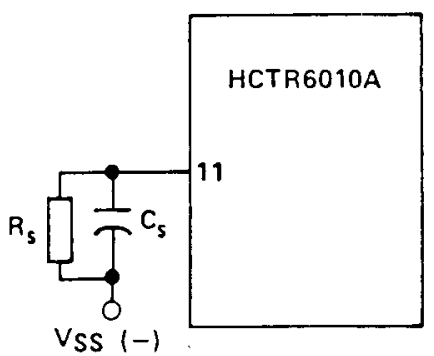

(c)

DECREASED DUTY CYCLE CONFIGURATION

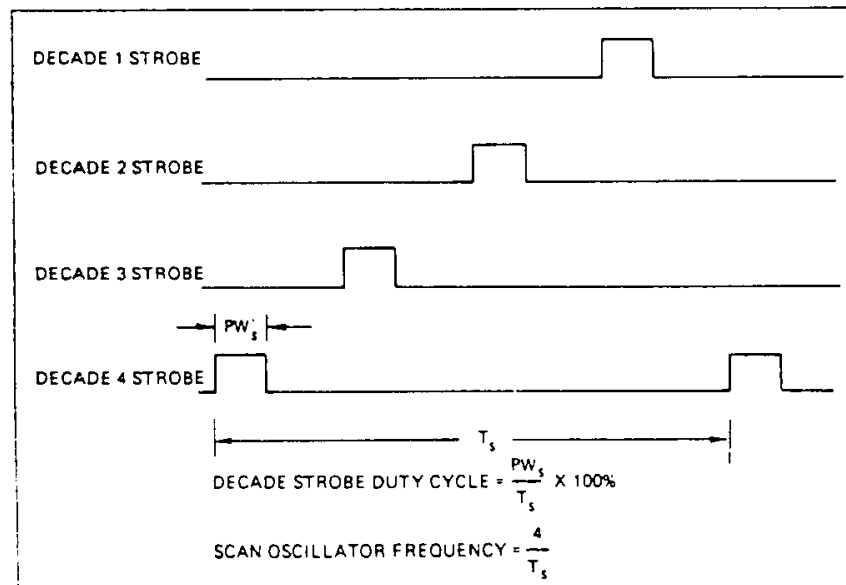

FIGURE ?(a) - DEFINITION OF SCAN OSCILLATOR FREOUENCY AND DECADE STROBE DUTY CYCLE

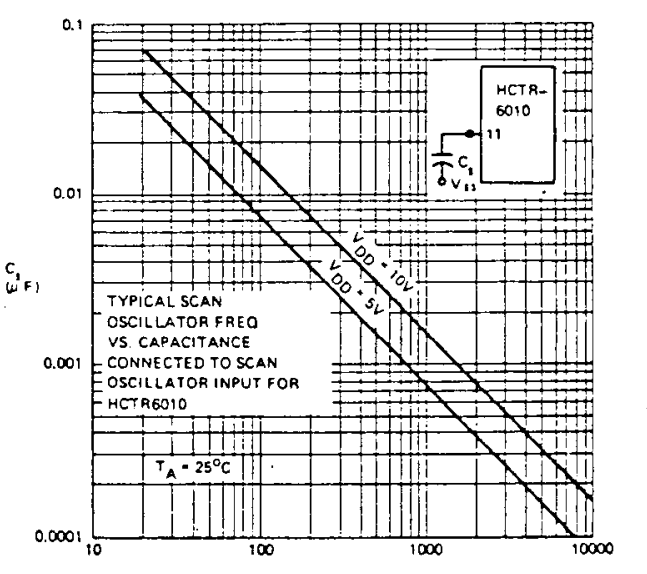

SCANOSCILLATOR FREOUENCY (HZ)

7101. TYPICAL HCT RGOIO SCAN OSCILLATDR FAEQUENCY CHARACTERISTICS
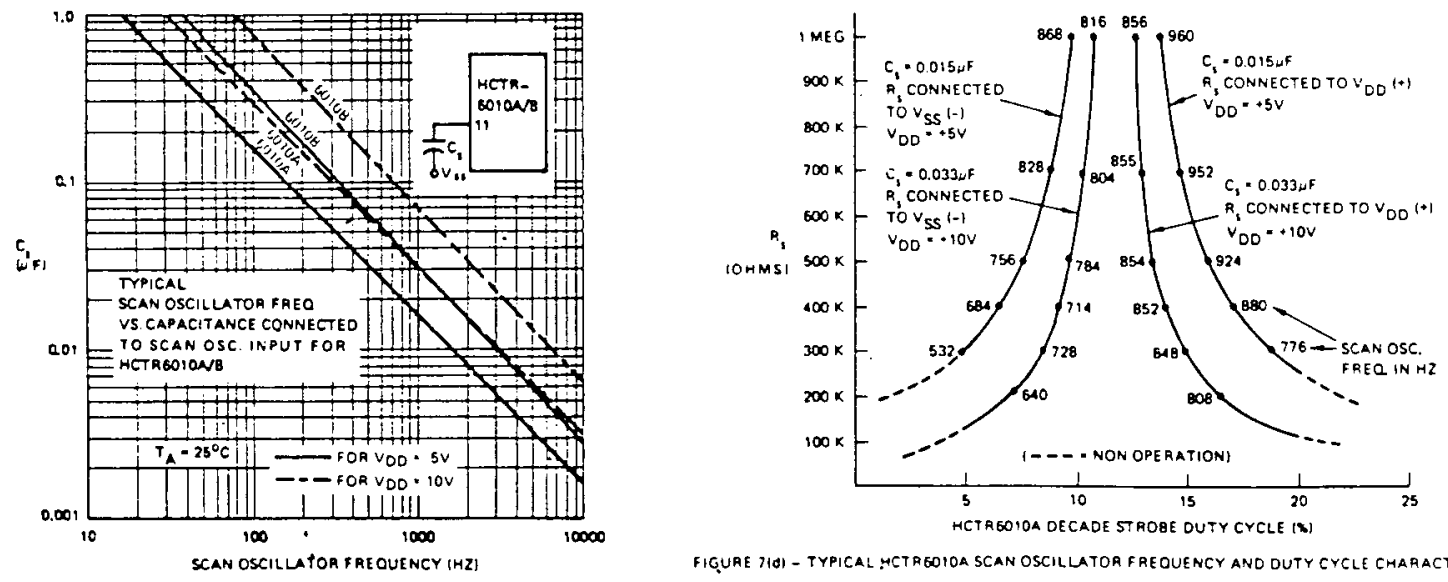

M(C) . TYPICAL MCTRGOIONE SCAN OSCILLATOR FREOUENCY CHAMACTERISTICS 


\section{GENERAL OPERATING CHARACTERISTICS}

As shown in the expanded block diagram of Figure 8 , the clock input frequency is counted by a series of four synchronous $B C D$ counters and a divide by two stage. The counter is advanced by the negative going transition of the clock input signal.

The BCD counter data is stored by loading the data into the deceje iacilies and the stored overfiow latch. A zero level on the Hold/Load Latches input will transfer data from the counter to the latches. The count is held in the latches when the Hold/Load Latches input is in a high state.

The BCD data from the decade latches is presented sequentially at the $B C D$ outputs by using time division multiplexing. The multiplex sequence is from the most significant decade to the least significant decade. A positive true output signal appears at each of the four Decade Strobe outputs 10 indicate which decade's BCD data is present at the BCD outputs. The latched overflow information is not multiplexed but connected to the Stored Overflow output through an output buffer stage.

Timinc sionals for the multiplexer and Decade Strobe signals are generated by an on chip scan oscillato and scan counter/de- coder. By connecting a resistor and capacitor to the Scan Oscillator control terminal, it is possible to control the duty cycle and frequency of the decade strobe outputs so that output characteristics can be made suitable for several different types of displays. The Scan Oscillator input may be driven by an external source if desired. The Scan Counter changes on the negative going lransition of the Scan Oscillator input.

A delay circuit on the Hold/Load latches input is present in the HCTR6010 bot not in the HCTR6010A and HCTR6010B. The delay is long enough to ensure that a count "rippling" through the counter will be stabilized and correctly loaded into the latches even if load latches and clock advance signals are applied simultaneously.

The clock out signai may be used for cascading devices by connecting it to the clock in of the following device. The clock out signal is a positive going pulse with leading edge coincident with the positive going transition of the clock input following count 9999. The trailing edge is coineident with the 1 - 0 transition of the clock iriput which drives the counter into state 10000.

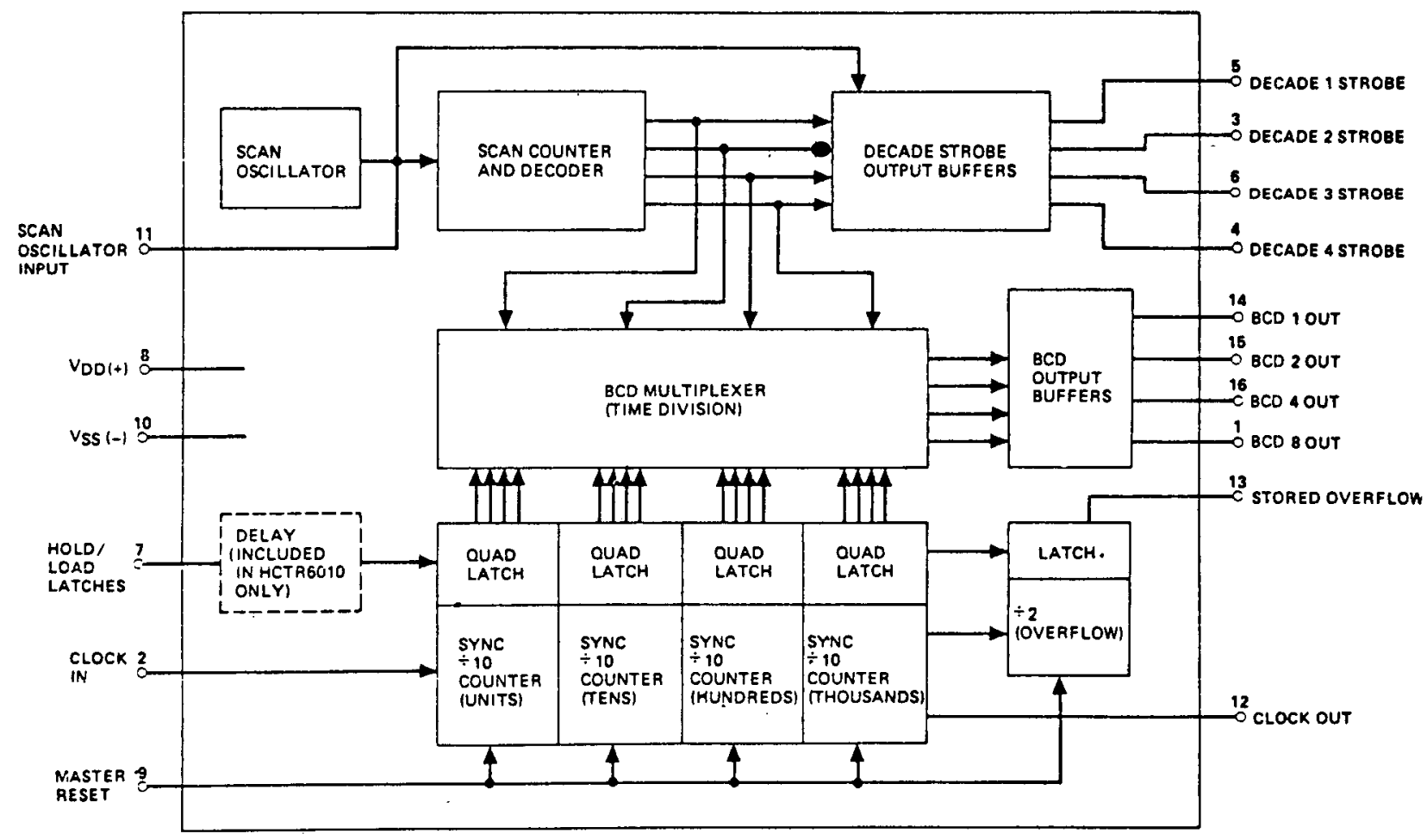

FIGURE B - EXPANDED BLOCK DIAGRAM OF HCTR6010/A/B

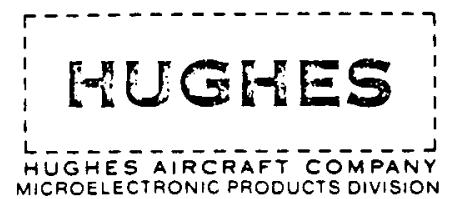

information furnished by Hughes is believed to be accurate and reliable. However, no responsibility is assumed by, Hughes for its use: nor for any infringements or patents or other rights of third parties which may result from its use. No license is granted by implication or otherwise under any parent or patent rights of Hughes. 


\section{(A) motorola}

\section{BIT RATE GENERATOR}

The MC14411 bit rate generator is constructed with comple. mentary MOS enhancement mode devices. It utitizes a frequency divider network to provide a wide range of output frequencies.

A crystal controlled oscillator is the clock source for the network. A two bit address is provided to select one of four multiple output clock rates.

Applications include a selectable frequency source for equipment in the data communications market, such as teleprinters, printers, CRT terminals, and microprocessor systems.

- Single 5.0 Vda (: $5 \%$ ) Power Supply

- Internal Oscillator Crystal Controlled for Stabitity (1.8432 MHz)

- Sixteen Different Output Clock Rates

- $50 \%$ Output Dutr Crcle

- Programmable Time Bases for One of Four Multiple Outpur Rates

- Buffered Outputs Compatible with Low Power TTL

- Noise Immunity $=45 \%$ of $V_{D D}$ Typical

- Diode Protection on All Inputs

- External Clock May be Applied to Pin 21

MAXIMUM RATINGS iVolioges ieferenced to VSS. Pin 12. .)

\begin{tabular}{|c|c|c|c|}
\hline Raring & Symbol & value & Unit \\
\hline DC Suppir Voltage Range & $v_{00}$ & 5.25 to- 0.5 & $v a c$ \\
\hline input Voltage. Ali inpuls & $v_{\text {in }}$ & $\begin{array}{c}V_{O D}+0.5 \text { to } \\
V_{S S}-0.5\end{array}$ & $v d c$ \\
\hline $0 \subset C_{1}$, ent Drain per P.n & 1 & 10 & mAdc \\
\hline Ouerating Temperature Range & $T_{A}$ & $-4010 \cdot 85$ & ${ }^{\circ} \mathrm{C}$ \\
\hline S.c.age Temes'alure Range & $T_{s 19}$ & $-65 t 0 \cdot 150$ & ${ }^{\circ} \mathrm{C}$ \\
\hline
\end{tabular}

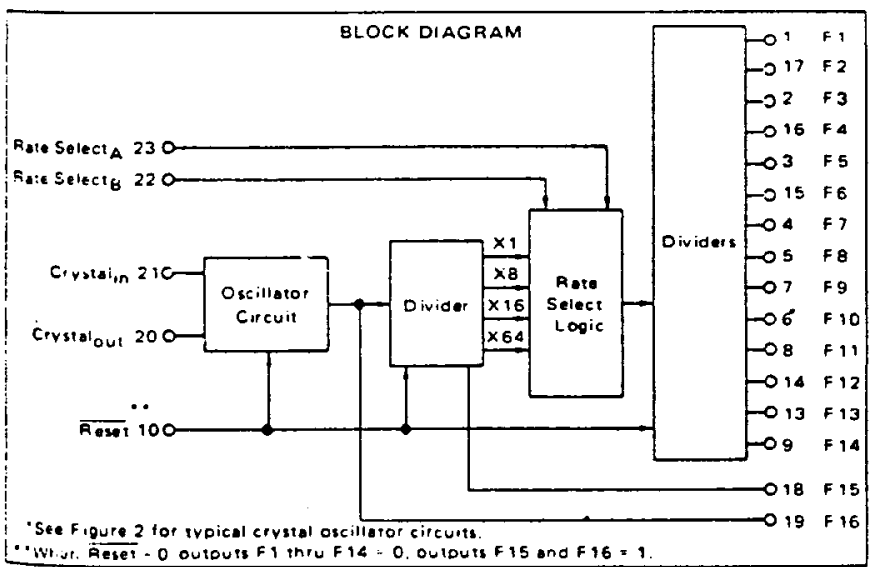

MC14411
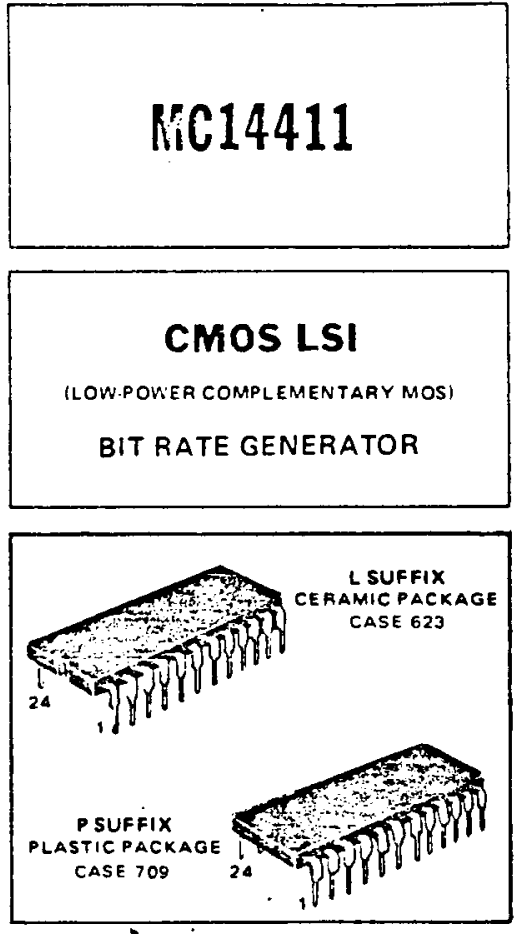

PIN ASSIGNMENT

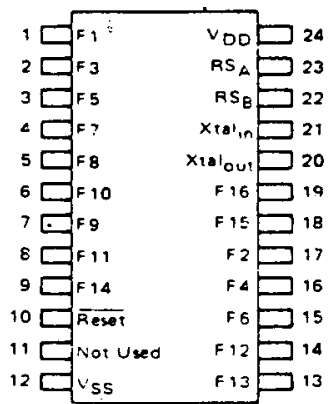

$V_{O D}=P_{\text {in }} 24$

$V_{S S}=P$ in 12

This device contains circuitry to protect the inpuis against damage due :c high static voltages or electric fields, however, it is acvised that normal precaulions be laken 10 avold. afplication of any vol:age highet than maximum raied voltages to this hizh impecance circuli, for prope: operation it is recommended that $v$ in ano $v_{\text {our }}$ be constrained io ine ion $v^{\prime}$ and be

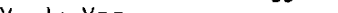
$v_{\text {out }}$ ! $=v_{D D}$

Unused inputs must always be tied to an aDP.ophate agic voltage leve le $a$ enther $\checkmark S S$ or $V D D$ 
MC14411

ELECTRICAL CHARACTERISTICS

\begin{tabular}{|c|c|c|c|c|c|c|c|c|c|c|}
\hline \multirow[b]{2}{*}{ Cheracteristic } & \multirow[b]{2}{*}{ Symbol } & \multirow{2}{*}{$\begin{array}{l}\text { VDD } \\
\text { Voc }\end{array}$} & \multicolumn{2}{|c|}{$-40^{\circ} \mathrm{C}$} & \multicolumn{3}{|c|}{$25^{\circ} \mathrm{C}$} & \multicolumn{2}{|c|}{$+85^{\circ} \mathrm{C}$} & \multirow[b]{2}{*}{ Unit } \\
\hline & & & $\operatorname{Min}$ & Max & $\operatorname{Min}$ & TYp & Max & $\operatorname{Min}$ & Max & \\
\hline Supply Voitage & $\overline{V_{D D}}$ & - & 4.75 & 5.25 & 4.75 & 5.0 & 5.25 & 4.75 & 5.25 & Vde \\
\hline \multirow[t]{2}{*}{ Oulput Voltege } & \multirow[t]{2}{*}{$\bar{v}_{\text {out }}$} & 5.0 & - & 0.05 & $=$ & 0 & 0.05 & - & 0.05 & Voc \\
\hline & & 5.0 & 4.95 & - & 4.95 & 5.0 & - & 4.95 & - & Voc \\
\hline $\begin{array}{l}\text { Input Vaitage } \\
\left(V_{O}=4.5 \text { or } c .5 \mathrm{Vdc}\right)\end{array}$ & $v_{I L}$ & 5.0 & - & 1.5 & - & 2.25 & 1.5 & - & 1.5 & $\overline{V d c}$ \\
\hline$\left(V_{0}=0.5\right.$ or $\left.4.5 \mathrm{Vdc}\right)$ & $v_{1 H}$ & 5.0 & 3.5 & - & 3.5 & 2.75 & - & 3.5 & - & Voc \\
\hline $\begin{array}{l}\text { Output Drive Current } \\
\qquad\left(\mathrm{V}_{\mathrm{OH}}=2.5 \mathrm{Vdc}\right)\end{array}$ & TOH & 5.0 & -0.23 & - & -0.20 & -1.7 & - & -0.16 & $=$ & madc \\
\hline$\left(\mathrm{V}_{\mathrm{O}_{L}}=0.1 \mathrm{Vds}\right)$ & $1 \mathrm{OL}$ & 5.0 & 0.23 & $\dot{-}$ & 0.20 & 0.78 & - & 0.16 & - & FACC \\
\hline Inputcurtent & Tin & $=$ & $=$ & 20.8 & $=$ & $=0.00001$ & $=0.1$ & $=$ & $=1.0$ & HAdc \\
\hline $\begin{array}{c}\text { Tnput Capaciance } \\
\left(V_{\text {in }}=0\right)\end{array}$ & $c_{\text {in }}$ & $=$ & - & $=$ & $\overline{-1}$ & 5.0 & - & $\overline{-}$ & - & pF \\
\hline Qulescent Dissipation & $P_{O}$ & 5.0 & - & 2.5 & $=$ & 0.015 & 2.5 & - & 15 & $m W$ \\
\hline $\begin{array}{l}\text { Power Dissipation " } T \\
\text { (Oynamic plus Quiescent) } \\
\left(C_{L}=15 \rho f\right)\end{array}$ & PD & 5.0 & & & $P_{D}=17$ & $5 \mathrm{~mW} / \mathrm{MH}$ & $T+P_{O}$ & & & $m W$ \\
\hline $\begin{array}{l}\text { Cutput Rise Timest } \\
\text { tTLH }=(3.0 \mathrm{~ns} / \mathrm{pF}) \mathrm{C}_{\mathrm{L}}+25 \mathrm{~ns}\end{array}$ & TLH & 5.0 & $=$ & - & - & 70 & 200 & - & - & $n s$ \\
\hline $\begin{array}{l}\text { OUtDut Fail Time' } \\
: T H L=(1.5 \mathrm{~ns} / \mathrm{pF}) \mathrm{C}_{L}+47 \mathrm{~ns}\end{array}$ & TTHL & 5.0 & $=$ & - & $=$ & 70 & 200 & $=$ & $\overline{-}$ & ns \\
\hline Thput Clock Frequency & ${ }^{\mathrm{C} L}$ & 50 & - & 1.85 & $=$ & $=$ & 1.85 & $=$ & 1.85 & $\mathrm{MHz}$ \\
\hline
\end{tabular}

\begin{tabular}{|c|c|c|c|c|}
\hline & & Rate Select & \multirow[t]{2}{*}{ Rate } & \\
\hline & $\vec{B}$ & A & & \\
\hline & c & 0 & $x 1$ & \\
\hline & c & 1 & $\times 8$ & \\
\hline & 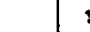 & 0 & $\times 16$ & \\
\hline & 1 & 1 & $\times 64$ & \\
\hline \multirow{2}{*}{$\begin{array}{l}\text { Output } \\
\text { Number }\end{array}$} & \multicolumn{4}{|c|}{ Output Rates $(\mathrm{Hz})$} \\
\hline & $\times 64$ & $\times 16$ & $x 8$ & $x_{1}$ \\
\hline$F 1$ & $614.4 k$ & $153.6 \mathrm{k}$ & $76.8 k$ & 9600 \\
\hline$F 2$ & $460.8 \mathrm{k}$ & $115.2 k$ & $57.6 \mathrm{k}$ & 7200 \\
\hline F 3 & $307.2 k$ & $76.8 k$ & $38.4 k$ & 4800 \\
\hline$F_{4}$ & $230.4 k$ & $57.6 k$ & $28.8 k$ & 3600 \\
\hline F5 & $153.6 \mathrm{k}$ & $38.4 k$ & $19.2 k$ & 2400 \\
\hline F6 & $115.2 k$ & $28.8 k$ & $14.4 k$ & 1800 \\
\hline F7 & $76.8 k$ & $19.2 k$ & 9600 & 1200 \\
\hline F 8 & $38.4 k$ & 5600 & 4800 & 600 \\
\hline F9 & $19.2 \mathrm{k}$ & 4800 & 2400 & 300 \\
\hline F 10 & $12.8 k$ & 3200 & 1600 & 200 \\
\hline FII & 9600 & 2400 & 1200 & 150 \\
\hline F 92 & 8613.2 & 2153.3 & $1076.6^{\circ}$ & 134.5 \\
\hline F13 & 7035.5 & 1758.8 & 8794 & 109.9 \\
\hline F 14 & 4800 & 1200 & 600 & 75 \\
\hline F 15 & $921.6 \mathrm{k}$ & $921.6 \mathrm{k}$ & $921.6 \mathrm{k}$ & $921.6 \mathrm{k}$ \\
\hline$F 16^{*}$ & $1.843 M$ & $1.843 \mathrm{M}$ & $1.843 \mathrm{M}$ & $1.843 M$ \\
\hline
\end{tabular}

Reproduced with permission from Motorola, Inc., Phoenix, AZ 85008. 
FIGURE 1 - DYNAMIC SIGNAL WAVEFORMS

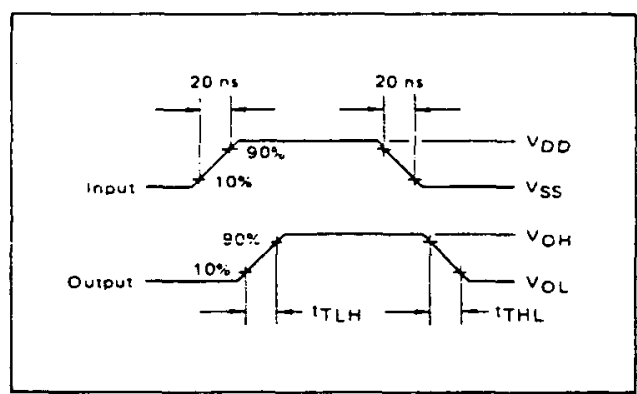

FIGURE 2 - TYPICAL CRYSTAL OSCILLATOR CIRCUIT

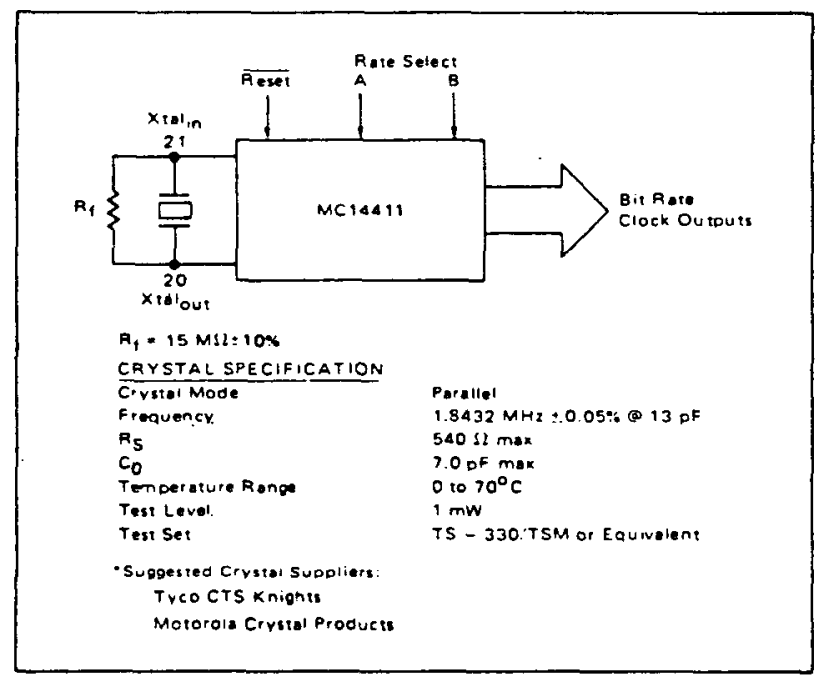

Circuit diegrams utilizang Motorcle products ore included as a means

of illustreting typics iemicanductor epolications: consequenuly,

complete intormetion sufticient for construction purdoses is nor

is believed to be entrety celisble. However. no responsibility is

necossority given. The information has deen carefulty checked and 


\section{(AA) nOTOFOEA}

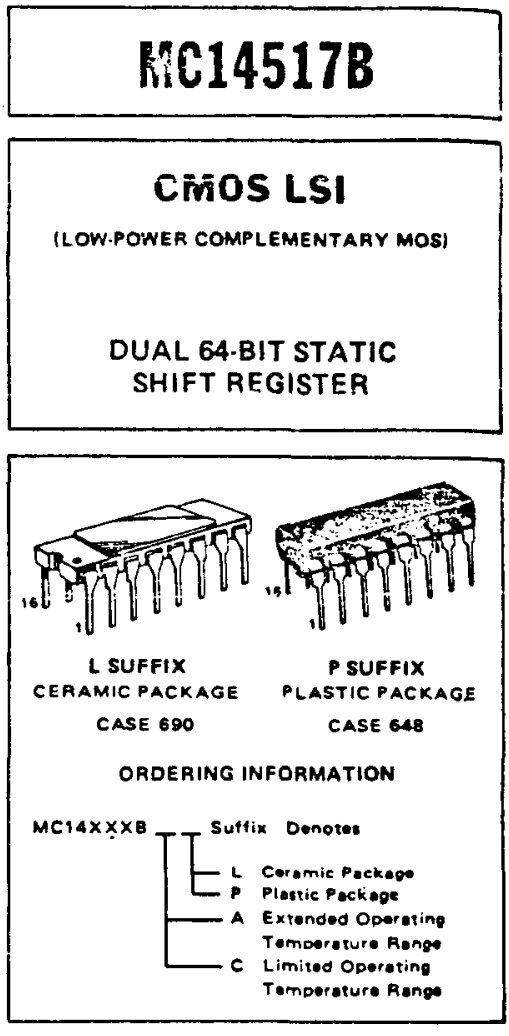

\section{DUAL 64-BIT STATIC SHIFT REGISTER}

The MC14517B dual 64-bit static shift register consists of two identical, independent, 64.bit registers. Each registcr has separate clock and write enable inputs, as well as outputs at bits $16,32,48$, and 64: Data at the data input is entered by clocking, regardiess of the state of the write enable input. An output is disabled lopen circuited) when the write enable input is high. During this time. data appearing at the data input as well as the 16.bit, 32-bit, and 48-bit taps may be entered into the device by application of a clock pulse. This feature permits the register to be loaded with 64 bits in 16 clock periods, and also permits bus logic to be used. This device is useful in time delay eircuits, temporary memory storage circuits, and other serial shift register applications.

- Quiescent Current $=10 \mathrm{nA} /$ package typical @ 5 Vdc

- Noise Immunity $=45 \%$ of $V^{\prime} D D$ typical

- Diode Protection on All Inputs

- Fully Static Operation

- Ouput Transitions Occur on the Rising Edge of the Clock Pulse

- 6.7 MHz Operation @ VDD $=10 \mathrm{Vdc}$

- Exceedingly Slow Input Transition Rates May Be Applied to the Clock input

- 3-State Output at 64 th.Bit Allows Use in Bus Logic Applications

- Shift Registers of any Length mày be Fully Loaded with 16 Clock Pulses

- Supply Voltage Range $=3.0 \mathrm{Vdc}$ to $18 \mathrm{Vdc}$

- Capable of Driving Two Low-power TTL Loads, One Low-power Schotiky TTL Load or Two HTL Loads Over the Rated Temper. ature Range

\begin{tabular}{|c|c|c|c|}
\hline Rating & Symbol & Volue & Unit \\
\hline DC Supp!y Voltage & $v_{D O}$ & -0.5 to +18 & $V d c$ \\
\hline input Voltsge. All Inputs & $v_{\text {in }}$ & -0.5 to $V_{D D}+0.5$ & Vde \\
\hline DC Current Drain per Pin & 1 & 10 & madc \\
\hline $\begin{array}{r}\text { Operating Temperature Aange - AL Device } \\
\text { CL/CP Device }\end{array}$ & $T_{A}$ & $\begin{array}{l}-5510+125 \\
-4010+85\end{array}$ & ${ }^{\circ} \mathrm{C}$ \\
\hline Storage Temperature Range & $T_{\text {stg }}$ & -65 to +150 & ${ }^{\circ} \mathrm{C}$ \\
\hline
\end{tabular}

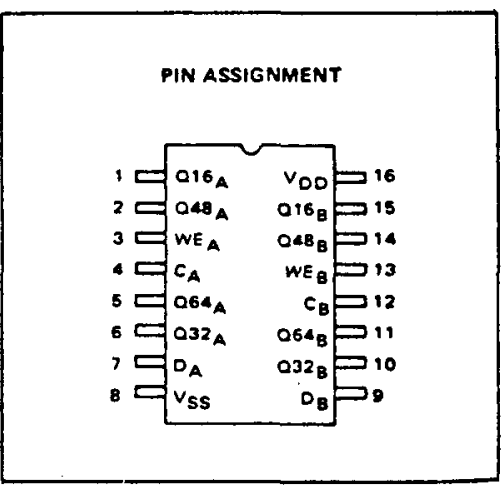

\begin{tabular}{|c|c|c|c|c|c|c|c|}
\hline \multirow{9}{*}{$\begin{array}{l}\text { FUNCTIONAL } \\
\text { TRUTH TABLE }\end{array}$} & CLOCK & $\begin{array}{c}\text { WRITE } \\
\text { ENABLE }\end{array}$ & DATA & 16-BIT TAP & 32.BIT TAP & 48.BIT TAP & 64.BIT TAP \\
\hline & 0 & 0 & $\cdot x$ & $\begin{array}{c}\text { Content of 16-Bit } \\
\text { Displeyed }\end{array}$ & $\begin{array}{c}\text { Content of 32-Bis } \\
\text { Displeyed }\end{array}$ & $\begin{array}{c}\text { Content of } 48 \text { - B it } \\
\text { Disolored }\end{array}$ & $\begin{array}{c}\text { Content of Ga.tin } \\
\text { Displored }\end{array}$ \\
\hline & 0 & 1 & $x$ & High Impodence & High Imomesence & High Impodence & High Impodence \\
\hline & 1 & 0 & $x$ & $\begin{array}{c}\text { Content of } 16 \text { - Bis } \\
\text { Displeyed }\end{array}$ & $\begin{array}{c}\text { Content ef } 32 \cdot \text { Bit } \\
\text { Disployed }\end{array}$ & $\begin{array}{c}\text { Content of } 48-B \text { it } \\
\text { Disployed }\end{array}$ & $\begin{array}{c}\text { Content of 64-Bit } \\
\text { Dimployed }\end{array}$ \\
\hline & 1 & 1 & $x$ & High Impedonces & High Impocance & High impodence & High Impedence \\
\hline & & 0 & \begin{tabular}{|l} 
Detuentered \\
into $18 t$ Bit \\
\end{tabular} & $\begin{array}{c}\text { Content of 16-Bit } \\
\text { Displaved }\end{array}$ & $\begin{array}{c}\text { Content cf 32-Bit } \\
\text { Displayed }\end{array}$ & $\begin{array}{c}\text { Conteni of } 48 \cdot B \text { it } \\
\text { Disolored }\end{array}$ & $\begin{array}{l}\text { Content of Ba-8it } \\
\text { Disolentid }\end{array}$ \\
\hline & & 1 & $\begin{array}{l}\text { Dote ontored } \\
\text { inta lor Bit }\end{array}$ & $\begin{array}{c}\text { Dete ot top } \\
\text { onvered into } 17 \text { - } 8 \text { it }\end{array}$ & \begin{tabular}{|c|} 
Dete st vop \\
entered into $33-8 \mathrm{it}$ \\
\end{tabular} & $\begin{array}{c}\text { Dote st 100 } \\
\text { entored inte } 49.8 \mathrm{sit}\end{array}$ & High Impedence \\
\hline & $ح$ & 0 & $x$ & $\begin{array}{c}\text { Content of 16- Bit } \\
\text { Displeyed }\end{array}$ & $\begin{array}{c}\text { Content of 32-Bit } \\
\text { Dieployed }\end{array}$ & $\begin{array}{c}\text { Content of } 4 \theta-B \text { it } \\
\text { Displered }\end{array}$ & $\begin{array}{c}\text { Content of 6a-8ht } \\
\text { Diploves }\end{array}$ \\
\hline & $\tau$ & 1 & $x$ & Migh Impedence & High imoes once & High Imperance & High Impedonea \\
\hline
\end{tabular}


MC14517B

ELECTIICAL CHARACTERISTICS

\begin{tabular}{|c|c|c|c|c|c|c|c|c|c|c|}
\hline \multirow[b]{2}{*}{ Charecteristic } & \multirow{3}{*}{$\frac{\text { Srmbol }}{V_{O L}}$} & \multirow{2}{*}{$\begin{array}{l}\text { VoD } \\
\text { Vdc }\end{array}$} & \multicolumn{2}{|c|}{$T_{\text {low }}$} & \multicolumn{3}{|c|}{$25^{\circ} \mathrm{C}$} & \multicolumn{2}{|c|}{$T_{\text {high }}{ }^{\circ}$} & \multirow[b]{2}{*}{ Unit } \\
\hline & & & Min & Max & Min & TYP & Max & Min & Max & \\
\hline \multirow{2}{*}{$\begin{array}{ll}\text { Outpul Voliage } & " 0 " \text { Level } \\
v_{\text {in }} & =V_{D D} \text { or } 0 \\
v_{\text {in }}=0 \text { or } V_{D D} & \end{array}$} & & $\begin{array}{l}5.0 \\
10 \\
15\end{array}$ & $\begin{array}{l}- \\
- \\
-\end{array}$ & $\begin{array}{l}0.05 \\
0.05 \\
0.05\end{array}$ & $\begin{array}{l}- \\
- \\
-\end{array}$ & $\begin{array}{l}0 \\
0 \\
0\end{array}$ & $\begin{array}{l}0.05 \\
0.05 \\
0.05 \\
\end{array}$ & $\overline{-}$ & $\begin{array}{l}0.05 \\
0.05 \\
0.05\end{array}$ & Voc \\
\hline & $\mathrm{VOH}_{\mathrm{OH}}$ & $\begin{array}{l}5.0 \\
10 \\
15 \\
\end{array}$ & $\begin{array}{r}4.95 \\
9.95 \\
14.95 \\
\end{array}$ & $\begin{array}{l}- \\
- \\
-\end{array}$ & $\begin{array}{r}4.95 \\
9.95 \\
14.95 \\
\end{array}$ & $\begin{array}{l}5.0 \\
10 \\
15 \\
\end{array}$ & - & $\begin{array}{r}4.95 \\
3.95 \\
14.95 \\
\end{array}$ & $\begin{array}{l}- \\
- \\
\end{array}$ & Vde \\
\hline \multirow{2}{*}{$\begin{array}{l}\text { Indut Voltage } \\
\left(V_{O}=4.5 \text { or } 0.5 \mathrm{Vdcl}\right. \\
\left(V_{0}=9.0 \text { or } 1.0 \mathrm{Vdc}\right) \\
\left(V_{O}=13.5 \text { or } 1.5 \mathrm{Vdc}\right) \\
\left(V_{O}=0.5 \text { or } 4.5 \mathrm{Vdc}\right)\end{array}$} & $V_{I L}$ & $\begin{array}{l}5.0 \\
10 \\
15 \\
\end{array}$ & $\begin{array}{l}- \\
-\end{array}$ & $\begin{array}{l}1.5 \\
3.0 \\
4.0 \\
\end{array}$ & $\begin{array}{l}- \\
- \\
-\end{array}$ & $\begin{array}{l}2.25 \\
4.50 \\
6.75 \\
\end{array}$ & $\begin{array}{l}1.5 \\
3.0 \\
4.0\end{array}$ & $\begin{array}{l}- \\
- \\
-\end{array}$ & $\begin{array}{l}1.5 \\
3.0 \\
4.0\end{array}$ & Vode \\
\hline & $V_{1 H}$ & $\begin{array}{l}5.0 \\
10 \\
15 \\
\end{array}$ & $\begin{array}{r}3.5 \\
7.0 \\
11.0 \\
\end{array}$ & $\begin{array}{l}- \\
- \\
-\end{array}$ & $\begin{array}{r}3.5 \\
7.0 \\
11.0 \\
\end{array}$ & $\begin{array}{l}2.75 \\
5.50 \\
8.25 \\
\end{array}$ & $\begin{array}{l}\overline{-} \\
\overline{-}\end{array}$ & $\begin{array}{r}3.5 \\
7.0 \\
11.0 \\
\end{array}$ & $\begin{array}{l}- \\
- \\
\end{array}$ & vole \\
\hline $\begin{array}{l}\text { Output Drive Current (AL Device) } \\
\text { (VOH }-2.5 \mathrm{Vde} \text { ) Source }\end{array}$ & TOH & $\begin{array}{l}5.0 \\
5.0 \\
10 \\
15 \\
\end{array}$ & $\begin{array}{l}-1.2 \\
-0.25 \\
-0.62 \\
-1.8 \\
\end{array}$ & $\begin{array}{l}- \\
- \\
-\end{array}$ & $\begin{array}{l}-1.0 \\
-0.2 \\
-0.5 \\
-1.5 \\
\end{array}$ & $\begin{array}{l}-1.7 \\
-0.36 \\
-0.9 \\
-3.5 \\
\end{array}$ & $\begin{array}{l}- \\
- \\
-\end{array}$ & $\begin{array}{l}-0.7 \\
-0.14 \\
-0.35 \\
-1.1 \\
\end{array}$ & $\begin{array}{l}- \\
- \\
- \\
\end{array}$ & mAdc \\
\hline $\begin{array}{l}\left(v_{\mathrm{OL}}=0.4 \mathrm{Vde}\right) \quad \text { Sink } \\
\left(v_{\mathrm{OL}}=0.5 \mathrm{Vde}\right) \\
\left(v_{\mathrm{OL}}=1.5 \mathrm{vdc}\right)\end{array}$ & IOL & $\begin{array}{l}5.0 \\
10 \\
15 \\
\end{array}$ & $\begin{array}{l}0.64 \\
1.6 \\
4.2 \\
\end{array}$ & $\begin{array}{l}- \\
- \\
-\end{array}$ & $\begin{array}{r}0.51 \\
1.3 \\
3.4 \\
\end{array}$ & $\begin{array}{c}0.88 \\
2.25 \\
8.8 \\
\end{array}$ & $\begin{array}{l}- \\
- \\
\end{array}$ & $\begin{array}{l}0.36 \\
0.9 \\
2.4 \\
\end{array}$ & $\begin{array}{l}- \\
- \\
-\end{array}$ & mAdc \\
\hline $\begin{aligned} \text { Output Drive Current }(C L / C P \text { Device }) \\
\left(V_{O H}=2.5 \mathrm{Vdc}\right) \quad \text { Source } \\
\left(V_{O H}=4.6 \mathrm{Vdc}\right) \\
-\left(V_{O H}=9.5 \mathrm{Vdc}\right) \\
\left(V_{O H}=13.5 \mathrm{Vdcl}\right.\end{aligned}$ & $\mathrm{TOH}$ & $\begin{array}{l}5.0 \\
5.0 \\
10 \\
15 \\
\end{array}$ & $\begin{array}{l}-1.0 \\
-0.2 \\
-0.5 \\
-1.4 \\
\end{array}$ & $\begin{array}{l}- \\
- \\
-\end{array}$ & $\begin{array}{l}-0.8 \\
-0.16 \\
-0.4 \\
-1.2 \\
\end{array}$ & $\begin{array}{l}-1.7 \\
-0.36 \\
-0.9 \\
-3.5\end{array}$ & $\begin{array}{l}- \\
- \\
-\end{array}$ & $\begin{array}{l}-0.6 \\
-0.12 \\
-0.3 \\
-1.0 \\
\end{array}$ & $\begin{array}{l}- \\
- \\
- \\
\end{array}$ & $\overline{\text { mAdc }}$ \\
\hline $\begin{array}{l}\left(\mathrm{V}_{\mathrm{OL}}=0.4 \mathrm{Vdd}\right) \quad \text { Sink } \\
\mid \mathrm{V}_{\mathrm{OL}}=0.5 \mathrm{Vdd} \\
\left(\mathrm{V}_{\mathrm{OL}}=1.5 \mathrm{Vdd}\right)\end{array}$ & 'OL & $\begin{array}{l}5.0 \\
10 \\
15 \\
\end{array}$ & $\begin{array}{c}0.52 \\
1.3 \\
3.6 \\
\end{array}$ & $\begin{array}{l}- \\
- \\
-\end{array}$ & $\begin{array}{c}0.44 \\
1.1 \\
3.0 \\
\end{array}$ & $\begin{array}{l}0.88 \\
2.25 \\
8.8 \\
\end{array}$ & $\begin{array}{l}- \\
- \\
-\end{array}$ & $\begin{array}{l}0.36 \\
0.9 \\
2.4 \\
\end{array}$ & $\begin{array}{l}\overline{-} \\
- \\
-\end{array}$ & mAdc \\
\hline Input Current (AL Device) & $I_{\text {in }}$ & 15 & - & $: 0.1$ & - & \pm 0.00001 & .0 .1 & - & $=1.0$ & $\mu A d c$ \\
\hline Input Current (CL/CP Device) & $I_{\text {in }}$ & 15 & - & 20.3 & - & $=0.00001$ & $: 0.3$ & $=$ & $: 10$ & MAdC \\
\hline $\begin{array}{c}\text { Inpu: Capeeitance } \\
\left(V_{\text {in }}=01\right.\end{array}$ & $c_{\text {in }}$ & - & - & - & - & 5.0 & 7.5 & - & - & pF \\
\hline $\begin{array}{l}\text { Ouresceni Current (AL Device) } \\
\text { (Per Package) }\end{array}$ & 100 & $\begin{array}{l}5.0 \\
10 \\
15 \\
\end{array}$ & $\begin{array}{l}\overline{-} \\
- \\
-\end{array}$ & $\begin{array}{l}5.0 \\
10 \\
20 \\
\end{array}$ & $\begin{array}{l}- \\
- \\
-\end{array}$ & $\begin{array}{l}0.010 \\
0.020 \\
0.030\end{array}$ & $\begin{array}{l}5.0 \\
10 \\
20 \\
\end{array}$ & $\begin{array}{l}- \\
- \\
-\end{array}$ & $\begin{array}{l}150 \\
300 \\
600 \\
\end{array}$ & $\mu A d c$ \\
\hline $\begin{array}{l}\text { Ouiesceni Curreni (CL/CP Device) } \\
\text { (Per Package) }\end{array}$ & 100 & $\begin{array}{l}5.0 \\
10 \\
15 \\
\end{array}$ & $\begin{array}{r}- \\
- \\
-\end{array}$ & $\begin{array}{c}50 \\
100 \\
200 \\
\end{array}$ & $\begin{array}{l}- \\
- \\
-\end{array}$ & $\begin{array}{l}0.010 \\
0.020 \\
0.030\end{array}$ & $\begin{array}{r}50 \\
100 \\
200 \\
\end{array}$ & $\begin{array}{l}- \\
- \\
-\end{array}$ & $\begin{array}{c}375 \\
750 \\
1500 \\
\end{array}$ & LAdC \\
\hline $\begin{array}{l}\text { Total Supply Current } \cdots \dagger \\
\text { Cornamic plus Quiescent, } \\
\text { Per Fackaget } \\
\text { (C }-50 \text { pF on all oulputs, all } \\
\text { butfers switchingl }\end{array}$ & IT & $\begin{array}{l}5.0 \\
10 \\
15\end{array}$ & & & $\begin{array}{l}I_{T}=14 \\
I_{T}=18 \\
T_{T}=19\end{array}$ & $\begin{array}{l}2 \mu \mathrm{A} / \mathrm{KHz} \\
8 \mathrm{MA} / \mathrm{kHz} \\
3.7 \mathrm{HA} / \mathrm{KHz}\end{array}$ & $\begin{array}{l}f+100 \\
+100 \\
+100\end{array}$ & & & MAdC \\
\hline $\begin{array}{l}\text { Three Srate Leakage Current } \\
\text { (AL Device) }\end{array}$ & ITL & 15 & - & 101 & - & $: 0.00001$ & \pm 0.1 & - & \pm 30 & MAdC \\
\hline $\begin{array}{l}\text { Three-Staie Leakage Current } \\
\text { (CL/CP Devicel }\end{array}$ & ITL & 15 & - & \pm 1.0 & - & .0 .00001 & $\geq 1.0$ & - & $\pm 7.5^{\circ}$ & MAdC \\
\hline
\end{tabular}

- T

low $=-55^{\circ} \mathrm{C}$ for AL Device, $40^{\circ} \mathrm{C}$ lor CL/CP Device.

eNoise immunity spetertied for worst-case input combination.

Noise Margin for both " 1 " and " 0 ; level $=1.0 \mathrm{Vac} \min \wp \mathrm{V}_{D D}=5.0 \mathrm{Vdc}$

$2.0 \mathrm{Vdc} \min \odot V_{D D}=10 \mathrm{Vdc}$

$2.5 \mathrm{Vdc} \min Q V_{D D}=15 \mathrm{Vdc}$

Tro calculate totat supoly current at lasds other than $50 \mathrm{DF}$

$I_{T}\left(C_{L}\right)=1 T(50 \mathrm{DF})+4 \times 10^{-3}\left(C_{L}-50\right) \vee D V^{t}$

where: $I_{T}$ is in $\mu A$ lper pock ogel, $C_{L}$ in $D F, V_{D D}$ in Voc, ond $f_{\text {in }} \mathrm{kHz}$ is input frequeney.

- The formulas given are for the irpical characteristics only at $25^{\circ} \mathrm{C}$. 


\section{MC14517B}

\begin{tabular}{|c|c|c|c|c|c|c|}
\hline Choroctorintic & Srmbol & $V_{D D}$ & Min & TYP & Max & Unit \\
\hline $\begin{array}{l}\text { Output Rise Time } \\
\text { tTLH }=(3.0 \mathrm{~ns} / \mathrm{pF}) C_{L} \cdot 30 \mathrm{~ns} \\
T L L H=(1.5 \mathrm{~ns} / \mathrm{pF}) C_{L}+15 \mathrm{~ns} \\
\text { TTLH }=(1.1 \mathrm{~ns} / \mathrm{pF}) C_{L}+10 \mathrm{~ns}\end{array}$ & TLH & $\begin{array}{l}5.0 \\
10 \\
15\end{array}$ & $\overline{-}$ & $\begin{array}{l}180 \\
90 \\
65\end{array}$ & $\begin{array}{l}360 \\
180 \\
130\end{array}$ & $\mathrm{~ms}$ \\
\hline $\begin{array}{l}\text { Output Fall Timo } \\
\text { THL }=(1.5 \mathrm{~ns} / \mathrm{pF}) C_{L}+25 \mathrm{~ns} \\
\text { TTHL }=10.75 \mathrm{~ns} / \mathrm{pF} / C_{L}+12.5 \mathrm{~ns} \\
\text { THHL }=10.55 \mathrm{~ns} / \mathrm{pF} / C_{L}+9.5 \mathrm{~ns}\end{array}$ & TTHL & $\begin{array}{l}5.0 \\
10 \\
15\end{array}$ & $\begin{array}{l}\overline{-} \\
-\end{array}$ & $\begin{array}{l}100 \\
50 \\
40\end{array}$ & $\begin{array}{l}200 \\
100 \\
80\end{array}$ & $n$ \\
\hline 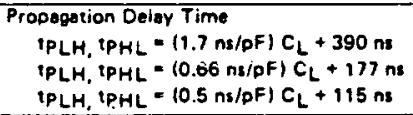 & $\begin{array}{l}\text { TPLH, } \\
\text { TPHLL }\end{array}$ & $\begin{array}{l}5.0 \\
10 \\
15\end{array}$ & $\overline{-}$ & $\begin{array}{l}475 \\
210 \\
140\end{array}$ & $\begin{array}{l}770 \\
300 \\
216\end{array}$ & $m$ \\
\hline Clock Pulse Width & WH & $\begin{array}{l}5.0 \\
10 \\
15\end{array}$ & $\begin{array}{l}330 \\
125 \\
100\end{array}$ & $\begin{array}{c}170 \\
75 \\
60\end{array}$ & $\bar{I}$ & $\overline{n s}$ \\
\hline Clock Pulse Frequency & $f_{\mathrm{cl}}$ & $\begin{array}{l}5.0 \\
10 \\
15\end{array}$ & $\begin{array}{l}- \\
\overline{-}\end{array}$ & $\begin{array}{l}3.0 \\
6.7 \\
8.3\end{array}$ & $\begin{array}{l}1.5 \\
4.0 \\
5.3\end{array}$ & $\mathrm{MHz}$ \\
\hline Clock Pulse Rise ond Fall Time & ITLH, 'THL & $\begin{array}{l}5.0 \\
10 \\
15\end{array}$ & \multicolumn{3}{|c|}{ " Soe Note } & - \\
\hline Date to Clock Setup Timo & isu & $\begin{array}{l}5.0 \\
10 \\
15\end{array}$ & $\begin{array}{c}0 \\
10 \\
15\end{array}$ & $\begin{array}{c}-40 \\
-15 \\
0\end{array}$ & $\bar{z}$ & $n+$ \\
\hline Dats to Clock Hold Time & 4 & $\begin{array}{l}5.0 \\
10 \\
15\end{array}$ & $\begin{array}{l}150 \\
75 \\
35\end{array}$ & $\begin{array}{l}75 \\
25 \\
10\end{array}$ & $\begin{array}{l}- \\
-\end{array}$ & $n$ \\
\hline Write Ensble to Clock Setup Timu & su & $\begin{array}{l}5.0 \\
10 \\
15\end{array}$ & $\begin{array}{l}400 \\
200 \\
110\end{array}$ & $\begin{array}{l}170 \\
65 \\
50\end{array}$ & $\begin{array}{l}\overline{-} \\
\overline{-}\end{array}$ & $\mathrm{ns}$ \\
\hline Write Enabie to Clock Releace Time & $i_{\text {rel }}$ & $\begin{array}{l}5.0 \\
10 \\
15\end{array}$ & $\begin{array}{l}380 \\
180 \\
100\end{array}$ & $\begin{array}{c}160 \\
55 \\
40\end{array}$ & $\begin{array}{l}\bar{z} \\
\overline{-}\end{array}$ & $n$ \\
\hline
\end{tabular}

-The formula given is for the typical characteristics only.

- When ghit register sections are cascaded, the maximum rise and fall time of the clock input thould be equal to or less than the rise and fell time of the date ou puts, driving data inputs, plus the propesstion delay of the output driving stape.

FIGURE 1 - POWEA DISSIPATION TEST CIRCUIT AND WAVEFOAM

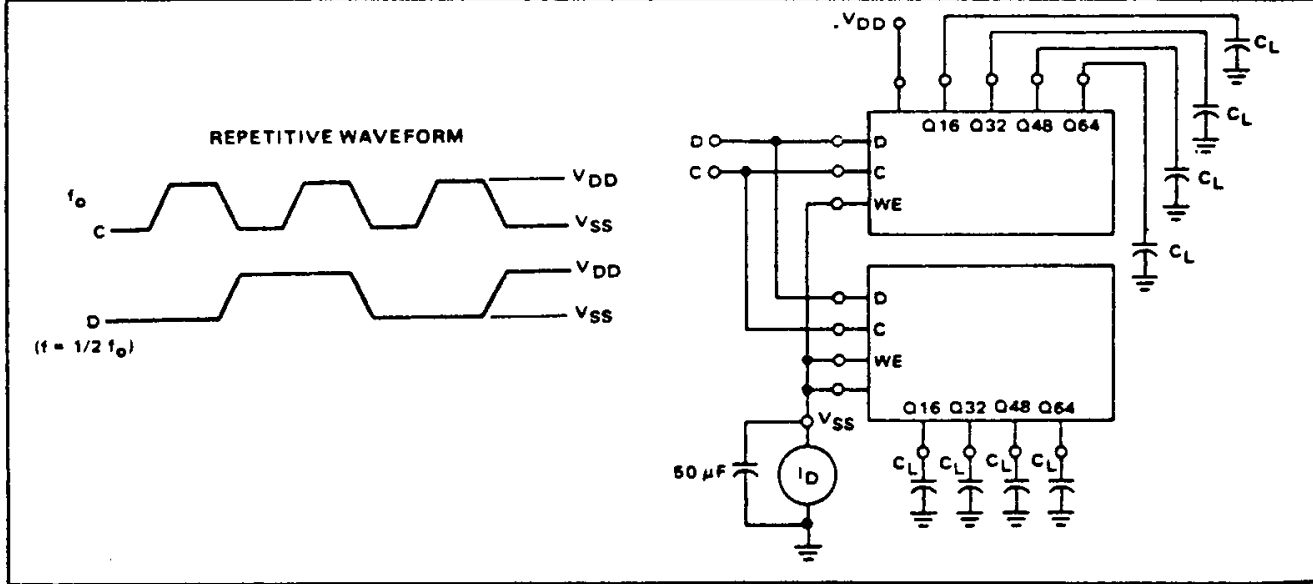




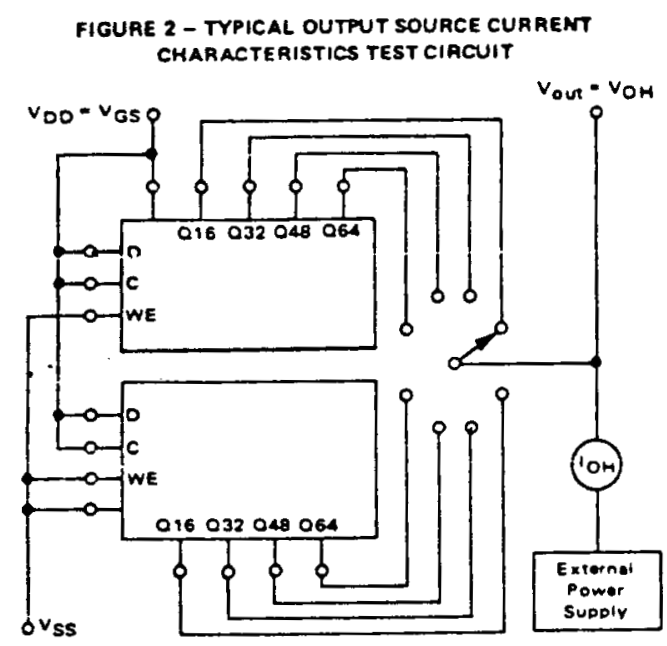

COutput being tested enould be in the Migh-logic statel.
FIGURE 3 - TYPICAL OUTPUT SINK CURRENT CHARACTERISTICS TEST CIRCUIT

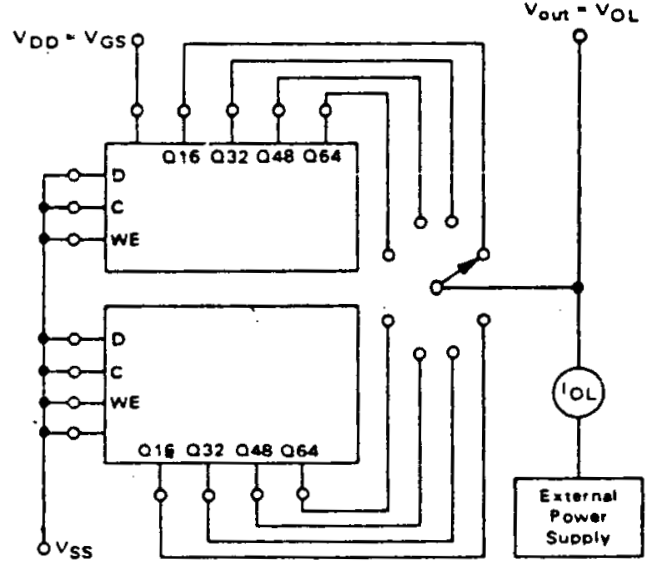

10y tout being terted thould be in the low logic statel.
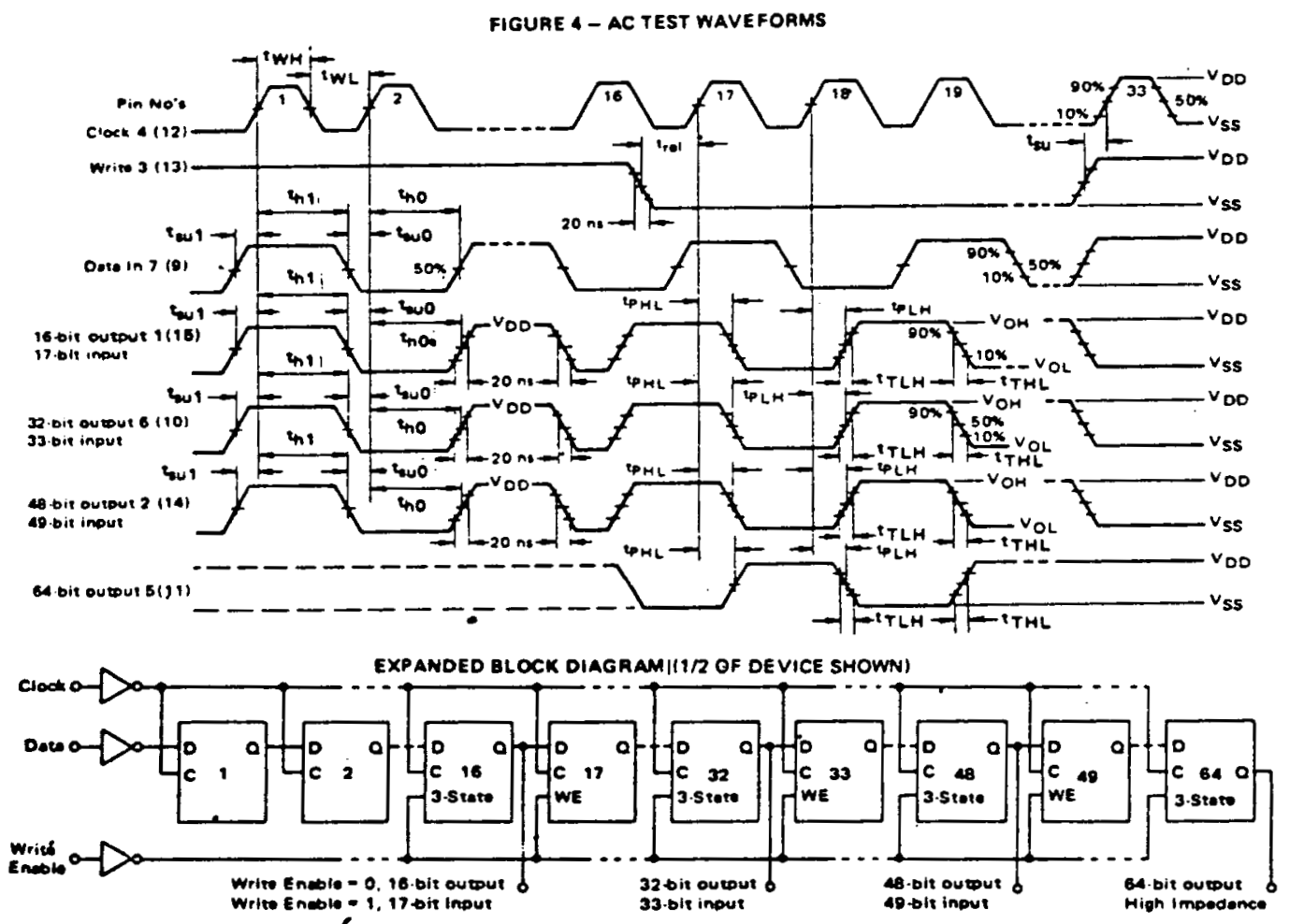
Write Endole - 1. 17 bit input 


\section{(AA) MOTOROLA}

\section{TO.64 BIT VARIABLE LENGTH SHIFT REGISTER}

The MC14557B is a static clocked serial shift register whose length nav be programmed to de any number of bits between 1 and 64 . The nutrter of bite selected is cqual to the sum of the substripts of the enabled Length Control inputs ( $L 1, L 2, L 4, L 8, L 16$, and $L 32$ ) plus one. Selial data may be selected from the $A$ or $B$ data inputs with the A B select input. This feature is useful for recirculation purposes. A Clock Enable (CE) input is provided to allow gating of the clock or nega tive edge clocking capability.

The device can be effectively used for variable digital delay lines or simpiy to implement odd length shift regisiers. characteristics can be found on the Family Data Sheet.

- Quiescent Current = $10 \mathrm{nA} /$ package typical @ 5 Vdc

- 1.64 Bit Programmable Length

- O and $\bar{O}$ Serial Buffered Outputs

- Asynchronous Master Reset

- All loputs Buffered

- No Limit On Clock Rise and Fail Times

- $8 \mathrm{MHz}$ Operation @ VDD $=10 \mathrm{Vdc} T$ Ypical

- Supply Voltage Range $=3.0 \mathrm{Vdc}$ to $18 \mathrm{Vdc}$

- Capsble of Driving Two Lov. power TTL Loads, One Low.power Schortky TTL Load or Two HTL Losds Over the Rated Temper. aturé Range

\begin{tabular}{|c|c|c|c|}
\hline Rating & Symbol & Value & Unit \\
\hline DC Supplv Vcleage & $V_{D D}$ & $-0510+18$ & $V d c$ \\
\hline inpu: Voltsout. All inputs & $v_{\text {in }}$ & $-0.510 V_{D O}+0.5$ & Vde \\
\hline DC Curreni Dian per Pin & 1 & 10 & $\mathrm{mAdc}$ \\
\hline $\begin{array}{r}\text { Ope'at:ng Temperalule Fange - A - Device } \\
C L \text { CP Device }\end{array}$ & $T_{A}$ & $\begin{array}{l}-55 \mathrm{x} \cdot 125 \\
-40 \mathrm{10} \cdot 85\end{array}$ & ${ }^{\circ} \mathrm{C}$ \\
\hline S:oroji Tenpesture Range & $T_{\text {sig }}$ & $-65+0-150$ & ${ }^{\circ} \mathrm{C}$ \\
\hline
\end{tabular}

\begin{tabular}{|c|c|c|c|c|c|c|}
\hline \multicolumn{7}{|c|}{ LENGTH SELECT TRUTH TABLE } \\
\hline L32 & $L 16$ & LE & 24 & L2 & L! & Reg:ster Lengih \\
\hline 0 & 0 & 0 & 0 & 0 & 0 & 1.818 \\
\hline 0 & 0 & 0 & 0 & 0 & 1 & 2. Bits \\
\hline 0 & 0 & 0 & 0 & 1 & 0 & 3. Bits \\
\hline 0 & 0 & 0 & 0 & 1 & 1 & 4. Bits \\
\hline 0 & 0 & 0 & 1 & 0 & 0 & 5 Bits \\
\hline 0 & 0 & 0 & 1 & 0 & 1 & 6 Bits \\
\hline " & . & . & . & $\cdot$ & & \\
\hline . & . & . & & & . & . \\
\hline 1 & 0 & 0 & 0 & 0 & 0 & 33 Bits \\
\hline 1 & 0 & 0 & 0 & 0 & 1 & 34'Bitg \\
\hline . & . & . & . & . & 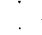 & \\
\hline - & . & . & . & . & $\cdot$ & . \\
\hline , & 1 & 1 & 1 & 0 & 0 & 61 Bits \\
\hline 1 & 1 & 1 & 1 & 0 & 1 & 62. Bits \\
\hline 1 & 1 & 1 & 1 & 1 & 0 & $63 \cdot \mathrm{Bits}$ \\
\hline 1 & $!$ & I & 1 & 1 & 1 & G4 Bits \\
\hline Note & $\begin{array}{l}\text { eng } \\
\text { ontt }\end{array}$ & & & & & length \\
\hline
\end{tabular}
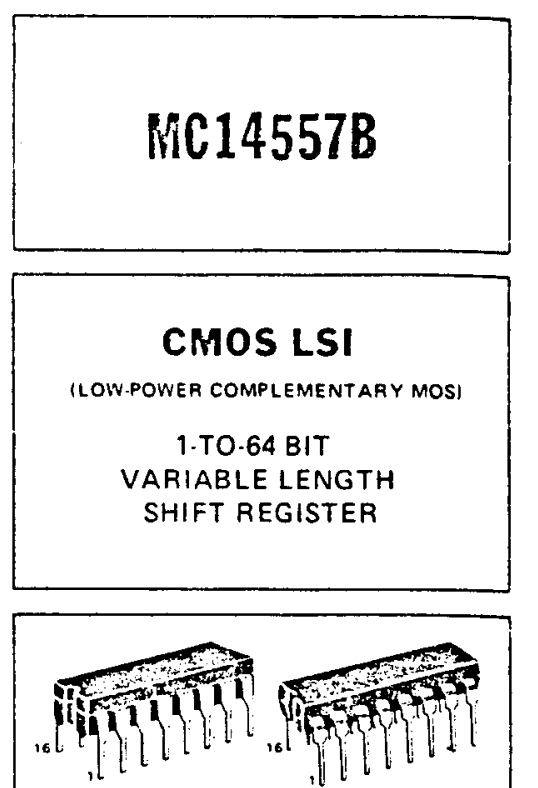

$$
\begin{array}{cc}
\text { LSUFFIX } & \text { PSUFFIX } \\
\text { CERAMICPACKAGE } & \text { PLASTICPACKAGE } \\
\text { CASE 620 } & \text { CASE 648 } \\
\text { ORDERING INFORMATION }
\end{array}
$$
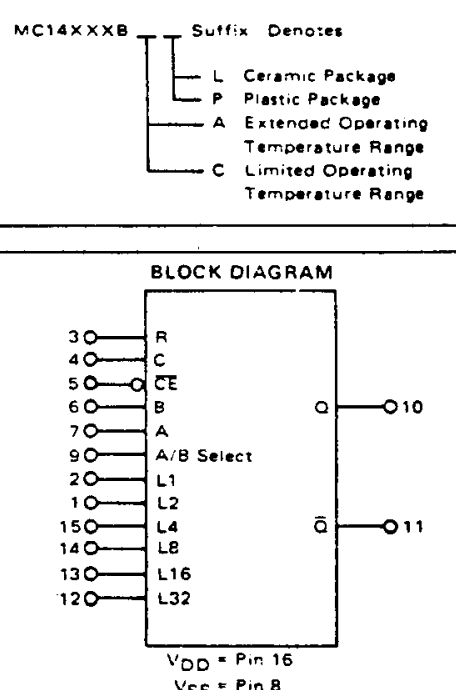

SS $=\operatorname{Pin} 8$

TRUTH TABLE

\begin{tabular}{|c|c|c|c|c|}
\hline \multicolumn{3}{|c|}{ Inputs } & Output \\
\hline$A$ & $A$ & Clock & $\overline{C E}$ & 2 \\
\hline 0 & 0 & - & 0 & $B$ \\
0 & 1 & - & 0 & $A$ \\
0 & 0 & 1 & - & $B$ \\
0 & 1 & 1 & $Z$ & $A$ \\
1 & $x$ & $x$ & $X$ & 0 \\
\hline
\end{tabular}
Q is the output of the first selected
shift rogister stege.
$x$

$x=$ Q

$x$ - Don't Care.

Reproduced with permission from Motorola, Inc., Phoenix, AZ 85008. 
$\operatorname{sic14557B}$

\begin{tabular}{|c|c|c|c|c|c|c|c|c|c|c|}
\hline \multirow{2}{*}{ Enaracieristic } & \multirow{2}{*}{ Symbol } & \multirow{2}{*}{$\begin{array}{l}V_{D D} \\
\text { Vde }\end{array}$} & \multicolumn{2}{|c|}{$T_{\text {low" }}$} & \multicolumn{3}{|c|}{$25^{\circ} \mathrm{C}$} & \multicolumn{2}{|c|}{ Thigh: } & \multirow[b]{2}{*}{ Unis } \\
\hline & & & $M, n$ & Mox & $M, n$ & TYp & Max & Min & Max & \\
\hline $\begin{array}{l}\text { Jilou: Voltage } \\
\text { Vin. VDD or } 0\end{array}$ & $v_{O L}$ & $\begin{array}{l}5.0 \\
10 \\
15 \\
\end{array}$ & $\begin{array}{l}- \\
- \\
-\end{array}$ & $\begin{array}{l}0.05 \\
0.05 \\
0.05 \\
\end{array}$ & $\begin{array}{l}- \\
- \\
-\end{array}$ & $\begin{array}{l}0 \\
0 \\
0 \\
\end{array}$ & $\begin{array}{l}0.05 \\
0.05 \\
0.05 \\
\end{array}$ & $\begin{array}{l}- \\
- \\
-\end{array}$ & $\begin{array}{l}0.05 \\
0.05 \\
0.05 \\
\end{array}$ & Vdc \\
\hline$\because n \cdot 0$ or $v_{D D}$ & $\mathrm{VOH}_{\mathrm{OH}}$ & $\begin{array}{l}5.0 \\
10 \\
15 \\
\end{array}$ & $\begin{array}{r}4.95 \\
9.95 \\
14.95 \\
\end{array}$ & - & $\begin{array}{r}4.95 \\
9.95 \\
14.95 \\
\end{array}$ & $\begin{array}{l}5.0 \\
10 \\
15 \\
\end{array}$ & $\begin{array}{l}- \\
- \\
-\end{array}$ & $\begin{array}{r}4.95 \\
9.95 \\
14.95 \\
\end{array}$ & $\begin{array}{l}- \\
- \\
\end{array}$ & Voc \\
\hline 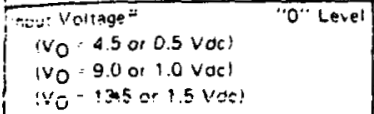 & $v_{11}$ & $\begin{array}{l}5.0 \\
10 \\
15 \\
\end{array}$ & $\begin{array}{l}- \\
-\end{array}$ & $\begin{array}{l}1.5 \\
3.0 \\
4.0 \\
\end{array}$ & $\begin{array}{l}- \\
- \\
\end{array}$ & $\begin{array}{l}2.25 \\
4.50 \\
6.75 \\
\end{array}$ & $\begin{array}{l}1.5 \\
3.0 \\
40 \\
\end{array}$ & $\begin{array}{l}- \\
-\end{array}$ & $\begin{array}{l}1.5 \\
3.0 \\
4.0 \\
\end{array}$ & $\bar{v} d c$ \\
\hline 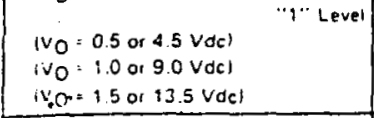 & $V_{1 H}$ & $\begin{array}{l}5.0 \\
10 \\
15 \\
\end{array}$ & $\begin{array}{r}3.5 \\
7.0 \\
11.0 \\
\end{array}$ & $\begin{array}{l}- \\
- \\
-\end{array}$ & $\begin{array}{r}3.5 \\
7.0 \\
11.0 \\
\end{array}$ & $\begin{array}{l}2.75 \\
5.50 \\
8.25 \\
\end{array}$ & $\begin{array}{l}- \\
- \\
-\end{array}$ & $\begin{array}{r}3.5 \\
7.0 \\
11.0 \\
\end{array}$ & $\begin{array}{l}- \\
- \\
\end{array}$ & Vdc \\
\hline 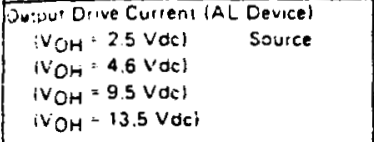 & $10 \mathrm{H}$ & $\begin{array}{l}5.0 \\
5.0 \\
10 \\
15 \\
\end{array}$ & $\begin{array}{l}-1.2 \\
-0.25 \\
-0.62 \\
-1.8 \\
\end{array}$ & $\begin{array}{l}- \\
- \\
- \\
\end{array}$ & $\begin{array}{l}-1.0 \\
-0.2 \\
-0.5 \\
-1.5 \\
\end{array}$ & $\begin{array}{l}-1.7 \\
-0.36 \\
-0.9 \\
-3.5 \\
\end{array}$ & $\begin{array}{l}- \\
- \\
-\end{array}$ & $\begin{array}{l}-0.7 \\
-0.14 \\
-0.35 \\
-1.1 \\
\end{array}$ & $\begin{array}{l}- \\
- \\
-\end{array}$ & madc \\
\hline $\begin{array}{l}\left(V_{O L}=0.4 \mathrm{Vdct}\right. \\
\left.N_{O L}=0.5 \mathrm{Vdc}\right) \quad \text { Sink } \\
V_{O L}=1.5 \mathrm{Vdcl}\end{array}$ & $10 L$ & $\begin{array}{l}5.0 \\
10 \\
15 \\
\end{array}$ & $\begin{array}{r}0.64 \\
1.6 \\
4.2 \\
\end{array}$ & $\begin{array}{l}- \\
- \\
\end{array}$ & $\begin{array}{r}0.51 \\
1.3 \\
3.4 \\
\end{array}$ & $\begin{array}{l}0.88 \\
2.25 \\
8.8 \\
\end{array}$ & $\begin{array}{l}- \\
- \\
\end{array}$ & $\begin{array}{l}0.36 \\
0.9 \\
2.4 \\
\end{array}$ & $\begin{array}{l}- \\
- \\
\end{array}$ & madc \\
\hline $\begin{array}{l}\text { Dutput Dive Curient }(C L / C P \text { Devicel } \\
\left(V_{O H}=2.5 \mathrm{Vocl} \quad \text { Source }\right. \\
{ }^{V_{O H}}=4.6 \mathrm{Vdcl} \\
\mathrm{V}_{\mathrm{OH}}=9.5 \mathrm{Vdcl} \\
\mathrm{V}_{\mathrm{OH}}=13.5 \mathrm{Vdcl}\end{array}$ & $\mathrm{TOH}$ & $\begin{array}{l}5.0 \\
5.0 \\
10 \\
15 \\
\end{array}$ & $\begin{array}{l}-1.0 \\
-0.2 \\
-0.5 \\
-1.4 \\
\end{array}$ & $\begin{array}{l}- \\
- \\
-\end{array}$ & $\begin{array}{l}-0.8 \\
-0.16 \\
-0.4 \\
-1.2 \\
\end{array}$ & $\begin{array}{l}-1.7 \\
-0.36 \\
-0.9 \\
-3.5 \\
\end{array}$ & $\begin{array}{l}- \\
- \\
-\end{array}$ & $\begin{array}{l}-0.6 \\
-0.12 \\
-0.3 \\
-1.0 \\
\end{array}$ & $\begin{array}{l}- \\
- \\
- \\
\end{array}$ & mads \\
\hline $\begin{array}{l}\left(V_{O L}=0.4 \vee d c\right) \\
\left(V_{O L}=0.5 \vee d c\right) \\
\left(V_{Q L}=1.5 \vee d c\right)\end{array}$ & 'OL & $\begin{array}{l}5.0 \\
10 \\
15 \\
\end{array}$ & $\begin{array}{c}0.52 \\
1.3 \\
3.6 \\
\end{array}$ & $\begin{array}{l}- \\
- \\
\end{array}$ & $\begin{array}{r}0.44 \\
1.1 \\
3.0 \\
\end{array}$ & $\begin{array}{c}0.88 \\
2.25 \\
8.8 \\
\end{array}$ & {$\left[\begin{array}{l}- \\
-\end{array}\right.$} & $\begin{array}{c}0.36 \\
0.9 \\
2.4 \\
\end{array}$ & $\begin{array}{l}- \\
- \\
\end{array}$ & made \\
\hline Inpur Curient IAL Devicel & $I_{\text {in }}$ & 15 & - & .0 .1 & - & \pm 000001 & .01 & - & $\therefore 10$ & $\angle A O C$ \\
\hline Imout Current $\mathrm{CL}, \mathrm{CP}$ Devicel & $\operatorname{lin}$ & 15 & $=$ & $: 0.3$ & - & $: 000001$ & $\therefore 0.3$ & - & $\therefore 10$ & $\mu$ Adc \\
\hline $\begin{array}{l}\text { inout Capacitance } \\
\left(\mathrm{V}_{\text {in }}-01\right.\end{array}$ & $\overline{C_{\text {in }}}$ & - & $\cdot$ & - & - & 50 & 7.5 & - & - & pF \\
\hline $\begin{array}{l}\text { Quiejcen! Curien! (AL Device) } \\
\text { (Pes Package) }\end{array}$ & 100 & $\begin{array}{l}5.0 \\
10 \\
15 \\
\end{array}$ & $\begin{array}{l}- \\
- \\
\end{array}$ & $\begin{array}{l}5.0 \\
10 \\
20 \\
\end{array}$ & $\begin{array}{l}- \\
- \\
\end{array}$ & $\begin{array}{l}0.010 \\
0.020 \\
0.030 \\
\end{array}$ & $\begin{array}{l}5.0 \\
10 \\
20 \\
\end{array}$ & $\overline{-}$ & $\begin{array}{l}150 \\
300 \\
600 \\
\end{array}$ & MAdC \\
\hline $\begin{array}{l}\text { Qu.esceni Culien: ICL:CP Device) } \\
\text { (Per Packagei }\end{array}$ & 'DD & $\begin{array}{l}5.0 \\
10 \\
15 \\
\end{array}$ & $\begin{array}{l}- \\
- \\
-\end{array}$ & $\begin{array}{c}50 \\
100 \\
200 \\
\end{array}$ & $\begin{array}{l}- \\
- \\
\end{array}$ & $\begin{array}{l}0.010 \\
0.020 \\
0.030 \\
\end{array}$ & $\begin{array}{c}50 \\
100 \\
200 \\
\end{array}$ & $\begin{array}{l}- \\
-\end{array}$ & $\begin{array}{c}375 \\
750 \\
1500 \\
\end{array}$ & $\mu A d c$ \\
\hline $\begin{array}{l}\text { Toial Supplv Curieni" } \\
\text { (Drnamic plus Ouiescent. } \\
\text { Per Dackage) } \\
\left(C_{\mathrm{i}}-50 \text { of on all outpuls. all }\right. \\
\text { rutteis switchinal }\end{array}$ & iT. & $\begin{array}{l}5.0 \\
10 \\
15\end{array}$ & & & $\begin{array}{l}I_{T}=11 \\
I_{T}=13 \\
I_{T}=15\end{array}$ & $\begin{array}{l}75 \mathrm{HA} / \mathrm{KHZ} \\
5 \mathrm{NA} / \mathrm{KHZ} \\
25 \mathrm{HA} / \mathrm{KHZ}\end{array}$ & $\begin{array}{l}211+100 \\
11+100 \\
211+100\end{array}$ & & & NAdC \\
\hline 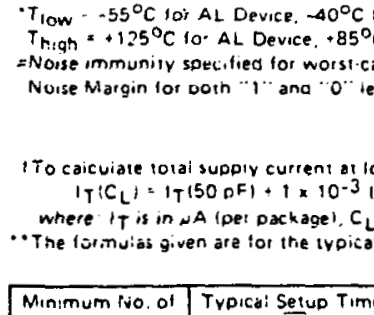 & 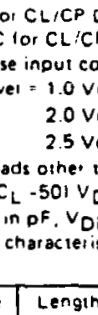 & $\begin{array}{l}\text { evice. } \\
\text { Device } \\
\text { noinsti } \\
\text { c min } \\
\text { c min } \\
\text { c min } \\
\text { man } 50 \\
0^{\prime} \\
\text { in vo } \\
\text { lics onl }\end{array}$ & $\begin{array}{l}D D=5 \\
D D= \\
D D= \\
\\
25^{\circ} \mathrm{C} \text {. } \\
{ }^{\circ} .\end{array}$ & $\begin{array}{l}\text { Voc } \\
\text { Voc } \\
\text { voc } \\
\text { is inou }\end{array}$ & 800 & & 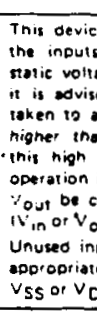 & $\begin{array}{l}\text { contains } \\
\text { ogainsi } \\
\text { ges or ele } \\
\text { o that no } \\
\text { oid appli } \\
\text { maxims } \\
\text { mpedance } \\
\text { is recom } \\
\text { nstesined } \\
1<\vee_{D O} \\
\text { uts must } \\
\text { logic voit } \\
\text { ol. }\end{array}$ & 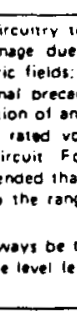 & 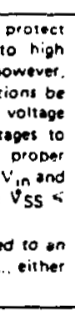 \\
\hline
\end{tabular}

\begin{tabular}{|c|c|c|}
\hline $\begin{array}{c}\text { Minimum ivo of } \\
\text { Bits Seiecied }\end{array}$ & $\begin{array}{c}\text { Tyoicai Setud Time } \\
a-\overline{C E} \text { ns }\end{array}$ & $\begin{array}{c}\text { Lengin Selest } \\
\text { Lines }=1\end{array}$ \\
\hline 1 & 180 & None \\
2 & 120 & $L 1$ \\
3 & 90 & $L 2$ \\
5 & 60 & $L 4$ \\
9 & 30 & $L B$ \\
17 & 0 & $L 16$ \\
33 & -30 & $L 32$ \\
\hline
\end{tabular}

\section{SETUP TIME CHART}

The nature of the length select logic causes the setup time to vary with the number of bis selected The following rable summarizes the ivpical variation at $V_{D D}=10 \mathrm{~V}, T_{A}=25^{\circ} \mathrm{C}$.

Reproduced with permission from Motorola, Inc., Phoenix, AZ 85008. 


\section{MC14557B}

SWITCHING CHARACTERISTICS* $\left\langle C_{L}=50 \mathrm{PF}, T_{A}=25^{\circ} \mathrm{C}\right)$

\begin{tabular}{|c|c|c|c|c|c|c|}
\hline Characteristic & Symbol & $V_{D D}$ & Min & Typ & Mex & Unit \\
\hline $\begin{array}{l}\text { OutDut Rise Time } \\
T T L H=(3.0 \mathrm{~ns} / \mathrm{pF}) \mathrm{C}_{L}+30 \mathrm{~ns} \\
T T L H=11.5 \mathrm{~ns} / \mathrm{pF}) C_{L}+15 \mathrm{~ns} \\
T T L H=11.1 \mathrm{~ns} / \mathrm{pF} / C_{L}+10 \mathrm{~ns}\end{array}$ & $t_{P L H}$ & $\begin{array}{l}5.0 \\
10 \\
15 \\
\end{array}$ & $\begin{array}{l}- \\
-\end{array}$ & $\begin{array}{l}180 \\
90 \\
65 \\
\end{array}$ & $\begin{array}{l}360 \\
180 \\
130 \\
\end{array}$ & $\mathrm{~ns}$ \\
\hline $\begin{array}{l}\text { Output Fal Time } \\
\text { TTHL }=\left(1.5 \mathrm{~ns} / \mathrm{pF} / C_{L}+25 \mathrm{~ns}\right. \\
{ }^{T} \mathrm{THL}=\left(0.75 \mathrm{~ns} / \mathrm{pF} / C_{L}+12.5 \mathrm{~ns}\right. \\
{ }_{T} T H L=10.55 \mathrm{~ns} / \mathrm{pF} / C_{L}+9.5 \mathrm{~ns}\end{array}$ & tPHL & $\begin{array}{l}5.0 \\
10 \\
15 \\
\end{array}$ & I & $\begin{array}{l}100 \\
50 \\
40 \\
\end{array}$ & $\begin{array}{l}200 \\
100 \\
80 \\
\end{array}$ & กs \\
\hline 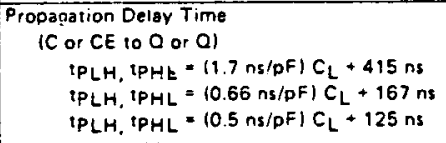 & $\begin{array}{l}\text { TPLH, } \\
\text { TPHL }\end{array}$ & $\begin{array}{l}5.0 \\
10 \\
15\end{array}$ & $\bar{z}$ & $\begin{array}{l}500 \\
200 \\
150\end{array}$ & $\begin{array}{l}1000 \\
400 \\
300\end{array}$ & $n s$ \\
\hline 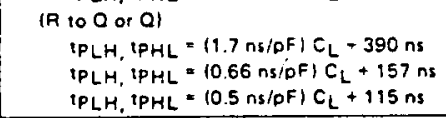 & & $\begin{array}{l}5.0 \\
10 \\
15 \\
\end{array}$ & - & $\begin{array}{l}475 \\
190 \\
140 \\
\end{array}$ & $\begin{array}{r}550 \\
380 \\
280 \\
\end{array}$ & nst \\
\hline Clock Pulse Width & $t_{W H(C)}$ & $\begin{array}{l}5.0 \\
10 \\
15 \\
\end{array}$ & $\begin{array}{l}440 \\
136 \\
100 \\
\end{array}$ & $\begin{array}{c}220 \\
68 \\
50\end{array}$ & $\begin{array}{l}\overline{-} \\
\overline{-}\end{array}$ & ns \\
\hline Reset Pulse Width & IWH(R) & $\begin{array}{l}5.0 \\
10 \\
15 \\
\end{array}$ & $\begin{array}{l}600 \\
180 \\
120 \\
\end{array}$ & $\begin{array}{l}300 \\
90 \\
60 \\
\end{array}$ & $\begin{array}{l}\bar{z} \\
\overline{-}\end{array}$ & $n s$ \\
\hline Clock Pulse Frequency & $\mathrm{fel}^{\mathrm{f}}$ & \begin{tabular}{|l|}
5.0 \\
10 \\
15 \\
\end{tabular} & $\begin{array}{l}- \\
- \\
-\end{array}$ & $\begin{array}{c}2.5 \\
8.0 \\
10.5 \\
\end{array}$ & $\begin{array}{l}1.7 \\
5.0 \\
6.7\end{array}$ & $\mathrm{MHz}$ \\
\hline Clock Pulse Rise and Fali Time & $\begin{array}{l}\text { TTLH, } \\
\text { TTHL }\end{array}$ & $\begin{array}{l}5.0 \\
10 \\
15 \\
\end{array}$ & & No Limit & & - \\
\hline $\begin{array}{l}\text { Oata to Clock Setup Time (A or } B \text { to } C \text { or } C E \text { ) } \\
\text { L1, L2, L4; L8, L 16, L32 }=0\end{array}$ & $t_{\text {su }}$ & $\begin{array}{l}5.0 \\
10 \\
15 \\
\end{array}$ & $\begin{array}{l}9000 \\
360 \\
170 \\
\end{array}$ & $\begin{array}{r}450 \\
180 \\
135 \\
\end{array}$ & $\begin{array}{l}\bar{z} \\
\overline{-}\end{array}$ & $\overline{n s}$ \\
\hline $\begin{array}{l}\text { Data to Clock Hold Time (A or B ro C or CE) } \\
\text { L1, L2, L4, LB, L 16, L32:0 }\end{array}$ & $t_{h}$ & $\begin{array}{l}5.0 \\
10 \\
15 \\
\end{array}$ & $\begin{array}{l}-225 \\
-90 \\
-60 \\
\end{array}$ & $\begin{array}{l}-450 \\
-180 \\
-135 \\
\end{array}$ & $\bar{z}$ & $n s$ \\
\hline Reset Fall Time & TTLH & $\begin{array}{l}5.0 \\
10 \\
15 \\
\end{array}$ & $\begin{array}{l}\bar{z} \\
\overline{-}\end{array}$ & $\begin{array}{l}- \\
=\end{array}$ & $\begin{array}{c}15 \\
5 \\
4 \\
\end{array}$ & us \\
\hline
\end{tabular}

"The formula given is for the typical characteristics only.

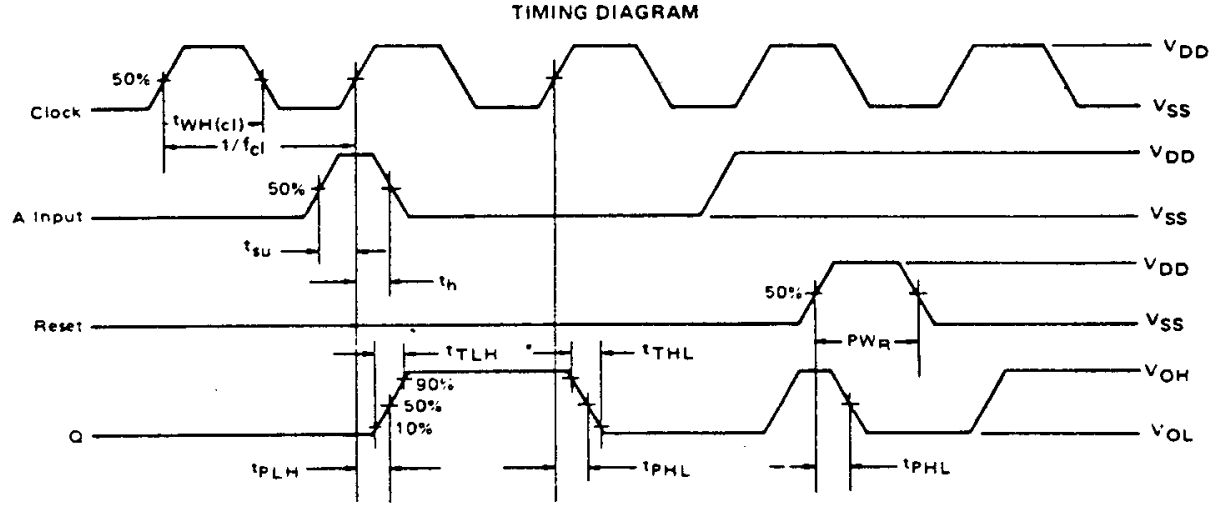

$1 \cdot$ bit length
$C, C=0$

$A / B=1$

$L 1=L 2=L 4=L 8=L 16=L 32=0$

Reproduced with permission from Motorola, Inc., Phoenix, AZ 85008. 
MC14557B

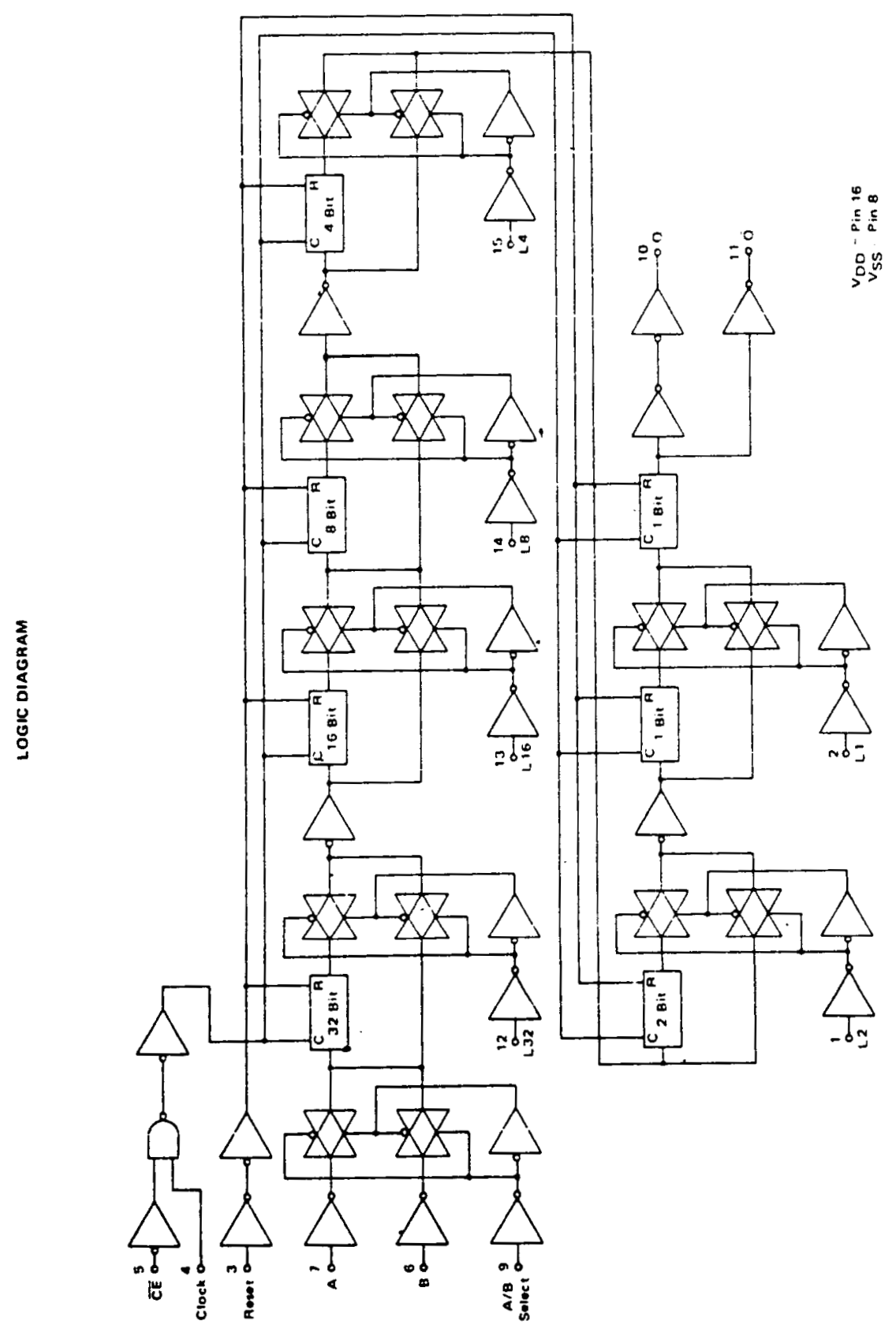

Reproduced with permission from Motorola, Inc., Phoenix, AZ 85008. 
MC6871A

full function microprocessor clock

$850 \mathrm{kHz}$ to $2.5 \mathrm{MHz}$

E

\section{specificications}

\begin{tabular}{l|c|c|c|}
\hline \multicolumn{1}{|c|}{ Rating } & Symbol & Value & Unit \\
\hline Supply Vollagge & $V_{c c}$ & $5.00 \pm 5 \%$ & Voc \\
\hline Operatina Temperature Range & $T_{A}$ & $0 t 0+70$ & ${ }^{\circ} \mathrm{C}$ \\
\hline Storage Temperature & $T_{119}$ & -55 to +125 & ${ }^{\circ} \mathrm{C}$ \\
\hline Power Suppiy Drain (max.) & $V_{\infty}$ & 100 & $\mathrm{~mA}$ \\
\hline
\end{tabular}

ELECTHICAL CHARACTERISTICS $\left(V_{c c}=5.0 \pm 5 \%, V_{u}=0 . T_{A}\right.$ $=0^{\circ}$ to $70^{\circ} \mathrm{C}$. unless otherwise noted)

\begin{tabular}{|c|c|c|c|c|c|}
\hline Characteristic & Symbol & Min & Typ & Max & Unit \\
\hline \multicolumn{6}{|l|}{ Frequency } \\
\hline Operating Frequency & $t_{=}$ & 850 & & 25 & $\mathrm{MHZ}$ \\
\hline $\begin{array}{l}\text { Frequency stability (inclusive } \\
\text { of calibration tolerance at } \\
+25^{\circ} \mathrm{C} \text {, operating temperature, } \\
\text { input voltage change, load } \\
\text { change, aging, shock and } \\
\text { vibration) }\end{array}$ & & & \pm .01 & & $\%$ \\
\hline \multicolumn{6}{|c|}{ NMOS Oulputs at $1.0 \mathrm{MHz}$ Operation * ** } \\
\hline $\begin{array}{l}\text { Pulse Width (meas. at } \\
V_{c t}=-3 V d c \text { level) }\end{array}$ & $\begin{array}{l}T \emptyset_{1} H \\
T \emptyset_{2} H \\
\end{array}$ & $\begin{array}{l}430 \\
450 \\
\end{array}$ & & & $\begin{array}{l}\text { ns } \\
\text { ns }\end{array}$ \\
\hline Logic Levels & $\begin{array}{l}V_{\text {OIC }} \\
V_{\text {OHC }}\end{array}$ & \begin{tabular}{|l|}
$V_{c u}-.1$ \\
$V_{c e}-.3$ \\
\end{tabular} & $\overline{-}$ & $\begin{array}{l}V_{11}+3 \\
V_{c c}+.1 \\
\end{array}$ & $\begin{array}{l}\text { Vdc } \\
V d c\end{array}$ \\
\hline Rise and Fall Times & tr & \begin{tabular}{|l|}
5 \\
5 \\
\end{tabular} & $\begin{array}{l}12 \\
12 \\
\end{array}$ & $\begin{array}{l}50 \\
50 \\
\end{array}$ & $\begin{array}{ll}\text { ns } \\
\text { ns }\end{array}$ \\
\hline $\begin{array}{c}\text { "Overshool/Undershoot } \\
\text { Logic "1" } \\
\text { Logic " } 0 \text { " }\end{array}$ & Vos & \begin{tabular}{|l}
$V_{a c}-5$ \\
$V_{t a}-5$ \\
\end{tabular} & & $\begin{array}{l}V_{c t}+5 \\
V_{31}+5 \\
\end{array}$ & $\begin{array}{l}\text { Vdc } \\
\text { Vdc }\end{array}$ \\
\hline $\begin{array}{l}\text { Fulse duration of any over- } \\
\text { shoot or undershool }\end{array}$ & Tos & & & 40 & ns \\
\hline Period@0.3V do Level & $l_{\text {crs }}$ & & 1.00 & & us \\
\hline Edge Timing @ $V_{c c}=0.3 \mathrm{Vdc}$ & $T x$ & 940 & & & $\mathrm{~ns}$ \\
\hline $\begin{array}{l}\text { NMOS Relationship } \\
\varrho+0.5 \mathrm{~V} d c \text { Level } \\
\end{array}$ & $t_{11}$ & $\begin{array}{l}0 \\
0 \\
\end{array}$ & & 8.0 & us \\
\hline \multicolumn{6}{|l|}{ TL Outputs } \\
\hline \multicolumn{6}{|l|}{ In ref. to $Q / 2$ NMOS@0.3V dc } \\
\hline \begin{tabular}{|ll}
$\Phi_{2}$ & $\pi L$ \\
$@$ & $1.4 \mathrm{~V} d c$
\end{tabular} & $T_{\hat{H}}$ & $\begin{array}{l}15 \\
10 \\
\end{array}$ & $\begin{array}{l}30 \\
25 \\
\end{array}$ & $\begin{array}{l}45 \\
40\end{array}$ & $\begin{array}{ll}\text { ns } \\
\text { ns }\end{array}$ \\
\hline $\begin{array}{l}\text { Memory Clock } \\
@ 1.4 \mathrm{Vdc}\end{array}$ & $\begin{array}{l}T_{e} \\
T_{j}\end{array}$ & $\begin{array}{l}30 \\
20\end{array}$ & $\begin{array}{l}50 \\
40\end{array}$ & $\begin{array}{l}70 \\
60\end{array}$ & $\begin{array}{l}\text { ns } \\
\text { ns }\end{array}$ \\
\hline $2 x i c @ 1.4 V d c$ & $T_{1}$ & 40 & 80 & 120 & ns \\
\hline Logic Levels & $\begin{array}{l}V_{\dot{o}_{+1}} \\
V_{O L}\end{array}$ & 2.4 & $\begin{array}{r}3.2 \\
.3 \\
\end{array}$ & .4 & $\begin{array}{l}V d c \\
V d c\end{array}$ \\
\hline $\begin{array}{c}\text { Rise and Fall Times } \\
.4 \mathrm{~V} \text { and } 2.4 \mathrm{~V} \\
2.4 \mathrm{~V} \text { and } .4 \mathrm{~V} \\
\end{array}$ & $\begin{array}{l}t_{1} \\
q_{1}\end{array}$ & & & $\begin{array}{l}15 \\
15 \\
\end{array}$ & $\begin{array}{l}\text { ns } \\
\text { ns }\end{array}$ \\
\hline Logic "0" Sink (/Gate) & $1 x$ & & & -1.6 & $m A$ \\
\hline Logic "1" Source (/Gate) & low & & & +40 & UA \\
\hline Current Output Shorted & Tse & -18 & & -57 & $\mathrm{~mA}$ \\
\hline \multicolumn{6}{|l|}{ !Load } \\
\hline NMOS-Load Capacity $\varnothing_{1}, \varnothing_{2}$ & $\mathrm{C}_{\text {NMOS }}$ & 80 & 120 & 160 & pf \\
\hline TTL-No. of Loads & & & & 5 & $\mathrm{ttl}$ \\
\hline$\pi L-L o a d$ Capacity & $\mathrm{C}_{T 76}$ & & & 50 & $\mathrm{pf}$ \\
\hline \multicolumn{6}{|c|}{ Logic Inputs" ("O" Level APflles HOLD or MEMORY READY) } \\
\hline $\begin{array}{l}\text { Holds } \varnothing_{1} \text { NMOS 'High', } \emptyset_{2} \\
\text { NMOS 'Low', } \varnothing_{2} T \Pi L \text { 'Low' }\end{array}$ & $\overline{\text { HOLD 1 }}$ & -.2 & & +.4 & Vdc \\
\hline $\begin{array}{l}\text { Holds } \square_{1} \text { NMOS 'LOW' } \varnothing_{2} \text { NMOS } \\
\text { 'High', } \bigotimes_{2} \text { TLL 'High', and } \\
\text { MENORY CLOCK 'High' }\end{array}$ & $\begin{array}{c}\text { MEM- } \\
\text { ORY } \\
\text { READY }\end{array}$ & -.2 & & +.4 & Vdc \\
\hline
\end{tabular}

- Into specified rest $10 \mathrm{ad}$

"Must be externally helo at "y" tevel (2.4V min., $5.0 \mathrm{~V}$ max.) it not used

-Apply the following parameters for frequencies other than $1 \mathrm{MHz}$

$T \phi_{1} H=0.5(P-140)$
ns

$T_{x=(P .60)}(P-100)$

$x=(P \cdot 60)$ ns

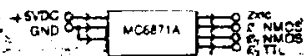

AOODI MEMOCO

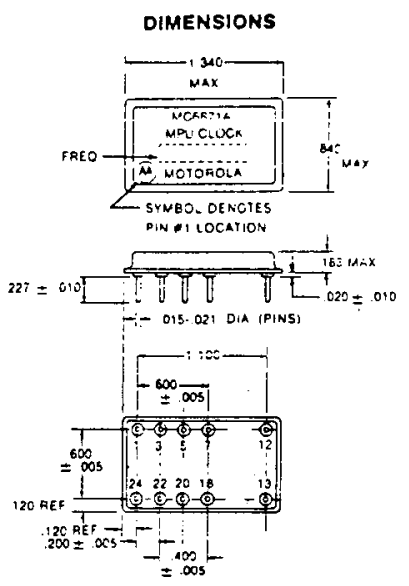

\begin{tabular}{|c|c}
\hline PIN & CONNECTION \\
\hline 1 & GND \\
\hline 3 & MEMORY CLOCK \\
\hline 5 & $C_{2}$ TTL \\
\hline 7 & $V_{c c}(+5 V D C)$ \\
\hline 12 & $\ell_{2}$ NMOS \\
\hline 13 & O NMOS $_{1}$ NMN \\
\hline 18 & GND \\
\hline 20 & HOLD 1 \\
\hline 22 & MEMORY READY \\
\hline 24 & $2 \times 1 C$ \\
\hline
\end{tabular}

Noie All dirtiensions are in inathes

WAVEFORM TIMING

ALL TIME IN NANOSECONDS

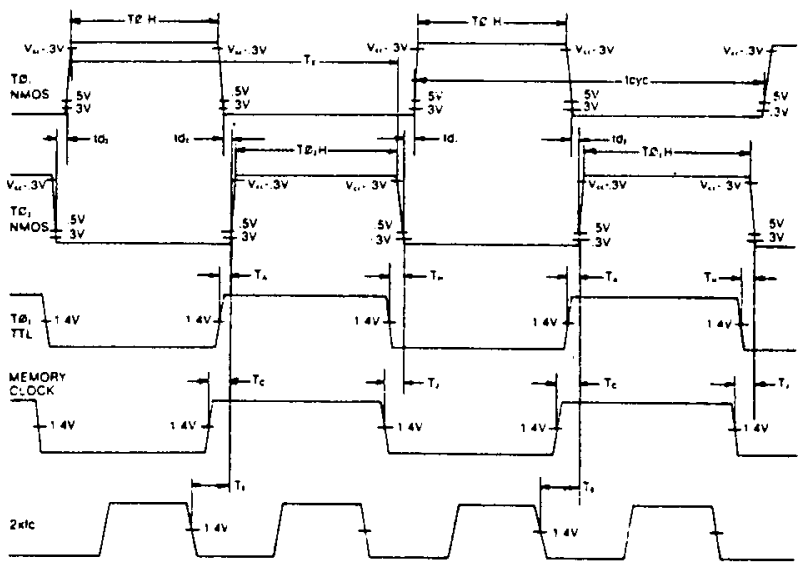

TEST CIRCUIT

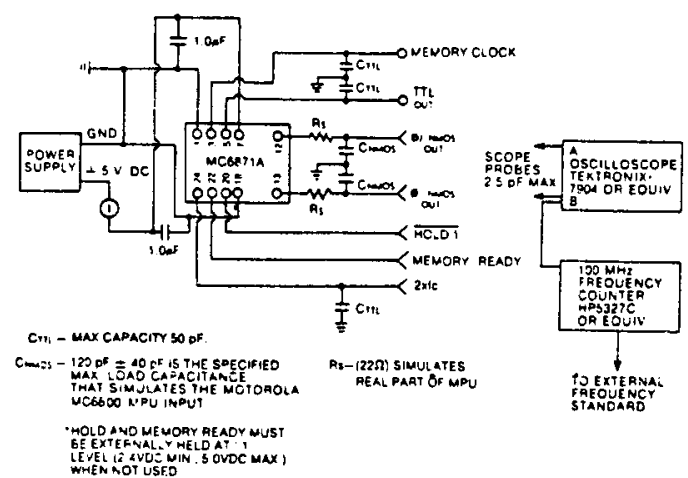

Reproduced with permission from Motorola, Inc., Phoenix, AZ 85008. 


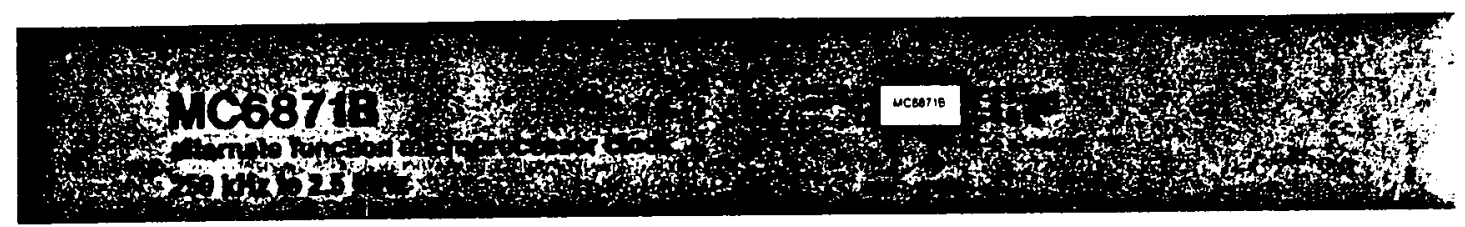

\section{specifications}

\begin{tabular}{|c|c|c|c|}
\hline Roting & 8ymbol & Valus & Unit \\
\hline Supply Voltage & $V_{\text {se }}$ & $5.00 \pm 5 \%$ & Vdc \\
\hline Operating Temperature Range & $T_{A}$ & $010+70$ & ${ }^{\circ} \mathrm{C}$ \\
\hline Storage Temperalure & $T_{\text {tin }}$ & -55 to +125 & ${ }^{\circ} \mathrm{C}$ \\
\hline Power Supply Drain (max.) & $I_{\text {m }}$ & 100 & $\mathrm{~mA}$ \\
\hline
\end{tabular}

ELECTRICAL CHARACTERISTICS $\left(V_{c e}=5.0 \pm 5 \%, V_{u}=0 . T_{A}\right.$ $=0^{\circ}$ to $70^{\circ} \mathrm{C}$, unless otherwise noted)

\begin{tabular}{|c|c|c|c|c|c|}
\hline Charactorialic & Bymbol & Min & Typ & $\max$ & $U$ \\
\hline \multicolumn{6}{|l|}{ Frequency } \\
\hline $\begin{array}{l}\text { Operating Frequency } \\
\text { Frequency stability (inclusive } \\
\text { of calibration tolerance at } \\
+25^{\circ} \mathrm{C} \text {, operating temperalure, } \\
\text { input voltage change, load } \\
\text { change, aging, shock and } \\
\text { vibration) }\end{array}$ & t. & .250 & \pm .01 & 2.5 & \\
\hline \multicolumn{6}{|c|}{ NMOS Outputs al 1.0 MHz Operation".." } \\
\hline $\begin{array}{l}\text { Pulse Width (meas at } \\
V_{c c}=-3 V \text { dc level) } \\
\end{array}$ & $\begin{array}{l}T \theta_{1} H \\
T \vartheta_{2} H \\
\end{array}$ & \begin{tabular}{|l|}
430 \\
450 \\
\end{tabular} & & & \\
\hline Logic Leveis & $\begin{array}{l}\text { Vac } \\
V_{\text {owc }}\end{array}$ & \begin{tabular}{|l|}
$V_{w-1}=.1$ \\
$V_{s c}=.3$
\end{tabular} & $\bar{z}$ & $\begin{array}{l}V_{u t}+.3 \\
V_{c s}+.1\end{array}$ & \\
\hline Rise and Fail Times & $\begin{array}{l}\mathrm{t} \\
\mathrm{t}_{1}\end{array}$ & \begin{tabular}{|l|}
5 \\
5 \\
\end{tabular} & $\begin{array}{l}12 \\
12 \\
\end{array}$ & $\begin{array}{r}50 \\
50 \\
\end{array}$ & \\
\hline $\begin{array}{c}\text { Overshoot/Undershoot } \\
\text { Logic "1" } \\
\text { Logic "0" }\end{array}$ & $V_{o s}$ & $\begin{array}{l}V_{\mathrm{cc}} \cdot .5 \\
V_{\mathrm{u}}-.5 \\
\end{array}$ & & $\begin{array}{l}V_{n}+.5 \\
V_{11}+.5 \\
\end{array}$ & \\
\hline $\begin{array}{l}\text { Pulse duration of any over- } \\
\text { shool or undershoot }\end{array}$ & $T_{\text {os }}$ & & & 40 & \\
\hline Period@0.3v dc Level & $t_{\mathrm{s}, \mathrm{c}}$ & & 1.00 & & \\
\hline Edge Timing @ $V_{c c}=0.3 V d c$ & $T x$ & 940 & & & \\
\hline $\begin{array}{l}\text { NMOS Relationshid } \\
@+0.5 \vee d c\end{array}$ & tal & $\begin{array}{l}0 \\
0 \\
\end{array}$ & & 8.0 & \\
\hline
\end{tabular}

\section{TTL Output:}

In rel. $10 \varnothing \varnothing_{2}$ NMOS @ $0.3 V$ dc

$\theta_{2} \pi \mathrm{TL} @ 1.4 \mathrm{~V} \mathrm{dc}$

\begin{tabular}{|c|c|c|c|c|c|}
\hline$\sigma_{2} \pi \mathrm{TL} @ 1.4 \mathrm{~V} d c$ & $T_{A}^{A}$ & $\begin{array}{l}15 \\
10\end{array}$ & $\begin{array}{l}30 \\
25\end{array}$ & $\begin{array}{l}45 \\
40\end{array}$ & $\begin{array}{l}\text { ns } \\
\text { ns }\end{array}$ \\
\hline$\varnothing_{2}$ Ungated @ 1.4V dc & $\begin{array}{l}T_{c} \\
T_{1}\end{array}$ & $\begin{array}{l}30 \\
20 \\
\end{array}$ & $\begin{array}{l}50 \\
40 \\
\end{array}$ & $\begin{array}{l}70 \\
60 \\
\end{array}$ & $\begin{array}{l}\mathrm{ns} \\
\mathrm{ns} \\
\end{array}$ \\
\hline $2 \times f c @ 1.4 \vee d c$ & $T_{1}$ & 40 & 80 & 120 & ns \\
\hline Logic Levele & $\begin{array}{l}V_{\alpha+1} \\
V_{\alpha}\end{array}$ & 2.4 & $\begin{array}{r}3.2 \\
.3 \\
\end{array}$ & .4 & $\begin{array}{l}\text { Vdc } \\
\text { Vdc }\end{array}$ \\
\hline \begin{tabular}{|c|} 
Rise and Fall Times \\
$.4 \mathrm{~V}$ and $2.4 \mathrm{~V}$ \\
$2.4 \mathrm{~V}$ and $.4 \mathrm{~V}$ \\
\end{tabular} & $\begin{array}{l}t \\
t \\
\end{array}$ & & & $\begin{array}{r}15 \\
15 \\
\end{array}$ & $\begin{array}{l}\text { ns } \\
\text { ns } \\
\end{array}$ \\
\hline Logic "0" Sink (/Gate) & la & & & -1.6 & $\mathrm{~mA}$ \\
\hline Logic "1" Source (/Gate) & Ion & & & +40 & UA \\
\hline Current Output Shorted & Ise & -18 & & -57 & $\mathbf{m A}$ \\
\hline
\end{tabular}

Current Output Shorted

Load

\begin{tabular}{|l|l|l|l|l|l|}
\hline NMOS-Load Capacity $\varnothing_{1}, \varnothing_{1}$ & $C_{\text {mas }}$ & 80 & 120 & 160 & pl \\
\hline
\end{tabular}

$\pi \mathrm{TL}-$ No. of Loads

TTL-Load Capacity

Logle Inputs" " "O" Level applies HOLO)

\begin{tabular}{|l|l|l|l|l|l|}
\hline Hoids $\emptyset_{1}$ NMOS 'High', $\emptyset_{2}$ \\
NMOS 'LOW', $\emptyset_{2}, \Pi L$ 'Low'
\end{tabular}

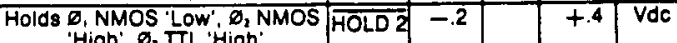
, TTL Migh

- Into specitied test loed

"Must be oxternally hald at " " " lovel (2.4v min., 5.0v max.) if not used

Appiy the following parameters for frequancies other than I MHE:

$T$ i.H $=0.5(P-140) n$

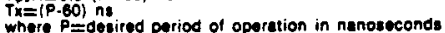

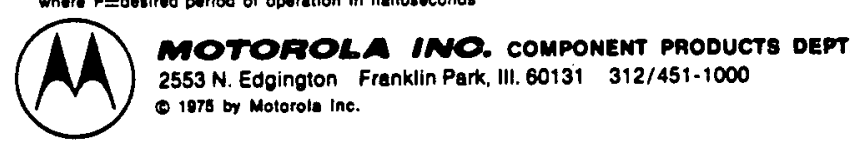

Franklin Park, III. $60131312 / 451-1000$

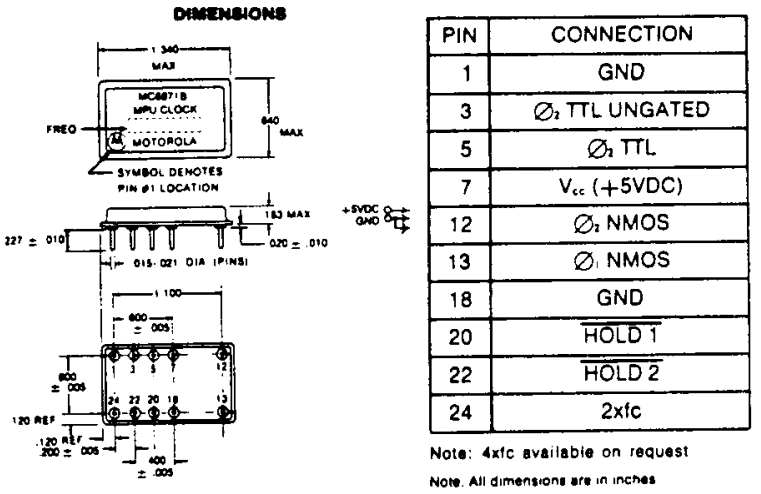

WAVEFoดM THINO.

ALL TIME IN MANOSECONOS

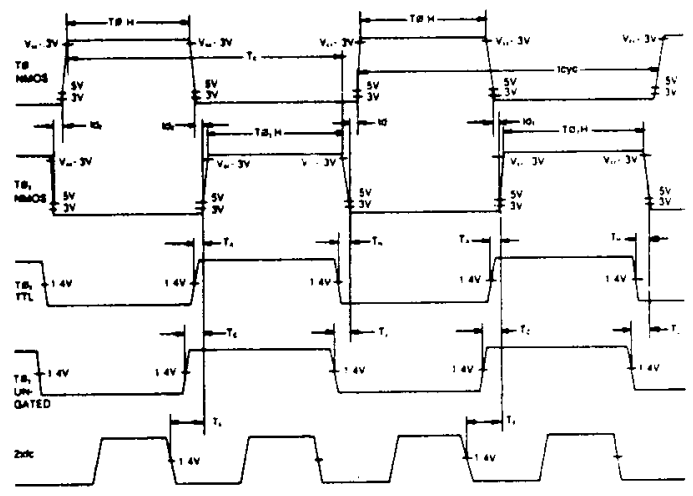

TET DLGRN

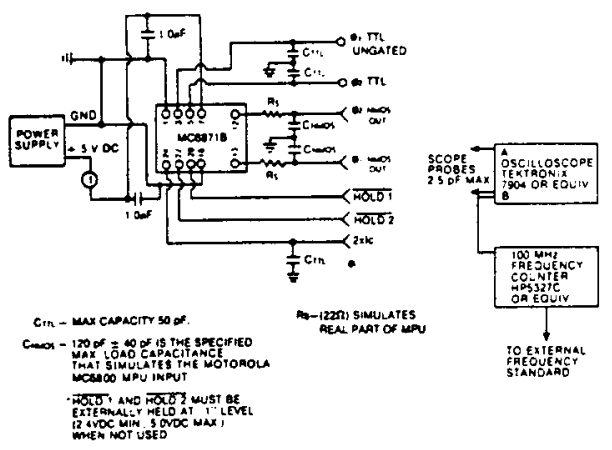
Soend moTOROLA are trademarke of Motorola, Inc. Printed in U.S.A. (750e). Meril

Reproduced with permission from Motorola, Inc., Phoenix, AZ 85008. 


\section{kos Counter Time-Base Circuit}

¿ Ion-implarited for ful! TTL/DTL compatibility

Internal ciock operates from:

External signal

External RC network

External crystal

] Operates $\mathrm{DC}$ to above $1 \mathrm{MHz}$

$\square$ Binary-encoded for frequency selection

\section{DESCRIPTION}

The NiK $5009 P$ is a highly versatile MOS oscillator and divicter chain manufactured by Mostek using its depletionload, ion-implantation process and $P$. - channel technology. The 16-pin DIP package provides frequency division ranges from 1 to $36 \times 10^{8}$. The circuit will operate from any of three frequency sources: the internal oscillator with an exiernal RC combination; the internal oscillator with an external crystal; or with an externally-applied TTL signal. Control inputs provide additional versatility and allow the circuit to be used in a variety of applications including instruments, timers, and clocks.

With an input frequency of 1
MHz, the MK $5009 \mathrm{P}$ provides the basic time periods necessary for most frequency measuring instruments, i.e., $1 \mu \mathrm{s}$ through 100 seconds. Oneminute, ten-minute, and onehour periods are also available using a $1 \mathrm{MHz}$ input. Using a $1 / 1.2 \mathrm{MHz}$ input, the MK $5009 \mathrm{P}$ can aiso provide a $50 / 60 \mathrm{~Hz}$ output for accurate generation of line frequencies in portable instruments or clocks.

The time-base output (TIME OUT) is a square wave, its frequency determined by the selected counter division, and by the oscillator frequency or external input. The falling edge of the output square wave should be used to control external gating. circuitry.

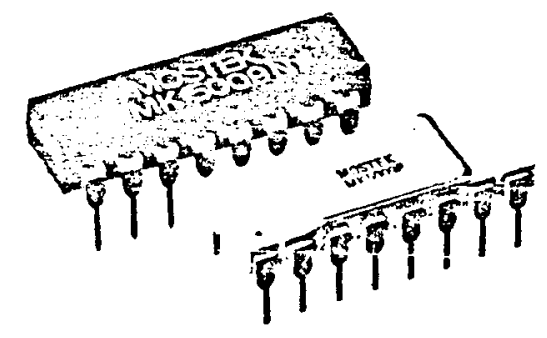

FUNCTIONAL DIAGRAM

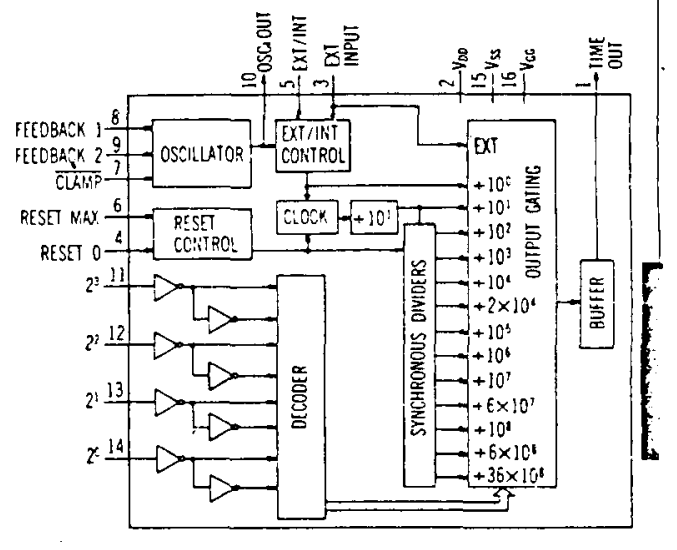

TIME OUT

\begin{tabular}{|c|c|c|c|c|c|c|c|c|c|}
\hline \multicolumn{4}{|c|}{ ADDRESS INPUTS } & \multirow{2}{*}{$\begin{aligned} \text { WITHOUT RESET } \\
R_{\operatorname{MAx}}=0 \\
R_{\odot}=0\end{aligned}$} & \multicolumn{2}{|c|}{ RESET } & \multicolumn{3}{|c|}{ BYPASS MODES (see page 3) } \\
\hline $2^{2}$ & 22 & 21 & $2^{\circ}$ & & $\begin{array}{l}\text { Reset Max. } \\
R_{\text {MAx }}=1 \\
R_{0}=0\end{array}$ & $\begin{array}{l}\text { Reset Min. } \\
R_{\max }=0 \\
R_{0}=1\end{array}$ & $\begin{aligned} \text { Mode } 1 & \\
R_{\operatorname{MAx}} & =V_{G G} . \\
R_{0} & =0\end{aligned}$ & $\begin{aligned} & \text { Mode } 2 \\
& R_{\text {max }}=0 \\
& R_{0}=V_{G G}\end{aligned}$ & $\begin{array}{c}\text { Mode } 3 \\
R_{\text {MAX }}=V_{G G} \\
R_{0}=V_{G G}\end{array}$ \\
\hline 0 & 0 & 0 & 0 & \multirow{2}{*}{$\begin{array}{l}\div 10^{\circ} \\
\div 10^{\prime}\end{array}$} & $\div 10^{\circ}$ & $\div 10^{\circ}$ & $\div 10^{\circ}$ & $\div 10^{\circ}$ & $\div 10^{\circ}$ \\
\hline 0 & 0 & 0 & 1 & & \multirow{3}{*}{ Resets } & \multirow{3}{*}{ Resets } & $\div 10^{\prime}$ & $\div 10^{\prime}$ & $\div 10^{1}$ \\
\hline 0 & 0 & 1 & 0 & $\div 10^{2}$ & & & $\div 10^{2}$ & $\div 10^{2}$ & $\div 10^{2}$ \\
\hline 0 & 0 & 1 & 1 & $\div 10^{3}$ & & & $\div 10^{3}$ & $\div 10^{3}$ & $\div 10^{3}$ \\
\hline 0 & 1 & 0 & 0 & $\div 10^{4}$ & \multirow[t]{2}{*}{ Counters } & \multirow[t]{2}{*}{ Counters } & $\div 10^{4}$ & $\div 10^{4}$ & $\div 10^{4}$ \\
\hline 0 & 1 & 0 & 1 & $\div 10^{5}$ & & & $\div 10^{2}$ & $\div 10^{5}$ & $\div 10^{2}$ \\
\hline 0 & 1 & 1 & 0 & $\div 10^{6}$ & \multirow[t]{2}{*}{ to their } & \multirow[t]{2}{*}{ to their } & $\div 10^{3}$ & $\div 10^{\circ}$ & $\div 10^{3}$ \\
\hline 0 & 1 & 1 & 1 & $\div 10^{7}$ & & & $\div 10^{4}$ & $\div 10^{7}$ & $\div 10^{4}$ \\
\hline 1 & 0 & 0 & 0 & $\div 10^{8}$ & \multirow[t]{2}{*}{ Highest } & \multirow[t]{2}{*}{ Lowest } & $\div 10^{5}$ & $\div 10^{5}$ & $\div 10^{2}$ \\
\hline 1 & 0 & 0 & 1 & $\div 6 \times 10^{7}$ & & & $\div 6 \times 10^{4}$ & $\div 6 \times 10^{4}$ & $\div 6 \times 10^{\prime}$ \\
\hline 1 & 0 & 1 & 0 & $\div 36 \times 10^{8}$ & \multirow[t]{3}{*}{ States } & \multirow[t]{3}{*}{ States } & $\div 36 \times 10^{5}$ & $\div 36 \times 10^{5}$ & $\div 36 \times 10^{2}$ \\
\hline 1 & 0 & 1. & 1 & $\div 6 \times 10^{8}$ & & & $\begin{array}{c}\div \quad 6 \times 10^{5} \\
-\end{array}$ & $\begin{array}{c}\div \quad 6 \times 10^{5} \\
-\end{array}$ & $\div 6 \times 10^{2}$ \\
\hline 1 & 1 & 1 & 0 & \multirow{2}{*}{$\begin{array}{l}\div 2 \times 10^{4} \\
\text { Ext. In. }\end{array}$} & & & $\div 2 \times 10^{1}$ & $\div 2 \times 101$ & $\div 2 \times 10^{\prime}$ \\
\hline 1 & 1. & 1 & 1 & & Ext. In. & Ext. In. & Ext. Int. & Ext. Int. & Ext, Int. \\
\hline
\end{tabular}

-Addresses 1100 and 1101 result in Logic 0 at the output regardless of the state of the Reset Max. and Reset 0 inputs. Logic $1=$ High $=V_{s s}$

Logic $0=$ LOW $=V_{D O}$ 


\section{ABSOLUTE MAXIMUM RATINGS}

Voltage on Any Terminal Relative to $V_{s s}$ Operating Temperature Range (Ambient)

Storage Temperature Range (Ambient)

$-0.3 \mathrm{~V}$ to $-20 \mathrm{~V}$

$0 . \mathrm{C} 10+70 \cdot \mathrm{C}$

$-55 \mathrm{C} 10+150^{\circ} \mathrm{C}$

\section{RECOMMENDED OPERATING CONDITIONS}

$\left(0^{\circ} \mathrm{C} \leq T_{\triangle} \leq 70^{\circ} \mathrm{C}\right)$

\begin{tabular}{|c|c|c|c|c|c|c|}
\hline & PARAMETER & MIN & TYP & MAXX & UNITS & NOTES \\
\hline$V_{s s}$ & Supply Voltage & $\div 4.5$ & & +5.5 & V & \\
\hline$V_{C D}$ & Supply Voltage & 0.0 & & 0.0 & $v$ & \\
\hline$V_{E G}$ & Supply Voltage & -9.6 & & -14.4 & v & \\
\hline$f_{X T A L}$ & Crystal Frequency & 0.1 & & 2.0 & $\mathrm{MHz}$ & \\
\hline$t_{R C}$ & RC Frequency & $D C$ & & 200 & $\mathrm{kHz}$ & \\
\hline$f_{\mathrm{EXT}}$ & External Frequency & $D C$ & & 2.0 & $\mathrm{MHz}$ & \\
\hline \multirow[t]{2}{*}{$t_{P L}$} & Logic O Pulse Width. $\overline{\text { CLAMP }}$ & - & & & & Note 5 \\
\hline & Ext. Input & 200 & & & nsec & \\
\hline \multirow[t]{3}{*}{$t_{P H}$} & Logic 1 Pulse Width. Ext. Input & 200 & & & nsec & \\
\hline & Reset Max & 10.0 & & & $i \mathrm{sec}$ & \\
\hline & Reset 0 & 10.0 & & & "sec & \\
\hline R & Feedback Resistance & .01 & & 2.5. & M!? & Fig. 1 \\
\hline$V_{12}$ & Input Voltage, Logic 0 . Reset Inputs & 0.0 & & 0.8 & $v$ & \\
\hline & $\begin{array}{l}\text { Reset (Bypass Mode) } \\
\text { All Other Logic Inputs }\end{array}$ & $V_{G G}$ & & $\begin{array}{c}v_{G G}+1.0 \\
0.8\end{array}$ & $v$ & Note 2 \\
\hline$V_{\mathbb{I H}}$ & Input Voltage, Logic 1, All Logic Inputs & $V_{s s}-1.0$ & $v_{s s}$ & $v_{s 5}+0.3$ & $v$ & \\
\hline
\end{tabular}

ELECTRICAL CHARACTERISTICS

$\left.3 V_{S S}=+5 \mathrm{~V}=10 \%: V_{D D} \quad 0 \mathrm{~V}: V_{G G} \quad-12.0 \mathrm{~V}-20 \%: 0 \mathrm{C} \leq \mathrm{T}_{A} \leq 70 \mathrm{C}\right)$

\begin{tabular}{|c|c|c|c|c|c|c|}
\hline 1 & PARAMETER & MIN & TYP & MAX & UNITS & NOTES \\
\hline $\begin{array}{l}l_{S S} \\
l_{G G}\end{array}$ & $\begin{array}{l}\text { Supply Current, } V_{S S} \\
\text { Supply Current. } V_{G G}\end{array}$ & & $\begin{array}{l}6.0 \\
6.0\end{array}$ & $\begin{array}{l}11.0 \\
11.0 \\
\end{array}$ & $m A$ & Note 1 \\
\hline$I_{I L}$ & Input Current, Logic 0 & & & -1.6 & $m A$ & $\begin{array}{l}\text { Note 2: } \\
V_{1}=0.4 \mathrm{~V}\end{array}$ \\
\hline $\begin{array}{l}V_{O L} \\
V_{O H}\end{array}$ & $\begin{array}{l}\text { Output Voltage, Logic } 0 \\
\text { Output Voltage. Logic } 1\end{array}$ & 2.4 & & 0.4 & $\begin{array}{l}V . \\
V\end{array}$ & $\begin{array}{l}\mathrm{I}_{\mathrm{OL}}=1.6 \mathrm{~mA} \\
\mathrm{I}_{\mathrm{OH}}=-4 \mathrm{O}^{\prime} \mathrm{A}\end{array}$ \\
\hline$f_{\text {STA }}$ & $\begin{aligned} \text { Frequency Stability wi Volt. Change, RC Mode } \\
\text { / Temp. Change. RC Mode } \\
\text { Crystal Mode }\end{aligned}$ & & $\begin{array}{r} \pm 3.0 \\
-0.2 \\
-\end{array}$ & & $\begin{array}{l}\% / \mathrm{V} \\
\% / \mathrm{C}\end{array}$ & $\begin{array}{l}\text { Note } 3 \\
\text { Note } 4\end{array}$ \\
\hline t. & Jitter, Edge-to-Edge Variation & & $<15$ & & nsec & $\begin{array}{l}\text { Temp. \& Sup- } \\
\text { ply Voltage } \\
\text { - Constant }\end{array}$ \\
\hline
\end{tabular}

-Tyoical values at $V_{S S}=+5 \mathrm{~V}, V_{C S}=\mathrm{OV} . V_{G G}=-: 2 \mathrm{~V}$. and $T . \ldots 25: \mathrm{C}$

1. Logic inpuls at $V_{s}$. Output open elicuited Each logic inful (see Nole 2) contribules an acditional $1.6 \mathrm{mh}$ (max.) lo lss when at logic 0

2 Legic Inpuis are: Reset Max: Resel O; Address Infuts: Ext. Input: ExI Int Seiecl: ano Clamp

3. Fiequency variations cue to Doxier sucoly changes only

4. Ciystat moce stabili:y is dependent upon ciystal.

5 Namum logie 0 time at clamp input is $50 \%$ of oseiliato: perioc.

- You, Voc apply only to Time Out 


\section{DESCFIPTION OF OPERATION}

The MK $5009 P$ consists basically of a series of counters. selectable via an internal multiplexer. The $\div 10^{\prime}$ counter output is used to generate an internal clock signal for the $10^{2}$ through $36 \times 10^{8}$ counter stages, which are fully synchronous with each other.

\section{OSCILLATOR CONTROLS}

Ooeration in the RC oscillator mode is achieved as shown in Figure 1. Frequency, $f$ is approximately 0.8 RC. The clamp circuit can be used in the RC mode to provide one-shot or accurate start-up operations. When Clamp goes io a iogic 0 . the internal circuitry is held at a reference level so that upon release of the Clamp (return to logic 1). the oscillator's first cycle will be a full cycle.

The ensta! oscillator mode is shown in Figure 2. Values for the resistors are chosen to bias the internal circuitry for optimum performance. The two capacitors are chosen to provide the loading capacitance $\left(C_{L}\right)$ specified for the selected crystal. It is recommended that $\mathrm{C} 1=\mathrm{C} 2=2 \mathrm{C}_{1}$.

\section{RESET/BYPASS CONTROLS}

The MK 5009 P provides two different reset conditions. A positive-going pulse of 10 :s or longer on Reset 0 will reset counters to their towest state. While a positive-going pulse at Reset Max will resei counters to their highest state. The Reset Max control enables the user to set up the counters to provide a falling edge at the next oscillator cycle or negative-going external input, regardless of which divider chain is selected.

In addition, taking one or both Reset Inputs to the most negative voltage. $V_{G G}$, allows bypassing portions of the divider chain for testing or other purposes (see table on page 1).

\section{EXTERNAL/INTERNAL FREQUENCY SOURCE}

When using an external signal source to operate the MK 5009 P, that signal should be applied at the External Input (Pin 3 ), and the External/Internal Select (Pin_5) should be brought to togic 1 .

For operation with an internal signal, the External Internal Select should be àt logic.0.

\section{OSCILLATOR OUTPUT}

The oscillator output, provided at $P$ in 10 , is not a true logic output, but may be used to drive a high impedance device such as a junction FET or other MOS circuitry.

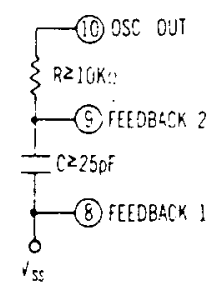

FIG. 1

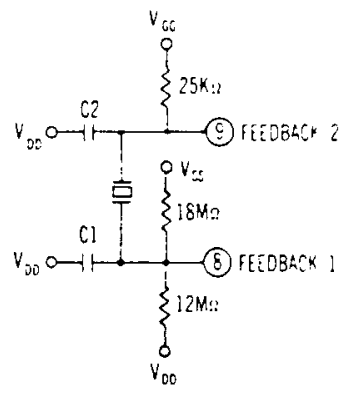

itive. 2

\section{PIN CONNECTIONS}
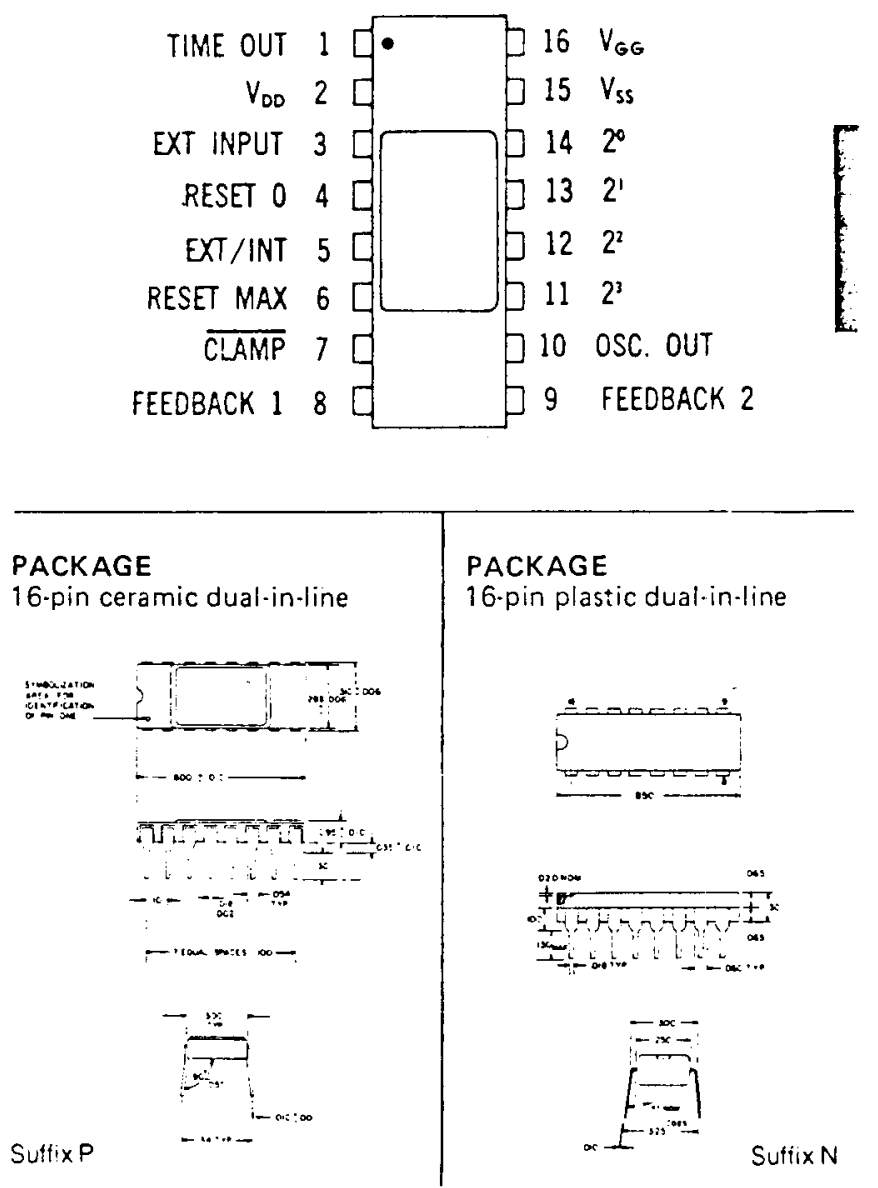

PACKAGE

16-pin plastic dual-in-line
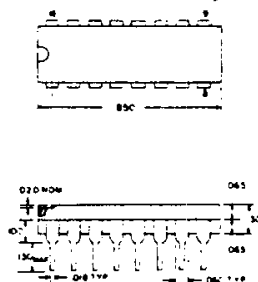

$=-\infty x$<smiles>c1ccncc1</smiles>

Sutitix $N$ 


\section{APPLICATION - $10 \mathrm{mHz}$ Frequency Counter}

The circuit shown below is a frequency counter capable of counting input rates up to $10 \mathrm{MHz}$. selected in four ranges. The MK 5009 P provides the time base intervals while the Mostek MK 5002 P counter circuit provices counting: storage, and display furictions. Two decades of prescaling using TTL are employed. TTL one-shots provide proper timing for the 5002 .

To replace the functions of the MK $5009 \mathrm{P}$, an active device and Schmitt trigger for the crystal osciltator would be needed, plus six 7490 's to achieve the correct time out. Replacing the functions of the MK 5002 would require four $7490^{\prime} \mathrm{s}$, four 7475 's, and four BCDto-seven-segment decoders.

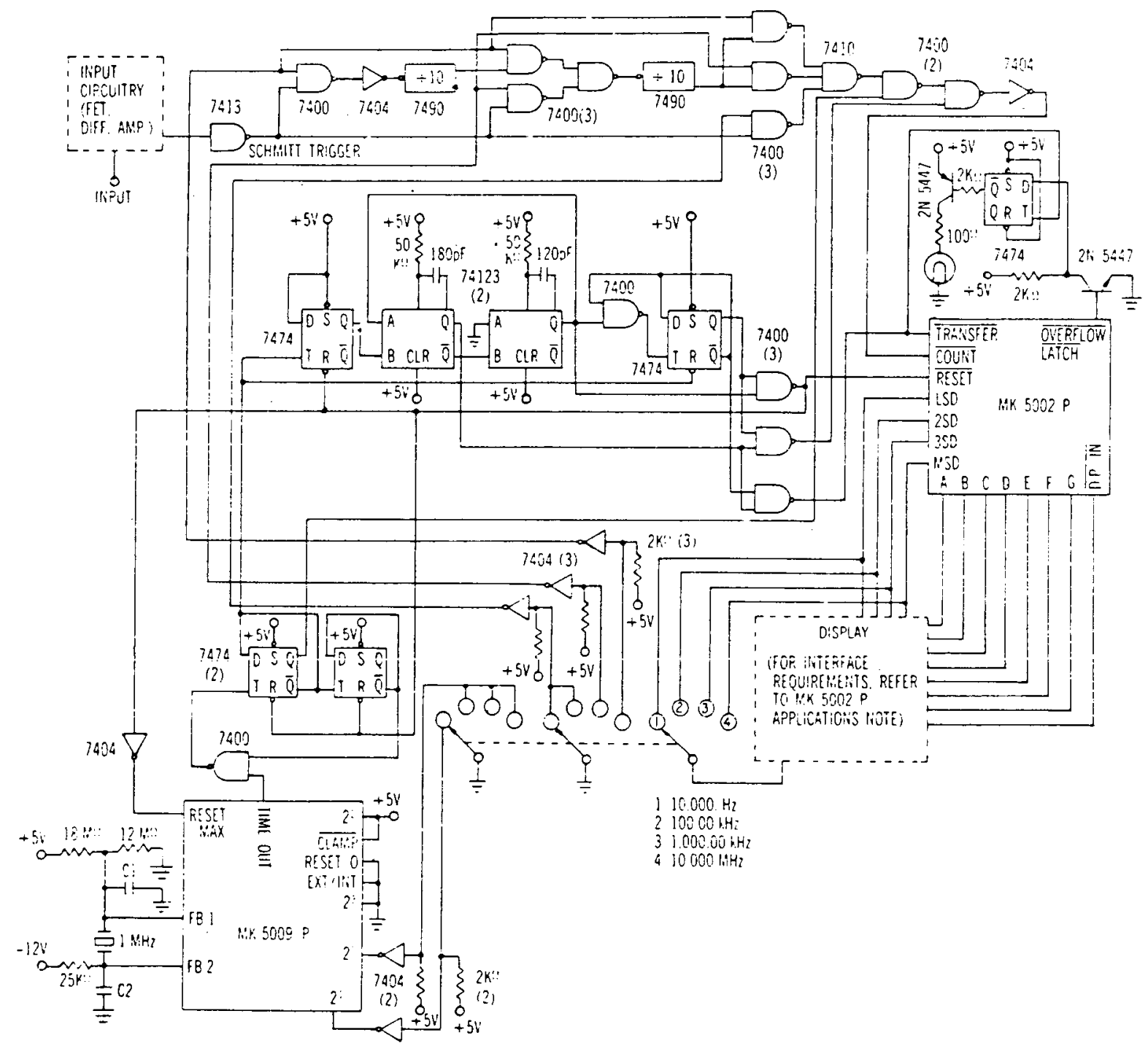

Reproduced with permission from Mostek, Carrollton, TX 75006. 


\section{SOLID STATE NUMERIC INDICATOR $5082-7430$ (7 Segment Monolithic) SERIES}

\section{Features}

- MOS COMPATIBLE

- Can be Driven Directly from many MOS Circuits

- LOW POWER

- Excellent Readability at Only $0.5 \mathrm{~mW}$ per Segment

- constRucted for stRobed OPERATION

- Minimizes Lead Connections

- STANDARD DIP PACKAGE

- End Stackable

- Integral Red Contrast Filter

- Rugged Construction

- CATEGORIZED FOR LUMINOUS INTENSITY

- Assures Uniformity of Light Output from Unit to Unit within a Single Category
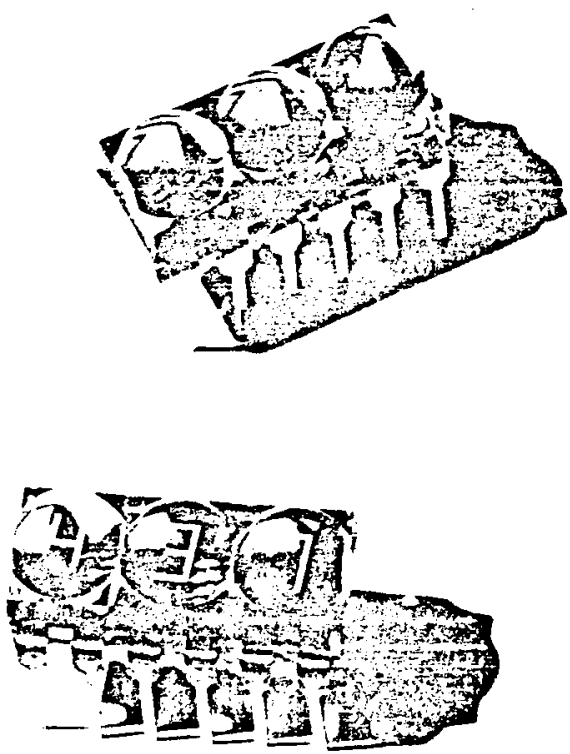

ters have the standard lower right hand decimal points.

Applications include hand-held calculators, portable instruments, digital thermometers, or any other product requiring low power, low cost, minimum space, and long lifetime indicators.

\section{Device Selection Guide}

\begin{tabular}{|c|c|c|c|}
\hline \multirow{2}{*}{$\begin{array}{c}\text { Digits per } \\
\text { Cluster }\end{array}$} & \multicolumn{2}{|c|}{ Configuration } & \multirow{2}{*}{ Part Number } \\
\hline & Device & Package & \\
\hline & $\theta$ & (Figure 5) & $5082 \cdot 7432$ \\
\hline 3 & $7 \mathrm{~B}$ & (Figure 5) & 5082.7433 \\
\hline
\end{tabular}




\section{Absolute Maxinnum Ratings}

\begin{tabular}{|c|c|c|c|c|}
\hline Paramezer & Symbol & Min. & Max. & Units \\
\hline Peak Forward Current per Segment or dp (Duration <500 $\mu s$ ) & IPEAK & & 50 & $\overline{\mathrm{mA}}$ \\
\hline Average Current per Segment or $d p$ & IAVG & & 5 & $\operatorname{mA}$ \\
\hline Poiver Dissipation per Digit [1] & $\overline{P_{D}}$ & & 80 & $\mathrm{~mW}$ \\
\hline Operating Temperature, Ambient & $T_{A}$ & -40 & 75 & ${ }^{\circ} \mathrm{C}$ \\
\hline Storage Temperature & $T_{5}$ & -40 & 100 & ${ }^{\circ} \mathrm{C}$ \\
\hline Reverse Voltage & $V_{R}$ & & 5 & $\mathrm{~V}$ \\
\hline Solder Temperature $1 / 16^{\prime \prime}$ below seating plane $(t \leqslant 5 \mathrm{sec} .)^{[2]}$ & & & 230 & ${ }^{\circ} \mathrm{C}$ \\
\hline
\end{tabular}

VOTES: 1. Derate linearly@1mW/ C above $25^{\circ} \mathrm{C}$ ambient. 2. See Nechanical section for recommended flux removal solvents.

\section{Electrical/Optical Characteristics at $\mathrm{T}_{\mathrm{A}}=25^{\circ} \mathrm{C}$}

\begin{tabular}{|l|l|l|c|c|c|c}
\hline Parameter & Symbol & Test Condition & Min. & Typ. & Max. & Units \\
\hline Luminous Intensity/Segment or $d p(3)$ & $L^{(3)}$ & $\begin{array}{l}I_{A V G}=500 \mu \mathrm{A} \\
\left(I_{P K}=5 \mathrm{~mA}\right. \\
d u t y c y c l e=10 \%)\end{array}$ & 10 & 40 & $\mu c d$ \\
\hline Peäk Wavelength & $\lambda_{\text {peak }}$ & & & 655 & & $\mathrm{~nm}$ \\
\hline Forward Voltage/Segment or dp & $V_{F}$ & $I_{F}=5 \mathrm{~mA}$ & & 1.55 & 2.0 & $\mathrm{~V}$ \\
\hline Reverse Current/Segment or dp & $I_{R}$ & $V_{R}=5 \mathrm{~V}$ & & & 100 & $\mu \mathrm{A}$ \\
\hline Rise and Fall Time & & & 10 & & $\mathrm{~ns}$ \\
\hline
\end{tabular}

NOTES: 3. The digits are categorized for luminous intensity. Intensity categories are designated by a letter located on the back side of the package. 4. Time for a $10 \%-90 \%$ change of light intensity for step change in current.

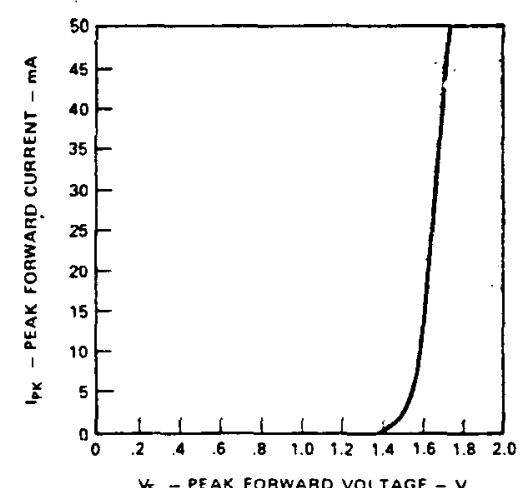

$V_{F}$ - PEAK FORWARO VOLTAGE - V

Figure 1. Peak Forward Current ws.

Peak Forward Voleage

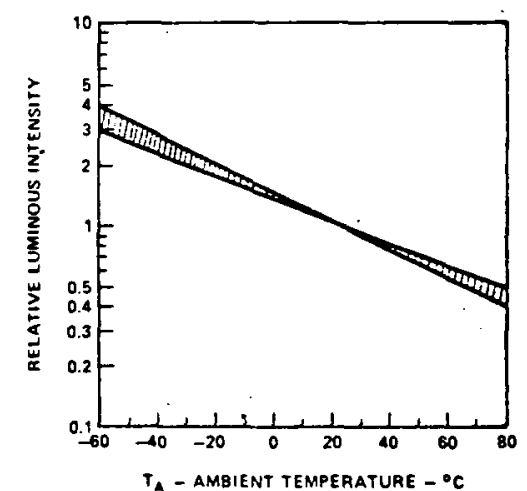

Figure 3. Relative Luminous Intensity v. Ambiem Temperature at Fixed Current Lovel

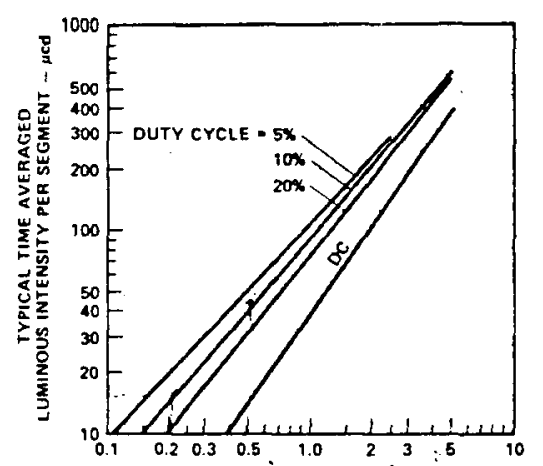

lavg - average cuaraent per segment - má

Figure 2. Typical Time Averaged Luminous Intensity per Segment vs. A verage Current per Segment

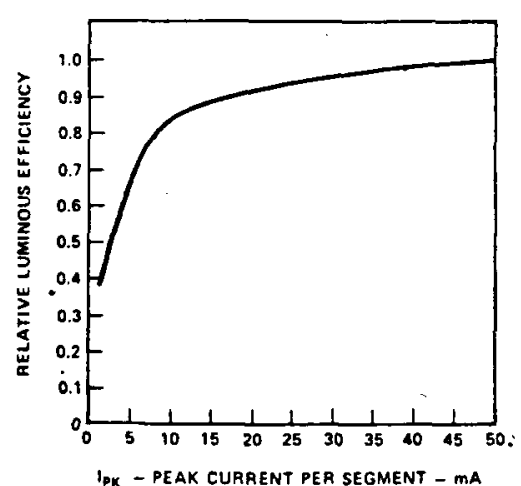

Figure 4. Relative Luminous Efficiency vs. Peak Current per Segment 
Package Description

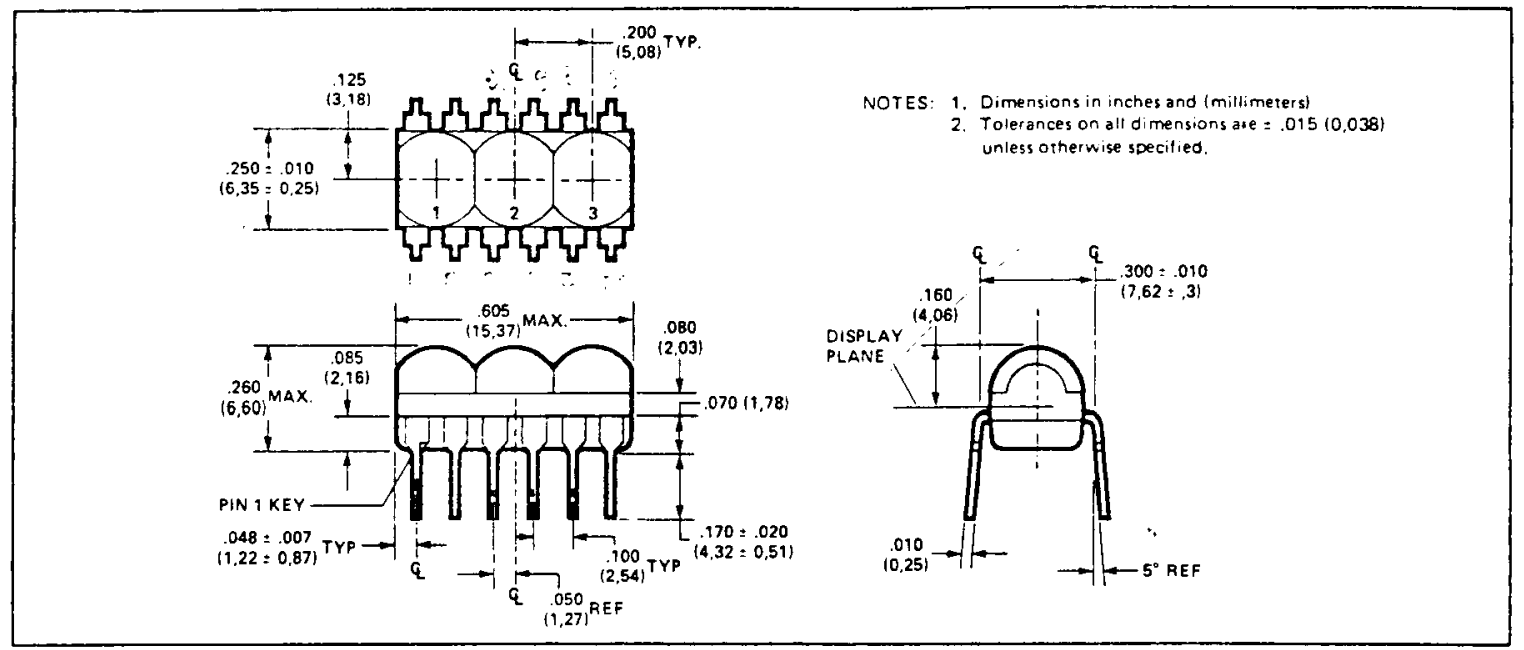

Figure 5.

\section{Magnified Character Font Description}

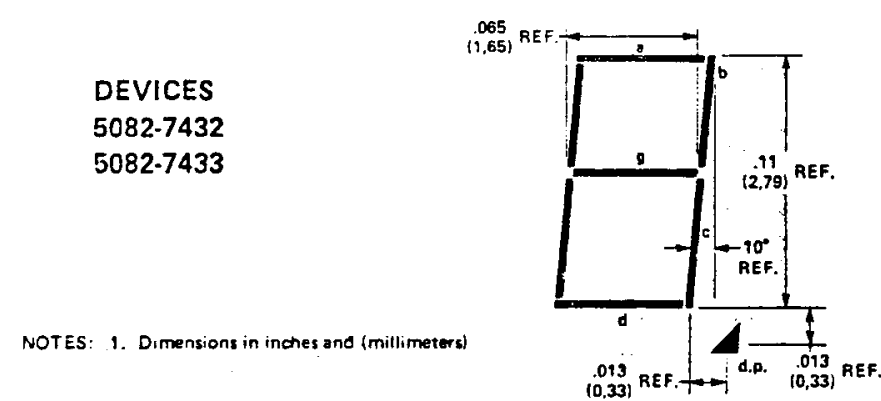

Figure 6.

Device Pin Description

\begin{tabular}{|c|c|c|}
\hline $\begin{array}{c}\text { PIN } \\
\text { NUMBER }\end{array}$ & $\begin{array}{c}5082-7432 \\
\text { FUNCTION }\end{array}$ & $\begin{array}{c}5082-7433 \\
\text { FUNCTION }\end{array}$ \\
\hline 1 & N/C & CATHODE 1 \\
\hline 2 & ANODE $\mathrm{e}$ & ANODE $\mathrm{C}$ \\
\hline 3 & ANODE d & ANODE d \\
\hline 4 & CATHODE 2 & CATHODE 2 \\
\hline 5 & ANODE $\mathrm{c}$ & ANODE $\mathrm{c}$ \\
\hline 6 & ANODE dp & ANODE dp \\
\hline 7 & CATHODE 3 & CATHODE 3 \\
\hline 8 & ANODE b & ANODE b \\
\hline 9 & ANODE $\mathrm{g}$ & ANODE g \\
\hline 10 & ANODE a & ANODE a \\
\hline 11 & ANODE $\mathrm{f}$ & ANODE $\mathrm{f}$ \\
\hline 12 & N/C & N/C \\
\hline
\end{tabular}

Reproduced with permission from Hewlett-Packard, Palo Alto, CA 94304. 


\section{Electrical/Optical}

The $5082-7430$ series devices utilize a monolithic GaAsP chip of 8 common cathode devices for each display digit. The segment anodes of each digit are interconnected, forming an $\delta$ by $\mathrm{N}$ line array, where $\mathrm{N}$ is the number of characters in the display. Each chip is positioned under an integrally molded lens giving a magnified character height of 0.11 inches. Satisfactory viewing will be realized within an angle of approximately $\pm 20^{\circ}$ from the centerline of the digit.

Character encoding on the $5082-7430$ series devices is performed by standard 7 segment decoder/driver circuits. Through the use of strobing techniques only one decoder/driver is required for very long multidigit displays.

A discussion of display circuits and drive techniques appears in Application Note 946.

\section{Mechanical}

The $5082-7430$ series package is a standard 12 Pin DIP consisting of a plastic encapsulated lead frame with integrally molded lenses. It is designed for plugging into DIP sockets or soldering into PC boards. Alignment problems are simplified due to the clustering of digits in a single package.

To imiprove display contrâst, the plastic encápsulant contains a red dye to reduce the reflected ambient light.

The devices can be soldered for up to 5 seconds at a maximum solder temperature of $230^{\circ} \mathrm{C}\left(1 / 16^{\prime \prime}\right.$ below the seating planel. The plastic encapsulant used in these displays may be damaged by some solvents commonly used for flux removal. It is recommended that only Freon TE, Freon TE-35, Freon TF, Isopropanol, or soap and water be used for cleaning operations.

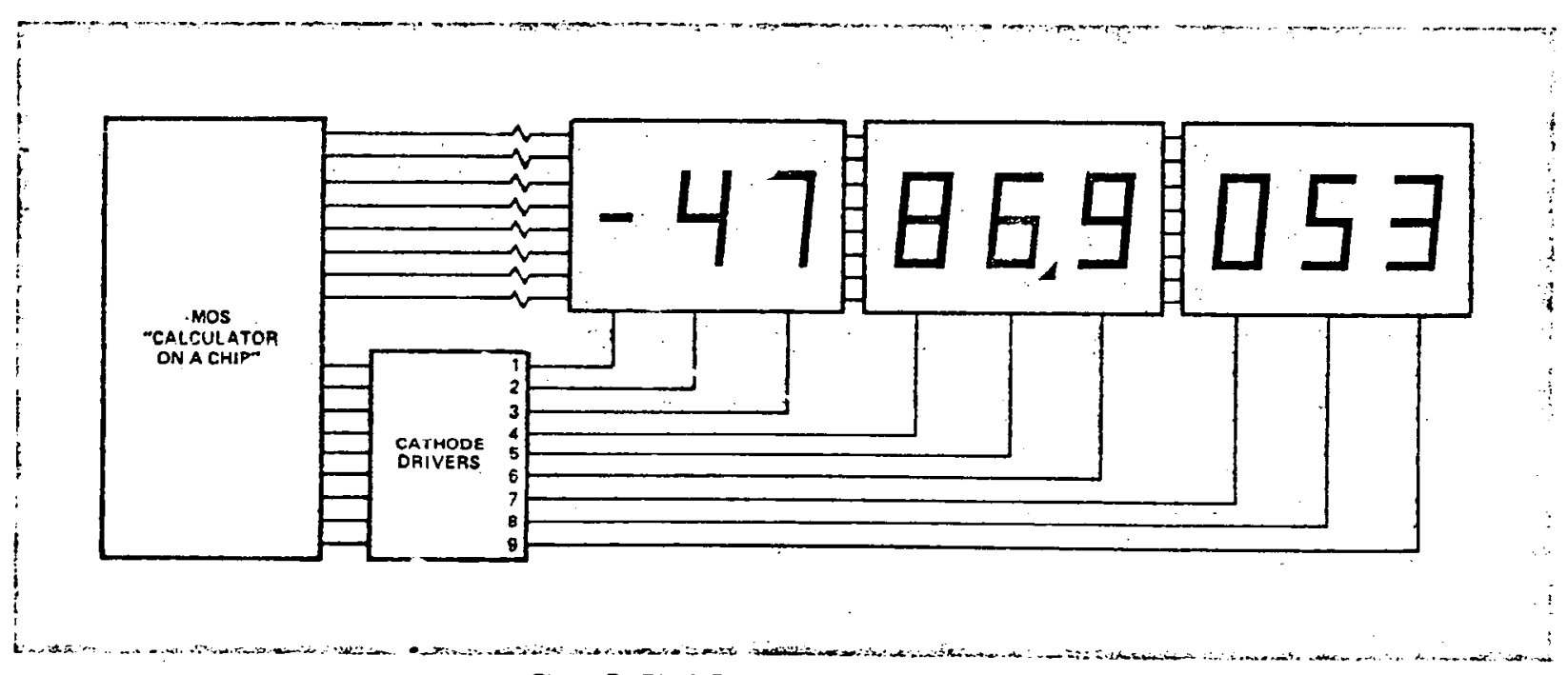

Figure 7. Block Diagram for Calculator Display 


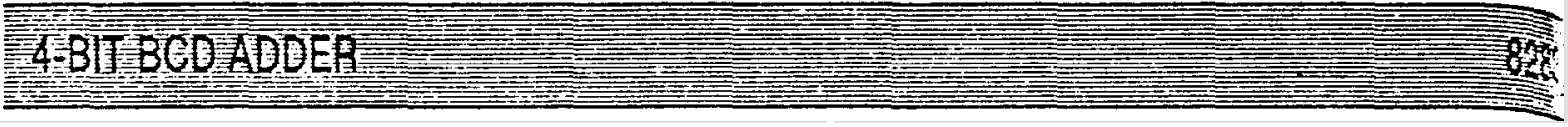

\section{SPEED/PACKAGE AVAILABILITY}

\section{DESCRIPTION}

The $82 S 83$ 4-bit binary coded (BCD) adder is a high speed -Schottky MSI circuit that has been designed for easy systems usage. This unit produces the $B C D$ sum of two decimal numbers presented in the 8-4-2-1 weighted BCD format. Carry-in and carry-out terms are provided for easy expansion to any number of decades. The $82 S 83$ BCD adder has been designed such that input and output logic levels including the carry are in their true logic form.

Compared to cumbersome hardware implementations previously at the designer's disposal that consist of binary addition followed by decimal correction, the $82 S 83$ BCD adder generates the BCD carry terms internally in the look-ahead mode and does $B C D$ addition directly. For valid $B C D$ numbers $(0$ through 9 ) at the $A$ and $B$ inputs the $B C D$ sum is formed at the output. If addition $(A+B+C I N)$ would yield a number greater than 9 , a valid $B C D$ number and a carry result.

Input codes above 9 are not defined except for binary to $B C D$ conversion. Binary to $B C D$ conversion is obtained by applying any 4-bit binary number to the $A N$ or $B N$ inputs while the remaining inputs are grounded. For input codes' 0 through 9 a $B C D$ number result at the output is usual. If binary inputs 10 through 15 are applied a carry term is generated and the carry output tooether with the sum out are the BCD equivalent of the binary input. Conversion of binary numbers greater than 16 can be achieved by cascading $82 S 83$ 's.

Subtraction can be done with the 82583 by using 9's complement adoition. Rather than implementing a 9's complement circuit with gates or ROM's, the $82 S 83$ BCD arithmetic unit should be-us^d. The 82583 incorporates the 9's complement feature and pertorms $B C D$ addition, $B C D$ subtraction, and number comparison.

\section{SWITCHING CHARACTERISTICS}

$T A=25^{\circ} \mathrm{C}$ and $\mathrm{VCC}=5.0 \mathrm{~V}$

\begin{tabular}{|c|c|c|c|c|c|}
\hline \multirow{2}{*}{\multicolumn{2}{|c|}{ PARAMETER }} & \multicolumn{4}{|c|}{ LIMITS } \\
\hline & & MIN. & TYP. & MAX. & UNITS \\
\hline \multicolumn{2}{|c|}{ Turn-On $/$ Turn-Off Delays } & & & & \\
\hline Any AN, BN, Cin & ion & & 20 & 35 & ns \\
\hline to SN & toff & & 20 & 35 & ns \\
\hline Any $\mathrm{AN}, \mathrm{BN}$, to & ton & & 33 & 40 & ns \\
\hline Cout & toff & & 17 & 25 & ns \\
\hline \multirow[t]{2}{*}{ Cin to Cout } & ton & & 17 & 25 & ns \\
\hline & toft & & 10 & 15 & ns \\
\hline
\end{tabular}

\section{PIN CONFIGURATION}

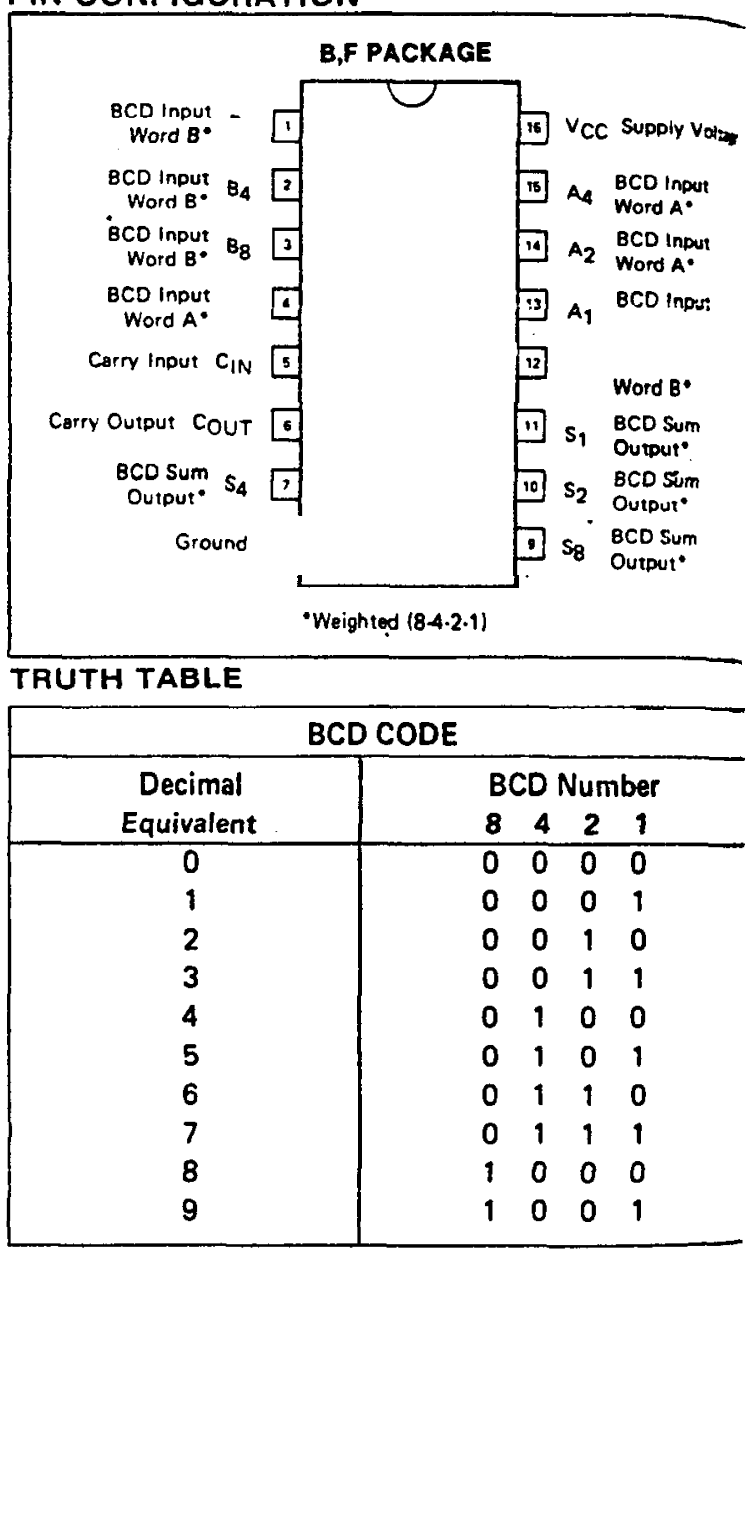




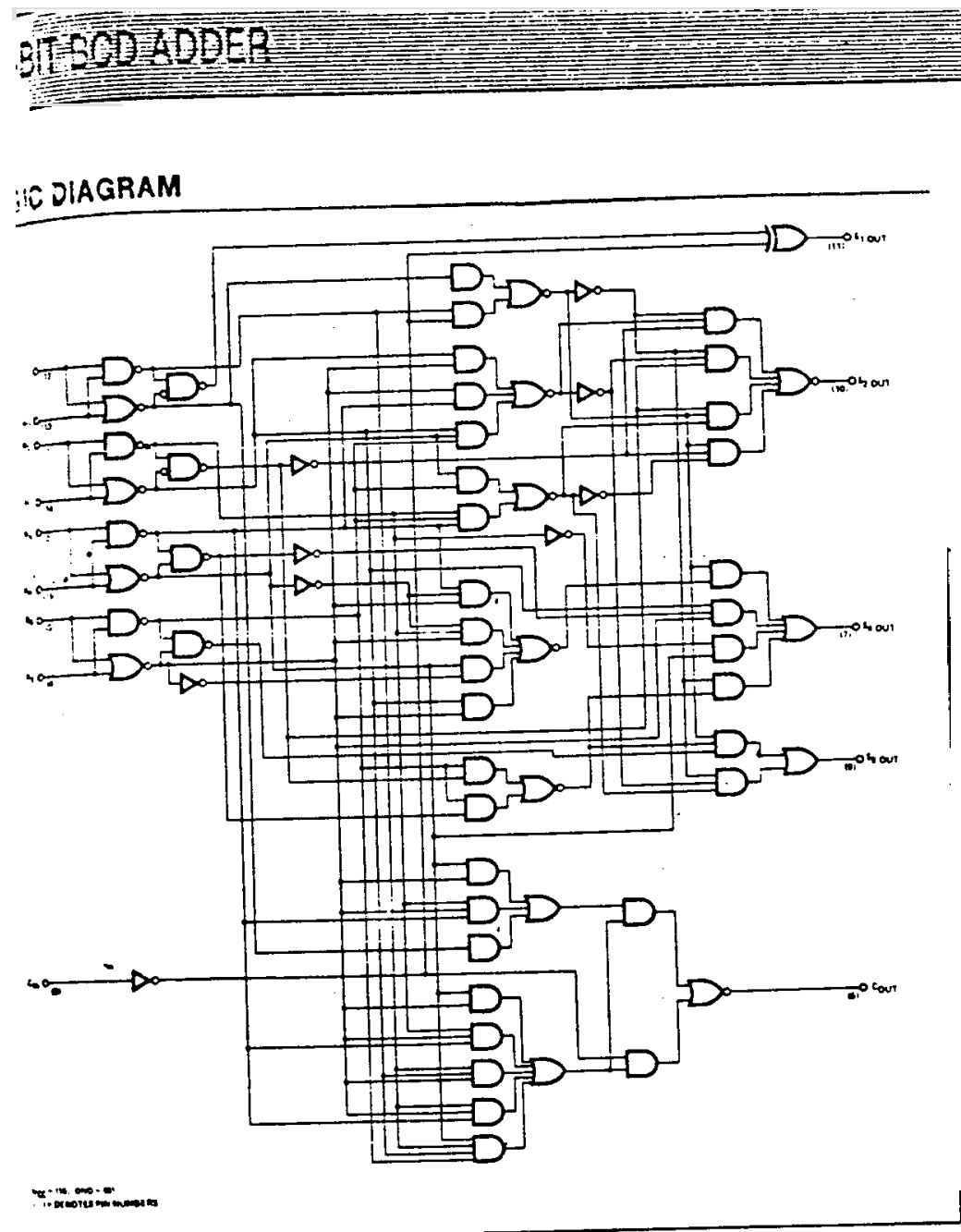

¿TEST FIGURE AND WAVEFORMS

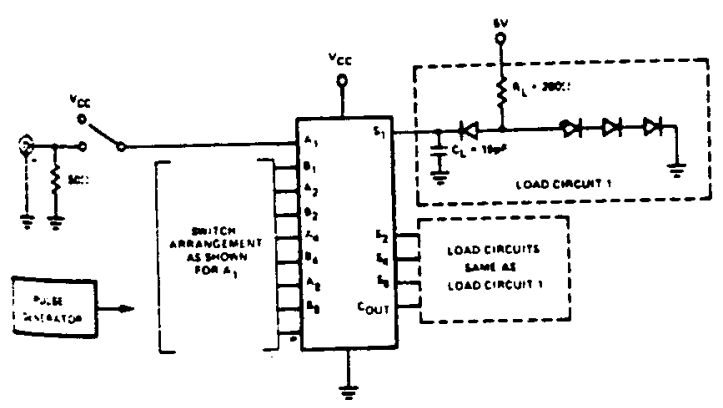

DUT PULSE:

PRA $=1$ MHZ

$\because:=11=5 \mathrm{~ns}$

L. OIODES ARE 1N3064

- INCLUDES PROBE AND Jig CapacitanCE

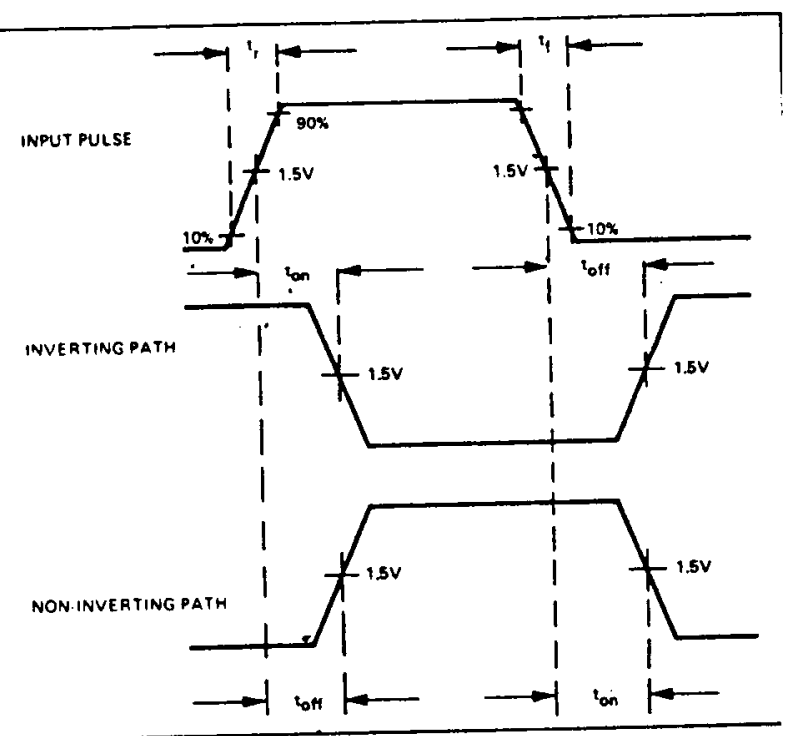

Reproduced with permission from Signetics, Sunnyvale, CA 94086. 
CAL APPLICATIONS (CONT'D.)

BINARY TO BCD CONVERSION USING $A_{i}$ INPUTS

\begin{tabular}{|c|cccc|cccc|cccc|c|}
\hline \multicolumn{10}{|c|}{ PARTIAL TRUTH TABLE FOR AI $>9$, BI } & $=0$ \\
\hline CIN & A1 & A2 & A4 & A8 & B1 & B2 & B4 & B3 & S1 & S2 & S4 & S8 & C0 \\
\hline 0 & 0 & 1 & 0 & 1 & 0 & 0 & 0 & 0 & 0 & 0 & 0 & 0 & 1 \\
0 & 1 & 1 & 0 & 1 & 0 & 0 & 0 & 0 & 1 & 0 & 0 & 0 & 1 \\
0 & 0 & 0 & 1 & 1 & 0 & 0 & 0 & 0 & 0 & 1 & 0 & 0 & 1 \\
0 & 1 & 0 & 1 & 1 & 0 & 0 & 0 & 0 & 1 & 1 & 0 & 0 & 1 \\
0 & 0 & 1 & 1 & 1 & 0 & 0 & 0 & 0 & 0 & 0 & 1 & 0 & 1 \\
0 & 1 & 1 & 1 & 1 & 0 & 0 & 0 & 0 & 1 & 0 & 1 & 0 & 1 \\
1 & 0 & 1 & 0 & 1 & 0 & 0 & 0 & 0 & 1 & 0 & 0 & 0 & 1 \\
1 & 1 & 1 & 0 & 1 & 0 & 0 & 0 & 0 & 0 & 1 & 0 & 0 & 1 \\
1 & 0 & 0 & 1 & 1 & 0 & 0 & 0 & 0 & 1 & 1 & 0 & 0 & 1 \\
1 & 1 & 0 & 1 & 1 & 0 & 0 & 0 & 0 & 0 & 0 & 1 & 0 & 1 \\
1 & 0 & 1 & 1 & 1 & 0 & 0 & 0 & 0 & 1 & 0 & 1 & 0 & 1 \\
1 & 1 & 1 & 1 & 1 & 0 & 0 & 0 & 0 & 0 & 1 & 1 & 0 & 1 \\
\hline
\end{tabular}

BINARY TO BCD CONVERSION USING B, INPUTS

\begin{tabular}{|c|c|c|c|c|c|c|c|c|c|c|c|c|c|c|c|}
\hline \multicolumn{16}{|c|}{ BINARY TO BCD CONVERSION USING B INPUTS } \\
\hline \multicolumn{14}{|c|}{ PARTIAL TRUTH TABLE FOR $B \mid>9, A I=0$} & & \\
\hline CIN & A1 & A2 & A4 & $A B$ & B1 & B2 & $\mathbf{B 4}$ & $\mathrm{Bg}$ & s1 & $\mathbf{5 2}$ & 54 & 58 & co & & \\
\hline 0 & 0 & 0 & 0 & 0 & 0 & 1 & 0 & 1 & 0 & 0 & 0 & 0 & 1 & & \\
\hline 0 & 0 & 0 & 0 & 0 & 1 & 1 & 0 & 1 & 1 & 0 & 0 & 0 & 1 & & \\
\hline 0 & 0 & 0 & 0 & 0 & 0 & 0 & 1 & 1 & 0 & 1 & 0 & 0 & 1 & & \\
\hline 0 & 0 & 0 & 0 & 0 & 1 & 0 & 1 & 1 & 1 & 1 & 0 & 0 & 1 & $\perp$ & \\
\hline 0 & 0 & 0 & 0 & 0 & 0 & 1 & 1 & 1 & 1 & 0 & 0 & 1 & 0 & $m$ & \\
\hline 0 & 0 & 0 & 0 & 0 & 1 & 1 & 1 & 1 & 1 & 0 & 1 & 0 & 1 & & $\begin{array}{c}\cos \\
\operatorname{tant}\end{array}$ \\
\hline 1 & 0 & 0 & 0 & 0 & 0 & 1 & 0 & 1 & 1 & 0 & 0 & 0 & 1 & ठ d & \\
\hline 1 & 0 & 0 & 0 & 0 & 1 & 1 & 0 & 1 & 0 & 1 & 0 & 0 & 1 & resentedcad & \\
\hline 1 & 0 & 0 & 0 & 0 & 0 & 0 & 1 & 1 & 1 & 1 & 0 & 0 & 1 & & \\
\hline 1 & 0 & 0 & 0 & 0 & 1 & 0 & 1 & 1 & 0 & 0 & 1 & 0 & 1 & & \\
\hline 1 & 0 & 0 & 0 & 0 & 0 & 1 & 1 & 1 & 1 & 0 & 1 & 0 & 1 & & \\
\hline 1 & 0 & 0 & 0 & 0 & 1 & 0 & 0 & 0 & 0 & 1 & 1 & 0 & 1 & & \\
\hline
\end{tabular}

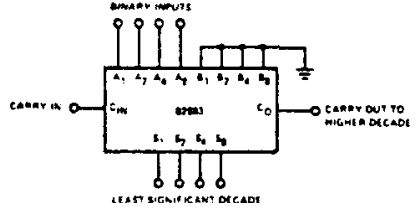

Reproduced with permission from Signetics, Sunnyvale, CA 94086. 


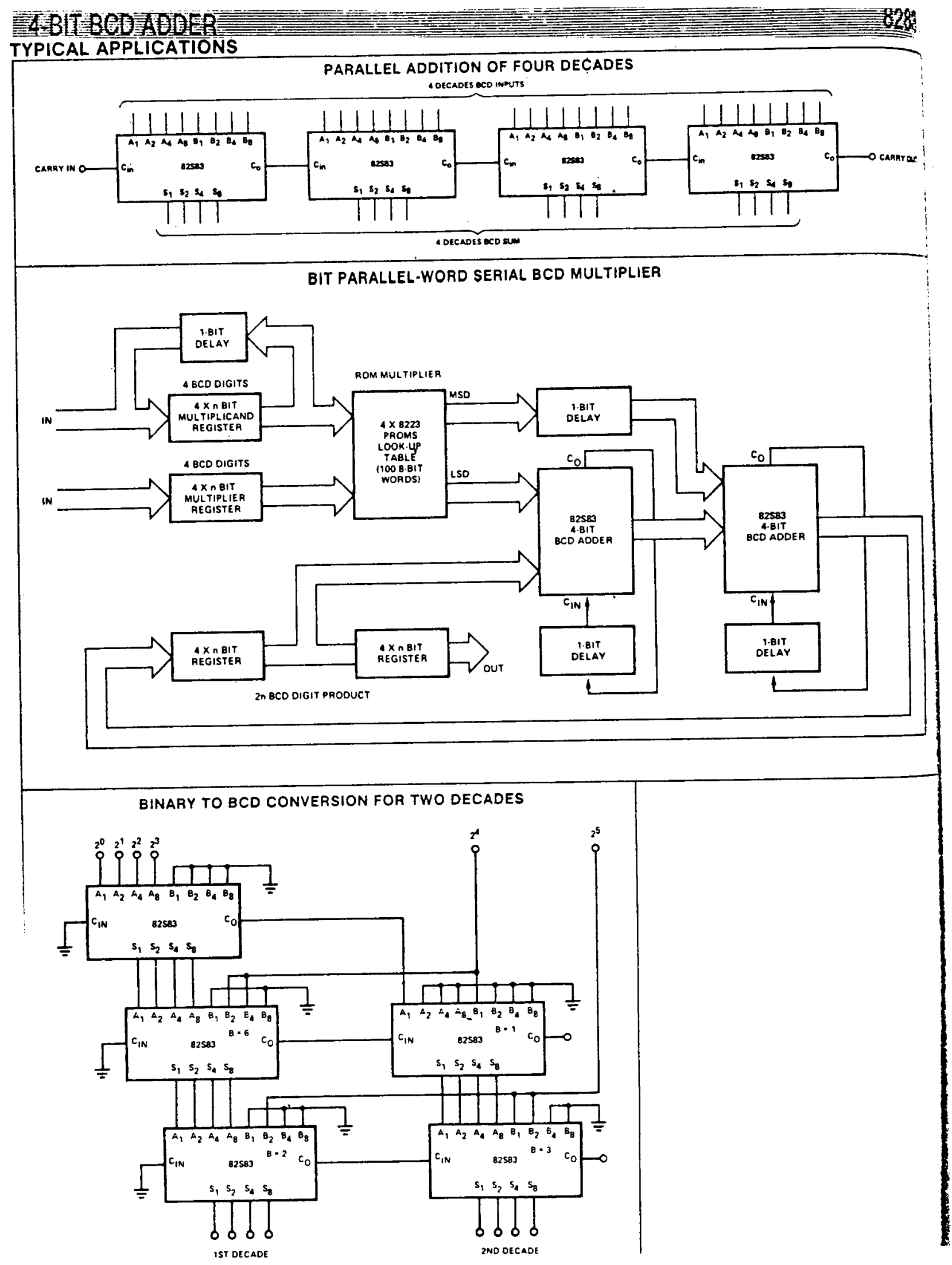

Reproduced with permission from Signetics, Sunnyvale, CA 94086. 


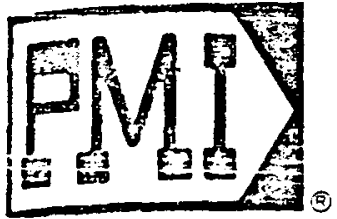

\section{FEATURES}

- Ultra.Low $V_{\text {OS }} \ldots \ldots \ldots \ldots \ldots \ldots \ldots \ldots \ldots, 10_{\mu} \mathrm{V}$ - Ultra.Low Vos Dril? ................. $0.2 \mu \mathrm{V} /{ }^{\circ} \mathrm{C}$ - Vitia.Ftable re Time $\ldots \ldots \ldots \ldots \ldots \ldots, 0.2_{\mu}$ VIMonth

- Uitra.Low Noise ................... 0.35 $\mathrm{V}_{\text {p.p }}$

- No External Components Required

- Replaces Chopper Amps at Lower Cost

- Single-Chip Monolithic Construction

- Large Input Voltage Range ............. $=14.0 \mathrm{~V}$

- Wide Supp!y Voltage Range .......... $\pm 3 \mathrm{~V}$ to $\pm 18 \mathrm{~V}$

- Fits, 725, 108A/308A, 741, AD510 Sockets

\section{GENERAL DESCRIPTION}

The OP.07 Series represents a breakthrough in monolithic operational amplifier performance $-V_{O S}$ of $10_{\mu} \mathrm{V}, \mathrm{TCV}$ OS of $0.2 \mu \mathrm{V} /{ }^{\circ} \mathrm{C}$ and long-term stability of $0.2 \mu \mathrm{V} /$ month are achieved zy a low-noise. chopper-less bipolar input transistor amplifier ci:cuit. Complete elimination of external components for offset nulling, frequency compensation and device protection osimits extreme miniaturization and optimization of system Near-Time-Qetween.Failure Rates in nigh-performance aerospace/defense and industrial applications. Excellent device inieichangeability provides reduced system assembiy time and eliminates field recalibrations.

True differential inputs with wide input voltage range and outstanding common mode rejection provide maximum flexibility and performance in high-noise environments and noninverting applications. Low bias currents and extremely-high input impedances are maintained over the entire temperature range.

ThE OP-07 provides unparalleled pertormance for low noise. high-accuracy amplification of very low-level signals in transducer applications. Devices are available in chip form lor use in hybrid circuitry. The OP-07 is a direct replacement for $725,108 \mathrm{~A} / 308 \mathrm{~A}^{*}$, and $O P-05$ amplifiers; 741 -types may be directly replaced by removing the 74 ''s nulling potentiometer. - T0.99 package only. For Matched Dual see OP.207.

\section{PIN CONNECTIONS \& ORDERING INFORMATION}

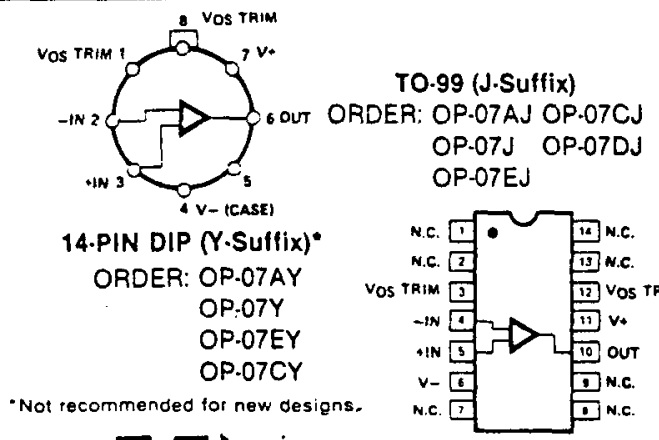

vos TaIm 0 ijvos TRIM EPOXY B MINI-DIP (P.Suffix)

ORDER: OP-OTCP

OP-OTEP

$\begin{array}{ll}V-4 & \text { OP-OTDP }\end{array}$

8.PIN DIP (Z.Sulfix)

ORDER: OP.OTAZ

OP-07Z

OP.07EZ

OP.07C2

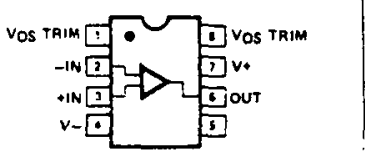

Military Temperature Range Devices with MIL.STD.883 Class B Processing

ORDER: OP07AJ/883 OP07AZ/883 OP07Y/883 OP07AY/883 OP07J/883 OP07Z/883

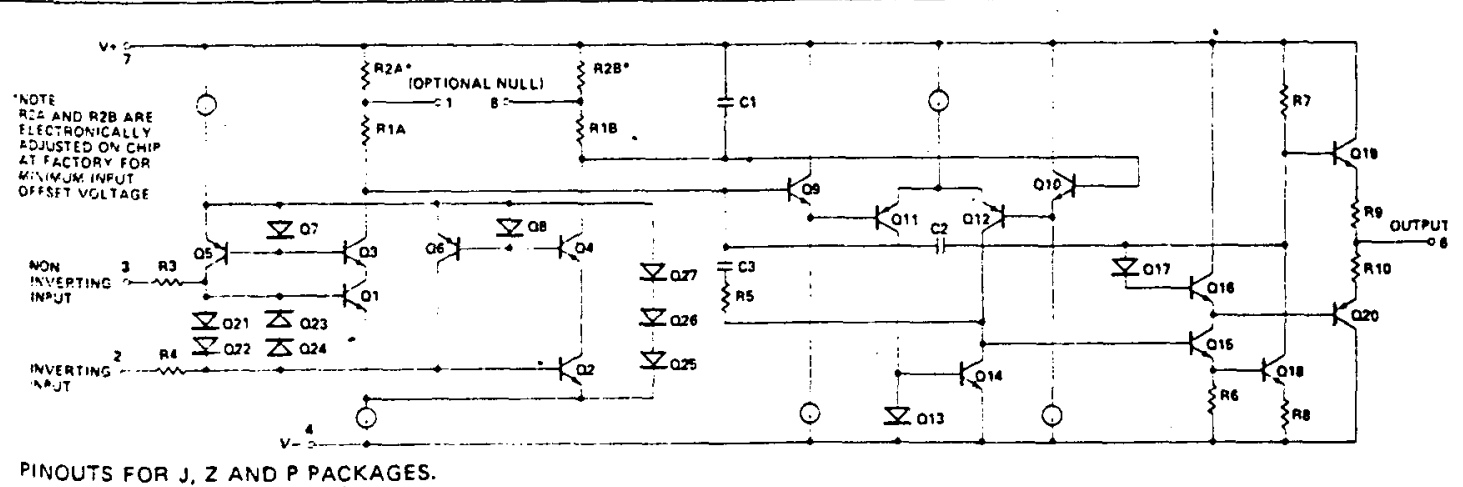
Reproduced with permission from Precision Monolithics, Inc., Santa Clara, CA
95050. 
ELECTRICAL CHARACTERISTICS at $V_{S}= \pm 15 \mathrm{~V},-55^{\circ} \mathrm{C} \leq T_{4} \leq+125^{\circ} \mathrm{C}$. unless otherwise noted.

\begin{tabular}{|c|c|c|c|c|c|c|c|c|c|}
\hline \multirow[b]{2}{*}{ PARKMETER } & \multirow[b]{2}{*}{ SYMBOL } & \multirow[b]{2}{*}{ CONDITIONS } & \multicolumn{3}{|c|}{ OP.C7A } & \multicolumn{3}{|c|}{ OP.07 } & \multirow[b]{2}{*}{ UNITS } \\
\hline & & & MIN & TYP & $\max$ & MiN & TYP & MAX & \\
\hline$\therefore \therefore$ Ot!set Voltage & Vos & (Nole 1$)$ & - & 25 & 60 & - & 60 & 200 & $\mu V$ \\
\hline \multirow{2}{*}{ 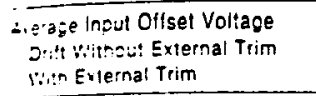 } & TCV OS & (Note 3) & - & 0.2 & 0.6 & - & 0.3 & 1.3 & \multirow{2}{*}{ uV: ${ }^{\circ} \mathrm{C}$} \\
\hline & TCV OS: & $R_{f}=20 \mathrm{k} n$ & - & 0.2 & 0.6 & - & 0.3 & 1.3 & \\
\hline On Otisei Current & los & & - & 0.8 & 4.0 & - & 1.2 & 5.6 & $n A$ \\
\hline $\begin{array}{l}\text { D.25aje Triput Offset Current } \\
\text { Drift }\end{array}$ & TClos & (Note 3) & - & 5 & 25 & - & 6 & 50 & $\mathrm{PA} \cdot{ }^{\bullet} \mathrm{C}$ \\
\hline :-su: Bias Current & $I_{B}$ & & $\dot{-}$ & \pm 1.0 & $=4.0$ & - & \pm 2.0 & $=6.0$ & nA \\
\hline Luerage Input Bias Current Dritt & $\mathrm{TCl}_{\mathrm{B}}$ & & - & 8 & 25 & - & 13 & 50 & $\mathrm{DA} /=\mathrm{C}$ \\
\hline - sut botioge Range & IVR & & \pm 13.0 & $=13.5$ & - & $=13.0$ & $=13.5$ & - & $v$ \\
\hline$C=T$ mon MoJe Rejection Ratio & CMRR & $v_{C M}= \pm 13 V$ & 106 & 123 & - & 106 & 123 & - & $\mathrm{AB}$ \\
\hline Dute: Sufply Pejection Ratio & PSRR & $V_{S}= \pm 3 V \quad 10=18 \mathrm{~V}$ & 94 & 106 & - & 94 & 106 & - & $A B$ \\
\hline 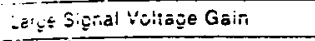 & Avo & $F_{L} \geq 2 \mathrm{k} ! n V_{O}= \pm i 0 \mathrm{~V}$ & 200 & 400 & - & 150 & 400 & - & vimv \\
\hline W3aritin Output Vollage Swing & $V_{O M}$ & $R_{L} \geq 2 \mathrm{k} !$ & $=12.0$ & $=12.6$ & - & \pm 12.0 & \pm 12.6 & - & $\bar{v}$ \\
\hline
\end{tabular}

TYPICAL OFFSET VOLTAGE TEST CIRCUIT

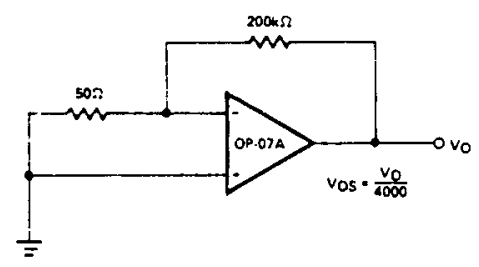

OPTIONAL OFFSET NULLING CIRCUIT

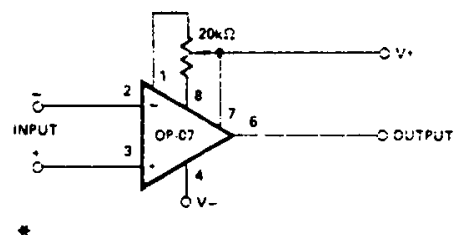

TYPICAL LOW FREQUENCY NOISE TEST CIRCUIT

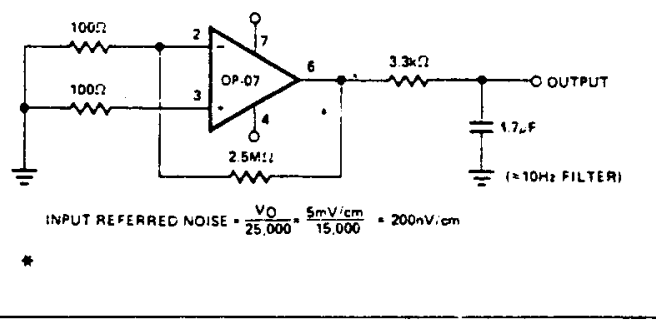

\section{BURN.IN CIRCUIT}

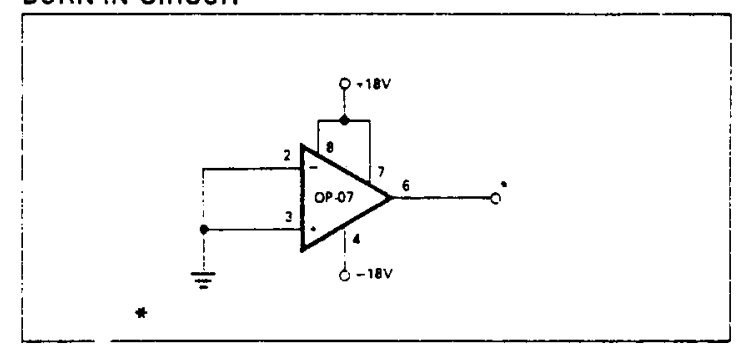


ELECTRICAL CHARACTERISTICS a: $V_{S}= \pm 15 \mathrm{~V}, T_{\Delta}=25^{\circ} \mathrm{C}$. uniess oinerwise noted.

\begin{tabular}{|c|c|c|c|c|c|c|c|c|c|c|c|c|}
\hline \multirow[b]{2}{*}{ PARAMETEA } & \multirow[b]{2}{*}{ SYMBOL } & \multirow[b]{2}{*}{ CONDITIONS } & \multicolumn{3}{|c|}{ OP.07E } & \multicolumn{3}{|c|}{ OP.07C } & \multicolumn{3}{|c|}{ OP.07D } & \multirow[b]{2}{*}{ UNITS } \\
\hline & & & MIN & TYP & $\operatorname{MAX}$ & MIN & & $\operatorname{MAX}$ & MIN & & $\operatorname{MAX}$ & \\
\hline $\begin{array}{l}\text { Inpul Offset } \\
\text { Vol:age }\end{array}$ & Vos & (Note 1) & - & 30 & 75 & - & 60 & 150 & - & 60 & 150 & ${ }_{\mu} V$ \\
\hline $\begin{array}{l}\text { Long Term Vos } \\
\text { Siatility }\end{array}$ & $v_{\text {os }} \pi$ ime & (Nole 2) & - & 0.3 & 1.5 & - & 0.4 & 2.0 & - & 0.5 & 3.0 & «VIMO \\
\hline $\begin{array}{l}\text { inpui Ottset } \\
\text { Current. }\end{array}$ & los & & - & 0.5 & 3.8 & - & 0.8 & 6.0 & - & 0.8 & 6.0 & nA \\
\hline Input Blas Current & $I_{B}$ & & - & $=1.2$ & $=4.0$ & - & $=1.8$ & $=7.0$ & - & $=2.0$ & \pm 12 & $\mathrm{nA}$ \\
\hline \multirow[t]{2}{*}{$\begin{array}{l}\text { inpur Noise } \\
\text { Vottage }\end{array}$} & $e_{\text {nD.D }}$ & $\begin{array}{l}0.1 \mathrm{~Hz} 1010 \mathrm{~Hz} \\
\text { (Note 3) }\end{array}$ & - & 0.35 & 0.6 & - & 0.38 & 0.65 & - & 0.38 & 0.65 & $\mu v_{D \cdot D}$ \\
\hline & & $t_{0}=10 \mathrm{~Hz}$ & - & 10.3 & 98.0 & - & 10.5 & 20.0 & - & 10.5 & 20.0 & \\
\hline \multirow[t]{2}{*}{$\begin{array}{l}\text { input Noise } \\
\text { Votrage Density }\end{array}$} & EniNote 3; & $; t_{n}=100 \mathrm{~Hz}$ & - & 10.0 & 13.0 & - & 10.2 & 13.5 & - & 10.3 & 13.5 & $n V_{1} \cdot \overline{\mathrm{Hz}}$ \\
\hline & & $1_{0}=1000 \mathrm{~Hz}$ & - & 9.6 & 11.0 & - & - 9.8 & 11.5 & - & 9.8 & 11.5 & \\
\hline \multirow[t]{2}{*}{$\begin{array}{l}\text { Input Noise } \\
\text { Current }\end{array}$} & $i_{n p-p}$ & $\begin{array}{l}0.1 \mathrm{~Hz} 1010 \mathrm{~Hz} \\
\text { (Note 3) }\end{array}$ & - & 14 & 30 & - & 15 &.$^{35}$ & - & 15 & 35 & $P A_{P \cdot P}$ \\
\hline & & $t_{0}=10 \mathrm{~Hz}$ & - & 0.32 & 0.80 & - & 0.35 & 0.90 & - & 0.35 & 0.90 & \\
\hline \multirow[t]{2}{*}{$\begin{array}{l}\text { Input Noise } \\
\text { Current Density }\end{array}$} & $i_{n}$ (Note 3) & $t_{0}=100 \mathrm{~Hz}$ & - & 0.14 & 0.23 & - & 0.15 & 0.27 & - & 0.15 & 0.27 & $D A N \sqrt{H 2}$ \\
\hline & & $t_{0}=1000 \mathrm{~Hz}$ & - & 0.12 & 0.17 & - & 0.13 & 0.18 & - & 0.13 & 0.18 & \\
\hline $\begin{array}{c}\text { inpuitresislance - } \\
\text { Ditlerential Mode }\end{array}$ & $R_{I N}$ & & 15 & so & - & 8 & 33 & - & 7 & 39 & - & $M \Omega$ \\
\hline $\begin{array}{l}\text { Input Resistance - } \\
\text { Common Mose }\end{array}$ & $R_{\text {inCM }}$ & & - & 160 & - & - & 120 & - & - & 120 & - & $G \Omega$ \\
\hline \multicolumn{3}{|c|}{ Input Voltage Range IVR } & \pm 13.0 & \pm 14.0 & - & \pm 13.0 & \pm 14.0 & - & \pm 13.0 & \pm 14.0 & - & $v$ \\
\hline $\begin{array}{l}\text { Common Mode } \\
\text { Aejectior, Ratio }\end{array}$ & CMPR & $V_{C M}==13 \mathrm{~V}$ & 106 & 123 & - & 100 & 120 & - & 94 & 110 & - & $d B$ \\
\hline \multirow[t]{2}{*}{$\begin{array}{l}\text { Power Supply } \\
\text { Rejection Patio }\end{array}$} & PSRA & $\begin{array}{l}V_{S}= \pm 3 v \\
10 \pm 18 \mathrm{~V}\end{array}$ & 94 & 107 & - & 90 & 104 & - & 90 & 104 & - & $d B$ \\
\hline & & $\begin{array}{l}A_{L} \geq 2 \times \Omega . \\
V_{O}= \pm 10\end{array}$ & 200 & 500 & - & 120 & 400 & - & 120 & 400 & - & \\
\hline \multirow[t]{2}{*}{$\begin{array}{l}\text { Large Signal } \\
\text { Voltage Gain }\end{array}$} & Avo & $\begin{array}{l}A_{L} \geq 500 \mathrm{n} . \\
V_{O}= \pm .5 \mathrm{~V}\end{array}$ & 150 & 500 & - & 100 & 400 & - & - & - & - & $\mathrm{V} / \mathrm{mV}$ \\
\hline & & $V_{S}= \pm 3 \mathrm{~V}$ & 150 & 500 & - & 100 & 400 & - & - & - & - & \\
\hline \multirow{3}{*}{$\begin{array}{l}\text { Maximum Voltage } \\
\text { Vollage Swing }\end{array}$} & & $R_{L} \geq 10 \mathrm{k} n$ & \pm 12.5 & $=13.0$ & - & \pm 12.0 & \pm 13.0 & - & \pm 12.0 & $=13.0$ & - & v \\
\hline & $V_{O M}$ & $R_{L} \geq 2 \mathrm{k} \Omega$ & \pm 12.0 & \pm 12.8 & - & \pm 11.5 & \pm 12.8 & - & \pm 11.5 & \pm 12.8 & - & $v$ \\
\hline & & $R_{L} \geq 1 \mathrm{k} \Omega$ & $=10.5$ & $=12.0$ & - & - & $\approx 12.0$ & - & - & - & - & $v$ \\
\hline Slewing Rate & $S R$ & $R_{L} \geq 2 \mathrm{~K} \Omega$ (Note 3 ) & -0.1 & 0.2 & - & 0.1 & 0.2 & - & 0.1 & 0.2 & - & $V / \mu s$ \\
\hline $\begin{array}{l}\text { Closed Loop } \\
\text { Bandwidin }\end{array}$ & BW & $\begin{array}{l}A_{\mathrm{V} C L}=+1.0 \\
\text { (Note } 3 \text { ) }\end{array}$ & 0.4 & 0.6 & - & 0.4 & 0.6 & - & 0.4 & 0.6 & - & $\mathrm{MHz}$ \\
\hline $\begin{array}{l}\text { Open Loop Output } \\
\text { Resistance }\end{array}$ & $R_{0}$ & $v_{0}=0, l_{0}=0$ & - & 60 & - & - & 60 & - & - & 60 & - & 8 \\
\hline $\begin{array}{l}\text { Power } \\
\text { Consumption }\end{array}$ & $P_{d}$ & $\begin{array}{l}V_{s}= \pm 15 \mathrm{~V} \\
v_{s}= \pm 3 \mathrm{~V}\end{array}$ & $\bar{z}$ & $\begin{array}{r}75 \\
4 \\
\end{array}$ & $\begin{array}{r}120 \\
6 \\
\end{array}$ & $=$ & $\begin{array}{r}80 \\
4\end{array}$ & $\begin{array}{r}150 \\
8 \\
\end{array}$ & $\bar{z}$ & $\begin{array}{r}80 \\
4\end{array}$ & $\begin{array}{r}150 \\
8 \\
\end{array}$ & $\mathrm{~mW}$ \\
\hline $\begin{array}{l}\text { Offsẹt Adjustment } \\
\text { Range }\end{array}$ & & $R_{F}=20 \mathrm{k} \Omega$ & - . & $=4$ & - & - & $=4$ & - & - & $=4$ & - & $m v$ \\
\hline
\end{tabular}

\section{NOTES:}

1. Input offset vollage measurements are perlormed by aulomated test equipment approximately 0.5 seconds alter application of power.

2. Long Term Input Otiset Voltage Stability refers to the averaged trend line of $V_{O S}$ vs. Time over extended periods after the first 30 days of operation. Exeluding the initial hour of operation. changes in $V_{0}$ during the tirst 30 operating cays are typically $2.5, \mathrm{~V}$ - e eler to typical pertor. mance curve on Page 5. Parameter is not $100 \%$ lested: $90 \%$ of units meet this specification.

3. Parameter is not $100 \%$ tesled; $90 \%$ of units meet this specification

Reproduced with permission from Precision Monolithics, Inc., Santa Clara, CA 95050. 
ELECTRICAL CHARACTERISTICS at $V_{S}= \pm 15 \mathrm{~V} \cdot 0^{\circ} \mathrm{C} \leq T_{A} \leq+70^{\circ} \mathrm{C}$, uniess otherwise noted.

\begin{tabular}{|c|c|c|c|c|c|c|c|c|c|c|c|c|}
\hline \multirow[b]{2}{*}{ DARAMETER } & \multirow[b]{2}{*}{ SYMBOL } & \multirow[b]{2}{*}{ CONDITIONS } & \multicolumn{3}{|c|}{ OP.07E } & \multicolumn{3}{|c|}{ OP.07C } & \multicolumn{3}{|c|}{ OP.07D } & \multirow[b]{2}{*}{ UNITS } \\
\hline & & & MIN & TYP & $\operatorname{MAX}$ & MIN & TYP & $\operatorname{MAX}$ & MIN & TYP & $\operatorname{MAX}$ & \\
\hline $\begin{array}{l}\text { Inpli Cliset } \\
\text { voltage }\end{array}$ & vos & (Note 1) & - & 45 & 130 & - & 85 & 250 & - & 85 & 250 & $\mu V$ \\
\hline \multirow{3}{*}{$\begin{array}{l}\text { sue:age input Off. } \\
\text { set Volage Drift } \\
\text { Wirnout External } \\
\text { Trim } \\
\text { With External } \\
\text { Trim }\end{array}$} & \multirow{2}{*}{\multicolumn{2}{|c|}{ TCVOS }} & \multirow[b]{2}{*}{-} & \multirow[b]{2}{*}{0.3} & \multirow[b]{2}{*}{1.3} & \multicolumn{3}{|c|}{ (Note 3) } & \multirow{2}{*}{\multicolumn{3}{|c|}{$0.7^{(\text {Note 3) }} 2.5$}} & \\
\hline & & & & & & - & 0.5 & 1.8 & & & & ${ }_{\mu} \mathrm{V} /{ }^{\circ} \mathrm{C}$ \\
\hline & $T C v_{\text {OSn }}$ & $R_{F}=20 \mathrm{k} \Omega$ & - & 0.3 & 1.3 & \multicolumn{3}{|c|}{$\begin{array}{l}0.4 \begin{array}{r}1.6 \\
\text { (Note 3) }\end{array} \\
\end{array}$} & \multicolumn{3}{|c|}{$\begin{array}{lr}0.7 & 2.5 \\
\text { (Note 3) }\end{array}$} & $\overline{{ }_{\mu} \mathrm{V} /{ }^{\circ} \mathrm{C}}$ \\
\hline $\begin{array}{l}\text { Inqut Oftsel } \\
\text { Current }\end{array}$ & los & & - & 0.8 & 5.3 & - & 1.6 & 8.0 & - & 1.6 & 8.0 & na \\
\hline $\begin{array}{l}\text { Ave:age input Off. } \\
\text { set Current Drift }\end{array}$ & TCios & (Note 3) & - & 8 & 35 & - & 12 & 50 & - & 12 & 50 & $\mathrm{pa} /{ }^{\circ} \mathrm{C}$ \\
\hline Inout Bias Current & $\mathrm{I}_{\mathrm{B}}$ & & - & \pm 1.5 & \pm 5.5 & - & \pm 2.2 & \pm 9.0 & - & $=3.0$ & \pm 14 & $n A$ \\
\hline $\begin{array}{l}\text { Average Inpul Bias } \\
\text { Curreht Drift }\end{array}$ & $\mathrm{TCl}_{8}$ & (Note 3) & - & 13 & 35 & - & 18 & 50 & - & 18 & 50 & $D A /{ }^{\circ} \mathrm{C}$ \\
\hline $\begin{array}{l}\text { Inpul Vollage } \\
\text { Range }\end{array}$ & IVR & & \pm 13.0 & \pm 13.5 & - & \pm 13.0 & \pm 13.5 & - & $=13.0$ & \pm 13.5 & - & $v$ \\
\hline $\begin{array}{l}\text { Common Hode } \\
\text { Rejection Ratio }\end{array}$ & CMRR & $V_{C M}= \pm 13 \mathrm{~V}$ & 103 & 123 & - & $1^{97}$ & 120 & - & 94 & 106 & - & $d B$ \\
\hline $\begin{array}{l}\text { Power Supply } \\
\text { Fejection Ratic }\end{array}$ & PSAR & $V_{S}= \pm 3 V$ to $=18 \mathrm{~V}$ & 90 & 104 & - & 86 & 100 & - & 86 & 100 & - & $A B$ \\
\hline $\begin{array}{l}\text { Lorge Signal } \\
\text { voltage Gain }\end{array}$ & Avo & $\begin{array}{l}R_{\mathrm{L}} \geq 2 \mathrm{kD}, \\
v_{\mathrm{O}}= \pm 10 \mathrm{~V}\end{array}$ & 180 & 450 & - & 100 & 400 & - & 100 & 400 & - & $\mathrm{V} / \mathrm{mV}$ \\
\hline $\begin{array}{l}\text { Maximum Output } \\
\text { Voltage Swing }\end{array}$ & $V_{O M}$ & $R_{L} \geq . . \mathrm{k} \Omega$ & \pm 12.0 & \pm 12.6 & - & \pm 11.0 & \pm 12.6 & - & \pm 11.0 & \pm 12.6 & - & $v$ \\
\hline
\end{tabular}

NOTES:

1. Input oftset voltage measurements are performed by automated test equipment approximateiy 0.5 seconds atter application of power.

2. Long Term Input Offset Voltage Stability refers to the averaged trend line of $V_{O S}$ ys. Time over extended periods after the first 30 days o operation. Exeluding the initial hour of operation, changes in Vos durlng the tirst 30 operating days are typically $2.5 \mu \mathrm{V}$ - refer 10 typical perfor. mance curve on Page 5. Parameter is not 100\% tested; $90 \%$ of units meet this specitication.

3. Parameter is not $100 \%$ tested; $90 \%$ of units meet this specification.

\section{TYPICAL PERFORMANCE CURVES}
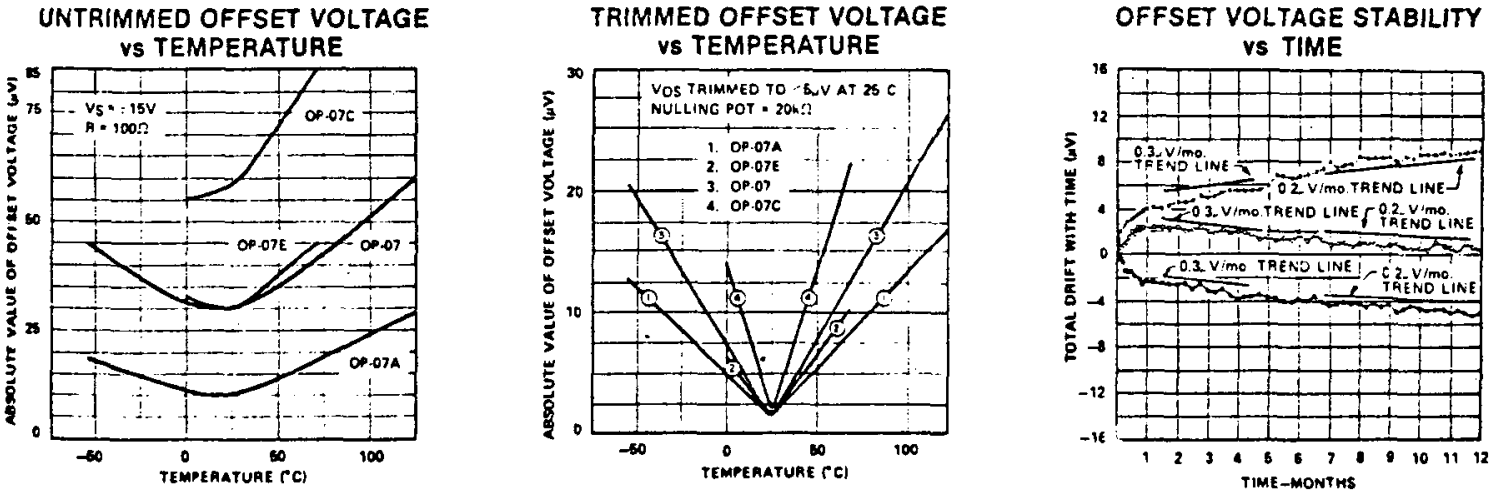

Reproduced with permission from Precision Monolithics, Inc., Santa Clara, CA 95050 . 
TYPICAL PERFORMANCE CURVES
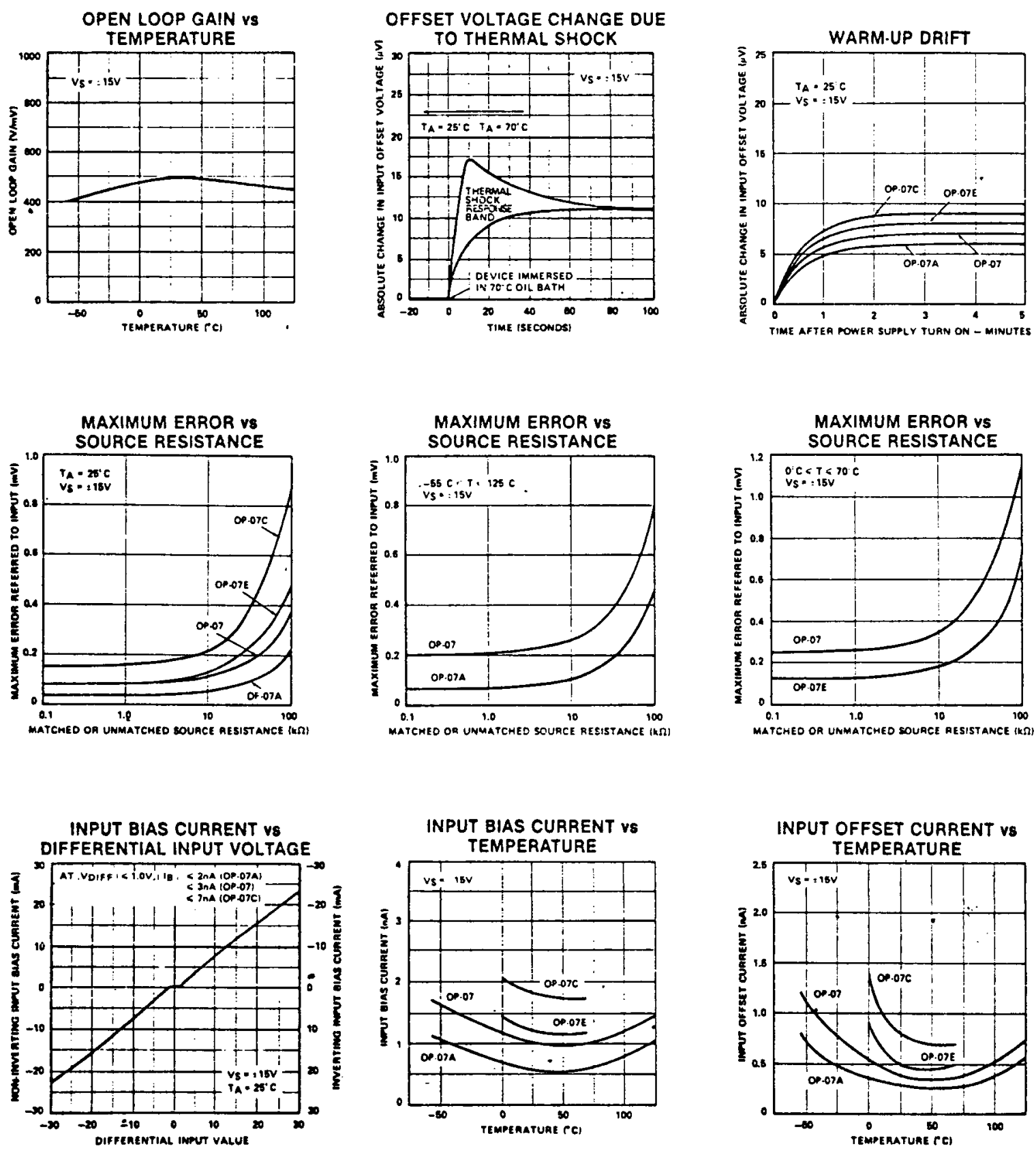

Reproduced with permission from Precision Monolithics, Inc., Santa Clara, CA 95050. 
TYPICAL PERFORMANCE CURVES

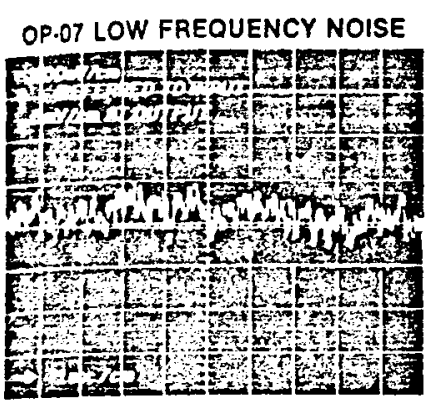

:SEE NOISE TEST CIRCUITI
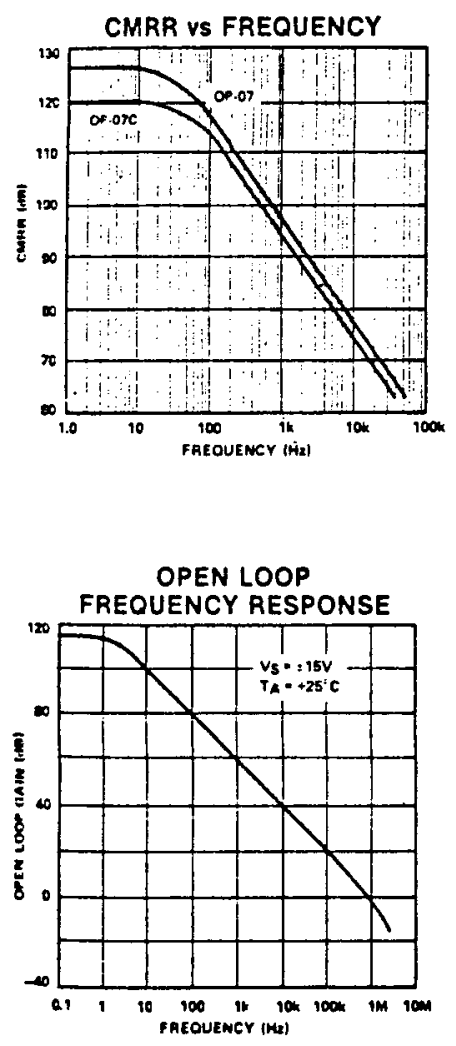
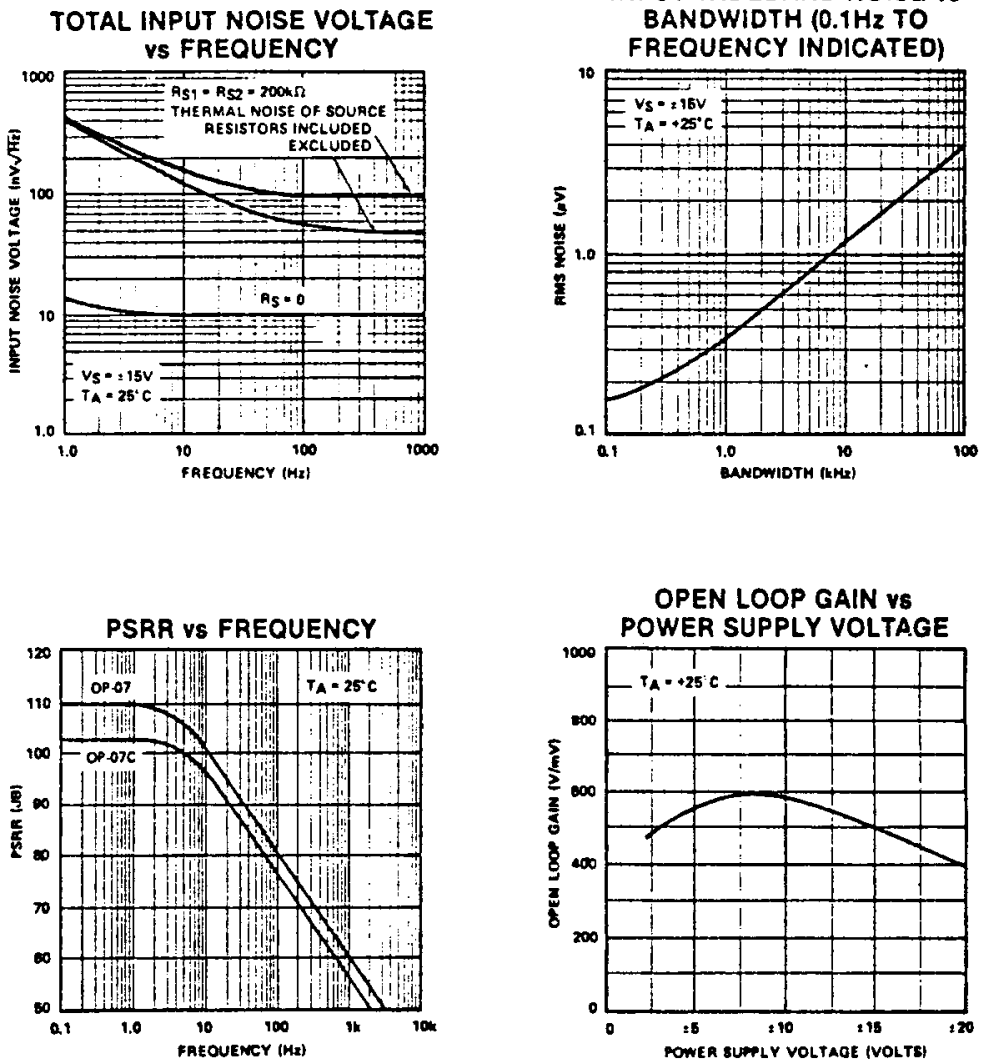

CLOSED LOOP RESPONSE FOR VARIOUS GAIN CONFIGURATIONS

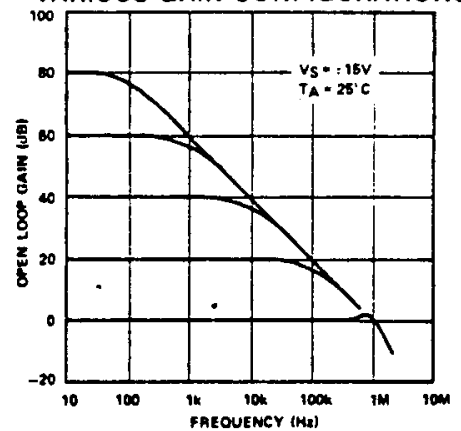

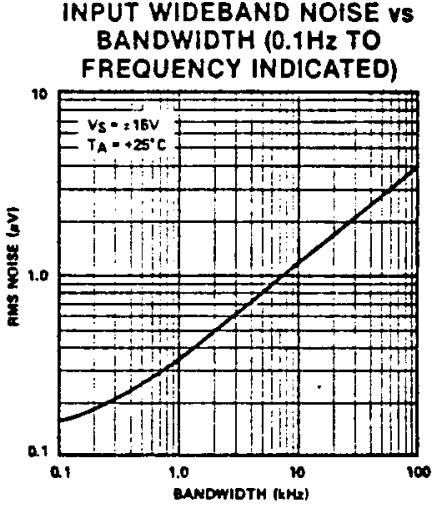

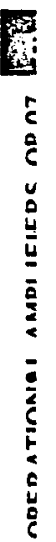

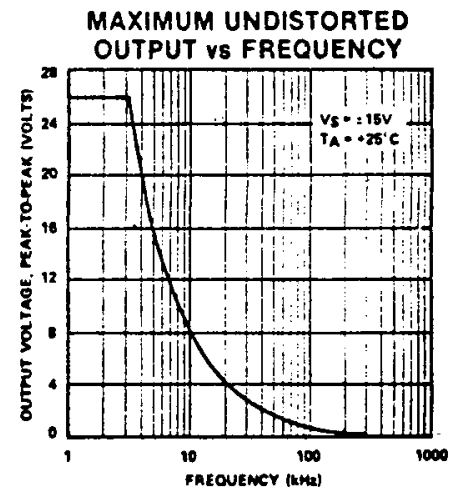

Reproduced with permission from Precision Monolithics, Inc., Santa Clara, CA 95050. 
TYPICAL PERFORMANCE CURVES
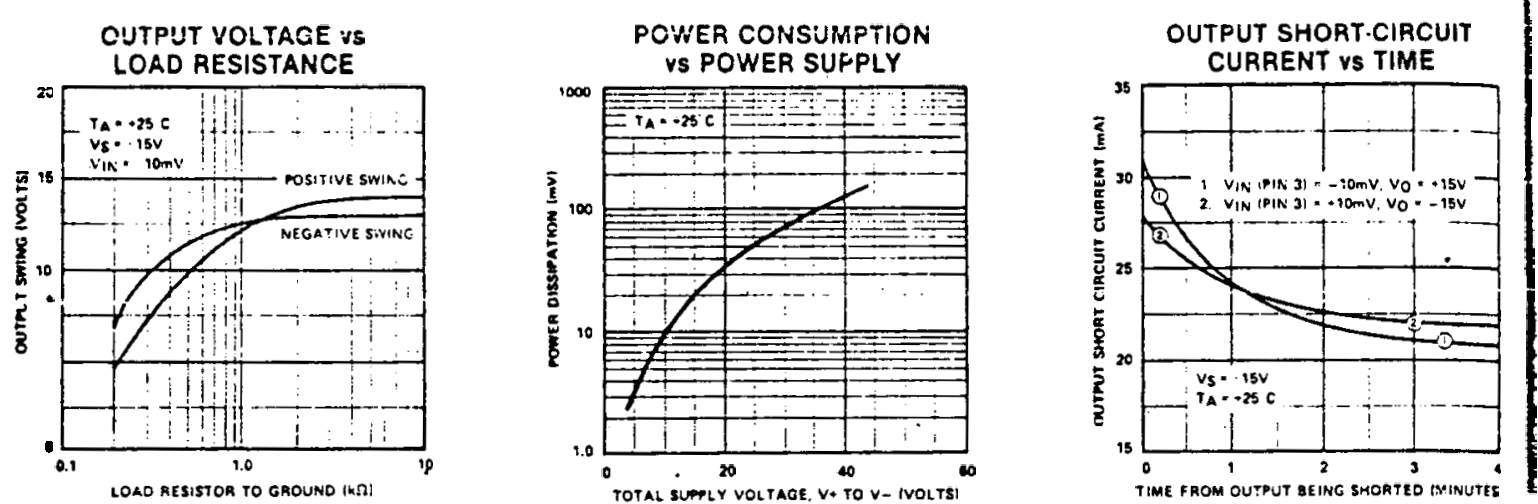

TYPICAL APPLICATIONS
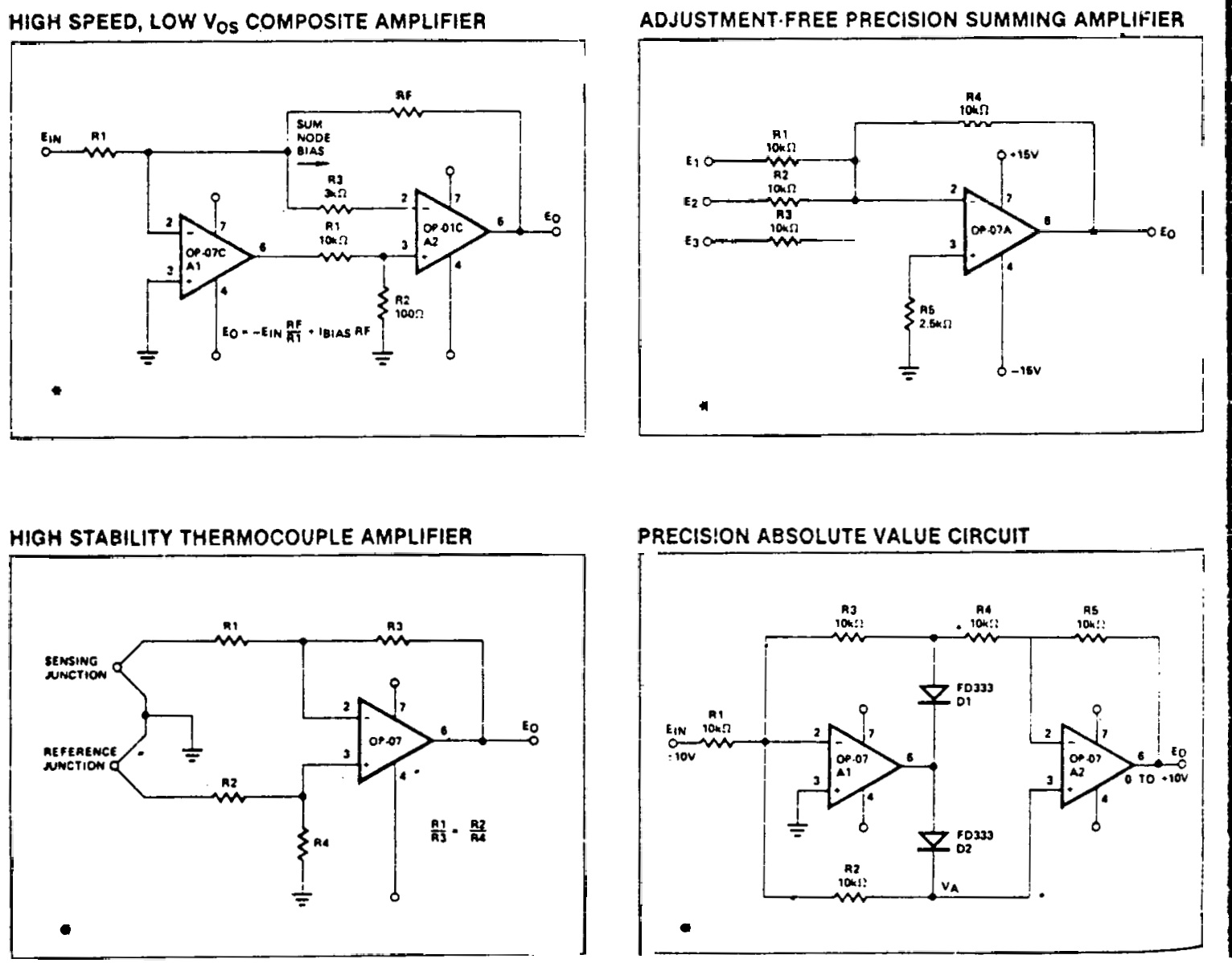

Reproduced with permission from Precision Monolithics, Inc., Santa Clara, CA 95050. 
APPLICATIONS INFORMATION

OP-07 Series units may be fitted directly to 725, 108A/308A* and OP-05 sockets with or without removal of external compensation or nulling components. Additionally, OP-07 may be fitted to unnulied 741-type sockets; however if conventronal 749 nulting circuitry is in use, it should be modified or removed to enable proper OP.07 operation. OP.07 offset voltage may be nulled to zero (or other desired setting) itrough use of a potentiometer (see diagram).

The OP.07 provides stable operation with load capacitance up to 500pF and $\pm 10 \mathrm{~V}$ swings; larger capacitances should be decoupled with $50 \Omega$ decoupling resistor. The designer is cautioned that stray thermoelectric voltages generated by dissimilar metals at the contracts to the input terminals can prevent realization of the drift performance indicated. Best operation will be obtained when both input contacts are maintained at the same temperature, preferably close to the temperature of the device's package.

DICE DIMENSIONS

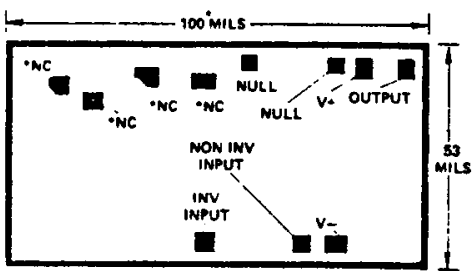


PHOTOMULTIPLIER POWER SUPPLY TYPE PMSB 2-025-1

SPECIFICATION

OUTPUT :

Voltage: $300 \mathrm{~V} \mathrm{DC}$ to $2000 \mathrm{~V}$ DC variable with input voltage. Load: 80N ohms Min.

Ripple: Less than $3 \mathrm{~V} \mathrm{p} / \mathrm{p}$ at $1800 \mathrm{~V}$ output and $180 \mathrm{M}$ load

Capacity: Less than $.002 \mathrm{mfd}$

Momentary Short Circuit Protected

Output isolated $2500 \mathrm{~V}$ to input ground

INPUT:

Voltage: (a) Approx. 3V tolov DC for required output voltage.

(b) with fixed output load $180 \mathrm{M}$ ohms input voltage shall be less than $8 V$ and input current less than 12 in ? for $1800 \mathrm{~V}$ output.

(c) Reverse polarity protected

ENVIRONMENTAL:

Operating temperature $-10^{\circ} \mathrm{C}$ to $40^{\circ} \mathrm{C}$

Temperature coefficient less than $+.028 / 0 \mathrm{C}$

CONFIGURATION:

As Drawing Al0740 Sheet 1

Reproduced with permission from Del Electronics Corporation, Mt. Vernon, NY 10550 . 


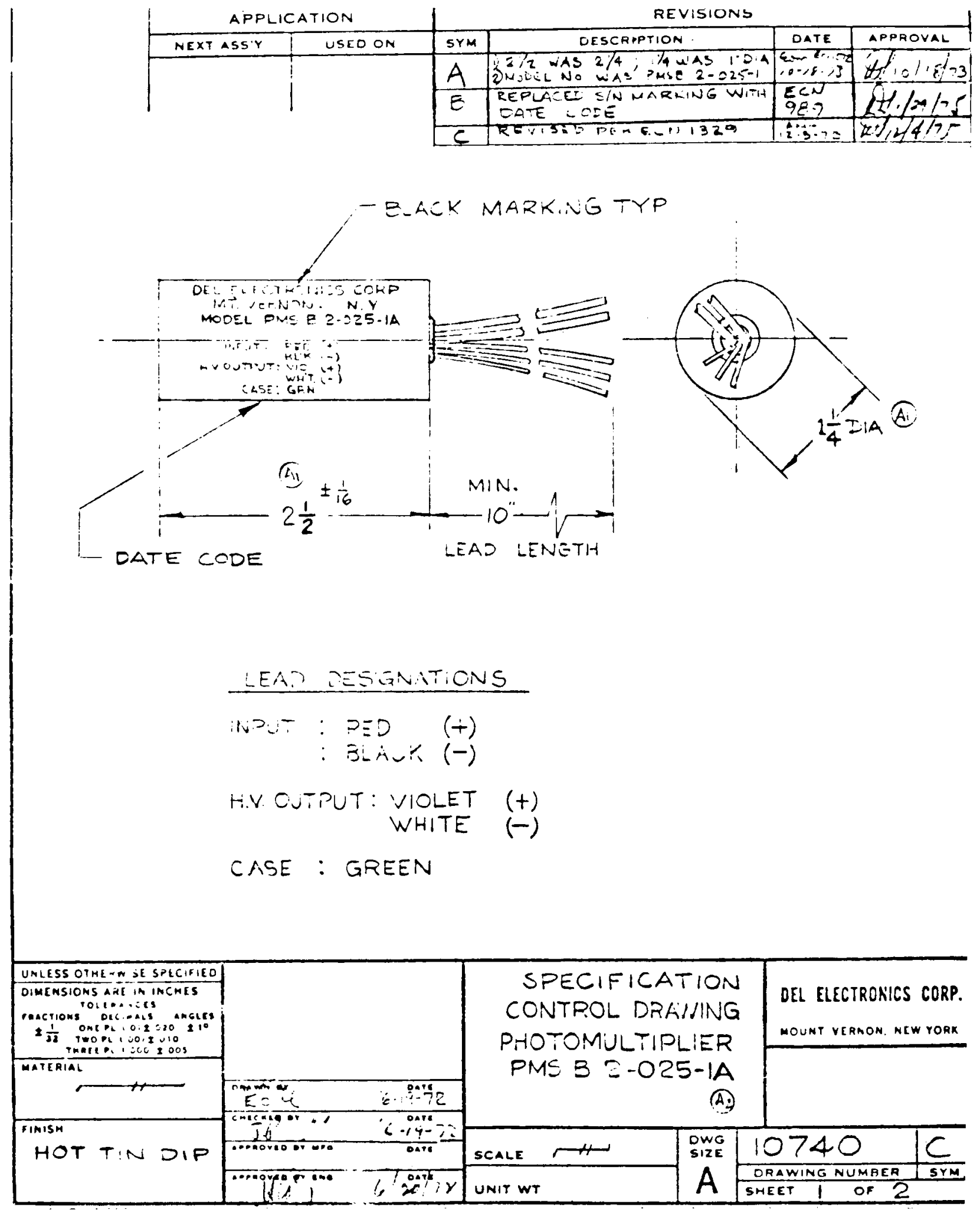

Reproduced with permission from Del Electronics Corporation, Mt. Vernon, NY 10550 . 


\section{Watts of Regulated Power (6.5V - 40VDC) INPUT High efficiency $\pm 15 \mathrm{~V},+5 \mathrm{~V}$ TRIPLE OUTPUT From a Single Module DC/DC CONVERTER That Accepts an Input From 6.5V to 40VDC POWER SUPPLY ${ }_{\text {WC Series }}^{\text {Indual }}$}

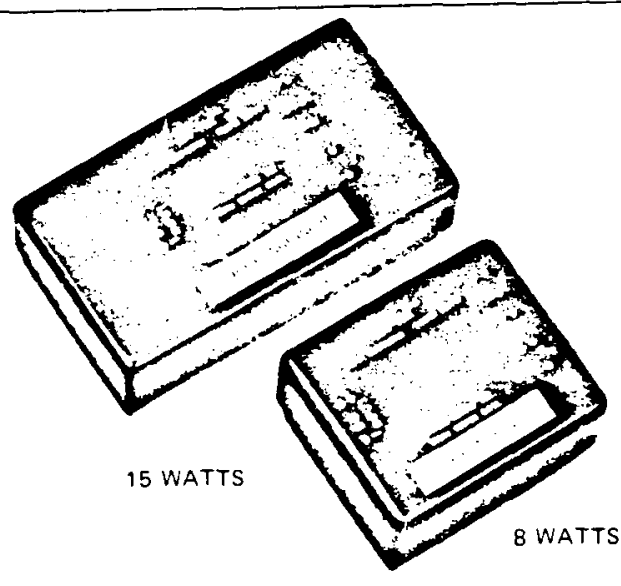

The Stevens-Arnold W'ide Range Input Triple Output DC to DC Converter advances the art by joining two well accepted power processing concepts, packaged in a single module for PCB mounting.

The Input voltage is applied directly to a step-down Switching Regulator. This con verts the $6.5 \mathrm{~V}$ to 40 volt $D C$ Input to a Well Regulated $(0.5 \%$ line. $0.2 \%$ load $)$ Low Noise $(40 \mathrm{mV}$ p-p) 5 volts DC Out put. This Output is not lsolated from the Input voltage since they must share com. mon negative refurns.

Within the module the 5 volts is addition. ally filtered and used to power a Fully lsolated DC to DC Converter of the Low Noise, High Efficiency design employed in our popular Series A Iso-Pak Converters. This delivers an extremely Well Regulated $(0.01 \%$ typical) \pm 15 volts DC (or \pm 12$) \mathrm{com}$ pletely Isolated from the Input and the 5 volt Output.

The result is a Single Module which can operate with virtually constant power Effi ciency (68 to $73 \%$ ) over and anywhere within the entire Input voltage range of $6.5 \mathrm{~V}$ to 40 volts DC. In addition, the High Efficiency produces a very Low Operating Case Temperature rise of $20^{\circ} \mathrm{C}$ thus im. proving the reliability of the module.

\section{Operating Considerations}

(CL) Capacitive Load Limits The 5VDC digital Output (sw. reg.) can be damaged or even destroyed if the Regulator sees a true effective Capacitive load greater than $100 \mu \mathrm{F}$.
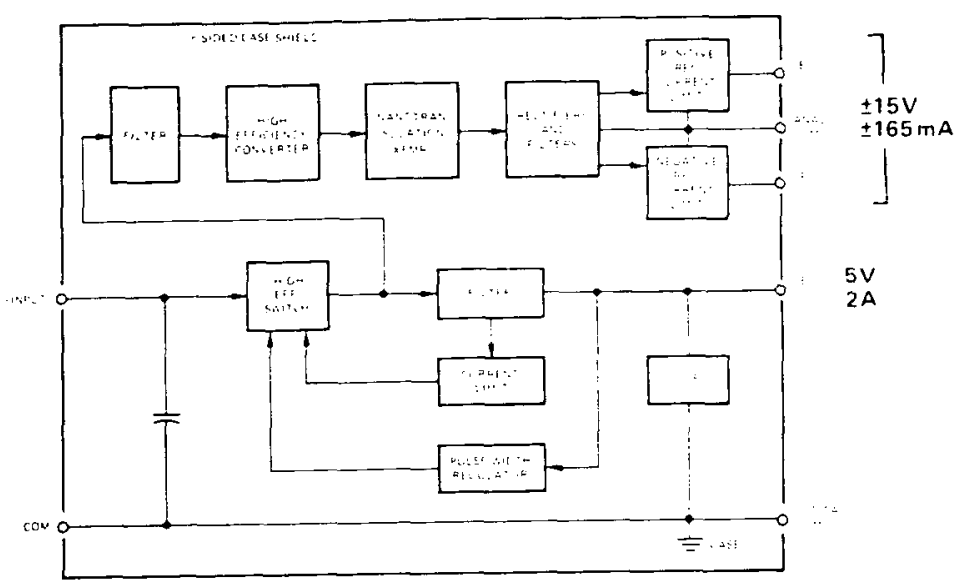

Figure 1. Block Diagram

( $R$ R in) Reflected Input Ripple Noise Current Swithing Regulators nurmally $e x$. hibit Inpu1 Current Pulses equal in nugnitude to the Load Current. We have prepar ed an Application Nole "Filtering Switching Regulators" which discusses a number of filtering techniques. An accessory huput Filter module. 10 be inserted between the Input source and the power Module. is available.

Fault Mode When the SVDC Single Out put is short circuited the Dual Output will also shut down.

Source Impedance The WC Series should be powered from a Source having a suffic iently Low Impedance to hold the voltage at the Input Terminals below I Volt p-p at $10 \mathrm{KHz}$ or above.

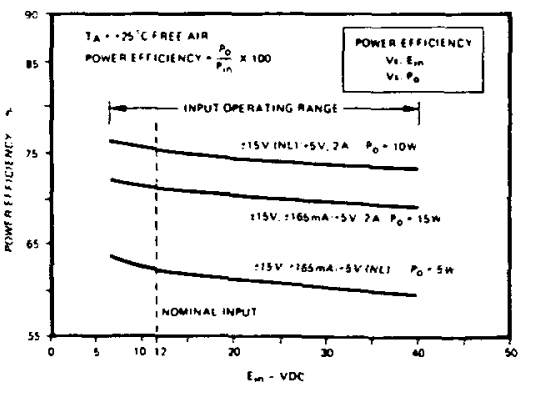

Figure 2. Typical Performan: : Curves

\section{FEATURES}

ONE MODEL for any DC Input Source from from $6.5 \mathrm{~V}$ to $40 \mathrm{VDC}$

LOW COST

- 15 watt output $\$ 114.00$ (9.9)

8 watt outpur $\$ 99.00(1-9)$

SHORT CIRCUIT PROTECTION AND O.V.P. (5VI

HIGH EFFICIENCY $70 \%$

LOW SURFACE TEMPERATURE RISE

FL Case Temp. Rise at $12 \mathrm{~V}$ Nominal InDu: $+20^{\circ} \mathrm{C}$

ISOLATION

- Input to Dual Output: $1 \times 10^{4}$ ohms 500VDC min.

- Input to Single Output: None

EMIRF: SHIELDING

- Transformer is Double Shielded

- Case is shielded 6 sides (continous)

LOW WIDEBAND NOISE (BW $=20 \mathrm{MHz}$ )

$1 m \vee$ rue RMS $-40 m V$ p.p

\section{APPLICATIONS}

U.P.S. (UNINTERRUPTABLE POWER SUPPLIESI

- Increases "Backup Power" operating time significantly

High Efficiency: 70\%

PORTABLE OR MCBILE SYSTEMS AND

INSTRUMENTS

Extends the voltage usable range of Low Cast dry cells

Extends the Voltage-usable range of 12 VDC Recluargeable celis and rectuces the Charge Duty Cycte

GENERAL

- Provides $0.1 \%$ Regulation from a $6.5 \mathrm{~V}$ to $40 \mathrm{VDC}$ Battery Input

Provides P.C. Board point-of-load Regulation from an Uniegulated main system Supply 
SPECIFICATIONS 1

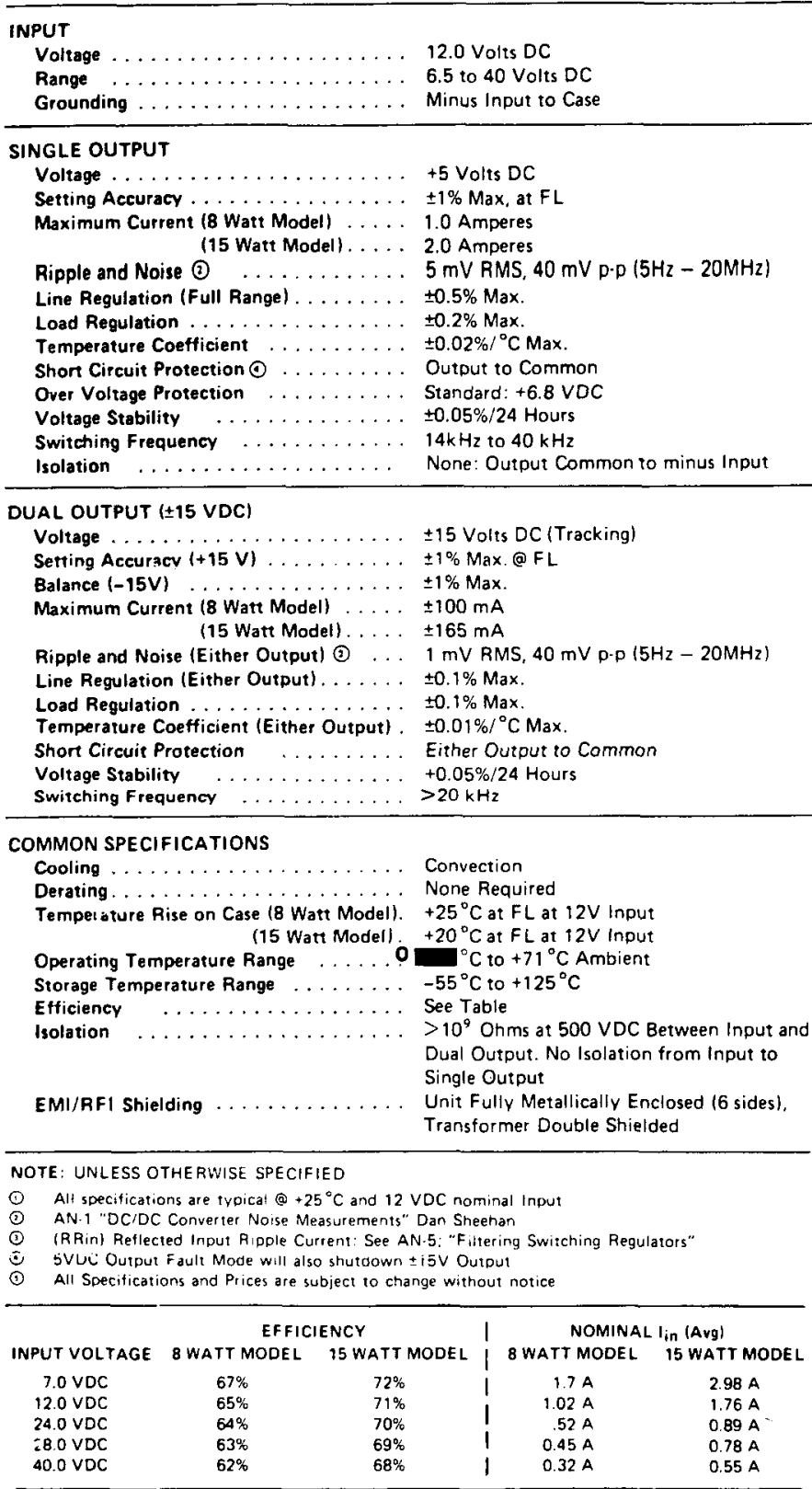

MODELS

All models have Triple Outputs consisting of I Dual Output feither $\pm 15 \mathrm{~V}$ or $\pm 12 \mathrm{~V}$ plus 1 Single Output. Other combinations are available on Special Order. The Single Output is always 5 volts, 2 Amp. in th: U Package and 1 Amp. in the $X$ Package.

TYPICAL ORDERING SPECIFICATION WC (7.40) T15/165 U

PRICES

$\begin{array}{ccccccc}\begin{array}{c}\text { Spec } \\ \text { Series }\end{array} & \begin{array}{c}\text { Input } \\ \text { Voltage } \\ \text { Range }\end{array} & \begin{array}{c}\text { Number } \\ \text { of } \\ \text { Outputs }\end{array} & \begin{array}{c}\text { Dual } \\ \text { Output } \\ \text { Voltage }\end{array} & \begin{array}{c}\text { Dual } \\ \text { Output } \\ \text { Current (mA) }\end{array} & \text { Pkg } & \begin{array}{c}\text { Price } \\ (1.9)\end{array} \\ \text { WC } & (7.40) & T & 15 & 165 & \text { U } & \$ 114.00 \\ \text { WC } & (7.40) & T & 12 & 165 & U & 114.00 \\ \text { WC } & (7.401 & T & 15 & 100 & \times & 99.00 \\ \text { WC } & \{7.40) & T & 12 & 100 & X & 99.00\end{array}$

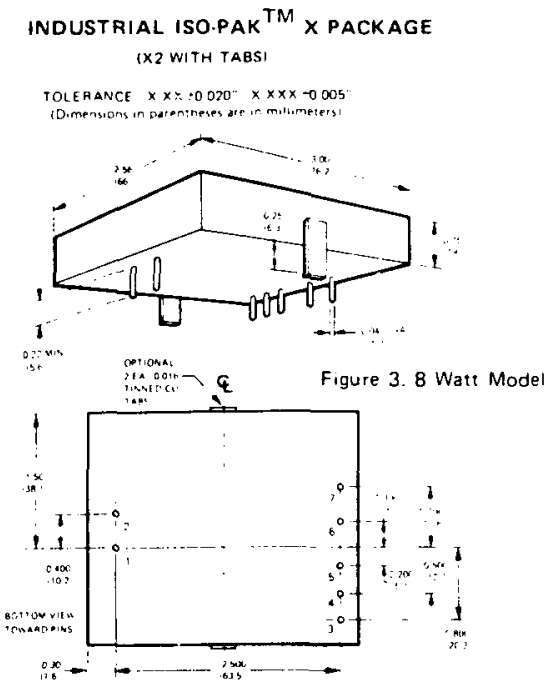

INDUSTRIAL ISOPAK TM UPACKAGE IUZ WITH TABS
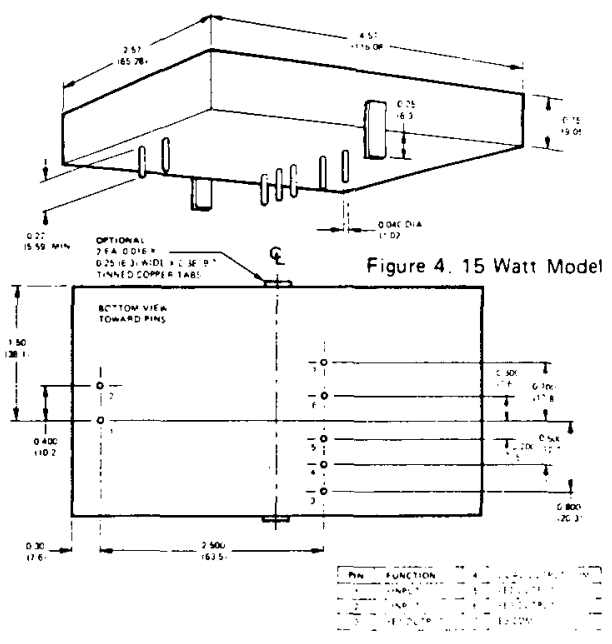

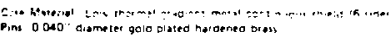

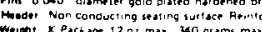

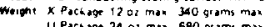

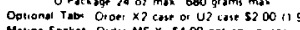

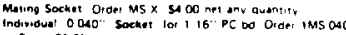

WARRANTY We watrant our products and Darts 10 be tree of detecis of materials and workmanship tor one vear from the dale of shipment Our liabilitr undet this war. tanty is limited to exchanging, repalisng or issuing credit 1.0.b our tactory, al out option, for any oroducts or Darts iustitiably returned or the customer. We assume no ALL OTHER WARAANTIES EXPAESSED OR IMPLIED. INCLUDING WARRANTIES FOR FITNESS FOA ANY PARTICULAA PURPOSE ANO MERCHANTABILITY. ARE EXCLUDEO

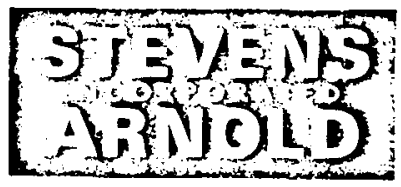

The Super Isolation People

7 Elkins Street, South $\mathrm{BC}$ TEL: $617.26 i$

Mass. 02127

Catalog No. SF-613 Cable Address Stearco

Printed in U.S.A. 


\section{APPENDIX E}

\section{SYSTEM-TIMING ILLUSTRATIONS}

The illustrations in this appendix show the data and clock-timing relationships in the HEC-100 (HLNCC) in both real-time and idealized form. Figures $E-1$ and -2 show overall data timing; Fig. E-3 shows coincidence gate timing; Figs. E-4, -5 , and -6 illustrate events in the coincidence summation circuitry with varying inputs; and Fig. E-7 illustrates clock-timing relationships. Figures $E-1,-2$, and -3 are real-time photographs, whereas Figs. $E-4$ through -7 are idealized figures.

Figures E-1 and -2 are photographs obtained with a Tektronix 475 oscilloscope, with a 20-MHz bandwidth set, and a C-30 oscilloscope camera. Each figure's horizontal scale is $100 \mathrm{~ns} /$ div. The top trace in both photos was the synchronization (triggering) signal; the signal is present at test point TA2 whenever there is a signal present at the shift-register input. In this case, the input was driven at a rate greater than $128 \mathrm{kHz}$ by the Los Alamos RP-501 random-pulse generator. The TAX and TBX designators at the immediate right of each photo refer to the test points found at the top of the shift-register board; each designator is followed by a signal label and, parenthetically, the voltage amplitude of the signal. The two photos are multiple exposures, with triggering as above, and vertical offsets from trace to trace, with each trace attenuated, as necessary, to produce a 1-graticule-division (vertical) transition for the logic levels. The test points and associated subcircuits (source and destination components) are schematically represented on Los Alamos Dwgs. D200734-1 and -2 .

Figure E-3 is a photograph made with the same equipment as was used for Figs. $E-1$ and -2 , including the signal source. The figure's horizontal scale is $50 \mathrm{~ns} /$ div. The vertical scale is $2 \mathrm{~V} / \mathrm{div}$. The oscilloscope trigger was the top trace $(R+A$ strobe, $T B 5)$. The bottom trace reflects logic transitions in the $R+A$ "1" bit board component location 3C, pin 11 (82S83 BCD adder) resulting from the interaction of the $R+A$ strobe, latch, and the UP/DOWN counter. Horizontal sweep rate was $50 \mathrm{~ns} /$ div. The photograph was originally taken to confirm that the circuitry met certain design requirements; namely, that 1atching, up-count, and down-count could all be done within one clock cycle and still meet component setup times conservatively. 


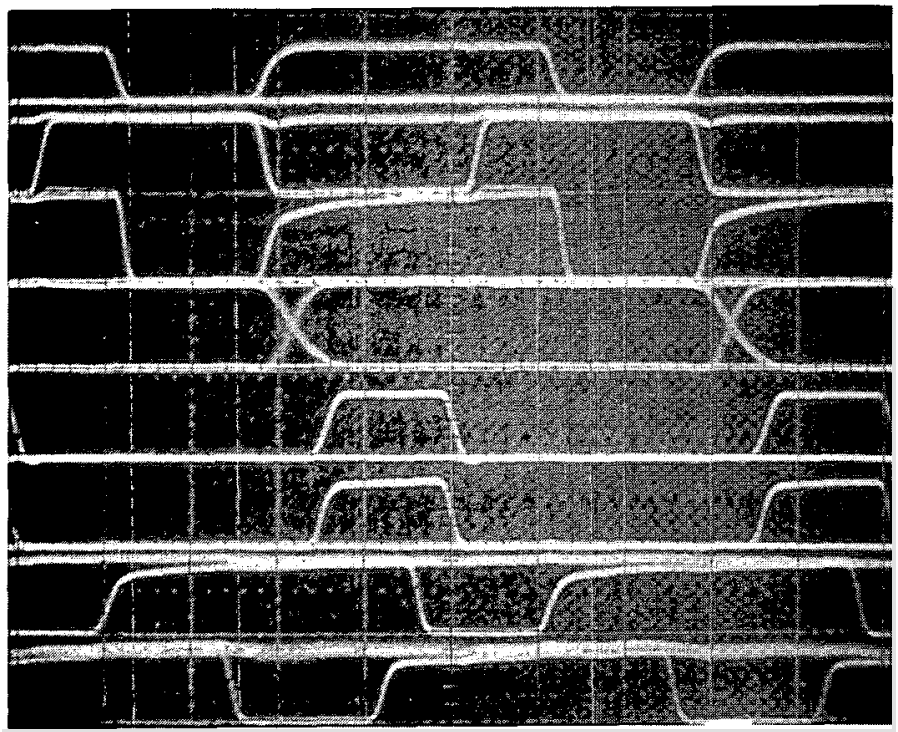

TA2 SYNC (5 V)

TB1 $\phi 1$ (12 V)

TB3 DELAY DATA IN (5 V)

TB4 DELAY DATA OUT ( $5 \mathrm{~V}$ )

TB5 $R+A(5 \mathrm{~V})$

TB7 A (5 V)

TB2 UP (5 V)

TA7 DOWN (5 V)

Fig. E-1. Data timing No. 1 .

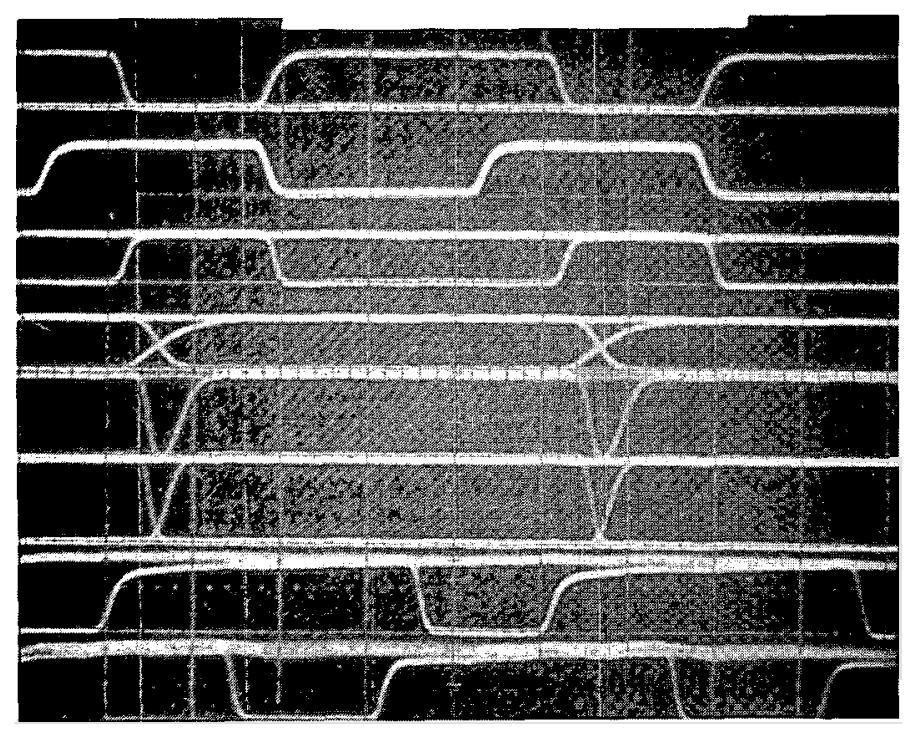

TA2 SYNC (5 V)

TA3 SRCK (12 V)

TA4 PREDELAY IN (12 V)

TA1 PREDELAY OUT (12 V)

TA6 PREDELAY OUT (5 V)

TA5 32 MS GATE (5 V)

TB2 UP (5 V)

TA7 DOWN (5 V)

Fig. E-2. Data timing No. 2 


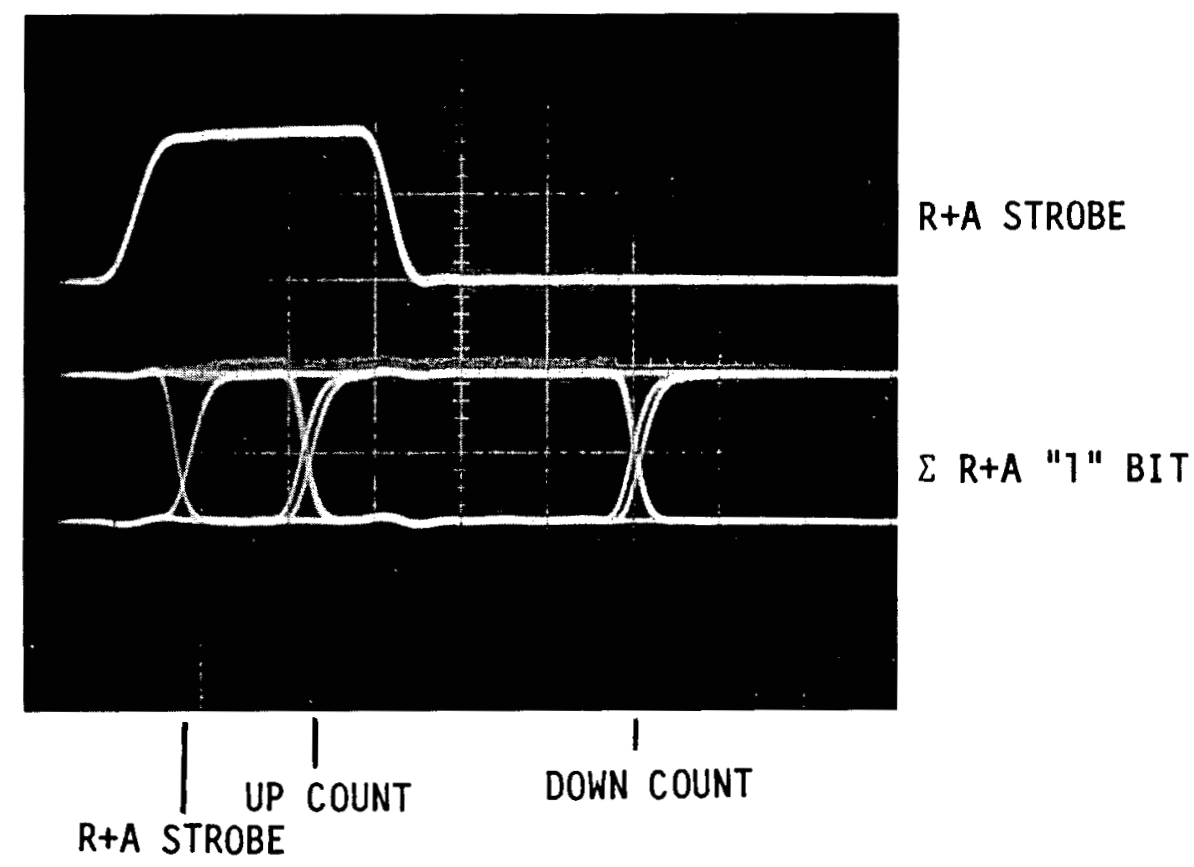

Fig. E-3. Coincidence gate timing. 
Figures E-4, -5 , and -6 are photographs obtained with a Tektronix 7001 logic analyzer (plug-in) module installed in a Tektronix 7603 oscilloscope mainframe using a C-50 camera. Each figure's horizontal scale is nominally $250 \mathrm{~ns} /$ div. The traces are idealized figures, rather than real time; the signals are available at the test points listed after the signal label with the exception of the (Sigma) $R+A 1$ signal, which is sourced at component $3 C$, pin 11 (shift-register board). The timing reference is the top trace, SRCK (Shift-Register lock) TA3. Triggering was generated by the SYNC OUT (Data Synchronizer Output) TA2. In Fig. E-4, the input to the shift register was a manually triggered single-shot pulse and in Figs. E-5 and -6 , a manually triggered pulse and (variable) delay pulse pair. The delay pulse in Fig. E-5 was delayed by less than the predelay setting from the initial pulse, whereas that of Fig. E- 6 was delayed by a time greater than that of the predelay setting.

Figure E-7 illustrates the various clock signals in the HEC-100. The photograph was taken with the same equipment as that used in Figs. E-4, -5 , and -6 ; again, these are idealized waveforms rather than real-time representations. The figure's horizontal scale is nominally $200 \mathrm{~ns} /$ div. Triggering was on the initial positive-going transition of Phase 2; this signal is available at (shift-register board) component location 8D, pin 11 (Phase 2 level shifter). The other signals aside from Phase 1 and SRCK, which are available at the listed test points, are sourced at the following locations.

CK

CK $1 / 2$

CK $1 / 2+150 \mathrm{~ns}$

CK $1 / 2+250 \mathrm{~ns}$

CK $1 / 2+350 \mathrm{~ns}$ component location 100, pin 2

component location 9D, pin 6

component location $6 \mathrm{D}$, pin 12

component location $6 \mathrm{D}$, pin 10

component location $6 \mathrm{D}$, pin 8

The clock sources are schematically represented on Los Alamos Drawing D200734-1. 


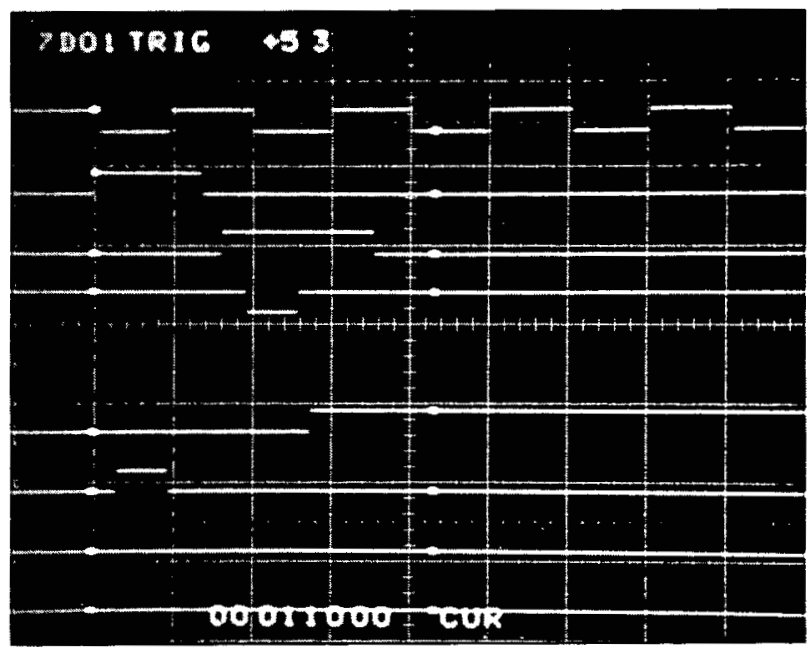

SRCK TA3

SYNC OUT TA2

PREDELAY OUT TA6

UP COUNT TB2

$\sum R+A 13 C 11$

$R+A$ STROBE TB5

Fig. E-4. Single pulse input.

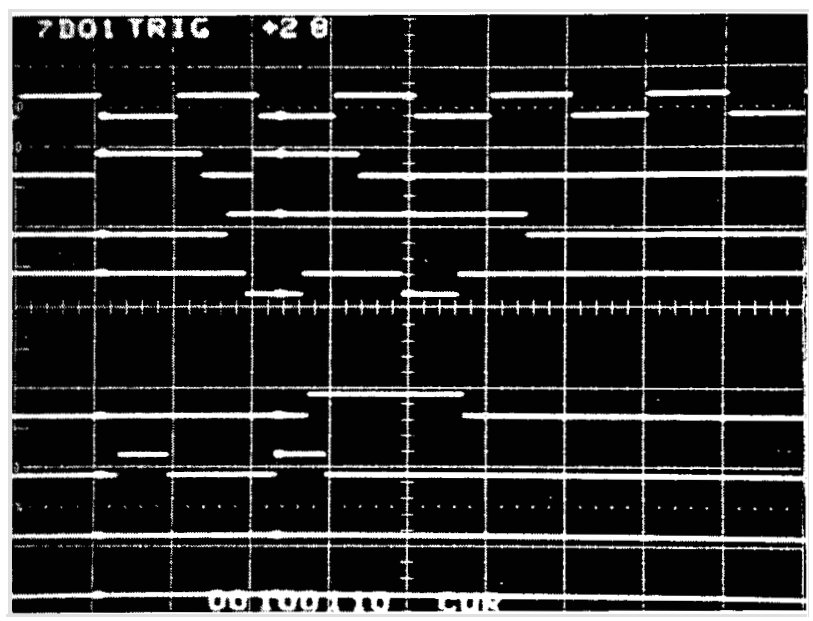

SRCK TA3

SYNC OUT TA2

PREDELAY OUT TA6

UP COUNT TB2

$\sum R+A 13 C 11$

R+A STROBE TB5

Fig. E-5. Double pulse input. (Delay period less than predelay).

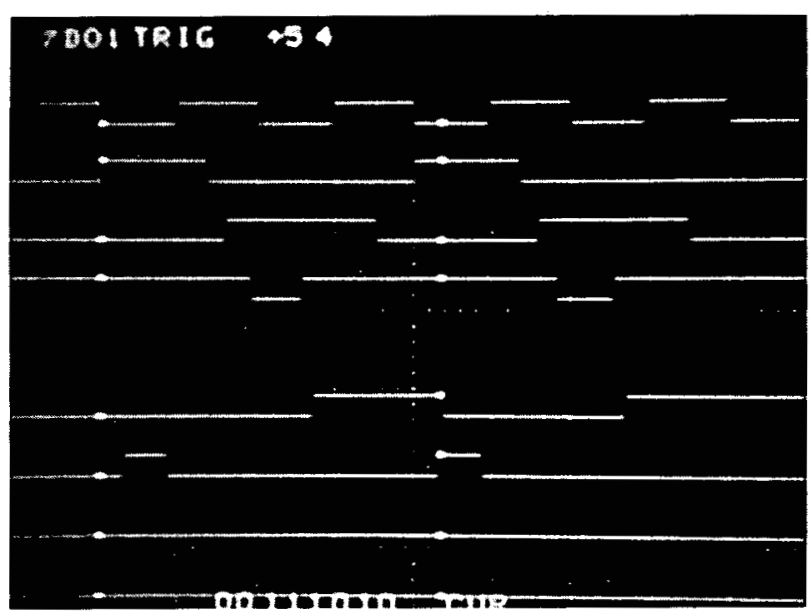

SRCK TA3

SYNC OUT TA2

PREDELAY OUT TA6

UP COUNT TB2

$\sum R+A>3 C 11$

$R+A$ STROBE TB5

Fig. E-6. Double pulse input. (Delay period greater than predelay). 


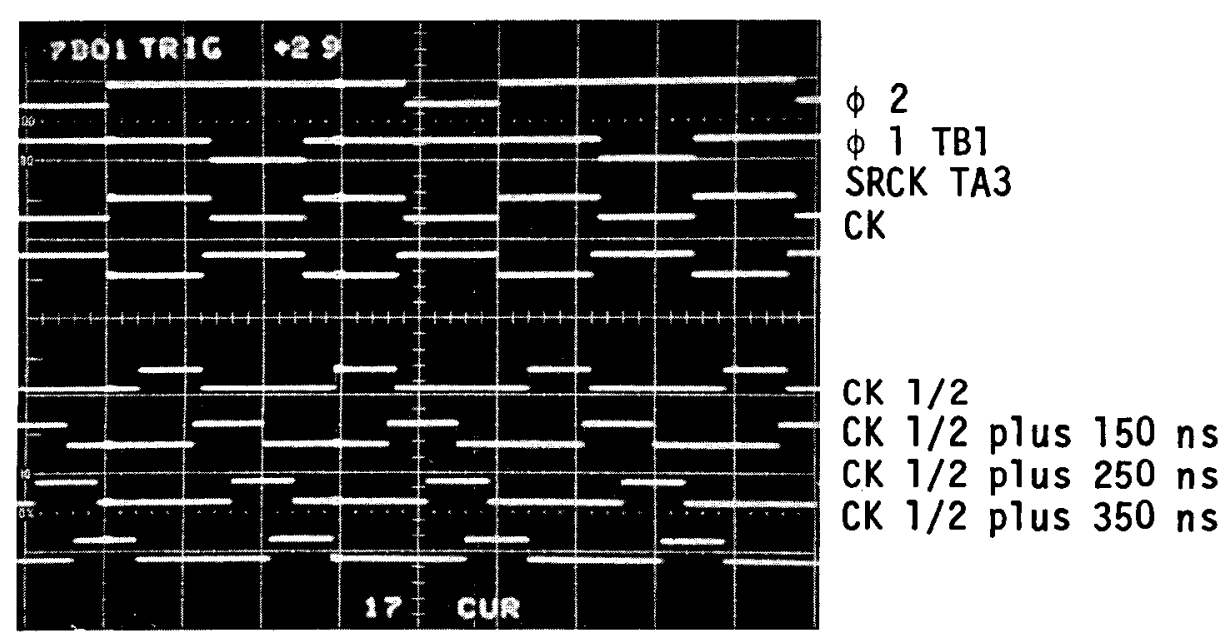

Fig. E-7. Clock Timing. 
APPENDIX F

HLNCC SCHEMATIC SET

The schematic sets in these manuals derive from the schematic set documenting the original (prototype) instrument. The title for that set is IAEA Thermal Neutron Coincidence Counter, Los Alamos Dwg. 68Y155362, sheets D1 through 011 . The schematic set was then redrawn and renumbered for publication ${ }^{2}$ by EG\&G in conjunction with their manufacture of the production prototype instrument. A listing of the schematic sets is shown below. The EG\&G drawing set has subsequently been retitled/renumbered by IRT Corporation for inclusion in their manual ${ }^{3}$ (issued with the current production instrument).

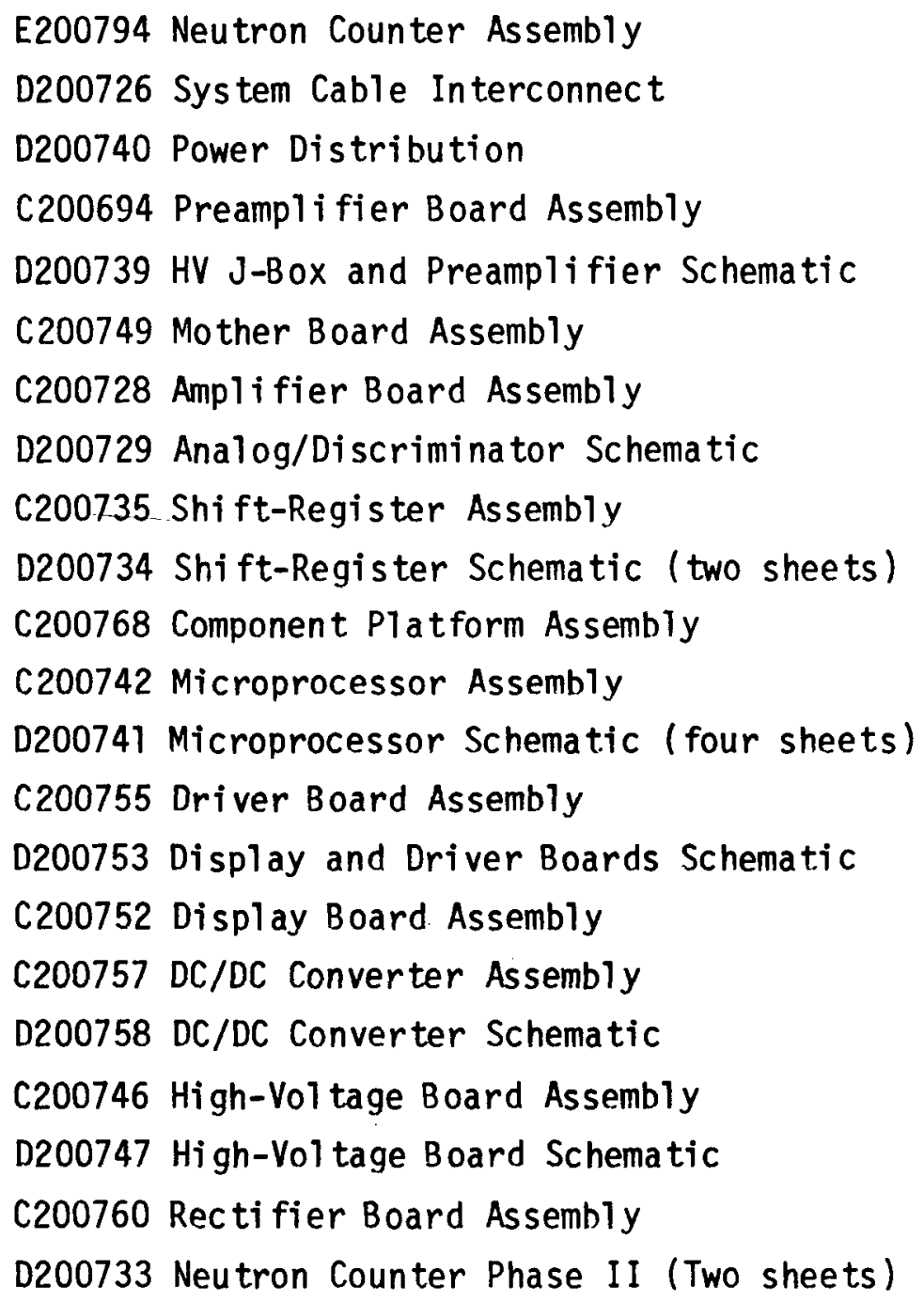



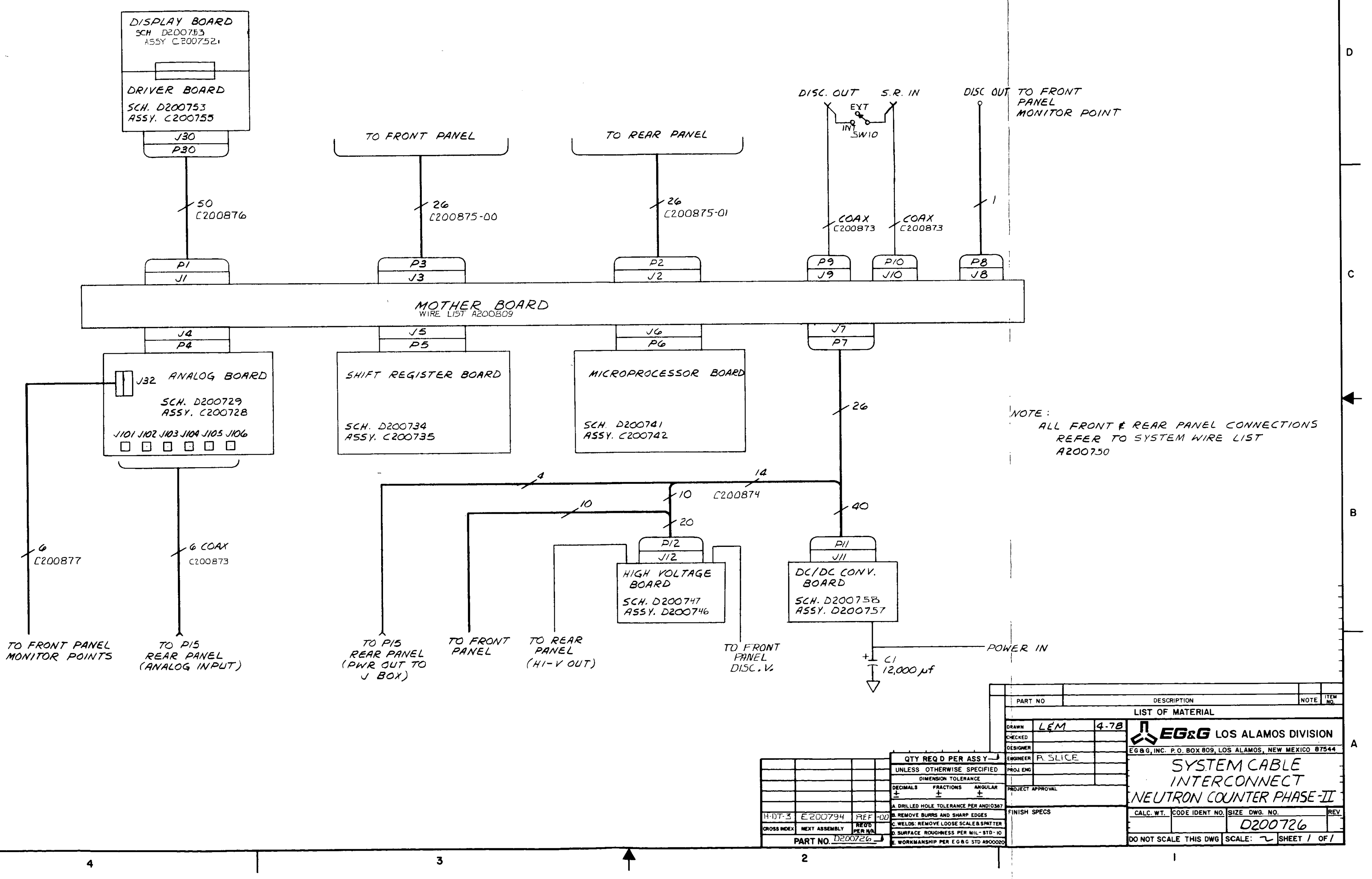


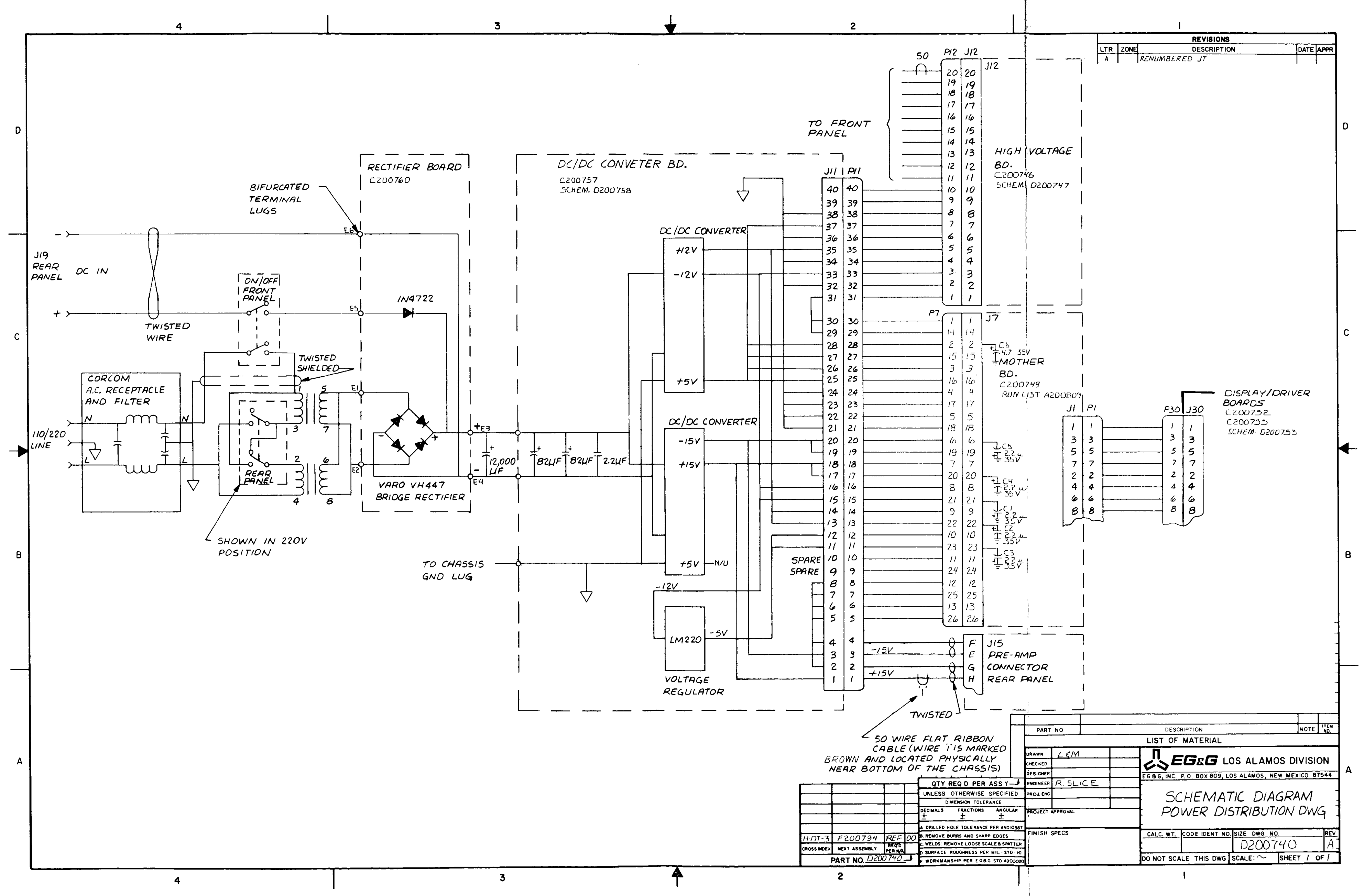




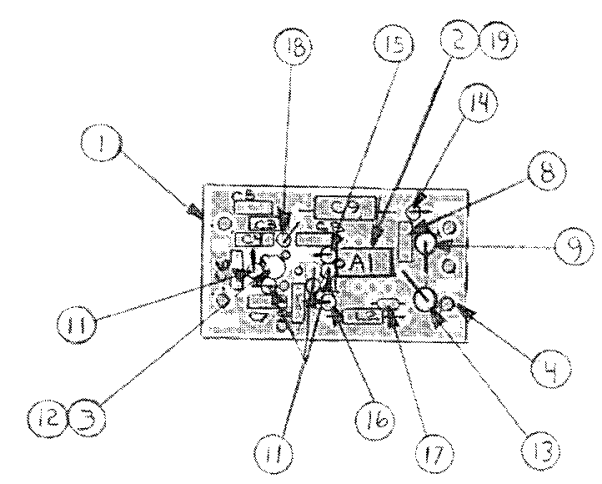

NOTES: UNLESS OTHERWISE SPECIFIED 1. FOR SHEMATIC SEE DWG NO. D200739 2. DIODES MOUNTED WITH CATHODE UP. 3. MOUNT WITH POSITIVE END DOWN. 


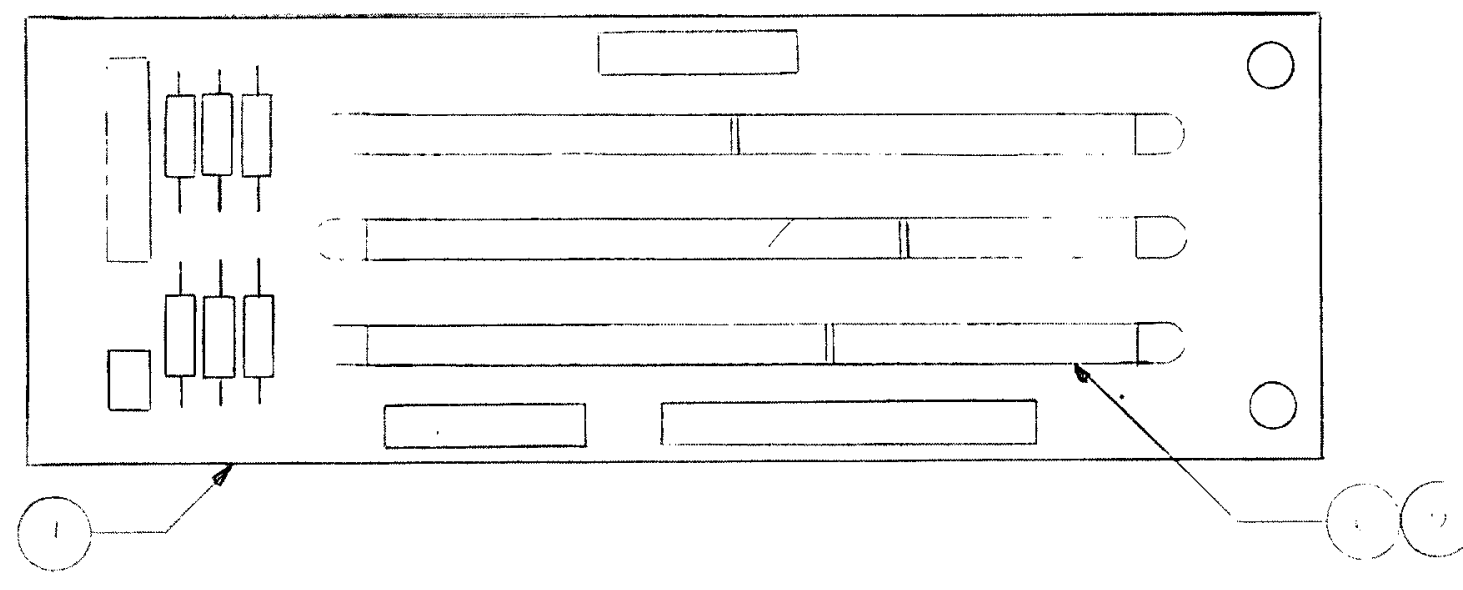

NOTES: UNLESS OTHERWISE SPECIFIED 1. FOR SCHENIATIC DIA. SEE DWG NO. AZ00809 2. CAPACITORS CI-CG ARE OPTIONAL

3. INSERT KEY ON UY BETWEEN PINS $26-27$ J5 BETWEEN PINS $15-16$

JG BETWEEN PINS $20-21$

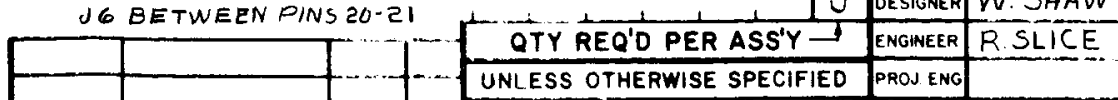

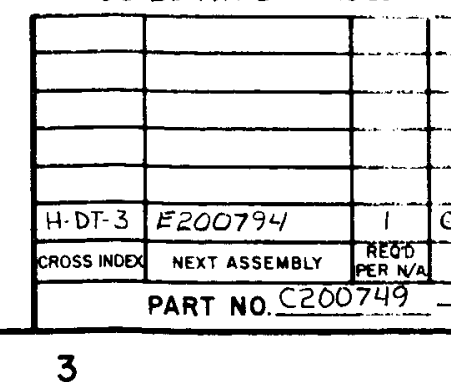

TECIMAIS TIMESSION TOLERANCE

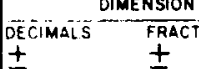
\begin{tabular}{l} 
\pm \\
\hline
\end{tabular}

\begin{tabular}{l} 
A ORILLED HOLE TOLERANCE PER ANDIOSQ7 \\
\hline REMOUE BURAS ANO SHARP EDGES
\end{tabular}

\begin{tabular}{l} 
B REMOUE BURAS ANO SHARP EDGES \\
\hline CWELOS REMOVEE LOOSE SCALE S SPATTER
\end{tabular}

D SURFACE ROUT TESS PER MLL-STO-10

4

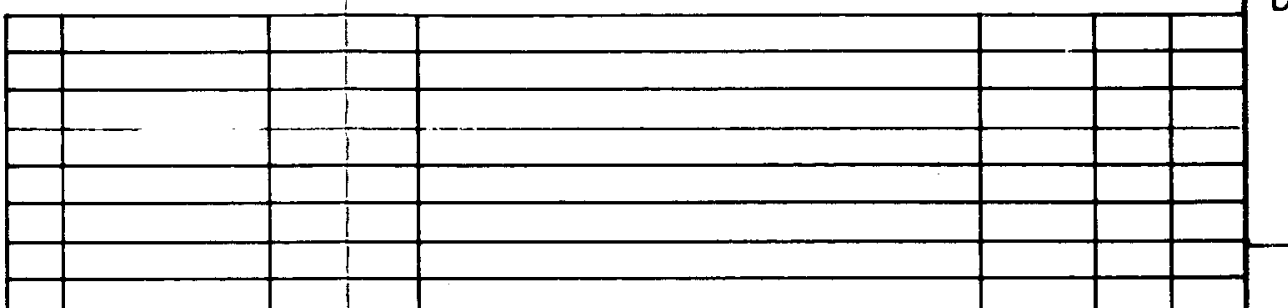

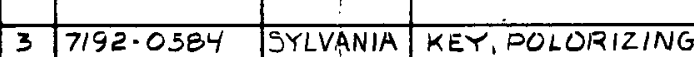

2 O31:0059-0001 MALCO CONNECTOR, COAXIAL, P.C.

\begin{tabular}{lll}
$65611-308$ & BERG & CONNECTOR, MINI-LATCH \\
\hline
\end{tabular}

3 6ADO4-50-1AZ-OOSYLVANIA CONINECTOR,CARD EDGE

\begin{tabular}{l|lll}
3 & $3493-2003$ & $3 \mathrm{M}$ & CONNECTOR, HEADER \\
\hline
\end{tabular}

\begin{tabular}{l|l|l|l}
$3456-2003$ & $3 \mathrm{M}$ & CONNECTOR, HEADER \\
\hline 1 & 3456 &
\end{tabular}

150D475X90358E' SPRAGUE CAP, TANTALUM, $4.7 \mu f, 35 \mathrm{WVDC}$

5 150D225 9905532 SPRAGUE CAP, TANTALUM, $2.2 \mu F_{2} 35 \mathrm{WVOC}$

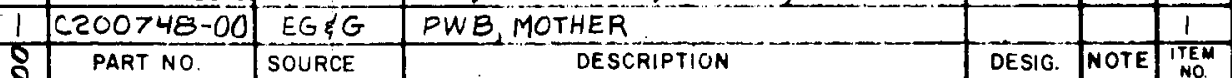

LIST OF MATERIAL

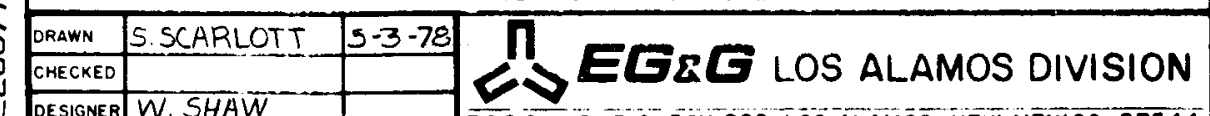

\begin{tabular}{l|l} 
R SLICE & ASSY, MOTHER BOARD
\end{tabular}

NEUTRON COUNTER II 


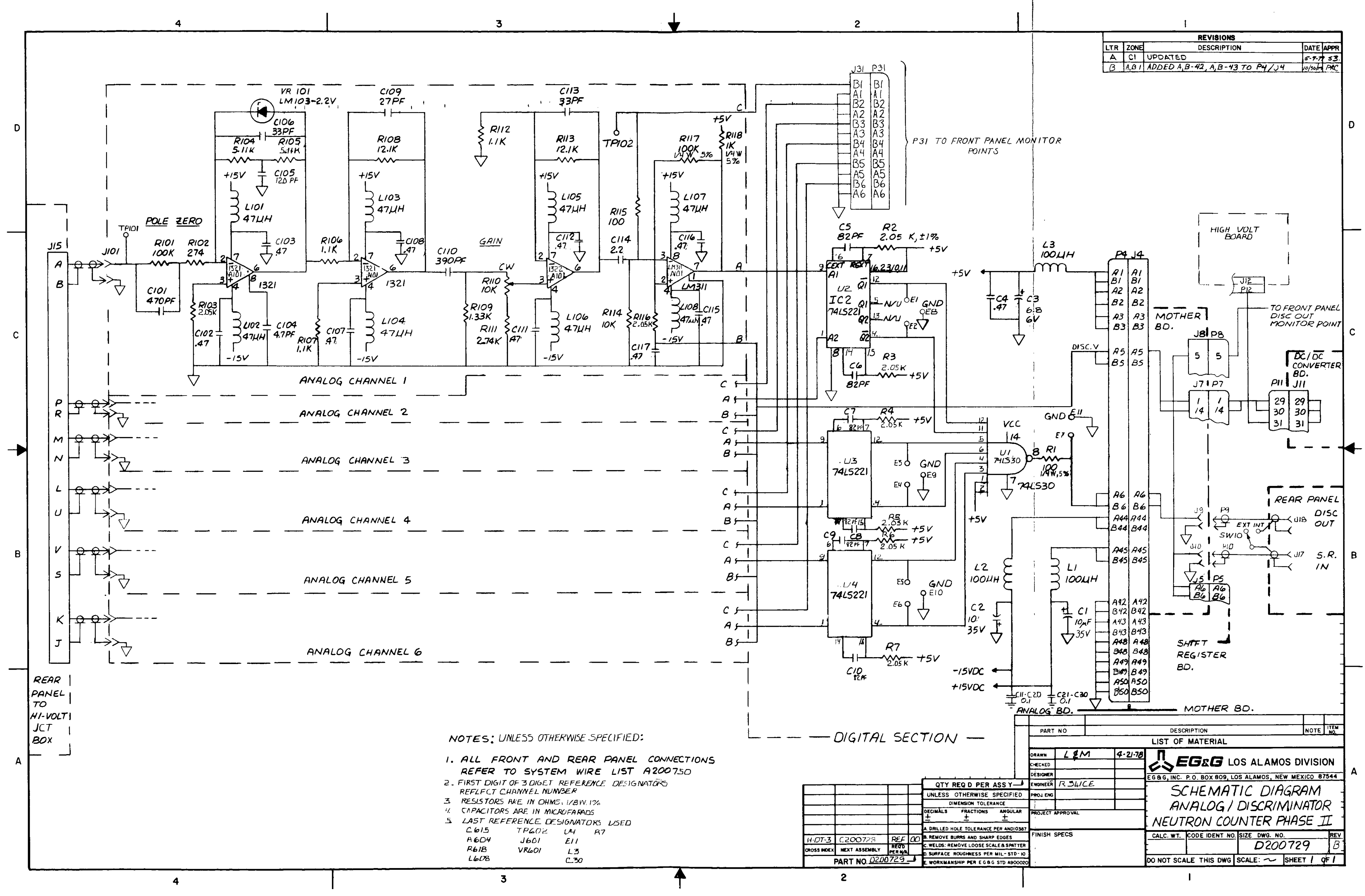



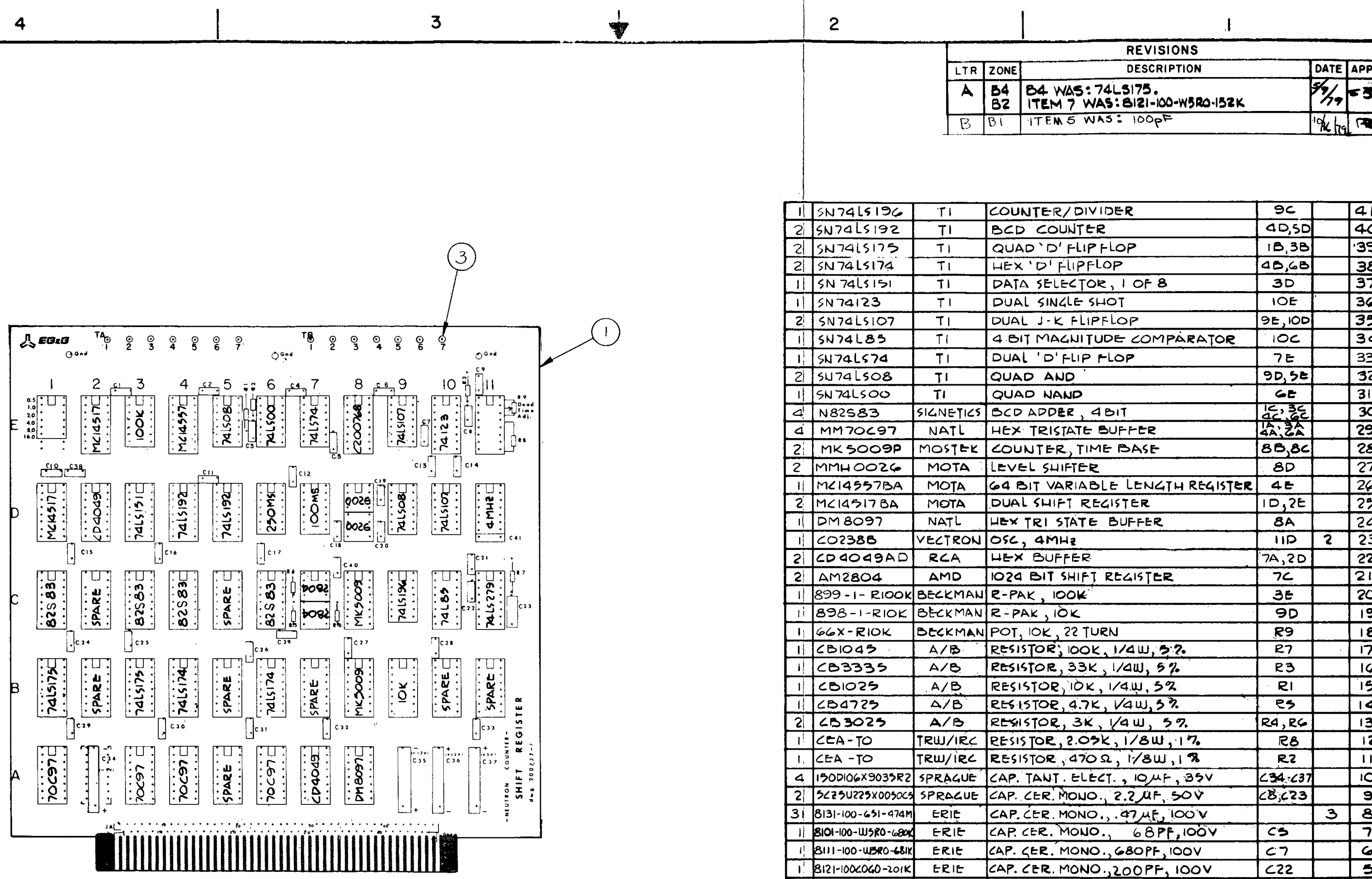

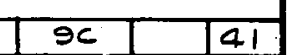

NOTES: UNLESS OTHERWISE SPECIFIED

1. FOR SCHEMATK DIA. SEE DWL, D200734

2. MOUT WITLOUT SOCKET

3. REFERENLE DESILNATORS FOR ITEM 8

$A R E \angle 1, \angle 2, \angle 4, \angle 6, \angle 9-\angle 21, \angle 24-\angle 33, \angle 38-\angle 41$

30 716-AG-2D AULAT SOCKET, DIP, 16 PIN $S$ 11714 -AL-2D AULAT SOCKET, DIP, 14 PIN

1 76PB-O6 CREYHILL DIP SUITCL

\begin{tabular}{l|l|l|l}
1 & $T T L-D M 2 S O$ & $E C^{2}$ & TIME DELAY \\
\hline 1 & $T T L-D M I O O$ & $E C^{2}$ & TME DELAY
\end{tabular}

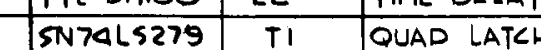

\begin{tabular}{l|l|l|}
\hline & STNTALSTY & TI \\
\hline
\end{tabular}
DESCRIPTION

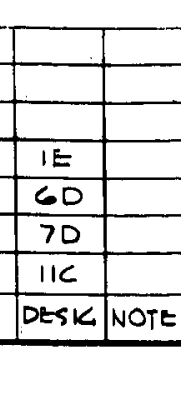

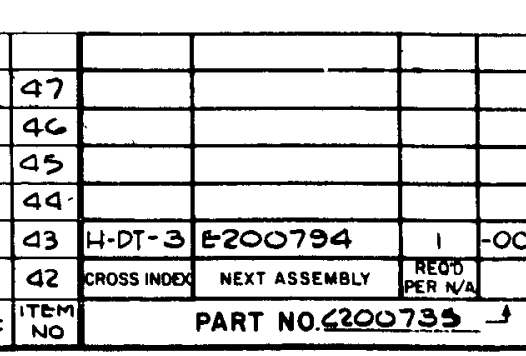

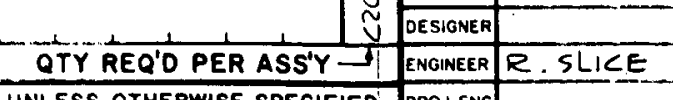
UNLESS OTHERWISE SPECIFIED DECTIMAIS OIMENSION TOLERANCE \pm FRACTIONS

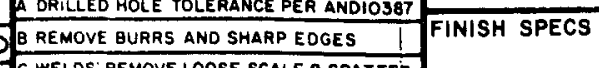
CWELOS REMOVE LOOSE SCALE Q SPATTER OS SURFACE ROUGHNESS PER MLL-STO-10 \begin{tabular}{|l|l|l|l|}
\hline 2 & SN74LSO8 & TI & QUAD AND \\
\hline 1 & SN74LSOO & TI & QUAD NAND \\
\hline 4 & N82S83 & SILNETICS & SCD ADDER, 4BIT \\
\hline
\end{tabular}

\begin{tabular}{|l|l|l|l}
\hline 482583 & SILNETLS & BCD ADDER, 4 BII \\
\hline 4 & $M M 70 C 97$ & NATL & HEX TRISTATE BUFFER \\
\hline
\end{tabular}

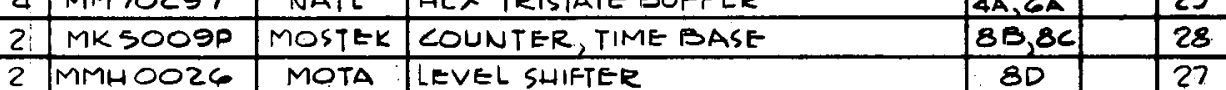

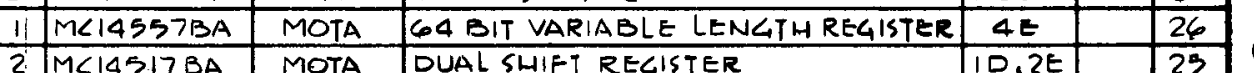

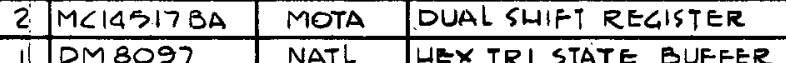

NATL HEX TRI STATE BUFFER

\begin{tabular}{|l|l|l|l|}
\hline 1 & CO238 & VECTRON & OSC, $4 M H z$ \\
\hline 2 & CD $\triangle O \triangle 9 A D$ & RCA & HEX BUFFER \\
\hline
\end{tabular}

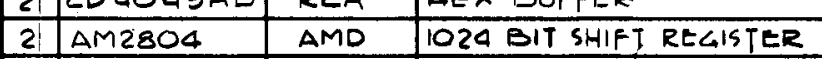

\section{899-1-RI0OK BECKMAN R-PAK, $100 \mathrm{~K}$}

1i $898-1-R I O K$ BECKMAN R-PAK, IOK

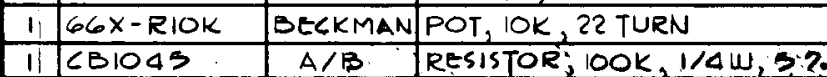

\begin{tabular}{|l|l|l|l}
\hline 11 & CBIO4S & $A / B$ & RESISTOR; $100 \mathrm{~K}, 1 / 4 W, 3 ; 2$ \\
\hline 1 & $C B 3335$ & $A / B$ & RESISTOR $333 \mathrm{~K}, 1 / 4 W_{1} 5 \%$
\end{tabular}

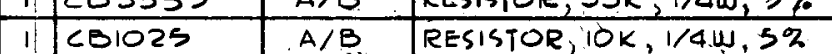

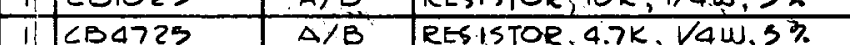

\begin{tabular}{l|l|l|l}
\hline 2 & $\angle B 3023$ & $A / B$ & $R E S I S T O R, 3 K, 1 / A W, 5 \%$
\end{tabular}

\begin{tabular}{l|l} 
1: CEA-TO & TRW/IRC \\
\hline
\end{tabular}

1. LEA -TO TRW/IRC RESISTOR, $470 \Omega, 1 / 8 W, 1 \mathrm{x}$

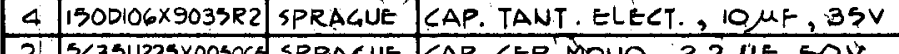

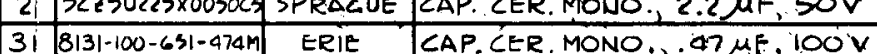

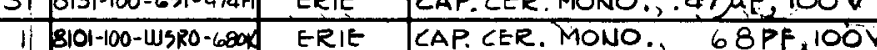

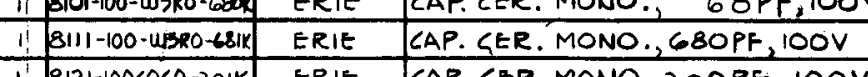

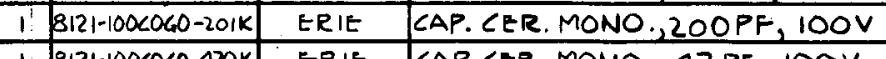

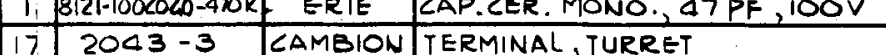

\begin{tabular}{l|l|l|l}
\hline 11 & $C 200768$ & $E L E L$ & $A S 5 Y$, COMPONENT PLATFORM
\end{tabular}

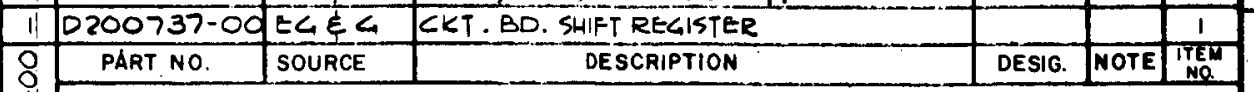

LIST OF MATERIAL

Thrar

EEERE LOS ALAMOS DIVISION

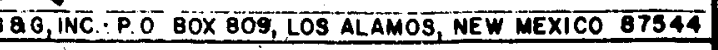
ASSY, SHIFT RELISTER 


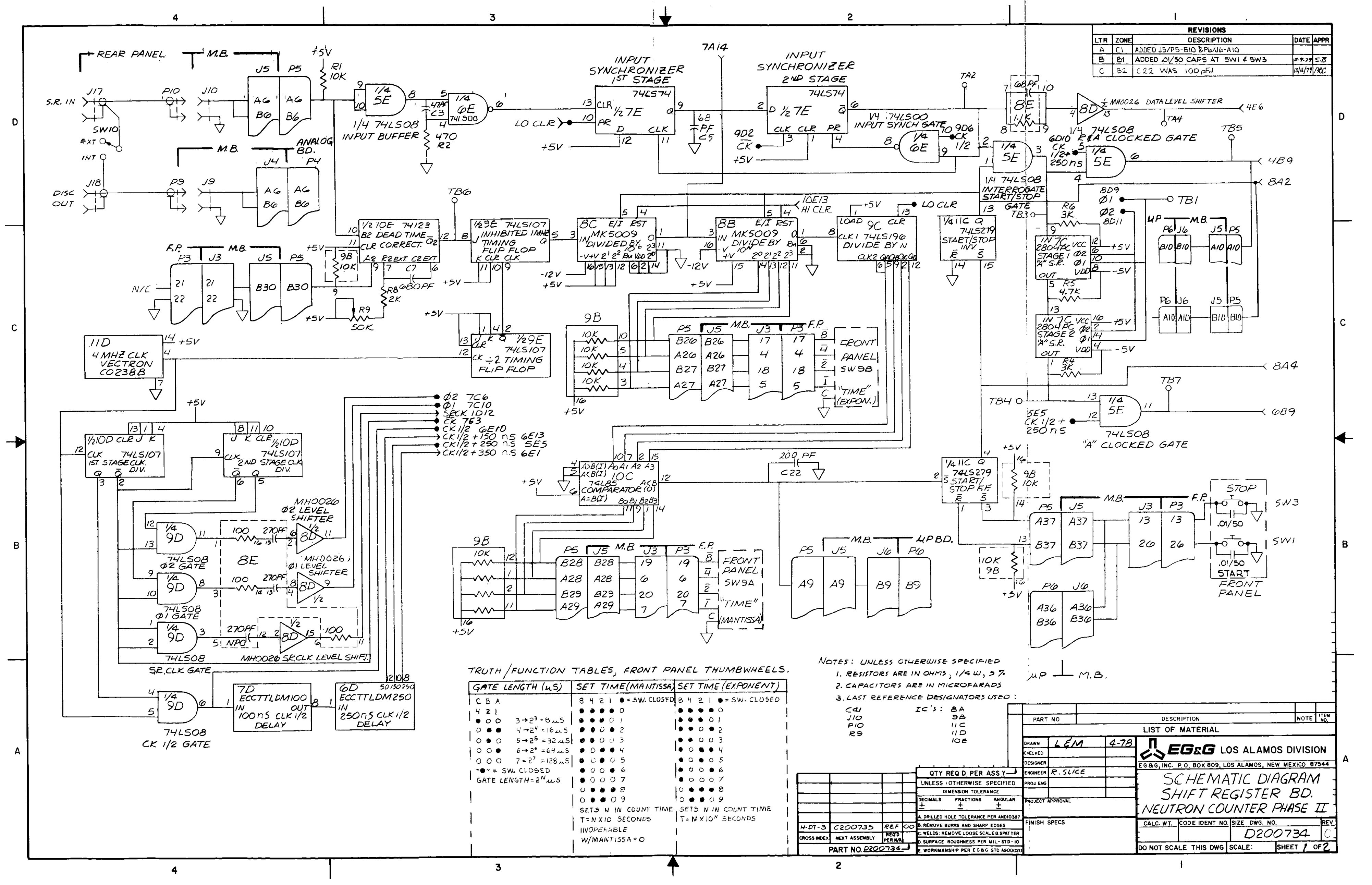




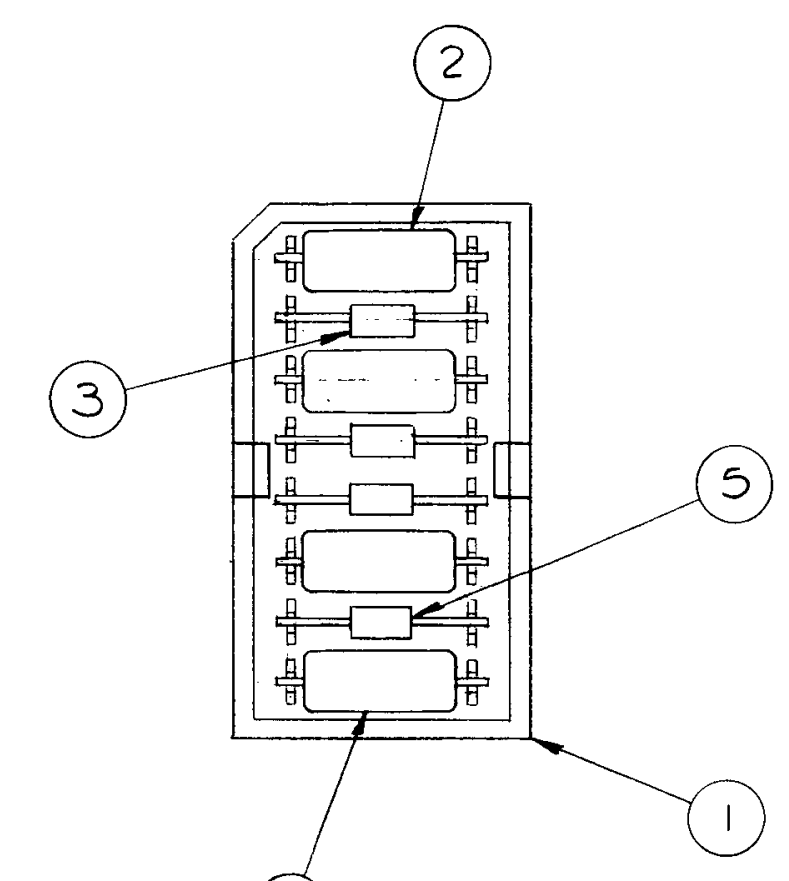

(4)

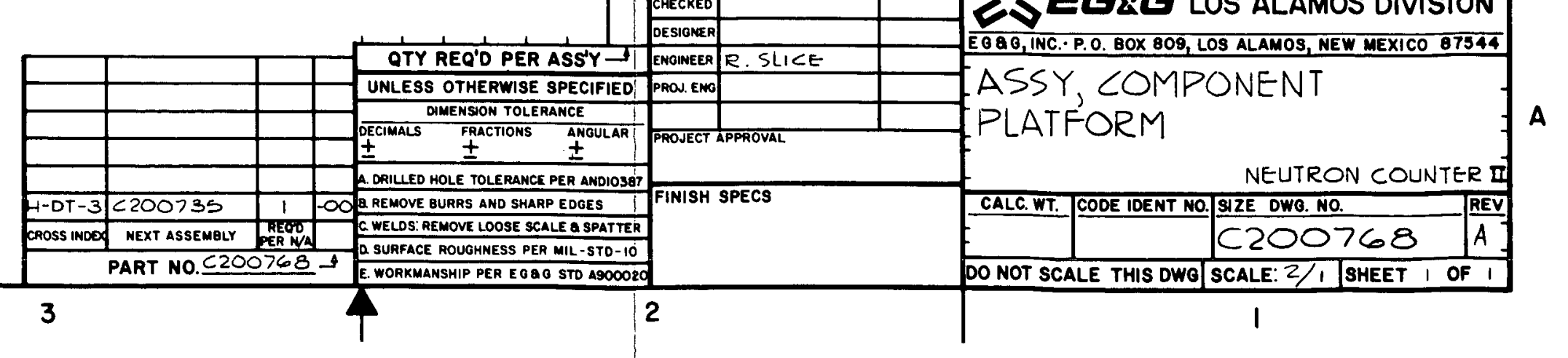




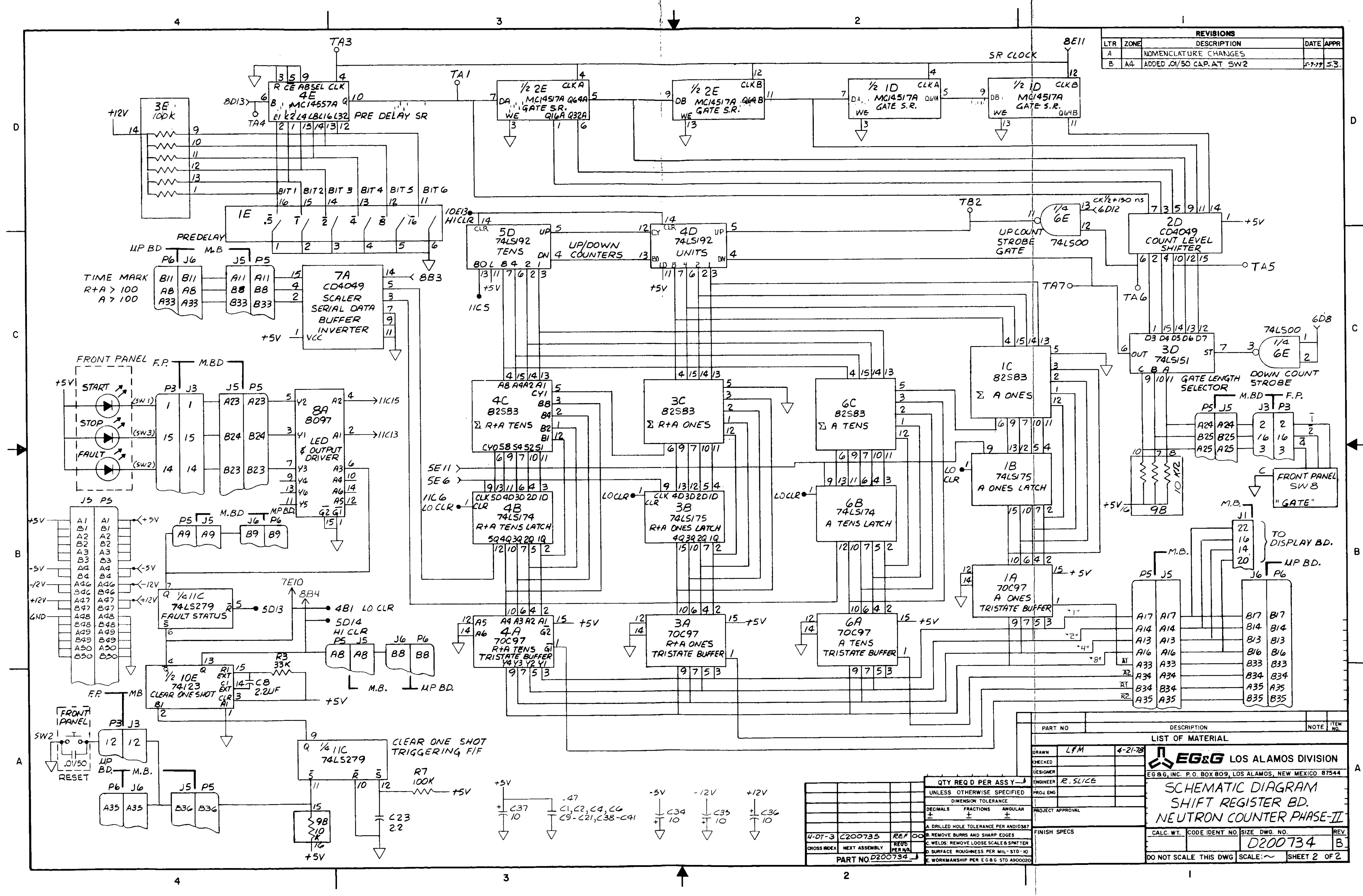


NOTES: UNLESS OTHERWISE SPECIFIED: 1. FOR SCHEMATIC DIA. SEE DWG D200741 2. REFERENCE DESIGNATORS FOR ITEM 7
ARE $C 1, C 2, C 5, C 8, C 9, C 12-C 15, C 17-C 29$

A

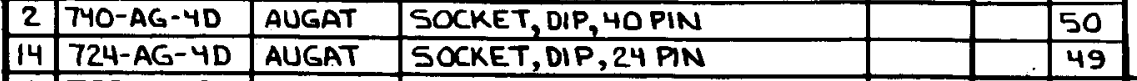
1 722-AG-2D ALGAT SOCKET, DIP, 22PIN

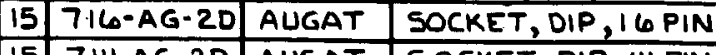
\begin{tabular}{l|l|l|l|}
\hline 15 & $714-A G-2 D$ & AUGAT & SOCKET, DIP, 14 PIN \\
\hline 5 & $2043-3$ & CAMBION
\end{tabular} \begin{tabular}{l|l|l|l}
\hline 5 & $2043-3$ & CAMBION & TERMINAL, TURRET \\
\hline
\end{tabular} \begin{tabular}{l|l|l|l}
1 1. & MFE-3002 & MOTOROLA FET, SWITCH \\
\hline 1 & . F9.
\end{tabular} 1 899-1-RIOK BECKMAN R-PAK, IOK 764-1-RIOOK BECKMAN R-PAK,10OK

\begin{tabular}{|l|l|l|l}
\hline OTY PART NO. & SOURCE & DESCRIPTION \\
\hline
\end{tabular}

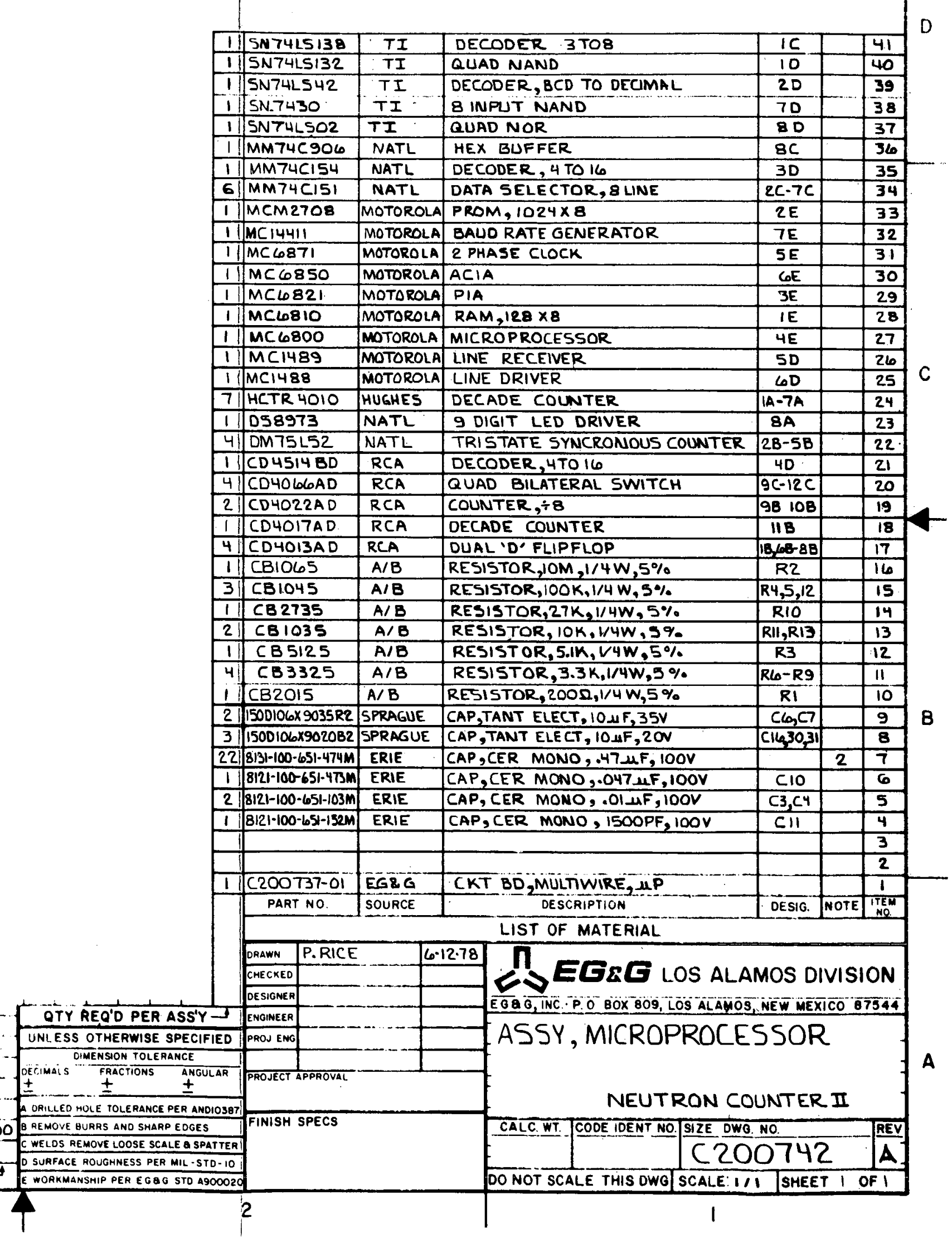




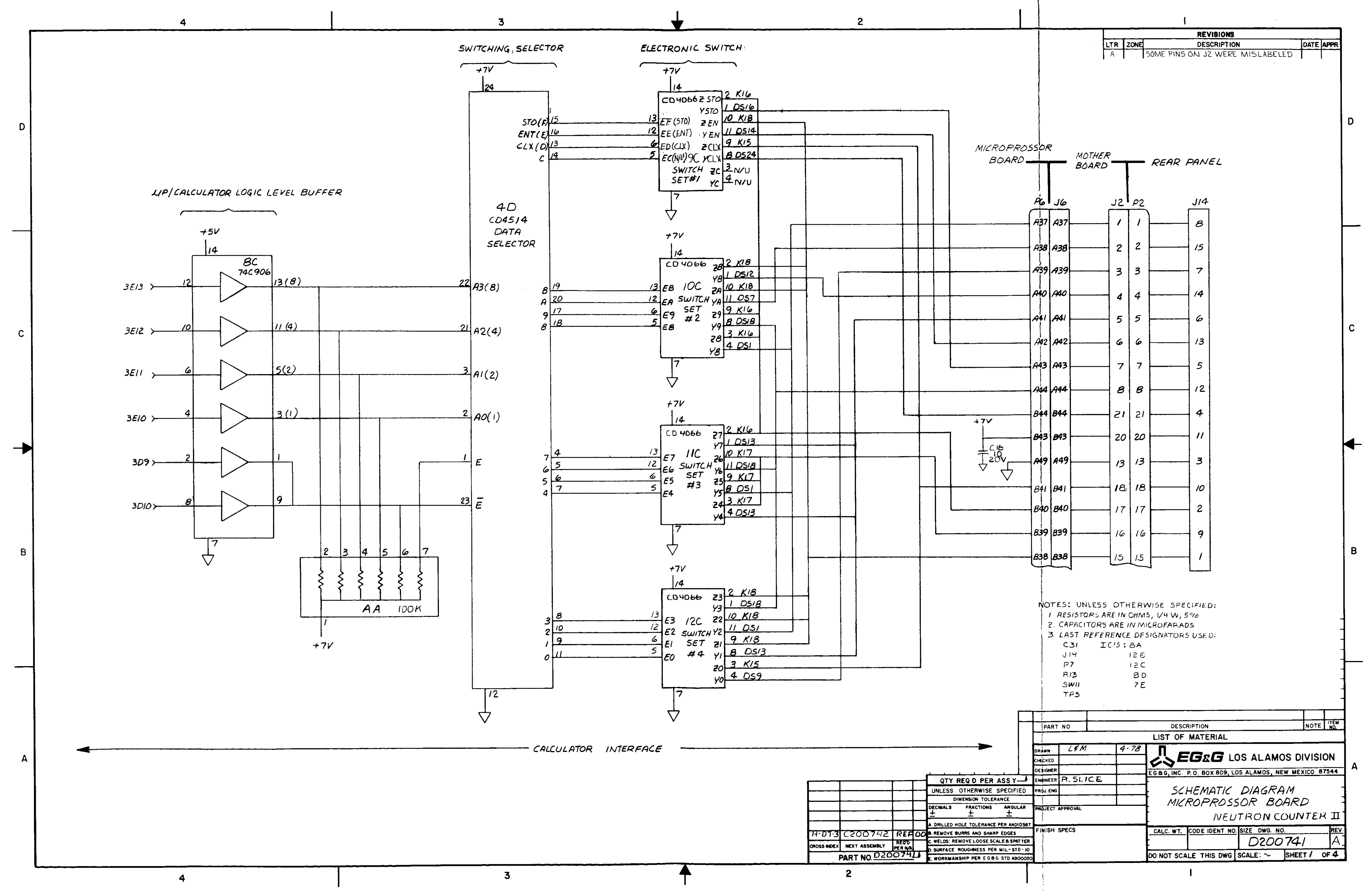




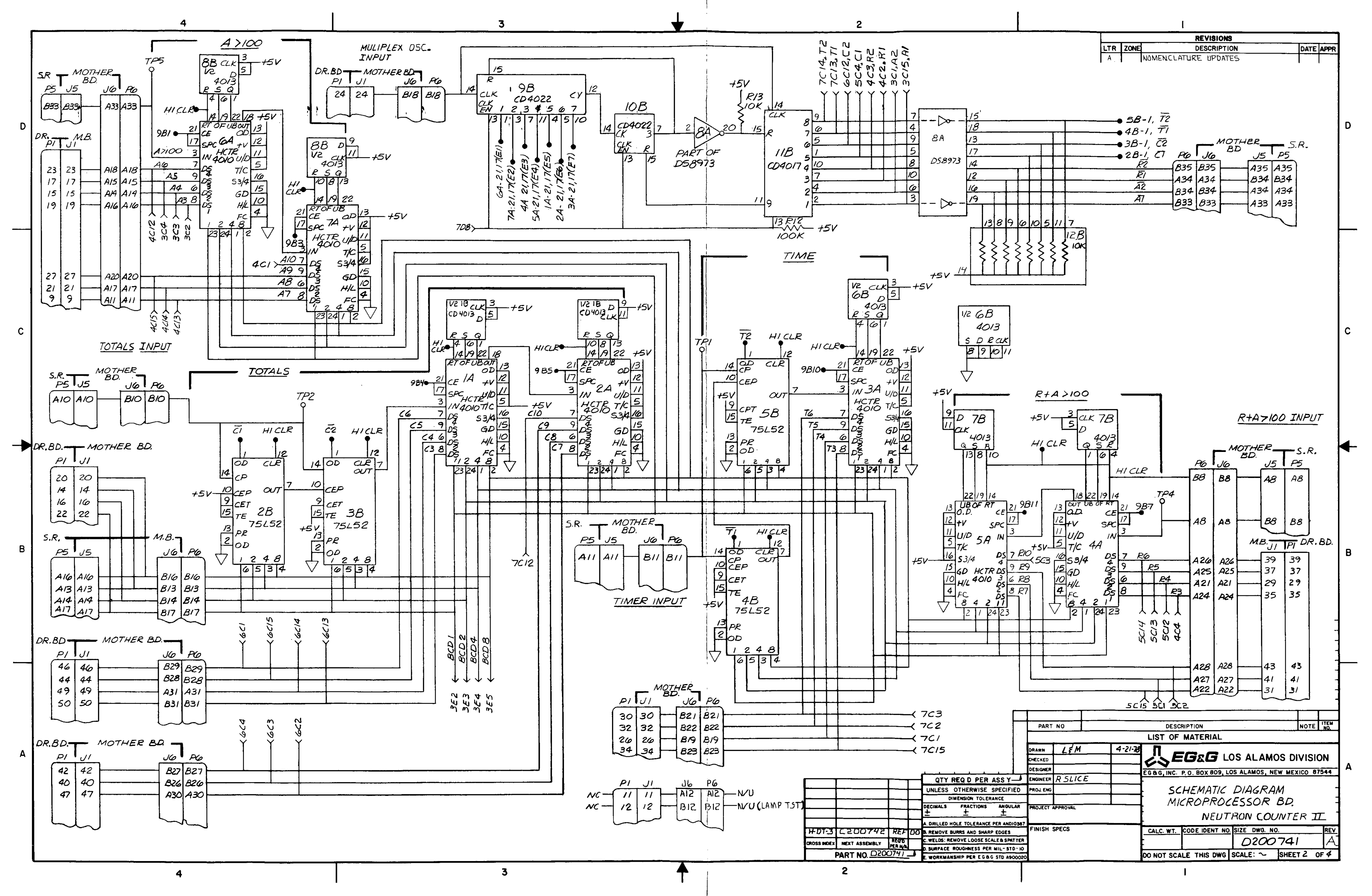




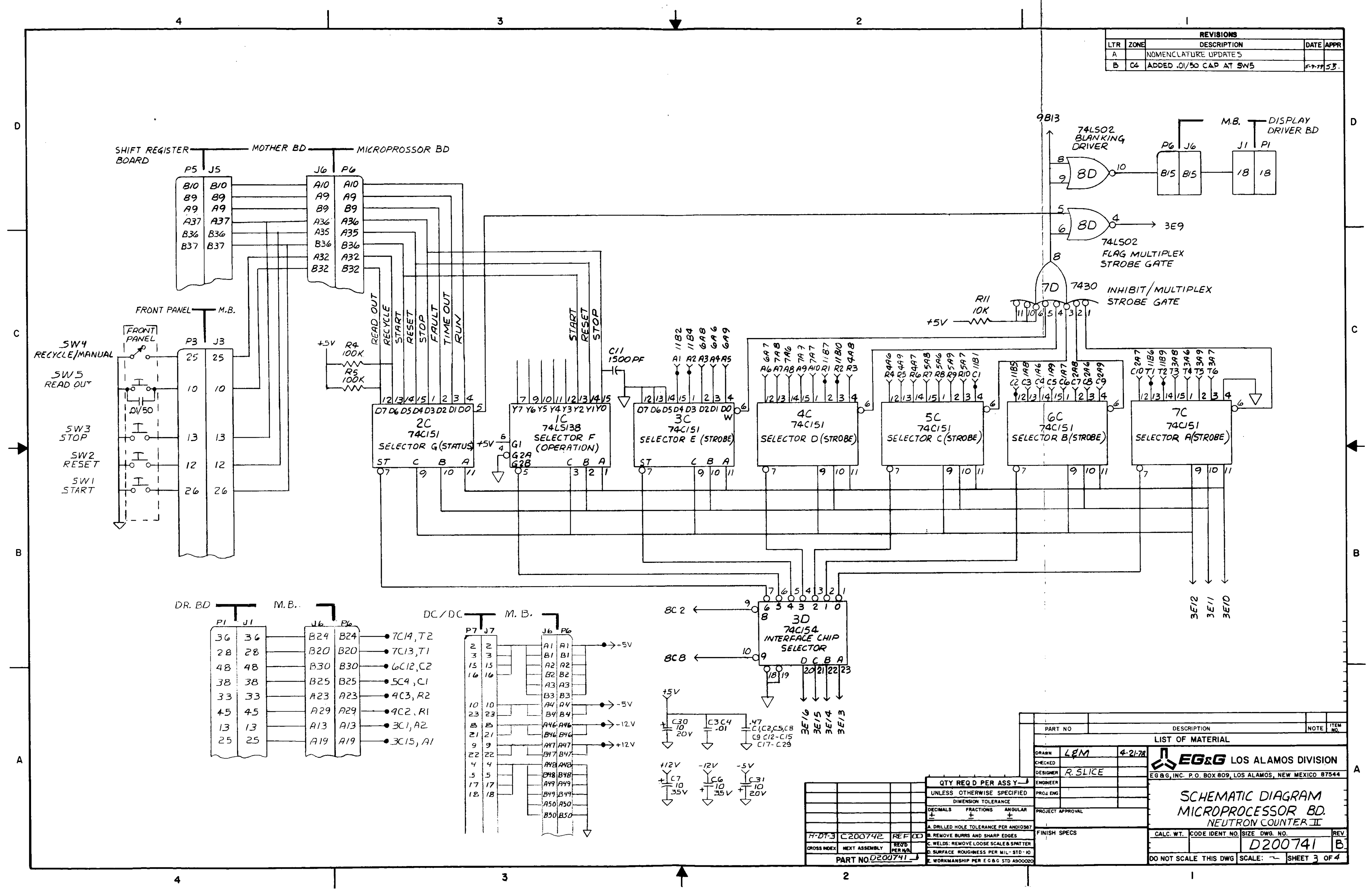




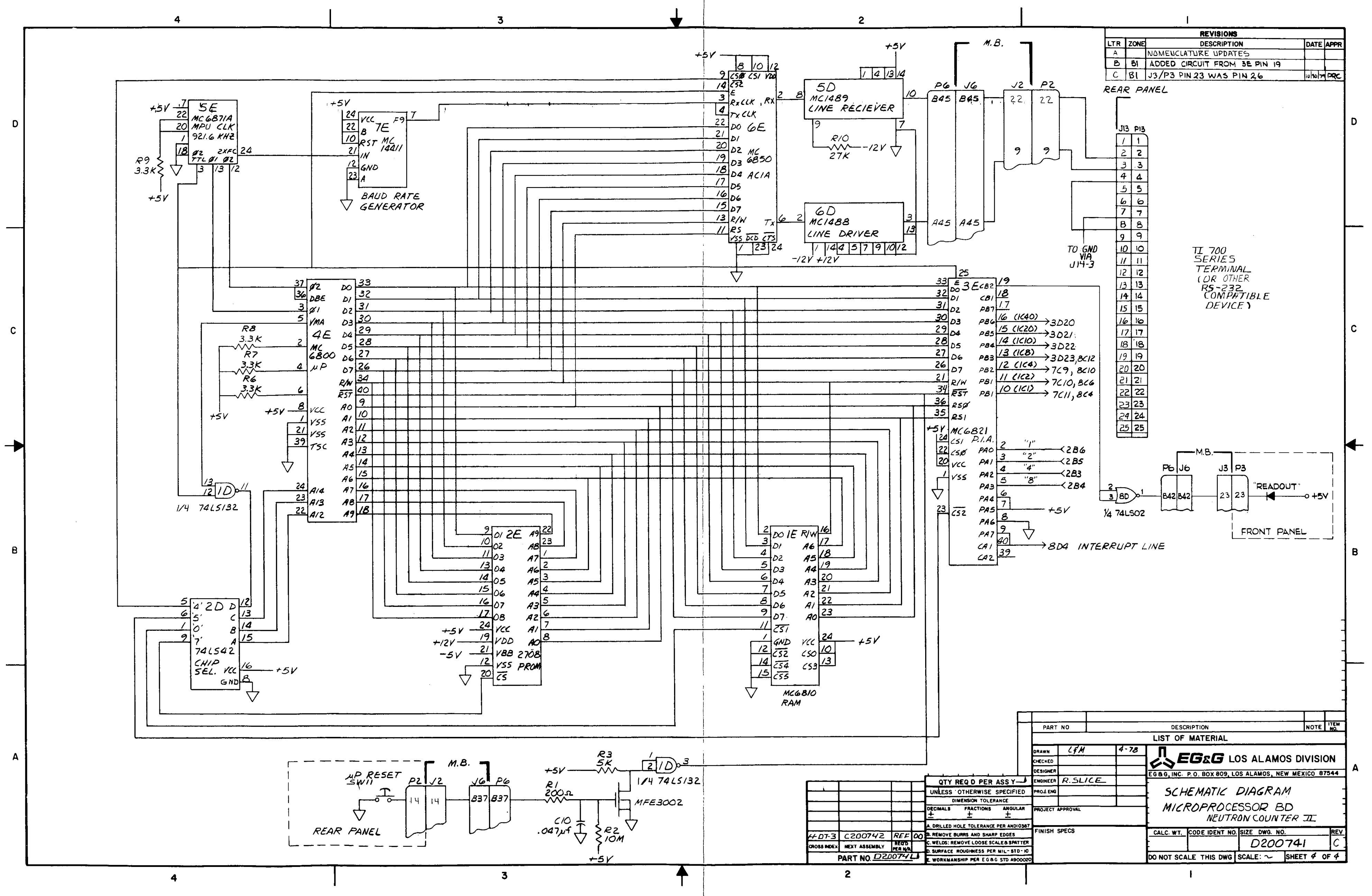




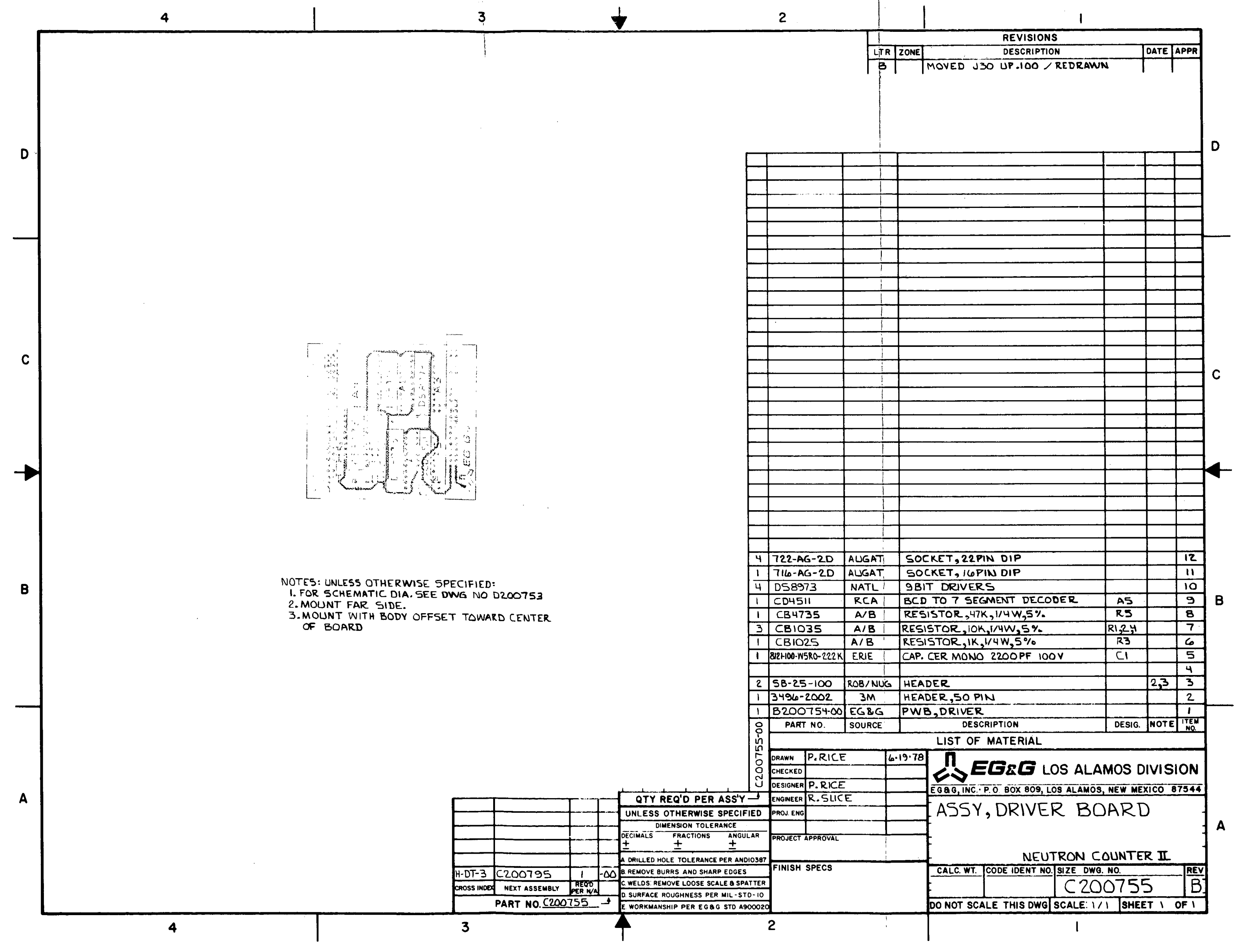




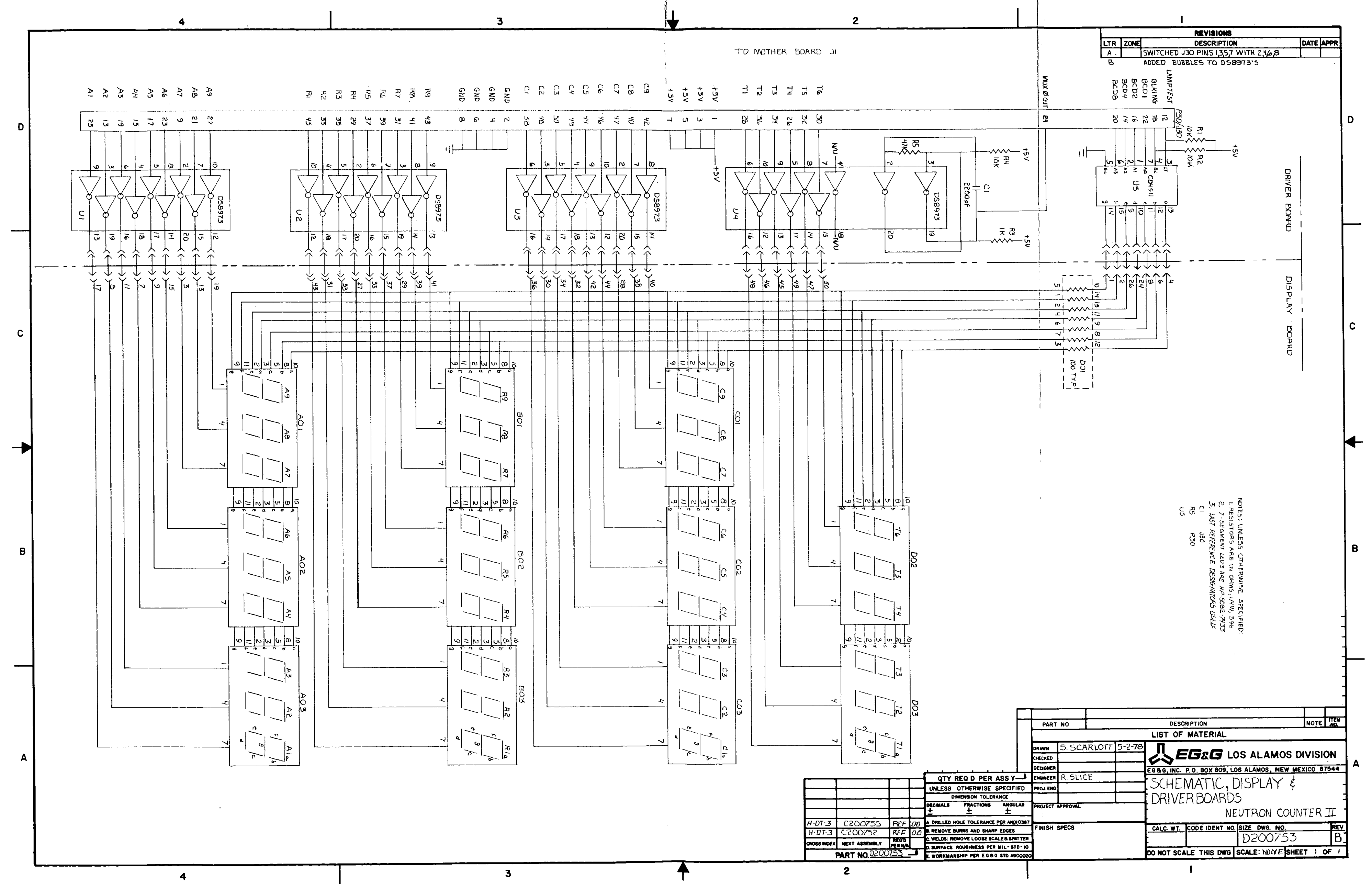




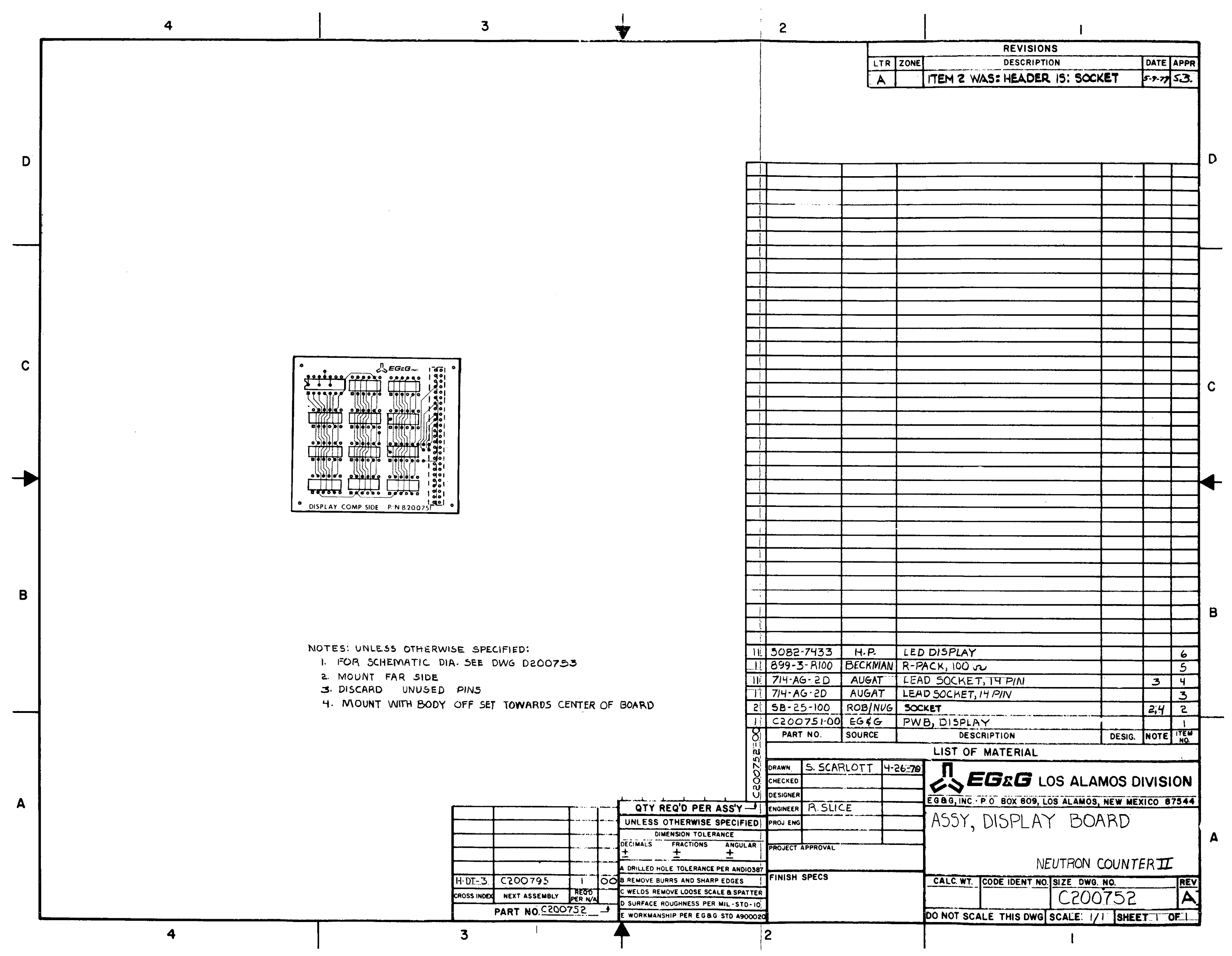



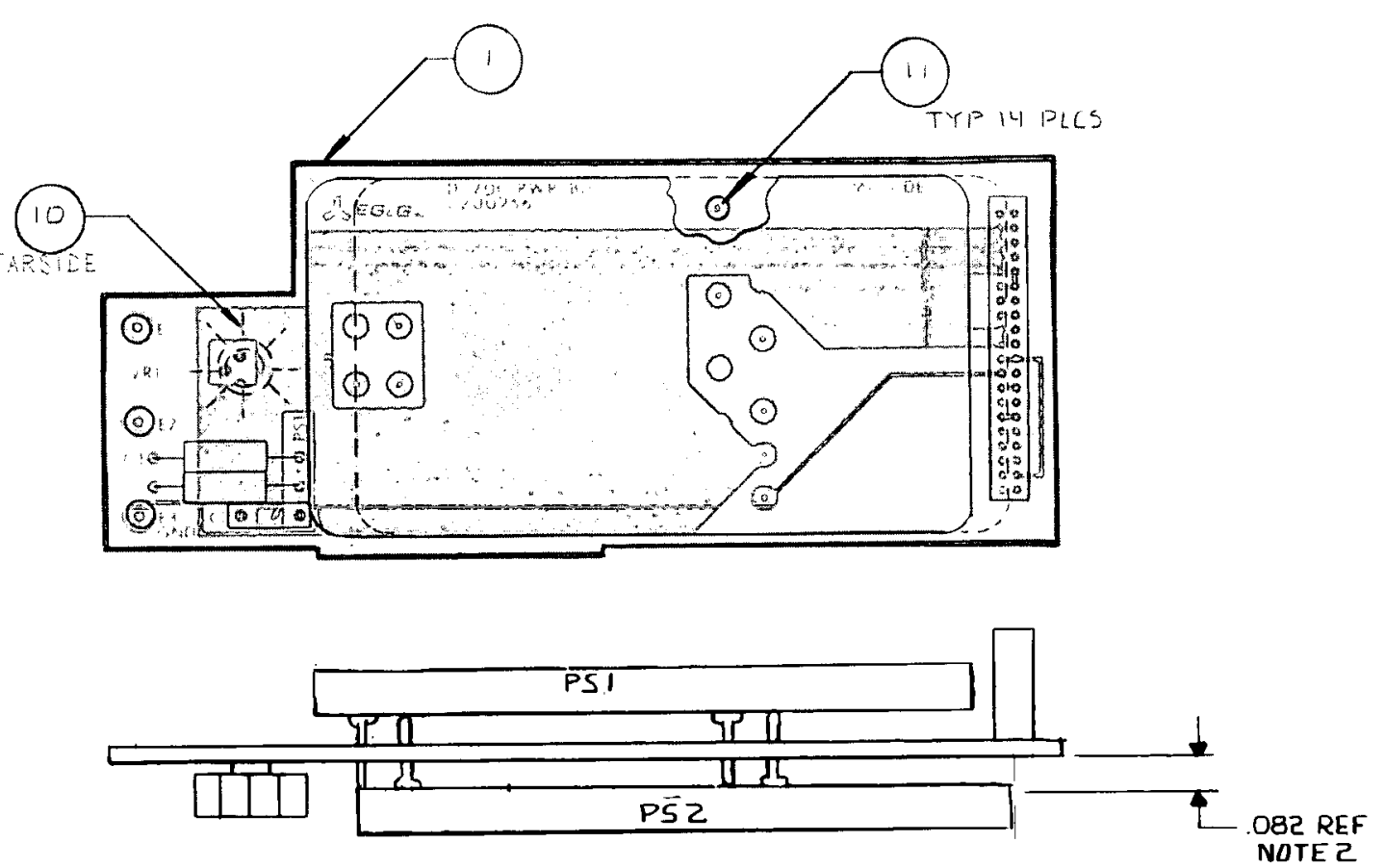

A

NOTES: UNLESS OTHEIRWISE SPECIFIED:

1. FOR SCHEMATIC DIAGRAM SEE IDWG D 200758

2. INSTAL ITEM II WITH FIXTURE B200763 TO

OBTAIN COARECT SOCHET PROTRUSION

ON BOTH SIDES OF P.W.B.

2

CONVERTER

SOCKET, PIN

HEATSINKK

REGULATOR, VOLTAGE

POWER SUPPLY, DC CONVERTER

CONNECTOR, 4OPIN HEADER

CONNECTOR, 4OPIN HEADER

CAP, CER.MONO, 2.2 $\mu, 50 \mathrm{~V}$

CAP, CER. MANT. ELECT., $82 \mu F, 50 \mathrm{~V}$

FIXTURE, PIN SPACING

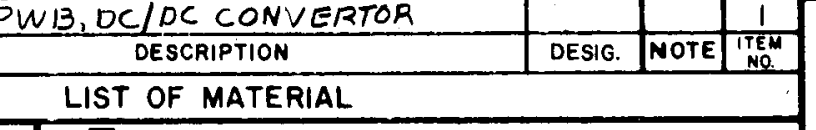

$\int_{\nabla}=\boldsymbol{G}_{\Sigma} \boldsymbol{E}$ los alamos dIVISION

EGQG, INC.P.O BOX 809, LOS ALAMOS, NEW MEXICO 87544 ASSY, DC/DC CONVERTER

NEUTRON CNTR II CALC. WT. CODE IDENT NO $\frac{\text { SIZE DWG. NO. }}{200757} \mid \frac{\mathrm{REV}}{\mathrm{A}}$ DO NOT SCALE THIS DWG SCALE: $I / 1$ SHEET I OF! 
NOTES

NLESS OTHERWISE SPECIFIEL

I FOR SCHEMATIC DIAGRAM SIE UWG \# D200747

2 MOUNT PSI, ITEM II TO DIMENSIONS SHOWN WIS HI ITEM 2

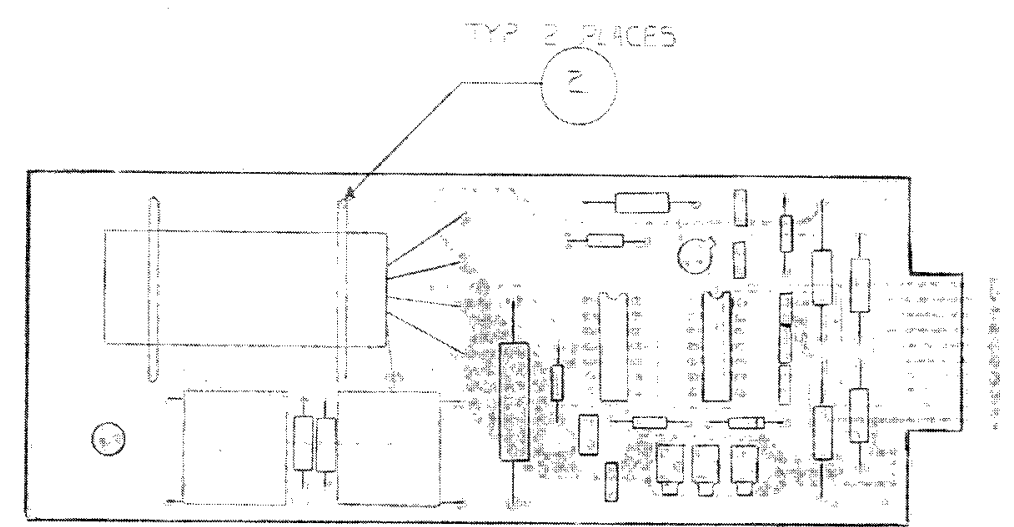

B

$.718 \pm .015$

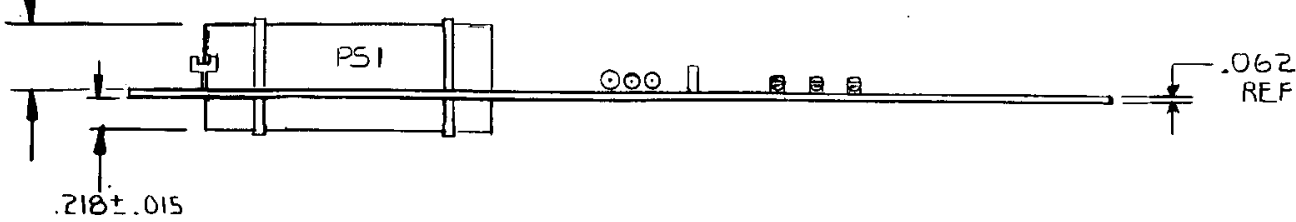

A
3

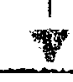

2
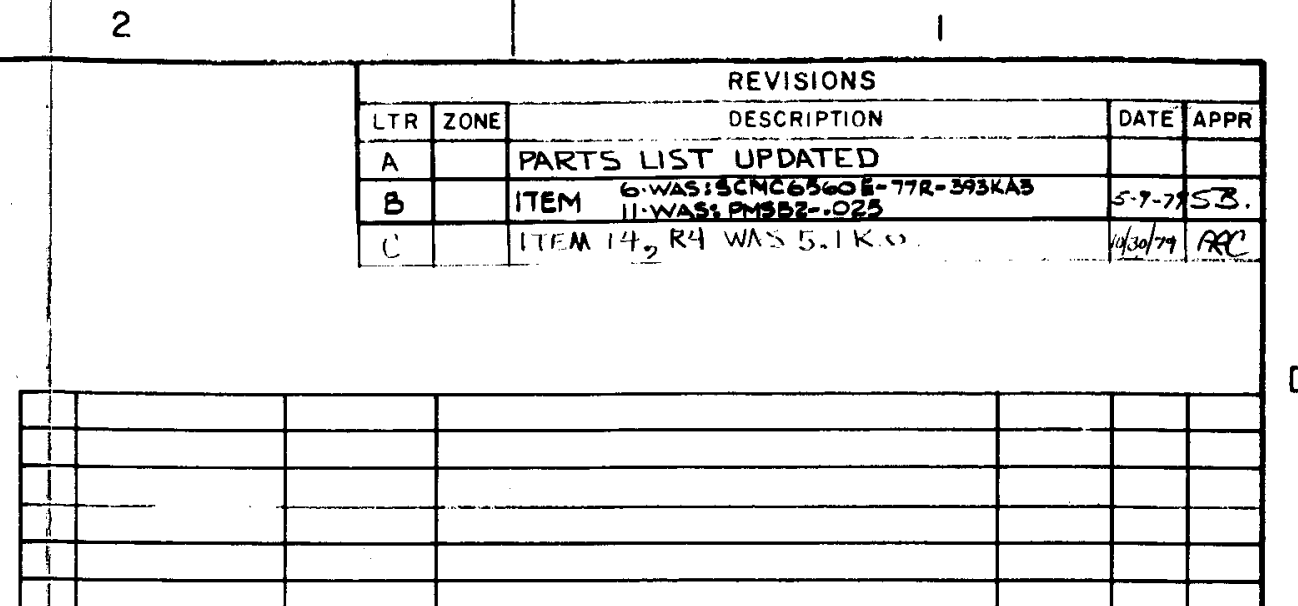

\begin{tabular}{|l|l|}
\hline 1 & -1 \\
\hline & \\
\hline 1 & \\
\hline
\end{tabular}

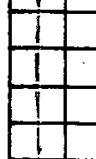

\begin{tabular}{c|c|c}
\hline 1 & \\
\hline 1 & -1 \\
\hline 1 &
\end{tabular}
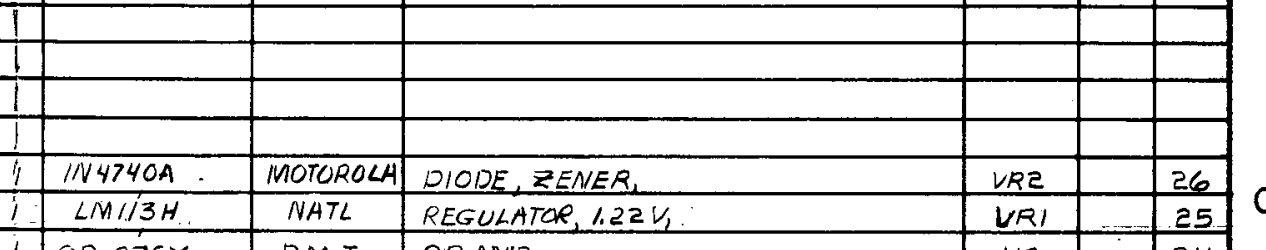

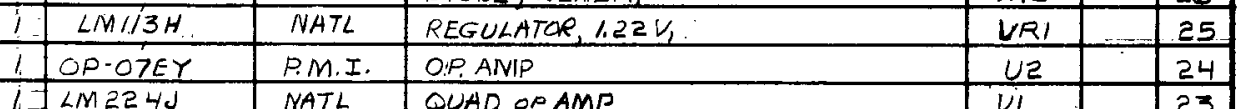

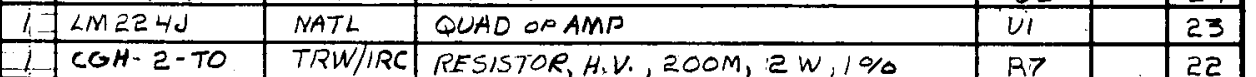

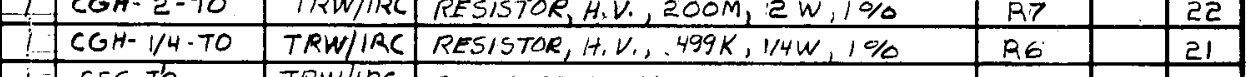

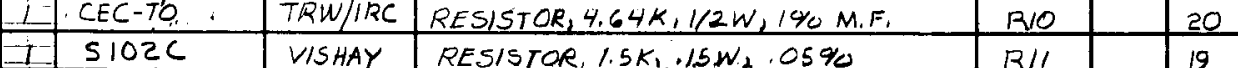

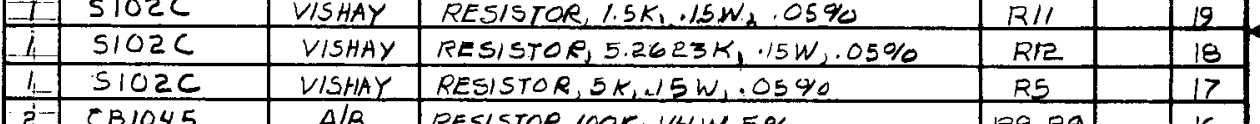

\begin{tabular}{l|l|l}
$C B 1035$ & $A / B$ & RESISTOE OK $144 W, 5 \%$ \\
\hline
\end{tabular}

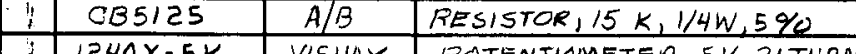

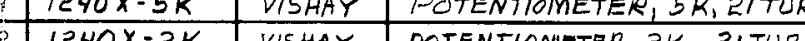

1 PMSB2-.025-1 DEL ELECT. POWER SUPPLY, HIGHVORAAGE, 2KV

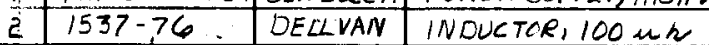

\begin{tabular}{|l|l|l|l|l}
\hline 1 & $1310 B$ & USECO & TERMINAL, BIFURCATED. \\
\hline
\end{tabular}

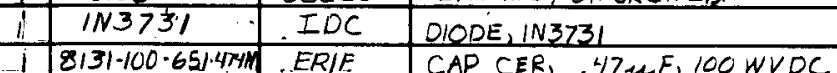

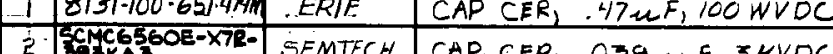

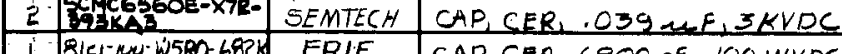

1

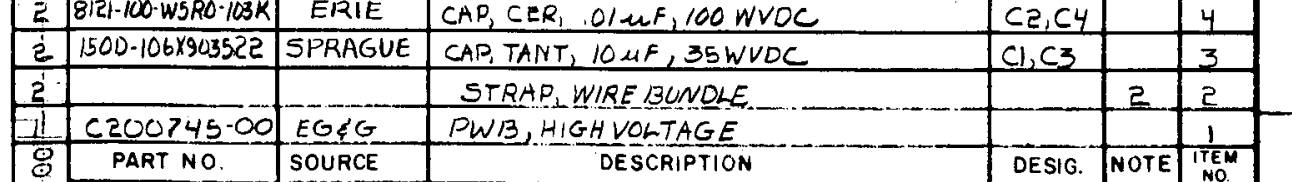

\begin{tabular}{l|l|l|l|l}
\hline$\%$ & PART NO. & SOURCE & DESCRIPTION \\
\cline { 2 - 4 } & & LIST OF MATERIAL \\
\hline
\end{tabular}

ORAWN S. SCARLOTT 5 5-8-78

CHECKEO

DESIGEER W. SHAW

\section{EG:G}

LOELOL LLAMOS DIVISION

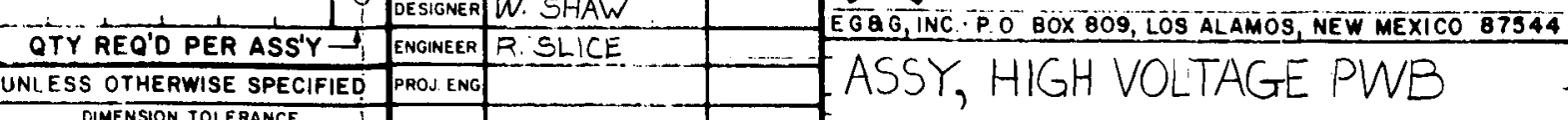
\begin{tabular}{c|c|c|c|c|}
\hline OMENSION TOLERANCE & \\
\hline
\end{tabular}

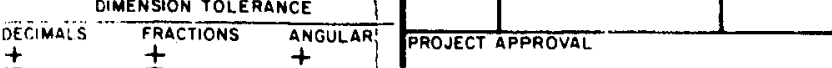
$\pm \ldots+ \pm \quad \pm$

NEUTRON COUNTER II 


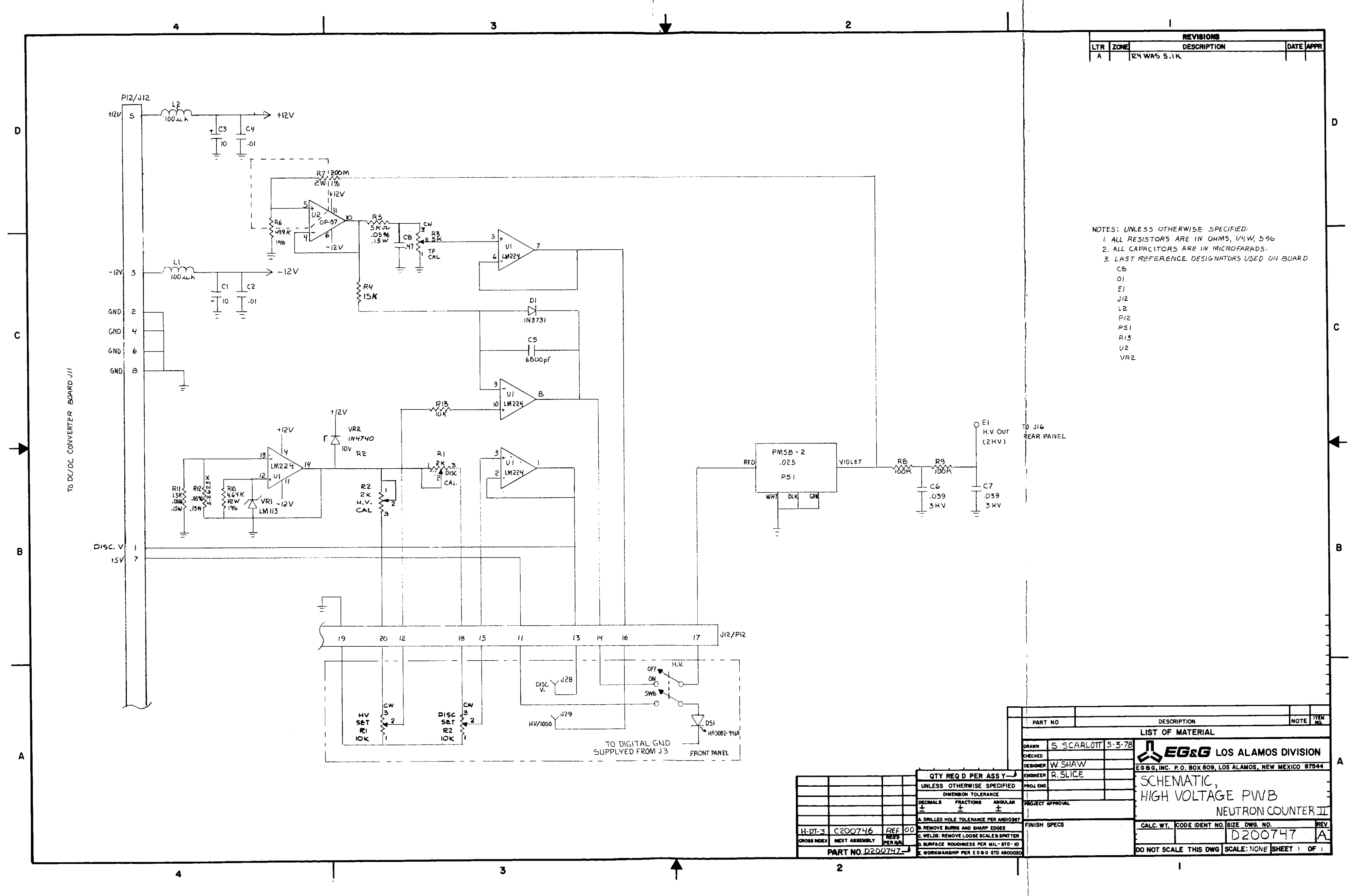


NOTES: UNLESS OTHEWISE SPECTFIED

T. FOR SCHEMATK DIA.SEE POWER. DISTRIBUTION DWG

NO. D200740:

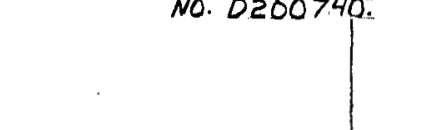

C
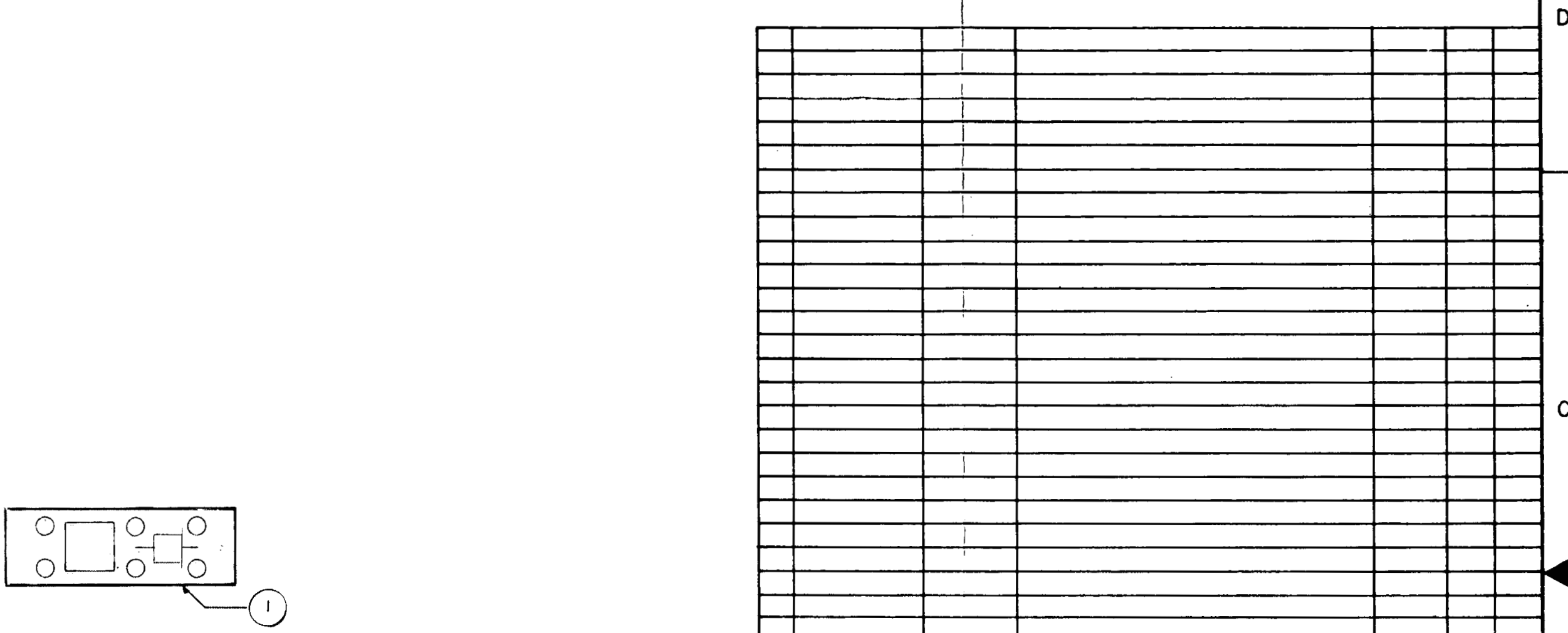

B

A $\pm$

DRILED HOLE TOLERANCE PER AMDOOS87

NEUTRON COUNTER II CALC. WT. COOE DOENT NO. SIZE DWO. NO 


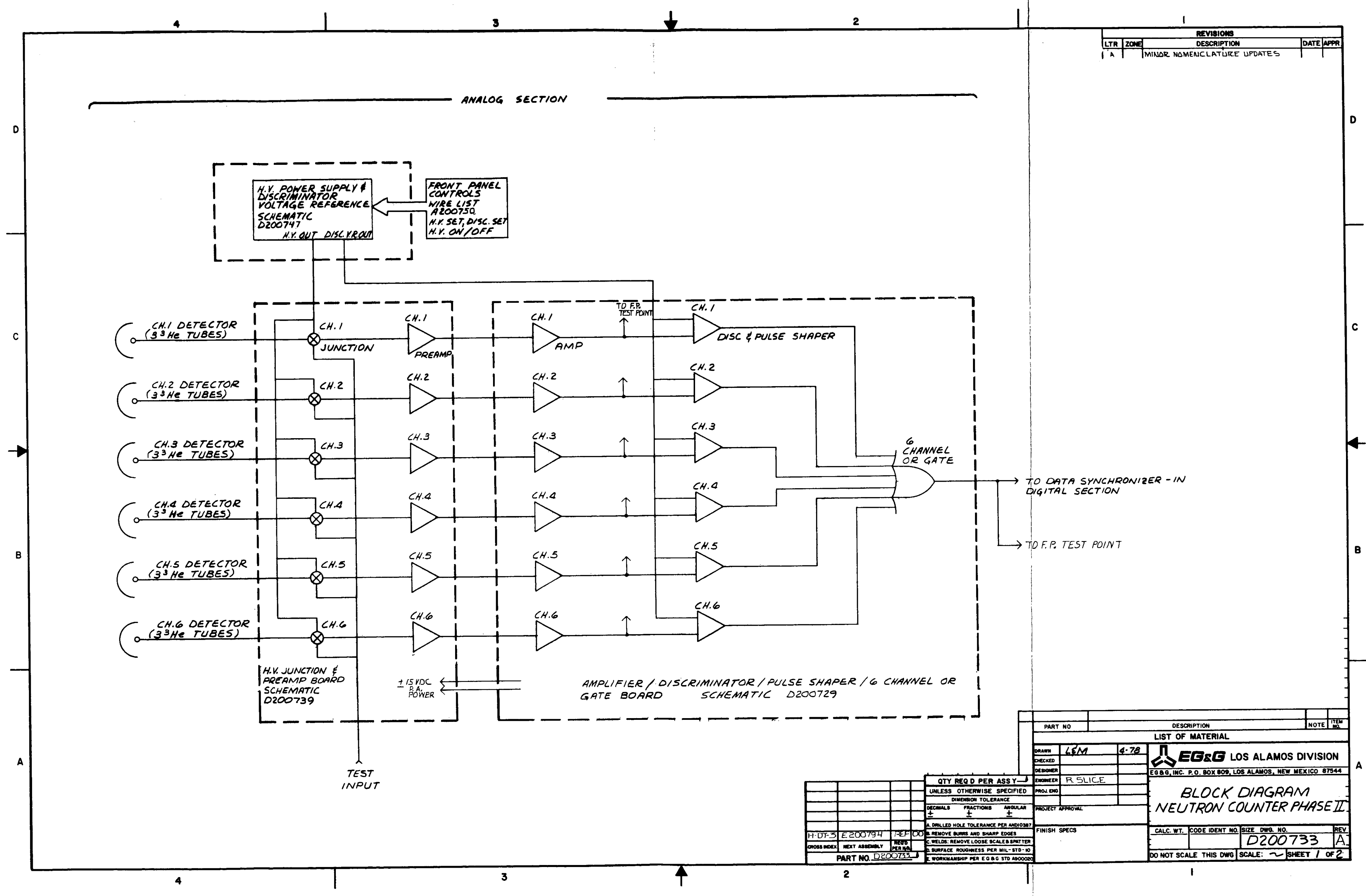




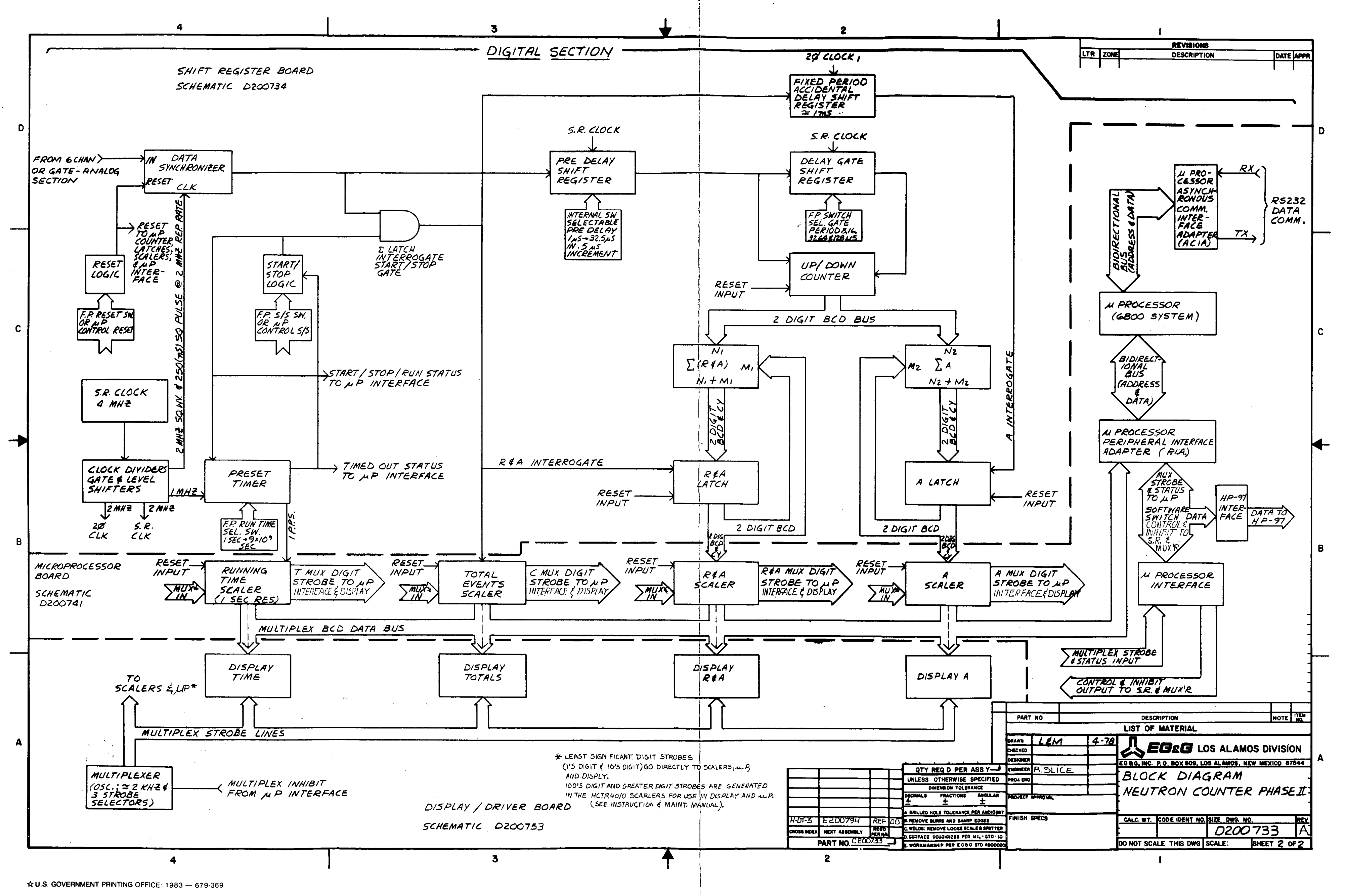

